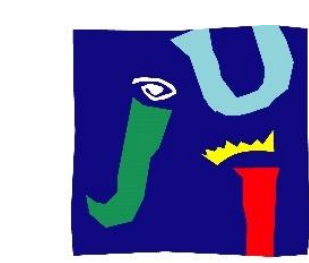

UNIVERSITAT

JAUME•I

Programa de Doctorado en Ciencias

Escola de Doctorat de la Universitat Jaume I

\title{
MOLECULAR NANOGELS AS NANOCARRIERS. INTRACELLULAR TRANSPORT OF PHOTOSENSITIZERS AND NITRIC OXIDE PROBES
}

Memoria presentada por Ana Torres Martínez para optar al grado de doctora por la Universitat Jaume I

Doctoranda

Ana Torres Martínez

$\begin{array}{ll}\text { ANA| } & \begin{array}{l}\text { Firmado } \\ \text { digitalmente por } \\ \text { ANA|TORRES| } \\ \text { MARTINEZ }\end{array} \\ \text { TORRES| } & \begin{array}{l}\text { Mecha: } 2021.01 .30 \\ \text { MA:24:41+01'00' }\end{array}\end{array}$

Juan F. Miravet Celades
$\begin{array}{ll}\text { JUAN FELIPE| } & \begin{array}{l}\text { Firmado digitalmente } \\ \text { por JUAN FELIPE }\end{array} \\ \text { MIRAVET| } & \text { MIRAVET|CELADES } \\ \text { CELADES } & \text { Fecha: } 2021.01 .31 \\ 10: 24: 08+0100^{\prime}\end{array}$

Directores

Francisco Galindo Honrubia

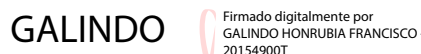

HONRUBIA

FRANCISCO

- 20154900T

Nombre de reconocimiento $(\mathrm{DN})$ :
$\mathrm{C}=\mathrm{ES}$,

serialNumber=IDCES-20154900T,

Cn=GALINDO HONRUBBA
FRANCISCO - 20154900T

Fecha: 2021.01.30 22:50:09+01'00'

Castelló de la Plana, enero 2021 



\section{Financiación recibida}

Esta tesis doctoral ha sido financiada por las siguientes fuentes:

- $\quad$ Ayuda para la formación de profesorado universitario (FPU14-05974) concedida por el Ministerio de Educación, Cultura y Deporte. Título del proyecto: Hidrogeles moleculares como materiales inteligentes con aplicaciones biomédicas.

- Ayuda complementaria de movilidad para estancias breves (EST17/00733), destinadas a beneficiarios del subprograma de formación del profesorado universitario, concedidas por el Ministerio de Educación, Cultura y Deporte.

- Proyecto concedido por el Ministerio de Economía y Competitividad: Nanogeles moleculares fotoactivos orientados a la solución de retos biomédicos (CTQ201571004-R)

- Proyecto concedido por el Ministerio de Economía y Competitividad: Nanogeles moleculares que responden a estímulos químicos como vectores de transporte y liberación de agentes de terapia fotodinámica y biosensores fluorescentes (RTI2018101675-B-100)

- Proyecto concedido por la Universitat Jaume I: Preparación y estabilidad de nanogeles moleculares. Estudio inicial de su aplicación en encapsulación y liberación controlada (P1·1B2015-50).

- Proyecto concedido por la Universitat Jaume I: Nanopartículas moleculares autoensambladas que responden a estímulos: un nuevo tipo de nanotransportadores con potenciales aplicaciones biomédicas (UJI-B2018-54). 

The main part of this work has been published up to date in the following papers:

In between molecules and self-assembled fibrillar networks: highly stable nanogel particles from a low molecular weight hydrogelator. Torres-Martínez, A.; Angulo-Pachón, C. A.; Galindo, F.; Miravet, J. F. Soft Matter 2019, 15 (17), 3565-3572.

Liposome-enveloped molecular nanogels. Torres-Martínez, A.; Angulo-Pachón, C. A.; Galindo, F.; Miravet, J. F. Langmuir 2019, 35 (41), 13375-13381.

Non-polymeric nanogels as versatile nanocarriers. Intracellular transport of the photosensitizers Rose Bengal and hypericin for photodynamic therapy. Torres-Martínez, A.; Bedrina, B.; Falomir, E; Marín, M. J.; Angulo-Pachón, C. A.; Galindo, F.; Miravet, J. F. 2020. Submitted.

Molecular nanogels for intracellular transport of nitric oxide probes. Torres-Martínez, A.; Bedrina, B.; Falomir, E; Marín, M. J.; Angulo-Pachón, C. A.; Galindo, F.; Miravet, J. F. 2021. Manuscript in preparation.

This word was also presented in conferences:

Oral communication: Self-assembled molecular nanoparticles as new delivery systems. Ana Torres-Martínez, Francisco Galindo, Juan F. Miravet. IV SEQT Young Researchers Symposium (SEQT: Sociedad Española de Química Terapéutica), Barcelona (Spain), May 2017.

Oral communication: Self-assembled molecular nanoparticles as new delivery systems. Ana Torres-Martínez, Francisco Galindo, Juan F. Miravet. VII Iberian Meeting on Colloids and Interfaces, Madrid (Spain), July 2017. Best young researcher oral presentation award.

Oral communication: Self-assembled molecular nanoparticles with potential biomedical applications. Ana Torres-Martínez, Francisco Galindo, Juan F. Miravet. XIV Simposio Investigadores Jóvenes de la Real Sociedad Española de Química, Badajoz (Spain), November 2017.

Oral communication: Self-assembled molecular nanogels with potential biomedical applications. Ana Torres-Martínez, Francisco Galindo, Juan F. Miravet. XXXVII Reunión Bienal de la Real Sociedad Española de Química, San Sebastián (Spain), May 2019. Best presentation award of "Simposio 18. De la Química a la Nanomedicina".

Poster: Self-assembled molecular nanogels as new nanocontainers. Ana Torres-Martínez, Francisco Galindo, Juan F. Miravet. ICREA Conference on Functional Nanocontainers, Tarragona (Spain), October 2016. 
Poster: Liposomes as containers for the self-assembly of molecular nanogels. Ana TorresMartínez, Francisco Galindo, Juan F. Miravet. International Conference on Self-Assembly in Confined Spaces (SACS16), San Sebastián (Spain), October 2016. 


\section{ACKNOWLEDGEMENTS}

La primera mención corresponde a mis directores de tesis, el Dr. Juan Felipe Miravet y el Dr. Francisco Galindo, por haberme dado la oportunidad de investigar y formarme en dos campos que me han apasionado tanto. Gracias por vuestra inestimable ayuda y la disponibilidad en cualquier momento que lo necesitara. Vuestro entusiasmo por la ciencia y vuestras diferentes formas de concebir la investigación han sido muy enriquecedoras. Gracias por habernos inspirado siempre a no ponernos límites. Habéis sido un ejemplo y un referente para mí, tanto en la parte científica y docente como en la personal, de los que he aprendido mucho. Muchísimas gracias.

También quiero agradecer a la Dra. María José Marín haberme acogido en su laboratorio de la University of East Anglia durante unos meses y haberme hecho sentir una más. No solo aprendí de ella muchísima ciencia, sino que me llevé de vuelta una amistad y un gran apoyo durante el final de la tesis.

Debo extender los agradecimientos a otras personas sin las que esta tesis no sería lo que es. A la Dra. Eva Falomir, por habernos brindado la oportunidad de utilizar su laboratorio para nuestros ensayos celulares y haberme hecho sentir que nunca había cambiado de planta. Siempre dispuesta a ayudar, es otro ejemplo a seguir. A Begoña Bedrina, pilar fundamental de la parte biológica desarrollada en la UJI. Al Dr. César A. Angulo, por siempre tener un vial de compuesto recién sintetizado para mí. Muchas gracias además por todo vuestro cariño.

Por último, agradecer a los compañeros de laboratorio y de departamento que he tenido durante estos años, amigos y familia. No podría haber tenido más suerte con vosotros. 



\begin{abstract}
The need for nanocarriers in medicine arises from the increasing number of therapeutic and diagnostic agents whose efficacy is affected by nonspecific cell and tissue biodistribution, systemic and organ toxicity, poor solubility and bioavailability, or rapid metabolization and excretion. Nanogels show advantages over other nanoparticles: improved flexibility and biocompatibility, simple preparation, high water content, high stability and high loading capacity for both hydrophilic and hydrophobic molecules. However, the use of polymeric nanogels presents challenges associated with stimuli-triggered release, biodegradation, polydispersity and batch-to-batch reproducibility in the preparation of polymers. Molecular gels, formed by low molecular weight molecules, may represent an exciting alternative considering their intrinsic characteristics: fully reversible stimuli-regulated assembly, easy biodegradation, excellent biocompatibility and possibility of tailored properties.

The preparation of a new type of nanoparticles, molecular nanogels, is reported in this thesis. In Chapter 3, molecular nanogels of $c a .50 \mathrm{~nm}$, obtained by self-assembly of a low molecular weight compound, were obtained in a reproducible manner by ultrasound promoted formation. These spherical nanogels, composed mainly of water, showed good temporal and kinetic stability. Moreover, the particles presented accessible hydrophobic domains in which a molecule can be entrapped and released at $\mathrm{pH}$ values above 11, partial solubilisation above $50^{\circ} \mathrm{C}$ and high cellular biocompatibility.

Molecular gels were also prepared using liposomes as a template. Novel hydrogel@liposome particles were obtained as intermediate by molecular gel formation inside liposomes. This type of nanocarriers merges the benefits of liposomal vehicles with the inherent characteristics of molecular gels. Additionally, these systems showed a release as for liposomes once loaded with doxorubicin. This foretells a potential use in the transport of bioactive substances and environmentally sensitive drug release of these hybrid systems.

Molecular nanogels were designed to present envisaged applications in biomedical issues related to drug delivery and sensing. The capability of molecular nanogels to incorporate and release actives and act as intracellular carriers was studied, aiming to improve the efficiency of the photoactive molecules in their biomedical use.
\end{abstract}


In Chapter 4, nanogels were loaded with photosensitizers and their use in photodynamic therapy for cancer was studied. The encapsulation of Rose Bengal and hypericin solved the aggregation-caused quenching of the excited states of these photosensitizers, which is detrimental for their use in photodynamic therapy. Additionally, in the case of hypericin, nanoparticles increased the solubility in water. The photosensitizers loaded in the nanogels showed enhanced cellular uptake and improved performance in photodynamic therapy.

Chapter 5 focuses on nanogels encapsulating nitric oxide probes. The signalling molecule nitric oxide is involved in a wide range of physiological functions, being its altered production implicated in a large number of pathologies. The encapsulation in the nanogels lead to an enhanced cellular uptake of DAF-2 and DAF-2 DA probes and an increased solubility in water of the probe DAQ.

Therefore, the use of molecular nanogels as vehicles to carry photoactive molecules has been demonstrated in this thesis. Moreover, the reported nanogel systems overcome some of the inherent unfavourable pharmacological properties of these molecules for a clinical application. The improvement of the intracellular performance observed for most assayed molecules could be a consequence of a different internalisation mechanism. The promising results of these first molecular nanogels show their great potential to be used as new nanocarriers for biomedical applications. 


\section{ABBREVIATIONS}

\begin{tabular}{|c|c|}
\hline ABMA & $\begin{array}{l}\text { 9,10-anthracenediyl- } \\
\text { bis(methylene) dimalonic acid }\end{array}$ \\
\hline AcOEt & Ethyl acetate \\
\hline AIE & Aggregation-induced emission \\
\hline a.u. & Arbitrary units \\
\hline cac & $\begin{array}{l}\text { Critical aggregation } \\
\text { concentration }\end{array}$ \\
\hline CLSM & $\begin{array}{l}\text { Confocal laser scanning } \\
\text { microscopy }\end{array}$ \\
\hline CMC & Critical micelle concentration \\
\hline СT & Charge-transfer \\
\hline DAF-2 & 4,5-Diaminofluorescein \\
\hline DAF2@1 & DAF-2-loaded nanogels \\
\hline DAF-2 DA & DAF-2 diacetate \\
\hline DAF2DA@1 & DAF-2 DA-loaded nanogels \\
\hline DAFs & Diaminofluoresceins \\
\hline DAF-2T & DAF-2 triazole derivative \\
\hline DAQ & 1,2-Diaminoanthraquinone \\
\hline DAQ@1 & DAQ-loaded nanogels \\
\hline DAQ-TZ & DAQ triazole derivative \\
\hline DCC & $N, N^{\prime}$-Dicyclohexylcarbodiimide \\
\hline DDS & Drug delivery system \\
\hline DHA & Dehydroascorbic acid \\
\hline DLS & Dynamic light scattering \\
\hline DMEM & $\begin{array}{l}\text { Dulbecco's Modified Eagle } \\
\text { Medium }\end{array}$ \\
\hline DMPC & Dimyristoyl-phosphatidylcholine \\
\hline DMSO & Dimethyl sulfoxide \\
\hline DNA & Deoxyribonucleic acid \\
\hline $\mathrm{d} n / \mathrm{d} C$ & Refractive index increment \\
\hline DSC & Differential scanning calorimetry \\
\hline EMA & European Medicines Agency \\
\hline EPR & $\begin{array}{l}\text { Enhanced permeability and } \\
\text { retention effect }\end{array}$ \\
\hline
\end{tabular}

\author{
EtOH Ethanol \\ FBS Fetal bovine serum \\ FDA Food and Drug Administration \\ FITC Fluorochrome fluorescein \\ isothiocyanate \\ FSC Forward scatter \\ HYP Hypericin \\ HYP@1 Hypericin-loaded nanogels \\ HOMO Highest occupied molecular \\ orbital \\ HPLC High-performance liquid \\ chromatography \\ HRMS High resolution mass \\ spectrometry \\ $I_{50}$ Half maximal inhibitory \\ concentration \\ INF- $\nu$ Interferon- $\nu$ \\ IR Infrared \\ ISC Intersystem crossing \\ $\lambda$ Wavelength \\ $\lambda_{\text {max }}$ Maximum absorption/emission \\ $\lambda_{\max }$ wavelength \\ $\lambda_{\text {ex/em }}$ Excitation/emission wavelength \\ LED Light-Emitting Diode \\ LMWGs Low molecular weight gelators \\ LPS Lipopolysaccharide \\ LUMO Lowest unoccupied molecular \\ orbital \\ mgc Minimum gelation \\ concentration \\ MGO Methylglyoxal \\ MRI Magnetic resonance imaging \\ MTT 3-(4,5-dimethylthiazol-2-yl)-2,5- \\ diphenyltetrazolium bromide \\ $M_{w} \quad$ Apparent molecular weight \\ MWCO Molecular weight cut-off \\ Nile Red@1 Nile red-loaded nanogels
}




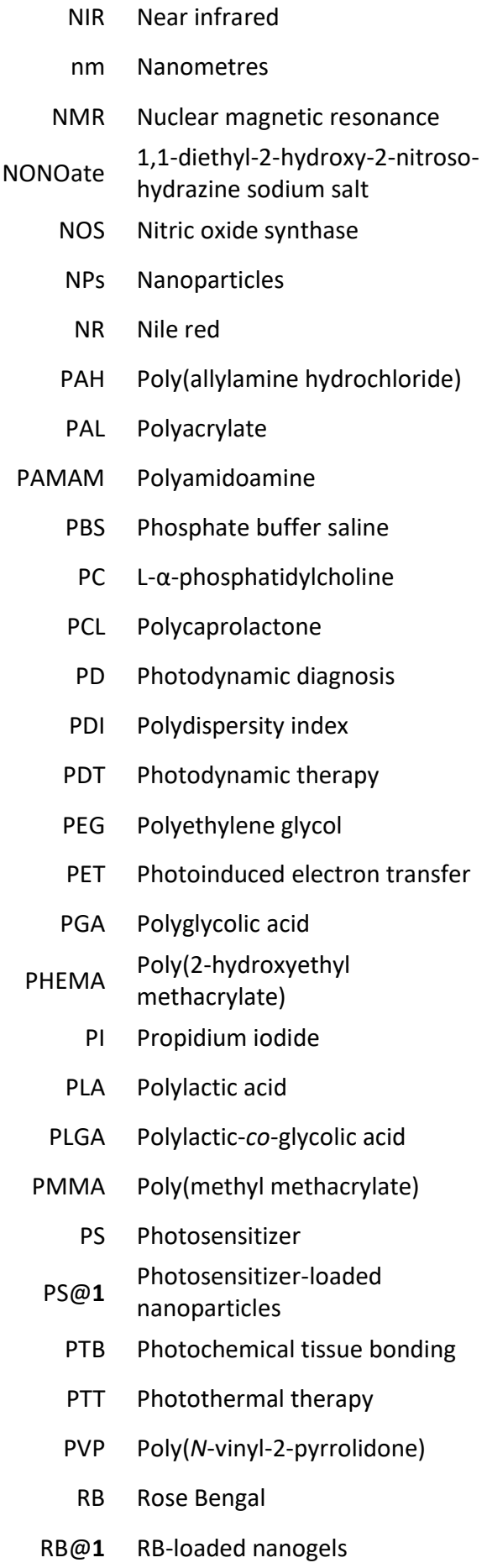

QDs Quantum dots

RES Reticuloendothelial system

RNA Ribonucleic acid

RNS Reactive nitrogen species

ROS Reactive oxygen species

rcf Relative centrifugal force

rpm Revolutions per minute

r.t. Room temperature

SALS Single angle static light scattering

SDS Sodium dodecyl sulfate

SEC Size exclusion chromatography

SLNs Solid-lipid nanoparticles

SSC Side scatter

Transmission electron microscopy

THF Tetrahydrofuran

UCNPs Upconverting nanoparticles

UV Ultraviolet

Vis Visible

w/w Weight/weight 


\section{TABLE OF CONTENTS}

$\begin{array}{ll}\text { CHAPTER 1. GENERAL INTRODUCTION } & 1\end{array}$

1.1. Nanoparticles as carriers 1

1.1.1. Nanocarriers in medicine 1

1.1.2. Types of nanoparticles $\quad 5$

$\begin{array}{lc}\text { 1.1.2.1. Organic nanoparticles } & 6\end{array}$

1.1.2.2. Inorganic nanoparticles $\quad 12$

1.1.3. Applications in nanomedicine 13

1.2. Molecular gels 16

$\begin{array}{ll}\text { 1.2.1. Gelation process } & 16\end{array}$

1.2.1. Stimuli responsiveness 17

1.2.2. Applications 19

1.3. References $\quad 20$

$\begin{array}{lr}\text { CHAPTER 2. OBJECTIVES OF THE THESIS } & 29\end{array}$

CHAPTER 3. NANOGEL PARTICLES FROM A LOW MOLECULAR WEIGHT $\begin{array}{ll}\text { HYDROGELATOR } & 33\end{array}$

3.1. Nanogels prepared by ultrasonication 33

3.1.1. Gelator synthesis and macroscopic gels $\quad 33$

3.1.2. Preparation and characterisation of nanogels 36

3.1.3. Optimisation of the preparation of nanogels 43

3.1.4. Nile red encapsulation into nanogels 44

3.1.5. Stimuli response of nanogels 48

3.1.5.1. Response to temperature changes 48

3.1.5.2. Response to $\mathrm{pH}$ changes 48

3.1.5.3. Response to ionic strength changes $\quad 51$

3.2. Nanogels prepared using liposomes as a template 52

3.2.1. Liposome formation and $\mathrm{pH}$ change studies 52

3.2.2. Preparation and characterisation of nanogel@liposomes and nanogels $\quad 54$

3.2.3. Nanogel@liposome particles as carriers 58

3.3. References $\quad 60$ 
CHAPTER 4. NANOGELS ENCAPSULATING PHOTOSENSITIZERS AND THEIR USE IN PHOTODYNAMIC THERAPY

4.1. Introduction to photodynamic therapy 65

4.2. Nanogels encapsulating Rose Bengal $\quad 74$

4.2.1. Introduction to Rose Bengal $\quad 74$

4.2.2. Results and discussion $\quad 79$

4.2.2.1. Loading studies $\quad 79$

4.2.2.2. Measurement of singlet oxygen photogeneration 84

4.3. Nanogels encapsulating hypericin $\quad 87$

4.3.1. Introduction to hypericin $\quad 87$

4.3.2. Results and discussion $\quad 94$

4.3.2.1. Loading studies $\quad 94$

4.3.2.2. Measurement of singlet oxygen photogeneration 98

4.4. Study of PS@1 as PDT agents in vitro 101

$\begin{array}{ll}\text { 4.4.1. General considerations } & 101\end{array}$

4.4.2. Study of nanogels toxicity 101

4.4.3. Rose Bengal 102

4.4.3.1. Sampling considerations 102

$\begin{array}{ll}\text { 4.4.3.2. Cellular uptake assays } & 103\end{array}$

4.4.3.3. Rose Bengal nanogels as PDT agents 107

4.4.4. Hypericin 113

4.4.4.1. Sampling considerations $\quad 113$

$\begin{array}{ll}\text { 4.4.4.2. Cellular uptake assays } & 114\end{array}$

$\begin{array}{ll}\text { 4.4.4.3. Hypericin nanogels as PDT agents } & 118\end{array}$

4.4.4.4. Discussion about in vitro hypericin results 125

$\begin{array}{ll}\text { 4.5. References } & 127\end{array}$

CHAPTER 5. NANOGELS ENCAPSULATING NITRIC OXIDE PROBES 141

$\begin{array}{ll}\text { 5.1. Introduction to nitric oxide probes } & 141\end{array}$

5.2. Nanogels encapsulating 4,5-diaminofluorescein (DAF-2) 152

5.2.1. Introduction 152

5.2.2. Aim of the research reported in this section 154

5.2.3. Results and discussion 154 
5.2.3.1. Spectroscopic characterization of DAF-2 and DAF-2T 154

5.2.3.2. DAF-2 and DAF-2 DA quantification 157

5.2.3.3. DAF-2 encapsulation into nanogels 159

5.2.3.4. DAF-2 DA encapsulation into nanogels 161

5.2.3.5. DAF2-loaded nanoparticles response to nitric oxide 163

5.2.3.6. Intracellular DAF-2 response to nitric oxide when internalised as DAF2/DAF2DA@1 166

5.3. Nanogels encapsulating 1,2-diaminoanthraquinone (DAQ) 170

$\begin{array}{ll}\text { 5.3.1. Introduction } & 170\end{array}$

5.3.2. Aim of the research reported in this section 171

5.3.3. Results and discussion 172

5.3.3.1. Spectroscopic characterization of DAQ and DAQ-TZ 172

5.3.3.2. DAQ quantification 176

5.3.3.3. DAQ encapsulation into nanogels 177

5.3.3.4. DAQ-loaded nanoparticles response to nitric oxide 183

5.3.3.5. Intracellular DAQ response to nitric oxide when internalised as DAQ@1 186

5.4. References 192

$\begin{array}{ll}\text { CHAPTER 6. CONCLUSIONS } & 201\end{array}$

$\begin{array}{ll}\text { CHAPTER 7. EXPERIMENTAL SECTION } & 207\end{array}$

ANNEXES

$\begin{array}{ll}\text { ANNEXE I. Resumen en castellano } & 231\end{array}$

ANNEXE II. Reprint of published papers $\quad 245$ 

CHAPTER 1

GENERAL INTRODUCTION 



\section{CHAPTER 1. GENERAL INTRODUCTION}

\subsection{NANOPARTICLES AS CARRIERS}

\subsubsection{NANOCARRIERS IN MEDICINE}

Nanotechnology is the science that studies and develops systems in the nanometric range. Nanoparticles are generally defined as any particulate material for which at least one dimension lies in the range of $1-100 \mathrm{~nm} .{ }^{1}$ To add context to the size of these systems, it has been said that the size of a typical nanoparticle is to a football as the football is to the Earth (Figure 1.1).

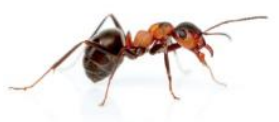

Ant 10.000.000 nm

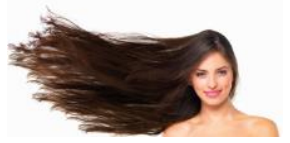

Human hair $80.000 \mathrm{~nm}$ width

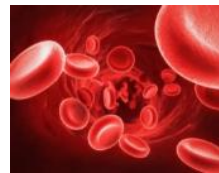

Red blood cells $7.000 \mathrm{~nm}$

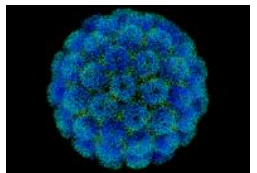

Polio virus $10 \mathrm{~nm}$

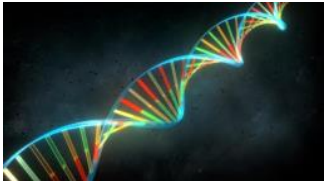

DNA molecule $2 \mathrm{~nm}$ width

Figure 1.1. Sizes of some general objects in nanometres.

Nanoparticles can exist in various shapes: spheres, ${ }^{2}$ rods, ${ }^{3}$ wires, ${ }^{4}$ planes, ${ }^{5}$ stars, $^{6}$ cages, $^{7}$ multipods, $^{8}$ etc. They have several unique properties not found in their bulk counterparts, which include high surface-to-volume ratio, high surface energy, unique mechanical, thermal, electrical, magnetic and optical behaviours, etc. These properties make them suitable for a wide range of applications, from electronics to energy harvesting and storage, communications, biology and medicine. ${ }^{9-11}$

Specifically, nanomedicine has emerged in the last years as an interdisciplinary area that seeks to address various medical challenges and shortcomings faced by conventional medicine. In addition to their unique properties, nanoparticles serve as excellent carriers of active molecular or macromolecular agents, which can be incorporated within their bulk and/or pore network within them or can be attached to the surface. The need for nanocarriers arises from the increasing number of therapeutic and diagnostic agents whose efficacy is affected by 
nonspecific cell and tissue biodistribution, systemic and organ toxicity, poor solubility and bioavailability, or rapid metabolization and excretion. ${ }^{1,12-14}$

Nanoparticles have several key advantages over conventional molecular agents in medicine: $:^{1,15,16}$

(i) They enable stable aqueous dispersions of poorly water-soluble agents, improving the solubility and stability of these actives. Their large surface area/volume ratio favours the loading of large amounts of payload. Additionally, the biodispersion, and thus the efficacy, of hydrophilic and macromolecular drugs can be improved by incorporation into nanoparticles.

(ii) Their composition, size, shape and surface properties can be exquisitely tailored so, when introduced in the biological milieu, they can protect the encapsulated agents from degradation by various endogenous defence mechanisms. These mechanisms include enzymatic degradation, immunodegradation, sequestration by the reticuloendothelial system (RES) in the bloodstream, acid hydrolysis in the stomach, mucociliary clearance in the lungs, etc. ${ }^{9}$ Encapsulation prolongs the systemic circulation time and enhances the biocompatibility and bioavailability of a drug, improving its pharmacokinetic profile. ${ }^{14,15}$ For example, coating the surface of nanoparticles with polyethylene glycol (PEG), "PEGylation", shields the surface from aggregation, opsonization and phagocytosis. ${ }^{17}$

(iii) Control of their size, shape and surface properties also allows nanoparticles to be targeted not only to specific organs/tissues in the body but also to achieve cellular and subcellular specificity. For instance, the local concentration of a drug can be increased at diseased sites, avoiding the toxicity seen towards healthy cells in cancer therapy. Passive targeting can be achieved in tumour tissues, where the enhanced permeability and retention (EPR) effect associated with these areas leads to an accumulation of circulating nanoparticles. ${ }^{18,19}$ This phenomenon is based on the combination of leaky vasculature, which enables enhanced extravasation of the particulate material from the surrounding vessel into the tumour, and poor lymphatic drainage at the tumour site, which leads to a prolonged retention time of nanoparticles. ${ }^{20}$ Active targeting relies on the specificity of appropriately surface-modified nanoparticles, decorated with targeting ligands to bind to receptors expressed on target cells/tissues or organelles. ${ }^{21-24}$ 
Moreover, particle size has great importance in the final localisation, and some guidelines for the proper particle size for specific applications can be found in the literature. For example, particles with a size larger than $1 \mu \mathrm{m}$ are immediately cleared from the circulation and accumulate in the liver and spleen, whereas particles with diameters in the range $200 \mathrm{~nm}-1 \mu \mathrm{m}$ are generally cleared by the spleen. Particles measuring 20-200 $\mathrm{nm}$ are ideal for drug delivery, with a high potential for prolonged circulation and efficient cellular internalization, being 20-100 $\mathrm{nm}$ the optimal range to take advantage of the so-called EPR effect. Finally, particles of 10-20 nm have the potential to cross the blood-brain barrier and particles with sizes $<10 \mathrm{~nm}$ are rapidly cleared through extravasation or renal clearance. ${ }^{25-27}$

(iv) Most nanomaterials are internalized, receptor-mediated or not, via endocytosis and then delivered to lysosomes, following two main pathways, phagocytosis and pinocytosis (Figure 1.2). ${ }^{15}$ Charge, shape, material composition and surface chemistry determine the cellular entry of nanomedicines through a definitive endocytic route. Particles as large as $20 \mu \mathrm{m}$ are ingested by the cell following phagocytosis, which is used as a defence mechanism and characteristic of professional phagocytes (macrophages, neutrophils, monocytes and dendritic cells). Extracellular fluid and small molecules are ingested by the cell via pinocytosis.

Endocytosis involves multiple stages. Simplistically, first, the cargo is engulfed in membrane invaginations that are pinched off to form membrane-bound vesicles. The endocytic vesicles fuse with the early endosomes $(\mathrm{pH}$ 6-6.5), which mature to late endosomes $(\mathrm{pH} \mathrm{5-6)}$ and then to lysosomes $(\mathrm{pH} 4-5.5)$. There, the proteins, lipids and other molecules are degraded by lysosomal hydrolases. The final products of the digestion are transported to the cytosol, where they are either excreted or reused to synthesise new molecules. ${ }^{28-30}$ 


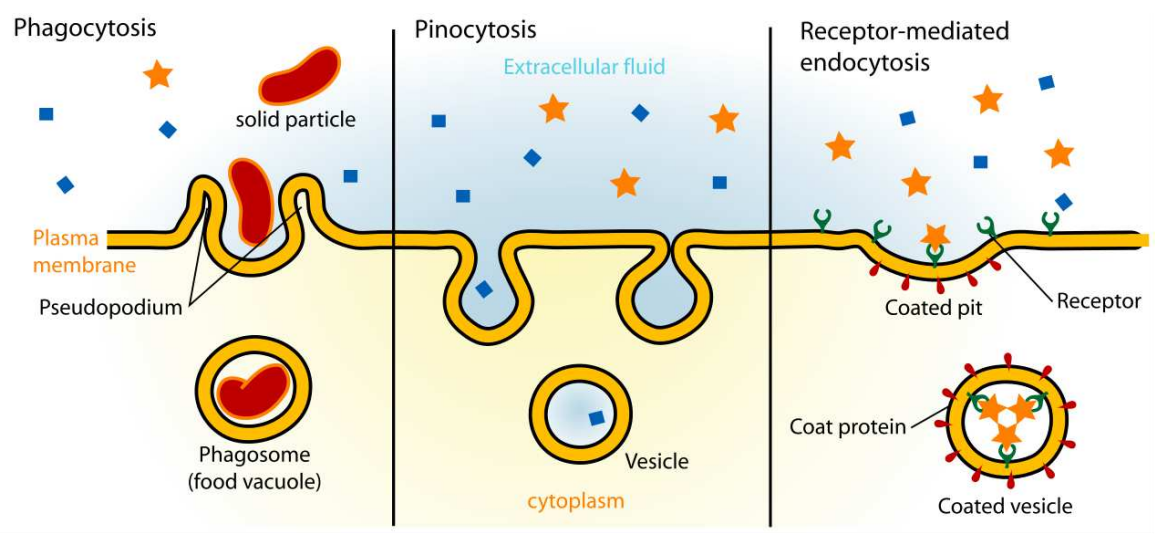

Figure 1.2. Different types of endocytosis.

(v) The nanocarrier matrix can be designed for the controlled release of drugs at target areas, $^{23}$ which includes sustained release, stimuli-sensitive release and externally activated release. In conventional administration, the drug concentration in blood plasma increases rapidly and decays exponentially during drug metabolism. However, sustained release maintains an approximately constant drug concentration for a specific period of time. A sustained release can be achieved using biodegradable carriers, whose matrix erodes progressively, or non-biodegradable carriers, which liberate drug molecules via slow diffusion through voids or channels in the carrier matrix. ${ }^{31,32}$ Stimulus-responsive drug delivery systems have been engineered for specific release to a target site. ${ }^{33}$ The stimulus can be endogenous or exogenous. The first exploits altered physiological processes occurring at or around target sites: changes in $\mathrm{pH}$, enzyme concentration or redox gradients. For example, at the tissue level, tumours have an extracellular $\mathrm{pH}$ of 6.57.2, which is slightly lower than the normal $\mathrm{pH}$ of $7.4 .{ }^{34}$ At the cellular level, the $\mathrm{pH}$ gradient of endosomes and lysosomes, which facilitates the hydrolysis of biomolecules and cellular debris, ${ }^{35}$ can be used for effective intracellular drug accumulation. Bacterial infections are also generally characterized by very low $\mathrm{pH}$ values because of anaerobic fermentation and subsequent inflammation. Other examples are the reduction-based cytosolic release that can be achieved as a higher glutathione concentration is found in the cytosol and nucleus $(2-10 \mathrm{mM})$ than in the extracellular fluids $(2-20 \mu \mathrm{M}),{ }^{26}$ or an increased level of certain enzymes such as matrix metalloproteinases.

On the other hand, an external stimulus (as variations in temperature, magnetic field, ultrasound intensity, light or electric pulses) could also trigger the release of the cargo 
once the carrier has arrived at the target site. For instance, nanoparticles containing thermosensitive polymers can be stable at body temperature $\left(37^{\circ} \mathrm{C}\right)$ and deliver the drug within a locally heated tumour $\left(40-42^{\circ} \mathrm{C}\right)$. This type of polymers has been used as a coating for magnetic nanoparticles, and the release is produced when an alternating magnetic field is applied and the temperature is increased in the nanoparticle. ${ }^{14,36}$ Photolabile bonds as the azobenzene group (from trans to cis on irradiation at 300-380 $\mathrm{nm}$, and from cis to trans by visible light) enable photoregulated control of drug release in a nanoparticle. ${ }^{1}$

(vi) Nanoparticles enable multimodality, which involves performing several diagnostic and/or therapeutic functions in tandem, being termed theranostic the combination of both. ${ }^{37,38}$ This can be achieved as different types of agents can be loaded into nanoparticles. In addition, some nanoparticles show unique properties for diagnosis and therapy, which would be summed to the ones of the carried species. ${ }^{23}$

(vii) Nanocarriers should exhibit no toxicity and be safely excreted from the body.

\subsubsection{TYPES OF NANOPARTICLES}

Owing to the impressive progress in materials science and pharmaceutics, a broad range of nanocarriers with diverse sizes, architectures and surface properties have been designed. Different classifications of nanoparticles can be found due to the overlap between different categories, but they are always divided into organic and inorganic. Some types of nanoparticles will be described in the following lines (Figure 1.3). 1,23,39 


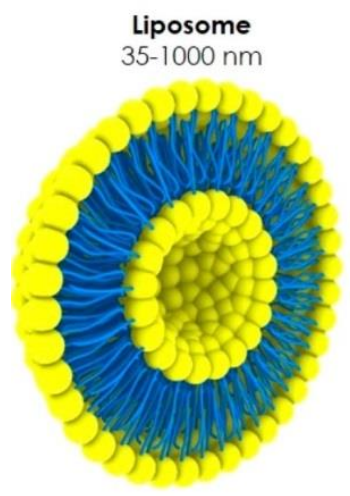

Inorganic materials

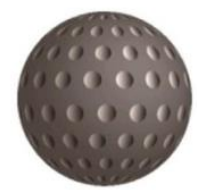

Mesoporous silica nanoparticle

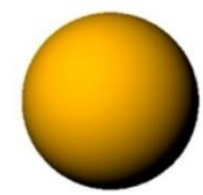

Metallic nanoparticle (e.g. Gold)

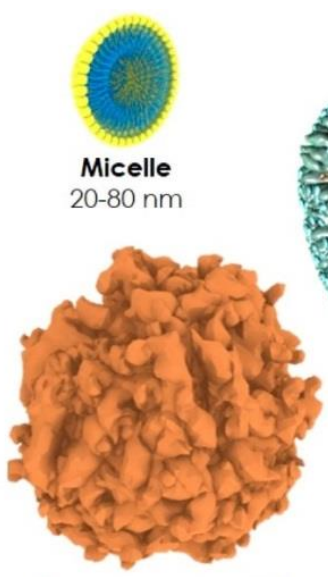

Albumin nanoparticle

$$
130 \mathrm{~nm}
$$

Organic materials

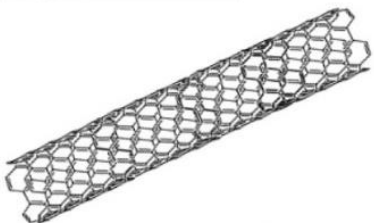

Carbon nanotube

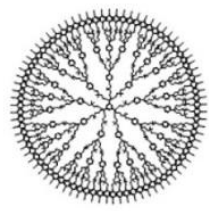

Dendrimer

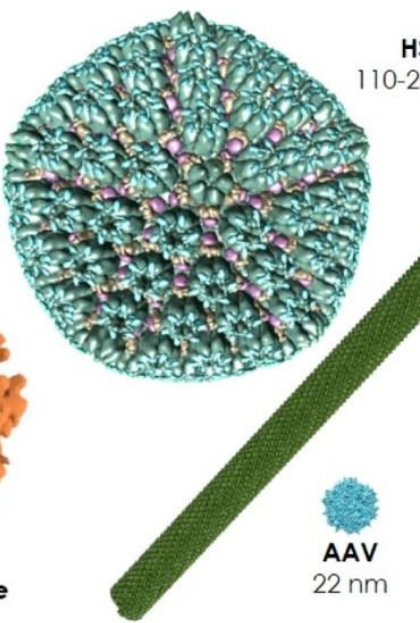

$22 \mathrm{~nm}$

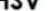

$-200 \mathrm{~nm}$

Drawn to scale

$\mathrm{m}$

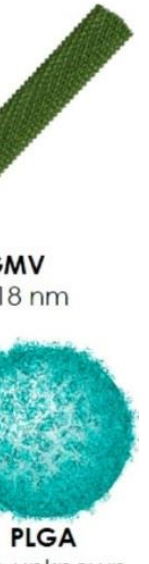

Size unknown

Not to scale

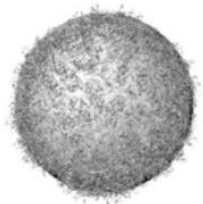

Natural polymer

(e.g. Chitosan)

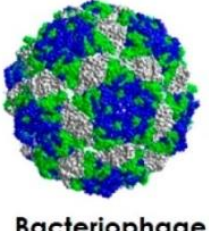

(e.g. QB)
Proteinaceous

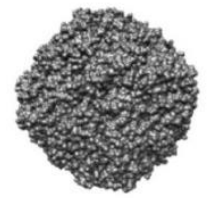

(e.g. Ferritin)

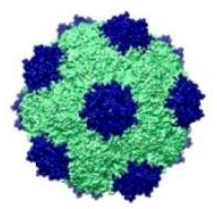

Plant virus (e.g. CPMV)

Figure 1.3. Size and structure of some nanocarriers, approved (top) and in development (bottom). HSV: herpes simplex virus, TMGMV: tobacco mild green mosaic virus, AAV: adeno-associated virus. ${ }^{39}$

\subsubsection{ORGANIC NANOPARTICLES}

Nanocarriers intended for medical use have drawn inspiration from the various natural nanoparticles discovered in the body, as nanosized vesicles, lipids, proteins and complex biomacromolecules, that regulate the natural functioning in the body and may act as carriers of active molecules. Therefore, most earlier examples of nanomedicine involved lipid- and polymer-based nanocarriers with encapsulated drugs for targeted and sustained drug delivery..$^{40,41}$ 
Polymeric nanoparticles. Polymeric nanoparticles are excellent drug carriers for many applications due to a great deal of flexibility in tailoring their chemical composition, size, biodegradability, morphology and surface functionality to control mechanical and physiological behaviour. ${ }^{32}$ Therefore, the drug release pattern can be completely specified via controlled polymer biodegradation or appropriate stimulus activation. Besides, they show high biocompatibility, high stability, efficient endocytosis and high drug loading efficiency. ${ }^{23}$ Some examples of the synthetic polymers tested include polyacrylate (PAL), PEG, polycaprolactone (PCL), polylactic acid (PLA), polyglycolic acid (PGA), polylactic-co-glycolic acid (PLGA), polyesters, polyurethanes and polyamino acids. Two polymeric nanocarriers have been approved by the U.S. Food and Drug Administration (FDA): a PLGA for cancer (Eligard) ${ }^{42}$ and an allylamine polymer for diabetes (Welchol). ${ }^{39,43}$ Natural polymers as proteins and sugars, with natural biocompatibility and biodegradability, have also been tested as inert shells: chitosan, sodium alginate, collagen, albumin, gelatin, etc. ${ }^{44}$ For example, the proteinaceous nanoshell Abraxane, consisting of albumin-bound paclitaxel for the treatment of cancer, is FDA approved. ${ }^{39}$ Also, several hydrophilic polymers, such as PEG, chitosan and dextran, are widely used as coating agents on other nanoparticles to enhance their aqueous dispersibility, bioavailability and targeting efficacy. ${ }^{25,45-47}$

Micellar Nanocarriers. Micelles are core-shell structures formed by the spontaneous selfassembly of amphiphilic molecules in an aqueous environment to leave an outer corona made of hydrophilic blocks. This phenomenon takes place for concentrations of the amphiphile above the so-called critical micelle concentration (CMC). The hydrophobic core of the micelle can sequester hydrophobic active ingredients. The size of the micelle (generally 20-80 nm for macromolecular amphiphiles) and, therefore, the amount of active ingredient that can be loaded in its core depends on the molecular size, geometry and polarity of the surfactant. ${ }^{48,49}$ Most micelles are made of block copolymers, with at least a hydrophilic and a lipophilic segment. As hydrophilic blocks, PEG, poly(N-vinyl-2- pyrrolidone) (PVP) and poly(vinyl alcohol) are the most popular, conferring the micelles good biocompatibility and reduced toxicity for cells. The hydrophobic blocks typically used are based on propylene oxide, protected L-lysine, b-benzoyl-L-aspartate, $\mathrm{\gamma}$-benzyl-L-glutamate caprolactone, protected D,L-lactic acid and spermine. ${ }^{23}$ Phospholipids can also be the lipophilic units to form phospholipid nanomicelles. ${ }^{50}$ Three micellar nanocarriers have been approved by the FDA: Estrasorb (a topical lotion for 
menopausal therapy) ${ }^{51}$ and Taxol ${ }^{52}$ and Taxotere ${ }^{53}$ (with paclitaxel and docetaxel, for cancer treatment). ${ }^{39}$

Liposomal Nanocarriers. Liposomes are spherical vesicles comprising one or more concentric lipid bilayers, with an aqueous polar core, a lipophilic bilayer compartment and a hydrophilic exterior. ${ }^{54,55}$ Hydrophobic ingredients can be inserted into the lipid bilayer or sequestered in the core, whereas water-soluble molecules can be encapsulated in the core. The lipid bilayer is usually composed of phospholipids and sterols such as cholesterol, the latter controlling membrane permeability and fluidity. The physicochemical properties of liposomes are determined by the lipid composition, sterol concentration, surface charge and nanoparticle size. ${ }^{56}$ For example, although liposomes enter the cell via endocytic processes, by adhering and fusing with the bilayer of the cell membrane, the overall surface charge tunes the cellular uptake: neutral liposomes do not readily interact with cells and release the content in the extracellular space, whereas positively charged liposomes readily interact with the negative charge on the cell surface via electrostatic forces. ${ }^{57}$ Also, the conjugation of hydrophilic polymers, such as PEG, has been used to address the rapid elimination from the bloodstream due to opsonization and clearance by the liver and the spleen, which limits their bioavailability. ${ }^{58}$ Ligand-targeted liposomes have also been engineered to promote sitespecific binding. ${ }^{59}$ Liposomal nanocarriers can be engineered to respond to temperature and/or $\mathrm{pH}^{60,61}$

Due to their biodegradable and biocompatible properties, liposomes undoubtedly represent the most successful type of drug delivery systems to enhance therapeutic potency. Liposome nanocarriers reached the market in 1995 with Doxil, a liposome containing doxorubicin. Since then, ten further products have been approved by the FDA/European Medicines Agency (EMA) for clinical use, mostly for combination cancer therapy. ${ }^{39}$ Exceptionally, AmBisome (carrying amphotericin B) is indicated for fungal infections, ${ }^{62}$ Curosurf (carrying poractant $\alpha$ ) is indicated for respiratory distress syndrome, Visudyne (carrying verteporfin) is indicated for macular degeneration $^{63}$ and Onpattro is an siRNA delivery formulation for the treatment of amyloidosis. $^{64}$

Dendrimers. Dendrimers are a particular class of highly branched polymeric nanocarriers with organized tree-like structures, a low polydispersity and size below $20 \mathrm{~nm}$. They comprise a central core that radiates a series of repeated branching units (generations), terminating with 
chemical groups available for functionalization. The active ingredient can be encapsulated in the core via hydrophobic/electrostatic interactions or conjugated to the surface. Most of the dendrimers used as nanocarriers are synthesized from hydrophilic polyamidoamine (PAMAM) or polypropylene imine units, which are not recognized by the immune system and show high biocompatibility. ${ }^{23,65-67}$

Solid-lipid nanoparticles (SLNs). SLNs are produced in the size range of 10-1000 nm using high-melting lipids, which are solid at body temperature. They are biocompatible and biodegradable, with considerably reduced side effects than other lipid/micellar nanostructures, more rigid, thus structurally more stable, and offer better protection to encapsulated drugs against chemical degradation. ${ }^{68,69}$

Virus-based Nanocarriers. Given the natural function of viruses, their capsid is used in the delivery of nucleic acids. The capsid structure is genetically programmed, so replication yields millions of identical particles, a level of monodispersity that cannot yet be achieved with synthetic nanoparticles. The FDA has approved two adeno-associated virus-based vectors for gene therapy. ${ }^{70}$

Polymer-drug conjugates. The conjugation of therapeutic agents to polymeric carriers, such as PEG, offers several advantages, including improved drug solubilization, prolonged circulation, reduced immunogenicity, controlled release and enhanced safety. The FDA and EMA have approved 17 PEGylated drugs, and the majority are indicated for cancer, hepatitis $\mathrm{C}$ or haemophilia. ${ }^{39}$ Despite its widespread use, the non-biodegradability is a considerable limitation of PEG and its subsequent utility in therapeutics, so natural biopolymers, such as polysaccharides, including dextran, polysialic acid and hyaluronic acid, as well as polypeptides, are being utilized for protein conjugation. ${ }^{71,72}$

Carbon nanostructures. Fullerenes, carbon nanotubes, carbon dots and graphene dots are characterized by possessing unique properties by themselves in addition to carrier properties.

Polymeric nanogels. Nanogels (nanohydrogels) are described as nanoparticles formed by three-dimensional polymeric networks capable of retaining large quantities of water. Different preparation procedures have been reported (Figure 1.4). ${ }^{73}$ Most of the described nanogels are constituted by covalently crosslinked networks, formed, for example, by emulsion polymerization, ${ }^{74}$ following the seminal work of Vinogradov with poly(ethylene glycol)- 
polyethyleneimine particles. ${ }^{75,76}$ Alternatively, nanogels can be prepared from polymer precursors, which are crosslinked with labile groups to improve biodegradability and stimuli responsiveness. ${ }^{77}$ Physically crosslinked nanogels have been reported based, for example, on the self-assembly of amphiphilic block copolymers, ${ }^{78}$ hydrophobized polysaccharides ${ }^{79}$ or DNA. ${ }^{80}$

A. Polymerization in Emulsion

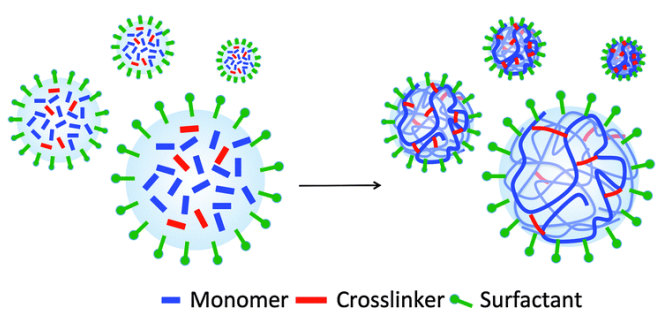

C. Self-Assembly through Physical interaction

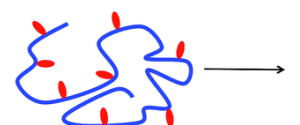

Polymer Hydrophobic moiety
B. Chemical/ Physical Crosslinking of Polymer in Emulsion

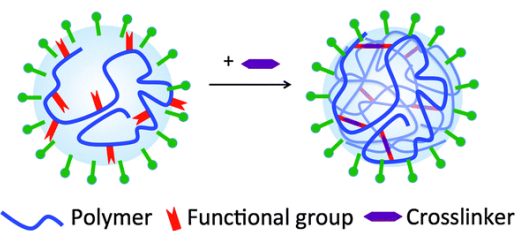

D. Self-Assembly through Electrostatic Interaction
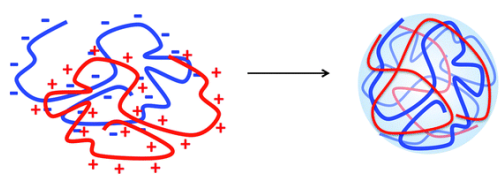

Negatively charged Polymer

$\sim$ Positively charged Polymer

Figure 1.4. Polymeric nanogel preparation strategies. ${ }^{81}$

The use of nanogels as vehicles is inspired by their macroscopic counterparts, which have been extensively studied in controlled release. ${ }^{82,83}$ Hydrogels can be used to load biotherapeutics, and stimuli such as temperature, $\mathrm{pH}$, ionic strength and enzyme activity can be used to control the rate of polymer degradation to achieve a slow and sustained release of the active ingredient. Examples include the FDA-approved intracanalicular implant Dextenza, a dexamethasone-loaded PEG hydrogel for the treatment of ocular pain following ophthalmic surgery. ${ }^{84-86}$

Nevertheless, nanogels can be injected in the circulation to reach target tissues and deliver their payloads locally and also intracellularly. ${ }^{87-90}$ Nanogels have recently received much attention as carriers due to their advantages over other nanoparticles, like improved flexibility and biocompatibility. ${ }^{22,87,91-99}$ Other advantages are their simple preparation, high water content and high stability. ${ }^{94}$ The characteristics of nanogels can be tailored by altering their size, crosslink density and surface properties. ${ }^{87,90}$ For example, the surface can be PEGylated or decorated with targeting ligands to shield the nanoparticle and elongate the circulation time 
with induced targeted delivery, which may significantly improve in vivo delivery and therapeutic efficacy. ${ }^{22,23,100}$

Nanogels have shown a high loading capacity for both hydrophilic and hydrophobic molecules due to the highly porous structure, which provides a large volume for loading drugs. The encapsulated species can be retained by different intermolecular forces. Species with low water solubility are adsorbed in hydrophobic domains of the polymeric network. Therapeutics can also be stably crosslinked. 22,77,87,90 Additionally, nanogels can be designed to release their payload as a response to environmental factors. A rapid swelling or degradation of nanogels, which in turn is associated with the intracellular release of the payload, can occur in response to external stimuli such as temperature, light and ultrasound, or to biological triggers upon cellular uptake, such as differences in $\mathrm{pH}$ or reduction potential. ${ }^{26,90,101}$ For example, reduction-responsible nanogels have been achieved including disulfide linkages, which are readily cleavable in reducing environments and converted to thiols. ${ }^{102} \mathrm{pH}$-responsive nanogels could be obtained by incorporation of acid-labile functional groups (as amine or carboxylic acid) within the polymer backbone or crosslinks. ${ }^{103,104}$

Nanogels formed by polymeric gels have been reported to deliver their payload inside cells and to increase drug delivery across biological barriers, enhancing oral and brain bioavailability. ${ }^{26,94}$ In addition to biomolecules (drugs, proteins, carbohydrates and DNA), nanogels can incorporate polymers or nanoparticles such as plasmonic, magnetic and carbonaceous nanoparticles, forming which are known as hybrid nanogels. ${ }^{103}$ These offer various potential applications in drug delivery, bioimaging and biosensing, ${ }^{93,105}$ and have been applied to anticancer therapies, ${ }^{106,107}$ diabetes, ${ }^{108}$ skin treatment, ${ }^{109}$ cosmetics, $^{110}$ lenses $^{111}$ or vaccines. ${ }^{112}$ Theranostic nanogels have also been reported with nanogels showing innate imaging characteristics, such as pH-responsive NIR-fluorescent nanogels. ${ }^{105,113}$

Lipogels. At the intersection of liposomes and nanogels, a growing interest has emerged in recent years regarding the development of hydrogel-filled liposomes, aiming to improve some aspects of conventional liposomal formulations. As several reviews state, the presence of a hydrogel core is intended to provide improved mechanical stability to the liposome, environmentally sensitive drug release and a greater loading capacity of the polymer network. ${ }^{114-120}$ On the other hand, the lipid bilayer around the nanogel is envisaged to enhance its bioavailability and biocompatibility. Different denominations have been used for 
hydrogel@liposome hybrid systems such as lipobeads, ${ }^{121}$ gel core liposomes, ${ }^{122}$ core-shell lipid-polymer nanoparticles, ${ }^{115}$ lipogels, ${ }^{123}$ liposome-camouflaged nanogels ${ }^{124}$ or gelliposomes. ${ }^{125}$ The first report on liposome-gel hybrids dates back to 1987 when Torchilin's group described the encapsulation of a cross-linked polyacrylamide gel in the inner compartment of vesicles. ${ }^{126}$ They prepared liposomes in the presence of a monomer and, after liposome separation by size exclusion chromatography (SEC), UV-promoted polymerization was carried out. UV polymerization is the most common approach for the preparation of lipogels that followed that initial work. The polymeric hydrogel@liposome particles prepared in this way include, for example, poly( $N$-isopropylacrylamide), ${ }^{127-130}$ cross-linked polymers derived from dextran, ${ }^{131,132}$ polyethylene glycol, ${ }^{133-135}$ polyacrylic acid, ${ }^{122,136}$ polyglycidol ${ }^{137}$ and ethyl methacrylate derivatives ${ }^{123}$ and hyaluronic acid. ${ }^{138}$ Other strategies include thermally initiated polymerization, which has been used to form a redox-responsive nanogel ${ }^{124}$ and a polymethacrylate derivative inside liposomes. ${ }^{139}$ Alternatively, hydrogels of alginate and polyacrylic acid were prepared into liposomes by the addition of $\mathrm{Ca}^{2+}$ and $\mathrm{pH}$ change, respectively. ${ }^{122,140}$ Moreover, temperature change provoked gel formation in liposomes containing poloxamer ${ }^{125}$ and, in a different approach, gel@liposomes were prepared by microfluidics. ${ }^{141}$

Significant results in the use of lipogels embrace, among others, the preparation of a synthetic mimic of the secretory granule for the delivery of doxorubicin, ${ }^{142}$ entrapment of hemoglobin, ${ }^{130}$ release of proteins, ${ }^{132}$ intramuscular delivery of a malaria antigen, ${ }^{122}$ enhancement of tumour immunotherapy ${ }^{134}$ and responsive intracellular release of doxorubicin. ${ }^{139}$ These systems have also been reported as an intermediate step in the preparation of nanogels using liposomes as a template. Nanogels are obtained after the solubilisation of the lipid bilayer by addition of a detergent. ${ }^{127,128,131-133,137,138,140,143}$

\subsubsection{INORGANIC NANOPARTICLES}

Inorganic nanocarriers include upconversion nanoparticles, silica-based nanocarriers, plasmonic nanocarriers, magnetic nanocarriers and semiconductor nanostructures (quantum dots). These types of nanoparticles as carriers offer the advantage of being extremely robust and thus very stable and highly resistant to enzymatic degradation. They can be prepared in ultrasmall sizes $(<20 \mathrm{~nm})$ that can be excreted via the renal route. ${ }^{9}$ Furthermore, their intrinsic electronic, optical and magnetic properties, which can be tailored by judicious control of their 
crystal phase, size, shape, composition and surface characteristics, provide capabilities for multifunctional imaging and therapy. ${ }^{23}$ Nine formulations have been approved by themselves (no cargo) to treat iron deficiency or as MRI contrast agents. ${ }^{39}$ However, toxicity is a significant concern with inorganic nanocarriers containing heavy metal atoms, which necessitate a biocompatible coating on the nanoparticle surface, together with the complete clearance of such injected inorganic nanocarriers from the human body in a reasonable amount of time. ${ }^{1}$

\subsubsection{APPLICATIONS IN NANOMEDICINE}

Three applications can be highlighted for nanoparticles in medicine: imaging, sensing and therapy. ${ }^{1}$

\section{BIOIMAGING}

Biomedical imaging and sensing play a critical role in diagnosing and staging human diseases as well as for research into the fundamental processes of life. ${ }^{144}$ Imaging methods include optical imaging, magnetic resonance imaging (MRI), X-ray computed tomography (CT scans) and plain radiography (radioisotope imaging). Optical, magnetic and radioactive functionalities to entail enhanced contrast between the region to be imaged and the surrounding background region can be provided by nanoparticle platforms or by a functional agent incorporated in their core/surface. Specifically, optical imaging plays a major role in clinical applications as it is non-invasive, rapid, highly sensitive and inexpensive. ${ }^{27}$ Most of the current photoluminescent nanoparticles for in vitro and in vivo optical bioimaging utilize fluorescence, as it not only enables diagnosis at the cellular or even at the single molecule level, ${ }^{10}$ but also provides imaging guidance for intraoperative surgical excision of tumors. ${ }^{145}$ Along with one kind of non-porous silica nanoparticles that has been approved by the FDA for clinical trials, organic fluorophores encapsulated in optically clear, soft nanoparticles or chemically conjugated to them have gained particular attention for fluorescence-based imaging. They overcome or ameliorate some drawbacks of fluorescent dyes, as improving cell delivery, reducing toxic effects, enhancing photostability and solubility, protecting from nonspecific interactions and photooxidation or obtaining brighter imaging agents (as every nanoparticle can carry a large number of fluorescent species). ${ }^{27,146}$ Fluorophores encapsulation often results in an increased fluorescence quantum yield. ${ }^{147}$ 


\section{BIOSENSING}

Moreover, in vitro biosensing determines specified analytes in biopsied cells, blood, urine, sweat, saliva and other body fluid samples collected from humans, which are potent indicators of any malfunctioning of the body and the spread of infectious diseases as can contain microbial organisms. For that objective, spectroscopic probes (based on absorption, fluorescence or chemiluminescence) are used, whose properties change when interacting with specified analytes. ${ }^{148,149}$ Again, fluorescence biosensors are the most used and utilize a range of fluorescence parameters to detect analytes, such as a change in the fluorescence intensity, a shift in the peak wavelength of fluorescence, a change of the fluorescence lifetime or a ratiometric variation of the intensities of two emission bands. As for imaging, fluorescence may be an intrinsic feature of the nanoparticle or come from a conjugated fluorophore.

\section{THERAPY}

Finally, one of the most versatile applications of nanoparticles in medicine is for treatment of diseases. ${ }^{1,150}$ As explained earlier, nanoparticles have several benefits that help to overcome the major drawbacks faced by conventional therapies. The first systems used in therapy involved inert nanomaterials that served as carriers of drug molecules for improved delivery and therapeutic outcome, but the advancement of nanoparticle research led to systems that can themselves act as drugs and the development of novel therapies yet unprecedented in medicine.

The various types of therapies offered by nanoparticles are: ${ }^{1}$

(i) Drug delivery: involves the incorporation of a drug within nanoparticulate carriers via encapsulation, absorption, adsorption or conjugation, for safe and stable administration in the body.

(ii) Photodynamic therapy (PDT): When a photosensitizer has targeted the diseased sites, its irradiation with light leads to the formation of reactive oxygen species (ROS), which destroy cancer cells, pathogenic microbes and unwanted tissue. ${ }^{151}$

(iii) Photothermal therapy (PTT): It involves the localization of metallic nanoparticles at cancerous areas and irradiation with near-infrared (NIR) or infrared (IR) light. The 
absorption by these particles generates local heating (up to $45^{\circ} \mathrm{C}$ ) and results in significant cell death through necrosis. ${ }^{152}$

(iv) Magnetocytolytic therapy: It involves the accumulation of magnetic nanoparticles at a diseased site and their activation by an external AC magnetic field to generate local heating for disease-specific toxicity. An external magnetic field can also guide a magnetic nanoparticle to a desired biological site in vitro and in vivo, allowing targeted delivery. ${ }^{153,154}$

(v) Antimicrobial therapy: Metallic nanoparticles (gold, silver, platinum), owing to their high surface energy, are well-known for their toxicity towards pathogenic microbes, such as bacteria, fungi, viruses, etc. ${ }^{9}$

(vi) Gene therapy: Gene therapy treats diseases at the genetic level by delivery of genetic material. Most diseases originate from the deficiency or malfunctioning of endogenous genes inside the body. Our immune system, which is our primary defence against invading foreign materials and pathogens, is also intricately controlled by specific genetic pathways. Nanoparticles would help, for example, in the prevention of enzymatic degradation of the external DNA or RNA and the efficient cellular uptake and delivery of these materials into the cytoplasm and nucleus. 


\subsection{MOLECULAR GELS}

\subsubsection{GELATION PROCESS}

The most comprehensive definition of gels was made in 1974 by Flory: "a substance is a gel if it has a continuous structure with macroscopic dimensions that is permanent on the time scale of an analytical experiment and is solid-like in its rheological behaviour". In other words, gels are a colloidal state of matter in which a small amount of a solid-like microphaseseparated component network (gelator) is able to immobilize the bulk flow of a larger amount of liquid-like phase. ${ }^{155,156}$

In opposition to polymeric gels formed by networks of macromolecules, molecular gels (also named supramolecular gels) are formed by low molecular weight molecules (100-1000 Da). These low molecular weight gelators (LMWGs) self-assemble most frequently into onedimensional, anisotropic, fibril-like aggregates which further evolve into 3D networks via noncovalent interactions, such as hydrogen bonding, metal-ligand coordination, van der Waals interactions, $\pi-\pi$ stacking interactions, solvophobic forces (hydrophobic forces for hydrogels), etc. ${ }^{157-163}$ (Figure 1.5) Other nano-structures like ribbons, sheets or spheres have also been described to create molecular gels. ${ }^{164}$

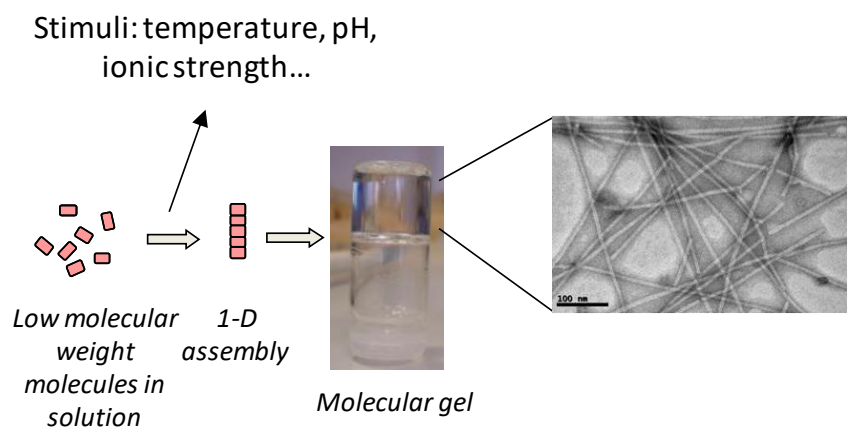

Figure 1.5. Schematic representation of the self-assembly process of LMWG.

The solution-to-gel transition occurs when the concentration of gelator remains above a critical value, the minimum gel concentration (mgc). Typically, a minimal amount of gelator, less than $1 \% \mathrm{w} / \mathrm{w}$, is required to immobilize the solvent and, most remarkably, molecules as small as 200 Dalton can form strong gels. Therefore, in contrast to polymeric gels, cheap and synthetically simple molecules with self-assembly information programmed at the molecular level can be converted into a valuable material following a bottom-up strategy. ${ }^{165}$ As the 
precise self-assembly process leads to aggregates in which molecules are highly organized, gelation has been referred quite often as an uncompleted crystallization. ${ }^{166}$

Up to date, more than 2000 gelators with varied different structural motifs have been reported, including dendritic systems, nucleobases, metallogels, sugars, amides/peptides and ureas (Figure 1.6). Standard structural units include, on the one hand, hydrogen-bonding groups such as amides, ureas and carbamates and, on the other hand, nonpolar moieties such as long alkyl tails to engage in solvophobic and van der Waals interactions, and extended aromatic surfaces to undergo $\pi-\pi$ stacking interactions. The relevance of the various intermolecular interactions, as expected, depends strongly on the solvent. For organic solvents of moderate to low polarity, hydrogen-bonding or ionic interactions are crucial. In contrast, in water, hydrophobic interactions direct the self-assembly (minimization of surfaces exposed to water, encompassing enthalpic interactions and entropic gain). ${ }^{167}$ However, for the successful formation of a hydrogel, an accurate balance between hydrophobic (which drive the selfassembly) and hydrophilic (which ensure compatibility with water) structural motifs is required. ${ }^{168}$
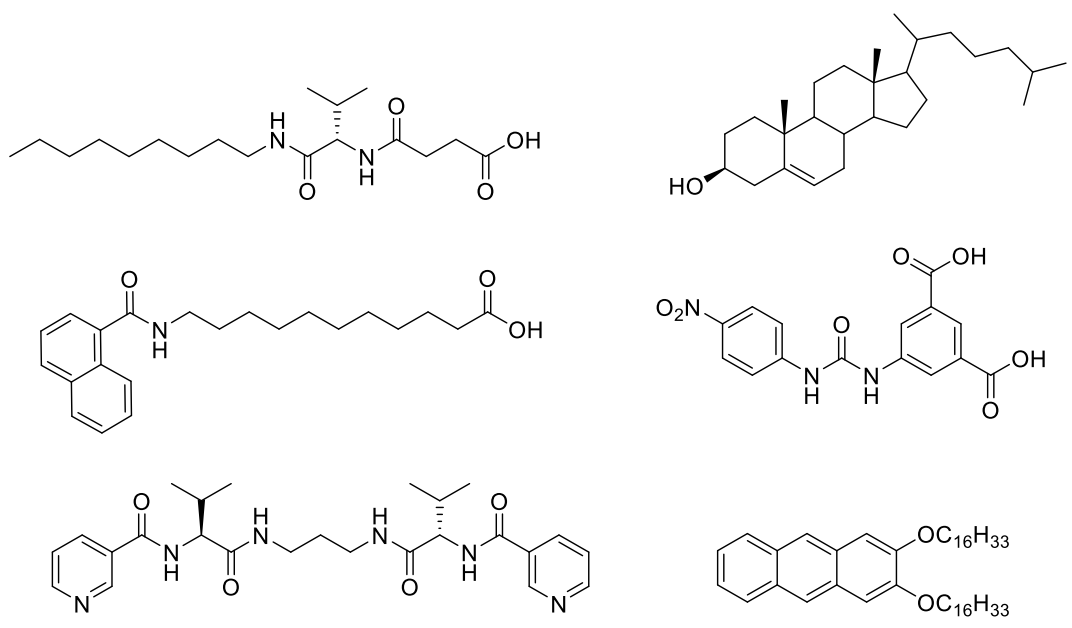

Figure 1.6. Structure of some reported low molecular weight gelators.

\subsubsection{STIMULI RESPONSIVENESS}

Furthermore, molecular gels are dynamic supramolecular systems in which free molecular entities and aggregates are in equilibrium governed by the solubility of the gel phase. ${ }^{169}$ This equilibrium is fast compared with polymer analogues and allows for a fast response to the 
environment, which, however, makes difficult the full characterization of gels. ${ }^{164}$ On the other hand, this dynamic nature of the non-covalent interactions and the fact that they are thermodynamically reversible, gives these supramolecular gels the inherent ability to respond to external stimuli, ${ }^{170-172}$ yielding the original free molecules. ${ }^{173}$

External stimuli can be classified into two main groups (Figure 1.7): physical and chemical. In the first group concentration, UV-vis light, ultrasound, mechanical forces, temperature and solvent polarity can be included as stimuli. ${ }^{174}$ Chemically sensitive gels can be prepared by introducing in the molecular design functional groups that may interact either by covalent or non-covalent forces with other molecules present in the medium. Once the gel is formed, such interaction may alter the gel properties, causing the gel disassembly by solubilisation or precipitation. Functional molecular gels have been reported to be sensitive to acids, bases, ions, redox-active compounds, neutral species, reactive compounds and enzymes. Furthermore, the combination of several stimuli may lead to multi-responsive systems in which individual or combined application of the chemical input may lead to complex responses. ${ }^{173}$

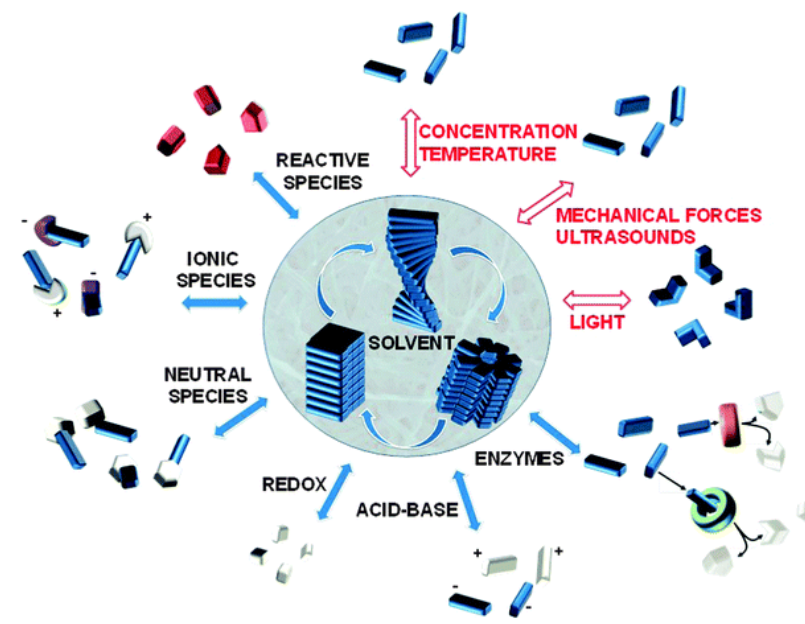

Figure 1.7. Classification of the different stimuli: physical (red) and chemical (black). ${ }^{173}$

Molecular gels are typically prepared by heating a solid gelator and a liquid component until a solution is formed and, subsequently, cooling the system below its sol-gel transition temperature, which is known as the heating-cooling protocol. ${ }^{175}$ However, the gel formation may also be the response to a stimulus by the design of pro-gelators that would be activated by a chemical input. The control of the gelation process can be found in an acid-base reaction by the design of gelators functionalised with ionisable groups. $\mathrm{pH}$ shifts are used to trigger the 
aggregation of gelators that are soluble when presenting neat charges and aggregate when neutral. ${ }^{176,177}$ The solution-to-gel transition has also been reported using sonication, ${ }^{178}$ solvent polarity shift, ${ }^{179}$ light irradiation, ${ }^{180}$ salt $^{173,181}$ and coordinating metals addition ${ }^{182}$ or enzymatic reactions, among others. ${ }^{183}$ In situ synthesis-gelation at room temperature has also been described. ${ }^{175}$

The properties of the gels are demonstrated to be highly process dependent, which means that it is possible to access materials with very different properties from a single gelator. For example, regarding triggering gelation by stimuli such as $\mathrm{pH}$, the physical properties of a gel can be quite sensitive to the rate of addition of the stimulus, with a poor reproducibility of the gel properties if the acidic stimulus is added too fast. ${ }^{184,185}$

\subsubsection{APPLICATIONS}

The study of these systems has blossomed in the last two decades, both from the fundamental and applied scientific fields. ${ }^{157-163}$ Although an extensive range of materials have been recognised since the 1860's to form molecular gels, the interest for these materials was eclipsed by their polymeric counterparts for many years. ${ }^{186}$ Overall, the singular self-assembly organization and the nature of the cohesive forces by which the molecular gels are formed confers to these materials fascinating properties, in contrast to covalent organizations: dynamic behaviour, high degrees of structural order, self-healing properties and error correction, intrinsic reversibility of the fibres to give the building blocks, stimuli responsiveness to multiple external stimuli, easy synthesis of supramolecular materials, easy functionalization for introducing desired functionalities at the molecular level to be translated (or even enhanced) to the gel, superior biodegradability and biocompatibility. ${ }^{165,168,173,187}$

Therefore, a plethora of exciting applications have raised fruit of the extensive studies on these soft materials. For example, molecular gels have been studied in a wide variety of fields, including templates for hard materials, ${ }^{188}$ photonics, magnetic (molecular-based ferromagnetism) and electronic (electronic conduction or semiconduction of donor-acceptor conjugated systems) materials, ${ }^{189}$ catalysis, $^{190}$ sensors (temperature, humidity and (bio)chemical agents), ${ }^{164,191}$ molecular and chiral recognition, ${ }^{192}$ controlled loading and release $^{193}$ and tissue engineering. ${ }^{159}$ In particular, special interest has grown regarding molecular hydrogels in a biomedical context. ${ }^{162}$ 


\subsection{REFERENCES}

Chen, G.; Roy, I.; Yang, C.; Prasad, P. N. Nanochemistry and Nanomedicine for Nanoparticle-Based Diagnostics and Therapy. Chem. Rev. 2016, 116 (5), 2826-2885.

Perrault, S. D.; Chan, W. C. W. Synthesis and Surface Modification of Highly Monodispersed, Spherical Gold Nanoparticles of 50-200 Nm. J. Am. Chem. Soc. 2009, 131 (47), 17042-17043.

Nikoobakht, B.; El-Sayed, M. A. Preparation and Growth Mechanism of Gold Nanorods (NRs) Using Seed-Mediated Growth Method. Chem. Mater. 2003, 15 (10), 1957-1962.

Cui, Y.; Wei, Q.; Park, H.; Lieber, C. M. Nanowire Nanosensors for Highly Sensitive and Selective Detection of Biological and Chemical Species. Science. 2001, 293 (5533), 1289-1292.

Coleman, J. N.; Lotya, M.; O’Neill, A.; Bergin, S. D.; King, P. J.; Khan, U.; Young, K.; Gaucher, A.; De, S.; Smith, R. J.; Shvets, I. V.; Arora, S. K.; Stanton, G.; Kim, H. Y.; Lee, K.; Kim, G. T.; Duesberg, G. S.; Hallam, T.; Boland, J. J.; Wang, J. J.; Donegan, J. F.; Grunlan, J. C.; Moriarty, G.; Shmeliov, A.; Nicholls, R. J.; Perkins, J. M.; Grieveson, E. M.; Theuwissen, K.; McComb, D. W.; Nellist, P. D.; Nicolosi, V. Two-Dimensional Nanosheets Produced by Liquid Exfoliation of Layered Materials. Science. 2011, 331 (6017), 568-571.

Hao, F.; Nehl, C. L.; Hafner, J. H.; Nordlander, P. Plasmon Resonances of a Gold Nanostar. Nano Lett. 2007, 7 (3), $729-732$.

Chen, J.; Saeki, F.; Wiley, B. J.; Cang, H.; Cobb, M. J.; Li, Z. Y.; Au, L.; Zhang, H.; Kimmey, M. B.; Li, X.; Xia, Y. Gold Nanocages: Bioconjugation and Their Potential Use as Optical Imaging Contrast Agents. Nano Lett. 2005, 5 (3), 473-477.

Teng, X.; Yang, H. Synthesis of Platinum Multipods: An Induced Anisotropic Growth. Nano Lett. 2005, 5 (5), 885-891.

Prasad, P. N. Introduction to Nanomedicine and Nanobioengineering; Hoboken, N. J., Ed.; John Wiley \& Sons, Ltd, 2012.

Prasad, P. N. Introduction to Biophotonics; Hoboken, N. J., Ed.; John Wiley \& Sons, Ltd, 2003.

Prasad, P. N. Nanophotonics; Hoboken, N. J., Ed.; John Wiley \& Sons, Ltd, 2004.

Riehemann, K.; Schneider, S. W.; Luger, T. A.; Godin, B.; Ferrari, M.; Fuchs, H. Nanomedicine - Challenge and Perspectives. Angew. Chemie - Int. Ed. 2009, 48 (5), 872-897.

Allen, T. M.; Cullis, P. R. Drug Delivery Systems: Entering the Mainstream. Science. 2004, 303 (5665), 1818-1822.

Mura, S.; Nicolas, J.; Couvreur, P. Stimuli-Responsive Nanocarriers for Drug Delivery. Nat. Mater. 2013, 12 (11), $991-1003$.

Polo, E.; Collado, M.; Pelaz, B.; Del Pino, P. Advances toward More Efficient Targeted Delivery of Nanoparticles in Vivo: Understanding Interactions between Nanoparticles and Cells. ACS Nano 2017, 11 (3), 2397-2402.

Peer, D.; Karp, J. M.; Hong, S.; Farokhzad, O. C.; Margalit, R.; Langer, R. Nanocarriers as an Emerging Platform for Cancer Therapy. Nat. Nanotechnol. 2007, 2 (12), 751-760.

Suk, J. S.; Xu, Q.; Kim, N.; Hanes, J.; Ensign, L. M. PEGylation as a Strategy for Improving Nanoparticle-Based Drug and Gene Delivery. Adv. Drug Deliv. Rev. 2016, 99, 28-51.

Matsumura, Y.; Maeda, H. A New Concept for Macromolecular Therapeutics in Cancer Chemotherapy: Mechanism of Tumoritropic Accumulation of Proteins and the Antitumor Agent Smancs. Cancer Res. 1986, 46, 6387-6392.

Duncan, R.; Sat, Y. N. Tumour Targeting by Enhanced Permeability and Retention (EPR) Effect. Ann. Oncol. 1998, No. 9, 39.

Maeda, H.; Tsukigawa, K.; Fang, J. A Retrospective 30 Years After Discovery of the Enhanced Permeability and Retention Effect of Solid Tumors: Next-Generation Chemotherapeutics and Photodynamic Therapy-Problems, Solutions, and Prospects. Microcirculation 2016, 23 (3), 173-182.

Bazak, R.; Houri, M.; El Achy, S.; Kamel, S.; Refaat, T. Cancer Active Targeting by Nanoparticles: A Comprehensive Review of Literature. J. Cancer Res. Clin. Oncol. 2015, 141 (5), 769-784.

Raemdonck, K.; Demeester, J.; De Smedt, S. Advanced Nanogel Engineering for Drug Delivery. Soft Matter 2009, 5 (4), 707715.

Jia, F.; Liu, X.; Li, L.; Mallapragada, S.; Narasimhan, B.; Wang, Q. Multifunctional Nanoparticles for Targeted Delivery of Immune Activating and Cancer Therapeutic Agents. J. Control. Release 2013, 172 (3), 1020-1034.

Wang, S.; Huang, P.; Chen, X. Stimuli-Responsive Programmed Specific Targeting in Nanomedicine. ACS Nano 2016, 10 (3), 2991-2994.

Banik, B. L.; Fattahi, P.; Brown, J. L. Polymeric Nanoparticles: The Future of Nanomedicine. Wiley Interdiscip. Rev. Nanomed. Nanobiotechnol. 2016, 8 (2), 271-299.

Li, D.; van Nostrum, C. F.; Mastrobattista, E.; Vermonden, T.; Hennink, W. E. Nanogels for Intracellular Delivery of Biotherapeutics. J. Control. Release 2017, 259, 16-28.

Peng, H.-S.; Chiu, D. T. Soft Fluorescent Nanomaterials for Biological and Biomedical Imaging. Chem. Soc. Rev. 2015, 44 (14), 4699-4722.

Sahay, G.; Alakhova, D.; Kabanov, A. V. Endocytosis of Nanomedicines. J. Control. Release 2010, 145 (3), $182-195$.

Doherty, G. J.; McMahon, H. T. Mechanisms of Endocytosis. Annu. Rev. Biochem. 2009, 78, 857-902.

Mukherjee, S.; Ghosh, R. N.; Maxfield, F. R. Endocytosis. Physiol. Rev. 1997, 77 (3), 759-803.

Okada, M. Chemical Syntheses of Biodegradable Polymers. Prog. Polym. Sci. 2002, 27, 87-133.

Uhrich, K. E.; Cannizzaro, S. M.; Langer, R. S.; Shakesheff, K. M. Polymeric Systems for Controlled Drug Release. Chem. Rev. 
1999, 99 (11), 3181-3198.

Wells, L. A.; Lasowski, F.; Fitzpatrick, S. D.; Sheardown, H. Responding to Change: Thermo- and Photo-Responsive Polymers as Unique Biomaterials. Crit. Rev. Biomed. Eng. 2010, 38 (6), 487-509. 444.

(35) Mindell, J. A. Lysosomal Acidification Mechanisms. Annu. Rev. Physiol. 2012, 74, 69-86.

(36) Huang, H.-Y.; Hu, S.-H.; Chian, C.-S.; Chen, S.-Y.; Lai, H.-Y.; Chen, Y.-Y. Self-Assembling PVA-F127 Thermosensitive Nanocarriers with Highly Sensitive Magnetically-Triggered Drug Release for Epilepsy Therapy in Vivo. J. Mater. Chem. 2012, 22 (17), 8566-8573.

(37) Ma, X.; Zhao, Y.; Liang, X. J. Theranostic Nanoparticles Engineered for Clinic and Pharmaceutics. Acc. Chem. Res. 2011, 44 (10), 1114-1122.

(38) Jokerst, J. V.; Gambhir, S. S. Molecular Imaging with Theranostic Nanoparticles. Acc. Chem. Res. 2011, 44 (10), 1050-1060.

(39) Chariou, P. L.; Ortega-Rivera, O. A.; Steinmetz, N. F. Nanocarriers for the Delivery of Medical, Veterinary, and Agricultural Active Ingredients. ACS Nano 2020, 14, 2678-2701.

(40) Müller, R. H.; Mäder, K.; Gohla, S. Solid Lipid Nanoparticles (SLN) for Controlled Drug Delivery - a Review of the State of the Art. Eur. J. Pharm. Biopharm. 2000, 50 (1), 161-177.

(41) Nicolas, J.; Mura, S.; Brambilla, D.; Mackiewicz, N.; Couvreur, P. Design, Functionalization Strategies and Biomedica Applications of Targeted Biodegradable/Biocompatible Polymer-Based Nanocarriers for Drug Delivery. Chem. Soc. Rev. 2013, 42 (3), 1147-1235.

(42) Sartor, O. Eligard: Leuprolide Acetate in a Novel Sustained-Release Delivery System. Urology 2003, 61, 25-31.

(43) Avitabile, N.; Banka, A.; Fonseca, V. A. Safety Evaluation of Colesevelam Therapy to Achieve Glycemic and Lipid Goals in Type 2 Diabetes. Expert Opin. Drug Saf. 2011, 10 (2), 305-310.

(44) Hawkins, M. J.; Soon-Shiong, P.; Desai, N. Protein Nanoparticles as Drug Carriers in Clinical Medicine. Adv. Drug Deliv. Rev. 2008, 60 (8), 876-885.

(45) Cho, W.-S.; Cho, M.; Jeong, J.; Choi, M.; Cho, H.-Y.; Han, B. S.; Kim, S. H.; Kim, H. O.; Lim, Y. T.; Chung, B. H.; Jeong, J. Acute Toxicity and Pharmacokinetics of 13 Nm-Sized PEG-Coated Gold Nanoparticles. Toxicol. Appl. Pharmacol. 2009, 236 (1), $16-$ 24

(46) Chronopoulou, L.; Massimi, M.; Giardi, M. F.; Cametti, C.; Devirgiliis, L. C.; Dentini, M.; Palocci, C. Chitosan-Coated PLGA Nanoparticles: A Sustained Drug Release Strategy for Cell Cultures. Colloids Surf. B. Biointerfaces 2013, 103, 310-317.

(47) Tassa, C.; Shaw, S. Y.; Weissleder, R. Dextran-Coated Iron Oxide Nanoparticles: A Versatile Platform for Targeted Molecular Imaging, Molecular Diagnostics, and Therapy. Acc. Chem. Res. 2011, 44 (10), 842-852.

(48) Croy, S. R.; Kwon, G. S. Polymeric Micelles for Drug Delivery. Curr. Pharm. Des. 2006, 12 (36), 4669-4684.

(49) Lu, Y.; Park, K. Polymeric Micelles and Alternative Nanonized Delivery Vehicles for Poorly Soluble Drugs. Int. J. Pharm. 2013 453, 198-214.

(50) Sawant, R. R.; Torchilin, V. P. Multifunctionality of Lipid-Core Micelles for Drug Delivery and Tumour Targeting. Mol. Membr. Biol. 2010, 27 (7), 232-246.

(51) Simon, J. A. Estradiol in Micellar Nanoparticles: The Efficacy and Safety of a Novel Transdermal Drug-Delivery Technology in the Management of Moderate to Severe Vasomotor Symptoms. Menopause 2006, 13 (2), 222-231.

(52) Scripture, C. D.; Szebeni, J.; Loos, W. J.; Figg, W. D.; Sparreboom, A. Comparative in Vitro Properties and Clinical Pharmacokinetics of Paclitaxel Following the Administration of Taxol and Paxene. Cancer Biol. Ther. 2005, 4 (5), 555-560.

(53) Hart, M.; Acott, S. Physical and Chemical Stability of Taxotere (Docetaxel) One-Vial (20 Mg/MI) Infusion Solution Following Refrigerated Storage. Ecancermedicalscience 2010, 4, 202.

(54) Torchilin, V. P. Recent Advances with Liposomes as Pharmaceutical Carriers. Nat. Rev. Drug Discov. 2005, 4 (2), 145-160.

(55) Allen, T. M.; Cullis, P. R. Liposomal Drug Delivery Systems: From Concept to Clinical Applications. Adv. Drug Deliv. Rev. 2013 65 (1), 36-48.

(56) Signorell, R. D.; Luciani, P.; Brambilla, D.; Leroux, J.-C. Pharmacokinetics of Lipid-Drug Conjugates Loaded into Liposomes. Eur. J. Pharm. Biopharm. 2018, 128, 188-199.

(57) Fröhlich, E. The Role of Surface Charge in Cellular Uptake and Cytotoxicity of Medical Nanoparticles. Int. J. Nanomedicine 2012, 7, 5577-5591.

(58) Duggan, S. T.; Keating, G. M. Pegylated Liposomal Doxorubicin: A Review of Its Use in Metastatic Breast Cancer, Ovarian Cancer, Multiple Myeloma and AIDS-Related Kaposi's Sarcoma. Drugs 2011, 71 (18), 2531-2558.

(59) Deshpande, P. P.; Biswas, S.; Torchilin, V. P. Current Trends in the Use of Liposomes for Tumor Targeting. Nanomedicine 2013, 8 (9), 1509-1528.

(60) Aryasomayajula, B.; Salzano, G.; Torchilin, V. P. Multifunctional Liposomes. In Cancer Nanotechnology. Methods in Molecular Biology; Zeineldin, R., Ed.; Springer New York: New York, NY, 2017; pp 41-61.

(61) Pattni, B. S.; Chupin, V. V.; Torchilin, V. P. New Developments in Liposomal Drug Delivery. Chem. Rev. 2015, 115 (19), 10938-10966.

(62) Stone, N. R. H.; Bicanic, T.; Salim, R.; Hope, W. Liposomal Amphotericin B (AmBisome ${ }^{\circledR}$ ): A Review of the Pharmacokinetics, Pharmacodynamics, Clinical Experience and Future Directions. Drugs 2016, 76 (4), 485-500. 
(63) Fenton, C.; Perry, C. M. Verteporfin: A Review of Its Use in the Management of Subfoveal Choroidal Neovascularisation. Drugs Aging 2006, 23 (5), 421-445.

(64) Weng, Y.; Xiao, H.; Zhang, J.; Liang, X.-J.; Huang, Y. RNAi Therapeutic and Its Innovative Biotechnological Evolution. Biotechnol. Adv. 2019, 37 (5), 801-825.

(65) Palmerston Mendes, L.; Pan, J.; Torchilin, V. P. Dendrimers as Nanocarriers for Nucleic Acid and Drug Delivery in Cancer Therapy. Molecules 2017, 22 (9), 1401.

(66) Kannan, R. M.; Nance, E.; Kannan, S.; Tomalia, D. A. Emerging Concepts in Dendrimer-Based Nanomedicine: From Design Principles to Clinical Applications. J. Intern. Med. 2014, 276 (6), 579-617.

(67) Sherje, A. P.; Jadhav, M.; Dravyakar, B. R.; Kadam, D. Dendrimers: A Versatile Nanocarrier for Drug Delivery and Targeting Int. J. Pharm. 2018, 548, 707-720.

(68) Scioli Montoto, S.; Muraca, G.; Ruiz, M. E. Solid Lipid Nanoparticles for Drug Delivery: Pharmacological and Biopharmaceutical Aspects. Front. Mol. Biosci. 2020, 7, 319

(69) Geszke-Moritz, M.; Moritz, M. Solid Lipid Nanoparticles as Attractive Drug Vehicles: Composition, Properties and Therapeutic Strategies. Mater. Sci. Eng. C 2016, 68, 982-994.

(70) Wen, A. M.; Steinmetz, N. F. Design of Virus-Based Nanomaterials for Medicine, Biotechnology, and Energy. Chem. Soc. Rev. 2016, 45 (15), 4074-4126.

(71) Ekladious, I.; Colson, Y. L.; Grinstaff, M. W. Polymer-Drug Conjugate Therapeutics: Advances, Insights and Prospects. Nat. Rev. Drug Discov. 2019, 18, 273-294.

(72) Greenwald, R. B.; Choe, Y. H.; McGuire, J.; Conover, C. D. Effective Drug Delivery by PEGylated Drug Conjugates. Adv. Drug Deliv. Rev. 2003, 55 (2), 217-250.

(73) Khoee, S.; Asadi, H. Nanogels: Chemical Approaches to Preparation. In Encyclopedia of Biomedical Polymers and Polymeric Biomaterials; Taylor \& Francis, 2016; pp 5266-5293.

(74) Landfester, K.; Musyanovych, A.; Mailänder, V. From Polymeric Particles to Multifunctional Nanocapsules for Biomedical Applications Using the Miniemulsion Process. J. Polym. Sci. Part A Polym. Chem. 2010, 48 (3), 493-515.

(75) Vinogradov, S.; Batrakova, E.; Kabanov, A. Poly(Ethylene Glycol)-Polyethyleneimine NanoGel(TM) Particles: Novel Drug Delivery Systems for Antisense Oligonucleotides. Colloids Surfaces B Biointerfaces 1999, 16, 291-304.

(76) Lupitskyy, R.; Minko, S. Robust Synthesis of Nanogel Particles by an Aggregation-Crosslinking Method. Soft Matter 2010, 6 (18), 4396-4402.

(77) Zhang, X.; Malhotra, S.; Molina, M.; Haag, R. Micro- and Nanogels with Labile Crosslinks - from Synthesis to Biomedical Applications. Chem. Soc. Rev. 2015, 44 (7), 1948-1973.

(78) Rösler, A.; Vandermeulen, G. W. M.; Klok, H. A. Advanced Drug Delivery Devices via Self-Assembly of Amphiphilic Block Copolymers. Adv. Drug Deliv. Rev. 2001, 53, 95-108.

(79) Akiyoshi, K.; Deguchi, S.; Tajima, H.; Nishikawa, T.; Sunamoto, J. Microscopic Structure and Thermoresponsiveness of a Hydrogel Nanoparticle by Self-Assembly of a Hydrophobized Polysaccharide. Macromolecules 1997, 30 (4), 857-861.

(80) Thelu, H. V. P.; Albert, S. K.; Golla, M.; Krishnan, N.; Ram, D.; Srinivasula, S. M.; Varghese, R. Size Controllable DNA Nanogels from the Self-Assembly of DNA Nanostructures through Multivalent Host-Guest Interactions. Nanoscale 2018, 10, 222-230.

(81) Chan, M.; Almutairi, A. Nanogels as Imaging Agents for Modalities Spanning the Electromagnetic Spectrum. Mater. Horizons 2016, 3, 21-40.

(82) Lin, C. C.; Metters, A. T. Hydrogels in Controlled Release Formulations: Network Design and Mathematical Modeling. Adv. Drug Deliv. Rev. 2006, 58, 1379-1408.

(83) Li, Q.; Ning, Z.; Ren, J.; Liao, W. Structural Design and Physicochemical Foundations of Hydrogels for Biomedical Applications. Curr. Med. Chem. 2018, 25 (8), 963-981.

(84) Peppas, N. A.; Hilt, J. Z.; Khademhosseini, A.; Langer, R. Hydrogels in Biology and Medicine: From Molecular Principles to Bionanotechnology. Adv. Mater. 2006, 18 (11), 1345-1360.

(85) Blizzard, C.; Desai, A.; Driscoll, A. Pharmacokinetic Studies of Sustained-Release Depot of Dexamethasone in Beagle Dogs. J. Ocul. Pharmacol. Ther. 2016, 32 (9), 595-600.

(86) Narayanaswamy, R.; Torchilin, V. P. Hydrogels and Their Applications in Targeted Drug Delivery. Molecules 2019,24 (3), 603.

(87) Soni, K. S.; Desale, S. S.; Bronich, T. K. Nanogels: An Overview of Properties, Biomedical Applications and Obstacles to Clinical Translation. J. Control. release 2016, 240, 109-126.

(88) Sasaki, Y.; Akiyoshi, K. Nanogel Engineering for New Nanobiomaterials: From Chaperoning Engineering to Biomedical Applications. Chem. Rec. 2010, 10 (6), 366-376.

(89) Thomann-Harwood, L. J.; Kaeuper, P.; Rossi, N.; Milona, P.; Herrmann, B.; McCullough, K. C. Nanogel Vaccines Targeting Dendritic Cells: Contributions of the Surface Decoration and Vaccine Cargo on Cell Targeting and Activation. J. Control. release 2013, 166 (2), 95-105.

(90) Wu, H.-Q.; Wang, C.-C. Biodegradable Smart Nanogels: A New Platform for Targeting Drug Delivery and Biomedical Diagnostics. Langmuir 2016, 32 (25), 6211-6225.

(91) Ramos, J.; Imaz, A.; Callejas-Fernández, J.; Barbosa-Barros, L.; Estelrich, J.; Quesada-Pérez, M.; Forcada, J. Soft Nanoparticles (Thermo-Responsive Nanogels and Bicelles) with Biotechnological Applications: From Synthesis to Simulation through Colloidal Characterization. Soft Matter 2011, 7 (11), 5067-5082. 
Hamidi, M.; Azadi, A.; Rafiei, P. Hydrogel Nanoparticles in Drug Delivery. Adv. Drug Deliv. Rev. 2008, 60 (15), 1638-1649. Oh, J. K.; Drumright, R.; Siegwart, D. J.; Matyjaszewski, K. The Development of Microgels/Nanogels for Drug Delivery Applications. Prog. Polym. Sci. 2008, 33 (4), 448-477.

Kabanov, A. V; Vinogradov, S. V. Nanogels as Pharmaceutical Carriers: Finite Networks of Infinite Capabilities. Angew. Chemie Int. Ed. 2009, 48 (30), 5418-5429.

Chacko, R. T.; Ventura, J.; Zhuang, J.; Thayumanavan, S. Polymer Nanogels: A Versatile Nanoscopic Drug Delivery Platform. Adv. Drug Deliv. Rev. 2012, 64 (9), 836-851.

Neamtu, I.; Rusu, A. G.; Diaconu, A.; Nita, L. E.; Chiriac, A. P. Basic Concepts and Recent Advances in Nanogels as Carriers for Medical Applications. Drug Deliv. 2017, 24 (1), 539-557.

Callejas-Fernández, J.; Estelrich, J.; Quesada-Pérez, M.; Forcada, J. Soft Nanoparticles for Biomedical Applications; Nanoscience \& Nanotechnology Series; Royal Society of Chemistry, 2014.

Vashist, A.; Kaushik, A. K.; Ahmad, S.; Nair, M. Nanogels for Biomedical Applications; Smart Materials Series; Royal Society of Chemistry, 2018.

Zha, L.; Banik, B.; Alexis, F. Stimulus Responsive Nanogels for Drug Delivery. Soft Matter 2011, 7 (13), 5908-5916.

Eckmann, D. M.; Composto, R. J.; Tsourkas, A.; Muzykantov, V. R. Nanogel Carrier Design for Targeted Drug Delivery. J. Mater. Chem. в 2014, 2 (46), 8085-8097.

Li, Y.; Maciel, D.; Rodrigues, J.; Shi, X.; Tomás, H. Biodegradable Polymer Nanogels for Drug/Nucleic Acid Delivery. Chem. Rev. 2015, 115 (16), 8564-8608.

Nuhn, L.; Kaps, L.; Diken, M.; Schuppan, D.; Zentel, R. Reductive Decationizable Block Copolymers for Stimuli-Responsive MRNA Delivery. Macromol. Rapid Commun. 2016, 37 (11), 924-933.

Molina, M.; Asadian-Birjand, M.; Balach, J.; Bergueiro, J.; Miceli, E.; Calderón, M. Stimuli-Responsive Nanogel Composites and Their Application in Nanomedicine. Chem. Soc. Rev. 2015, 44 (17), 6161-6186.

Shu, T.; Shen, Q.; Zhang, X.; Serpe, M. J. Stimuli-Responsive Polymer/Nanomaterial Hybrids for Sensing Applications. Analyst 2020, 145 (17), 5713-5724.

Vijayan, V. M.; Vasudevan, P. N.; Thomas, V. Polymeric Nanogels for Theranostic Applications: A Mini-Review. Curr. Nanosci. 2020, 16, 392-398.

Maya, S.; Sarmento, B.; Nair, A.; Rejinold, N. S.; Nair, S. V; Jayakumar, R. Smart Stimuli Sensitive Nanogels in Cancer Drug Delivery and Imaging: A Review. Curr. Pharm. Des. 2013, 19 (41), 7203-7218.

Yallapu, M. M.; Jaggi, M.; Chauhan, S. C. Design and Engineering of Nanogels for Cancer Treatment. Drug Discov. Today 2011, 16, 457-463.

Wu, W.; Zhou, S. Responsive Materials for Self-Regulated Insulin Delivery. Macromol. Biosci. 2013, 13 (11), 1464-1477.

Dasgupta, S.; Ghosh, S. K.; Ray, S.; Kaurav, S. S.; Mazumder, B. In Vitro \& in Vivo Studies on Lornoxicam Loaded Nanoemulsion Gels for Topical Application. Curr. Drug Deliv. 2014, 11, 132-138.

Somasundaran, P.; Mehta, S. C.; Rhein, L.; Chakraborty, S. Nanotechnology and Related Safety Issues for Delivery of Active Ingredients in Cosmetics. MRS Bull. 2007, 32 (10), 779-786.

Kim, H.-J.; Zhang, K.; Moore, L.; Ho, D. Diamond Nanogel-Embedded Contact Lenses Mediate Lysozyme-Dependent Therapeutic Release. ACS Nano 2014, 8 (3), 2998-3005.

Ferreira, S. A.; Gama, F. M.; Vilanova, M. Polymeric Nanogels as Vaccine Delivery Systems. Nanomedicine 2013, 9 (2), 159173.

Vijayan, V. M.; Shenoy, S. J.; Victor, S. P.; Muthu, J. Stimulus Responsive Nanogel with Innate near IR Fluorescent Capability for Drug Delivery and Bioimaging. Colloids Surf. B. Biointerfaces 2016, 146, 84-96.

Hadinoto, K.; Sundaresan, A.; Cheow, W. S. Lipid-Polymer Hybrid Nanoparticles as a New Generation Therapeutic Delivery Platform: A Review. Eur. J. Pharm. Biopharm. 2013, 85, 427-443.

Mandal, B.; Bhattacharjee, H.; Mittal, N.; Sah, H.; Balabathula, P.; Thoma, L. A.; Wood, G. C. Core-Shell-Type Lipid-Polymer Hybrid Nanoparticles as a Drug Delivery Platform. Nanomedicine Nanotechnology, Biol. Med. 2013, 9 (4), 474-491.

Tan, S.; Li, X.; Guo, Y.; Zhang, Z. Lipid-Enveloped Hybrid Nanoparticles for Drug Delivery. Nanoscale 2013, 5 (3), $860-872$.

Raemdonck, K.; Braeckmans, K.; Demeester, J.; De Smedt, S. C. Merging the Best of Both Worlds: Hybrid Lipid-Enveloped Matrix Nanocomposites in Drug Delivery. Chem. Soc. Rev. 2014, 43, 444-472.

Kazakov, S. Liposome-Nanogel Structures for Future Pharmaceutical Applications: An Updated Review. Curr. Pharm. Des. 2016, 22 (10), 1391-1413.

Kazakov, S.; Levon, K. Liposome-Nanogel Structures for Future Pharmaceutical Applications. Curr. Pharm. Des. 2006, 12 (36), 4713-4728.

Petralito, S.; Paolicelli, P.; Nardoni, M.; Trilli, J.; Di Muzio, L.; Cesa, S.; Relucenti, M.; Matassa, R.; Vitalone, A.; Adrover, A.; Casadei, M. A. Gelation of the Internal Core of Liposomes as a Strategy for Stabilization and Modified Drug Delivery I. Physico-Chemistry Study. Int. J. Pharm. 2020, 585, 119467.

Jin, T.; Pennefather, P.; Lee, P. I. Lipobeads: A Hydrogel Anchored Lipid Vesicle System. FEBS Lett. 1996, 397, 70-74.

Tiwari, S.; Goyal, A. K.; Mishra, N.; Khatri, K.; Vaidya, B.; Mehta, A.; Wu, Y.; Vyas, S. P. Development and Characterization of Novel Carrier Gel Core Liposomes Based Transmission Blocking Malaria Vaccine. J. Control. Release 2009, 140 (2), $157-165$. 
(123) Homyak, C. C.; Fernandez, A.; Touve, M. A.; Zhao, B.; Anson, F.; Hardy, J. A.; Vachet, R. W.; Gianneschi, N. C.; Ross, J. L.; Thayumanavan, S. Lipogels for Encapsulation of Hydrophilic Proteins and Hydrophobic Small Molecules. Biomacromolecules 2018, 19, 132-140.

(124) Ma, J.; Deng, H.; Zhao, F.; Deng, L.; Wang, W.; Dong, A.; Zhang, J. Liposomes-Camouflaged Redox-Responsive Nanogels to Resolve the Dilemma between Extracellular Stability and Intracellular Drug Release. Macromol. Biosci. 2018, $18,1800049$.

(125) Zhang, B.; Chen, J.; Lu, Y.; Qi, J.; Wu, W. Liposomes Interiorly Thickened with Thermosensitive Nanogels as Novel Drug Delivery Systems. Int. J. Pharm. 2013, 455, 276-284.

(126) Torchilin, V. P.; Klibanov, A. L.; Ivanov, N. N.; Ringsdorf, H.; Schlarb, B. Polymerization of Liposome-Encapsulated Hydrophilic Monomers. Die Makromol. Chemie, Rapid Commun. 1987, 8 (9), 457-460.

(127) Kazakov, S.; Kaholek, M.; Teraoka, I.; Levon, K. UV-Induced Gelation on Nanometer Scale Using Liposome Reactor. Macromolecules 2002, 35 (5), 1911-1920.

(128) Kazakov, S.; Kaholek, M.; Kudasheva, D.; Teraoka, I.; Cowman, M. K.; Levon, K. Poly(N-Isopropylacrylamide-Co-1Vinylimidazole) Hydrogel Nanoparticles Prepared and Hydrophobically Modified in Liposome Reactors: Atomic Force Microscopy and Dynamic Light Scattering Study. Langmuir 2003, 19 (19), 8086-8093.

(129) Patton, J. N.; Palmer, A. F. Photopolymerization of Bovine Hemoglobin Entrapped Nanoscale Hydrogel Particles within Liposomal Reactors for Use as an Artificial Blood Substitute. Biomacromolecules 2005, 6, 414-424.

(130) Patton, J. N.; Palmer, A. F. Engineering Temperature-Sensitive Hydrogel Nanoparticles Entrapping Hemoglobin as a Novel Type of Oxygen Carrier. Biomacromolecules 2005, 6 (4), 2204-2212.

(131) Van Thienen, T. G.; Lucas, B.; Flesch, F. M.; Van Nostrum, C. F.; Demeester, J.; De Smedt, S. C. On the Synthesis and Characterization of Biodegradable Dextran Nanogels with Tunable Degradation Properties. Macromolecules 2005, 38 (20), 8503-8511.

(132) Van Thienen, T. G.; Raemdonck, K.; Demeester, J.; De Smedt, S. C. Protein Release from Biodegradable Dextran Nanogels. Langmuir 2007, 23 (19), 9794-9801.

(133) An, S. Y.; Bui, M. P. N.; Nam, Y. J.; Han, K. N.; Li, C. A.; Choo, J.; Lee, E. K.; Katoh, S.; Kumada, Y.; Seong, G. H. Preparation of Monodisperse and Size-Controlled Poly(Ethylene Glycol) Hydrogel Nanoparticles Using Liposome Templates. J. Colloid Interface Sci. 2009, 331, 98-103.

(134) Park, J.; Wrzesinski, S. H.; Stern, E.; Look, M.; Criscione, J.; Ragheb, R.; Jay, S. M.; Demento, S. L.; Agawu, A.; Licona Limon, P.; Ferrandino, A. F.; Gonzalez, D.; Habermann, A.; Flavell, R. A.; Fahmy, T. M. Combination Delivery of TGF- $\beta$ Inhibitor and IL-2 by Nanoscale Liposomal Polymeric Gels Enhances Tumour Immunotherapy. Nat. Mater. 2012, 11 (10), 895-905.

(135) Petralito, S.; Spera, R.; Pacelli, S.; Relucenti, M.; Familiari, G.; Vitalone, A.; Paolicelli, P.; Casadei, M. A. Design and Development of PEG-DMA Gel-in-Liposomes as a New Tool for Drug Delivery. React. Funct. Polym. 2014, 77, 30-38.

(136) Wang, Y.; Tu, S.; Pinchuk, A. N.; Xiong, M. P. Active Drug Encapsulation and Release Kinetics from Hydrogel-in-Liposome Nanoparticles. J. Colloid Interface Sci. 2013, 406, 247-255.

(137) Lockhart, J. N.; Beezer, D. B.; Stevens, D. M.; Spears, B. R.; Harth, E. One-Pot Polyglycidol Nanogels via Liposome Master Templates for Dual Drug Delivery. J. Control. Release 2016, 244, 366-374.

(138) Hachet, E.; Sereni, N.; Pignot-Paintrand, I.; Ravaine, V.; Szarpak-Jankowska, A.; Auzély-Velty, R. Thiol-Ene Clickable Hyaluronans: From Macro-to Nanogels. J. Colloid Interface Sci. 2014, 419, 52-55.

(139) Zhao, X.; Deng, L.; Deng, H.; Dong, A.; Wang, W.; Zhang, J. In Situ Template Polymerization to Prepare Liposome-Coated PDMAEMA Nanogels with Controlled Size, High Stability, Low Cytotoxicity, and Responsive Drug Release for Intracellular DOX Release. Macromol. Chem. Phys. 2018, 219, 1800071.

(140) Monshipouri, M.; Rudolph, A. S. Liposome-Encapsulated Alginate: Controlled Hydrogel Particle Formation and Release. J. Microencapsul. 1995, 12 (2), 117-127.

(141) Hong, J. S.; Stavis, S. M.; Depaoli Lacerda, S. H.; Locascio, L. E.; Raghavan, S. R.; Gaitan, M. Microfluidic Directed SelfAssembly of Liposome-Hydrogel Hybrid Nanoparticles. Langmuir 2010, 26 (13), 11581-11588.

(142) Kiser, P. F.; Wilson, G.; Needham, D. A Synthetic Mimic of the Secretory Granule for Drug Delivery. Nature 1998, 394, 459462.

(143) Hong, J. S.; Vreeland, W. N.; DePaoli Lacerda, S. H.; Locascio, L. E.; Gaitan, M.; Raghavan, S. R. Liposome-Templated Supramolecular Assembly of Responsive Alginate Nanogels. Langmuir 2008, 24 (8), 4092-4096.

(144) Nagano, T. Development of Fluorescent Probes for Bioimaging Applications. Proc. Japan Acad. Ser. B Phys. Biol. Sci. 2010, 86 (8), 837-847.

(145) Orringer, D. A.; Koo, Y. E.; Chen, T.; Kopelman, R.; Sagher, O.; Philbert, M. A. Small Solutions for Big Problems: The Application of Nanoparticles to Brain Tumor Diagnosis and Therapy. Clin. Pharmacol. Ther. 2009, 85 (5), 531-534.

(146) Kerr, C. a.; de la Rica, R. Photoluminescent Nanosensors for Intracellular Detection. Anal. Methods 2015, 7 (17), 7067-7075.

(147) Muddana, H. S.; Morgan, T. T.; Adair, J. H.; Butler, P. J. Photophysics of Cy3-Encapsulated Calcium Phosphate Nanoparticles. Nano Lett. 2009, 9 (4), 1559-1566.

(148) Zhou, J.; Ma, H. Design Principles of Spectroscopic Probes for Biological Applications. Chem. Sci. 2016, 7 (10), $6309-6315$.

(149) Li, X.; Gao, X.; Shi, W.; Ma, H. Design Strategies for Water-Soluble Small Molecular Chromogenic and Fluorogenic Probes. Chem. Rev. 2014, 114, 590-659.

(150) Decuzzi, P.; Peer, D.; Di Mascolo, D.; Palange, A. L.; Manghnani, P. N.; Moein Moghimi, S.; Shadi Farhangrazi, Z.; Howard, K. 
A.; Rosenblum, D.; Liang, T.; Chen, Z.; Wang, Z.; Zhu, J. J.; Gu, Z.; Korin, N.; Letourneur, D.; Chauvierre, C.; van der Meel, R.; Kiessling, F.; Lammers, T. Roadmap on Nanomedicine. Nanotechnology 2021, 32 (1), 012001

(151) Abrahamse, H.; Hamblin, M. R. New Photosensitizers for Photodynamic Therapy. Biochem. J. 2016, 473 (4), 347-364.

(152) Hirsch, L. R.; Gobin, A. M.; Lowery, A. R.; Tam, F.; Drezek, R. A.; Halas, N. J.; West, J. L. Metal Nanoshells. Ann. Biomed. Eng. 2006, 34 (1), 15-22.

(153) Dutz, S.; Hergt, R. Magnetic Particle Hyperthermia - a Promising Tumour Therapy? Nanotechnology 2014,25 (45), 452001.

(154) Schütt, W.; Grüttner, C.; Häfeli, U.; Zborowski, M.; Teller, J.; Putzar, H.; Schümichen, C. Applications of Magnetic Targeting in Diagnosis and Therapy - Possibilities and Limitations: A Mini-Review. Hybridoma 1997, 16 (1), 109-117.

(155) Almdal, K.; Dyre, J.; Hvidt, S.; Kramer, O. Towards a Phenomenological Definition of the Term 'Gel.' Polym. Gels Networks 1993, 1 (1), 5-17.

(156) Flory, P. J. Introductory Lecture. Faraday Discuss. Chem. Soc. 1974, 57, 7-18.

(157) Terech, P.; Weiss, R. G. Low Molecular Mass Gelators of Organic Liquids and the Properties of Their Gels. Chem. Rev. 1997, $97(8), 3133-3159$.

(158) Estroff, L. A.; Hamilton, A. D. Water Gelation by Small Organic Molecules. Chem. Rev. 2004, 104 (3), $1201-1217$.

(159) Hirst, A. R.; Escuder, B.; Miravet, J. F.; Smith, D. K. High-Tech Applications of Self-Assembling Supramolecular Nanostructured Gel-Phase Materials: From Regenerative Medicine to Electronic Devices. Angew. Chemie - Int. Ed. 2008, 47 (42), 8002-8018.

(160) Banerjee, S.; Das, R. K.; Maitra, U. Supramolecular Gels “in Action.” J. Mater. Chem. 2009, 19 (37), 6649-6687.

(161) Steed, J. W. Supramolecular Gel Chemistry: Developments over the Last Decade. Chem. Commun. 2011, 47 (5), $1379-1383$.

(162) Du, X.; Zhou, J.; Shi, J.; Xu, B. Supramolecular Hydrogelators and Hydrogels: From Soft Matter to Molecular Biomaterials. Chem. Rev. 2015, 115 (24), 13165-13307.

(163) Draper, E. R.; Adams, D. J. Low-Molecular-Weight Gels: The State of the Art. Chem 2017, 3 (3), $390-410$.

(164) Yu, G.; Yan, X.; Han, C.; Huang, F. Characterization of Supramolecular Gels. Chem. Soc. Rev. 2013, 42 (16), $6697-6722$.

(165) Dawn, A.; Shiraki, T.; Haraguchi, S.; Tamaru, S.; Shinkai, S. What Kind of "Soft Materials" Can We Design from Molecular Gels? Chem. Asian J. 2011, 6 (2), 266-282.

(166) Menger, F. M.; Caran, K. L. Anatomy of a Gel. Amino Acid Derivatives That Rigidify Water at Submillimolar Concentrations. J. Am. Chem. Soc. 2000, 122 (47), 11679-11691.

(167) Chandler, D. Interfaces and the Driving Force of Hydrophobic Assembly. Nature 2005, 437 (7059), 640-647.

(168) Miravet, J. F.; Escuder, B. Chapter 11. Supramolecular Gels for Pharmaceutical and Biomedical Applications. In Supramolecular systems in biomedical fields; Schneider, H.-J., Ed.; RSC Publishing, 2013.

(169) Hirst, A. R.; Coates, I. A.; Boucheteau, T. R.; Miravet, J. F.; Escuder, B.; Castelletto, V.; Hamley, I. W.; Smith, D. K. LowMolecular-Weight Gelators: Elucidating the Principles of Gelation Based on Gelator Solubility and a Cooperative SelfAssembly Model. J. Am. Chem. Soc. 2008, 130 (28), 9113-9121.

(170) Haines, S. R.; Harrison, R. G. Novel Resorcinarene-Based PH-Triggered Gelator. Chem. Commun. 2002, No. 23, $2846-2847$.

(171) Kohsaka, Y.; Koyama, Y.; Takata, T. Graft Polyrotaxanes: A New Class of Graft Copolymers with Mobile Graft Chains. Angew. Chemie Int. Ed. 2011, 50 (44), 10417-10420.

(172) Ogoshi, T.; Takashima, Y.; Yamaguchi, H.; Harada, A. Chemically-Responsive Sol-Gel Transition of Supramolecular SingleWalled Carbon Nanotubes (SWNTs) Hydrogel Made by Hybrids of SWNTs and Cyclodextrins. J. Am. Chem. Soc. 2007, 129 (16), 4878-4879.

(173) Segarra-Maset, M. D.; Nebot, V. J.; Miravet, J. F.; Escuder, B. Control of Molecular Gelation by Chemical Stimuli. Chem. Soc. Rev. 2013, 42 (17), 7086-7098.

(174) Yang, X.; Zhang, G.; Zhang, D. Stimuli Responsive Gels Based on Low Molecular Weight Gelators. J. Mater. Chem. 2012, 22 (1), 38-50.

(175) Fontanillo, M.; Angulo-Pachón, C. A.; Escuder, B.; Miravet, J. F. In Situ Synthesis-Gelation at Room Temperature vs. HeatingCooling Procedure. Fine Tuning of Molecular Gels Derived from Succinic Acid and L-Valine. J. Colloid Interface Sci. 2013, 412, 65-71.

(176) Angulo-Pachón, C. A.; Miravet, J. F. Sucrose-Fueled, Energy Dissipative, Transient Formation of Molecular Hydrogels Mediated by Yeast Activity. Chem. Commun. 2016, 52 (31), 5398-5401.

(177) Miravet, J. F.; Escuder, B. Pyridine-Functionalised Ambidextrous Gelators: Towards Catalytic Gels. Chem. Commun. 2005, No. 46, 5796-5798.

(178) Cravotto, G.; Cintas, P. Molecular Self-Assembly and Patterning Induced by Sound Waves. The Case of Gelation. Chem. Soc. Rev. 2009, 38 (9), 2684-2697.

(179) Raeburn, J.; Mendoza-Cuenca, C.; Cattoz, B. N.; Little, M. A.; Terry, A. E.; Zamith Cardoso, A.; Griffiths, P. C.; Adams, D. J. The Effect of Solvent Choice on the Gelation and Final Hydrogel Properties of Fmoc-Diphenylalanine. Soft Matter 2015, 11 (5), 927-935.

(180) Draper, E. R.; Adams, D. J. Photoresponsive Gelators. Chem. Commun. 2016, 52 (53), 8196-8206.

(181) Nebot, V. J.; Ojeda-Flores, J. J.; Smets, J.; Fernández-Prieto, S.; Escuder, B.; Miravet, J. F. Rational Design of Heat-Set and Specific-Ion-Responsive Supramolecular Hydrogels Based on the Hofmeister Effect. Chem. Eur. J. 2014, 20 (44), 14465- 
14472.

(182) Piepenbrock, M.-O. M.; Lloyd, G. O.; Clarke, N.; Steed, J. W. Metal- and Anion-Binding Supramolecular Gels. Chem. Rev. 2010, 110 (4), 1960-2004.

(183) Ulijn, R. V. Enzyme-Responsive Materials: A New Class of Smart Biomaterials. J. Mater. Chem. 2006, 16 (23), $2217-2225$.

(184) Díaz-Oltra, S.; Berdugo, C.; Miravet, J. F.; Escuder, B. Study of the Effect of Polymorphism on the Self-Assembly and Catalytic Performance of an L-Proline Based Molecular Hydrogelator. New J. Chem. 2015, 39 (5), 3785-3791.

(185) Colquhoun, C.; Draper, E. R.; Schweins, R.; Marcello, M.; Vadukul, D.; Serpell, L. C.; Adams, D. J. Controlling the Network Type in Self-Assembled Dipeptide Hydrogels. Soft Matter 2017, 13 (9), 1914-1919.

(186) Weiss, R. G. The Past, Present, and Future of Molecular Gels. What Is the Status of the Field, and Where Is It Going? J. Am. Chem. Soc. 2014, 136 (21), 7519-7530.

(187) Sun, Z.; Huang, Q.; He, T.; Li, Z.; Zhang, Y.; Yi, L. Multistimuli-Responsive Supramolecular Gels: Design Rationale, Recent Advances, and Perspectives. ChemPhysChem 2014, 15 (12), 2421-2430.

(188) Roy, G.; Miravet, J. F.; Escuder, B.; Sanchez, C.; Llusar, M. Morphology Templating of Nanofibrous Silica through PH-Sensitive Gels: "In Situ" and "Post-Diffusion" Strategies. J. Mater. Chem. 2006, 16 (19), 1817-1824.

(189) Babu, S. S.; Praveen, V. K.; Ajayaghosh, A. Functional $\pi$-Gelators and Their Applications. Chem. Rev. 2014, 114 (4), $1973-$ 2129.

(190) Escuder, B.; Rodríguez-Llansola, F.; Miravet, J. F. Supramolecular Gels as Active Media for Organic Reactions and Catalysis. New J. Chem. 2010, 34 (6), 1044-1054.

(191) Wang, S.; Xue, P.; Wang, P.; Yao, B. Emission Enhanced Two-Component Gels for the Detection of Organic Amine Vapors. New J. Chem. 2015, 39 (9), 6874-6881.

(192) Dickert, F. L.; Haunschild, A. Sensor Materials for Solvent Vapor Detection - Donor-Acceptor and Host-Guest Interactions. Adv. Mater. 1993, 5 (12), 887-895.

(193) Vintiloiu, A.; Leroux, J.-C. Organogels and Their Use in Drug Delivery - a Review. J. Control. release 2008, 125 (3), $179-192$. 


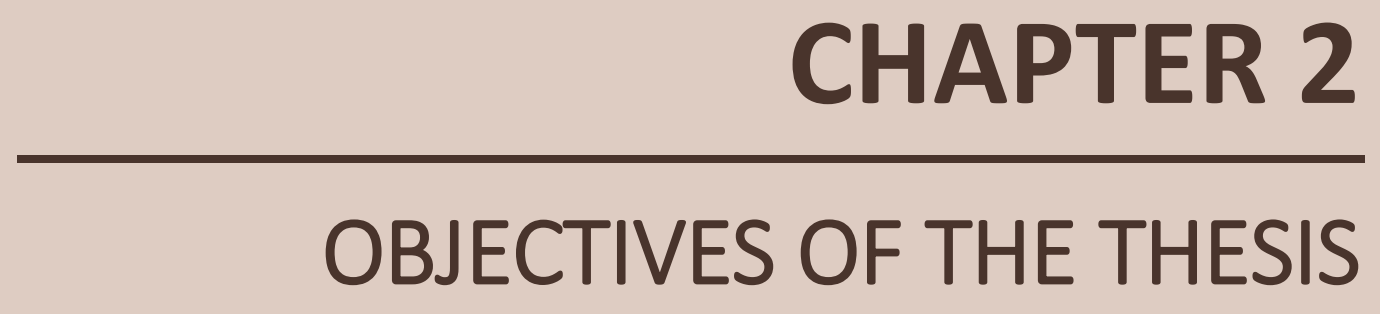





\section{CHAPTER 2. OBJECTIVES OF THE THESIS}

The main objective of this thesis has been to develop a new type of nanoparticles: molecular nanogels. Molecular nanogels merge the interest in polymeric nanogels as nanocarriers and in molecular gels as soft materials. These particles, obtained by self-assembly of a low molecular weight compound, were intended to be prepared with appropriate thermodynamic or kinetic stability and in a reproducible manner, and to present envisaged applications in biomedical issues related to drug delivery and sensing.

Although many polymeric nanogels have been reported in the literature, there are no references to the study of molecular nanogels. The present work is aimed to be the first report on their preparation and detailed characterization. The use of polymeric nanogels in drug delivery presents challenges associated with stimuli-triggered release, biodegradation, polymer polydispersity and batch-to-batch reproducibility in the preparation of polymers. ${ }^{1}$ Molecular nanogels may represent an exciting alternative to polymeric analogues taking into account their intrinsic characteristics: (i) a fully reversible assembly to give the original free molecules that can be stimuli regulated, (ii) easy biodegradation because low molecular weight molecules compose them, and (iii) excellent biocompatibility as they are mainly built from natural products. Additionally, the extensive library of molecular gelators available paves the way for the preparation of molecular nanogels with tailored properties, such as stimuli responsiveness or the presence of a desired functional group in their structure.

Two different strategies are assayed in this work to prepare molecular nanogels: ultrasoundpromoted nanogel formation and using liposomes as a template (Chapter 3 ). This latter method includes the preparation of novel hydrogel@liposome particles as intermediate, obtained by molecular gel formation inside liposomes. All the lipogels reported in literature are constituted by polymers, the interaction of molecular gels and liposomes being only described for liposome formation within a macroscopic supramolecular gel. ${ }^{2}$ Again, the inherent characteristics of molecular gels would improve the polymeric version of lipogels as drug delivery systems, with stimuli responsiveness, ideal for the preparation of environmentsensitive carriers, and enhanced biocompatibility. 
Finally, another crucial objective of this work has been the study of the capability of molecular nanogels to incorporate and release actives and act as intracellular carriers. Specifically, fluorescent species were selected for this purpose: nitric oxide (NO) probes (Chapter 5) and photosensitizers for anticancer photodynamic therapy (Chapter 4). Commercial fluorophores with different nature regarding the solubility in water have been chosen. The study of the properties of the new loaded systems in solution and in vitro (sensing and photoactivity) was set as an additional objective in this work, aiming to improve the efficiency of the photoactive molecules in their biomedical use.

(1) Vinogradov, S. V. Nanogels in the Race for Drug Delivery. Nanomedicine 2010, 5 (2), 165-168.

(2) Boekhoven, J.; Brizard, A. M.; Stuart, M. C. A.; Florusse, L.; Raffy, G.; Del Guerzo, A.; Van Esch, J. H. Bio-Inspired Supramolecular Materials by Orthogonal Self-Assembly of Hydrogelators and Phospholipids. Chem. Sci. 2016, 7 (9), 60216031. 
NANOGEL PARTICLES FROM A LOW MOLECULAR WEIGHT HYDROGELATOR 



\section{CHAPTER 3. NANOGEL PARTICLES FROM A LOW MOLECULAR WEIGHT HYDROGELATOR 3.1. NANOGELS PREPARED BY ULTRASONICATION}

This section is mainly based on the "Results and Discussion" part of the paper "TorresMartínez, A.; Angulo-Pachón, C. A.; Galindo, F.; Miravet, J. F. In between molecules and selfassembled fibrillar networks: Highly stable nanogel particles from a low molecular weight hydrogelator. Soft Matter 2019, 15, 3565-3572".

\subsubsection{GELATOR SYNTHESIS AND MACROSCOPIC GELS}

The synthesis of (S)-4-((3-methyl-1-(nonylamino)-1-oxobutan-2-yl)amino)-4-oxobutanoic acid, compound 1 , is outlined in Figure 3.1. Compound 1 consists of L-valine modified as nonylamide at the carboxylic acid unit and acylated with succinic acid at the amine function. This gelator can be easily prepared on a gram scale from the amino acid by $\mathrm{N}$-acylation with succinic anhydride and amide formation with nonylamine.

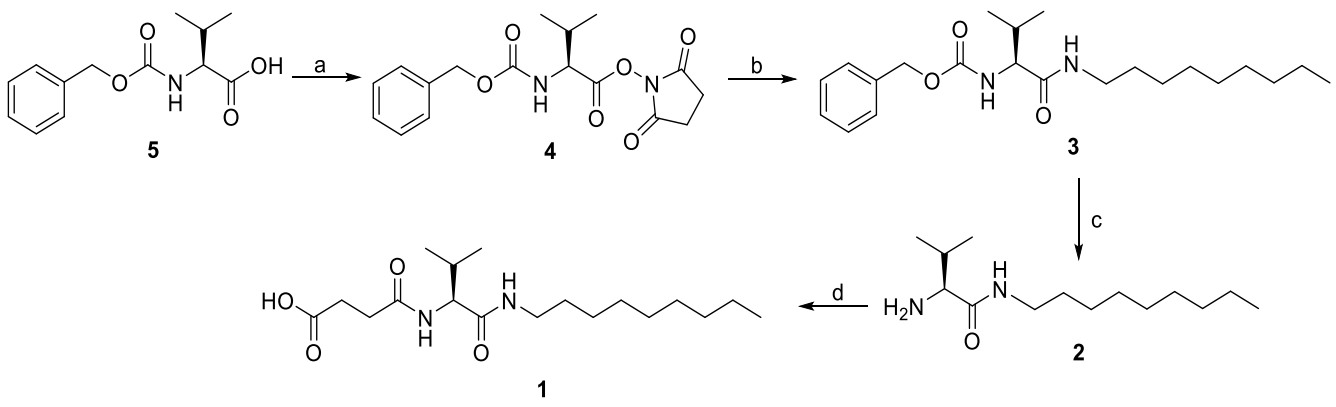

Figure 3.1. Synthetic route for the synthesis of 1. Reagents and conditions: (a) DCC, $\mathrm{N}$ hydroxysuccinimide, THF, 2 h, 94\%; (b) n-nonylamine, THF, 5 h, 77\%; (c) Pd/C, $\mathrm{H}_{2}, \mathrm{CH}_{3} \mathrm{OH}, 4$ h, 94\%; (d) succinic anhydride, $\mathrm{K}_{2} \mathrm{CO}_{3}$, THF, $16 \mathrm{~h}, 97 \%$.

The presence of an ionizable carboxylic unit in $\mathbf{1}$ results in $\mathrm{pH}$-dependent gelation properties, being the neutral species responsible for gelation (Figure 3.2). Potentiometric titrations were carried out to evaluate the $\mathrm{pH}$ range of existence of neutral species of 1, revealing an apparent $\mathrm{p} K_{\mathrm{a}}$ of 7.6 (Figure 3.3). As has been previously reported, this $\mathrm{p} K_{\mathrm{a}}$ value is considerably shifted when compared to soluble, non-aggregating carboxylic acids, which present $\mathrm{p} K_{\mathrm{a}}$ values around $3-5 .{ }^{1-3}$ The reduced acidity of 1 could be ascribed to the thermodynamic stabilisation of neutral species gained from the aggregation process. 


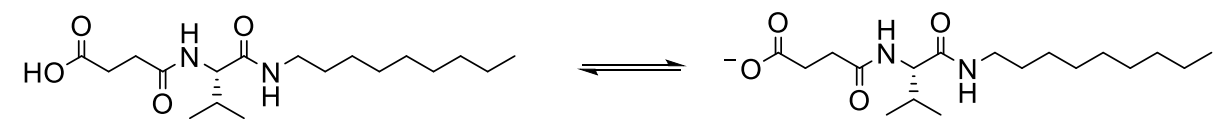

Figure 3.2. Acid/base equilibrium for compound 1 .

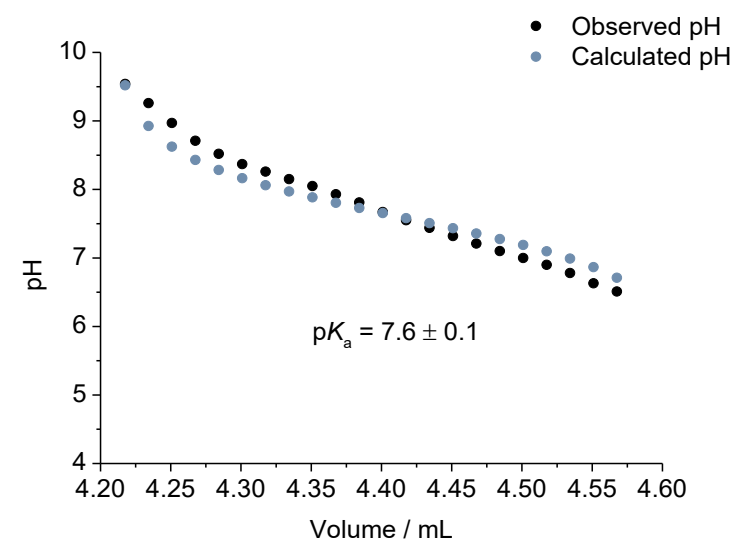

Figure 3.3. Calculated (HYPERQUAD_2008, blue) vs. observed pH (black) for the potentiometric titration of 1 with $\mathrm{HCl} 0.1 \mathrm{M}$.

The aggregation in water of this type of molecule should be based on hydrophobic forces complemented with multiple $\mathrm{H}$-bonds as reported for related systems. ${ }^{4} \mathrm{~A}$ tentative aggregation model is shown in Figure 3.4, based on those proposed for closely related molecules previously described. ${ }^{5,6}$

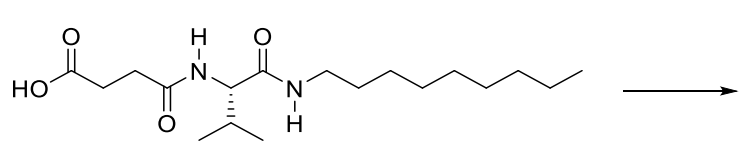

1

SOLUTION

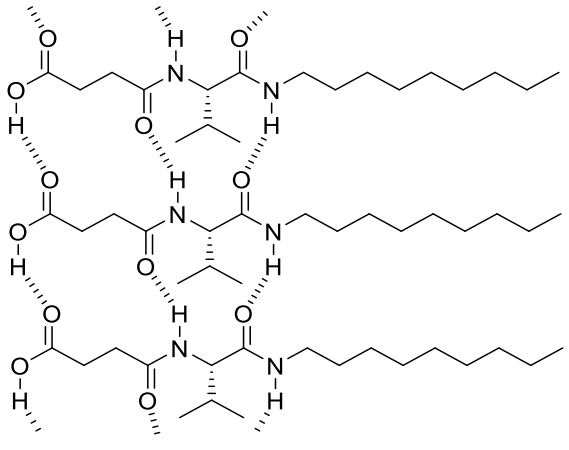

GEL

Figure 3.4. Proposed aggregation model for compound 1.

Gels of 1 could be formed in water by cooling down hot solutions of the gelator, with a minimum gelation concentration value $(\mathrm{mgc})$ of $5 \mathrm{mg} / \mathrm{mL}(16 \mathrm{mM})$, or by $\mathrm{pH}$ change (hydrochloric acid is added to a solution of 1 in sodium hydroxide), with a mgc of $2 \mathrm{mg} / \mathrm{mL}$ (5 
$\mathrm{mM}$ ). Additionally, gels can also be prepared in different organic solvents by cooling down hot solutions of the gelator, with $\mathrm{mgc}$ in the range 3-38 mM (see Table 3.1).

Table 3.1. Minimum gelation concentration of compound 1 in different solvents using the heating-cooling process.

\begin{tabular}{|c|c|c|}
\hline Solvent & $\mathrm{mgc} / \mathrm{mg} \cdot \mathrm{mL}^{-1}$ & $\mathrm{mgc} / \mathrm{mM}$ \\
\hline Acetonitrile & 6 & 16 \\
\hline Dichloromethane & 6 & 18 \\
\hline Toluene & 1 & 3 \\
\hline Water & 5 & 16 \\
\hline Ethyl acetate & 13 & 38 \\
\hline Chloroform & \multicolumn{2}{|c|}{ Soluble } \\
\hline Tetrahydrofuran & \multicolumn{2}{|c|}{ Soluble } \\
\hline Ethanol & \multicolumn{2}{|c|}{ Soluble } \\
\hline Methanol & \multicolumn{2}{|c|}{ Soluble } \\
\hline
\end{tabular}

The transmission electron microscopy (TEM) images of the xerogel in water and toluene (Figure 3.5) showed a fibrillar structure, as commonly described for molecular gels. Noticeably the fibres in the xerogel in water are straight and monodisperse, with a diameter of $c a .20 \mathrm{~nm}$, while those obtained in toluene show more flexibility, and are curved and entangled. These morphological differences probably reflect the different arrangements of the gelator molecules in the fibres, being hydrophobic forces dominant for the aggregation in water and polar interactions, namely hydrogen bonding, predominant in organic solvents. Indeed, differential scanning calorimetry (DSC) reveals that the xerogels obtained in water and toluene are polymorphic, presenting different melting points and endothermic polymorphic transitions

(Figure 3.6).

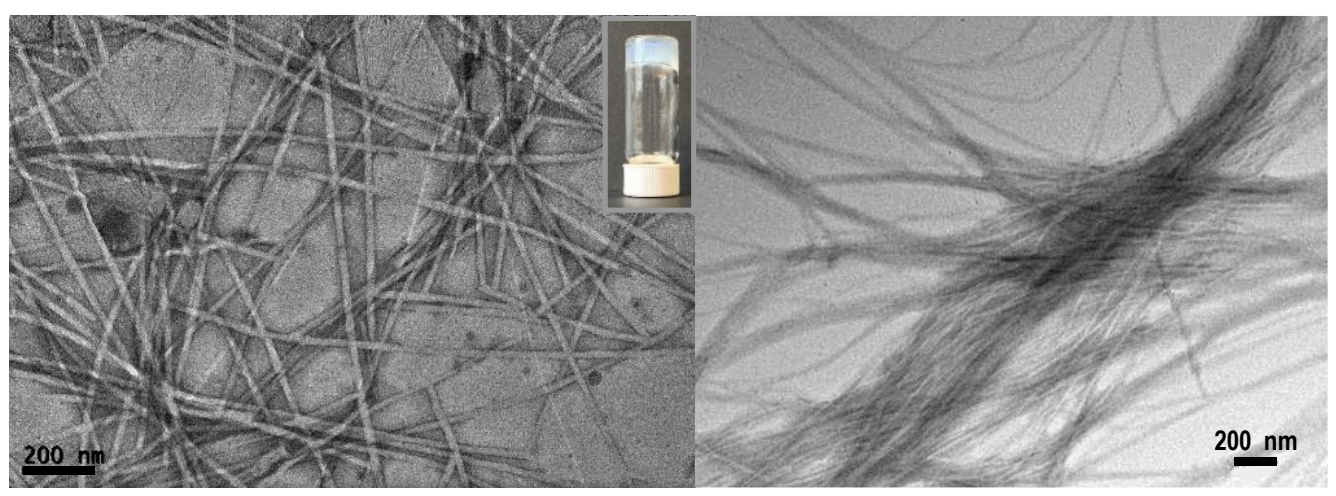

Figure 3.5. TEM image of the xerogels obtained from 1 in water (left) and toluene (right). Inset: Macroscopic image of the hydrogel. 

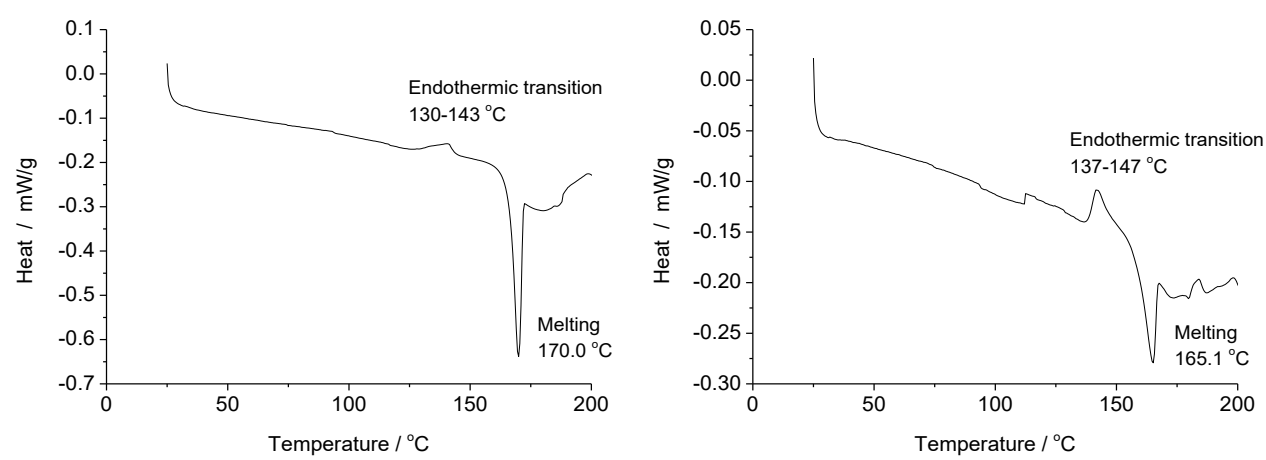

Figure 3.6. DSC traces of the xerogels from toluene (left) and water (right).

\subsubsection{PREPARATION AND CHARACTERISATION OF NANOGELS}

Following a report on the formation of hybrid peptide-quantum dot colloidal spheres, ${ }^{7}$ nanogel particles were prepared by sonication of a xerogel of 1 suspended in aqueous medium (Figure 3.7). For this purpose, a gel was prepared in toluene, and the corresponding xerogel was obtained by solvent evaporation under vacuum. Upon sonication in phosphate buffer saline (PBS, $10 \mathrm{mM}, \mathrm{pH}$ 7), a colloidal suspension of the nanoparticles with a final $\mathrm{pH}$ of 6.4 was obtained. Centrifugation to remove large fragments of the xerogel particles afforded an optically clear suspension of the nanogels (Figure $\mathbf{3 . 8}$ ).

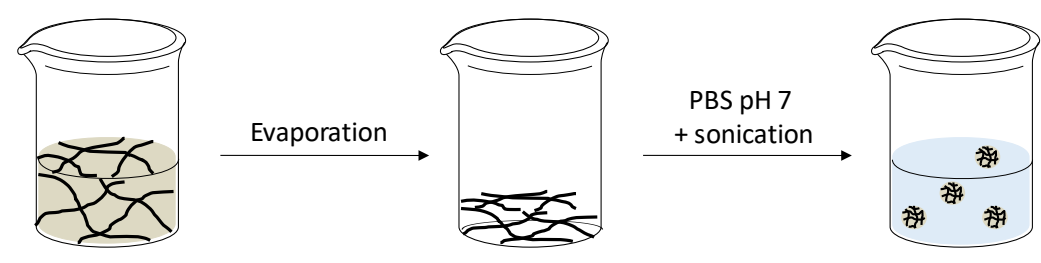

Figure 3.7. Scheme of the preparation of the nanogels of 1 by sonication.

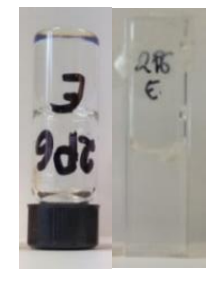

Figure 3.8. Toluene gel (left) and a sample of nanogels (right) of 1.

The analysis by dynamic light scattering (DLS) of the obtained suspension revealed a bimodal distribution of intensity averaged diameters $\left(D_{\mathrm{i}}\right)$ with maxima at $c a .200$ and $50 \mathrm{~nm}$ (Figure 3.9). The conversion of these data to number averaged diameters $\left(D_{n}\right)$ shows that particles with a diameter around $50 \mathrm{~nm}$ are predominant. The procedure for the preparation of the 
nanoparticles was found to be quite reproducible, and a set of 29 preparations afforded similar results, as shown in Figure 3.10. The polydispersity index (PDI) was around 0.3, which is indicative of a moderate polydispersity (PDI is calculated as [std_dev/mean_size] ${ }^{2}$, with values between 0 (monodisperse sample) and 1).
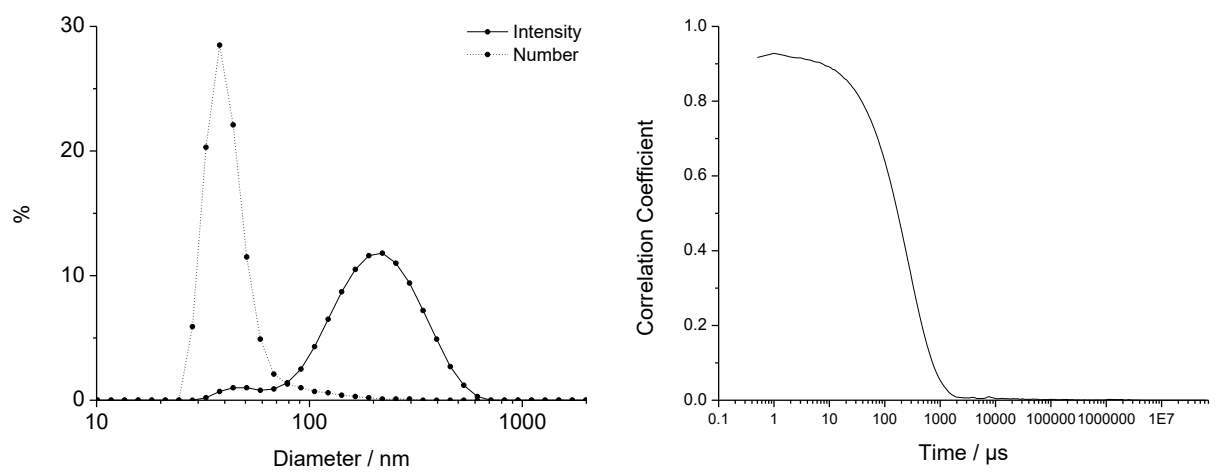

Figure 3.9. Left: Size distribution by intensity (solid line) and by number (dotted line) of a representative sample of nanogel particles obtained by DLS analysis. Right: Correlation function of the same DLS measurement.

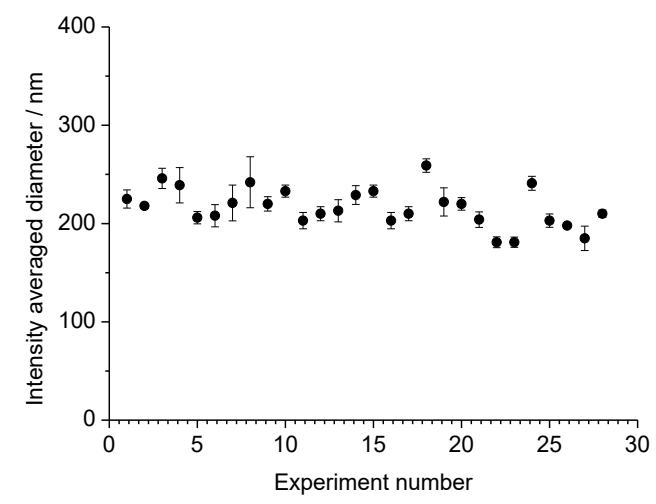

Figure 3.10. Intensity averaged diameters of 29 different nanogel samples.

In agreement with DLS results, TEM analysis revealed the presence of irregular spherical objects with diameters around $50 \mathrm{~nm}$ (Figure 3.11 top). The particles were also observed by cryo-TEM (Figure 3.11 bottom), showing diameters of $c a .200 \mathrm{~nm}$. The cryo-TEM images show a sponge-like structure with dark dots that might correspond to non-vitreous water trapped inside the particles. ${ }^{8}$ The smaller particle diameter observed by TEM when compared to that by cryo-TEM could be ascribed to drying effects associated with the former technique. 


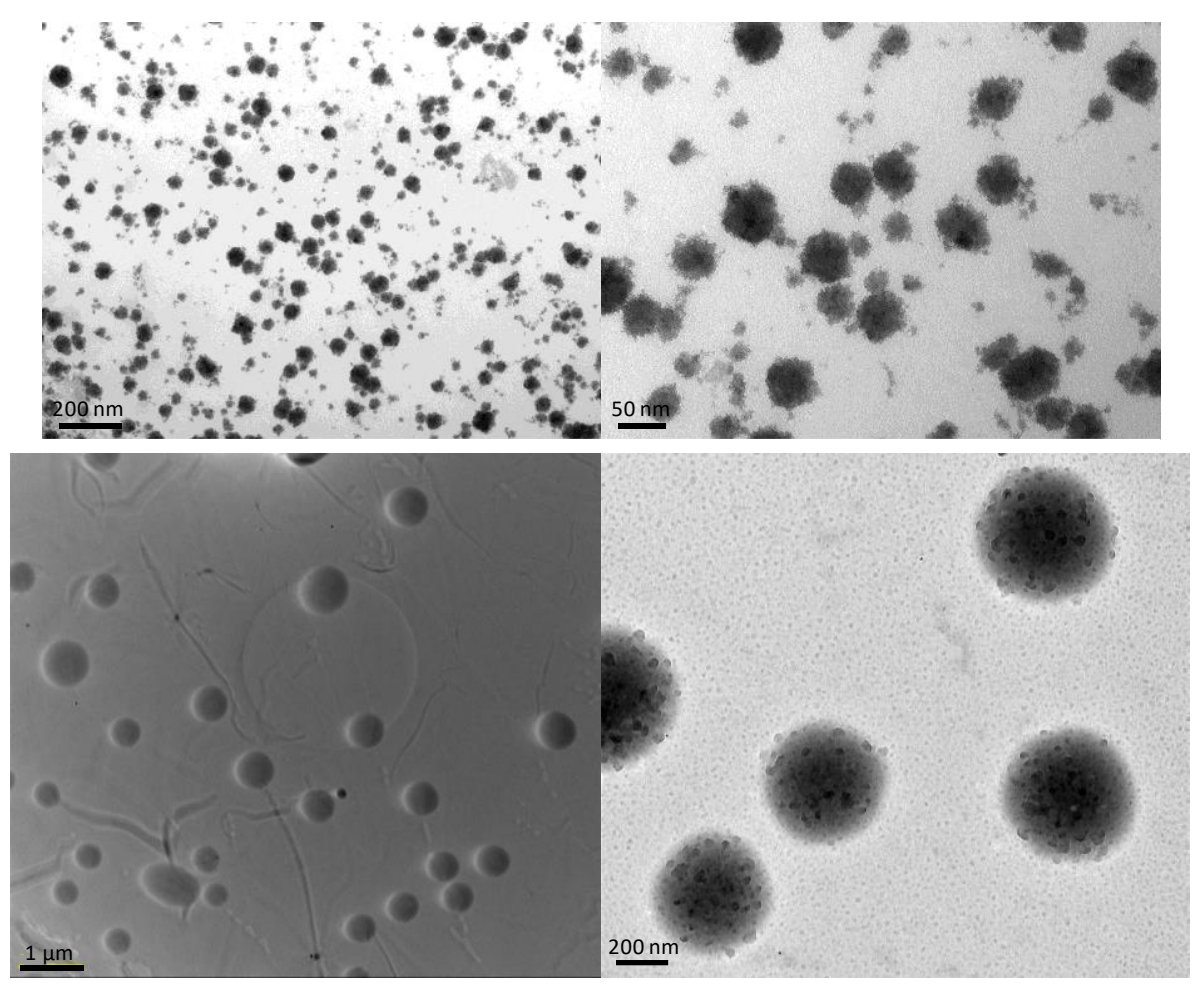

Figure 3.11. Electron microscopy images of nanogel particles formed by 1: TEM (top) and cryo-TEM (bottom).

The efficiency of the transformation of the xerogel into nanoparticles was evaluated determining the concentration of $\mathbf{1}$ in the colloids by ${ }^{1} \mathrm{H}$ NMR. For this purpose, nanoparticles were solubilised in an organic solvent and the signals were integrated using a calibrated electronic signal (ERETIC). ${ }^{9}$ The concentration of the nanogel particles was found to be reproducible. For a set of 10 experiments, using an initial concentration of xerogel of 1.2 $\mathrm{mg} / \mathrm{mL}$, an average concentration of 1 in the sample of $0.71 \pm 0.14 \mathrm{mg} / \mathrm{mL}$, namely $2.1 \pm 0.4 \mathrm{mM}$, was obtained. Thus, $60 \%$ of the added gelator was obtained in final samples.

Z-potential is the electrical potential at the interface that separates the mobile fluid from the fluid that remains attached to the surface of a particle. This potential is a crucial indicator of the stability of colloidal dispersions, and its magnitude indicates the degree of electrostatic repulsion between adjacent, similarly charged particles in a dispersion. Particles in suspension with a considerable zeta potential tend to repel each other, and there is no tendency to flocculate. In contrast, particles with low zeta potential values flocculate as there is no force to prevent the particles from coming together. ${ }^{11}$ For nanoparticles of 1 this value was measured 
to be $-65 \mathrm{mV}$, which is in the range of those reported for highly stable colloids. ${ }^{12}$ In agreement, nanogels exhibited good temporal stability: similar DLS results were obtained when stored at room temperature for 4 days, whereas the samples stored at $4^{\circ} \mathrm{C}$ were stable after ten days (Figure 3.12).

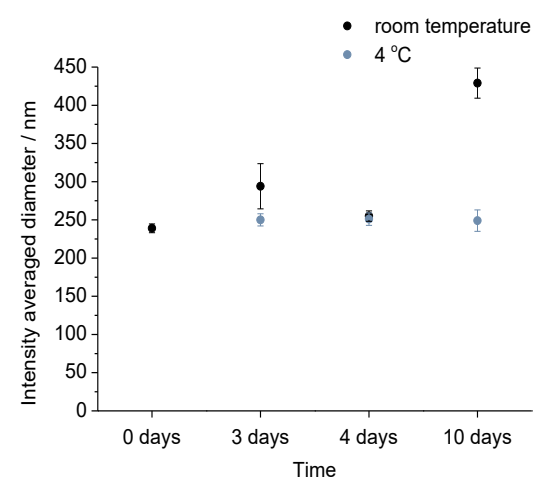

Figure 3.12. Size changes of nanogels samples over time at room temperature (black) and at $4^{\circ} \mathrm{C}$ (blue).

The critical aggregation concentration (cac) was also determined. Unlike mgc, which determines the amount of compound required to form a sample spanning network, cac indicates the onset of self-assembly and represents the dilution limit for thermodynamically stable aggregates. Determination of cac in an acidic medium for 1 was carried out using pyrene as a fluorescent probe. The incorporation of pyrene into hydrophobic environments leads to an increase of intensity of its emission band III relative to band I (Figure 3.13 right). ${ }^{10}$ Fluorescence spectra were recorded for samples with increasing concentrations of 1. As can be seen in Figure $\mathbf{3 . 1 3}$ left, the measured fluorescence ratio $\left(l_{1} / l_{111}\right)$ shows a moderate decrease with concentration up to a point where a dramatic tendency change is observed, with a much steeper slope, resulting in a cac value of $0.22 \mathrm{mM}$.
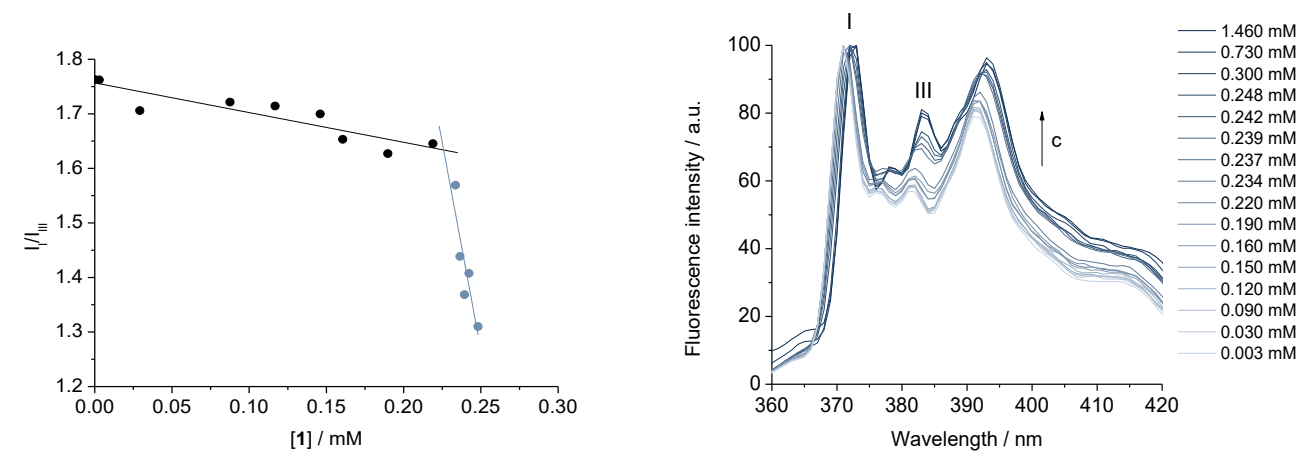

Figure 3.13. Variation of the relative intensity of emission bands I and III of pyrene in the presence of increasing concentrations of compound 1 in water $\left(\lambda_{\text {ex }} 334 \mathrm{~nm}\right)$. 
The non-covalent nature of molecular gels makes them sensible to concentration. Therefore, self-assembled nanoparticles upon dilution would be a system in a metastable state, whose equilibrium state is the formation of a gelator solution uniformly distributed by all the available solvent. However, kinetic phenomena are critical in the formation of molecular gels and it is relatively common to have a slow disassembly kinetics. In our case, the particles are stable upon dilution with PBS in the concentration range $0.25-2 \mathrm{mM}$, with no diameter variation (Figure 3.14). The stability of the nanoparticles at concentrations below cac $(0.22$ $\mathrm{mM}$ ) was also studied. Several samples were diluted at $\mathrm{pH} 7$ and their size over time was monitored. Figure $\mathbf{3 . 1 5}$ shows the results for a representative example, with the higher values meaning a disassembly of the nanogels. It was found that nanogels are kinetically trapped and are stable for $24 \mathrm{~h}$ even at a concentration of $0.02 \mathrm{mM}$, whereas they are immediately disassembled at $0.002 \mathrm{mM}$.

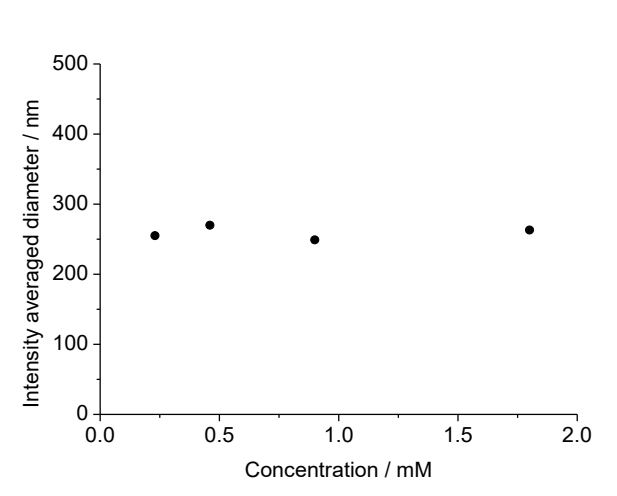

Figure 3.14. Influence of the concentration on the size of the nanogels.

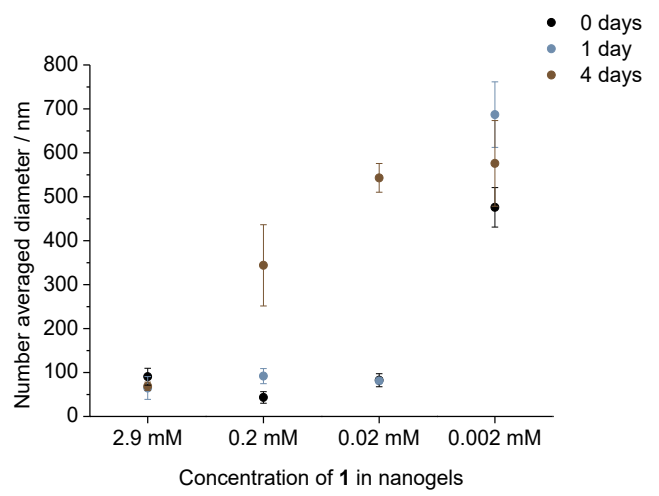

Figure 3.15. Influence of the concentration and time on the size of the nanogels.

To evaluate the gel-like nature of the nanoparticles, an average concentration of $\mathbf{1}$ within the particle $\left([1]_{\mathrm{NP}}\right)$ can be calculated using Equation (1), where $M_{\mathrm{w}}$ is the apparent molecular weight of the particle, $r$ is the radius of the particle and $N_{\mathrm{A}}$ is Avogadro's number. ${ }^{13-16}$

$$
[\mathbf{1}]_{\mathrm{NP}}=M_{\mathrm{w}} / N_{\mathrm{A}}\left(4 / 3 \pi r^{3}\right)^{-1}
$$

$M_{\mathrm{w}}$ was obtained by single angle static light scattering (SALS) measurements performed using a DLS equipment. ${ }^{17}$ The light scattering intensity was measured at different concentrations and analysed using the Debye-Zimm relation (see Equation (2)). ${ }^{18}$ In Equation (2), $K$ is an experimental constant (see Equation (3)) that depends on the wavelength of the incident light 
$\left(\lambda_{0}\right)$, the refractive index of the solvent $\left(n_{0}\right)$ and the variation of the refractive index with particle concentration $(\mathrm{d} n / \mathrm{d} c)$. Additionally, $c$ is the sample concentration, $R_{\theta}$ is the ratio of scattered light to incident light, $A_{2}$ is the thermodynamic 2 nd virial nonideality coefficient, and $P(\theta)$ is the angular dependence of scattering intensity.

$$
\begin{aligned}
& K c / R_{\theta}=\left(1 / M_{\mathrm{w}}+2 A_{2} c\right) P(\theta) \\
& K=\left(4 \pi^{2} / \lambda_{0} 4 N_{\mathrm{A}}\right)\left(n_{0} \mathrm{~d} n / \mathrm{d} c\right)^{2}
\end{aligned}
$$

For the study of the nanogels of $1, \mathrm{dn} / \mathrm{dc}$ was found to be $0.063 \mathrm{~mL} / \mathrm{g}$ using a flow field-flow fractionation (AF4) platform and injecting the sample directly to analyse in the refractive index detector. The scattering intensity of five aliquots of a sample at different concentrations was measured (Table 3.2). It has to be noted that given the supramolecular nature of the nanoparticles and their $\mathrm{pH}$-sensitivity, the concentration of $\mathbf{1}$ in the form of nanoparticles should be less than the total amount of 1 in the sample. The presence of the ionised compound $(9.5 \%$ of the total amount at $\mathrm{pH} 6.4$, calculated considering that $50 \%$ of the compound is ionised at $\mathrm{p} K_{\mathrm{a}}$ value, 7.6) and free 1 corresponding to the cac value was considered (see Table 3.3). The graphical representation of the value $K c / R_{\theta} v s . c$ is the socalled Debye plot, the intercept of the linear fitting at $c=0$ being the value of $1 / M_{w}$ (Figure 3.16). A good correlation was obtained, and the apparent $M_{\mathrm{w}}$ was calculated to be $13.8 \times 10^{6} \pm$ $8.3 \times 10^{6} \mathrm{Da}$. The error of this result was estimated as we are using SALS, which requires the simplification of considering isotropic scattering (use of a value of 1 for $P(\theta)$ in Equation (2)). This assumption usually generates an error of $60 \%$ in the $M_{w}$ value for particles with a diameter of $200 \mathrm{~nm}$ like ours. ${ }^{19}$ Using this calculated apparent $M_{\mathrm{w}}$ value and the diameter obtained by DLS for the studied samples $(210 \mathrm{~nm})$, an average concentration of 1 in the particles of $4.7 \pm 2.8 \mathrm{mg} / \mathrm{mL}$ is calculated using Equation (1). This result reveals the gel-like nature of the nanoparticles, with water being the main component. The concentration of $\mathbf{1}$ in the particles is similar to that described for polyethylene glycol with $M_{\mathrm{w}}=2 \times 10^{6} \mathrm{Da}, 2.9$ $\mathrm{mg} / \mathrm{mL}^{20}$ and comparable to that found, for example, in microgels ${ }^{14,21}$ or pullulan nanogels, ${ }^{16,22}$ which show a polymer concentration in the range $10-30 \mathrm{mg} / \mathrm{mL}$.

To assess the reliability of SALS in the determination of nanoparticles $M_{w}$, the measurements were carried out for a standard of monodisperse polystyrene latex nanoparticles (diameter = $100 \mathrm{~nm}$ ). Using a value of $\mathrm{d} n / \mathrm{d} c$ of $0.159 \mathrm{~mL} / \mathrm{g},{ }^{23}$ the $M_{\mathrm{w}}$ of the polystyrene nanoparticles was calculated to be $6.1 \times 10^{5} \pm 2 \times 10^{5} \mathrm{kDa}$ (Figure 3.17). This value is in reasonable agreement with 
that obtained considering solid-like particles with a density of $1.04 \mathrm{~g} / \mathrm{mL}$ and a diameter of 100 $\mathrm{nm}$, which results in $M_{\mathrm{w}}=3.3 \times 10^{5} \mathrm{kDa}$.

Table 3.2. Light scattering intensity obtained for aqueous dispersions of nanoparticles of 1 at different concentrations.

\begin{tabular}{ccc}
\hline$[\mathbf{1}] / \mathbf{~} \mathbf{m g} \cdot \mathrm{mL}^{-1}$ & Count rate $/ \mathbf{~ k c p s}$ & $\mathbf{1 0}^{\mathbf{4}} \boldsymbol{K c} / \mathbf{R}_{\boldsymbol{\theta}} / \mathbf{k D a}^{-1}$ \\
\hline 0.54 & 1058 & 1.36 \\
0.45 & 922 & 1.30 \\
0.36 & 781 & 1.24 \\
0.27 & 654 & 1.11 \\
0.17 & 527 & 0.88 \\
\hline
\end{tabular}

Table 3.3. Calculated concentration of aggregated $\mathbf{1}$ in the samples used in SALS. [1 $]_{\text {ionized }}$ is calculated for a system with $\mathrm{pH}=6.4$ and $\mathrm{p} K_{\mathrm{a}}=7.6$; [1 $]_{\text {free, neutral }}$ corresponds to the cac value: $0.2 \mathrm{mM}, 0.075 \mathrm{mg} / \mathrm{mL}$.

\begin{tabular}{cccc}
\hline$[1]_{\text {total }} / \mathbf{m g} \cdot \mathrm{mL}^{-1}$ & {$[1]_{\text {ionized }} / \mathbf{m g} \cdot \mathrm{mL}^{-1}$} & {$[1]_{\text {free, neutral }} / \mathbf{m g} \cdot \mathrm{mL}^{-1}$} & {$[1]_{\text {nanoparticles }} / \mathbf{m g} \cdot \mathrm{mL}^{-1}$} \\
\hline 0.274 & 0.026 & 0.075 & 0.173 \\
0.377 & 0.036 & 0.075 & 0.266 \\
0.480 & 0.046 & 0.075 & 0.359 \\
0.582 & 0.055 & 0.075 & 0.452 \\
0.685 & 0.065 & 0.075 & 0.545 \\
\hline
\end{tabular}

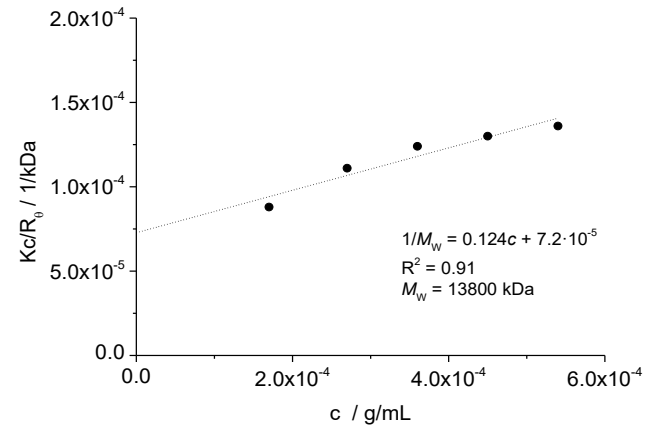

Figure 3.16. Debye plot obtained for the SALS study of the nanoparticles.

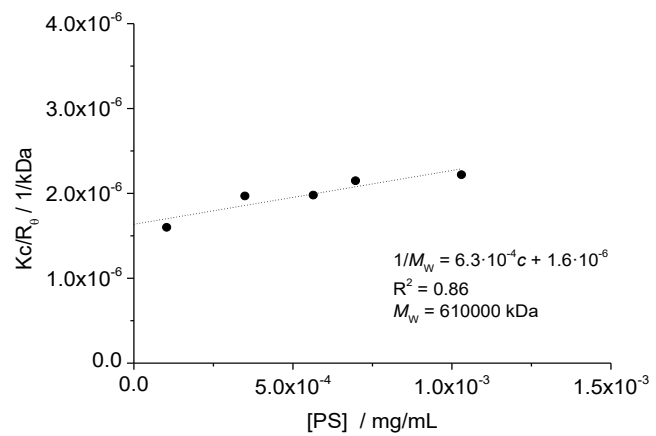

Figure 3.17. Debye plot for determination of $M_{w}$ of standard polystyrene latex particles $(\mathrm{d}=100 \mathrm{~nm})$.

The average concentration of the gelator in the particles is closely related to the so-called overlap concentration, which is used in polymer chemistry and is defined as the point where the concentration within a given polymer particle is equal to the solution concentration. ${ }^{24} \mathrm{By}$ analogy, [1] NP would represent the total sample concentration required for the onset of 
interparticle interactions, leading to gelation. Notably, there is a reasonable agreement between $[1]_{\mathrm{NP}}, 4.7 \pm 2.8 \mathrm{mg} / \mathrm{mL}$, and $\mathrm{mgc}, 5 \mathrm{mg} / \mathrm{mL}$.

The TEM images shown above do not permit distinguishing a fibrillar structure within the particles, such as that observed in the parent macroscopic gels (Figure 3.5). Indeed, the formation of such small nanoparticles by fibres of width as that found in the xerogels does not seem feasible. Consequently, the sonication, rather than fracturing the xerogel fibres, should provoke solubilisation of the monomers, affording local concentrations high enough to form seminal fibrils, which are described as precursors of fibres in the formation of macroscopic gels. ${ }^{25}$ The gel-like nature of the particles should be ascribed to the entanglement of these seminal fibrils into spherical particles. Therefore, the nanogel particles would correspond to the initial stages of the aggregation of molecular gels, and constitute an intermediate state between free molecules and fibrillar objects. In this regard, kinetic studies usually demonstrate that molecular gels are formed by a nucleation-growth mechanism, ${ }^{3,26,27}$ and it has been recently reported that fibres could be formed by scrolling of supramolecular lamellae that present asymmetric surfaces. ${ }^{28}$

Finally, the biocompatibility of the gelator and the nanoparticles in human lung carcinoma cells (A549) was found to be high, with IC 50 values above $250 \mu \mathrm{M}$. IC 50 value is the half maximal inhibitory concentration or compound concentration capable of inhibit the $50 \%$ of cell proliferation. These values were obtained using the MTT (3-(4,5-dimethylthiazol-2-yl)-2,5diphenyltetrazolium bromide) dye reduction assay, a colorimetric assay based in the metabolic reduction of MTT (yellow) to formazan (violet) by a mitochondrial enzyme. In this assay, cells were incubated with serial dilutions of the tested compounds for $48 \mathrm{~h}$ and MTT assay revealed the quantity of viable cells per cell population. ${ }^{29,30}$

\subsubsection{OPTIMISATION OF THE PREPARATION OF NANOGELS}

The procedure reported in the previous section for nanogels preparation was obtained after an optimisation process, but was not the only path that lead to nanogels. For example, if ethyl acetate was used instead of toluene for gel preparation, nanoparticles with similar sizes were obtained. DLS analysis of a representative sample of these nanoparticles is shown in Figure 3.18. An average of $207 \pm 5 \mathrm{~nm}$ was obtained as intensity averaged diameter $\left(D_{\mathrm{i}}\right)$ and of $45 \pm 20$ 
$\mathrm{nm}$ as number averaged diameter $\left(D_{\mathrm{n}}\right)$. However, the PDI of these particles is higher (around 0.4). Similar size distribution and gelator concentration were also obtained in the case of using a xerogel obtained in an aqueous medium, Figure 3.19, with $D_{\mathrm{i}}=149 \pm 10 \mathrm{~nm}$ and $D_{\mathrm{n}}=51 \pm 7$ $\mathrm{nm}$. These results highlight that the solvent in which the xerogel is obtained is not, a priori, a key parameter in the preparation of the nanoparticles. However, sonication of a finely powered solid gelator did not afford the nanoparticles, revealing that the high surface ratio of the fibrillar xerogels is essential for their transformation into nanogels. It was decided to focus on the study of the particles obtained from the xerogel in toluene because, for future envisaged studies, the use of an organic solvent should be favourable for the entrapment of actives which are poorly soluble in water.

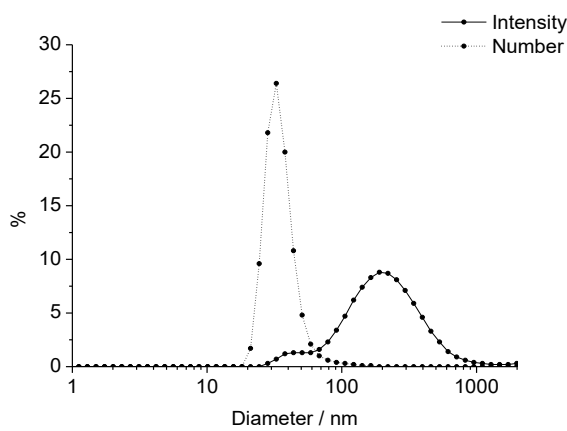

Figure 3.18. DLS analysis of size distribution by intensity (solid line) and by number (dotted line) of a representative sample of nanogel particles obtained from a xerogel from ethyl acetate.

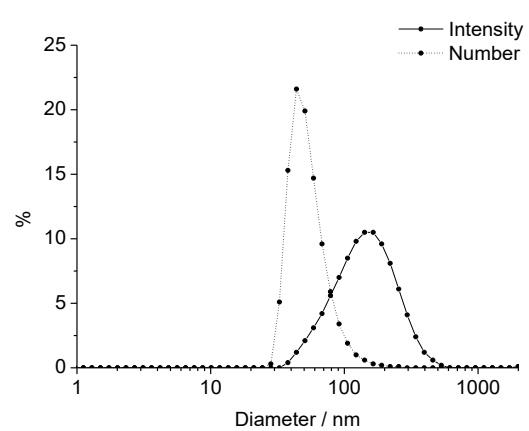

Figure 3.19. DLS analysis of size distribution by intensity (solid line) and by number (dotted line) of a representative sample of nanogel particles obtained from a xerogel from water.

Other aspects evaluated in the optimisation process were the initial gelator concentration, which does not affect to nanogels size, or sonication time, which leads to aggregation when increased. The $\mathrm{pH}$ of the suspension medium was also determined as key parameter. Neutral $\mathrm{pH}$ values were optimum, whereas below $\mathrm{pH} 5$ aggregates were obtained. Also, there is a need for ionic strength in the medium to obtain stable nanoparticles over time, otherwise they tend to aggregate with time.

\subsubsection{NILE RED ENCAPSULATION INTO NANOGELS}

The results mentioned above on the determination of cac demonstrate that the initial aggregates formed upon increasing concentration of 1 can entrap hydrophobic species such as 
pyrene, but no nanogel preparation was carried out in that study. To test the accessibility of the hydrophobic domains of the nanogel particles, experiments using the fluorescent dye Nile red were carried out. This polarity-sensitive probe is almost nonfluorescent in water and other polar solvents but shows intense fluorescence emission in nonpolar environments. Additionally, the absorption and emission maximum of this probe is strongly affected by the polarity of the medium (Figure 3.20 and Table 3.4). ${ }^{31}$ For example, the emission maximum wavelength experiments a dramatically change from $641 \mathrm{~nm}$ in water to $532 \mathrm{~nm}$ in hexane. This property is explained by dipole moment of the excited state of Nile red, originated by charge separation between the diethyl amine as an electron donor and the quinoid part of the molecule as an electron acceptor. ${ }^{32}$ For this reason, Nile red has been used to estimate the local environment of the pockets available in nanoparticles or aggregates. ${ }^{32,33}$
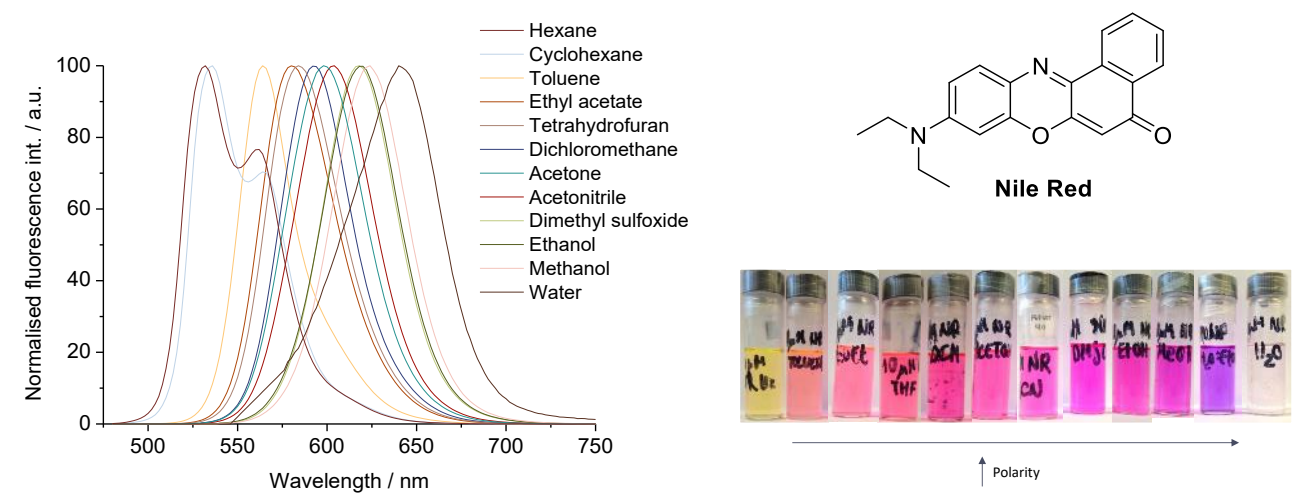

Figure 3.20. $10 \mu \mathrm{M}$ Nile red in different solvents and their normalised fluorescence emission spectra $\left(\lambda_{\mathrm{ex}}\right.$ at the corresponding absorption maximum) and Nile red molecule.

Table 3.4. Absorption and fluorescence emission maximum for Nile red in different solvents.

\begin{tabular}{lcc}
\hline \multicolumn{1}{c}{ Solvent } & Absorption $\boldsymbol{\lambda}_{\max } / \mathbf{n m}$ & Fluorescence emission $\boldsymbol{\lambda}_{\max } / \mathbf{n m}$ \\
\hline Hexane & 507 & 532 \\
Cyclohexane & 511 & 535 \\
Toluene & 522 & 564 \\
Ethyl acetate & 523 & 581 \\
Tetrahydrofuran & 527 & 584 \\
Dichloromethane & 538 & 593 \\
Acetone & 532 & 598 \\
Acetonitrile & 534 & 604 \\
Dimethyl sulfoxide & 551 & 618 \\
Ethanol & 548 & 619 \\
Methanol & 552 & 624 \\
Water & 544 & 641 \\
\hline
\end{tabular}


Two strategies were followed to encapsulate a hydrophobic molecule as Nile red into nanogels of 1 (Nile red@1): (a) preparation of nanogels in the presence of the cargo, or (b) addition of the cargo once nanoparticles were formed (Figure 3.21). In the first case, the molecule was added in the suspension of the gelator before gel preparation, expecting an interaction with the fibres once formed. In the second case, the preference of the probe to interact with the hydrophobic spaces of the nanogels rather than being in water was contemplated to lead its encapsulation.

(a) Preparation of nanogels in the presence of the cargo

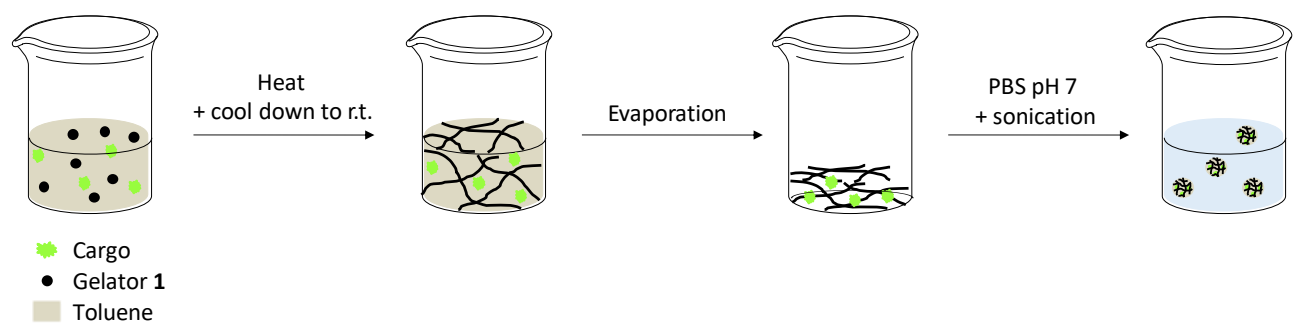

(b) Addition to nanogels sample

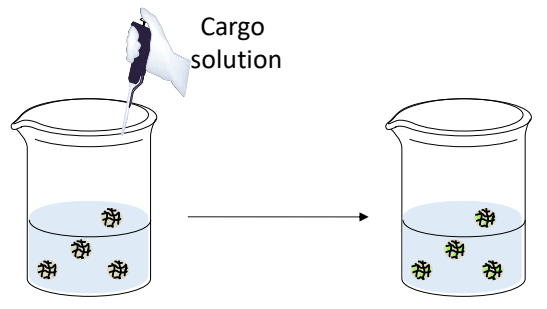

Figure 3.21. Strategies followed to encapsulate molecules into nanogels of $\mathbf{1}$ by sonication method.

In the first strategy, Nile red was added as a solution in toluene to obtain a nominal $5 \mu \mathrm{M}$ concentration in nanogel. For comparison purposes, a control experiment (NR control) of the procedure without gelator was also performed. In the second approach, $1.5 \mathrm{~mL}$ of a $10 \mu \mathrm{M}$ Nile red solution in PBS-0.4\%EtOH were mixed with $1.5 \mathrm{~mL}$ of nanogels sample (or $1.5 \mathrm{~mL}$ of PBS for NR control) and $20 \mathrm{~min}$ of stabilisation were required to obtain encapsulated Nile red. ${ }^{32}$ Identical fluorescence emission spectra were obtained for both strategies. As can be seen in Figure 3.22, a notable increase in fluorescence was observed compared with the control in the absence of nanogels and the emission maximum of Nile red@1 was shifted from $641 \mathrm{~nm}$ to $587 \mathrm{~nm}$, indicating a quite hydrophobic environment for the probe. Therefore, these results highlight the presence of accessible hydrophobic domains in the nanogel 
particles, demonstrating their potential use for the entrapment of poorly soluble organic actives.

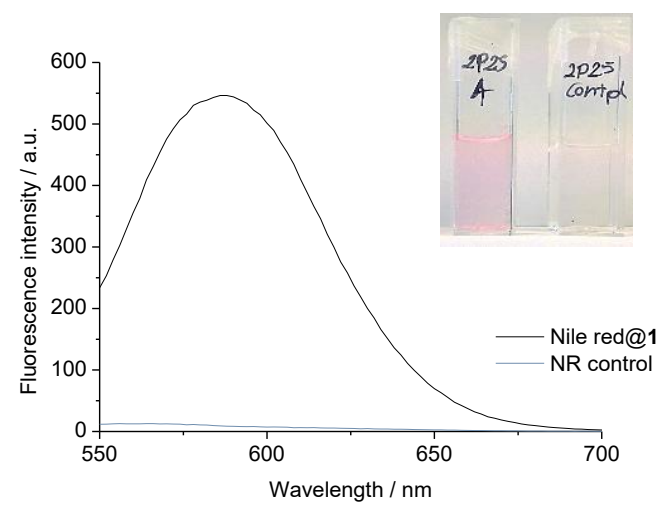

Figure 3.22. Fluorescence emission spectra $\left(\lambda_{\mathrm{ex}} 530 \mathrm{~nm}\right.$ ) of a representative Nile red@1 sample (black) and the corresponding control (blue). Inset: Cuvettes containing nanogel particles loaded with Nile red (left) and free Nile red (right).

In addition, Nile red encapsulation does not interfere with the size of nanogels. According to DLS results for a representative example in Figure 3.23, an average of $263 \pm 21 \mathrm{~nm}$ was obtained as intensity averaged diameter $\left(D_{\mathrm{i}}\right)$ and of $99 \pm 9 \mathrm{~nm}$ as number averaged diameter $\left(D_{n}\right)$.

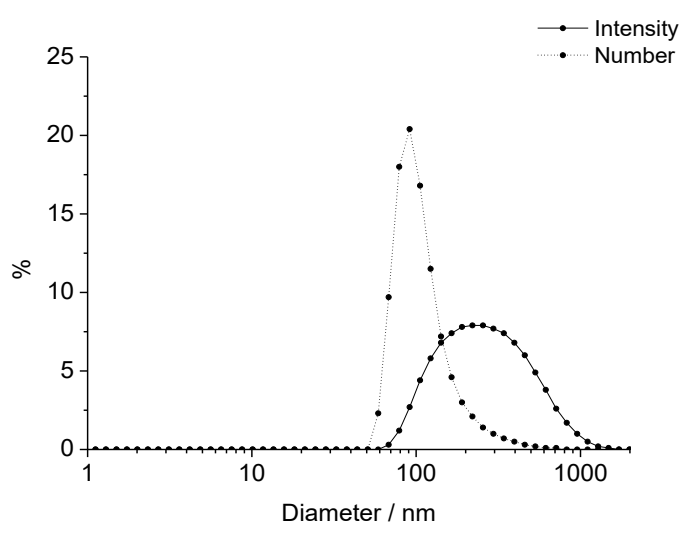

Figure 3.23. Size distribution by intensity (black line) and by number (dotted line) of a representative Nile red@1 sample obtained by DLS analysis. 


\subsubsection{STIMULI RESPONSE OF NANOGELS}

Molecular gels have been reported to respond to stimuli and assemble/disassemble depending on the environment. This behaviour was studied in nanogels of 1 .

\subsubsection{RESPONSE TO TEMPERATURE CHANGES}

The stability towards temperature between 30 and $75^{\circ} \mathrm{C}$ was assayed by DLS (Figure 3.24). The intensity of scattered light is measured in DLS by the number of photons per second arriving at the detector, which is known as count rate. The higher signal strength usually indicates higher concentration or larger particles. Attenuators are used before measuring to fit this light intensity in the adequate range of detection and derived count rate is the theoretical count rate one would obtain with zero attenuation. As shown in Figure 3.24, the intensity averaged diameter was not affected by temperature changes but, on the other hand, the intensity of scattered light dropped significantly at $50^{\circ} \mathrm{C}$, suggesting partial solubilisation of 1.
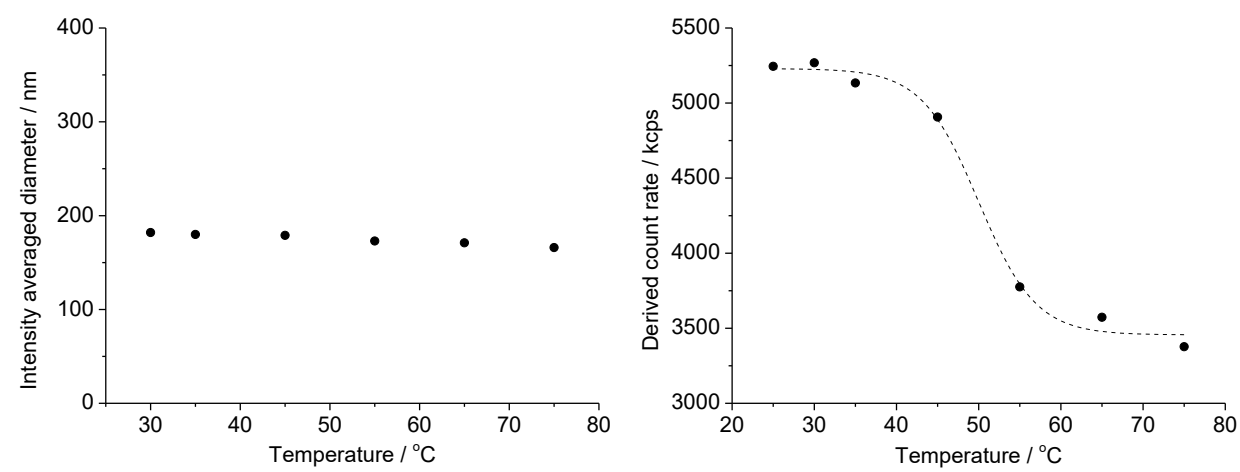

Figure 3.24. Influence of temperature on the size of the nanogels (left) and on the intensity of scattered light (right).

\subsubsection{RESPONSE TO pH CHANGES}

As explained earlier, the presence of an ionizable carboxylic unit in 1 results in $\mathrm{pH}$-dependent gelation properties, being only the protonated neutral species responsible for gelation (Figure 3.2). Therefore, we hypothesised that at $\mathrm{pH}$ values above its $\mathrm{p} K_{\mathrm{a}} 7.6$, nanogels of 1 would disassemble. Nile red@1 nanogels were used to study this response to $\mathrm{pH}$ changes: at $\mathrm{pH}$ values above 7.6, a release of the cargo and, then, a change in Nile red emission spectra, were expected from the disassembly of the nanogels. A Nile red@1 sample was divided in four 
aliquots and $\mathrm{pH}$ was changed to 7,11, 12 and 13. Fluorescence emission intensity at the $\lambda_{\max }$ (ca. $570 \mathrm{~nm}$ ) for each aliquot over time was plotted in Figure 3.25. A decrease of the Nile red emission of almost an $80 \%$ was observed from day 1 for $\mathrm{pH} 13$, and of $35 \%$ for $\mathrm{pH} 11$ and 12 , whereas just a $4 \%$ decrease was observed for $\mathrm{pH} 7$ after 7 days. In that cases in which the maximum decreases, a peak at $640 \mathrm{~nm}$ corresponding to Nile red in water appeared with time (Figure 3.26). Therefore, it seems that Nile red is being released from the hydrophobic pockets when nanogels are at $\mathrm{pH}$ values above 11. Besides, it also seems that nanoparticles can entrap and keep non-polar substances at $\mathrm{pH}$ 7. Therefore, the release seems to be only produced in response to a stimulus and not due to dye diffusion towards the solution, which is a good characteristic for nanocarriers.

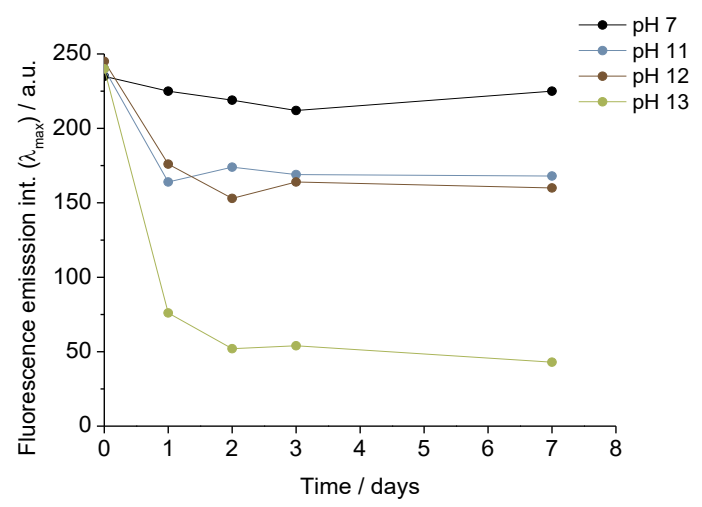

Figure 3.25. Fluorescence emission intensity at the maximum wavelength for aliquots of a NR@1 sample at different $\mathrm{pH}$ over time $\left(\lambda_{\mathrm{ex}} 530 \mathrm{~nm}\right)$.

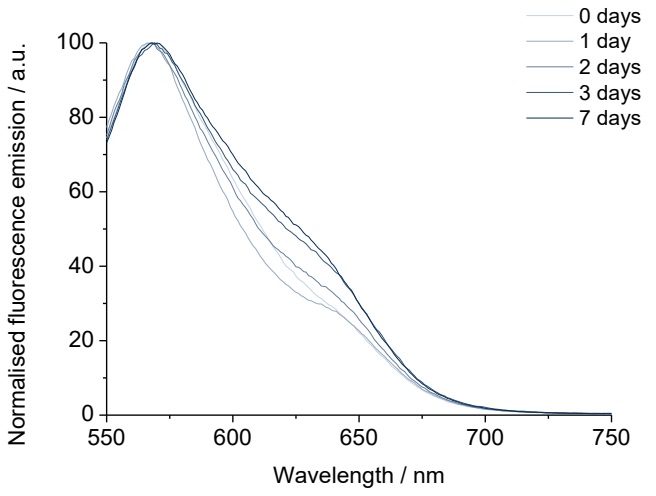

Figure 3.26. Normalised fluorescence emission spectra for a NR@1 sample at pH 11 over time $\left(\lambda_{\mathrm{ex}} 530 \mathrm{~nm}\right)$.

This effect was also studied by DLS. The $\mathrm{pH}$ of an aliquot of a nanogels sample was changed to 13 by addition of $1 \mathrm{M} \mathrm{NaOH}$ and size was measured after $24 \mathrm{~h}$. Results for two representative examples can be found in Figure 3.27, together with the ones for the corresponding original nanogels sample after $24 \mathrm{~h}$, as a control. Although no significant changes were observed in number $\left(D_{n}\right)$ averaged diameter, an increase of polydispersity index, which was also reflected in an increased intensity averaged diameter $\left(D_{\mathrm{i}}\right)$ and its error, was observed. For example, PDI of example $A$ goes from 0.25 to 0.74 and, for example $B$, from 0.41 to 0.77 . Studies with different samples and $\mathrm{pH}$ values showed that this increase is produced from $\mathrm{pH} 11$. The reason for these alterations at basic $\mathrm{pH}$ could be found in a structural change of nanogels to objects with similar size, but with a lower hydrophobic encapsulation power that would release Nile red. 

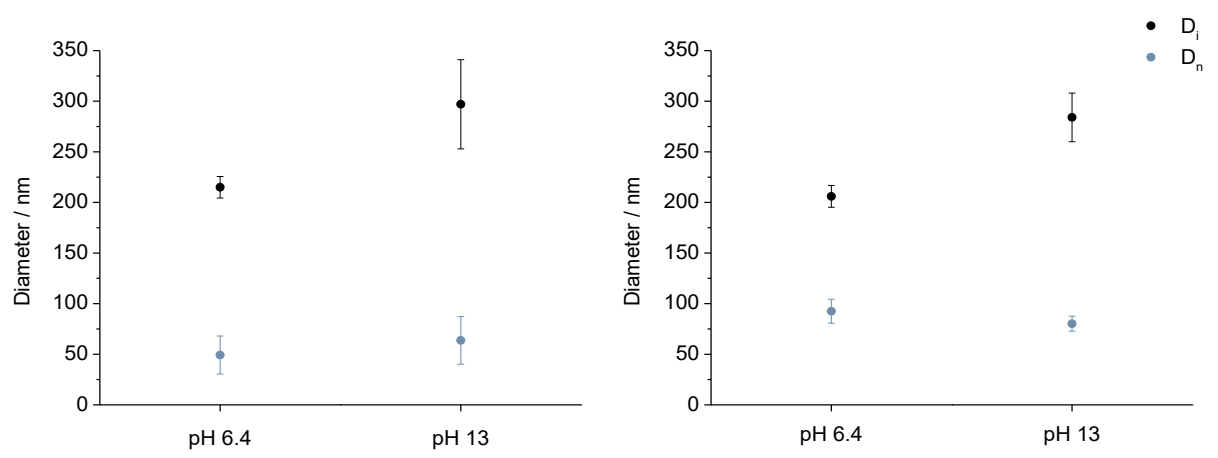

Figure 3.27. Size measurement after $24 \mathrm{~h}$ of the original nanogels sample and an aliquot at $\mathrm{pH} 13$ for two representative examples.

These objects formed by ionised compound $\mathbf{1}$ have been labelled as micellar aggregates in the scheme of Figure 3.28, which summarises the forms adopted depending on $\mathrm{pH}$ and gelator concentration. The ionic form of compound $\mathbf{1}$ has an amphiphilic character and would tend to form micelles in water. These micelles (with size of few nanometres) could be forming, for example, micellar aggregates when increasing gelator concentration. ${ }^{34}$ The critical micelle concentration (CMC) for compound $\mathbf{1}$ at two basic $\mathrm{pH}$ values was calculated by pyrene fluorescence changes and found to be $0.7 \mathrm{mM}(\mathrm{pH} 11.6)$ and $1.6 \mathrm{mM}(\mathrm{pH}$ 13). Solutions of compound 1 ( $3 \mathrm{mM}$ ) at different basic $\mathrm{pH}$ were analysed by DLS and again structures with sizes in the range of those found to nanogels were obtained, although the dispersity of these samples was much higher. These structures were also studied by TEM without conclusive results.

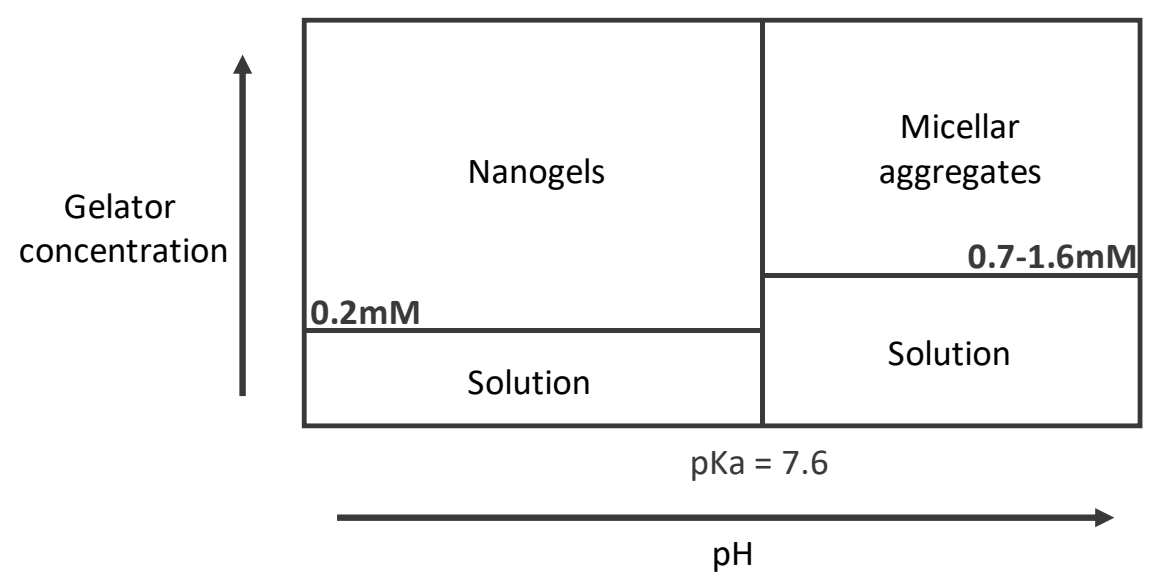

Figure 3.28. Scheme of the forms adopted by compound $\mathbf{1}$ depending on $\mathrm{pH}$ and concentration. 


\subsubsection{RESPONSE TO IONIC STRENGTH CHANGES}

It has been reported that ions can have an influence on the solubility of molecular hydrogels and cause their disassembly. ${ }^{35}$ The effect of the concentration of sodium chloride found in physiological media was studied to see the biocompatibility with our systems. No changes in particle size was observed after $154 \mathrm{mM} \mathrm{NaCl}$ addition (Figure 3.29) to nanogels prepared in $10 \mathrm{mM}$ phosphate buffer, even at different nanoparticles concentrations.
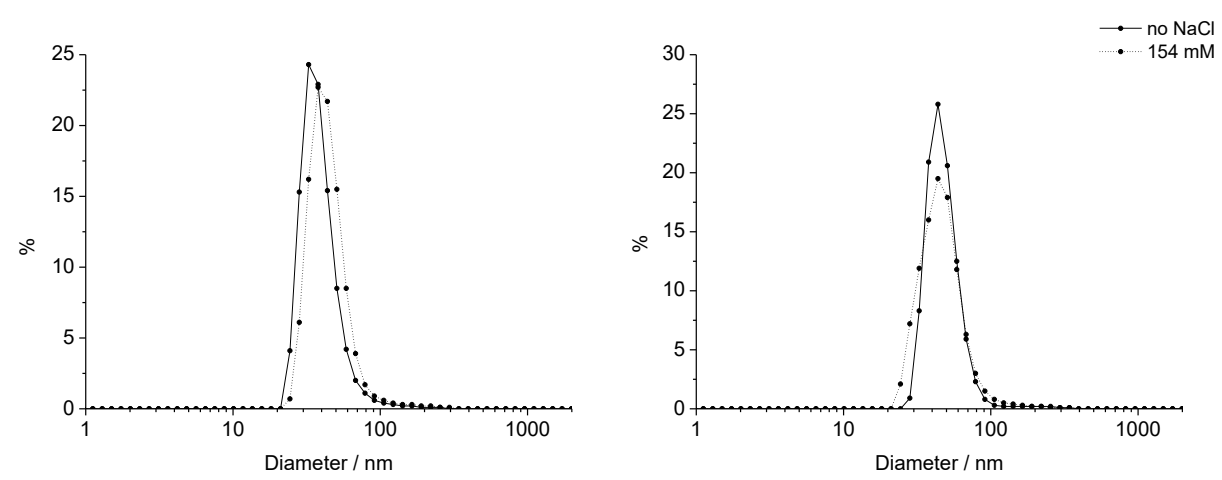

Figure 3.29. Size distribution by number obtained by DLS of a nanogels sample before (solid line) and after addition of $154 \mathrm{mM} \mathrm{NaCl}$ (dotted line). Left: $150 \mu \mathrm{M}$ of 1 (from 42 to $56 \mathrm{~nm}$ ), right: $100 \mu \mathrm{M}$ of 1 (from 47 to $45 \mathrm{~nm}$ ). 


\subsection{NANOGELS PREPARED USING LIPOSOMES AS A TEMPLATE}

This section is mainly based on the "Results and Discussion" part of the paper "TorresMartínez, A.; Angulo-Pachón, C. A.; Galindo, F.; Miravet, J. F. Liposome-Enveloped Molecular Nanogels. Langmuir 2019, 35, 13375-13381".

The strategy followed to prepare nanogels of compound $\mathbf{1}$ using liposomes as a template is depicted in Figure 3.30. The process starts with the formation of liposomes in a solution of the gelator at $\mathrm{pH} 9$ and their isolation from molecules outside the obtained vesicles. Then, hydrogel@liposome particles are obtained by pH-triggered molecular gel formation inside of liposomes after acidification to $\mathrm{pH}$ 5. Removal of the lipid bilayer yields naked nanogel particles.

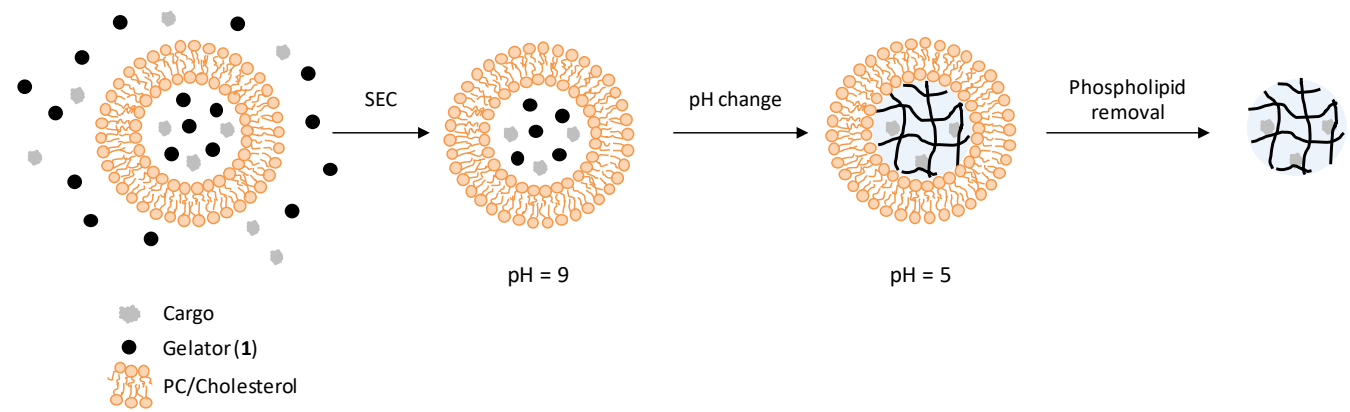

Figure 3.30. Outline of the preparation of nanogels of 1 using liposomes as a template and entrapping a cargo.

\subsubsection{LIPOSOME FORMATION AND pH CHANGE STUDIES}

Before nanogel@liposome development, liposome formation and changes in their internal pH were assayed. Liposomes were made using L- $\alpha$-phosphatidylcholine (PC) and cholesterol (8:2 molar ratio) (Figure 3.31). The addition of cholesterol provides a more robust bilayer, reducing leakage from the liposomes. ${ }^{36}$ The preparation process involved the hydration of the dry lipid film with a pH 9 phosphate buffer and sonication. ${ }^{37,38}$ Small unilamellar vesicles were obtained after size-exclusion chromatography (SEC), as could be visualized by transmission electron microscopy (TEM, Figure 3.32). 
<smiles>[R]C(=O)OC[C@H](COP(=O)([O-])OCC[N+](C)(C)C)OC([R])=O</smiles>

Phosphatidylcholine $R, R^{\prime}=$ fatty acid residues

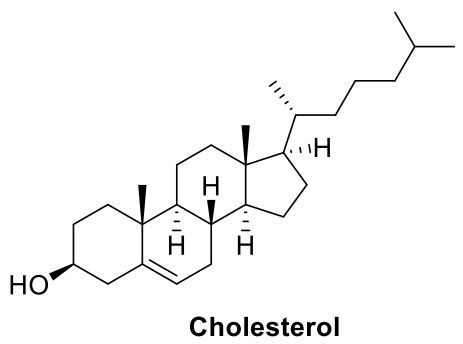

Cholesterol

Figure 3.31. Structure of the components of liposomes.
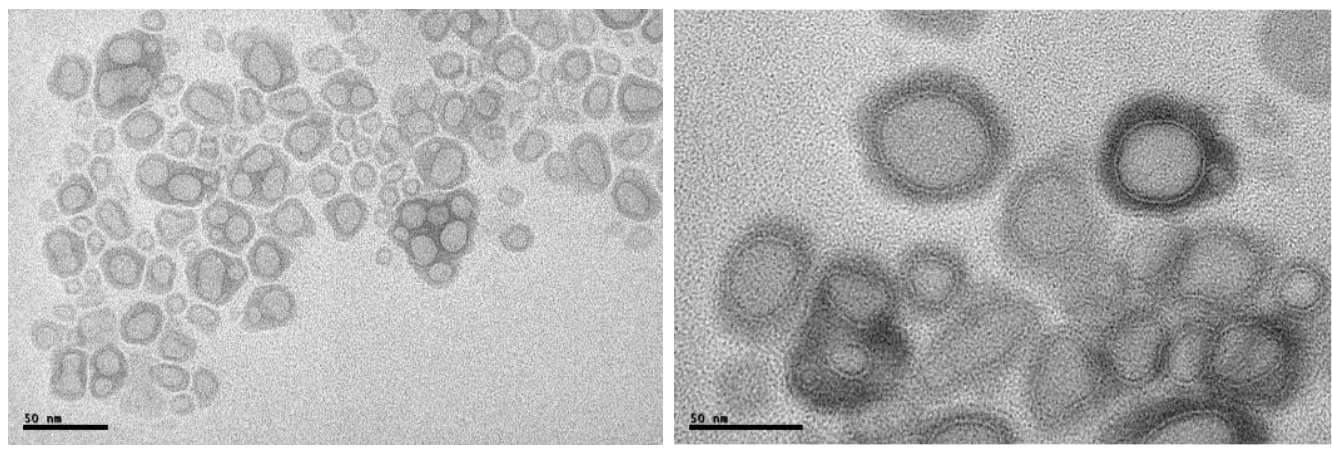

Figure 3.32. TEM images (no staining) of the liposomes prepared at $\mathrm{pH}=9$ (without hydrogelator).

The changes in the intraliposomal pH were studied using pyranine, a water-soluble, membrane-impermeable fluorescent molecule. Pyranine-loaded liposomes were prepared at $\mathrm{pH} 9$ and isolated by SEC, in which pyranine was used as a marker to follow the separation process of the vesicles. Then, the system was acidified by the addition of D-glucono-1,5lactone, which affords progressive, smooth acidification, useful in molecular gel formation. ${ }^{39}$ The changes of $\mathrm{pH}$ inside the liposomes could be evaluated from the pyranine excitation spectrum, which consists of two independent contributions: the neutral molecule (400 nm) and its deprotonated conjugate base $(450 \mathrm{~nm}$ ) (Figure $3.33 \mathrm{left}$ ). The ratio of fluorescence excitation at 460 and $415 \mathrm{~nm}$, monitoring emission at $511 \mathrm{~nm}$, thereby gives a ratiometric $\mathrm{pH}$ measure. ${ }^{40-42} \mathrm{~A}$ pyranine $\mathrm{pH}$ calibration curve (Figure 3.33 right), obtained from standard solutions at different $\mathrm{pH}$, was used to determine that, after $2 \mathrm{~h}$, the $\mathrm{pH}$ inside the vesicles changed from 9 to 6.3 by D-glucono-1,5-lactone acidification (see Figure 3.34). This pH value is compatible with gel formation in the case of compound $1\left(p K_{a}=7.6\right)$, as was checked by the formation of macroscopic gels of $\mathbf{1}$ under these conditions. After $\mathrm{pH}$ change, liposomes were stable regarding their size. 

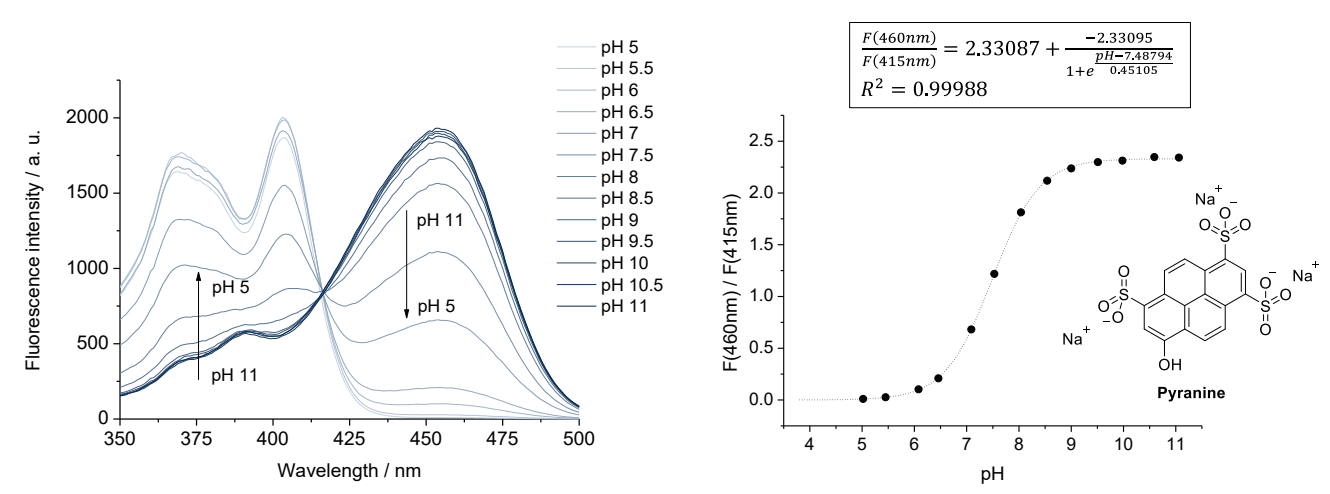

Figure 3.33. Fluorescence excitation spectra $\left(\lambda_{\mathrm{em}} 511 \mathrm{~nm}\right)$ of pyranine standards $(1 \mu \mathrm{M}$ in phosphate buffer $0.1 \mathrm{M}$ ) at different $\mathrm{pH}$ (from 5 to 11 ) (left) and the obtained pH calibration curve (right). Inset: pyranine chemical structure.

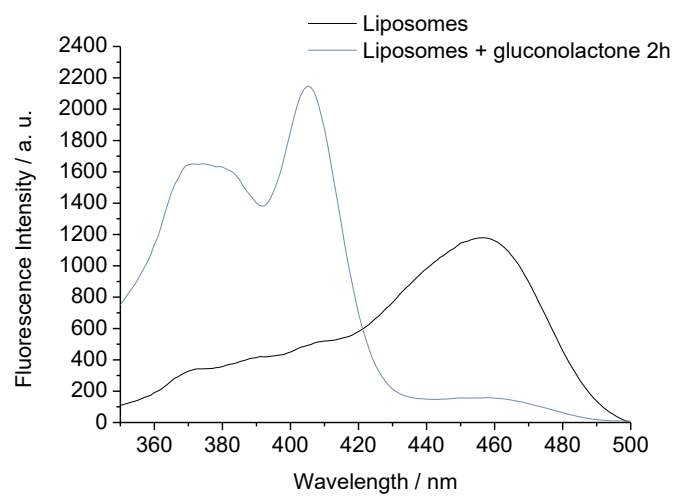

Figure 3.34. Fluorescence excitation spectra $\left(\lambda_{\mathrm{em}} 511 \mathrm{~nm}\right)$ of pyranine@liposomes before (black, pH 9) and after $2 \mathrm{~h}$ of external $\mathrm{pH}$ change with gluconolactone (blue, $\mathrm{pH} 6.3$ ).

\subsubsection{PREPARATION AND CHARACTERISATION OF NANOGEL@LIPOSOMES AND NANOGELS}

Next, following the preparation of nanogel@liposomes outlined in Figure 3.30, liposomes were assembled at pH 9 in the presence of the ionized, soluble form of gelator $\mathbf{1}$ and pyranine. After separation of the loaded liposomes from the non-encapsulated molecules by SEC, dynamic light scattering (DLS) revealed the formation of particles with a number averaged diameter of $41 \pm 24 \mathrm{~nm}$ (Figure $3.38 \mathrm{top}$ ). Then, the system was acidified to protonate gelator 1, promoting self-assembly and affording gel@liposome hybrid particles. The particles were analysed by TEM (Figure 3.35) and DLS (Figure 3.38 centre), revealing a very similar size 
distribution to that found for the initial liposomes, with number averaged diameters obtained by DLS and TEM (Figure 3.39), respectively, of $37 \pm 10 \mathrm{~nm}$ and $95 \pm 19 \mathrm{~nm}$. Additionally, cryoTEM images could be obtained (Figure 3.36), revealing larger diameters with an average value of $293 \pm 90 \mathrm{~nm}$. Such differences between diameters obtained by DLS, TEM, and cryo-TEM might be caused by aggregation phenomena associated with sample preparation in the electron microscopy techniques.
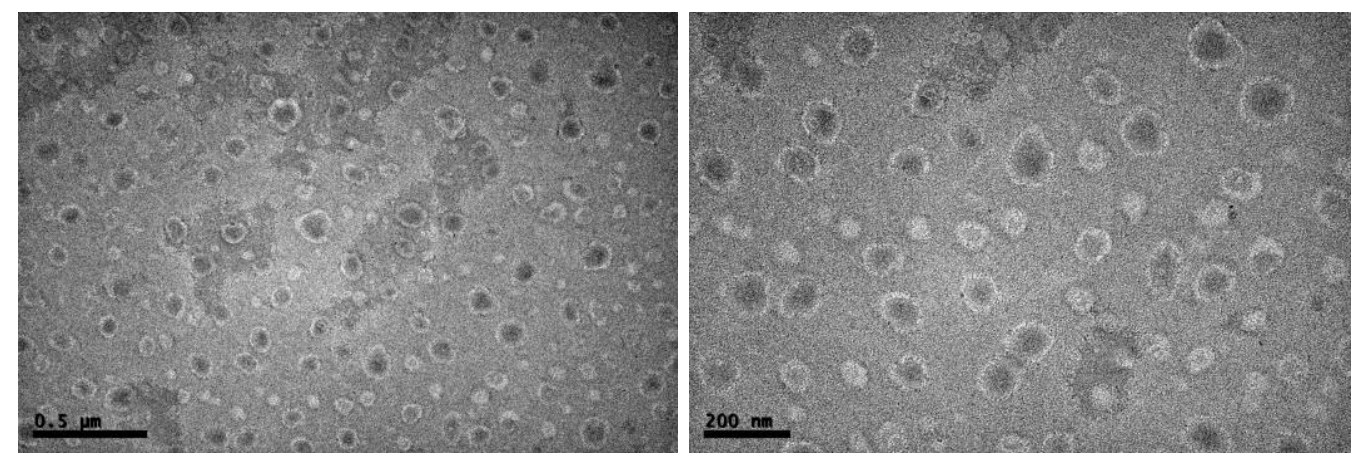

Figure 3.35. TEM image (no staining) of gel@liposome particles.

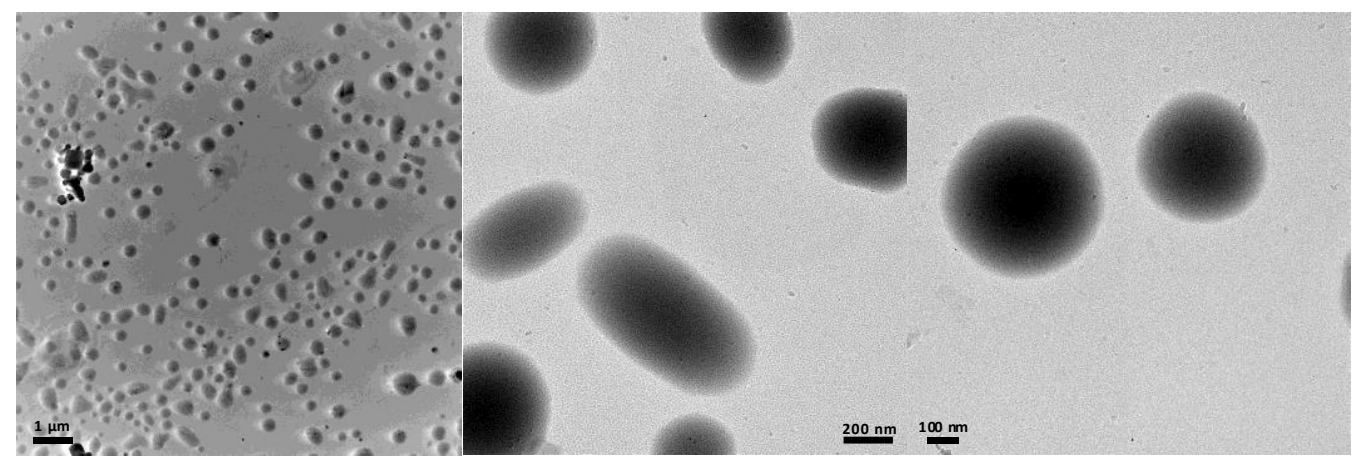

Figure 3.36. Cryo-TEM images of gel@liposome particles.

Liposome disassembly permitted to obtain naked nanogel particles. For this purpose, sodium dodecyl sulfate (SDS) was added, and dialysis against water was performed to remove the mixed micelles of SDS-PC and any free gelator that could have leaked from the particles. TEM images corroborated the formation of nanogel particles (Figure 3.37), and DLS revealed a number averaged diameter of $51 \pm 25 \mathrm{~nm}$ as the average of ten samples, presenting the size distribution a broad tail in the region of big particles (Figure $\mathbf{3 . 3 8}$ bottom). These larger particles may arise from aggregates formed between SDS and the phospholipid ${ }^{43}$ which were not removed by dialysis, or from the interaction of SDS with the nanogel particles. ${ }^{44}$ 


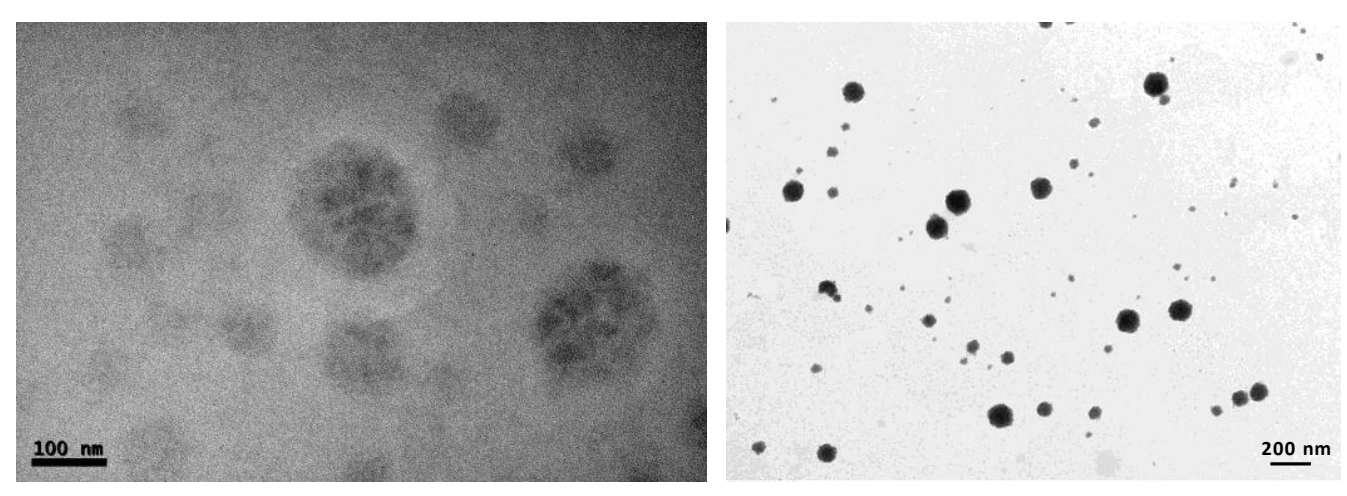

Figure 3.37. TEM images of nanogel particles. Left: no staining; right: $\mathrm{OsO}_{4}$ staining.
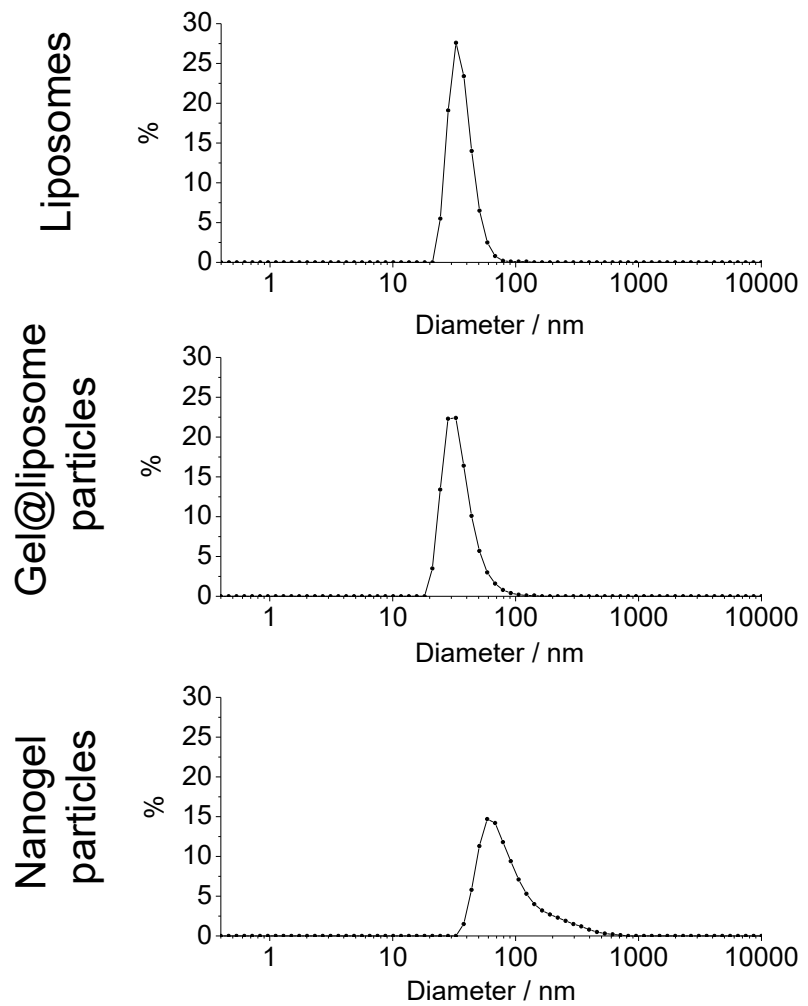

Figure 3.38. Number size distribution of a representative sample of gelator@liposomes (pH 9), gel@liposome particles, and nanogel particles obtained by DLS analysis. 

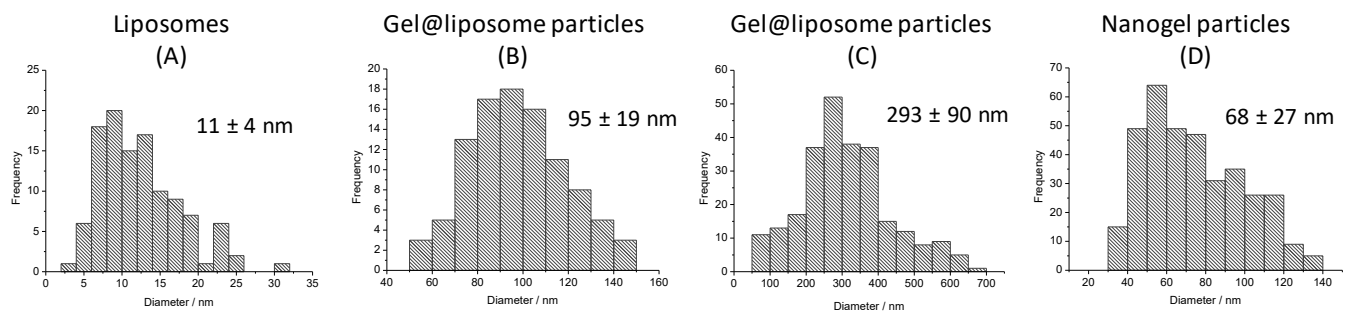

Figure 3.39. Size distribution of the particles obtained from TEM and cryo-TEM images: Figure 3.32 (A), Figure 3.35 (B), Figure 3.36 (C) and Figure 3.37 (D). Mean particle size indicated for each histogram. At least 100 particles measured for each histogram.

The quantification of the concentration of the gelator in the aqueous samples containing naked hydrogel particles was found to be $0.7 \mathrm{mM}$. This determination was carried out dissolving a lyophilized sample in $\mathrm{CDCl}_{3}$ and measuring the integration of the signals in the ${ }^{1} \mathrm{H}$ NMR spectrum against an external standard. Furthermore, the study of different samples using pyranine revealed pH values of 5-6 for both lipogels and nanogel particles (Figure 3.40).

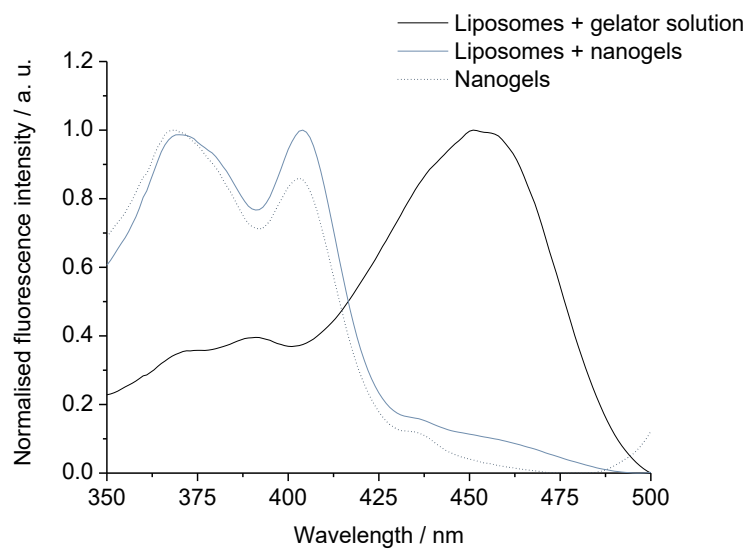

Figure 3.40. Normalized fluorescence excitation spectra $\left(\lambda_{\mathrm{em}}=511 \mathrm{~nm}\right)$ of a gelator@liposomes sample (black solid line, pH 8.2), gel@liposome particles (blue line, pH 6.5) and nanogel particles (dotted line, pH 6.4).

Regarding stability, gel@liposome particles and nanogels were stable at least for a month at $4^{\circ} \mathrm{C}$ according to DLS measurements, which revealed a similar size distribution although slightly shifted towards larger particles, presumably because of aggregation (Figure 3.41). 

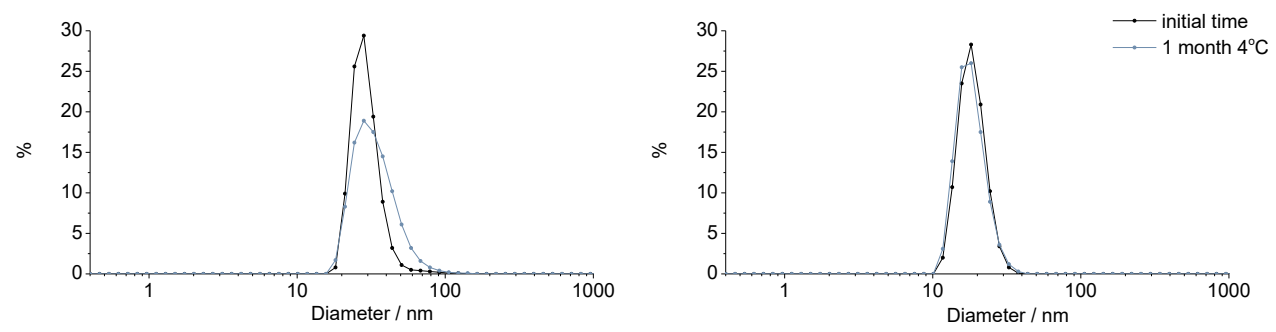

Figure 3.41. Number averaged size distribution of a representative sample of gel@liposome particles (left) and nanogels of $\mathbf{1}$ (right) obtained by DLS analysis: immediately after preparation (black) and one month later kept at $4^{\circ} \mathrm{C}$ (blue).

\subsubsection{NANOGEL@LIPOSOME PARTICLES AS CARRIERS}

The ability of the novel gel@liposome particles to entrap and release doxorubicin was tested. Doxorubicin is the most effective chemotherapeutic drug developed against a broad range of cancers. ${ }^{45}$ Because of its cardiotoxicity, doxorubicin is loaded into liposomes (MyocetV) and pegylated liposomes (DOXIL) in clinically approved formulations. Aiming to improve its therapeutic efficiency, hundreds of papers have explored the use of different nanocarriers for doxorubicin, including, among others, dextran, polylactic acid, solid-lipid nanoparticles or polymeric nanogels. ${ }^{46}$ The preparation of the lipogel particles was carried out using a $1 \mathrm{mM}$ doxorubicin solution as hydration medium. UV-vis and fluorescence spectroscopy revealed its incorporation into the lipogel particles. As can be seen in Figure 3.42, fluorescence spectra for free and lipogel-loaded doxorubicin are quite similar, but the emission of doxorubicin in the lipogel is slightly red-shifted compared to that of plain liposomes at the same $\mathrm{pH}$. It has to be considered that the hydrophilic nature of the drug probably precludes its complete adsorption on the nanogel network, being solvated in the aqueous pools. However, this shift implies a small difference in solvation, which would indicate that there is an interaction with the gel fibres, although the environment remains essentially aqueous. Additionally, doxorubicin release from the lipogels and liposomes was studied using a dialysis membrane against a $\mathrm{pH} 7$ solution. ${ }^{47-50}$ The amount of doxorubicin outside the dialysis tubing was quantified by UV-vis and fluorescence spectroscopy, revealing a rather similar release from both systems (ca. 20\% after $24 \mathrm{~h}$, see Figure 3.43). Therefore, the lipogels presented here maintain the capability of entrapping doxorubicin found in pure liposomes. It seems reasonable that the gel does not alter the doxorubicin release considering the hydrophilic nature of this drug and its low 
molecular weight, which does not limit the diffusion through the gel network. Also, it has to be considered that the dialysis process at pH 7 may, at least partially, disassemble the nanogel core considering that the $\mathrm{p} K_{\mathrm{a}}$ of the gelator is 7.6.

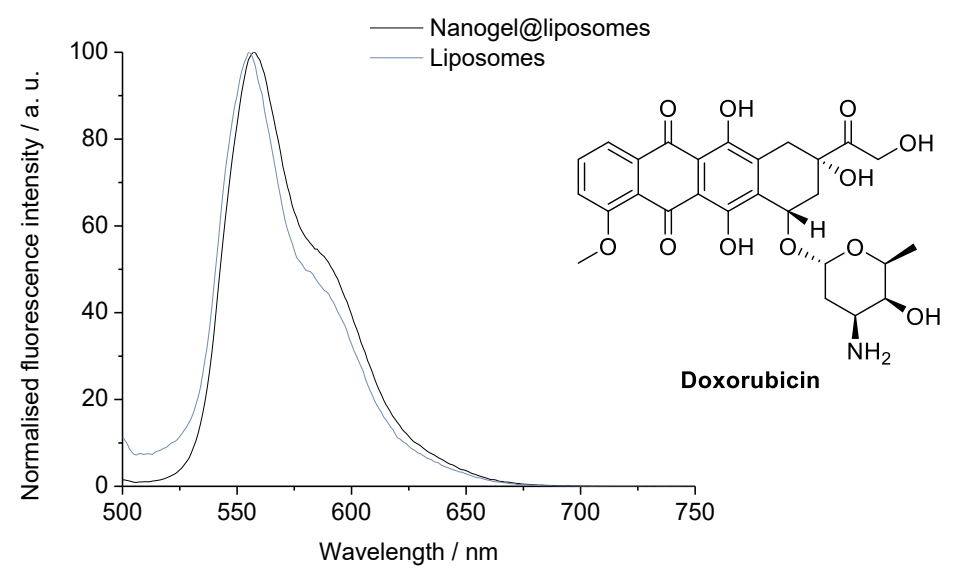

Figure 3.42. Comparison of doxorubicin emission spectra $\left(\lambda_{\mathrm{ex}} 480 \mathrm{~nm}\right)$ in liposomes (blue) and lipogels (black). Inset: doxorubicin structure.

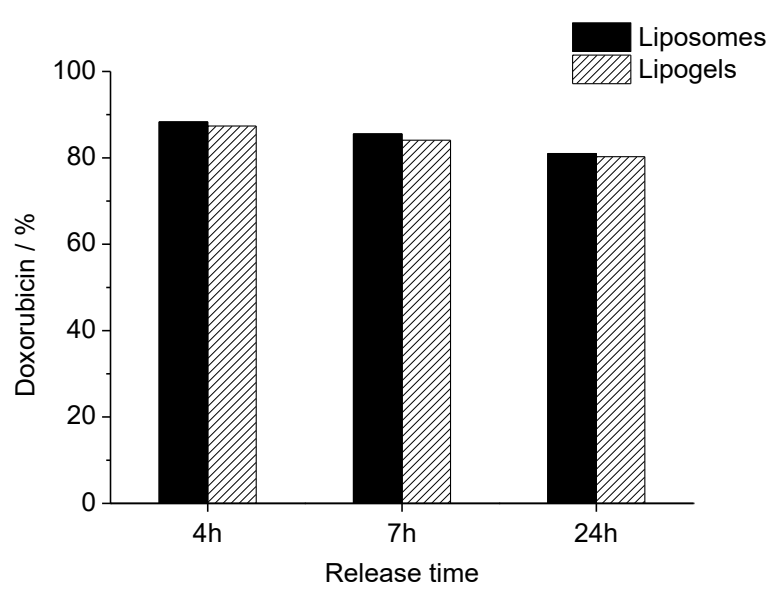

Figure 3.43. Percentage of doxorubicin kept in the dialysis membrane after the indicated times of dialysis for doxorubicin@liposomes (black) and doxorubicin@lipogels (pattern). 


\subsection{REFERENCES}

Tang, C.; Smith, A. M.; Collins, R. F.; Ulijn, R. V.; Saiani, A. Fmoc-Diphenylalanine Self-Assembly Mechanism Induces Apparent PKa Shifts. Langmuir 2009, 25 (16), 9447-9453.

Chen, L.; Revel, S.; Morris, K.; Serpell, L. C.; Adams, D. J. Effect of Molecular Structure on the Properties of NaphthaleneDipeptide Hydrogelators. Langmuir 2010, 26 (16), 13466-13471.

Tena-Solsona, M.; Escuder, B.; Miravet, J. F.; Casttelleto, V.; Hamley, I. W.; Dehsorkhi, A. Thermodynamic and Kinetic Study of the Fibrillization of a Family of Tetrapeptides and Its Application to Self-Sorting. What Takes so Long? Chem. Mater. 2015, 27 (9), 3358-3365.

Nebot, V. J.; Armengol, J.; Smets, J.; Fernández-Prieto, S.; Escuder, B.; Miravet, J. F. Molecular Hydrogels from Bolaform Amino Acid Derivatives: A Structure-Properties Study Based on the Thermodynamics of Gel Solubilization. Chem. - A Eur. J. 2012, 18 (13), 4063-4072.

Fontanillo, M.; Angulo-Pachón, C. A.; Escuder, B.; Miravet, J. F. In Situ Synthesis-Gelation at Room Temperature vs. HeatingCooling Procedure. Fine Tuning of Molecular Gels Derived from Succinic Acid and L-Valine. J. Colloid Interface Sci. 2013, 412, 65-71.

Angulo-Pachón, C. A.; Miravet, J. F. Sucrose-Fueled, Energy Dissipative, Transient Formation of Molecular Hydrogels Mediated by Yeast Activity. Chem. Commun. 2016, 52 (31), 5398-5401.

Yan, X.; Cui, Y.; Qi, W.; Su, Y.; Yang, Y.; He, Q.; Li, J. Self-Assembly of Peptide-Based Colloids Containing Lipophilic Nanocrystals. Small 2008, 4 (10), 1687-1693.

Sone, E. D.; Weiner, S.; Addadi, L. Biomineralization of Limpet Teeth: A Cryo-TEM Study of the Organic Matrix and the Onset of Mineral Deposition. J. Struct. Biol. 2007, 158 (3), 428-444.

Akoka, S.; Barantin, L.; Trierweiler, M. Concentration Measurement by Proton NMR Using the ERETIC Method. Anal. Chem. 1999, 71 (13), 2554-2557.

Burguete, M. I.; Galindo, F.; García-Verdugo, E.; Karbass, N.; Luis, S. V. Polymer Supported lonic Liquid Phases (SILPs) versus Ionic Liquids (ILs): How Much Do They Look Alike. Chem. Commun. 2007, No. 29, 3086-3088.

Zetasizer Nano Series User Manual, Malvern, MAN0485-1.1; 2013.

Stachurski, J.; Michalek, M. The Effect of the $\zeta$ Potential on the Stability of a Non-Polar Oil-in-Water Emulsion. J. Colloid Interface Sci. 1996, 184 (2), 433-436.

Akiyoshi, K.; Deguchi, S.; Tajima, H.; Nishikawa, T.; Sunamoto, J. Microscopic Structure and Thermoresponsiveness of a Hydrogel Nanoparticle by Self-Assembly of a Hydrophobized Polysaccharide. Macromolecules 1997, 30 (4), 857-861.

Wu, C.; Zhou, S. Light Scattering Study of Spherical Poly(N-Isopropylacrylamide) Microgels. J. Macromol. Sci. - Phys. 1997,36 (3), 345-355.

Pelton, R. Unresolved Issues in the Preparation and Characterization of Thermoresponsive Microgels. Macromol. Symp. 2004, 207, 57-65.

Morimoto, N.; Hirano, S.; Takahashi, H.; Loethen, S.; Thompson, D. H.; Akiyoshi, K. Self-Assembled PH-Sensitive Cholesteryl Pullulan Nanogel as a Protein Delivery Vehicle. Biomacromolecules 2013, 14, 56-63.

Islam, S.; Inglefield, D. L.; Velev, O. D. Revisiting the Colloidal Fundamentals of Water-Dispersible Polyesters: Interactions and Self-Assembly of Polymer Nanoaggregates in Water. Soft Matter 2018, 14 (11), 2118-2130.

Zimm, B. H. The Scattering of Light and the Radial Distribution Function of High Polymer Solutions. J. Chem. Phys. 1948, 16 (12), 1093-1099.

Zetasizer Nano Application Note: Molecular Weight Measurements with the Zetasizer Nano System.

Ziębacz, N.; Wieczorek, S. A.; Kalwarczyk, T.; Fiałkowski, M.; Hołyst, R. Crossover Regime for the Diffusion of Nanoparticles in Polyethylene Glycol Solutions: Influence of the Depletion Layer. Soft Matter 2011, 7 (16), 7181-7186.

Peng, S.; Wu, C. Surfactant Effect on PH and Temperature Sensitivities of Poly(N-Vinylcaprolactam-Co-Sodium Acrylate) Microgels. Macromolecules 2001, 34 (3), 568-571.

Sekine, Y.; Endo, H.; Iwase, H.; Takeda, S.; Mukai, S. A.; Fukazawa, H.; Littrell, K. C.; Sasaki, Y.; Akiyoshi, K. Nanoscopic Structural Investigation of Physically Cross-Linked Nanogels Formed from Self-Associating Polymers. J. Phys. Chem. B 2016, 120 (46), 11996-12002.

Jacobs, J.; Byrne, A.; Gathergood, N.; Keyes, T. E.; Heuts, J. P. A.; Heise, A. Facile Synthesis of Fluorescent Latex Nanoparticles with Selective Binding Properties Using Amphiphilic Glycosylated Polypeptide Surfactants. Macromolecules 2014, 47 (21), 7303-7310.

Ying, Q.; Chu, B. Overlap Concentration of Macromolecules in Solution. Macromolecules 1987, 20 (2), 362-366.

Aggeli, A.; Nyrkova, I. A.; Bell, M.; Harding, R.; Carrick, L.; McLeish, T. C. B.; Semenov, A. N.; Boden, N. Hierarchical SelfAssembly of Chiral Rod-like Molecules as a Model for Peptide $\beta$-Sheet Tapes, Ribbons, Fibrils, and Fibers. Proc. Natl. Acad. Sci. U. S. A. 2001, 98 (21), 11857-11862. 
Molecular Gels Using Synchrotron FT-IR. CrystEngComm 2015, 17 (42), 8085-8092.

Jones, C. D.; Kennedy, S. R.; Walker, M.; Yufit, D. S.; Steed, J. W. Scrolling of Supramolecular Lamellae in the Hierarchical Self-Assembly of Fibrous Gels. Chem 2017, 3, 603-628.

Conesa-Milián, L.; Falomir, E.; Murga, J.; Carda, M.; Meyen, E.; Liekens, S.; Marco, J. A. Synthesis and Biological Evaluation of Carbamates Derived from Aminocombretastatin A-4 as Vascular Disrupting Agents. Eur. J. Med. Chem. 2018, 147, $183-193$. Rodriguez-Nieto, S.; Medina, M. A.; Quesada, A. R. A Re-Evaluation of Fumagillin Selectivity towards Endothelial Cells. Anticancer Res. 2001, 21 (5), 3457-3460.

Fletcher, K. A.; Storey, I. A.; Hendricks, A. E.; Pandey, S.; Pandey, S. Behavior of the Solvatochromic Probes Reichardt's Dye, Pyrene, Dansylamide, Nile Red and 1-Pyrenecarbaldehyde within the Room-Temperature lonic Liquid BmimPF6. Green Chem. 2001, 3 (5), 210-215.

Mishra, R.; Sjölander, D.; Hammarström, P. Spectroscopic Characterization of Diverse Amyloid Fibrils in Vitro by the Fluorescent Dye Nile Red. Mol. Biosyst. 2011, 7 (4), 1232-1240.

Behnke, T.; Würth, C.; Hoffmann, K.; Hübner, M.; Panne, U.; Resch-Genger, U. Encapsulation of Hydrophobic Dyes in Polystyrene Micro- and Nanoparticles via Swelling Procedures. J. Fluoresc. 2011, 21 (3), 937-944.

Lombardo, D.; Munaò, G.; Calandra, P.; Pasqua, L.; Caccamo, M. T. Evidence of Pre-Micellar Aggregates in Aqueous Solution of Amphiphilic PDMS-PEO Block Copolymer. Phys. Chem. Chem. Phys. 2019, 21 (22), 11983-11991.

Nebot, V. J.; Ojeda-Flores, J. J.; Smets, J.; Fernández-Prieto, S.; Escuder, B.; Miravet, J. F. Rational Design of Heat-Set and Specific-Ion-Responsive Supramolecular Hydrogels Based on the Hofmeister Effect. Chem. Eur. J. 2014, 20 (44), 1446514472.

McIntosh, T. J. The Effect of Cholesterol on the Structure of Phosphatidylcholine Bilayers. Biochim. Biophys. Acta Biomembr. 1978, 513, 43-58.

Patil, Y. P.; Jadhav, S. Novel Methods for Liposome Preparation. Chem. Phys. Lipids 2014, 177, 8-18.

Akbarzadeh, A.; Rezaei-Sadabady, R.; Davaran, S.; Joo, S. W.; Zarghami, N.; Hanifehpour, Y.; Samiei, M.; Kouhi, M.; NejatiKoshki, K. Liposome: Classification, Preparation, and Applications. Nanoscale Res. Lett. 2013, 8 (102).

Adams, D. J.; Butler, M. F.; Frith, W. J.; Kirkland, M.; Mullen, L.; Sanderson, P. A New Method for Maintaining Homogeneity during Liquid-Hydrogel Transitions Using Low Molecular Weight Hydrogelators. Soft Matter 2009, 5 (9), 1856-1862.

Avnir, Y.; Barenholz, Y. PH Determination by Pyranine: Medium-Related Artifacts and Their Correction. Anal. Biochem. 2005, 347, 34-41.

Clement, N. R.; Gould, J. M. Pyranine (8-Hydroxy-1,3,6-Pyrenetrisulfonate) as a Probe of Internal Aqueous Hydrogen Ion Concentration in Phospholipid Vesicles. Biochemistry 1981, 20 (6), 1534-1538.

Kano, K.; Fendler, J. H. Pyranine as a Sensitive PH Probe for Liposome Interiors and Surfaces. PH Gradients across Phospholipid Vesicles. Biochim. Biophys. Acta - Biomembr. 1978, 509 (2), 289-299.

Chen, Y.; Qiao, F.; Fan, Y.; Han, Y.; Wang, Y. Interactions of Phospholipid Vesicles with Cationic and Anionic Oligomeric Surfactants. J. Phys. Chem. B 2017, 121 (29), 7122-7132. Responsive Soft Matter Systems with Tunable Properties. Langmuir 2013, 29 (30), 9544-9550.

Sun, J.; Wei, Q.; Zhou, Y.; Wang, J.; Liu, Q.; Xu, H. A Systematic Analysis of FDA-Approved Anticancer Drugs. BMC Syst. Biol. 2017, 11, 87. Updated Insight. J. Drug Target. 2018, 26 (4), 296-310.

Sun, H.; Guo, B.; Cheng, R.; Meng, F.; Liu, H.; Zhong, Z. Biodegradable Micelles with Sheddable Poly(Ethylene Glycol) Shells for Triggered Intracellular Release of Doxorubicin. Biomaterials 2009, 30 (31), 6358-6366.

Shuai, X.; Ai, H.; Nasongkla, N.; Kim, S.; Gao, J. Micellar Carriers Based on Block Copolymers of Poly ( $\varepsilon$-Caprolactone) and Poly(Ethylene Glycol) for Doxorubicin Delivery. J. Control. Release 2004, 98 (3), 415-426.

Fugit, K. D.; Xiang, T.-X.; Choi, D. H.; Kangarlou, S.; Csuhai, E.; Bummer, P. M.; Anderson, B. D. Mechanistic Model and Analysis of Doxorubicin Release from Liposomal Formulations. J. Control. Release 2015, 217, 82-91.

Russell, L. M.; Hultz, M.; Searson, P. C. Leakage Kinetics of the Liposomal Chemotherapeutic Agent Doxil: The Role of Dissolution, Protonation, and Passive Transport, and Implications for Mechanism of Action. J. Control. Release 2018, 269, 171-176. 
REFERENCES 


\section{CHAPTER 4}

NANOGELS ENCAPSULATING PHOTOSENSITIZERS AND THEIR USE IN PHOTODYNAMIC THERAPY 



\section{CHAPTER 4. NANOGELS ENCAPSULATING PHOTOSENSITIZERS AND THEIR USE IN PHOTODYNAMIC THERAPY}

Some of the results presented in this chapter were outlined and performed under the supervision of Dr María J. Marín during a research stay (September - December 2018) in the School of Chemistry at the University of East Anglia (Norwich, UK).

\subsection{INTRODUCTION TO PHOTODYNAMIC THERAPY}

\section{PHOTODYNAMIC THERAPY AND CANCER}

Photosensitization to produce reactive oxygen species (ROS) is widely used in a variety of fields, as photocatalysis, photodynamic inactivation of microorganisms ${ }^{1}$ or bacteria ${ }^{2}$ or photodynamic therapy (PDT) in medicine. Although Indians and Egyptians already had made use of PDT in ancient times, ${ }^{3}$ their scientific bases were not understood until $1900 .{ }^{4}$ The first large series of patients successfully treated with PDT was reported in 1978. A hematoporphyrin derivative was used, followed by exposure to red light. ${ }^{5}$ Nowadays, cancer is the primary area of use of PDT in medicine. Still, there is a growing interest in this therapy for the treatment of infectious diseases, ${ }^{6}$ for causing focal thrombosis ${ }^{7}$ and for dermatology and cosmetic practice. In this latter, PDT increases the production of collagen, ${ }^{5}$ which is interesting for the treatment of photodamage or for photorejuvenation, and is also used for the treatment of inflammatory skin disorders as acne ${ }^{8}$ or psoriasis. ${ }^{9}$

Cancer remains one of the deadliest diseases and causes millions of deaths every year. PDT has been considered a clinical option for the treatment of solid tumours for the past 25 years. ${ }^{10}$ PDT requires three components: a photosensitizer (PS), light and oxygen present in the tissue. ${ }^{8,11}$ The general process involves a systemically or topically administered PS and then site-specific irradiation of the PS with the appropriate wavelength, which generates reactive oxygen species (ROS) that cause cancerous cells to perish. ${ }^{12-15}$ Since PSs only produce ROS upon irradiation with a particular type of light and ROS diffusion is limited, the cytotoxic damage is only caused in the specific irradiated region and the immediate surrounding area. Therefore, PDT may serve as a "magic bullet" to selectively disrupt malignant tumours, while sparing healthy organs. ${ }^{16-20}$ For this reason, PDT has become an emerging solution for cancer therapy that avoids the frequent 
severe systemic toxicity and adverse effects of the two primary clinical treatments, chemotherapy and radiotherapy. ${ }^{21-23} \mathrm{~A}$ list of photosensitizers approved and in ongoing clinical trials for cancer PDT can be found in Table 4.1, Figure 4.1 and Figure 4.2.

Table 4.1. List of photosensitizers approved and in ongoing clinical trials for PDT cancer. ${ }^{24}$

\begin{tabular}{|c|c|c|c|c|}
\hline Status & PS & Uses & Irradiation & Formulation \\
\hline \multirow{5}{*}{ Approved } & Photofrin & $\begin{array}{l}\text { Bladder cancer (Canada), lung } \\
\text { cancer (Japan), esophageal cancer } \\
\text { and lung cancer (USA) }\end{array}$ & $630 \mathrm{~nm}$ & Water-soluble \\
\hline & Foscan/temoporfin & $\begin{array}{l}\text { Head and neck squamous cell } \\
\text { carcinoma (Europe) }\end{array}$ & $652 \mathrm{~nm}$ & $\begin{array}{l}40 \% \text { ethanol- } 60 \% \\
\text { propylene glycol }\end{array}$ \\
\hline & Verteporfin & $\begin{array}{l}\text { Wet age-related macular } \\
\text { degeneration (USA) }\end{array}$ & $689 \mathrm{~nm}$ & Liposomal preparation \\
\hline & Tookad/padeliporfin & Prostate cancer & $753 \mathrm{~nm}$ & Water-soluble \\
\hline & Talaporfin sodium & Lung cancer (Japan) & LED light & Water-soluble \\
\hline \multirow{5}{*}{$\begin{array}{l}\text { Clinical } \\
\text { trials }\end{array}$} & HPPH/Photochlor & $\begin{array}{l}\text { Malignant pleural mesothelioma, } \\
\text { esophageal cancer, lung cancer, oral } \\
\text { cancer and cancer of the larynx }\end{array}$ & - & With Tween 80 \\
\hline & Redaporfin & Head and neck cancer & - & $\begin{array}{l}\text { Pluronic 123-based } \\
\text { micelles }\end{array}$ \\
\hline & Fimaporfin & Extrahepatic cholangiocarcinoma & $652 \mathrm{~nm}$ & Water-soluble \\
\hline & $\begin{array}{l}\text { Silicon } \\
\text { phthalocyanine PC4 }\end{array}$ & Cutaneous malignancies & $675 \mathrm{~nm}$ & $\begin{array}{l}\text { PEG-polycaprolactone } \\
\text { micelles }\end{array}$ \\
\hline & TLD1433 & Bladder cancer & $530 \mathrm{~nm}$ & Water-soluble \\
\hline
\end{tabular}

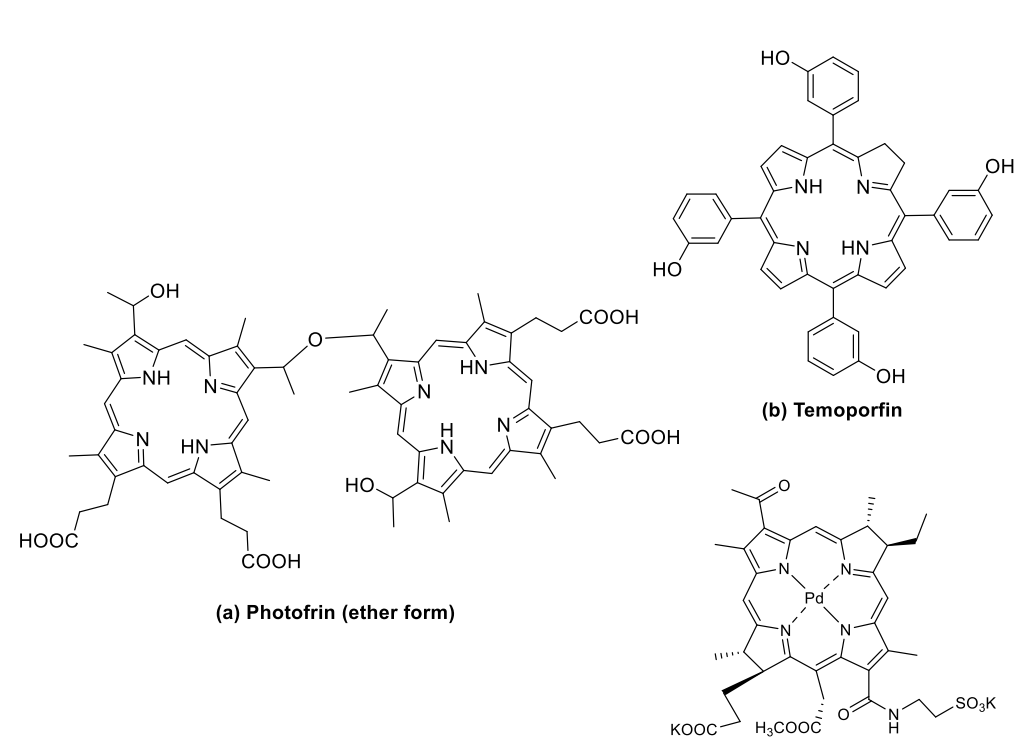

(d) Padeliporfin

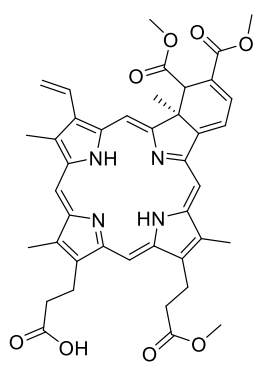

(c) Verteporfin

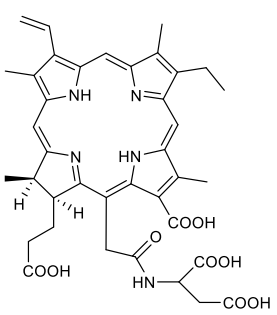

(e) Talaporfin

Figure 4.1. Chemical structures of approved PS for PDT cancer ${ }^{24}$ : (a) Photofrin (ether-linked dimer), (b) Temoporfin (Foscan), (c) Verteporfin (Visudyne), (d) Padeliporfin (Tookad soluble), (e) Talaporfin (LS11). 


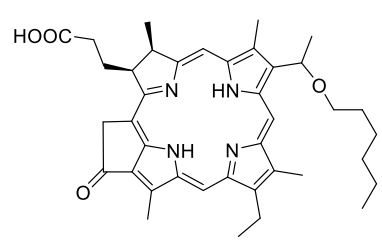

(a) Photochlor

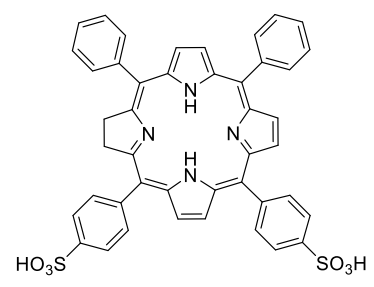

(c) Fimaporfin

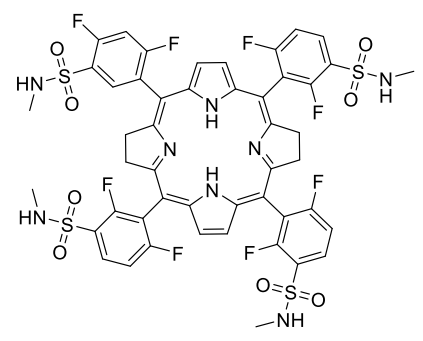

(b) Redaporfin

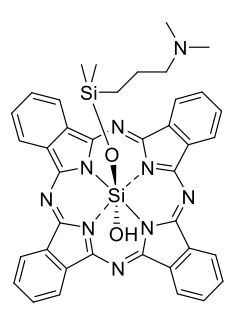

(d) PC4

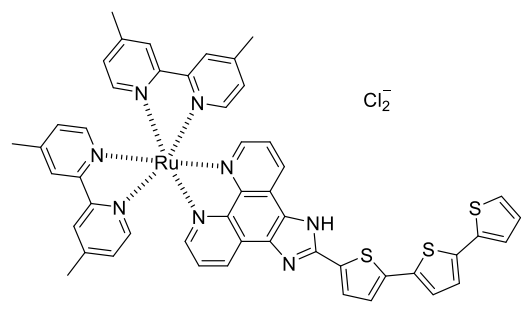

(e) TLD1433

Figure 4.2. Chemical structures of PS in ongoing clinical trials ${ }^{24}$ : (a) Photochlor (HPPH), (b) Redaporfin (LUZ11), (c) Fimaporfin (Amphinex), (d) Silicon phthalocyanine (PC4), (e) TLD1433.

\section{TYPES OF PHOTODYNAMIC THERAPY}

Based on the different photochemical reaction processes, PDT can be divided into two types: type I PDT and type II PDT (Figure 4.3). After light activation, the PS is transformed from the ground singlet state $\left(\mathrm{S}_{0}\right)$ to the excited singlet state $\left(\mathrm{S}_{1}\right)$. Then, the chromophore can relax back to the ground state by emitting light (fluorescence) or undergo intersystem crossing (ISC) leading to an electronically excited triplet state $\left(\mathrm{T}_{1}\right) \cdot{ }^{17} \mathrm{~T}_{1}$ triggers the photochemical reaction via two different (type I and type II) paths. ${ }^{25}$ For type I PDT, $T_{1}$ participates in a hydrogen- or electron-transfer process to react directly with a biological substrate to form free radicals, which can interact with triplet oxygen $\left({ }^{3} \mathrm{O}_{2}\right)$ and water to produce superoxide anions $\left(\mathrm{O}_{2}{ }^{\circ}\right)$ and hydroxyl radicals $\left(\mathrm{OH}^{\circ}\right)$, respectively. ${ }^{26,27}$ During the type II PDT process, $\mathrm{T}_{1}$ undergoes a type II photochemical reaction to convert the surrounding ${ }^{3} \mathrm{O}_{2}$ into cytotoxic singlet oxygen $\left({ }^{1} \mathrm{O}_{2}\right)$ via direct energy transfer. ${ }^{28-30}$ Therefore, type II PDT dominates in well-oxygenated environments, while type I PDT can occur under hypoxic conditions. 


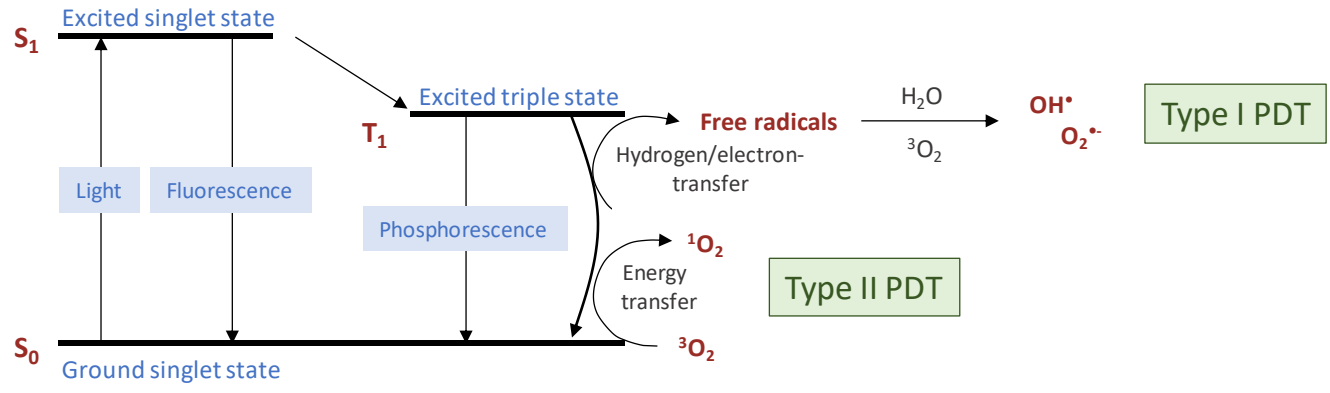

Figure 4.3. A Jablonski diagram showing the photochemical reactions for type I and type II PDT. ${ }^{31}$

\section{THE IDEAL PHOTOSENSITIZER}

A good photosensitizer, as critical element involved in PDT, should meet some essential requirements that include: 1) a high absorption coefficient in the spectral region of the excitation light and a maximum absorption at a long wavelength; 2) a high intersystem crossing yield; 3 ) a triplet state of appropriate energy $\left(E_{\mathrm{T}}>95 \mathrm{~kJ} / \mathrm{mol}\right)$ to allow for efficient energy transfer to ground-state oxygen; 4$)$ a high quantum yield of the triplet state $\left(\Phi_{T}>0.4\right)$ and long tripletstate lifetimes ( $\left.\tau_{\top}>1 \mu \mathrm{s}\right) ; 5$ ) a high yield of ${ }^{1} \mathrm{O}_{2}$ generation; 6 ) a high photostability; 7) no dark toxicity and minimized phototoxic damage to normal tissues. ${ }^{29,32,33}$

\section{MECHANISM OF ACTION OF PDT IN CANCER}

The anti-tumour effect of PDT is mediated by a combination of three main actions: a) direct cytotoxic effect on the cancer cells, b) the destruction of the tumour blood vessels, and c) the stimulation of anti-tumour immunity. ${ }^{10}$

The process starts when, in the presence of oxygen, the photoactivated photosensitizer leads to the formation of reactive oxygen species. These ROS are very short-lived (less than $200 \mathrm{~ns}$ ) and have a limited diffusion ability $(<20 \mathrm{~nm}),{ }^{34}$ reacting with biomolecules in its immediate vicinity. Therefore, the localization of the photosensitizer at the time of irradiation, both in the body and/or cellular compartments, largely determines where the oxidative damage will occur. For instance, hydrophobic photosensitizers tend to accumulate in the membranes of the cellular organelles. They can be observed in the endoplasmic reticulum (ER), Golgi apparatus (GA) and/or mitochondria, where the oxidative damage is deemed the most lethal. On the other hand, hydrophilic photosensitizers are more often observed in locations related to the endocytic pathway. ${ }^{10}$ 
- $\quad$ The destruction of the tumour blood vessels

Low intervals between PS administration and irradiation principally target tumour vasculature, with the destruction of the network usually associated with extensive necrosis. Platelet aggregation and vascular occlusion also produce the antivascular action. ${ }^{9}$ The depleting of nutrients and oxygen supply leads to cell death at the site of the tumour.

\section{- Direct cytotoxic effect on the cancer cells}

Long intervals between PS administration and irradiation lead to an optimal redistribution of the compound in cellular compartments of the cancer cells. Once cells enter in contact with ROS, different mechanisms of cell death are triggered, being necrosis, apoptosis and autophagy the best known (Figure 4.4). Cell death is not only produced by the damage inflicted by ROS on different biomolecules, but also to the oxidative stress derived from the cells trying to remove/repair this damaged material.

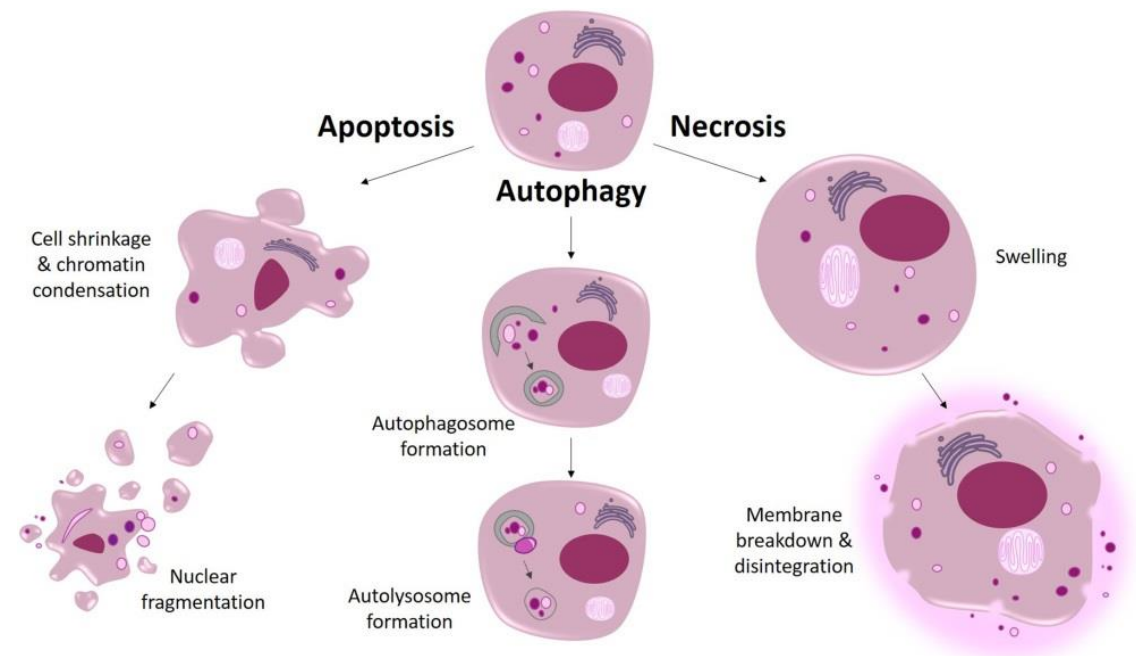

Figure 4.4. The three most noted pathways of cell death. ${ }^{10}$

Apoptosis, or programmed cell death, is the process that cells undergo when they become damaged or are no longer needed. This normal physiological process occurs during embryonic development, as well as in maintenance of tissue homeostasis, under pathological conditions and in aging. Apoptosis is an organized phenomenon in which a cell actively participates in its own destructive processes. ${ }^{35}$ It is characterized by certain morphological features, such as changes in the plasma membrane (such as loss of membrane symmetry and loss of membrane 
attachment), plasma membrane blebbing (formation of apoptotic bodies), a condensation of the cytoplasm and nucleus and internucleosomal cleavage of DNA. In the final stages of the process, plasma membrane integrity is always maintained in vivo, and dying cells are eliminated by phagocytes and degraded within lysosomes, whereas in vitro, membrane breakdown occurs, typically designated as secondary necrosis. ${ }^{36-38}$

On the other hand, necrosis, or accidental cell death, is a form of cell injury which results in the premature death of cells in living tissue by autolysis (destruction of a cell through the action of its own enzymes). Necrosis is caused by internal or external stresses, such as mechanistic injuries, chemical agents or pathogens. This process leads to increased membrane permeability resulting in water and ion influx, cellular and organelle swelling and, finally, membrane breakdown and content release to the extracellular space. This latter process causes inflammatory responses and activates leukocytes, lymphocytes and macrophages that phagocytose the remnants of dead cells. ${ }^{39}$

Autophagy is a catabolic process which can act as a pro-survival mechanism through the clearance of damaged cellular material or as a death mechanism. A double layer membrane vesicle is formed and engulfs damaged material (unfolded proteins, damaged organelles, microorganisms), separating it from the cytoplasm. Then, it is degraded by lysosomal hydrolases and the recycled nutrients are reused for normal cellular processes. ${ }^{10}$

In addition to the intracellular localisation of the photosensitive compound, the type of cell death induced by PDT depends on its concentration and incubation time, on the wavelength of light radiation, fluence rate, light dose and post-irradiation time, as well as on the histological origin of the tissue and oxygen pressure in it. ${ }^{40-42}$ The same photosensitizer may induce the three cell death mechanisms, and sometimes even overlap, depending on the treatment conditions. For instance, high photo-damage (high photosensitizer concentration and/or high dose light) induces necrosis, moderate damage is expected to cause regulated cell death modalities as apoptosis, and minor damage induces autophagy (which can further trigger other mechanisms of regulated cell death). ${ }^{10}$ Therefore, it is likely that different forms of cell death simultaneously occur within a tumour submitted to PDT in vivo. ${ }^{10}$ 
- $\quad$ The stimulation of anti-tumour immunity

Finally, besides the immediate local impact, the action of these ROS may subsequently elicit immune responses with systemic implications. The resultant cell debris from tumour cell death at the site of targeted therapy promotes localised inflammation, which attracts macrophages, neutrophils and dendritic cells, allowing tumour cell antigens to be exposed to the host immune system. ${ }^{9,43}$ This contributes to the long-term control of the disease and constitutes a significant advantage over traditional therapies, including surgery or chemotherapy, which are immunologically silent or even immunosuppressive. ${ }^{10}$

\section{DISADVANTAGES OF PDT THERAPY IN CANCER}

Although PDT is a promising strategy to treat tumours, there are still three main challenges in its clinical practice with a common solution. ${ }^{31}$

The first one is hypoxia, a distinct pathophysiological feature in most types of solid tumours, especially in the central region, due to the rapid tumour progression. ${ }^{44,45}$ Two useful strategies have been developed to enhance the efficacy of hypoxic deep PDT: tumour oxygenation (for example, direct oxidation of endogenous $\mathrm{H}_{2} \mathrm{O}_{2}$ into $\left.\mathrm{O}_{2}\right)^{46}$ and type I PDT. However, multifunctional nanomedicines are required in both cases: a synergistic therapy between the $\mathrm{O}_{2}$ generation specie and type II PDT is needed in the former case, and a cooperative treatment between type I PDT and other drugs, in the latter. ${ }^{47-50}$

Moreover, the tissue penetration depth of excitation light is another critical parameter for PDT. ${ }^{51,52}$ The majority of currently available photosensitizers are excited using UV/visible light, whose tissue penetration depth is $c a$. 1-3 mm (tissue components as melanin and haemoglobin absorb this light). ${ }^{53}$ To overcome this issue, which has become the Achilles' heel in treating deep-seated tumours below the skin, deep PDT has been developed. It is based on the Förster resonance energy transfer (FRET) from photoconverting nanoparticles to PS after using excitation sources as near-infrared (NIR) light (lies in the "optical transparency window" of biological tissues) or X-ray radiation (high live-body permeability), or without external excitation based on internal self-luminescence (for example, luminol as a chemiluminescent donor ${ }^{54}$ ). ${ }^{31}$

Finally, the last challenge is found in the chemical nature of the compounds used as PS. There have been three generations of organic PSs. Porphyrins and their analogues are considered the first one. ${ }^{55}$ Some drawbacks of this first set of PSs, such as low selectivity towards tumours, high 
accumulation in skin (causing cutaneous photosensitivity) ${ }^{56}$ and the requirement of excitation wavelengths around $630 \mathrm{~nm}$ (with low tissue penetration), ${ }^{57,58}$ lead to the use of other compounds. The so-called second generation of PSs for PDT is constituted mainly by phthalocyanines, chlorines and some natural dyes. Nevertheless, there are still some physicochemical properties of these new PSs that limit their activity in PDT. For example, hydrophobic PSs are more potent compared to water-soluble compounds because they can be efficiently internalised by cells through interaction with the lipid layers of the cell membrane. ${ }^{26,59}$ However, they from aggregates in solution, restricting their medical applications as the singlet oxygen production decreases dramatically with aggregation. ${ }^{60,61}$ On the other hand, water-soluble PSs can be easily administered intravenously and significantly improve tumour killing. ${ }^{62,63}$ However, they poorly accumulate in tumour cells, due both to their difficulty in crossing cell membranes owing to their charge and to their fast removal from the cells once they have been internalised (cellular transport systems in cancer cells are slowly accelerated for hydrophilic drugs to pass through when compared to normal cells). ${ }^{64}$

All these cited inconveniences, which compromise the therapeutic effect of PS, boosted the third generation of PSs, constituted by a wide range of inorganic/organic nanocarriers developed to carry PS and those other components necessary to achieve a proper PDT therapy. ${ }^{65-70}$ In addition to the generic ideal characteristics of a drug delivery system such as high loading capacity, biocompatibility, biodegradability or minimum toxicity, nanocarriers should incorporate the photosensitizer without loss or alteration of the sensitizer activity and provide an environment where the photosensitizer can be administered in monomeric form. ${ }^{71}$ Besides the solubilisation of the PS, they are required to increase the selectivity towards the tumour (to avoid generalised photosensitivity), either coating the nanoparticle with ligands with strong affinities towards specific cell receptors overexpressed on the surface of the tumours, ${ }^{72}$ or either via the EPR effect by choosing the appropriate nanoparticle size. ${ }^{37}$ Nanoencapsulation may also potentially increase the excited-state lifetime of a photosensitizer, enhance intersystem crossing and subsequently improve singlet oxygen yield. ${ }^{73}$ Photosensitizers formulated using biodegradable polymeric nanoparticles, polymeric micelles and liposomes have been used for PDT drug delivery. ${ }^{74}$ However, the release of the PDT agent is not a necessity for its therapeutic action and optically transparent and porous nanomatrix, for unimpeded transmission of light as well as for to-and-fro diffusion of molecular and singlet oxygen, are also desirable. ${ }^{75}$ 
In summary, it seems that the future of PDT as a promising cancer therapeutic strategy lies in the development of new carriers to solve the cited disadvantages. The use of nanoparticles as vehicles for the selective delivery of PS in PDT has received significant attention over the past 10-15 years ${ }^{76}$ and lots of examples can be found in the literature. Some of these systems will be found in next sections. 


\subsection{NANOGELS ENCAPSULATING ROSE BENGAL}

\subsubsection{INTRODUCTION TO ROSE BENGAL}

\section{SPECTRAL CHARACTERISTICS AND APPLICATIONS}

Rose Bengal (abbreviated as RB; 4,5,6,7-tetrachloro-2' $4^{\prime}, 5^{\prime}, 7^{\prime}$-tetraiodofluorescein disodium, Figure 4.5) belongs to the family of xanthenes. The compounds of this family were unsuccessfully designed as dyes, as they show photobleaching, and their first use was as acidbase indicators, as their colour changes with $\mathrm{pH}$. Then, their promising photochemical properties in the context of photodynamic action were discovered and, nowadays, they are also widely used as biomolecular tracers and imaging markers for live cells. ${ }^{77}$

Rose Bengal was initially synthesized during the period $1880-1884$ as a fabric dye to mimic the red colours in Bengali clothes, with its name connected to the symbolic red spot worn by Bengali women to symbolize marriage. ${ }^{78,79}$ At neutral and basic $\mathrm{pH}$ values, it is a water-soluble dianion which presents an intense absorption band in the visible region of the spectrum at $c a .550 \mathrm{~nm}$ (associated to transition $\mathrm{S}_{0}-\mathrm{S}_{1}$ ), with a shoulder at $525 \mathrm{~nm}$ (attributed to transition $\mathrm{S}_{0}-\mathrm{S}_{2}$ ), and a fluorescence quantum yield of $0.02 .{ }^{80,81}$ Rose Bengal has a low price, wide availability and is versatile and applicable to a wide range of fields. ${ }^{82}$ For instance, RB is a red additive permitted as an artificial food dye in Japan ${ }^{83}$ and has also been applied for decades, with minimal sideeffects, in the systemic diagnosis of hepatic function. ${ }^{84-86} \mathrm{RB}$ is also FDA-approved for diagnosis of ocular surface damage, mainly dry eye syndrome. It is sold as strips or solution ${ }^{87}$ (Figure 4.6) and stains the nuclei and cell walls of dead or degenerated epithelial cells of the cornea and conjuctiva, and the mucus of the precorneal tear film..$^{88-91}$<smiles>[N+]O[N+](=O)c1c(I)cc2c(-c3c(Cl)c(Cl)c(Cl)c(Cl)c3C(=O)O)c3cc(I)c(=O)c(I)c-3oc2c1I</smiles>

Figure 4.5. Chemical structure of Rose Bengal.
Figure 4.6. Rose Bengal ophthalmological strips. 
This dye can also be combined with light for other applications in the medical field. RB is a potent and well-known photosensitizer ${ }^{78,92}$ for type II PDT: when irradiated with green light shows an intersystem crossing quantum yield approaching unity ( $\left.\Phi_{\text {isc }}=0.98\right)$, a high triplet quantum yield $\left(\Phi_{T}=0.76\right)$, a reasonably long-lived triplet state $\left(t_{1 / 2}=0.1-0.3 \mathrm{~ms}\right)$ and a high singlet oxygen quantum yield $\left(\Phi^{1} \mathrm{O}_{2}=0.75\right.$ under $540 \mathrm{~nm}$ light irradiation)..$^{78,81,93-96}$ Therefore, RB is capable of producing singlet oxygen with high efficiently upon irradiation. Additionally, its long history of safe use as a systemic diagnostic of hepatic function as well as a topical ophthalmic diagnostic suggest that, in marked contrast to many PDT agents, RB should have minimal potential for side effects, such as prolonged photosensitivity. ${ }^{97}$

The mentioned photophysical properties are very appealing for the use of RB in PDT and, therefore, it is one of the most commonly used synthetic PS in PDT, mainly as an anticancer and antimicrobial agent. ${ }^{98}$ For example, the antimicrobial effect of photoactivated RB has been used to kill microorganism such as viruses, ${ }^{99,100}$ Gram-positive bacterial species, ${ }^{101}$ fungi ${ }^{102}$ and protozoa. ${ }^{103}$ Additionally, RB is used in PDT for cutaneous skin disorders. The minimally penetrating nature of the green light required for its excitation (which in fact is one of the disadvantges of this probe for photodynamic processes), the negligible effect exhibited in normal skin, and the suggested self-limiting photodynamic impact (as RB readily photobleaches ${ }^{104}$ ), make this RB-PDT particularly safe and useful for the treatment of skin. ${ }^{97} \mathrm{An}$ example is PH-10, a non-steroidal, small molecule-based hydrogel formulation of RB (0.002$0.1 \%$ ) for topical administration. This gel has undergone Phase 2 clinical trials for the treatment of inflammatory dermatoses, such as plaque psoriasis and atopic dermatitis, using ambient exposure to visible light for photoactivation. ${ }^{105,106}$ PDT is useful for inflammatory disorders (immune-mediated and also characterized by the uncontrolled proliferation of invading microbes, which perturbs the wound-healing process) or autoimmune diseases. In this regard, sublethal, low-dose PDT can be used to suppress the immune response, as it provokes apoptosis, changes cytokine release or alters surface receptor expression of the activated immune cells, while keeping the cell viability intact. In addition to this immunomodulatory activity, the antimicrobial and wound-healing properties (stimulates growth factor synthesis) of PDT and that photosensitizers are preferentially taken up by rapidly dividing cells (such as tumour cells in cancer or activated immune cells in immune-mediated diseases) are also useful. ${ }^{9}$

Another application of photoactivated RB for skin treatment is photochemical tissue bonding (PTB). This nanosuturing technique uses light energy to activate RB that has been applied to the 
wound surfaces. The excited-state dye interacts with collagen to form free radicals that couple to form new covalent crosslinks (nanosutures) between collagen molecules in the apposed tissue surfaces (Figure 4.7). PTB was developed in the Kochevar and Redmond laboratories over the last decades ${ }^{107}$ and tested in clinical studies for excisional wound healing. ${ }^{106} 0.1 \%$ RB in PBS and green light $(532 \mathrm{~nm})$ for $200 \mathrm{~s}$ provided an immediate watertight seal, with no adverse responses and the scars had a better appearance compared with traditional suturing. ${ }^{108,109}$ Due to this positive outcome, the introduction of PTB in daily clinical practice is currently being studied. ${ }^{82}$

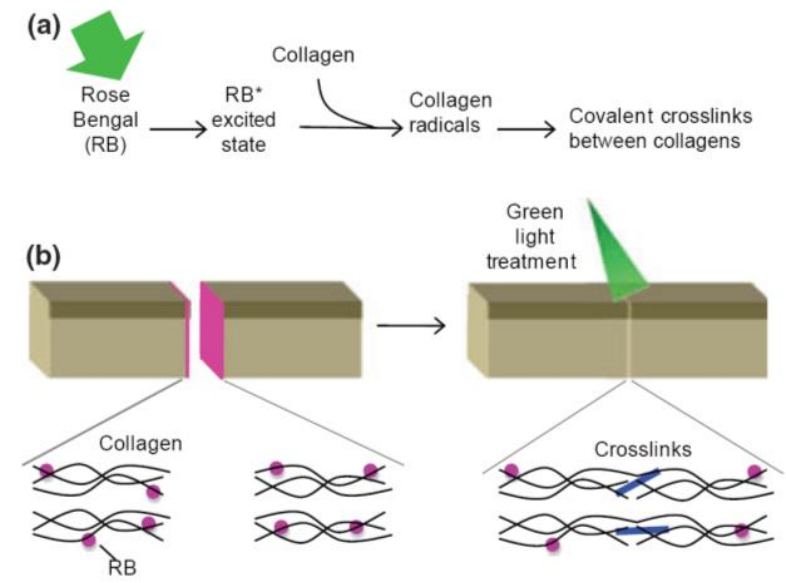

Figure 4.7. Proposed mechanism for tissue sealing using photoactivated tissue bonding (PTB). ${ }^{109}$

Following with the biomedical applications of photoactivated RB, it has been used in tissue engineering, as a photo-crosslinker in collagen-based hydrogels, to improve stability, stiffness and resistance of the gel. ${ }^{82}$ It has also shown to inhibit $\beta$-amyloid self-assembly at an early-stage Alzheimer's disease. ${ }^{110}$ Photoactivated RB is also used in vivo for occlusion of blood vessels in a procedure called photothrombosis. This process, in which RB is intravenously delivered $\left(10^{-4}\right.$ to $\left.5 \times 10^{-3} \mathrm{M}\right)$ and immediately irradiated, is a non-mechanical and non-invasive methodology to create models of reproducible brain infarction. ${ }^{83,111}$

As an alternative to light, RB can also be activated by ultrasound for sonodynamic therapy in cancer treatment, which comes with the advantage that allows delivering energy to deep tissues. RB induces ultrasonic cavitation, which is the generation and oscillation of gas bubbles that may cause irreversible cell damage and modify membrane structure and functional properties of cells to induce lysis, necrosis or apoptosis. ${ }^{112,113}$ 
Finally, RB by itself has also gained some attention as accumulating evidence suggests that RB has preferential and intrinsic toxicity towards cancer cells rather than normal cells, presenting the potential for tumour ablation with minimal side effects. ${ }^{114-116}$ PV-10 is a $10 \%$ sterile, nonpyrogenic saline solution of $\mathrm{RB}^{114}$ which is used for treatment of unresectable local metastases accessible for intralesional injection. ${ }^{105,106}$ Examples include clinical trials for the treatment of melanoma, for cancer metastatic to the liver and recurrent breast cancer carcinoma, ${ }^{106}$ and preclinical for pediatric cancers. ${ }^{117}$ This formulation has a unique mechanism of action producing chemical tumour ablation for local disease control and, additionally, the tumour necrosis induces a tumour-specific systemic immune response, which prevents the progression of distant metastases.

\section{PDT FOR CANCER TREATMENT}

RB has the photophysical properties required to be a useful PS for PDT and has shown promising results in in vitro and in vivo studies. For example, it has demonstrated selectiveness when killing cancer cells over healthy cells in a broad range of cancer cell types, such as skin, breast, prostate and lymphoma. Despite that, RB inherent unfavourable pharmacological properties have hindered its clinical development as anticancer PDT agent. In particular, due to its anionic nature at physiological pH and low lipophilicity, RB is inhibited from crossing cell membranes and interacting with some organelles such as mitochondria, which has a net negative charge potential. Thus, RB suffers from low intracellular uptake ability. ${ }^{118-121}$ Moreover, its tendency to aggregate in dimers and higher multimers in solution under physiological conditions decreases the yield of reactive oxygen species ${ }^{122,123}$ and causes problems for intravenous administration.

Therefore, it is highly desirable to have an appropriate formulation for the delivery of this photosensitizer in therapeutic levels and to favour its intracellular accumulation. One of the solutions has been to increase its lipophobicity, by developing hydrophobic derivatives as the diacetate. ${ }^{124,125}$ The addition of acetate groups to the xanthene ring converts the RB molecule into a fluorogenic substrate, being RB recovered once it enters in the cell. However, the most effective solution to increase the transmembrane transport of RB has been the use of nanoparticles as vehicles, with an increasing number of reported systems. Selected examples have been included in Table 4.2. 
Table 4.2. Selected examples of nanocarriers containing RB, chronologically ordered from oldest to newest.

\begin{tabular}{|c|c|}
\hline System & Advantages \\
\hline Albumin nanoparticles ${ }^{126}$ & Controlled drug release, protection against enzymatic degradation \\
\hline Liposomes $^{119,127}$ & $\begin{array}{l}\text { Enhanced photostability; in vitro: enhanced intracellular photodynamic } \\
\text { efficacy, good intracellular uptake and increased cell death rate }\end{array}$ \\
\hline Chitosan (covalent bond) $)^{128}$ & In vitro (breast cancer): low dark toxicity, enhanced phototoxic effect \\
\hline $\begin{array}{l}\text { Polyamidoamine (PAMAM) dendrimers } \\
(20 \mathrm{~nm})^{129}\end{array}$ & $\begin{array}{l}\text { In vitro (Dalton's lymphoma ascites cells): decreased dark toxicity, } \\
\text { enhanced photodynamic efficacy, efficient delivery of RB }\end{array}$ \\
\hline Silica nanoparticles ${ }^{130}$ & $\begin{array}{l}\text { In vitro (oral and breast cancer cells): enhanced uptake, enhanced } \\
\text { toxicity compared to free RB }\end{array}$ \\
\hline Gold nanoparticles (covalent bond) ${ }^{131}$ & $\begin{array}{l}\text { In vitro (oral cancer cells): enhanced cellular uptake, enhanced PDT } \\
\text { effect, enhanced therapeutic effects }\end{array}$ \\
\hline $\begin{array}{l}\text { Mesostructured silica nanoparticles (150- } \\
180 \mathrm{~nm} \text { ) (covalent bond) })^{132,133}\end{array}$ & $\begin{array}{l}\text { Enhanced singlet oxygen generation, in vitro (melanoma cells) } \\
\text { phototoxicity }\end{array}$ \\
\hline Semiconductor zinc oxide NPs $(24 \mathrm{~nm})^{134}$ & Increased ROS generation, in vitro (HeLa cells) toxicity \\
\hline $\begin{array}{l}\mathrm{Fe}_{3} \mathrm{O}_{4} @ \mathrm{Au} @ \mathrm{mSiO}_{2} \text { nanoplatform }(60 \mathrm{~nm}) \\
\text { (covalent bond) })^{135}\end{array}$ & Enhanced singlet oxygen generation \\
\hline Cationic phosphorous dendrimer ${ }^{136}$ & $\begin{array}{l}\text { Enhanced singlet oxygen generation, in vitro (basal carcinoma cells): } \\
\text { enhanced phototoxic effect, increased cellular uptake, enhanced PDT } \\
\text { activity }\end{array}$ \\
\hline $\begin{array}{l}\text { Light-triggered liposomes containing gold } \\
\text { nanoparticles }{ }^{137}\end{array}$ & In vitro: good cell-killing efficacy of RB combined with gold \\
\hline $\begin{array}{l}\text { Bis(pyrene)@RB-Hemoglobin } \\
\text { nanocomplex }(150 \mathrm{~nm})^{138}\end{array}$ & $\begin{array}{l}\text { In vitro, in vivo: increased treatment depth, avoiding of tumour hypoxia, } \\
\text { improved PDT efficiency }\end{array}$ \\
\hline $\begin{array}{l}\mathrm{NaGdF}_{4}: \mathrm{Yb}^{3+} / \mathrm{Er}^{3+} \text { UCNPs (covalent } \\
\text { bond) }\end{array}$ & In vitro (lung cancer cells) phototoxicity \\
\hline $\begin{array}{l}\text { Barium titanate and RB composite } \\
\text { nanoparticles (BT@PAH/RB/PAH) } \text { (140 }^{14}\end{array}$ & $\begin{array}{l}\text { Enhanced singlet oxygen generation, in vitro (cervical cancer cells): } \\
\text { increased PDT effect }\end{array}$ \\
\hline Carboxymethyl chitosan ${ }^{141}$ & In vitro (oral cancer cells): enhanced PDT efficacy \\
\hline
\end{tabular}

\section{INTRACELLULAR LOCALISATION AND CELL DEATH IN CANCER PDT}

$\mathrm{RB}^{142-145}$ and $\mathrm{RB}$ acetate ${ }^{146,147}$ phototoxicity has been reported in several cell lines. However, the localisation into the cell and the cell death differ for both molecules. On the one hand, RB at concentrations lower than ca. $10^{-4} \mathrm{M}$ accumulates mainly in the cell membrane (at low concentrations is inhibited from crossing the cell membrane and entering cells), where the singlet oxygen causes cytotoxicity, impairing membrane functions through damage to plasma membrane components. ${ }^{121,148,149}$ At higher concentrations, RB has intrinsic toxicity towards tumour cells as it accumulates in lysosomes (the $\mathrm{pH}$ of 4.5-5 drives the conversion of soluble RB disodium salt into the insoluble RB lactonic form), which triggers the intracellular release of lysosomal enzymes that results in autophagy ${ }^{114}$ or apoptosis. ${ }^{117}$ 
On the other hand, once RB acetate is internalised, restored RB molecules early localize in endosomes and then undergo intracellular redistribution, firstly, in the perinuclear region, and finally in the Golgi apparatus and endoplasmic reticulum, except in mitochondria. ${ }^{146}$ At these spots, the irradiation of RB can efficiently and independently induce multiple cell death types. Indeed, RB is considered as a very promising photosensitising drug as it ensures long-term cytotoxic effects by induction of relevant percentage of apoptosis and autophagy in a timerelated manner after PDT. Apoptosis is the preferred mechanism for cell death, being necrosis negligible. ${ }^{147} \mathrm{RB}$ internalized with a carrier is expected to undergo a similar process.

\subsubsection{RESULTS AND DISCUSSION}

\subsubsection{LOADING STUDIES}

\section{ROSE BENGAL ENCAPSULATION INTO NANOGELS}

Rose Bengal was loaded into nanogels of 1 (RB@1) prepared by sonication method (Section 3.1). A RB suspension in toluene was used to prepare the gel. Although RB is a red suspension in toluene, it becomes a solution when heating, and pink homogeneous gels of $\mathbf{1}$ are obtained. ${ }^{150}$

Firstly, the preparation of a series of RB@1 samples at different RB concentrations was carried out to optimize the loading procedure. For comparison purposes, control experiments of the procedure of RB@1 samples preparation without gelator were also performed (from now on, RB-prep control; see Figure 4.8). Therefore, for each batch of RB@1/RB-prep control, $1 \mathrm{~mL}$ of a $\mathrm{X} \mu \mathrm{M}$ RB suspension in toluene was added to a vial containing the gelator and to an empty vial. After the nanogels preparation protocol, $2 \mathrm{~mL}$ RB@1 sample and $2 \mathrm{~mL}$ RB-prep control were obtained with a nominal X/2 $\mu \mathrm{M}$ RB concentration. The size of the RB@1 particles was analysed by DLS, and no significant changes from neat nanogels were observed.
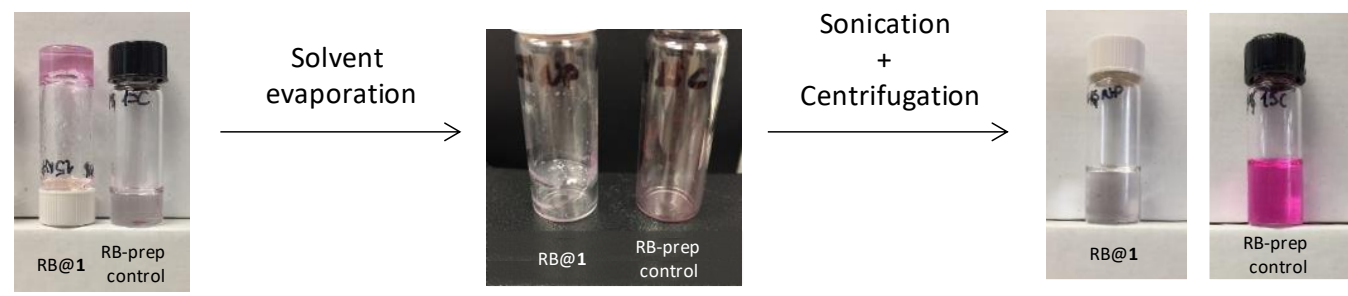

Figure 4.8. Preparation of RB@1 and the corresponding RB-prep control. 


\section{ABSORPTION AND FLUORESCENCE EMISSION SPECTRA}

The absorption and fluorescence emission spectra for a representative batch with a nominal 10 $\mu \mathrm{M} R B$ concentration is shown in Figure 4.9. Similar spectra were obtained for all tested samples (2.5-40 $\mu \mathrm{M})$. Although absorption spectra are normalised, considering that the same quantity of RB is used to prepare the nanoparticle sample and the control, it is noticeable in Figure $\mathbf{4 . 8}$ that RB-prep controls are highly coloured, whereas RB@1 samples are almost colourless to the naked eye. A rationale for this observation is that a significant amount of $\mathrm{RB}$ remains trapped in the xerogel that has not been dispersed by sonication in PBS during nanoparticles formation. Indeed, full solubilization of this remaining xerogel in $0.1 \mathrm{M}$ aqueous $\mathrm{NaOH}$ afforded a pink solution, revealing that a substantial amount of RB was trapped there.

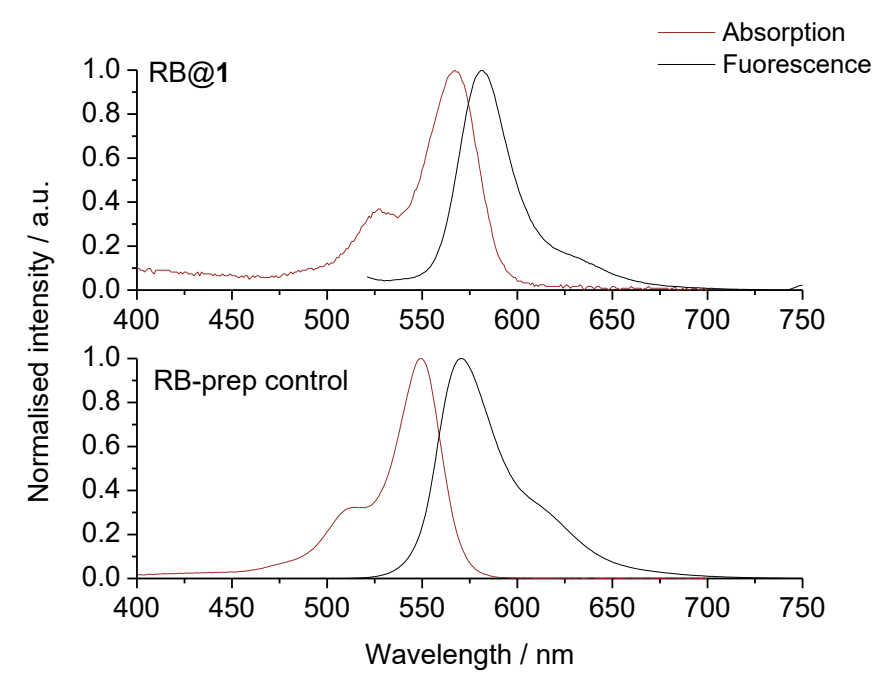

Figure 4.9. Normalised absorption (red) and fluorescence emission (black, $\lambda_{\mathrm{ex}} 500 \mathrm{~nm}$ ) spectra for a representative RB@1 sample (top) and its corresponding RB-prep control (bottom) (10 $\mu \mathrm{M}$ nominal concentration).

The absorption maximum for RB@1 $\left(\lambda_{\max } 569 \mathrm{~nm}\right)$ is red-shifted compared to the one of RBprep control ( $\lambda_{\max } 549 \mathrm{~nm}$ ). Emission maxima also shows this shift to longer wavelengths, from ca. $570 \mathrm{~nm}$ for RB-prep controls to ca. $580 \mathrm{~nm}$ for RB@1. Figure 4.10 represents absorption $\lambda_{\max }$ for 13 different batches, the shift found to be reproducible in the range of 17-21 nm. This bathochromic shift has been previously reported in the literature and some examples are collected in Table 4.3. For example, similarly than for our system, upon adpsortion of $5 \mu M$ RB on PAMAM dendrimers the absorption maximum shifts from $549 \mathrm{~nm}$ to $560 \mathrm{~nm} .{ }^{151}$ The red-shift experienced by RB should be ascribed to decreasing polarity in the environment of encapsulated 
RB. The strong solute-solvent interactions in protic media (as water), such as hydrogen bonds, which stabilize the ground state of RB, are missed in hydrophobic environments. This effect diminishes the energy gap in the absorption spectra leading to $\lambda_{\max }$ red-shifts. ${ }^{120,152}$ Indeed, the same bathochromic effect is produced when RB changes its environment going from water to solvents with reduced polarity as can be seen in Table 4.4. ${ }^{153}$

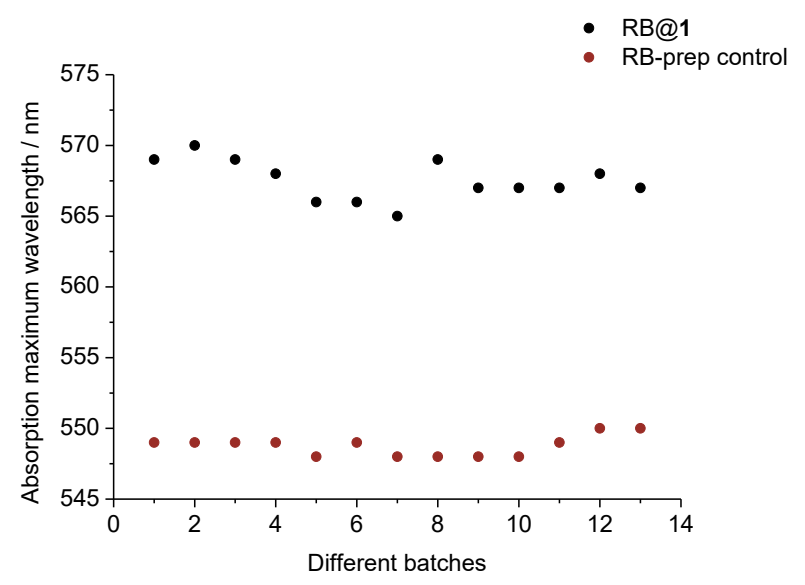

Figure 4.10. Absorption maximum wavelengths for RB@1 (black) and the corresponding RB-prep controls (red) for 13 different batches.

Table 4.3. Some reported bathochromic shifts of absorption and fluorescence emission maxima of RB in different systems.

\begin{tabular}{|c|c|c|}
\hline \multirow{2}{*}{ Systems } & \multicolumn{2}{|c|}{$\lambda_{\max }$ red-shift / nm } \\
\hline & Absorption & Emission \\
\hline RB in DMPC liposomes ${ }^{119}$ & 15 & 13 \\
\hline Multivesicular liposomes loaded with RB 127 & 22 & - \\
\hline RB and lipoprotein complex ${ }^{121}$ & - & 5 \\
\hline RB adsorbed to $A \beta_{42}{ }^{110}$ & 12 & - \\
\hline RB adsorption to collagen-like peptide ${ }^{123}$ & 20 & - \\
\hline RB adsorbed to PAMAM dendrimers ${ }^{151}$ & 11 & 11 \\
\hline RB adsorbed on microgranular cellulose ${ }^{154}$ & 12 & - \\
\hline RB aggregated in alumina-coated silica nanoparticles ${ }^{155}$ & 16 & - \\
\hline RB adsorbed on PDDA polymer ${ }^{156}$ & $\begin{array}{c}10 \text { (solution), } \\
23 \text { (film) }\end{array}$ & $\begin{array}{c}10 \text { (solution) } \\
23 \text { (film) }\end{array}$ \\
\hline RB dimer/aggregates adsorbed in PAH-RB films ${ }^{157}$ & 21 & - \\
\hline RB covalent linked to mesostructured silica nanoparticles ${ }^{132}$ & 17 & 25 \\
\hline $\mathrm{RB}$ bound to $\mathrm{ZnO}$ nanoparticles ${ }^{134}$ & 5 & - \\
\hline
\end{tabular}


Table 4.4. Absorption and fluorescence emission maxima of RB in various solvents (form more to less polar). ${ }^{153}$

\begin{tabular}{lcc}
\hline \multicolumn{1}{c}{ Solvent } & \multicolumn{2}{c}{$\boldsymbol{\lambda}_{\max } / \mathbf{n m}$} \\
\hline Water & 546 & Emission \\
Methanol & 556 & 567 \\
Ethanol & 558 & 573 \\
Isopropanol & 562 & 571 \\
Benzyl alcohol & 570 & 573 \\
Acetonitrile & 558 & 585 \\
Dimethyl sulfoxide & 566 & 571 \\
Dimethylformamide & 562 & 579 \\
Acetone & 562 & 575 \\
Tetrahydrofuran & 560 & 573 \\
1,4-dioxane & 554 & 575 \\
\hline
\end{tabular}

Finally, is also worthy discussing if RB is aggregated or not in our systems, as the formation of aggregates when RB is encapsulated has been often discussed in the literature. ${ }^{154,155} \mathrm{RB}$ tends to aggregate in solution, driven by hydrophobic effects, and does not follow Beer's law at concentrations above $5 \times 10^{-5} \mathrm{M} \cdot{ }^{158-161}$ When RB dimers are formed, a change in the ratio of the peak $(c a .549 \mathrm{~nm})$ to the shoulder $(c a .514 \mathrm{~nm})$ is produced. It has been reported that these RB absorption bands correspond to the monomer and the dimer species, respectively. ${ }^{160,162,163}$ Therefore, the relative intensity of the peak to the shoulder is usually used as a measure of the aggregation of RB (the larger ratio value indicating lesser aggregation). ${ }^{160,164}$ For example, a value of 2.88 was reported for RB partially aggregated in water and of 3.45-3.91, for monomeric RB encapsulated into liposomes. ${ }^{119}$ RB covalently linked to mesostructured silica nanoparticles showed a 1.60-1.92 ratio due to aggregates formation, wheareas a 3.42 ratio was determined for monomeric RB in water. ${ }^{132}$ In our case, the intensity ratios of the absorption of longer to shorter wavelength were found to be 3.1 for RB-prep control (average of 10 samples) and 2.9 for RB@1 (average of 29 samples at different nominal concentrations; correcting the baseline due to nanoparticles light dispersion effect). Therefore, according to the literature precedents, it could be stated that RB in RB@1 samples is found mainly in a monomeric, not aggregated form. This is a favourable fact for the use of RB@1 as singlet oxygen generation systems for PDT, as aggregates are usually found to have lower singlet oxygen quantum yield and, therefore, a lower photodynamic efficacy. ${ }^{120,154}$ 


\section{ROSE BENGAL QUANTIFICATION}

A calibration curve to quantify the concentration of RB was obtained after measuring the absorbance at $549 \mathrm{~nm}$ of a series of RB standard solutions in PBS (0.1-15 $\mu \mathrm{M}$ ) (Figure 4.11). The quantification of RB in the nanogel samples was done measuring the absorbance value of the maximum, assuming the same molar extinction coefficient $(\varepsilon)$ for RB and RB@1. This assumption was proved to be reasonable because upon basification of RB@1 samples, giving free $\mathrm{RB}$, the intensity of the absorption maximum remained essentially the same.

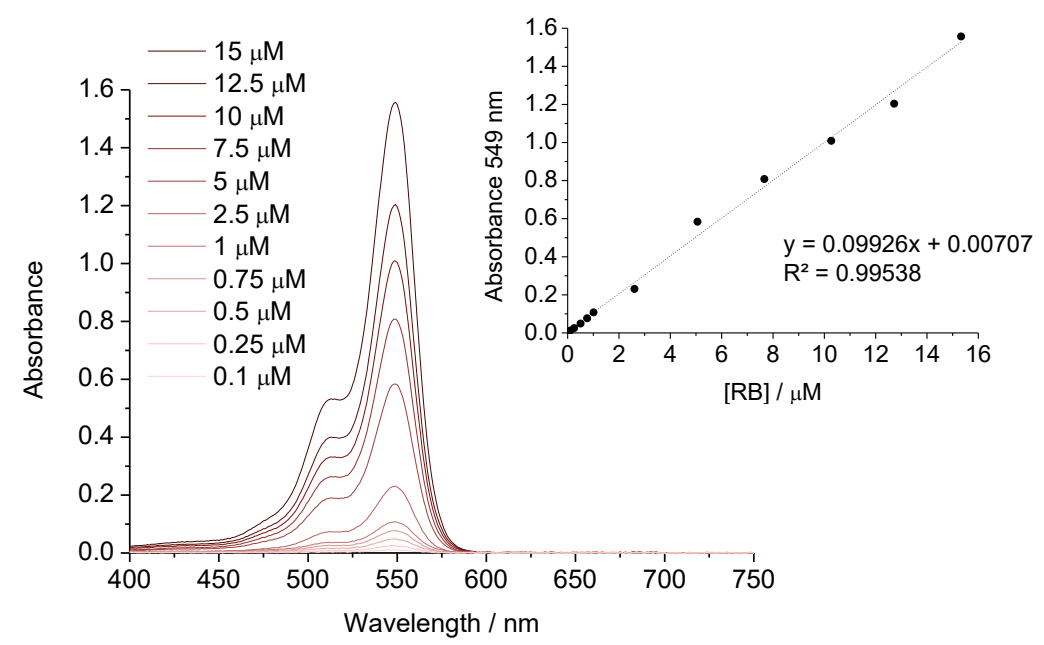

Figure 4.11. Absorption spectra of RB standard solutions in PBS and calibration curve at $549 \mathrm{~nm}$.

The quantified concentration of RB was found to be 0.7-6.9 $\mu \mathrm{M}$ for RB@1 and 1.2-28.5 $\mu \mathrm{M}$ for RB-prep controls with different nominal concentrations (Figure 4.12). Aiming to reach the highest loading, a $20 \mu \mathrm{M}$ expected final concentration was chosen to prepare samples for in vitro assays. The results for 26 different RB@1 samples can be seen in Figure 4.13, with an average concentration of $6 \pm 3 \mu \mathrm{M}$ RB. A $30 \% \mathrm{~mol}$ was found as average entrapment efficiency (ratio of obtained RB concentration to the originally added) of these RB@1 samples. The drug loading was also determined to be $0.8 \% \mathrm{w} / \mathrm{w}$, considering an average concentration of 1 in the nanoparticle suspension of $0.71 \mathrm{mg} / \mathrm{mL}$ (see Section 3.1.2). 


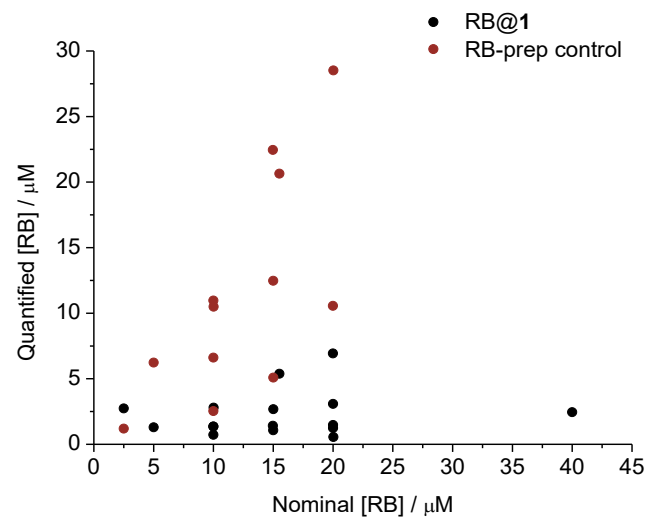

Figure 4.12. Quantified Rose Bengal concentration $v s$. the expected Rose Bengal concentration regarding the quantity of RB added for several RB@1 samples (black) and RB-prep controls (red).

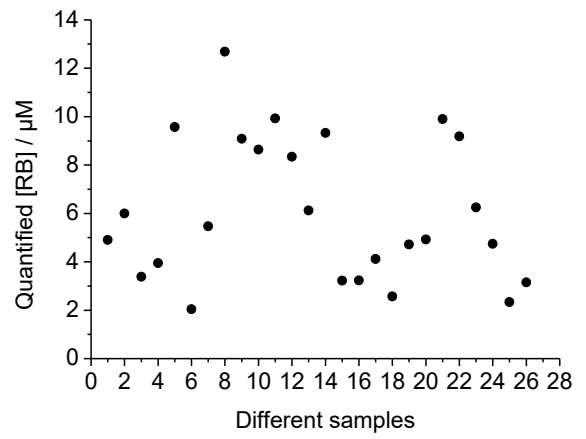

Figure 4.13. Quantification of RB in 26 RB@1 samples of $20 \mu \mathrm{M}$ nominal concentration.

\subsubsection{MEASUREMENT OF SINGLET OXYGEN PHOTOGENERATION}

To confirm whether the RB@1 system could be used for photodynamic therapy, the production of reactive oxygen species by encapsulated RB upon irradiation needed to be proved. As RB is a type II photosensitiser, the production of singlet oxygen $\left({ }^{1} \mathrm{O}_{2}\right)$ was spectroscopically studied using the benchmark method of the probe 9,10-anthracenediyl-bis(methylene) dimalonic acid (ABMA or ABDA). ${ }^{165-167}$ This water-soluble, fluorescent anthracene derivative selectively reacts with ${ }^{1} \mathrm{O}_{2}$, which leads to the formation of a non-fluorescent 9,10-endoperoxide product (Figure 4.14). Therefore, the decay of the absorbance/fluorescence of $A B M A$ is a direct measure of ${ }^{1} \mathrm{O}_{2}$ in the solution, i.e. the generation of ${ }^{1} \mathrm{O}_{2}$ by Rose Bengal.<smiles>O=C(O)C(Cc1c2ccccc2c(CC(C(=O)O)C(=O)O)c2ccccc12)C(=O)O</smiles>

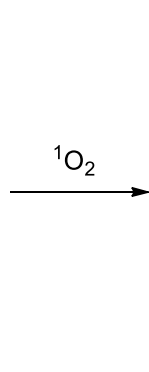

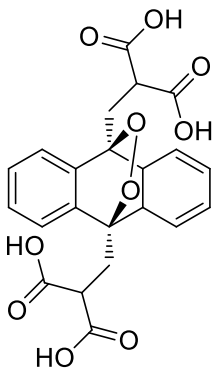

ABMA

Figure 4.14. Reaction between $A B M A$ and ${ }^{1} \mathrm{O}_{2}$ to give the corresponding non-fluorescent 9,10-endoperoxide product. 
For this assay, a RB@1 sample (0.9 $\mu \mathrm{M}$ RB) containing ABMA $(1 \mu \mathrm{M})$ was irradiated with a visible light LED bulb (8.6W) in a cuvette with vigorous stirring (Figure 4.15). The evolution of the photoreaction was monitored over time using fluorescence spectroscopy ( $\lambda_{\text {ex }} 380 \mathrm{~nm}$ ) and studying the decrease of ABMA fluorescence at $407 \mathrm{~nm}$. To check the differences with free photosensitiser, the production of ${ }^{1} \mathrm{O}_{2}$ by a RB solution in PBS at a higher concentration $(5.2 \mu \mathrm{M}$, RB control) was also assayed. The emission spectra at each irradiation time and the decay curves of the normalised ABMA emission at $407 \mathrm{~nm}$ as a function of the irradiation time for both samples are reported in Figure 4.16.

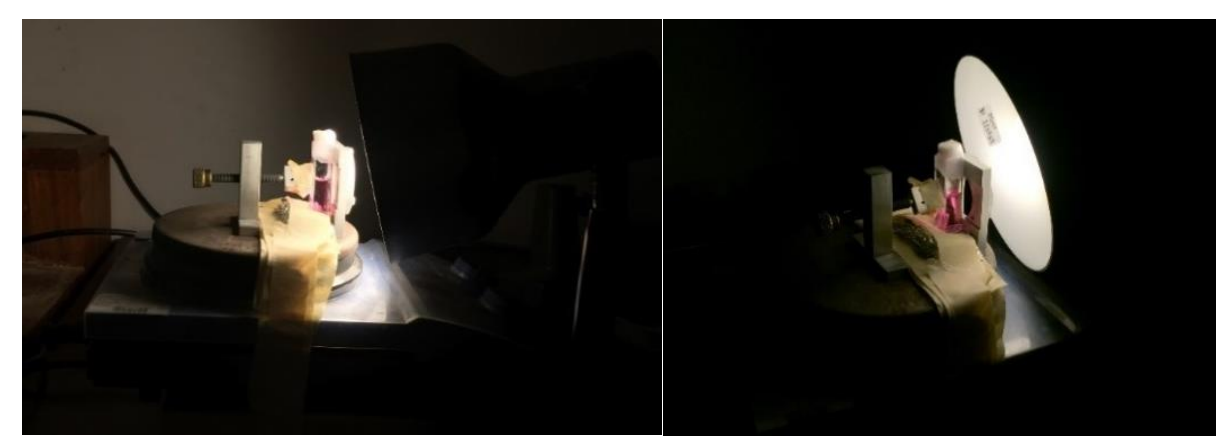

Figure 4.15. Irradiation set up.
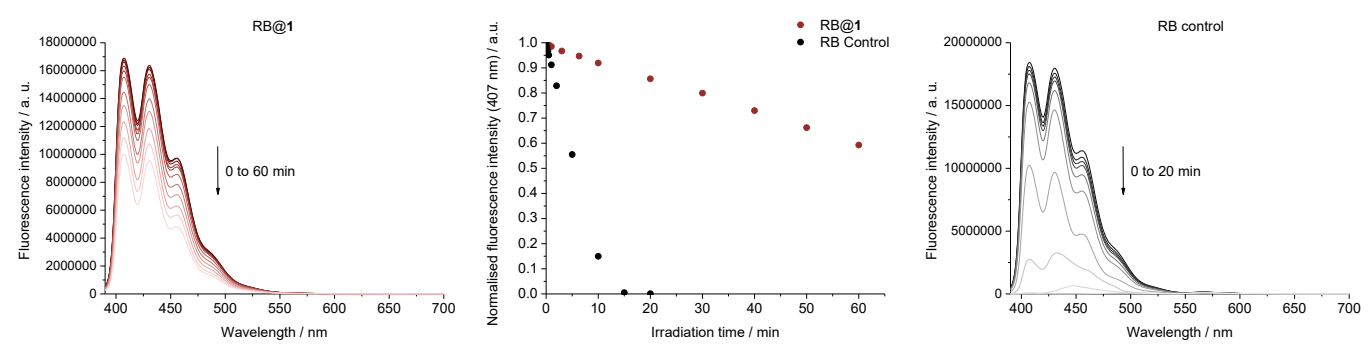

Figure 4.16. Photobleaching of ABMA in the presence of RB@1 (red) and RB control (black) after irradiation. Ends: Fluorescence emission spectra of $A B M A$ as a function of irradiation time for RB@1 (left) and RB control (right) $\left(\lambda_{\text {ex }} 380 \mathrm{~nm}\right.$ ). Centre: Decay curves of the normalized ABMA emission intensity at $407 \mathrm{~nm}$ as a function of the irradiation time in the presence of RB@1 and RB control.

The results for both samples show a decrease in ABMA fluorescence (typical of the photobleaching caused by the formation of the non-fluorescent 9,10-endoperoxide product) upon irradiation of $\mathrm{RB}$, meaning that ${ }^{1} \mathrm{O}_{2}$ is being produced.

In order to compare, the initial points of the kinetic traces (fluorescence intensity at the maximum peak, $407 \mathrm{~nm}$ ) were fitted to a pseudo-first order model $\left(\ln C / C_{0}=-k_{\text {obs }} t\right.$, where $C$ is 
the concentration of $A B M A$ at a certain time $t$ and $C_{0}$ is the initial concentration of $A B M A$ ), considering that for low concentrations can be assumed that fluorescence intensity is proportional to concentration. ${ }^{150,167,168}$ A good fit was obtained. The first-order rate constants kobs were determined from the slope of the linear plots for both systems (Table 4.5) and corrected constants $\left(k_{\mathrm{obs}} /[\mathrm{RB}]\right)$ were calculated to compare avoiding the concentration factor. The corrected $k_{\text {obs }}$ for Rose Bengal in solution is 2.6 times higher than that for encapsulated RB. Namely, the photobleaching of ABMA, i.e., the production of singlet oxygen by irradiated RB, is 2.6 times faster for RB control than for RB@1 samples. A possible rationale for this behaviour is that the nanogel environment lowers the diffusion rate of $\mathrm{O}_{2}$ inside the nanogel, and consequently, the diffusion of ${ }^{1} \mathrm{O}_{2}$ outside of the nanoparticle. ${ }^{169}$ Also, it has to be noted that the efficiency of ${ }^{1} \mathrm{O}_{2}$ generation in the cuvette, hence in the absence of cells, could not be reflecting the situation in the biological context since once the RB@1 system crosses the cellular membrane, the PS could be released due to the disassembly of the nanoparticle.

Table 4.5. Rate constants $k_{\mathrm{obs}}$ obtained for ABMA reaction with singlet oxygen after irradiation of RB@1 and RB control samples. Corrected $k_{\mathrm{obs}}=k_{\mathrm{obs}} /[\mathrm{RB}]$.

\begin{tabular}{lccc}
\hline Sample & $\boldsymbol{k}_{\text {obs }} / \mathbf{~ m i n}^{-1}$ & Corrected $\boldsymbol{k}_{\text {obs }} / \mathbf{L} \cdot \boldsymbol{\mu} \mathbf{m o l}^{-1} \cdot \mathbf{m i n}^{-1}$ & $\mathbf{R}^{\mathbf{2}}$ \\
\hline RB@1 & $0.00790 \pm 0.00016$ & 0.00878 & 0.99677 \\
RB control & $0.11706 \pm 0.00448$ & 0.02251 & 0.99271 \\
\hline
\end{tabular}




\subsection{NANOGELS ENCAPSULATING HYPERICIN}

\subsubsection{INTRODUCTION TO HYPERICIN}

\section{HYPERICIN IN MEDICINE}

Hypericin (HYP) is a natural compound belonging to a class of phenanthroperylenequinones extracted from the plant Hypericum perforatum (commonly known as St. John's wort, Figure 4.17), a perennial herb with golden yellow flowers that can be found worldwide. Although the function of these extracted compounds in the own plant is not very well known (it is thought they could function as deterrent allelochemicals to defend the host plant against insects and other pests), Hypericum species have been of scientific interest for many years due to their widespread use in folk medicine..$^{40,170-173}$ Nowadays, Hypericum extract is still widely used for the treatment of mild and moderate depression $(500 \mathrm{mg}$ extract are used as an oral daily dose, corresponding to a total amount of $1-2 \mathrm{mg}$ of hypericin) ${ }^{174,175}$ and clinical trials have demonstrated that a low dosage has a positive effect on mood and short-term verbal memory. ${ }^{176}$

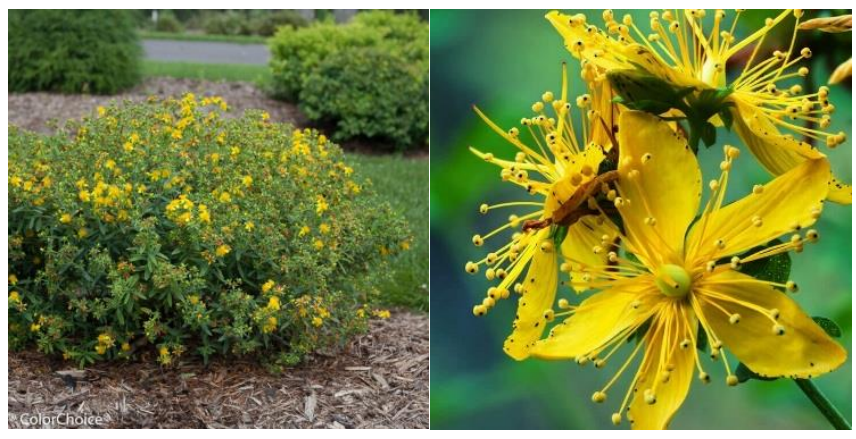<smiles></smiles>

Figure 4.17. Hypericum perforatum (St. John's wort) and the structure of hypericin.

Hypericin itself has also been intensively studied for its broad pharmacological spectrum. Hypericin consists of aromatic rings that form a planar molecule (Figure 4.17). Its delocalised $\pi$ electron system is responsible for its photoactive behaviour, and it generates reactive oxygen species, with a high singlet oxygen quantum yield, ${ }^{94,177}$ in the presence of light (at wavelengths around $600 \mathrm{~nm}$ ) and oxygen. ${ }^{178}$ Additionally, it possesses minimal or no toxicity in the dark ${ }^{179-}$ 183 and accumulates preferentially in neoplastic tissues. ${ }^{184,185}$ It also shows some advantages over usual photosensitizers, as a low photobleaching, a high extinction coefficient and a large excitation range in the visible region. These properties make hypericin a powerful 
photosensitizer that is suitable for photodynamic therapy, with attractive applications for the treatment of tumours. Moreover, its light-dependent fungicidal, ${ }^{186,187}$ bactericidal ${ }^{188,189}$ and antiviral effects $40,190,191$ have also been reported. Without light, hypericin has also been used to treat abdominal pain and headache due to its anti-inflammatory activity. ${ }^{192,193}$ Hypericin exhibits bright fluorescence so, as its accumulation is significantly higher in neoplastic tissue than in normal tissue, it can also be used in cancer photodynamic diagnosis (PD) as an effective fluorescence marker for tumour detection and visualization. ${ }^{40,194}$ Fluorescence diagnosis using hypericin has been clinically tested in bladder ${ }^{195}$ (even using nanocarriers, such as hypericin noncovalent bonded to polyvinylpyrrolidone ${ }^{196}$ ), head and neck cancers ${ }^{197,198}$ or gliomas. ${ }^{199}$

The exact mechanisms of hypericin cellular uptake are still unclear. They require further investigation, but results indicate that it might be transported into or through cells via temperature-dependent diffusion, ${ }^{179,200}$ partitioning, pinocytosis or endocytosis. ${ }^{201}$ Concerning its subcellular redistribution, cellular localization studies in cancer cell lines ${ }^{180,202,203}$ revealed that this compound, with pronounced hydrophobic character, accumulates in cytoplasmic membranes, particularly in the membranes of the endoplasmic reticulum, the Golgi complex, lysosomes and mitochondria. ${ }^{40,204-206}$ However, the cellular uptake and subcellular localization of hypericin also appears to be influenced by the cell type (for example, it is reported to accumulate in nuclear membranes in colon carcinoma Caco-2 cells ${ }^{200}$ ), incubation concentrations and/or interaction with serum lipoproteins. ${ }^{194}$

The antineoplastic photodynamic efficacy of hypericin can be directly correlated to the intracellular accumulation of the drug. Membranes and, specifically, mitochondria, ${ }^{40,207}$ have been postulated as the principal cellular targets of hypericin. ${ }^{202,203,208}$ Upon light-activation, hypericin is efficient primarily in the generation of singlet oxygen (type II mechanism) ${ }^{94}$ and superoxide anion (type I mechanism). ${ }^{178,179}$ Additional mechanisms may contribute, as the formation of hypericin radicals or a hypericin-induced $\mathrm{pH}$ drop have been suggested as potential mediators of its photocytotoxicity. ${ }^{40}$ The final response of hypericin-mediated PDT (HYP-PDT) might also be affected by the ability of cells to overcome oxidative stress through the activity of various cytoprotective mechanisms, including cellular redox systems. ${ }^{206,209}$ The exact mechanisms of action and the cellular aspects of HYP-PDT have been outlined and summarized in several reviews. ${ }^{40,171,210,211}$ In any case, the process can ultimately lead to necrosis, ${ }^{212-214}$ apoptosis, ${ }^{204,214}$ autophagy-associated cell death ${ }^{215,216}$ or even to immunogenic cell death. ${ }^{217}$ 
Preclinical and clinical studies have assessed the hypericin antineoplastic efficacy upon irradiation. On the one hand, many in vitro studies have demonstrated the potent cytotoxicity of photoactivated hypericin in various cancer cell types. ${ }^{213,218-222}$ In vivo studies of HYP-PDT ${ }^{194}$ showed the inhibition of tumour growth, prolonged survival time of the treated animals and tumour necrosis, apoptosis or damage to the tumour vasculature in, just to cite some examples, epidermoid, ${ }^{223}$ nasopharyngeal, ${ }^{224}$ squamous $^{219}$ and bladder ${ }^{184,225}$ carcinoma, prostate adenocarcinoma ${ }^{218}$ or pancreatic cancer. ${ }^{226}$ Finally, three clinical trials of HYP-PDT applied to various skin disorders have been published for the treatment of (i) basal and squamous cell carcinoma, ${ }^{227}$ (ii) non-melanoma skin cancers, ${ }^{228}$ and (iii) lymphocyte-mediated skin disorders (mycosis fungoides, psoriasis). ${ }^{229}$ All these clinical data indicate that topically applied hypericin, combined with its photoactivation, might be a promising and safe alternative for the treatment of some cancerous and non-cancerous skin disorders.

\section{HYPERICIN AGGREGATION}

Hypericin is readily dissolved in polar organic solvents, like dimethyl sulfoxide (DMSO), acetone, acetonitrile, tetrahydrofuran, dioxane, ethyl acetate or alcohols, to give red solutions. ${ }^{230}$ HYP dissolved in these solvents remains in its monomeric form up to a concentration of $c a .10^{-3} \mathrm{M}$, with a relatively high quantum yield of fluorescence (ca. 0.2 ) and a high quantum yield of singlet oxygen formation. ${ }^{231,232}$ In an aqueous environment and some nonpolar organic solvents (as toluene, carbon tetrachloride, chloroform, hexane or cyclohexane), HYP is almost insoluble and forms aggregates. ${ }^{233-235}$ The aggregation in water solutions strongly depends on the $\mathrm{pH}$. Neutral, non-ionic HYP, which predominates at very acidic $\mathrm{pH}$ values, and the monoanionic form, which predominates in the $\mathrm{pH}$ range $2-8$, are prone to aggregation. The dianion, the main species in the $\mathrm{pH}$ window 8-11, is considerably less susceptible to aggregation and presents higher water solubility (Figure 4.18 and Figure 4.19). ${ }^{235,236}$ Two different mechanism for neutral and/or monoanionic HYP self-association have been proposed: (i) stacked (face-to-face) association forming $\mathrm{H}$-aggregates and (ii) planar association comprising J-aggregates. ${ }^{231,235,237}$ The stacked association is due to the hydrophobic effect of the aromatic core of HYP molecules, which leads to $\pi-\pi$ stacking interaction between molecules. A model in which the neighbouring molecules within the stack are rotated against each other by about $180^{\circ}$ was proposed. ${ }^{234,236,238}$ Planar association involves intermolecular hydrogen bonding between the hydroxyl and carbonyl groups of HYP molecules. ${ }^{238-240}$ 


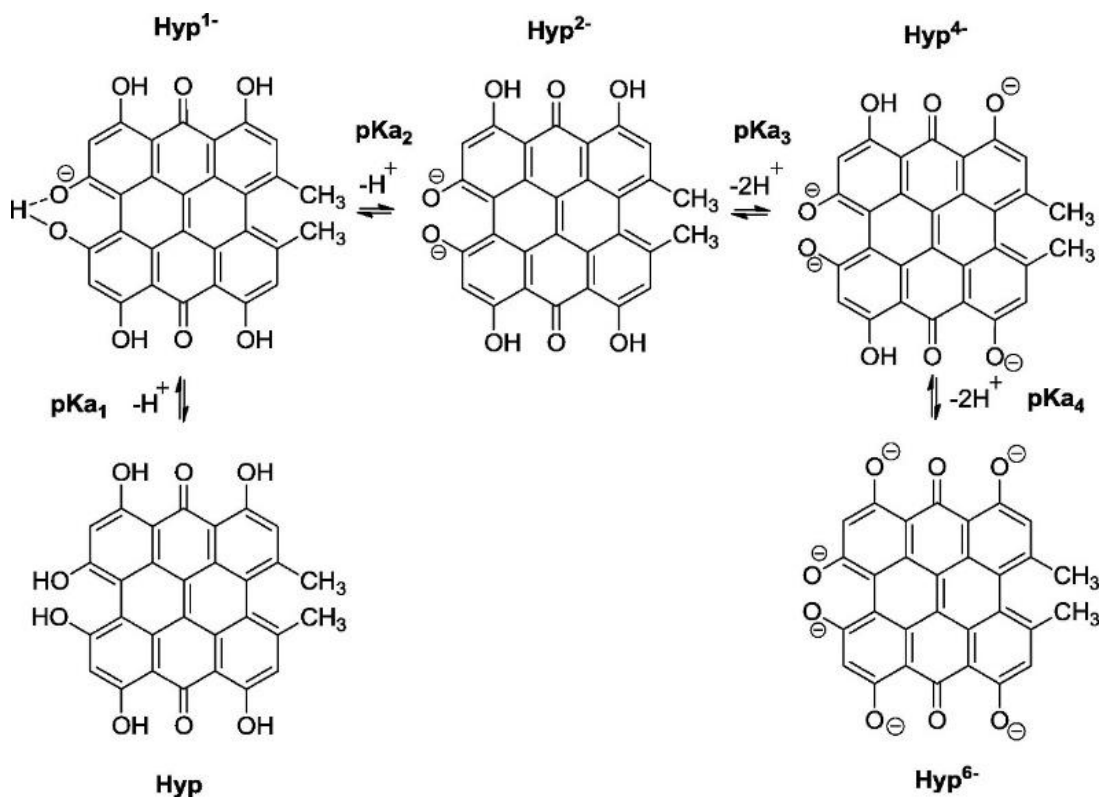

Figure 4.18. Protolytic equilibriums of hypericin. ${ }^{241}$

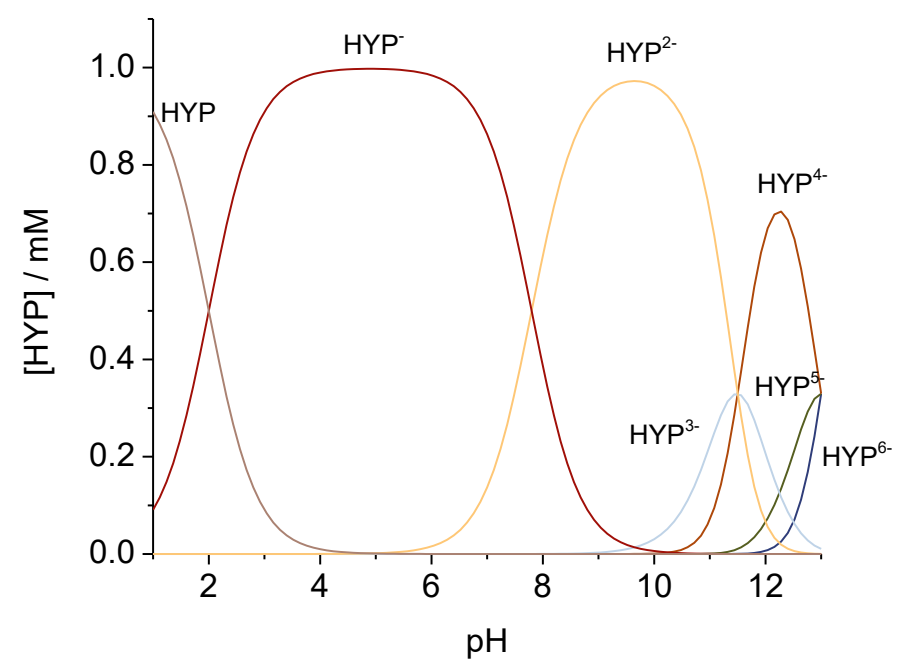

Figure 4.19. $\mathrm{pH}$ range of existence of different hypericin forms.

Aggregation modifies hypericin spectral and energetic characteristics, affects its biological activity and influences its efficacy in applications. For instance, the absorption spectrum of hypericin is solvent dependent. ${ }^{236,240,242}$ It contains two major visible transitions, $\mathrm{S}_{0} \rightarrow \mathrm{S}_{1}\left(\lambda_{\max }=\right.$ $500-600 \mathrm{~nm}$, with three vibronic levels) and $\mathrm{S}_{0}->S_{2}\left(\lambda_{\max }=485-425 \mathrm{~nm}\right)$, and two major UV 
bands. ${ }^{230}$ In polar organic solvents (Figure 4.20 left, corresponding to monomeric hypericin), the spectrum is characterised by well-resolved absorption bands ${ }^{240}$ and the dominance of the redmost band. On the other hand, in water and in nonpolar organic solvents, spectra (Figure 4.20 right) exhibit some changes associated with hypericin aggregation: large reductions in extinction coefficients (although the relative absorbance of bands in the UV region is increased compared to that in the red region), broadening of visible bands, less vibronic structure and changes in the relative intensity of the peaks associated with the $S_{1}$ transition (the second peak with lower energy becomes the most intense peak (water, cyclohexane, toluene) or these two peaks are nearly equal in intensity (hexane, chloroform, carbon tetrachloride)). ${ }^{230}$ In all cases, the peak maxima of both the first $\left(\mathrm{S}_{0}->\mathrm{S}_{1}\right)$ and the second $\left(\mathrm{S}_{0}>\mathrm{S}_{2}\right)$ electronic transitions shift with solvent: to the red region on increasing the solvent polarity and to the blue region in hydrogen-bonding solvents. The same shifts have been seen for fluorescence emission. ${ }^{230,240}$ For a detailed data of absorption maxima for hypericin in various solvents, see Table 4.6. For example, monomeric hypericin in DMSO (Figure 4.20 left) shows two narrow absorption peaks at 555 and $599 \mathrm{~nm}$. In contrast, hypericin aggregates in an aqueous environment (10 $\mu \mathrm{M}$ suspension in PBS-10\%DMSO, Figure 4.20 right) show a bathochromic shift, with wider bands at 564 and $603 \mathrm{~nm}$ and decreased intensity.
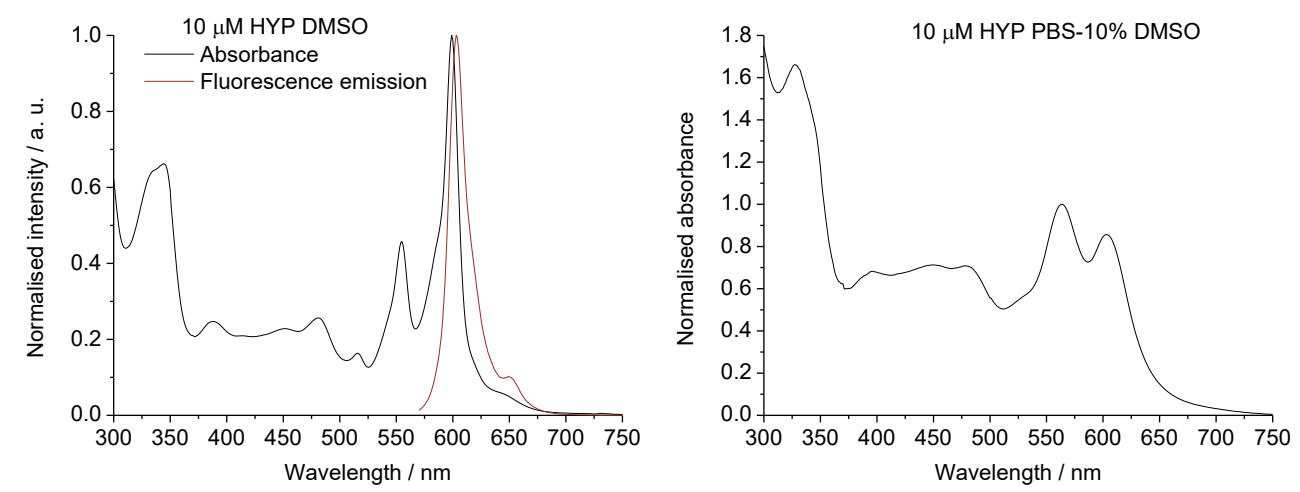

Figure 4.20. Left: Normalised absorption (black) and fluorescent emission (red, $\lambda_{\mathrm{ex}} 550 \mathrm{~nm}$ ) spectra of a $10 \mu \mathrm{M}$ hypericin solution in DMSO. Right: Normalised absorption spectra of a $10 \mu \mathrm{M}$ hypericin solution in PBS-10\%DMSO. In agreement with other publications. ${ }^{243}$ 
Table 4.6. Absorption maxima for hypericin in various solvents. ${ }^{230}$

\begin{tabular}{lcc}
\hline \multicolumn{1}{c}{ Solvent } & Absorption $\boldsymbol{\lambda}_{\max } / \mathbf{~} \mathbf{m}$ \\
\hline Tetrahydrofuran & 600 & 555 \\
Dimethylformamide & 598 & 554 \\
Benzyl alcohol & 596 & 551 \\
Acetone & 596 & 551 \\
Acetonitrile & 594 & 550 \\
Butanol & 592 & 549 \\
Ethyl acetate & 592 & 549 \\
Ethanol & 591 & 547 \\
Dioxane & 590 & 547 \\
Methanol & 588 & 545 \\
Ether & 580 & 540 \\
Acetic acid & 579 & 538 \\
Formic acid & 578 & 537 \\
Cyclohexane & 613 & 570 \\
Hexane & 613 & 570 \\
Toluene & 609 & 570 \\
Carbon tetrachloride & 609 & 568 \\
Water & 598 & 560 \\
Chloroform & 595 & 570 \\
\hline
\end{tabular}

Aggregates of hypericin are non-fluorescent, as emission is quenched due to intermolecular charge-transfer. ${ }^{234}$ For example, if the proportion of water increases in a water-DMSO mixture, no fluorescence emission is obtained at an $80 \%$ water content (100 $\mu \mathrm{M}$ HYP $).{ }^{237}$ Thus, fluorescence intensity variations can be used to detect the aggregation of HYP. Aggregates also show a suppressed photodynamic activity ${ }^{231,234,244}$ and, regarding the bioactivity, it has been seen that HYP aggregation exerts poor phototoxicity on cells ${ }^{245}$ or changes the biodistribution. ${ }^{237,243}$

\section{HYPERICIN ENCAPSULATION}

The majority of in vitro and in vivo studies imply handling hypericin as solutions in ethanol or organic solvents to preclude aggregation. ${ }^{246,247}$ Also, different formulations of HYP have been investigated ${ }^{193,248}$ using nontoxic pharmaceutical additives like plasma proteins, ${ }^{195}$ ion pairs, polyethyleneglycol, ${ }^{249}$ Beeler basis, ointments, Carbopol ${ }^{\mathrm{TM}}$ gel, Cetomacrogol ${ }^{\mathrm{TM}} \mathrm{cream}$ or petrolatum, ${ }^{250}$ among others.

The use of delivery nanosystems for hypericin has also been receiving increasing attention. ${ }^{237}$ Several examples of hypericin-loaded nanosystems are listed in Table 4.7. Most of them encapsulate hypericin by non-covalent interactions, otherwise indicated. Hypericin has been 
encapsulated into liposomes, polymeric nanoparticles, monoclonal antibodies, polymeric micelles, lipoproteins, solid lipid NPs, NPs from block copolymers, UCNPs, lipid nanocapsules, iron oxide NPs, magnetic nanospheres or calcium phosphate NPs, among others.

Table 4.7. Examples of hypericin-loaded systems chronologically listed (PD: photodetection, NPs: nanoparticles, DDS: drug delivery system, UCNP: Upconverting nanoparticles).

\begin{tabular}{|c|c|c|c|c|}
\hline Type of nanocarrier & Size / nm & Drug loading & Objective & Reported \\
\hline $\begin{array}{l}\text { Transferrin- } \\
\text { conjugated PEG- } \\
\text { liposomes }^{251}\end{array}$ & 150 & $\begin{array}{c}20000 \\
\text { molecules/liposome }\end{array}$ & Cancer PDT & $\begin{array}{l}\text { Similar in vitro (HeLa cells) } \\
\text { phototoxicity and intracellular } \\
\text { accumulation than free drug }\end{array}$ \\
\hline $\begin{array}{l}\text { Polymeric polylactic } \\
\text { acid (PLA) NPs }{ }^{61}\end{array}$ & $200-300$ & $0.03-0.15 \% \mathrm{w} / \mathrm{w}$ & $\begin{array}{l}\text { PD and PDT of } \\
\text { ovarian cancer }\end{array}$ & $\begin{array}{l}\text { Higher in vitro (ovarian cancer } \\
\text { cells) photoactivity than free } \\
\text { drug }\end{array}$ \\
\hline Polymeric PLA NPs ${ }^{248}$ & 268 & $3.7 \% \mathrm{w} / \mathrm{w}$ & $\begin{array}{l}\text { PD of ovarian } \\
\text { cancer }\end{array}$ & $\begin{array}{l}\text { Higher in vivo accumulation } \\
\text { than free drug in malignant } \\
\text { ovarian tissues }\end{array}$ \\
\hline $\begin{array}{l}\text { Biodegradable PLA } \\
\text { NPs }^{252}\end{array}$ & $240-270$ & $0.04 \%-0.15 \% \mathrm{w} / \mathrm{w}$ & $\begin{array}{l}\text { Fluorescence and } \\
\text { photoactivity } \\
\text { quenching into } \\
\text { NPs to obtain an } \\
\text { "activatable" DDS }\end{array}$ & $\begin{array}{l}\text { In vitro (SK-OV-3 ovarian } \\
\text { carcinoma cells) fluorescence } \\
\text { and photodynamic activity } \\
\text { recovery upon cell } \\
\text { internalization }\end{array}$ \\
\hline $\begin{array}{l}\text { Anti-HSA (anti- } \\
\text { hepatocyte specific } \\
\text { antigen) monoclonal } \\
\text { antibodies }{ }^{253}\end{array}$ & - & $0.2 \mu \mathrm{M}$ & Cancer PDT & $\begin{array}{l}\text { Increased in vitro (HepG2 cells) } \\
\text { phototoxicity }\end{array}$ \\
\hline $\begin{array}{l}\text { MPEG-hexPLA } \\
\text { copolymer } \\
\text { micelles }^{254}\end{array}$ & $<32$ & $\begin{array}{l}0.69 \text { and } 2 \mathrm{mg} / \mathrm{mL} \\
(1.7 \text { and } 9.8 \% \mathrm{w} / \mathrm{w})\end{array}$ & $\begin{array}{l}\text { PD of ovarian } \\
\text { cancer }\end{array}$ & $\begin{array}{l}\text { Higher in vivo accumulation } \\
\text { than free drug in tumours }\end{array}$ \\
\hline $\begin{array}{l}\text { Dextran-coated low- } \\
\text { density lipoproteins } \\
(\mathrm{LDL})^{255}\end{array}$ & 22 & $0.4 \mu \mathrm{M}$ & Cancer PDT & $\begin{array}{l}\text { Improved in vitro (U87-MG } \\
\text { cells) carrying versus uncoated } \\
\text { LDL }\end{array}$ \\
\hline Solid lipid NPs ${ }^{256}$ & $200-300$ & $0.8 \%$ & Cancer PDT & $\begin{array}{l}\text { In vitro (HepG2 cells) inhibition } \\
\text { of the photoactivity }\end{array}$ \\
\hline $\begin{array}{l}\text { Biodegradable PCL- } \\
\text { PEG block copolymer } \\
\text { NPs }^{247}\end{array}$ & 50 & $0.2 \mu \mathrm{M}$ & $\begin{array}{l}\text { Antimicrobial PDT } \\
\text { and wound } \\
\text { healing }\end{array}$ & $\begin{array}{l}\text { Improved in vitro and in vivo } \\
\text { antimicrobial PDT and } \\
\text { wound healing }\end{array}$ \\
\hline $\begin{array}{l}\text { Silica-coated UCNP } \\
\text { (NaYF } 4: Y b / E r) \\
\text { (covalent bond) }{ }^{257}\end{array}$ & 10 & - & Cancer PDT & $\begin{array}{l}\text { In vitro (HeLa and HepG2 cells) } \\
\text { outstanding induction of } \\
\text { apoptosis }\end{array}$ \\
\hline $\begin{array}{l}\text { Lipid nanocapsule } \\
\text { formulation }^{258}\end{array}$ & $25-100$ & $0.02-0.04 \% \mathrm{w} / \mathrm{w}$ & Cancer PDT & $\begin{array}{l}\text { In vitro (HeLa and HEK cells) } \\
\text { photoactivity }\end{array}$ \\
\hline Solid lipid NPs ${ }^{259}$ & 153 & $5.2 \% \mathrm{w} / \mathrm{w}$ & Cancer PDT & $\begin{array}{l}\text { Higher in vitro (HEp-2 and B16- } \\
\text { F10 cells) cellular uptake (30\%) } \\
\text { and phototoxicity (26\%) than } \\
\text { free drug }\end{array}$ \\
\hline $\begin{array}{l}\text { Dextran-coated iron } \\
\text { oxide NPs (covalent } \\
\text { bond) }{ }^{260}\end{array}$ & $55-85$ & $6.9 \mu \mathrm{g} / \mathrm{mL}$ & Cancer PDT & $\begin{array}{l}\text { In vitro (Jurkat T-cells) } \\
\text { photoactivity }\end{array}$ \\
\hline
\end{tabular}




\begin{tabular}{|c|c|c|c|c|}
\hline $\begin{array}{l}\text { Biodegradable PLA } \\
\text { NPs }^{261}\end{array}$ & 205 & $1.1 \mathrm{mg} / 150 \mathrm{mg}$ & $\begin{array}{l}\text { Study of } \\
\text { localisation in } \\
\text { cancer cells }\end{array}$ & $\begin{array}{l}\text { In vitro (A549 cancer cells) } \\
\text { study of intracellular dynamics } \\
\text { associated with NP uptake }\end{array}$ \\
\hline $\begin{array}{l}\text { Hyaluronic acid- } \\
\text { coated PLGA-PEI } \\
\text { NPs }^{262}\end{array}$ & 160 & $8 \mu \mathrm{g} / \mathrm{mL}$ & $\begin{array}{l}\text { Gene therapy and } \\
\text { PD for cancer }\end{array}$ & $\begin{array}{l}\text { In vivo enhance of transfection } \\
\text { efficiency and accumulation in } \\
\text { tumours }\end{array}$ \\
\hline $\begin{array}{l}\text { Core-shell magnetic } \\
\text { nanospheres } \\
\left(\mathrm{Fe}_{3} \mathrm{O}_{4} @ \mathrm{PDA} \mathrm{NSs}\right)^{263} \\
\end{array}$ & 560 & 18 mg/g & $\begin{array}{l}\text { Selective } \\
\text { recognition of HYP }\end{array}$ & - \\
\hline $\begin{array}{l}\text { Calcium phosphate } \\
\text { NPs }^{264}\end{array}$ & 43 & $0.1 \mu \mathrm{g} / \mu \mathrm{g}$ & $\begin{array}{l}\text { PDT for parasitic } \\
\text { diseases }\end{array}$ & $\begin{array}{l}\text { In vitro antileishmanial } \\
\text { response and photoactivity }\end{array}$ \\
\hline $\begin{array}{l}\text { Lipid nanocapsules } \\
\text { (with two PS) }^{265}\end{array}$ & 30 & $0.04 \% w / w$ & Cancer PDT & $\begin{array}{l}\text { Increased in vitro (HeLa and } \\
\text { MDA-MB- } 231 \text { cells) } \\
\text { phototoxicity, in vivo tumour } \\
\text { growth retardation }\end{array}$ \\
\hline $\begin{array}{l}\text { Fusogenic, DOTAP } \\
\text { and DSPE-PEG } \\
\text { liposomes }^{266} \\
\end{array}$ & 120 & $200 \mu \mathrm{M}$ & Antimicrobial PDT & $\begin{array}{l}\text { In vitro improved bacterial } \\
\text { uptake and reduction of } \\
\text { bacteria }\end{array}$ \\
\hline $\begin{array}{l}\text { HYP-cyclodextrin } \\
\text { complex into } \\
\text { liposomes }^{267}\end{array}$ & 200 & $150 \mu \mathrm{g} / \mathrm{mL}$ & Antimicrobial PDT & $\begin{array}{l}\text { In vitro photodynamic } \\
\text { reduction of bacteria }\end{array}$ \\
\hline DPPC liposome ${ }^{268}$ & 154 & $2.1 \mu \mathrm{M}$ & $\begin{array}{l}\text { Study of } \\
\text { interactions with } \\
\text { liposomes } \\
\text { as biomimetic } \\
\text { system }\end{array}$ & $\begin{array}{l}\text { Study of the loading, interaction } \\
\text { and localization of HYP in DPPC } \\
\text { liposomes }\end{array}$ \\
\hline $\begin{array}{l}\text { HYP-cyclodextrin } \\
\text { complex into } \\
\text { liposomes }{ }^{269}\end{array}$ & $127-212$ & - & Cancer PDT & $\begin{array}{l}\text { In vitro (SK-OV-3 cells) severe } \\
\text { phototoxicity }\end{array}$ \\
\hline
\end{tabular}

\subsubsection{RESULTS AND DISCUSSION}

\subsubsection{LOADING STUDIES}

\section{HYPERICIN ENCAPSULATION INTO NANOGELS}

The hypericin-loaded nanogels (HYP@1) were prepared following the method described in Section 3.1. The procedure requires the preparation of gels of 1 using a HYP suspension in toluene (Figure 4.21). Firstly, a series of HYP@1 samples with different concentrations were prepared to optimize the loading. For comparison purposes, a control experiment (HYP-PBS control) following the same protocol without gelator was also performed. Noticeably, HYP@1 samples are slightly purple to the naked eye, while HYP-PBS ones are colourless. 


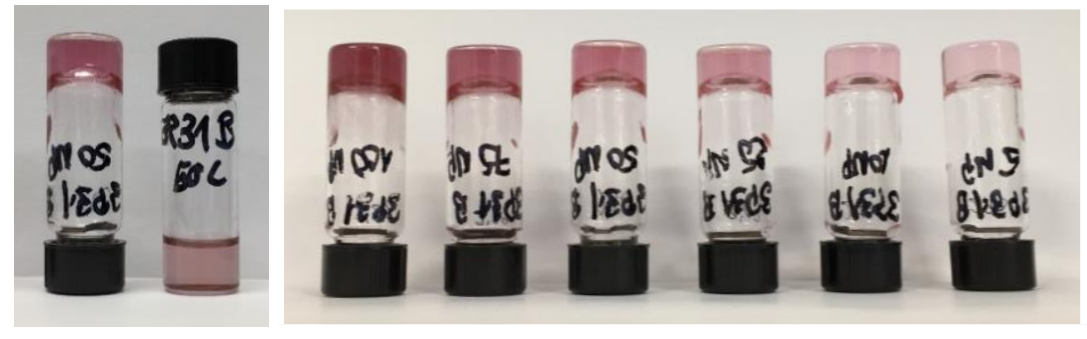

Figure 4.21. Gels of 1 with hypericin in toluene. Left: $100 \mu \mathrm{M}$ HYP gel and the corresponding HYP-PBS control. Right: gels in a hypericin concentration from 200 to $10 \mu \mathrm{M}$.

\section{ABSORPTION AND FLUORESCENCE EMISSION SPECTRA}

The absorption and fluorescence emission $\left(\lambda_{\text {ex }} 550 \mathrm{~nm}\right)$ spectra of a representative pair HYP@1/HYP-PBS control (75 $\mu \mathrm{M}$ nominal concentration) can be seen in Figure 4.22.
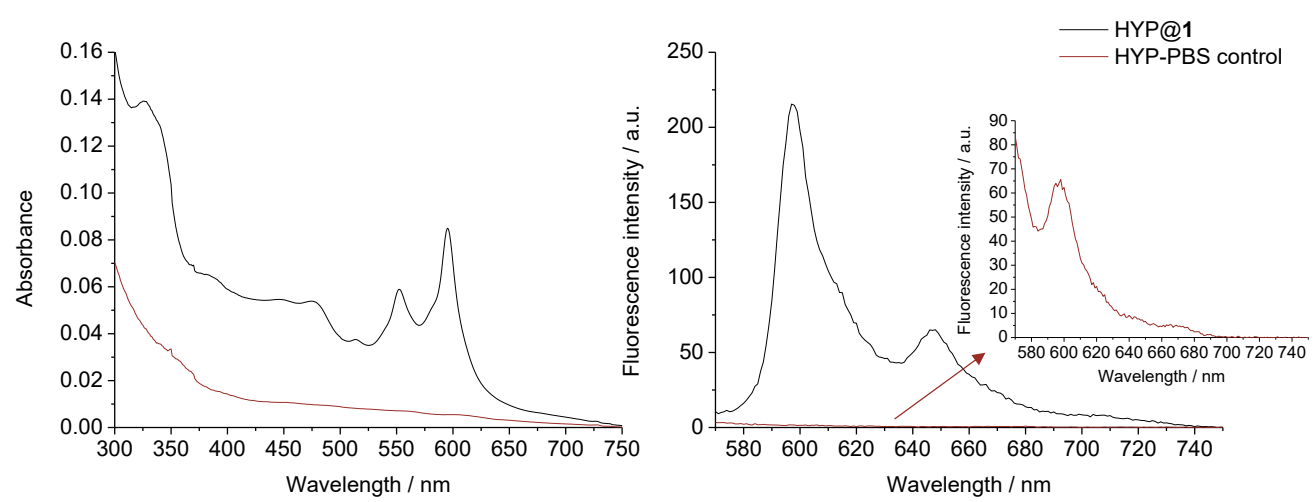

Figure 4.22. Absorption (left) and fluorescence emission (right, $\lambda_{\text {ex }} 550 \mathrm{~nm}$ ) spectra of a representative pair HYP@1 (black) and HYP-PBS control (red) (75 $\mu \mathrm{M}$ nominal concentration). Inset: Fluorescence emission ( $\lambda_{\mathrm{ex}} 550 \mathrm{~nm}$ ) spectra of HYP-PBS control using different acquisition parameters.

The absorption and fluorescence emission of hypericin are almost negligible for HYP-PBS controls. This result comes from the very low water solubility of HYP. However, the presence of nanogel particles helps to solubilise HYP, resulting in a dramatic enhancement of absorption and fluorescence emission. As expected, the spectra of HYP-PBS controls in the visible region show the characteristics of aggregated hypericin in water: broad absorption bands at 560 and $605 \mathrm{~nm}$ and low-intensity fluorescence emission bands at 597 and $670 \mathrm{~nm}$. On the other hand, as shown in Figure 4.23, the shape of the absorption spectrum of HYP@1 samples (maxima at 552 and $596 \mathrm{~nm}$ ) is identical to that of hypericin in a DMSO solution, with a shift of $3 \mathrm{~nm}$ towards shorter wavelengths. So it is the emission spectrum (maxima at 597 and $648 \mathrm{~nm}$ ), with a $\lambda_{\max }$ 
shift of $6 \mathrm{~nm}$, again towards the blue region. Therefore, these data suggest that hypericin is mainly in a monomeric, non-aggregated form in HYP@1. Additionally, it has been described that the absorption peak maxima of hypericin shifts to the red on increasing the solvent polarity. ${ }^{230,240,265}$ Therefore, hypericin in the nanogels $\left(\lambda_{\max } 596 \mathrm{~nm}\right)$ is in a more hydrophobic environment compared to both PBS $\left(\lambda_{\max } 605 \mathrm{~nm}\right)$ and DMSO $\left(\lambda_{\max } 599 \mathrm{~nm}\right)$, probably being located in hydrophobic pockets of the nanoparticles.

Having hypericin in a monomeric state, essential for effective PDT, and at a higher concentration than that attainable in water (HYP-PBS control) is an encouraging result. The monomeric form of hypericin has been previously reported into lipid membrane structures ${ }^{270}$ and nanoparticles. ${ }^{265}$ The absorption spectrum of monomeric HYP has also been observed for hypericin in other systems such as biodegradable PLA NPs, ${ }^{261}$ anti-HSA monoclonal antibodies, ${ }^{253}$ solid lipid nanoparticles ${ }^{259}$ or lipid nanocapsules. ${ }^{258,265}$ In this latter example, a 3 nm hypsochromic shift regarding DMSO $\lambda_{\max }$, as in HYP@1, was described. ${ }^{258,265}$
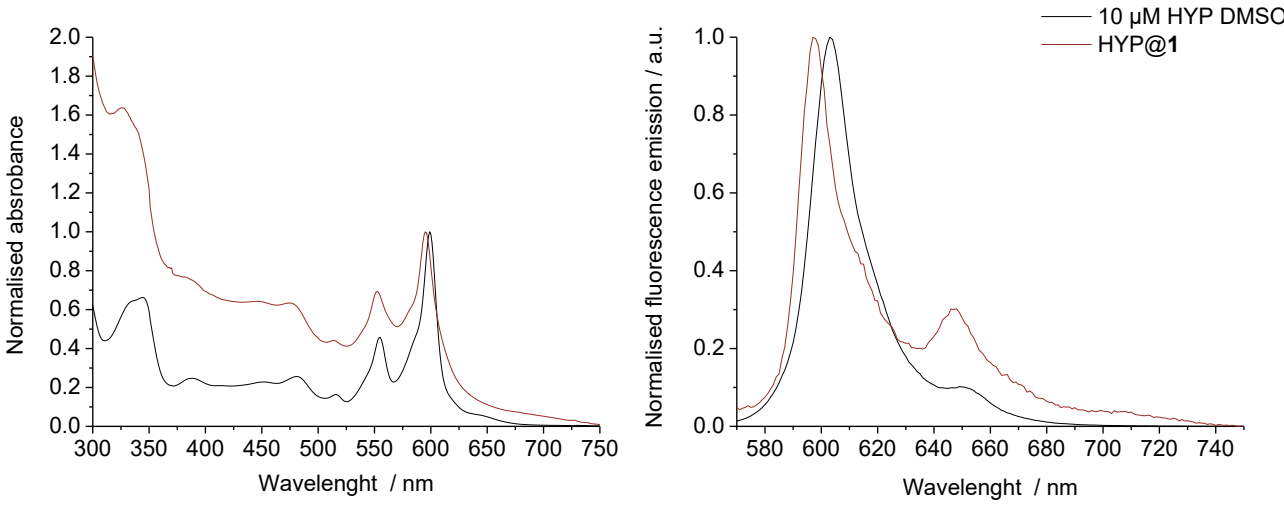

Figure 4.23. Overlap of the normalised absorption (left) and fluorescence emission (right, $\lambda_{\mathrm{ex}} 550 \mathrm{~nm}$ ) spectra of a $10 \mu \mathrm{M}$ hypericin solution in DMSO (black) and a representative HYP@1 sample (red) (75 $\mu \mathrm{M}$ nominal concentration).

\section{HYPERICIN QUANTIFICATION}

A calibration curve to quantify the concentration of HYP was built from the absorbance at 599 $\mathrm{nm}$ of a series of standard hypericin solutions in DMSO (0.1-10 $\mu \mathrm{M})$ (Figure 4.24). The concentration of hypericin obtained for those batches with different nominal concentrations (5$100 \mu \mathrm{M})$ is summarized in Figure 4.25. The determined concentration values were 0.16-3.2 $\mu \mathrm{M}$ for HYP@1 (average entrapment efficiency of 2-3\%mol) and <0.1-0.19 $\mu \mathrm{M}$ for HYP-PBS controls. A value of $0.01-0.23 \%$ w/w was calculated as drug loading in HYP@1, which is quite similar to 
some examples of hypericin-loaded systems in Table 4.7. It also seems that there is a correlation between the initial hypericin quantity and the quantified one. As a trend, increasing HYP total amount in the system results in higher loading into the nanogels. For nominal concentrations of $100 \mu \mathrm{M}$, a concentration of $c a .3 \mu \mathrm{M}$ of loaded HYP is achieved, a value 20-30 times higher than that reached for the solubilization in PBS.

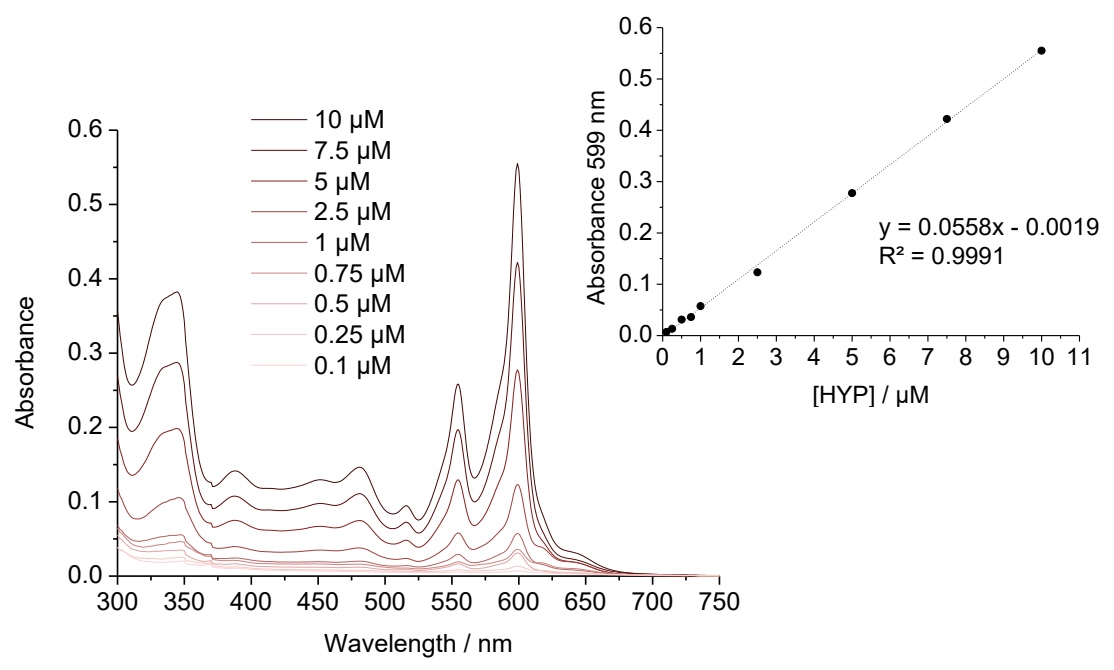

Figure 4.24. Absorption spectra of hypericin standard solutions in DMSO and calibration curve at $599 \mathrm{~nm}$.
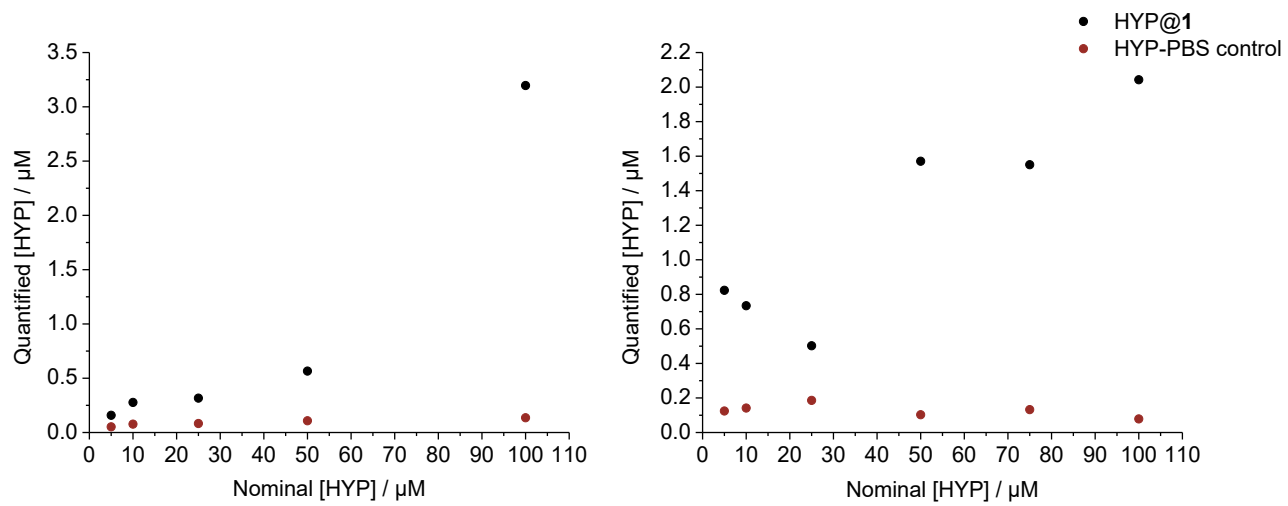

Figure 4.25. Results of the quantification of the concentration of hypericin in HYP@1 samples (black) and HYP-PBS controls (red) for two different sets of experiments.

HYP@1 samples were prepared using a $25 \mu \mathrm{M}$ nominal concentration for in vitro experiments. The quantification results can be seen in Figure 4.26, with an average of $0.7 \pm 0.3 \mu \mathrm{M}$ of hypericin for 22 different HYP@1 samples (0.05\% w/w drug loading) and of 0.09 $\pm 0.05 \mu \mathrm{M}$ for 9 HYP-PBS 
controls. Therefore, a ca. 10 times higher solubilization of hypericin is achieved when using nanoparticles. The dispersion of values seen in Figure $\mathbf{4 . 2 6}$ most likely reveals that the loading efficiency depends on several experimental variables that are not easily controlled, such as the degree of aggregation of hypericin, the particle size of the nanogels or the variability in sonication efficiency.

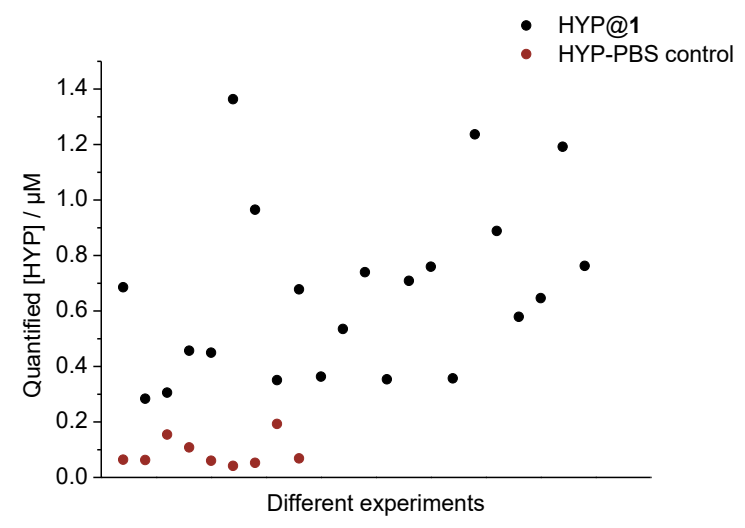

Figure 4.26. Quantification of HYP in 22 HYP@1 samples (black) and 9 HYP-PBS controls (red) of a $25 \mu \mathrm{M}$ nominal concentration.

\subsubsection{MEASUREMENT OF SINGLET OXYGEN PHOTOGENERATION}

The production of reactive oxygen species by encapsulated hypericin upon irradiation was tested using $A B M A$ as ${ }^{1} \mathrm{O}_{2}$ probe, as in Section 4.2.2.2. For each assay, a sample containing ABMA (1 $\mu \mathrm{M})$ was irradiated with visible light using two LED lamps (11W each one, ca. 400-700 $\mathrm{nm}$ emission output) in a cuvette with vigorous stirring. The lamps were placed perpendicular to each other at $1 \mathrm{~cm}$ from the cuvette (see assembly in Figure 4.27). The photoreaction evolution was monitored over time using fluorescence spectroscopy $\left(\lambda_{\mathrm{ex}} 380 \mathrm{~nm}\right)$ and studying the decrease of ABMA fluorescence at $406 \mathrm{~nm}$. 

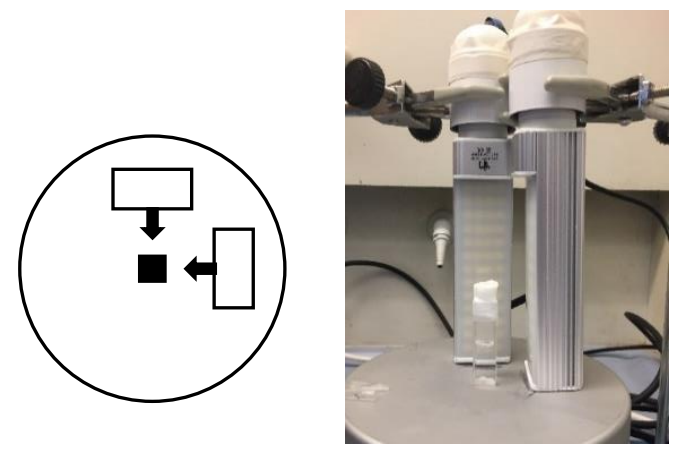

Figure 4.27. Schematic top view and a picture of the assembly used for irradiation experiments. The sample in a $10 \times 10 \mathrm{~mm}$ cuvette (black square) is placed in a magnetic stirrer and irradiated with two LED lamps (white rectangles) placed perpendicular to each other at $1 \mathrm{~cm}$ from the cuvette.

The production of ${ }^{1} \mathrm{O}_{2}$ by HYP@1 samples (0.6, 0.8, $1.2 \mu \mathrm{M}$ hypericin) and HYP-PBS controls $(0.05,0.07,0.19 \mu \mathrm{M}$ hypericin) was tested. A control experiment in the absence of the photosensitizer (using just PBS) was also performed. The emission spectra at each irradiation time and the decay curves of the normalised ABMA emission at $406 \mathrm{~nm}$ as a function of the irradiation time for representative examples (HYP@1 $0.8 \mu \mathrm{M}$, HYP-PBS control $0.05 \mu \mathrm{M}$ and PBS control) are reported in Figure 4.28 .
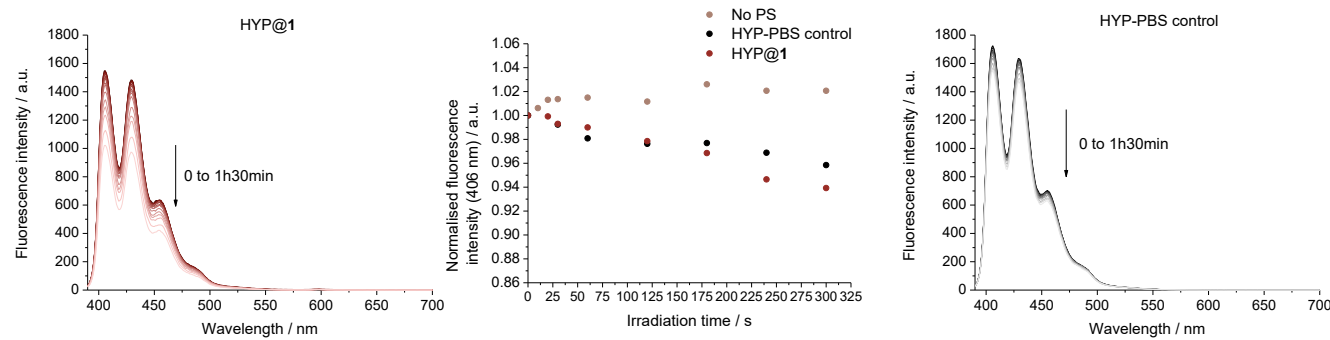

Figure 4.28. A representative example of photobleaching of ABMA in the presence of a HYP@1 sample and a HYP-PBS control after irradiation. Ends: Fluorescence emission spectra of ABMA as a function of irradiation time for a HYP@1 sample (left) and HYP-PBS control (right) $\left(\lambda_{\mathrm{ex}} 380 \mathrm{~nm}\right)$. Centre: Decay curves of the normalized ABMA emission intensity at $406 \mathrm{~nm}$ as a function of the irradiation time in the presence of a HYP@1 sample (red), a HYP-PBS control (black) and without photosensitizer (light brown).

The results show decreased ABMA fluorescence upon irradiation, meaning that ${ }^{1} \mathrm{O}_{2}$ is being produced in the solution. The initial points of the kinetic traces (fluorescence intensity at the maximum peak, $406 \mathrm{~nm})$ were fitted to a pseudo-first-order model $\left(\ln C / C_{0}=-k_{\mathrm{obs}} t\right)$, as in Section 4.2.2.2, and the first-order rate constants $k_{o b s}$ were determined from the slope of the linear plots (Table 4.8). Corrected $k_{\mathrm{obs}}\left(k_{\mathrm{obs}} /[\mathrm{HYP}]\right)$ were calculated to compare $k_{\mathrm{obs}}$ avoiding the 
concentration factor, and an average of 6.2 times faster singlet oxygen production was found for HYP-PBS controls. This reduction of photoactivity for HYP@1 may be caused by the interaction of hypericin with the gelator. However, the average uncorrected $k_{\text {obs }}$ for encapsulated hypericin is 1.8 times higher than for HYP-PBS, meaning that a more efficient singlet oxygen production is achieved for HYP@1 samples than for the maximum quantity of solubilized hypericin in PBS. Overall, a higher PDT activity is achieved in HYP@1 systems due to enhanced hypericin solubility but the photosensitization activity of HYP is reduced by encapsulation.

Table 4.8. Rate constants $k_{\text {obs }}$ obtained for ABMA reaction with singlet oxygen after irradiation of HYP@1 and HYP-PBS control samples. Corrected $k_{\mathrm{obs}}=k_{\mathrm{obs}} /[\mathrm{HYP}]$.

\begin{tabular}{lcccc}
\hline Sample & {$[\mathrm{HYP}] / \boldsymbol{\mu M}$} & $\mathbf{1 0}^{-\mathbf{4}} \boldsymbol{k}_{\text {obs }} / \mathbf{s}^{\mathbf{- 1}}$ & $\mathbf{1 0 ^ { - 4 }}$ Corrected $\boldsymbol{k}_{\text {obs }} / \mathbf{L} \cdot \boldsymbol{\mu} \mathbf{m o l}^{-\mathbf{1}} \cdot \mathbf{s}^{\mathbf{- 1}}$ & $\mathbf{R}^{\mathbf{2}}$ \\
\hline \multirow{3}{*}{ HYP@1 } & 0.6 & $1.95 \pm 0.25$ & 3.25 & 0.90 \\
& 0.8 & $2.16 \pm 0.12$ & 2.70 & 0.98 \\
& 1.2 & $1.63 \pm 0.19$ & 1.36 & 0.92 \\
\hline \multirow{2}{*}{ HYP-PBS } & 0.05 & $1.20 \pm 0.17$ & 24 & 0.91 \\
control & 0.07 & $1.17 \pm 0.15$ & 16.7 & 0.94 \\
& 0.19 & $0.85 \pm 0.18$ & 4.47 & 0.84 \\
\hline
\end{tabular}




\subsection{STUDY OF PS@1 AS PDT AGENTS IN VITRO}

\subsubsection{GENERAL CONSIDERATIONS}

The activity of the photosensitizer-loaded nanoparticles (PS@1) prepared in previous sections was evaluated in vitro with human colon adenocarcinoma HT-29 cells. This cell line was chosen due to its extensive use in biological and cancer research. After assuring that our nanogels show no toxicity to this cell line, the first goal was to determine if the photosensitizer cellular uptake is improved with the use of the nanogels. The internalisation of the probes after $24 \mathrm{~h}$ was studied, taking advantage of their fluorescence, using flow cytometry and confocal microscopy. A direct correlation between the concentration of the probe and fluorescence was assumed. Then, the use of PS@1 as agents for photodynamic therapy was studied. The outcome of cell irradiation was evaluated in terms of cell viability and the presence of apoptosis.

\subsubsection{STUDY OF NANOGELS TOXICITY}

The first objective was to test the potential toxicity of the nanogel particles to the HT-29 cell line after $24 \mathrm{~h}$ of incubation. Samples of nanogels were diluted $1: 2$ or 1:3 in the cell culture medium. After the incubation period, cells were resuspended in fresh media and the viability was evaluated using the Trypan blue assay. Cells with a damaged cell membrane internalize this blue dye, whereas the stain cannot cross the cell membrane of viable cells. Using a Neubauer chamber and a microscope, the concentration of viable and non-viable cells could be calculated.

Results of cell viability are reported in Figure 4.29, expressed as the average of three incubation replicas. The average of viable cells for the negative controls (samples vehicle, PBS) is $97.2 \pm 0.2$ $\%$ and $97.0 \pm 0.9 \%$ for $1: 2$ and $1: 3$ dilutions, respectively. For cell populations incubated with the nanoparticles, cell viability was $90.5 \pm 3.0 \%$ and $96.7 \pm 0.6 \%$ for $1: 2$ and $1: 3$ dilutions, respectively. Therefore, the effect of nanogel particles over cell viability is not relevant at both concentrations.

However, not all the cell populations had the same density (sum of viable and non-viable cells). As shown in Figure 4.30, the number of resuspended cells after incubation with nanoparticles in a $1: 2$ dilution was $55 \%$ less than the number of cells for the negative control. The reduction in the density was only of $20 \%$ in the case of cells incubated with the nanoparticles sample in a 
1:3 dilution. It should be noted that the same number of cells was initially seeded in each well, so the population differences are most likely due to cells that died and lysed during incubation. Therefore, it seems that cells incubated with nanogels suffer some kind of concentrationdependent cell death, perhaps related to the surfactant-like nature of the gelator. For example, SDS is known to produce cell lysis by incorporating the detergent into the cell membrane, solubilizing lipids and proteins and creating pores. ${ }^{271}$ Therefore, if we consider this process to occur, cells incubated with a 1:3 dilution would cause less damage to cell membranes. Accordingly, it was concluded that a 1:3 dilution of nanoparticles would be used in following experiments.

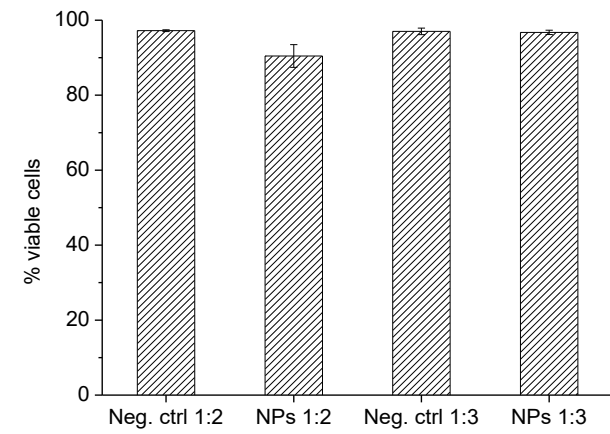

Figure 4.29. Viability of the HT-29 cells incubated for $24 \mathrm{~h}$ with the indicated samples and dilutions in cell culture medium $(n=3)$.

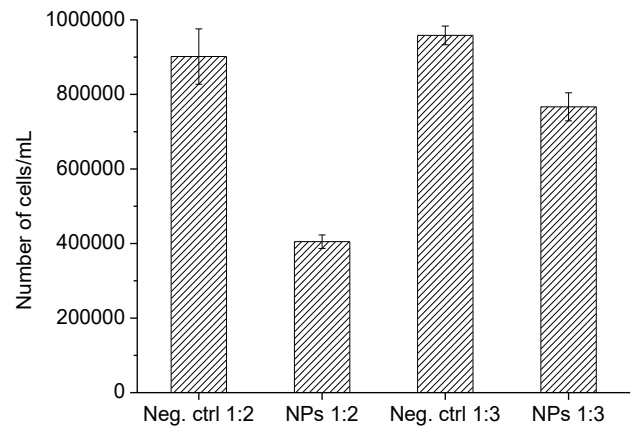

Figure 4.30. Total number of HT-29 cells (viable and non-viable) after incubation for $24 \mathrm{~h}$ with the indicated samples and dilutions in cell culture medium $(n=3)$.

\subsubsection{ROSE BENGAL}

\subsubsection{SAMPLING CONSIDERATIONS}

Due to the variability seen in Section 4.2.2.1 for the quantity of RB encapsulated, several RB@1 samples were tested in each experiment. The pH of RB@1 samples was adjusted to pH 7. For each RB@1 sample, a control solution of Rose Bengal in PBS at a higher concentration (RB control) was used. As negative control, the vehicle of nanoparticle preparation, PBS, was tested. All samples were tested right after their preparation to avoid the effect of aging. As determined in Section 4.4.2, all tested samples were added to cells in a 1:3 dilution with cell culture medium to minimise toxicity from the NPs. Phenol red-free medium was used in all experiments to avoid fluorescence interferences. 


\subsubsection{CELLULAR UPTAKE ASSAYS}

\section{FLOW CYTOMETRY}

The internalisation of three RB@1 samples in HT-29 cells was studied measuring intracellular RB fluorescence with flow cytometry. Flow cytometry is a single-cell analysis method that simultaneously characterizes the fluorescence emission and physical properties of the cell. Figure 4.31 illustrates the parts and setup of a typical flow cytometer. The cell population flows past the interrogation point, where interacts with laser light, one cell at a time. Light excites all fluorophores in the cell, and the fluorescence emission is split and collected by different detectors that filter specific wavelengths (bandpass filters). Light scatter caused by the interaction with the cells is simultaneously measured by two optical detectors. Forward scatter (FSC) allows for the discrimination of cells by size (FSC intensity is primarily due to light diffraction around the cell, so it is proportional to the diameter of the cell). The other detector measures the side scatter ( $\mathrm{SSC}$ ) $\left(90^{\circ}\right.$ angle relative to the laser) and provides information about the internal complexity (i.e., granularity) of a cell, as cells has some cellular components that increase side scatter (for example, granules and the nucleus). Results of SSC vs. FSC for a heterogeneous population of cells are usually represented as dot plots to differentiate and gate the cells of interest based on characteristic differences in cell size and granularity (for example, debris show low forward-and side-scatter). The fluorescence emission of these selected cells is then studied. ${ }^{272-274}$

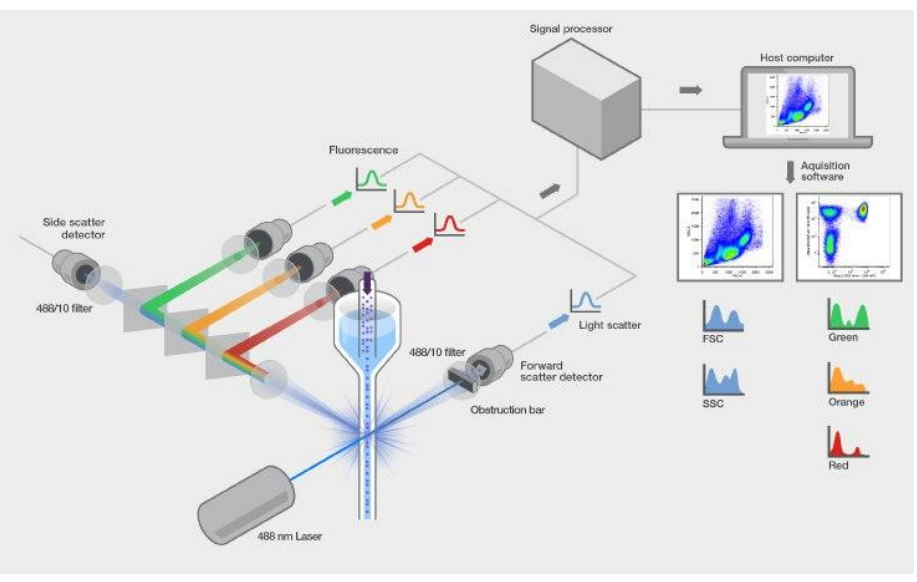

Figure 4.31. Schematic overview of a typical flow cytometer setup. ${ }^{273}$ 
For each internalisation experiment, cells were separately incubated with a RB@1 sample, its RB control and PBS (negative control). The samples were diluted 1:3 with FBS-free medium to stimulate cell starvation. The incubation time was set to $24 \mathrm{~h}$, as it was seen that a 50 -fold intracellular fluorescence intensity was obtained versus $1 \mathrm{~h}$ of incubation. Then, cells were washed with PBS and suspended in FBS-free medium. Flow cytometry $\left(\lambda_{\text {ex }} 488 \mathrm{~nm}\right.$ and emission collected above $670 \mathrm{~nm}$ ) was used to quantify the average fluorescence intensity/cell of the internalised Rose Bengal in each tested cell population. In Figure 4.32, a representative example of the emission spectra $\left(\lambda_{\mathrm{ex}} 488 \mathrm{~nm}\right.$ ) of a RB@1 sample and the flow cytometry acquisition conditions are depicted. After excitation at $488 \mathrm{~nm}$, the emission maximum for RB@1 samples and RB controls (573 nm and $564 \mathrm{~nm}$, respectively) overlaps with HT-29 cells autofluorescence. For this reason, a 670LP filter was chosen to detect RB internalisation as the overlapping is minimised in this range of detection.

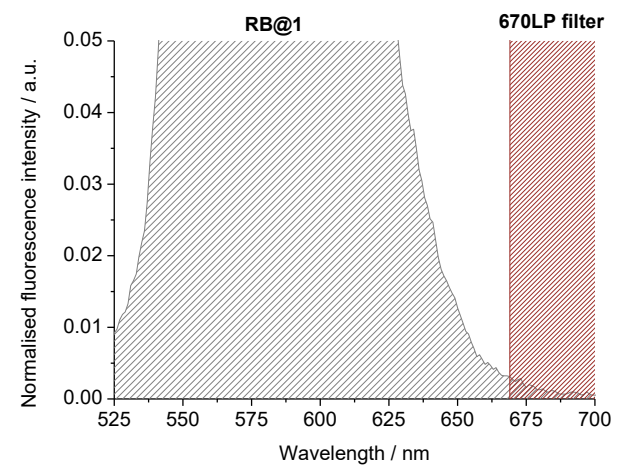

Figure 4.32. Overlap of fluorescence emission spectra of a RB@1 sample $\left(\lambda_{\mathrm{ex}} 488 \mathrm{~nm}\right)$ and the range of detection of the flow cytometer using the 670LP filter.

Figure 4.33 shows the results for cells incubated with the three RB@1 samples and the three RB controls. Results are normalised as the percentage of fluorescence intensity/cell over the negative control. Error bars indicate the average of two replicas. Results for each category are ordered by increasing concentration, with the numbers above each bar showing the RB concentration ( $\mu \mathrm{M})$ tested. Original RB@1 samples had a RB concentration of 4.8, 5.6 and 7.1 $\mu \mathrm{M}$ and RB controls of 6.7, 7.6 and 8.2 $\mu \mathrm{M}$. The percentage of fluorescence intensity/cell over the negative control is approximately 70 times higher for cells incubated with RB@1 samples (with an average of ca. 2000\%) versus the cells incubated with RB controls (with an average of ca. $30 \%)$. 


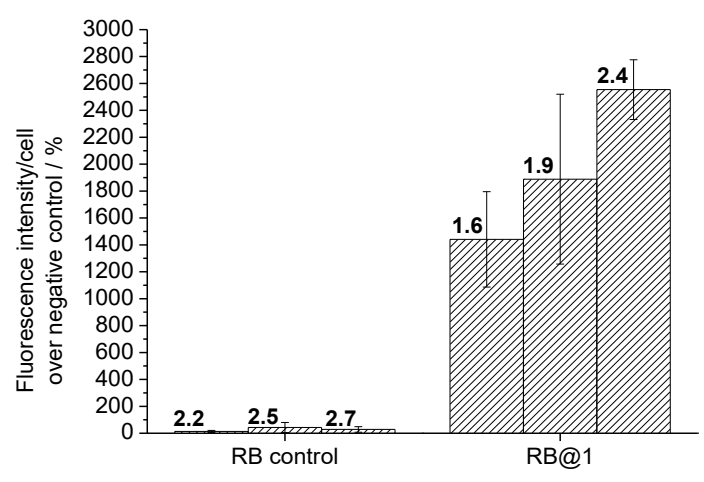

Figure 4.33. Percentage of fluorescence intensity/cell over negative control of cells incubated with three Rose Bengal-loaded nanoparticles samples and three controls of Rose Bengal solution in PBS. The numbers above each bar indicate the RB concentration $(\mu M)$ tested $(n=2)$.

\section{CONFOCAL MICROSCOPY}

The internalization of RB@1 into HT-29 cells was also studied by confocal microscopy. Cells were incubated overnight with a RB@1 sample (3.4 $\mu \mathrm{M} R B, 1.1 \mu \mathrm{M}$ after dilution) in a 1:3 dilution with FBS-free medium to stimulate cell starvation. Then, cells were washed with PBS and imaged by confocal microscopy $\left(\lambda_{\text {ex }} 514 \mathrm{~nm}\right.$ ) in fresh FBS-free medium to avoid interferences. For control purposes, cells incubated under the same conditions with PBS (negative control) and RB control (5.5 $\mu \mathrm{M} \mathrm{RB}, 1.8 \mu \mathrm{M}$ after dilution) were also imaged. Representative images of the different populations of cells are shown in Figure 4.34. For RB@1, the assay was performed twice with the same results. 


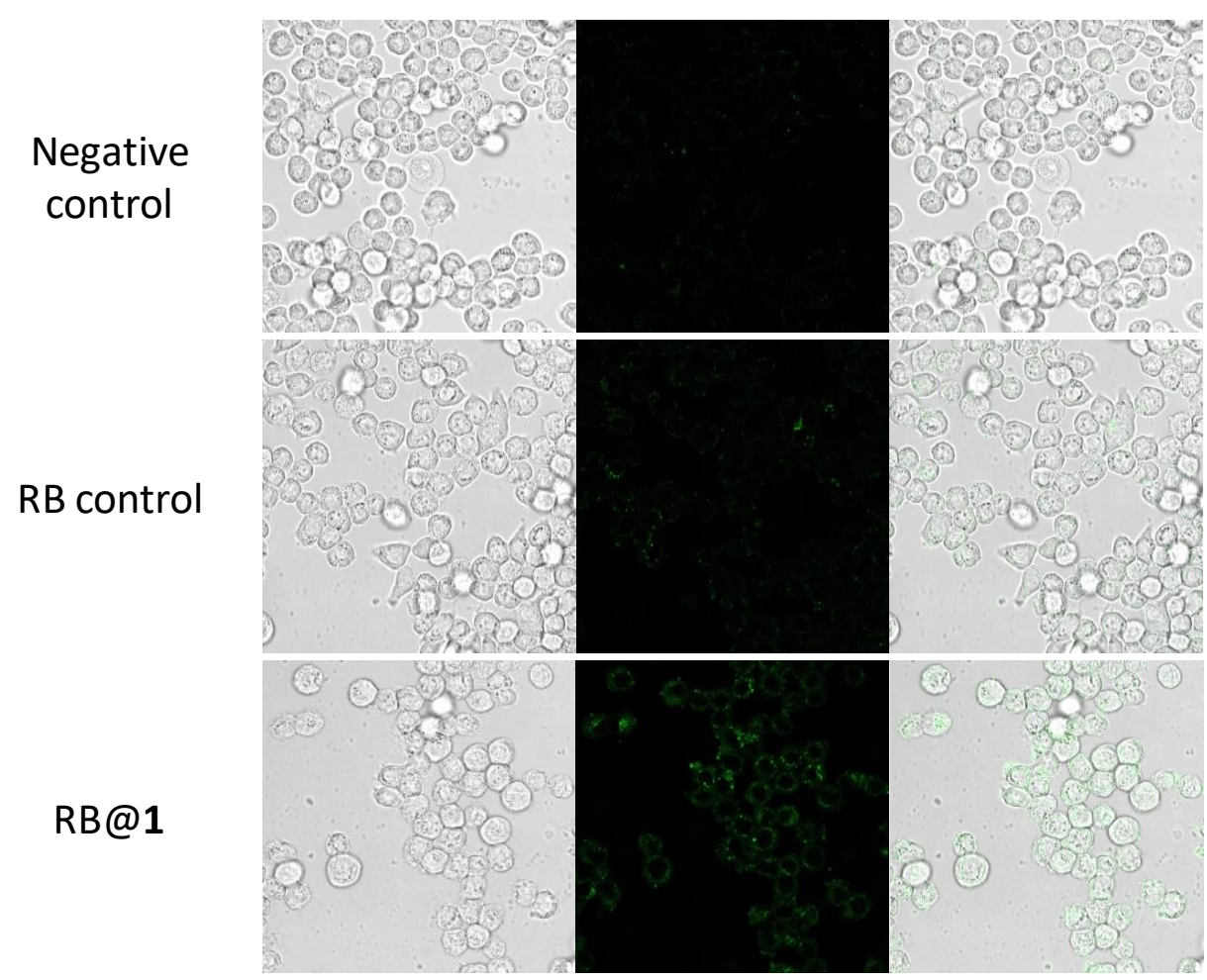

Figure 4.34. Confocal microscopy images of cells incubated overnight with RB@1 (1.1 $\mu \mathrm{M})$, RB control $(1.8 \mu \mathrm{M})$ and a negative control. Bright field (left), green channel (centre), merged (right).

The quantification of the fluorescence intensity of imaged cells is expressed in Figure 4.35 as the average of fluorescence intensity/cell (at least 50 cells per sample). The fluorescence intensity, thus the internalization of RB, is higher when RB is carried by nanoparticles ( 5.2 times higher than negative control) than when it is in solution (2.2 times higher than negative control), even when that concentration is higher than the one loaded in the NPs. From the green channel images, it seems that only the cytoplasm is stained in both cases (RB@1 and RB control), showing a non-specific distribution. 


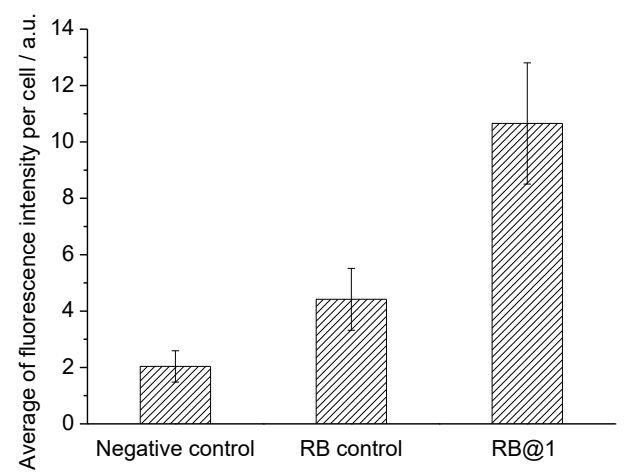

Figure 4.35. Quantification of the fluorescence intensity of the confocal microscopy images of cells (at least 50 cells per sample) incubated overnight with RB@1 $(1.1 \mu \mathrm{M}), \mathrm{RB}$ control $(1.8 \mu \mathrm{M})$ and negative control.

\subsubsection{ROSE BENGAL NANOGELS AS PDT AGENTS}

\section{GENERAL CONSIDERATIONS}

The use of Rose Bengal-loaded nanoparticles as agents for photodynamic therapy was studied in HT-29 cells. After incubation with RB and visible light irradiation, the number of viable/apoptotic cells was determined. This parameter was chosen as it has been reported that apoptosis is usually the preferred mechanism for cell death in RB-PDT. ${ }^{147}$ The percentage of cells actively undergoing apoptosis was measured by flow cytometry using a commercial kit that detects the membrane early changes (FITC Annexin V apoptosis detection kit I, BD Pharmingen $\left.{ }^{\mathrm{TM}}\right) \cdot{ }^{36,275}$

Annexin $V$ is a phospholipid-binding protein with a high affinity for phosphatidylserine $\left(K_{d}\right.$ of $\left.\sim 5 \times 10^{-10} \mathrm{M}\right)$. Under normal physiological conditions, the membrane phospholipid phosphatidylserine is predominantly placed in the cytoplasmic surface of the cell membrane (see Figure 4.36). In apoptotic cells, phosphatidylserine losses its asymmetric distribution and is translocated from the inner to the outer leaflet of the plasma membrane, thereby exposing phosphatidylserine to the external cellular environment, where it can be detected by annexin $\mathrm{V}$ conjugates. ${ }^{276}$ 


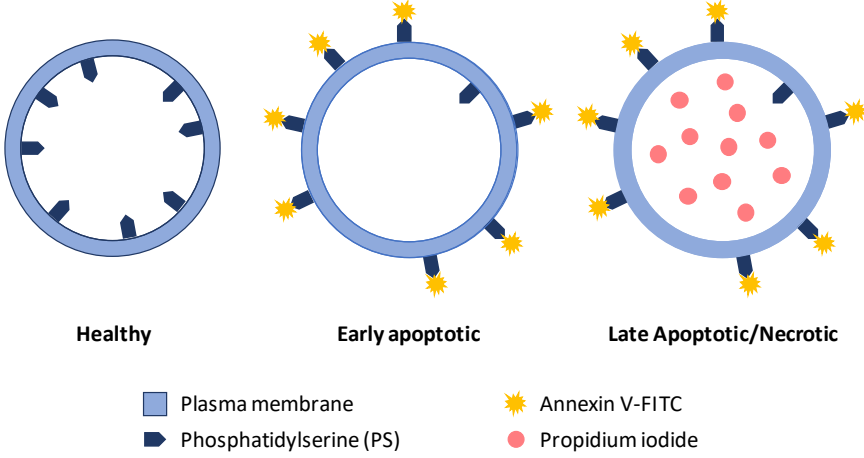

Figure 4.36. Diagram showing healthy and apoptotic cells with markers for detection of apoptosis. ${ }^{36}$

In the detection kit mentioned above, Annexin $\mathrm{V}$ is conjugated to the fluorochrome fluorescein isothiocyanate (FITC), one of the most popular amine-reactive fluorescein derivatives for fluorescent protein labelling. FITC consist of a mixture of isomers at the 5- and 6-positions of the carboxyphenyl ring (Figure 4.37). FTIC-functionalized Annexin V preserves a high affinity for phosphatidylserine, and FITC excitation maximum (494 nm) closely matches the $488 \mathrm{~nm}$ spectral line of the argon-ion laser used in flow cytometry. ${ }^{277}$ The standard viability probe propidium iodide (PI, Figure 4.38) is used in conjunction with FITC-Annexin V to differentiate early and late apoptotic cells (Figure 4.36)..$^{278}$ Viable cells or those in the early stages of apoptosis, with intact membranes, exclude PI. In contrast, the membranes of non-viable cells are permeable to PI, where it stains DNA by intercalation. Non-viable cells include dead cells and those in necrosis or late apoptosis processes (since no phagocytes are present in monocultures in vitro, the final stages of apoptosis involve necrotic-like disintegration of the complete cell). Therefore, cells that are considered viable are FITC-Annexin V and PI negative; cells in early apoptosis are FITCAnnexin V positive and PI negative; and cells in late apoptosis, undergoing necrosis or already dead are PI positive. In Figure 4.39, there is an example of the dot plot obtained after representing the PI vs. FITC fluorescence emission for a double-stained cell population analysed by flow cytometry. Quadrant A (FITC-Annexin V and PI negative) corresponds to viable cells, quadrant B to cells in early apoptosis (FITC-Annexin V positive and PI negative), and cells in quadrant $C$ and D are non-viable (PI positive). This assay does not distinguish between cells in late apoptosis and cells that have undergone apoptotic or necrotic death. In either case, the dead cells will stain both annexin V and PI (due to membrane disintegration during necrosis, Annexin V also binds to intracellularly located PS in necrotic cells). ${ }^{279,280}$ 
<smiles>O=C(O)c1cc(N=C=S)ccc1-c1c2ccc(=O)cc-2oc2cc(O)ccc12</smiles>

5-FITC

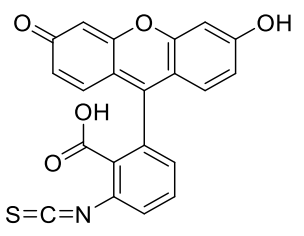

6-FITC

Figure 4.37. Chemical structures of fluorescein-5-isothiocyanate (5-FITC) and fluorescein-6-isothiocyanate (6-FITC).

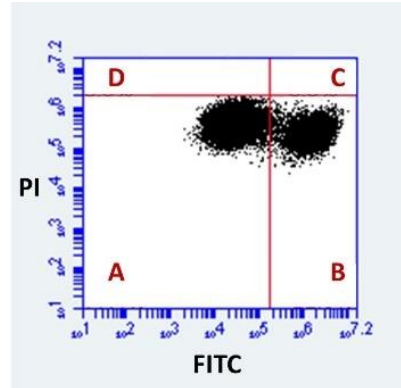

Figure 4.39. Example of a PI vs. FITC fluorescence emission dot plot for a double-stained population of cells analysed by flow cytometry.

Both FITC and propidium iodide were excited with $488 \mathrm{~nm}$ light. FITC green fluorescence $\left(\lambda_{\max }\right.$ $520 \mathrm{~nm}$ ) was measured in the range 518-548 nm (533/30 nm bandpass filter), and propidium iodide red fluorescence ( $\lambda_{\max } 617 \mathrm{~nm}$ when bound) was recorded in the range 600-620 nm (610/20 nm bandpass filter), according to the manufacturer's instructions (see Figure 4.40).

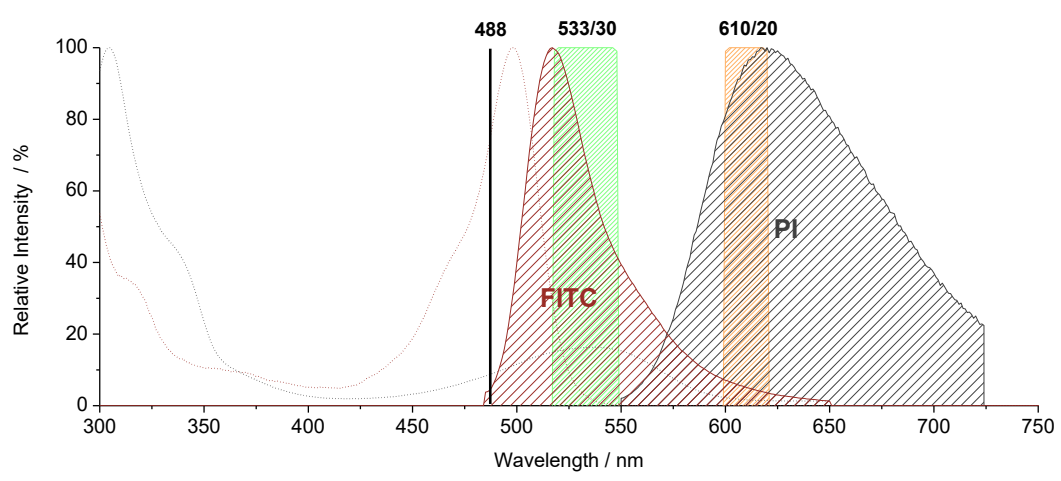

Figure 4.40. FITC (red) and PI (grey) excitation (dot line) and emission (solid patterned curve) spectra and flow cytometry excitation and detection wavelengths (adapted from BD Spectrum Viewer). 


\section{RESULTS AND DISCUSSION}

PDT assays were carried out with HT-29 cells incubated for $24 \mathrm{~h}$ with RB@1 in a 1:3 dilution with cell culture medium. The cells were irradiated for $2 \mathrm{~min}$ with two LED lamps (11W each one, ca. 400-700 nm emission output) placed as close to the lid of the cells plate as possible (see Figure 4.41). After additional $24 \mathrm{~h}$, the state of the cells was evaluated by flow cytometry after staining with FITC-Annexin V and PI. Incubations were performed in all cases using FBS in the medium to avoid cell death as a consequence of starvation. The conditions of the PDT study were selected according to previous studies and after optimisation. ${ }^{165}$ For each RB@1 sample tested, a RB control and PBS as negative control were also tested in the same conditions. Also, as control of dark toxicity, the samples were tested precisely in the same way in another plate covered with aluminium foil and kept next to the other during irradiation (Figure 4.41). Therefore, a complete PDT experiment included testing a RB@1 sample and the RB and negative controls, both irradiating and without irradiation.

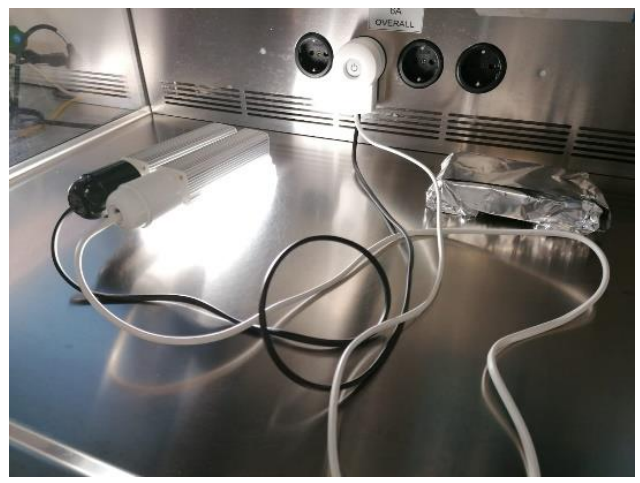

Figure 4.41. Irradiation of a cells plate and the corresponding non-irradiated control covered in aluminium foil under the same conditions.

Three different sets of experiments were performed with three different RB@1 samples (RB concentrations shown in Table 4.9).

Table 4.9. RB concentrations of samples tested.

\begin{tabular}{lcccccc}
\hline & \multicolumn{2}{c}{ RB@1 } & \multicolumn{2}{c}{ [RB] / $\mu$ M } & \multicolumn{2}{c}{ RB Control } \\
\cline { 2 - 3 } \cline { 5 - 7 } & Sample & Cells & & Sample & Cells \\
\hline Experiment A & 4.7 & 1.6 & & 6.8 & 2.3 \\
Experiment B & 7.0 & 2.3 & & 10.2 & 3.4 \\
Experiment C & 7.6 & 2.5 & & 8.0 & 2.7 \\
\hline
\end{tabular}


The flow cytometry results of a representative experiment (2.5 $\mu \mathrm{M}$ RB for RB@1, $2.7 \mu \mathrm{M}$ RB for RB control) can be seen in Figure 4.42. For each sample and irradiation conditions tested, the upper row shows the dot plot corresponding to the light scatter. In the bottom row, the dot plot corresponds to the fluorescence emission of PI (Y-axis) and FITC (X-axis) from the gated cells. For irradiated or non-irradiated cells, cells show no propidium iodide internalization (no cells in the upper quadrants), meaning that their membrane is not permeant to $\mathrm{PI}$ and cells are viable. Regarding FITC staining, two populations can be observed: viable (FITC and PI negative) and early apoptotic cells (FITC positive and PI negative). The apoptotic cells population shows an increase both in irradiated RB control and RB@1. This increase in apoptotic cells is also reflected by changes in the light scatter (SSC vs. FSC dot plot). During apoptosis, a decrease in cell volume (cell shrinkage associated with cytoskeletal breakdown) occurs, associated with a reduction in forward scatter (size). Further, the formation of apoptotic vesicles in the cells ("blebbing" of the plasma membrane) leads to an increased side scatter profile (granularity). ${ }^{36,281}$

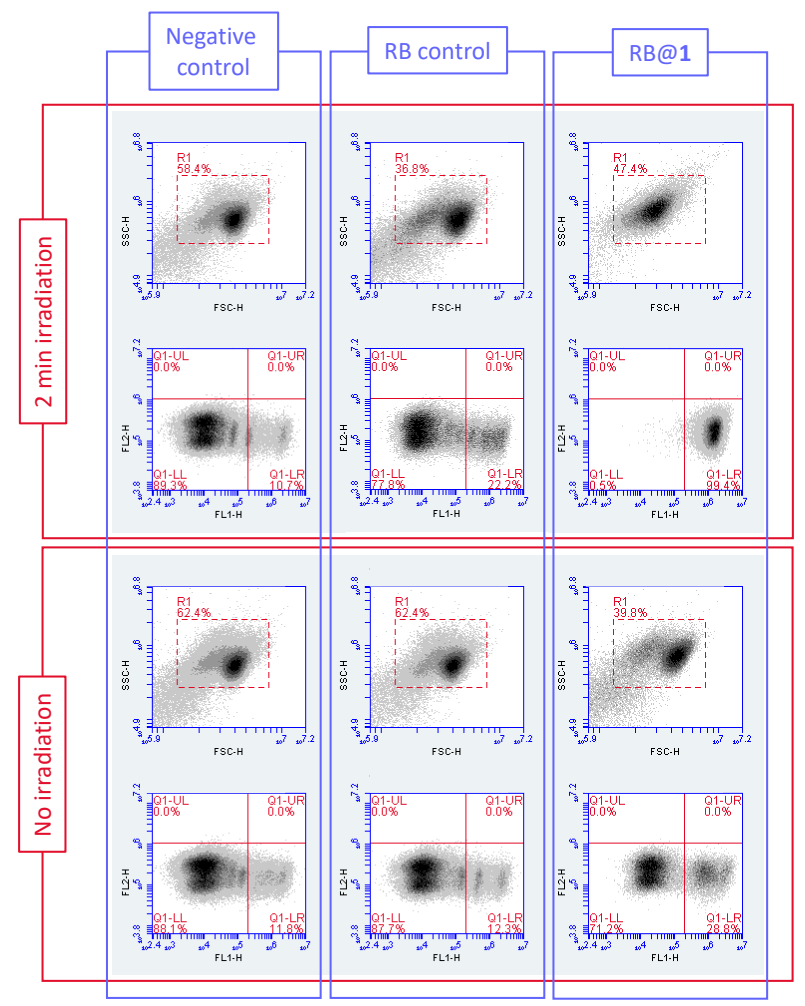

Figure 4.42. Dot plots obtained after flow cytometry analysis of the cell populations of a PDT experiment (2.5 $\mu \mathrm{M}$ RB@1, $2.7 \mu \mathrm{M}$ RB control) concurrent stained with FITC-Annexin V and PI. For each cell population, top row: light scatter dot plot, bottom row: PI vs. FITC fluorescence emission. 
The percentage of cells in early apoptosis for the three different experiments is shown in Figure

4.43. Results are expressed as the average of two replicas (error bar in each column, even though the smallest are not seen). It should be noted that, even in the absence of induced apoptosis, most cell populations contain a minor percentage of cells that are positive for apoptosis. On this regard, it is known that the separation of the adherent cells from culture flasks can cause phosphatidylserine flipping, ${ }^{282}$ so the untreated population is used to define

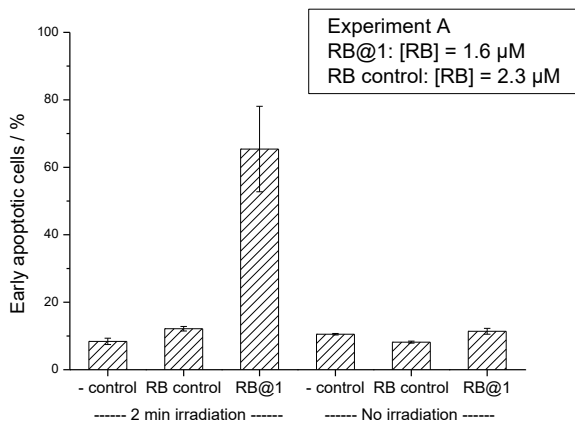
the basal level of apoptotic cells. In Figure 4.43, for a given experiment, the three first bars correspond to irradiated cells and the others to non-irradiated controls. A dramatic increase in the apoptotic population can be seen after the irradiation of cells incubated with the three RB@1 samples. From a basal apoptotic level of 8-32\% for the different control experiments, a

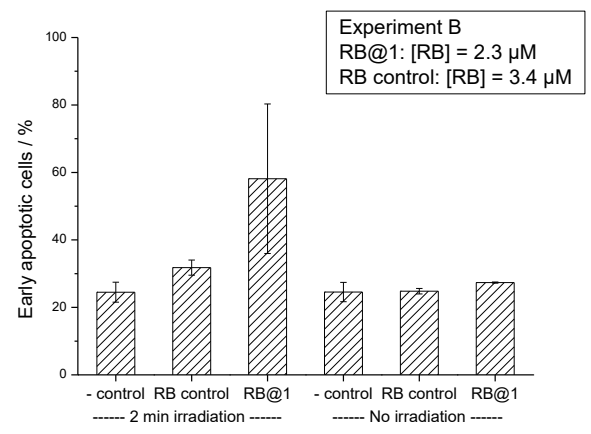
percentage of $58-99 \%$ of apoptotic cells is obtained for RB@1 in irradiated cells. It can be concluded that singlet oxygen production has been much more efficient for cells incubated with RB@1. This result should be ascribed to the higher internalization of RB in cells when incubated as RB@1. It is noticeable that no dark toxicity is observed for RB@1 samples. Also, the light stimulation of the cells in the absence of RB is not affecting for cell viability.

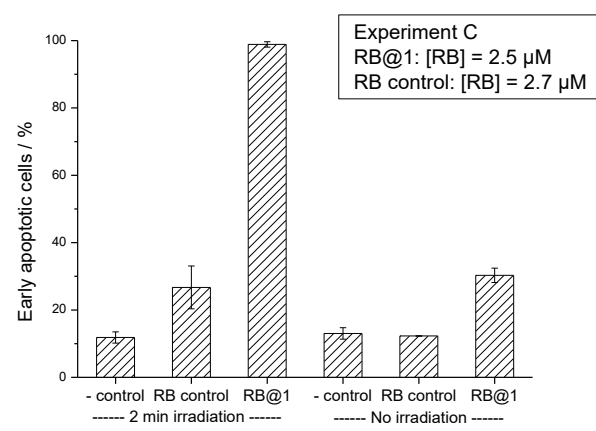

Figure 4.43. Percentage of cells in early apoptosis for the three PDT experiments carried out $(n=2)$.

Unloaded nanoparticles were tested to discard the idea that the RB@1 effect is due to nanogels. Two control PDT experiments with neat nanoparticle samples were performed following the same conditions used for RB@1 samples. The pH of NP samples was set to 7 as done for RB@1 samples. Dot plots (data not shown) were similar to those obtained for negative controls in 
Figure 4.42, with only two main populations, viable and early apoptotic cells. In Figure 4.44, the percentage of early apoptotic cells for negative controls and nanogels samples is shown. A basal level of $20-23 \%$ of cells in early apoptosis was obtained for the controls, and a $23-44 \%$ was obtained for nanoparticles, a value distant of that obtained for RB@1 samples.

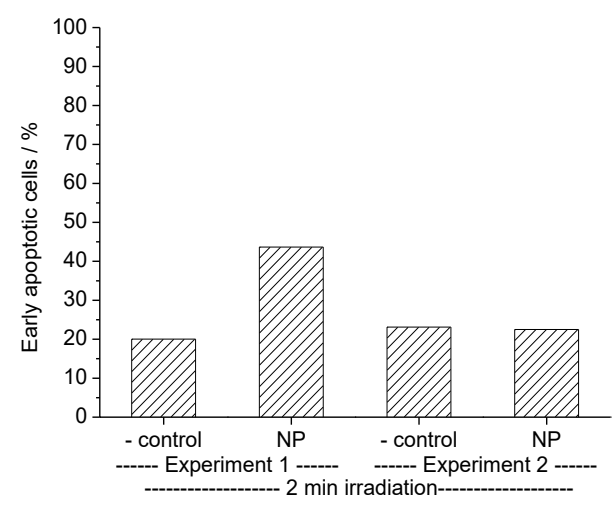

Figure 4.44. Percentage of cells in early apoptosis after incubation with two unloaded nanogels samples and irradiation.

\subsubsection{HYPERICIN}

\subsubsection{SAMPLING CONSIDERATIONS}

Several samples were tested in each experiment due to differences in the hypericin loading found for different HYP@1 samples (Section 4.3.2.1). For each HYP@1 sample, a hypericin solution at the same concentration in PBS-1\%DMSO (HYP-DMSO control) was tested. Additionally, experiments were also carried out with the controls of the preparation process (HYP-PBS control), which are dispersions that contain the maximum quantity of hypericin that can be solubilised in PBS. In all cases, the pH was kept as obtained after preparation: 6.4 for HYP@1 samples and 7 for HYP controls. As negative control, the vehicle of nanoparticle preparation, PBS, was tested. All samples were assayed right after their preparation to avoid the effect of aging. As determined in Section 4.4.2, all tested samples were added to cells in a 1:3 dilution with medium to reduce the toxicity from the NPs. Phenol red-free medium was used in all experiments to avoid fluorescence interferences. 


\subsubsection{CELLULAR UPTAKE ASSAYS}

\section{FLOW CYTOMETRY}

The internalisation of hypericin in HT-29 cells was studied through its fluorescence with flow cytometry. For each internalisation experiment, cells were incubated for 24 h with a HYP@1 sample, the corresponding HYP-DMSO control and PBS as negative control. The samples were 1:3 diluted with FBS-free medium to stimulate cell starvation. After washing cells with PBS, each tested cell population was resuspended in fresh FBS-free medium. The average fluorescence intensity/cell of internalised hypericin was quantified by flow cytometry ( $\lambda_{\text {ex }} 488 \mathrm{~nm}$ and emission collected above 670 nm). HYP@1 samples show emission maxima at ca. 600 and 650 $\mathrm{nm}$ (ca. 600 and $670 \mathrm{~nm}$ for free hypericin, $\lambda_{\text {ex }} 550 \mathrm{~nm}$ ). As for RB, the fluorescence emission is collected with the 670LP filter due to the high autofluorescence of HT-29 cells, which overlaps with our probe emission at lower wavelengths.

Six different HYP@1/HYP-DMSO batches were tested $([\mathrm{HYP}]=0.4,0.5,0.7,0.8,0.9,1.2$ $\mu \mathrm{M})$. A representative example of the plots obtained after analysing with flow cytometer can be seen in Figure 4.45. The light scatter dot plot shows that cells remain unchanged after incubation. To compare different experiments, Figure 4.46 shows the results normalised as the percentage of fluorescence intensity/cell over the corresponding negative control. For each experiment, the ratio of this percentage for HYP@1 to HYP-DMSO control is indicated in the legend in parenthesis, together with the tested concentration. Error bars indicate the average of two replicas.

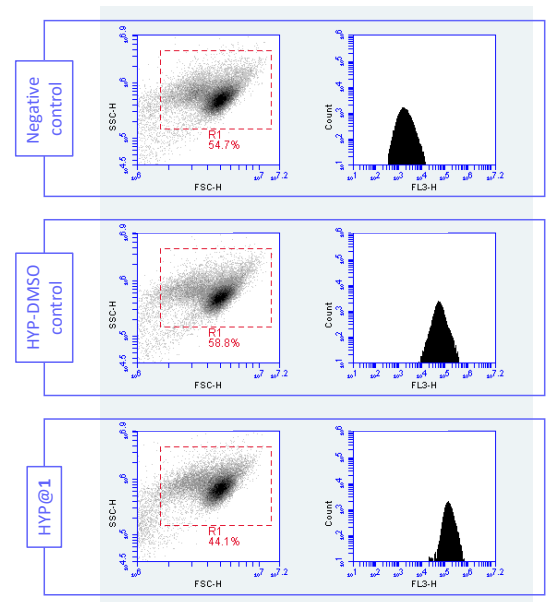

Figure 4.45. Light scatter dot plots and counts vs. fluorescence intensity plot for a representative experiment of cell incubation with HYP@1 and the corresponding controls. 


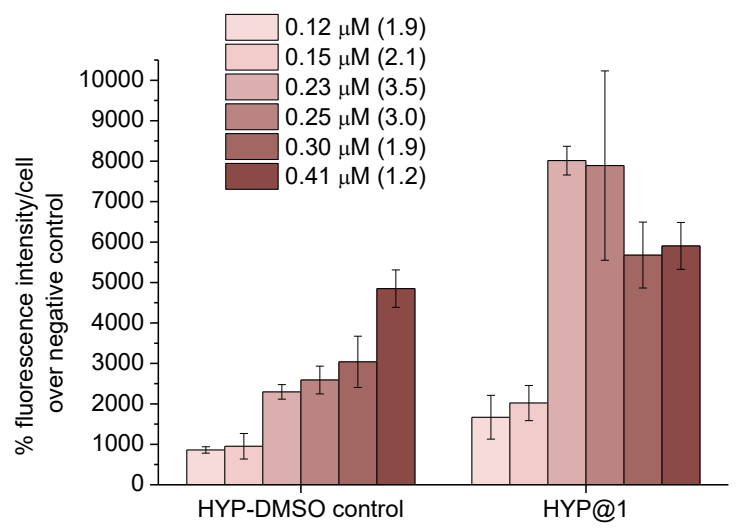

Figure 4.46. Percentage of fluorescence intensity/cell over negative control of cells incubated with six HYP@1 samples and the corresponding HYP-DMSO controls $(n=2)$. In parenthesis in the legend: ratio \%HYP@1/\%HYP-DMSO for the given concentration.

The percentage of fluorescence intensity/cell over negative control for cells incubated with HYP@1 is 1.2-3.5 times higher than HYP-DMSO controls. Therefore, nanogels have a positive effect on the internalization of the probe. Moreover, it should be highlighted that the reported hypericin concentrations can only be achieved when using nanoparticles or DMSO to increase the solubility. Otherwise, the maximum concentration obtained in PBS (HYP-PBS controls) is around 10 times lower, and HYP internalization achieved with HYP@1 is up to 14.4 times higher than for HYP-PBS (data not shown).

\section{CONFOCAL MICROSCOPY}

The internalization of HYP@1 into HT-29 cells was also studied by confocal microscopy. Cells were incubated overnight with a HYP@1 sample (0.6 $\mu \mathrm{M}$ HYP, $0.2 \mu \mathrm{M}$ after dilution), in a 1:3 dilution with FBS-free medium to stimulate cell starvation. Then, cells were washed with PBS and imaged by confocal microscopy ( $\lambda_{\text {ex }} 561 \mathrm{~nm}$, emission 585-700 nm) in fresh FBS-free medium to avoid interferences. For control purposes, cells incubated with the corresponding HYP-DMSO control (0.2 $\mu \mathrm{M}$ after dilution), a HYP-PBS control (0.06 $\mu \mathrm{M}$ HYP, $0.02 \mu \mathrm{M}$ after dilution) and PBS as negative control were also imaged. Representative images of the different populations of cells are shown in Figure 4.47. 


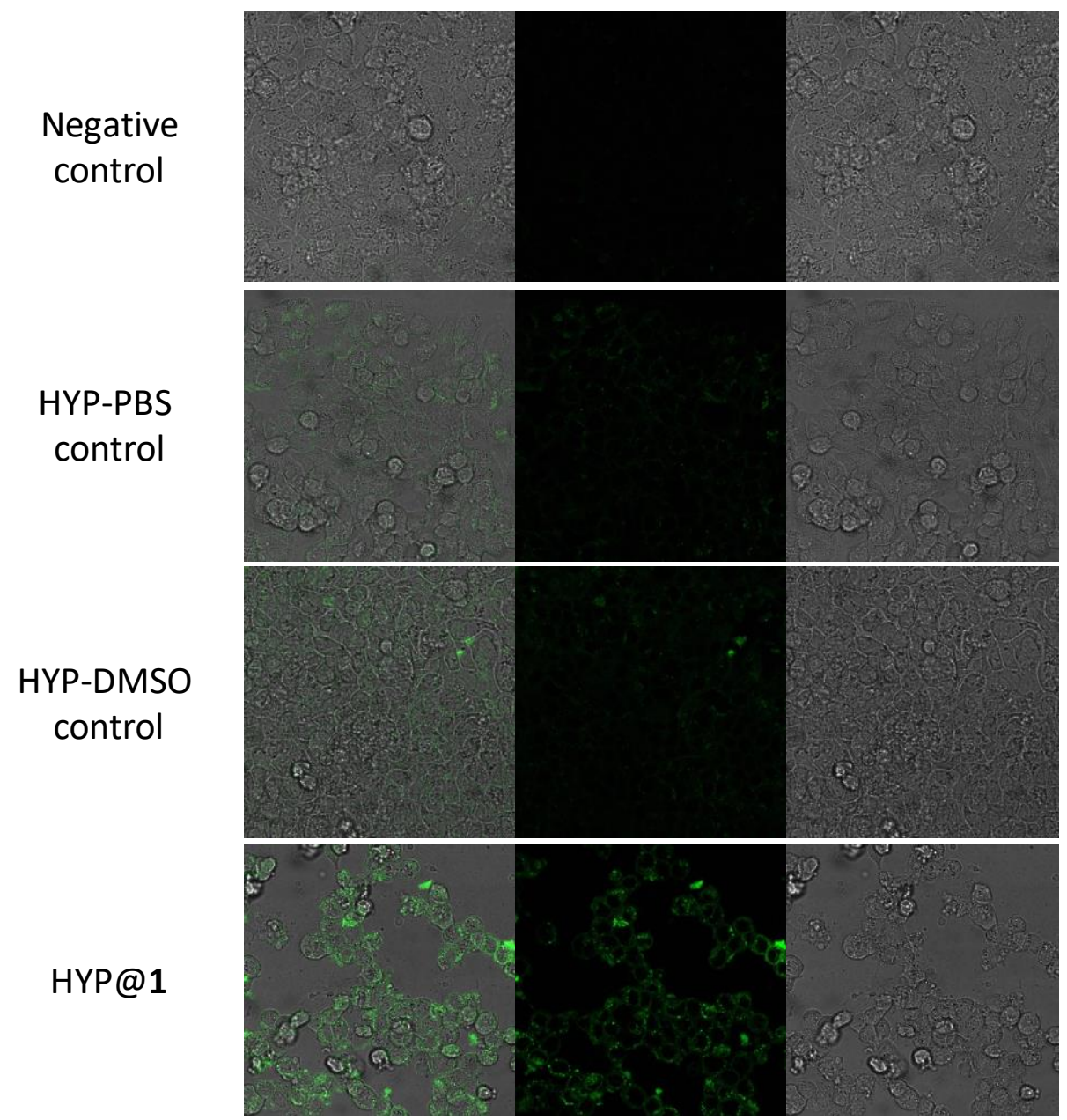

Figure 4.47. Confocal microscopy images of cells incubated overnight with a HYP@1 sample (0.2 $\mu \mathrm{M})$, HYP-DMSO control $(0.2 \mu \mathrm{M})$, HYP-PBS control $(0.02 \mu \mathrm{M})$ and a negative control. Bright field (right), green channel (centre), merged (left).

The fluorescence intensity of imaged cells was weighed out in Figure 4.48 as the average fluorescence intensity/cell (at least 140 cells per sample). The fluorescence intensity was twice when nanoparticles carried HYP (7.2 times higher than negative control) than when HYP was in a 1\% DMSO solution at the same concentration (HYP-DMSO control, 3.6 times higher than negative control). This result agrees with that obtained from flow cytometry. The internalization of hypericin carried by nanoparticles was 3.5 times the one of HYP-PBS (2.1 times higher than negative control), again in agreement with the results obtained by flow cytometry. 


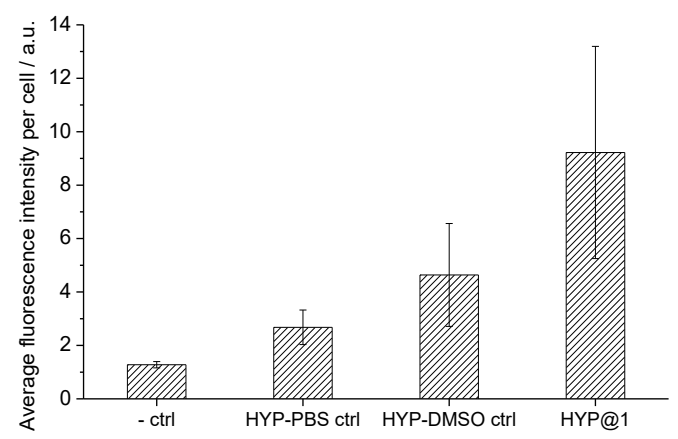

Figure 4.48. Quantification of the fluorescence intensity of the confocal microscopy images of cells (at least 140 cells/sample) incubated overnight with a HYP@1 sample (0.2 $\mu \mathrm{M})$, HYP-DMSO control $(0.2 \mu \mathrm{M})$, HYP-PBS control $(0.02 \mu \mathrm{M})$ and a negative control.

The intracellular spectra of imaged cells were acquired $\left(\lambda_{\text {ex }} 561 \mathrm{~nm}\right)$ and reported in Figure 4.49. Cells incubated with HYP@1, HYP-PBS control and HYP-DMSO control show a spectrum that matches monomeric hypericin (probably because it interacts with some cellular components ${ }^{283}$ ) with identical maxima at 599 and $648 \mathrm{~nm}$, thus confirming that we are observing its fluorescence for all samples. Besides, it shows that we still have a fluorescent emission above $670 \mathrm{~nm}$ when HYP is inside the cell, confirming that the 670LP bandpass was an appropriate flow cytometry detection option.

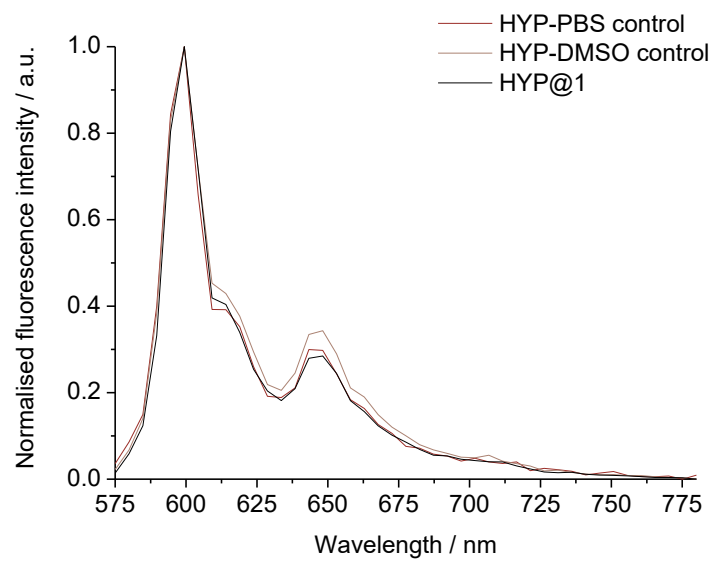

Figure 4.49. Normalised intracellular fluorescence emission spectra $\left(\lambda_{\text {ex }} 561 \mathrm{~nm}\right)$ of cells incubated overnight with a HYP@1 sample (black) and the corresponding HYP-DMSO control (light brown, $0.2 \mu \mathrm{M}$ ) and a HYP-PBS control (red, $0.02 \mu \mathrm{M})$. 
Finally, the localisation of the probe was also studied. In Figure 4.50, the fluorescence intensity was software-enhanced to visualize the differences. It seems that hypericin is accumulated in the membrane when cells are incubated with the free probe and in the cytoplasm in a nonspecific way when nanogels are used in the incubation.

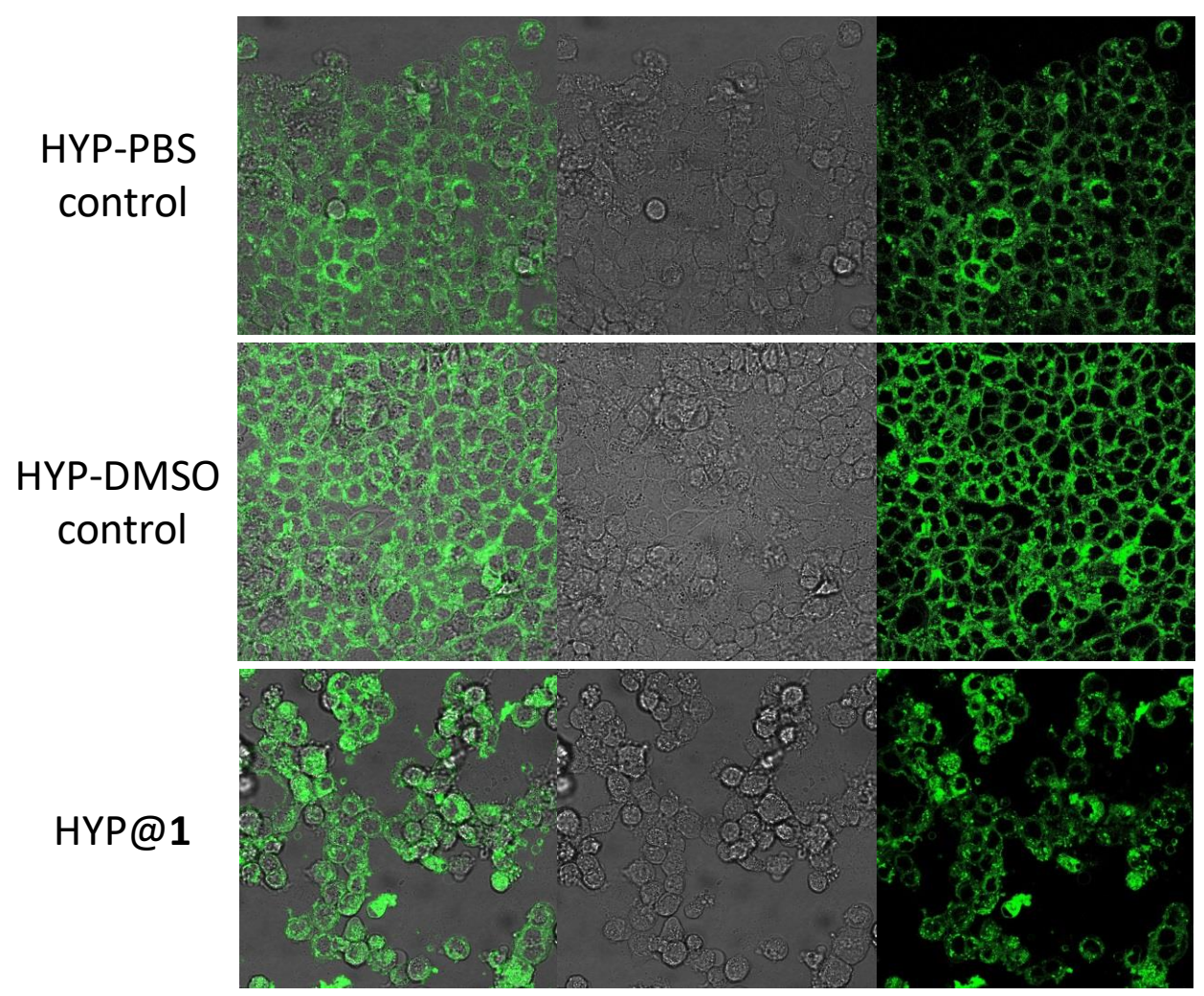

Figure 4.50. Confocal microscopy images of cells incubated overnight with a HYP@1 sample (0.2 $\mu \mathrm{M})$, HYP-DMSO control $(0.2 \mu \mathrm{M})$, HYP-PBS control $(0.02 \mu \mathrm{M})$. Bright field (centre), green channel (right), merged (left). Software-enhanced intensity of the green channel.

\subsubsection{HYPERICIN NANOGELS AS PDT AGENTS}

\section{GENERAL CONSIDERATIONS}

The use of hypericin-loaded nanoparticles as agents for PDT was studied in HT-29 cells. After the internalisation of HYP, the effect of irradiation was evaluated. A commercial kit that detects apoptosis and cell death based on changes in the permeability of cell membrane was employed (Vybrant $^{\mathrm{TM}}$ Apoptosis Assay Kit \#4, V13243 Invitrogen ThermoFisher). ${ }^{284}$ 
During apoptosis, the cytoplasmic membrane becomes slightly permeant. YO-PRO ${ }^{\circledR}-1$ (Figure 4.51) can selectively pass through the plasma membrane and enter apoptotic cells. ${ }^{285-289}$ Its relatively large size $(630 \mathrm{Da})$ prevents this dye from penetrating the intact plasma membrane of living cells. ${ }^{290}$ Due to its nucleic acid-binding nature as cyanine, this molecule exhibits a large degree of fluorescence enhancement upon binding to DNA (the fluorescence of the unbound dye is negligible). ${ }^{291-293}$

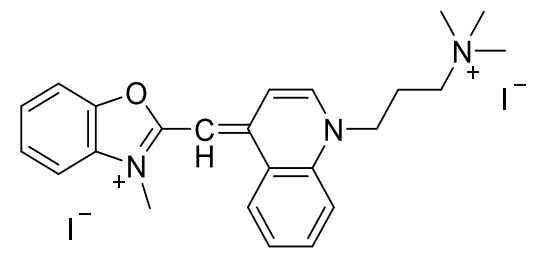

Figure 4.51. Chemical structure of $\mathrm{YO}-\mathrm{PRO}^{\circledR}-1$.

As in the kit used for RB@1 samples (Section 4.4.3.3), the standard dead-cell stain propidium iodide (Figure 4.38) is also used. Again, the permeability to YO-PRO ${ }^{\circledR}-1$ is an early apoptosis event and occurs well before the loss of membrane integrity and permeability to $\mathrm{PI}$ due to cell death. So, after staining a cell suspension with $\mathrm{YO}-\mathrm{PRO}^{\circledR}-1$ and $\mathrm{PI}$, three populations of cells can be observed after flow cytometry analysis. As in Figure 4.39, but plotting PI vs. YO-PRO ${ }^{\circledR}-1$ fluorescence emission, quadrant A corresponds to viable cells (negative to both labels), quadrant $B$ to apoptotic cells (YO-PRO ${ }^{\circledR}-1$ positive), and cells in quadrants $C$ and $D$ are non-viable (PI positive). ${ }^{280,294-296}$ The limitations of distinguishing death and late apoptosis/necrosis are the same as explained in Section 4.4.3.3.

The detection and quantification of the cells with the different labels were performed by flow cytometry, as depicted in Figure 4.52. Both species were excited at $488 \mathrm{~nm}$, being collected the YO-PRO ${ }^{\circledR}-1$ green fluorescence emission $\left(\lambda_{\max } 509 \mathrm{~nm}\right.$ when bound to DNA) in the range 518$548 \mathrm{~nm}\left(533 / 30 \mathrm{~nm}\right.$ bandpass filter) and the propidium iodide red fluorescence emission $\left(\lambda_{\max }\right.$ $617 \mathrm{~nm}$ when bound to DNA) recorded above $670 \mathrm{~nm}$ (670LP bandpass filter). 


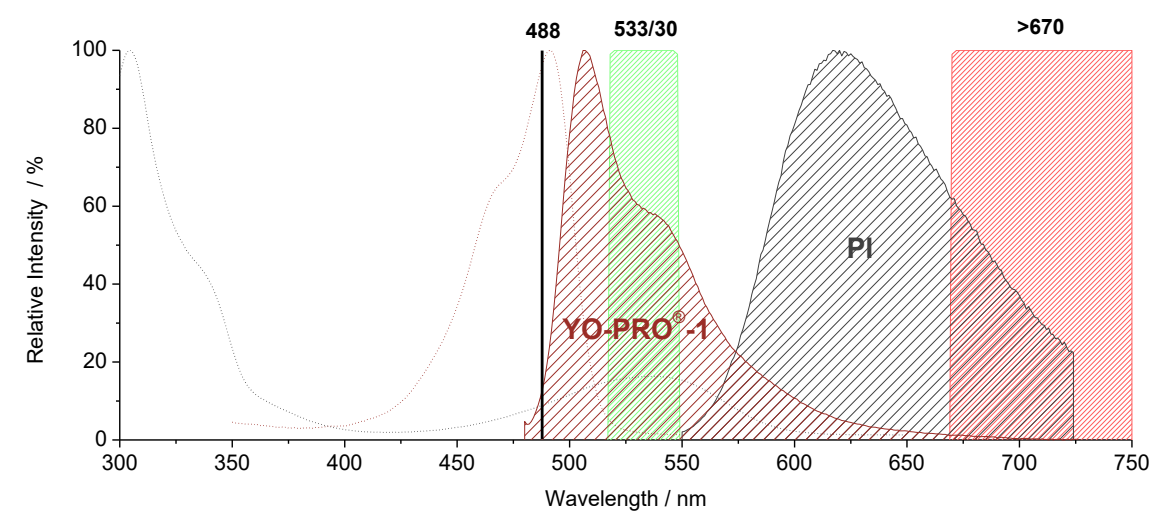

Figure 4.52. $\mathrm{YO}^{\mathrm{PRO}}{ }^{\circledR}-1$ (red) and propidium iodide (grey) excitation (dot line) and emission (solid patterned curve) spectra and flow cytometry excitation and detection wavelengths (adapted from BD Spectrum Viewer).

\section{RESULTS AND DISCUSSION}

HT-29 cells were incubated for $24 \mathrm{~h}$ with the HYP@1 sample in a 1:3 dilution with cell culture medium. Hypericin singlet oxygen production was stimulated by irradiation for 2 min with two LED lamps (11W each one, ca. 400-700 nm emission output) placed as close to the lid of the cells plate as possible (see Figure 4.41). The state of the cells was evaluated $24 \mathrm{~h}$ after irradiation by flow cytometry. Incubations were performed in all cases using FBS in the medium to avoid cell death as a consequence of starvation. For each experiment, a HYP@1 sample, a HYP-DMSO control at the same concentration and PBS as the negative control were assayed in the same conditions. Also, as control of dark toxicity, the samples were tested precisely in the same way in another plate covered with aluminium foil and kept next to the other during irradiation

(Figure 4.41). Doxorubicin was used as positive apoptosis control for internal control purposes (data not shown).

Four HYP@1 samples were tested, with hypericin concentrations of 0.4, 0.45, 0.6 and $0.8 \mu \mathrm{M}$ (after dilution: $0.12,0.15,0.20$ and $0.25 \mu \mathrm{M}$ ). The results of the flow cytometry analysis are shown in Figure $\mathbf{4 . 5 3}$ for two representative experiments. For each sample and irradiation conditions tested, the upper row shows the light scatter dot plot. In the bottom row, the dot plot corresponding to the fluorescence emission of $\mathrm{PI}$ (Y-axis) vs. YO-PRO ${ }^{\circledR}-1$ (X-axis) from the gated cells is represented. From the latter PI vs. YO-PRO ${ }^{\circledR}-1$ dot plot, the percentage of viable (beige), apoptotic (red) and non-viable (pink) cells was represented in the stacked bar charts of Figure $\mathbf{4 . 5 4}$ for the four experiments. For an experiment, the three first bars correspond to 
irradiated cells and the others to non-irradiated controls. Results are expressed as the average of two replicas.

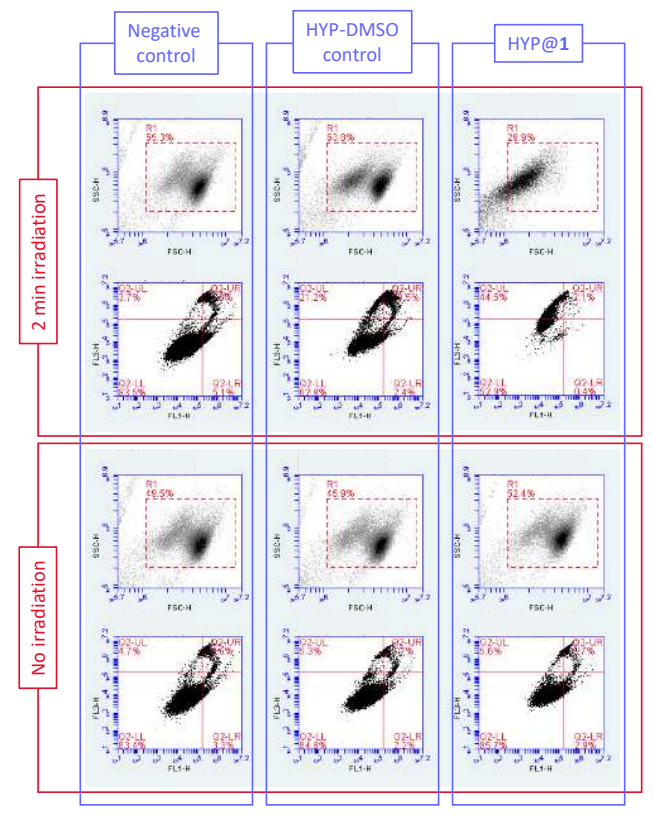

$[\mathrm{HYP}]=0.12 \mu \mathrm{M}$

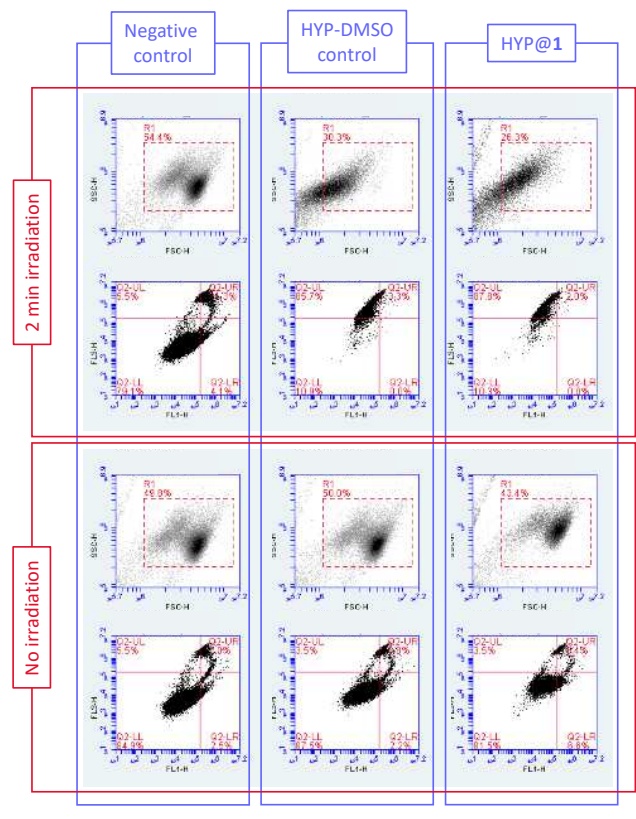

$[\mathrm{HYP}]=0.25 \mu \mathrm{M}$

Figure 4.53. Dot plots obtained after flow cytometry analysis of the cell populations of two representative experiments (left: $0.12 \mu \mathrm{M}$ HYP, right: $0.25 \mu \mathrm{M}$ HYP) concurrent stained with YO-PRO ${ }^{\circledR}-1$ and $\mathrm{PI}$. For each cell population, the top row corresponds to light scatter dot plot and the bottom one to $\mathrm{PI}$ vs. YO-PRO ${ }^{\circledR}-1$ fluorescence emission. 

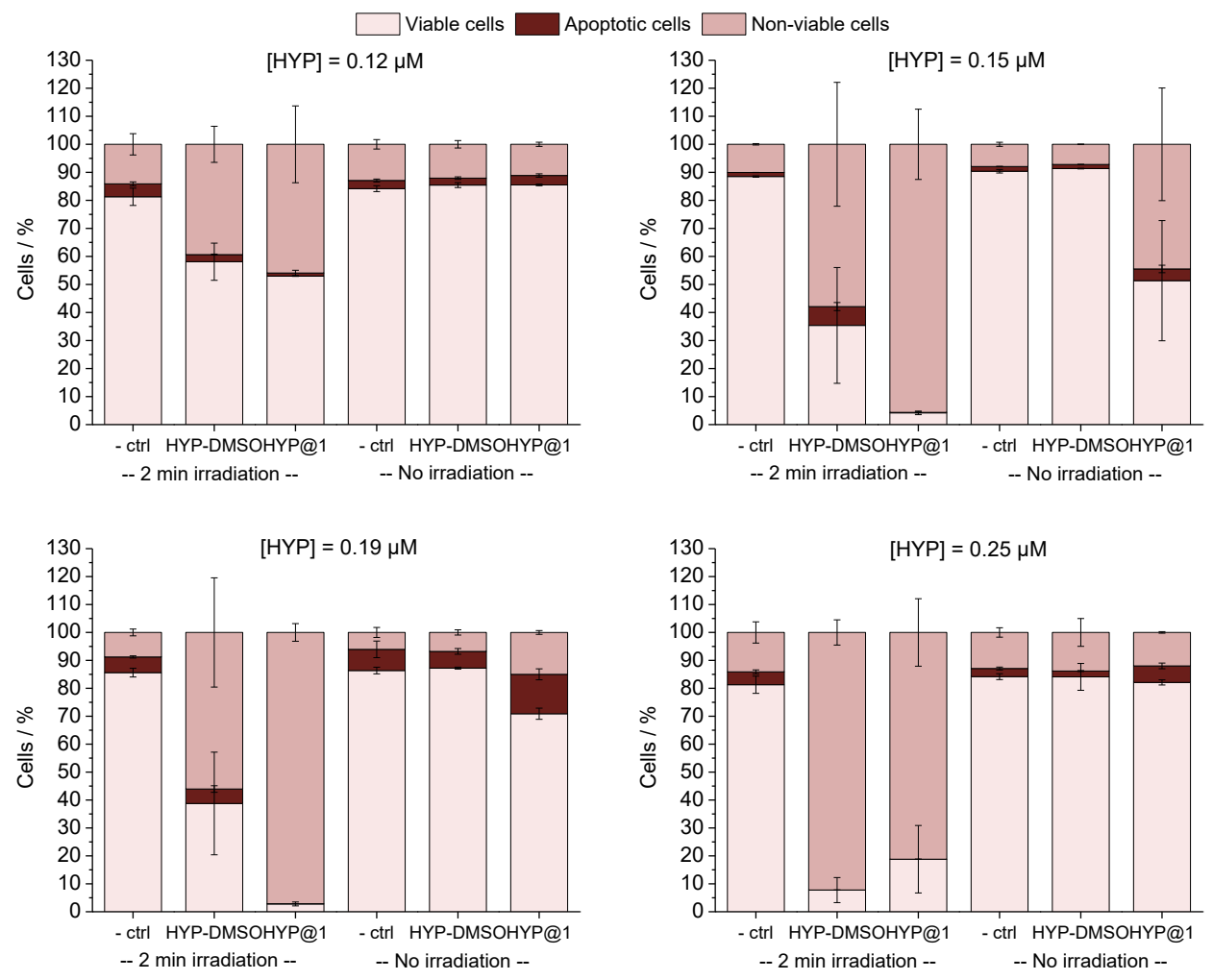

Figure 4.54. Distribution of cell populations in viable (beige), apoptotic (red) and non-viable (pink) cells for four different PDT experiments with HYP@1 samples and HYP-DMSO controls $(n=2)$.

Generally speaking, the results reveal that cells are mainly viable or dead at the analysis time, being the number of cells in apoptosis low. Therefore, to simplify, we will speak about cell viability to compare results. Regarding experiments without irradiation, a basal level of $84-91 \%$ of cell viability was obtained for the negative controls. The level of viable cells is, in general, similar for populations incubated with HYP@1 and HYP-DMSO controls. Therefore, the particles toxicity is low without irradiation (low dark toxicity), making them suitable for clinical applications. Regarding experiments with irradiation, again, the light stimulation of the cells is not significant for cell viability. As a result of reactive oxygen species production, cell viability is decreased for cells incubated with both HYP@1 and HYP-DMSO controls.

The potential effect on PDT efficiency of moderate changes in HYP concentration in the medium was evaluated. The percentage of viable cells after irradiation is shown for the different HYPDMSO controls and HYP@1 samples in Figure 4.55. No clear trends are obtained, considering the margin of error of the results. 

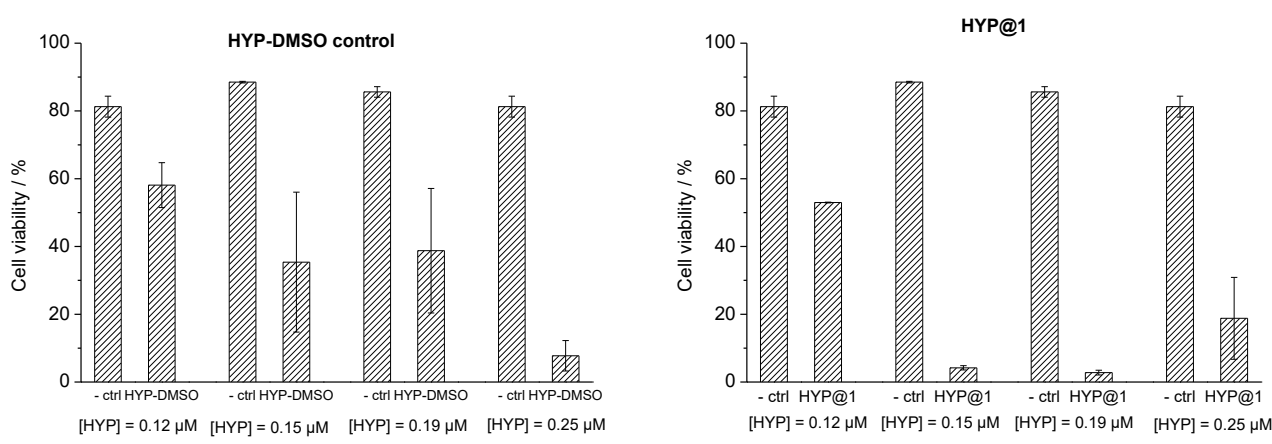

Figure 4.55. Cell viability vs. hypericin concentration of cells incubated with HYP@1 (right) or HYP-DMSO control (left), irradiated for $2 \mathrm{~min}$ and analysed after $24 \mathrm{~h}(\mathrm{n}=2)$.

HYP-PBS samples were also tested, with HYP concentrations of 0.04, 0.06 and $0.06 \mu \mathrm{M}$ hypericin (after dilution, $0.014,0.020,0.021 \mu \mathrm{M}$ ). The results of the flow cytometry analysis are shown in Figure 4.56 for a representative experiment. The data extracted from PI vs. YO-PRO ${ }^{\circledR}-1$ dot plot can be seen in Figure 4.57. The two first bars correspond to irradiated cells and the others to non-irradiated controls for a given experiment. Results are expressed as the average of two replicas.

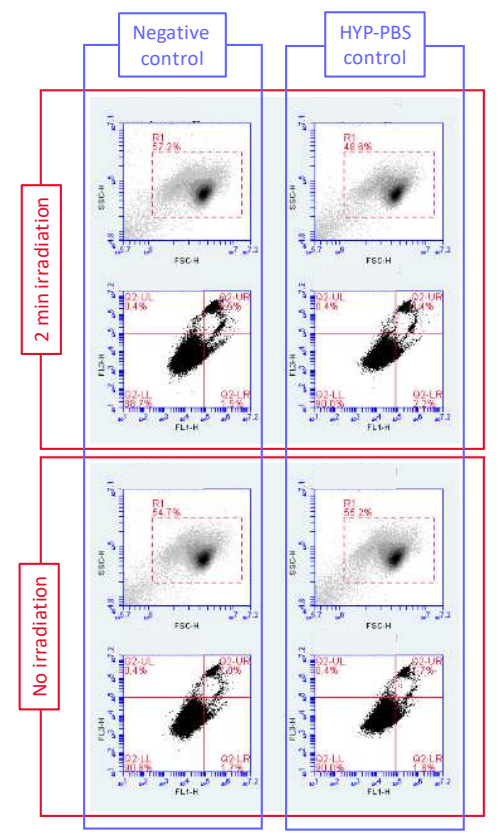

Figure 4.56. Dot plots obtained after flow cytometry analysis of the cell populations of a representative experiment of HYP-PBS control concurrent stained with YO-PRO ${ }^{\circledR}-1$ and PI. For each cell population, top row: light scatter dot plot, bottom row: PI vs. YO-PRO ${ }^{\circledR}-1$ fluorescence emission. 

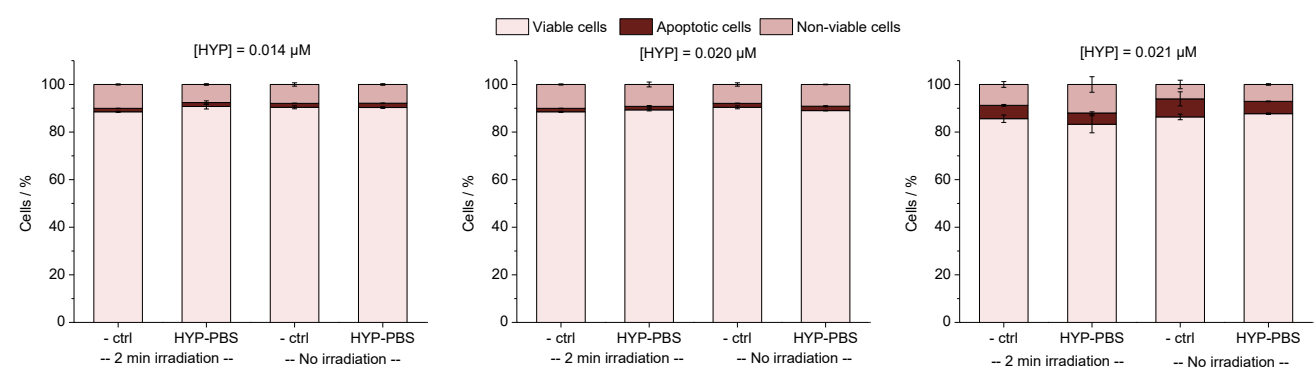

Figure 4.57. Distribution of cell populations in viable (beige), apoptotic (red) and non-viable (pink) cells for three different PDT experiments with HYP-PBS controls $(n=2)$.

Results are relatively homogeneous for the three different experiments, and the percentages of cell viability are near the basal level for all cell populations. Expressed as the mean of the three experiments, percentages of viability are $88 \pm 2 \%$ for the irradiated negative control, $88 \pm 4 \%$ for irradiated HYP-PBS control, $89 \pm 2 \%$ for non-irradiated negative control, and $89 \pm 1 \%$ for the nonirradiated HYP-PBS control. Thus, no singlet oxygen or ROS are produced after irradiation of cells incubated with HYP-PBS controls.

Although the kit used cannot distinguish the cause of death, as in Section 4.4.3.3, alterations in the cells light scattering provides more insight. During apoptosis, a decrease of cell volume due to cell shrinkage (decrease in forward scatter) and the formation of apoptotic vesicles in the cells (increase in side scatter profile) occur. ${ }^{36,281}$ On the other hand, necrosis finishes with the cell disintegration, disseminating its internal content. Therefore, the dot plot looks less focused and more dispersed, with a tendency to low FSC and SSC due to dispersed cellular material. ${ }^{281,295}$ In Figure 4.53, we can see the SSC vs. FSC dot plots for $0.12 \mu \mathrm{M}$ (also representative for 0.15 and $0.19 \mu \mathrm{M})$ and for $0.25 \mu \mathrm{M}$ HYP. For the lowest concentrations, the cells that have been irradiated after incubation with HYP@1 are necrotic, and those with HYP-DMSO control have part of the cell population in late apoptosis. On the other hand, cells show necrosis after incubation with both HYP@1 and HYP-DMSO control and irradiation for the highest HYP concentration. Furthermore, another confirmation of these observations is the percentage of cells in the gate (red dotted rectangle). In necrosis cases, a decrease in the cell count can be seen in SSC vs. FSC dot plots. The gate is always in the same position and has the same area. So, a lower proportion of cells indicates that cells have disintegrated, and there is more cell debris than in other samples. If apoptosis occurs, cells are still in the sample, and their percentage does not change. 
It has been reported (Section 4.1) that the type of cell death induced by PDT depends on PS subcellular localisation and concentration. High PS concentration induces necrosis, whereas a lower concentration gives apoptosis. ${ }^{297,298}$ Also, the ROS effect in the membrane is less lethal than in other organelles, such as lysosomes, where it produces necrosis. Thus, we can think of two possibilities. Firstly, hypericin internalization using HYP@1 is up to 3.5 times higher than for hypericin in PBS-1\%DMSO solution, resulting in the former case in necrosis induction. Secondly, confocal microscopy has revealed that hypericin localizes in the membrane for cells incubated with the free probe, where the photodamage is lower. In contrast, hypericin after HYP@1 internalization localizes in the cytoplasm, maybe in some organelle, where the damage is more lethal. Therefore, it seems that we are observing the sum of both effects in our cell death results. PDT with HYP@1 would always induce necrosis due to a higher subcellular concentration and a cytoplasmic localisation. In contrast, PDT with hypericin in a PBS-1\%DMSO solution would induce apoptosis or necrosis, depending on the concentration. It must also be highlighted that, although hypericin is known to induce apoptosis with high efficiency in most of the examined cell lines, ${ }^{297,299,300}$ a prevalence of necrosis has been observed in HT-29 cells treated with hypericin and exposed to an extensive range of PDT doses. ${ }^{213}$ Nevertheless, even if protocols favouring apoptosis are recommended when PDT is applied for curative treatment of in situ neoplasia, necrosis accompanied by inflammatory reaction may be recommended for curing infiltrative tumours possibly already spreading beyond the margins of the illumination spot. ${ }^{301}$

\subsubsection{DISCUSSION ABOUT IN VITRO HYPERICIN RESULTS}

The study of the cellular uptake of hypericin in HT-29 cells confirms that its internalization depends on the assay and formulation conditions as it has been reported in the literature. ${ }^{302}$ The highest internalization corresponds to hypericin loaded into the nanoparticles. It is even 3.5 times higher than for hypericin in a PBS-1\%DMSO solution at the same concentration. So, nanogels would be helping to internalize the probe, possibly due to a higher local concentration per particle and the difference in the mechanism of hypericin cellular entrance. Diffusion likely participates in hypericin uptake, whereas for nanoparticles, endocytosis is the most common pathway into the cell. ${ }^{258}$ Besides, a 14 times lower intracellular accumulation was obtained for the maximum quantity of hypericin that can be solubilized in water (HYP-PBS), which is 10 times lower in concentration. The localization of the probe also shows changes associated with the use of nanogels as vehicles. Hypericin is accumulated in the membrane when cells are incubated 
with the free probe and in the cytoplasm in a non-specific way when nanogels are used in the incubation. It should be mentioned here that these conclusions assume that internalized hypericin shows fluorescent emission. However, it cannot be wholly discarded that intracellular aggregation occurs, resulting in "silent" hypericin species. ${ }^{302}$ Nevertheless, the most sensible interpretation of the results, using Occam's razor reasoning, is that hypericin fluorescence activity in HT-29 cancer cells increases when using the nanoparticles as vehicles. It would be interesting to explore our nanoparticles potential as hypericin vehicles to be used in photodynamic cancer diagnosis (PD).

As for the phototoxicity of hypericin internalized in HT-29 cells, the singlet oxygen or ROS production of HYP@1 and HYP-DMSO control gives cell viability below 10\%. On the other hand, HYP-PBS shows no phototoxicity, likely because of low cell uptake or intracellular aggregation. It has to be highlighted that the therapeutic use of the DMSO containing solution would be limited, as the proportion of a cosolvent can change in cellular media, causing hypericin aggregation. Additionally, the use of solvents in cell cultures, commonly ethanol and DMSO, has some drawbacks that should not be neglected. For example, water-DMSO systems with 0.25 and $0.5 \%$ of the organic solvent induce inhibitory or stimulatory effects in some cell types. ${ }^{303}$ Therefore, HYP@1 represent a safer alternative to solubilize hypericin and achieve good intracellular delivery and phototoxicity, avoiding cosolvents. 


\subsection{REFERENCES}

Felip-León, C.; Arnau Del Valle, C.; Pérez-Laguna, V.; Millán-Lou, M. I.; Miravet, J. F.; Mikhailov, M.; Sokolov, M. N.; RezustaLópez, A.; Galindo, F. Superior Performance of Macroporous over Gel Type Polystyrene as a Support for the Development of Photo-Bactericidal Materials. J. Mater. Chem. B 2017, 5 (30), 6058-6064.

Oda, D. F.; Duarte, M. A. H.; Andrade, F. B.; Moriyama, L. T.; Bagnato, V. S.; de Moraes, I. G. Antimicrobial Action of Photodynamic Therapy in Root Canals Using LED Curing Light, Curcumin and Carbopol Gel. Int. Endod. J. 2019, 52 (7), 10101019.

Abdel-Kader, M. H. History of Photodynamic Therapy. In Photodynamic Therapy; Abdel-Kader, M. H., Ed.; Springer: Berlin, Heidelberg, Germany, 2014; pp 3-22.

Raab, O. Uber Die Wirkung Fluorescirender Stoffe Auf Infusorien. Ztg. Biol. 1900, 39, 524-546.

Dougherty, T. J.; Kaufman, J. E.; Goldfarb, A.; Weishaupt, K. R.; Boyle, D.; Mittleman, A. Photoradiation Therapy for the Treatment of Malignant Tumors. Cancer Res. 1978, 38, 2628-2635.

Shi, X.; Zhang, C. Y.; Gao, J.; Wang, Z. Recent Advances in Photodynamic Therapy for Cancer and Infectious Diseases. Wiley Interdiscip. Rev. Nanomedicine Nanobiotechnology 2019, 11, e1560.

Dietrich, W. D.; Watson, B. D.; Busto, R.; Ginsberg, M. D.; Bethea, J. R. Photochemically Induced Cerebral Infarction - I. Early Microvascular Alterations. Acta Neuropathol. 1987, 72, 315-325.

Calzavara-Pinton, P. G.; Rossi, M. T.; Aronson, E.; Sala, R.; Arpaia, N.; Burtica, E. C.; Amerio, P.; Virgili, A.; Rossi, R.; Buggiani, G.; Zanca, A.; Bugatti, L.; Fai, D.; Cervadoro, E.; Cavicchini, S.; Fantini, F.; Fabbrocini, G.; Piaserico, S.; Teoli, M.; Eibenschutz, L.; Arcangeli, F.; Broganelli, P.; Schena, D. A Retrospective Analysis of Real-Life Practice of off-Label Photodynamic Therapy Using Methyl Aminolevulinate (MAL-PDT) in 20 Italian Dermatology Departments. Part 1: Inflammatory and Aesthetic Indications. Photochem. Photobiol. Sci. 2013, 12 (1), 148-157.

Reinhard, A.; Sandborn, W. J.; Melhem, H.; Bolotine, L.; Chamaillard, M.; Peyrin-Biroulet, L. Photodynamic Therapy as a New Treatment Modality for Inflammatory and Infectious Conditions. Expert Rev. Clin. Immunol. 2015, 11 (5), 637-657.

Donohoe, C.; Senge, M. O.; Arnaut, L. G.; Gomes-da-Silva, L. C. Cell Death in Photodynamic Therapy: From Oxidative Stress to Anti-Tumor Immunity. Biochim. Biophys. Acta-Rev. Cancer 2019, 1872 (2), 188308.

Wang, C.; Cheng, L.; Liu, Z. Upconversion Nanoparticles for Photodynamic Therapy and Other Cancer Therapeutics. Theranostics 2013, 3 (5), 317-330.

Triesscheijn, M.; Baas, P.; Schellens, J. H. M.; Steward, F. A. Photodynamic Therapy in Oncology. Oncologist 2006, 11, 10341044.

Karunakaran, S. C.; Babu, P. S. S.; Madhuri, B.; Marydasan, B.; Paul, A. K.; Nair, A. S.; Rao, K. S.; Srinivasan, A.; Chandrashekar, T. K.; Rao, C. M.; Pillai, R.; Ramaiah, D. In Vitro Demonstration of Apoptosis Mediated Photodynamic Activity and NIR Nucleus Imaging through a Novel Porphyrin. ACS Chem. Biol. 2013, 8 (1), 127-132.

Liu, K.; Liu, X.; Zeng, Q.; Zhang, Y.; Tu, L.; Liu, T.; Kong, X.; Wang, Y.; Cao, F.; Lambrechts, S. A. G.; Aalders, M. C. G.; Zhang, H. Covalently Assembled NIR Nanoplatform for Simultaneous Fluorescence Imaging and Photodynamic Therapy of Cancer Cells. ACS Nano 2012, 6 (5), 4054-4062.

Sharman, W. M.; Allen, C. M.; Van Lier, J. E. Photodynamic Therapeutics: Basic Principles and Clinical Applications. Drug Discov. Today 1999, 4 (11), 507-517.

Sharman, W. M.; Van Lier, J. E.; Allen, C. M. Targeted Photodynamic Therapy via Receptor Mediated Delivery Systems. Adv. Drug Deliv. Rev. 2004, 56, 53-76.

Ethirajan, M.; Chen, Y.; Joshi, P.; Pandey, R. K. The Role of Porphyrin Chemistry in Tumor Imaging and Photodynamic Therapy. Chem. Soc. Rev. 2011, 40, 340-362.

Huang, P.; Li, Z.; Lin, J.; Yang, D.; Gao, G.; Xu, C.; Bao, L.; Zhang, C.; Wang, K.; Song, H.; Hu, H.; Cui, D. Photosensitize rConjugated Magnetic Nanoparticles for in Vivo Simultaneous Magnetofluorescent Imaging and Targeting Therapy. Biomaterials 2011, 32 (13), 3447-3458.

Huang, P.; Xu, C.; Lin, J.; Wang, C.; Wang, X.; Zhang, C.; Zhou, X.; Guo, S.; Cui, D. Folic Acid-Conjugated Graphene Oxide Loaded with Photosensitizers for Targeting Photodynamic Therapy. Theranostics 2011, 1, 240-250.

Huang, P.; Lin, J.; Wang, X.; Wang, Z.; Zhang, C.; He, M.; Wang, K.; Chen, F.; Li, Z.; Shen, G.; Cui, D.; Chen, X. Light-Triggered Theranostics Based on Photosensitizer-Conjugated Carbon Dots for Simultaneous Enhanced-Fluorescence Imaging and Photodynamic Therapy. Adv. Mater. 2012, 24 (37), 5104-5110.

Yang, P.; Gai, S.; Lin, J. Functionalized Mesoporous Silica Materials for Controlled Drug Delivery. Chem. Soc. Rev. 2012, 41 (9), 3679-3698.

Li, L.; Tang, F.; Liu, H.; Liu, T.; Hao, N.; Chen, D.; Teng, X.; He, J. In Vivo Delivery of Silica Nanorattle Encapsulated Docetaxel for Liver Cancer Therapy with Low Toxicity and High Efficacy. ACS Nano 2010, 4 (11), 6874-6882.

Hogle, W. P. The State of the Art in Radiation Therapy. Semin. Oncol. Nurs. 2006, 22 (4), 212-220.

Hamblin, M. R. Photodynamic Therapy for Cancer: What's Past Is Prologue. Photochem. Photobiol. 2020, X(X), X.

Foote, C. S. Definition of Type I and Type II Photosensitized Oxidation. Photochem. Photobiol. 1991, 54 (5), 659.

Plaetzer, K.; Krammer, B.; Berlanda, J.; Berr, F.; Kiesslich, T. Photophysics and Photochemistry of Photodynamic Therapy: 
Fundamental Aspects. Lasers Med. Sci. 2009, 24 (2), 259-268.

(27) Kamkaew, A.; Lim, S. H.; Lee, H. B.; Kiew, L. V.; Chung, L. Y.; Burgess, K. BODIPY Dyes in Photodynamic Therapy. Chem. Soc Rev. 2013, $42(1), 77-88$.

(28) Zhao, J.; Wu, W.; Sun, J.; Guo, S. Triplet Photosensitizers: From Molecular Design to Applications. Chem. Soc. Rev. 2013, 42 (12), 5323-5351.

(29) DeRosa, M. C.; Crutchley, R. J. Photosensitized Singlet Oxygen and Its Applications. Coord. Chem. Rev. 2002, 233, 351-371.

(30) Macdonald, I. J.; Dougherty, T. J. Basic Principles of Photodynamic Therapy. J. Porphyr. Phthalocyanines 2001, No. 5, $105-129$.

(31) Fan, W.; Huang, P.; Chen, X. Overcoming the Achilles' Heel of Photodynamic Therapy. Chem. Soc. Rev. 2016, 45 (23), 64886519.

(32) Jori, G. Tumour Photosensitizers: Approaches to Enhance the Selectivity and Efficiency of Photodynamic Therapy. J. Photochem. Photobiol. B Biol. 1996, 36, 87-93.

(33) Castano, A. P.; Demidova, T. N.; Hamblin, M. R. Mechanisms in Photodynamic Therapy: Part Three - Photosensitize Pharmacokinetics, Biodistribution, Tumor Localization and Modes of Tumor Destruction. Photodiagnosis Photodyn. Ther. 2005, 2, 91-106.

(34) Hatz, S.; Lambert, J. D. C.; Ogilby, P. R. Measuring the Lifetime of Singlet Oxygen in a Single Cell: Addressing the Issue of Cell Viability. Photochem. Photobiol. Sci. 2007, 6, 1106-1116.

(35) Kerr, J. F. R.; Wyllie, A. H.; Currie, A. R. Apoptosis: A Basic Biological Phenomenon with Wide-Ranging Implications in Tissue Kinetics. Br. J. Cancer 1972, 26, 239-257.

(36) Hingorani, R.; Deng, J.; Elia, J.; McIntyre, C.; Mittar, D. Application Note - BD Biosciences: Detection of Apoptosis Using the BD Annexin V FITC Assay on the BD FACSVerse ${ }^{T M}$ System; 2011.

(37) Fuchs, Y.; Steller, H. Live to Die Another Way: Modes of Programmed Cell Death and the Signals Emanating from Dying Cells. Nat Rev Mol Cell Biol 2015, 16 (6), 329-344.

(38) Chapter 15 - Assays for Cell Viability, Proliferation and Function. In The Molecular Probes ${ }^{\circledR}$ Handbook-A Guide to Fluorescent Probes and Labeling Technologies; Invitrogen, ThermoFisher Scientific, 2010.

(39) Proskuryakov, S. Y.; Konoplyannikov, A. G.; Gabai, V. L. Necrosis: A Specific Form of Programmed Cell Death? Exp. Cell Res. 2003, 283, 1-16.

(40) Agostinis, P.; Vantieghem, A.; Merlevede, W.; De Witte, P. A. M. Hypericin in Cancer Treatment: More Light on the Way. Int J. Biochem. Cell Biol. 2002, 34 (3), 221-241.

(41) Mansoori, B.; Mohammadi, A.; Amin Doustvandi, M.; Mohammadnejad, F.; Kamari, F.; Gjerstorff, M. F.; Baradaran, B.; Hamblin, M. R. Photodynamic Therapy for Cancer: Role of Natural Products. Photodiagnosis Photodyn. Ther. 2019, 26, 395404.

(42) Mroz, P.; Yaroslavsky, A.; Kharkwal, G. B.; Hamblin, M. R. Cell Death Pathways in Photodynamic Therapy of Cancer. Cancers (Basel). 2011, 3, 2516-2539.

(43) Yanovsky, R. L.; Bartenstein, D. W.; Rogers, G. S.; Isakoff, S. J.; Chen, S. T. Photodynamic Therapy for Solid Tumors: A Review of the Literature. Photodermatol. Photoimmunol. Photomed. 2019, 35, 295-303.

(44) Harris, A. L. Hypoxia - A Key Regulatory Factor in Tumour Growth. Nat. Rev. Cancer 2002, 2, 38-47.

(45) Vaupel, P. Tumor Microenvironmental Physiology and Its Implications for Radiation Oncology. Semin. Radiat. Oncol. 2004, 14 (3), 198-206.

(46) Chen, H.; Tian, J.; He, W.; Guo, Z. H2O2-Activatable and O2-Evolving Nanoparticles for Highly Efficient and Selective Photodynamic Therapy against Hypoxic Tumor Cells. J. Am. Chem. Soc. 2015, 137 (4), 1539-1547.

(47) Brown, J. M.; Wilson, W. R. Exploiting Tumour Hypoxia in Cancer Treatment. Nat. Rev. Cancer 2004, 4, $437-447$.

(48) Carmeliet, P.; Jain, R. K. Molecular Mechanisms and Clinical Applications of Angiogenesis. Nature 2011, 473, $298-307$.

(49) Dewhirst, M. W.; Cao, Y.; Moeller, B. Cycling Hypoxia and Free Radicals Regulate Angiogenesis and Radiotherapy Response. Nat. Rev. Cancer 2008, 8, 425-437.

(50) Yao, C.; Wang, W.; Wang, P.; Zhao, M.; Li, X.; Zhang, F. Near-Infrared Upconversion Mesoporous Cerium Oxide Hollow Biophotocatalyst for Concurrent PH-/H2O2-Responsive O2-Evolving Synergetic Cancer Therapy. Adv. Mater. 2018, 30, 1704833.

(51) Brancaleon, L.; Moseley, H. Laser and Non-Laser Light Sources for Photodynamic Therapy. Lasers Med. Sci. 2002, 17, 173186.

(52) Mang, T. S. Lasers and Light Sources for PDT: Past, Present and Future. Photodiagnosis Photodyn. Ther. 2004, 1, $43-48$.

(53) Frangioni, J. V. In Vivo Near-Infrared Fluorescence Imaging. Curr. Opin. Chem. Biol. 2003, 7, 626-634.

(54) Zhang, Y.; Pang, L.; Ma, C.; Tu, Q.; Zhang, R.; Saeed, E.; Mahmoud, A. E.; Wang, J. Small Molecule-Initiated Light-Activated Semiconducting Polymer Dots: An Integrated Nanoplatform for Targeted Photodynamic Therapy and Imaging of Cancer Cells. Anal. Chem. 2014, 86, 3092-3099.

(55) Paszko, E.; Ehrhardt, C.; Senge, M. O.; Kelleher, D. P.; Reynolds, J. V. Nanodrug Applications in Photodynamic Therapy. Photodiagnosis Photodyn. Ther. 2011, 8, 14-29.

(56) Wöhrle, D.; Hirth, A.; Bogdahn-Rai, T.; Schnurpfeil, G.; Shopova, M. Photodynamic Therapy of Cancer: Second and Third Generations of Photosensitizers. Russ. Chem. Bull. 1998, 47 (5), 807-816. 
(57) Josefsen, L. B.; Boyle, R. W. Unique Diagnostic and Therapeutic Roles of Porphyrins and Phthalocyanines in Photodynamic Therapy, Imaging and Theranostics. Theranostics 2012, 2 (9), 916-966.

(58) O'Connor, A. E.; Gallagher, W. M.; Byrne, A. T. Porphyrin and Nonporphyrin Photosensitizers in Oncology: Preclinical and Clinical Advances in Photodynamic Therapy. Photochem. Photobiol. 2009, 85, 1053-1074.

(59) García Calavia, P.; Marín, M. J.; Chambrier, I.; Cook, M. J.; Russell, D. A. Towards Optimisation of Surface Enhanced Photodynamic Therapy of Breast Cancer Cells Using Gold Nanoparticle-Photosensitiser Conjugates. Photochem. Photobiol. Sci. 2018, 17 (3), 281-289.

(60) Orenstein, A.; Kostenich, G.; Roitman, L.; Shechtman, Y.; Kopolovic, Y.; Ehrenberg, B.; Malik, Z. A Comparative Study of Tissue Distribution and Photodynamic Therapy Selectivity of Chlorin E6, Photofrin II and ALA-Induced Protoporphyrin IX in a Colon Carcinoma Model. Br. J. Cancer 1996, 73, 937-944.

(61) Zeisser-Labouèbe, M.; Lange, N.; Gurny, R.; Delie, F. Hypericin-Loaded Nanoparticles for the Photodynamic Treatment of Ovarian Cancer. Int. J. Pharm. 2006, 326, 174-181.

(62) Moore, C. M.; Pendse, D.; Emberton, M. Photodynamic Therapy for Prostate Cancer - A Review of Current Status and Future Promise. Nat. Clin. Pract. Urol. 2009, 6 (1), 18-30.

(63) Vrouenraets, M. B.; Visser, G. W. M.; Stigter, M.; Oppelaar, H.; Snow, G. B.; van Dongen, G. A. M. S. Comparison of Aluminium (III) Phthalocyanine Tetrasulfonate- and Meta-Tetrahydroxyphenylchlorin-Monoclonal Antibody Conjugates for Their Efficacy in Photodynamic Therapy in Vitro. Int. J. Cancer 2002, 98, 793-798.

(64) Kessel, D. Transport and Binding of Hematoporphyrin Derivative and Related Porphyrins by Murine Leukemia L1210 Cells. Cancer Res. 1981, 41, 1318-1323.

(65) Couleaud, P.; Morosini, V.; Frochot, C.; Richeter, S.; Raehm, L.; Durand, J.-O. Silica-Based Nanoparticles for Photodynamic Therapy Applications. Nanoscale 2010, 2, 1083-1095.

(66) Nishiyama, N.; Morimoto, Y.; Jang, W. D.; Kataoka, K. Design and Development of Dendrimer Photosensitizer-Incorporated Polymeric Micelles for Enhanced Photodynamic Therapy. Adv. Drug Deliv. Rev. 2009, 61, 327-338.

(67) Chen, H.; Xiao, L.; Anraku, Y.; Mi, P.; Liu, X.; Cabral, H.; Inoue, A.; Nomoto, T.; Kishimura, A.; Nishiyama, N.; Kataoka, K. Polyion Complex Vesicles for Photoinduced Intracellular Delivery of Amphiphilic Photosensitizer. J. Am. Chem. Soc. 2014, 136, 157163.

(68) Derycke, A. S. L.; De Witte, P. A. M. Liposomes for Photodynamic Therapy. Adv. Drug Deliv. Rev. 2004, 56, 17-30

(69) Hofman, J. W.; Carstens, M. G.; Van Zeeland, F.; Helwig, C.; Flesch, F. M.; Hennink, W. E.; Van Nostrum, C. F. Photocytotoxicity of MTHPC (Temoporfin) Loaded Polymeric Micelles Mediated by Lipase Catalyzed Degradation. Pharm. Res. 2008, 25 (9), 2065-2073.

(70) Van Nostrum, C. F. Polymeric Micelles to Deliver Photosensitizers for Photodynamic Therapy. Adv. Drug Deliv. Rev. 2004, 56, 9-16.

(71) Konan, Y. N.; Gurny, R.; Allémann, E. State of the Art in the Delivery of Photosensitizers for Photodynamic Therapy. J. Photochem. Photobiol. B Biol. 2002, 66, 89-106.

(72) Chatterjee, D. K.; Fong, L. S.; Zhang, Y. Nanoparticles in Photodynamic Therapy: An Emerging Paradigm. Adv. Drug Deliv. Rev. 2008, 60, 1627-1637.

(73) Chen, G.; Roy, I.; Yang, C.; Prasad, P. N. Nanochemistry and Nanomedicine for Nanoparticle-Based Diagnostics and Therapy. Chem. Rev. 2016, 116 (5), 2826-2885.

(74) Schmitt, F.; Juillerat-Jeanneret, L. Drug Targeting Strategies for Photodynamic Therapy. Anticancer. Agents Med. Chem. 2012 12 (5), 500-525.

(75) Roy, I.; Ohulchanskyy, T. Y.; Pudavar, H. E.; Bergey, E. J.; Oseroff, A. R.; Morgan, J.; Dougherty, T. J.; Prasad, P. N. Cera micBased Nanoparticles Entrapping Water-Insoluble Photosensitizing Anticancer Drugs: A Novel Drug-Carrier System for Photodynamic Therapy. J. Am. Chem. Soc. 2003, 125 (26), 7860-7865.

(76) Marín, M. J.; Russell, D. A. Upconversion Nanomaterials for Photodynamic Therapy. In Near-infrared Nanomaterials: Preparation, Bioimaging and Therapy Applications; Zhang, F., Ed.; The Royal Society of Chemistry: Croydon, 2016; p 192.

(77) Neckers, D. C.; Valdes-Aguilera, O. M. Photochemistry of the Xanthene Dyes. In Advances in Photochemistry; 1993; Vol. 18, pp 315-394.

(78) Neckers, D. C. Rose Bengal. J. Photochem. Photobiol. A Chem. 1989, 47, 1-29.

(79) Neckers, D. C. The Indian Happiness Wart in the Development of Photodynamic Action. Journrl Chem. Educ. 1987, 64 (8), 649656.

(80) Kochevar, I. E.; Lambert, C. R.; Lynch, M. C.; Tedesco, A. C. Comparison of Photosensitized Plasma Membrane Damage Caused by Singlet Oxygen and Free Radicals. Biochim. Biophys. Acta-Biomembr. 1996, 1280 (2), 223-230.

(81) Fleming, G. R.; Knight, A. W. E.; Morris, J. M.; Morrison, R. J. S.; Robinson, G. W. Picosecond Fluorescence Studies of Xanthene Dyes. J. Am. Chem. Soc. 1977, 99 (13), 4306-4311.

(82) Vanerio, N.; Stijnen, M.; De Mol, B. A. J. M.; Kock, L. M. Biomedical Applications of Photo- and Sono-Activated Rose Bengal: A Review. Photobiomodulation, Photomedicine, Laser Surg. 2019, 37 (7), 383-394.

(83) Tseng, S. C.; Feenstra, R. P.; Watson, B. D. Characterization of Photodynamic Actions of Rose Bengal on Cultured Cells. Invest Ophthalmol. Vis. Sci. 1994, 35 (8), 3295-3307.

(84) Delprat, G. D.; Epstein, N. N.; Kerr, W. J. A New Liver Function Test: The Elimination of Rose Bengal When Injected into the 
Circulation of Human Subjects. Arch. Intern. Med. 1924, 34 (4), 533-541.

(85) Taplin, G. V; Meredith, O. M. J.; Winter, C. C.; Johnson, D. Rose Bengal and lodopyracet as Radiodiagnostic Agents in Liver and Kidney Diseases. Ann. N. Y. Acad. Sci. 1959, 78, 872-893.

(86) Leevy, C. M. Diagnostic Procedures in the Evaluation of Hepatic Diseases. Functional Evaluation of the Biliary System. Lab. Res. Meth. Biol. Med. 1983, 7, 43e57.

(87) DrugBank https://www.drugbank.ca/ (accessed Jan 15, 2019).

(88) Lansche, R. K. Vital Staining in Normal Eyes and in Keratoconjunctivitis Sicca. Am. J. Ophthalmol. 1965, 60 (3), $520-525$.

(89) Doughty, M. J. Rose Bengal Staining as an Assessment of Ocular Surface Damage and Recovery in Dry Eye Disease - A Review. Contact Lens Anterior Eye 2013, 36 (6), 272-280.

(90) Khan-Lim, D.; Berry, M. Still Confused about Rose Bengal? Curr. Eye Res. 2004, 29 (4-5), 311-317.

(91) Feenstra, R. P. G.; Tseng, S. C. G. What Is Actually Stained by Rose Bengal? Arch. Ophthalmol. 1992, 110 (7), $984-993$.

(92) Paczkowski, J.; Lamberts, J. J.; Paczkowska, B.; Neckers, D. C. Photophysical Properties of Rose Bengal and Its Derivatives (XII). J. Free Radic. Biol. Med. 1985, 1 (5-6), 341-351.

(93) Gandin, E.; Lion, Y.; Van de Vorst, A. Quantum Yield of Singlet Oxygen Production by Xanthene Derivatives. Photochem. Photobiol. 1983, 37 (3), 271-278.

(94) Redmond, R. W.; Gamlin, J. N. A Compilation of Singlet Oxygen Yields from Biologically Relevant Molecules. Photochem Photobiol. 1999, 70 (4), 391-475.

(95) Zhao, T.; Shen, X.; Li, L.; Guan, Z.; Gao, N.; Yuan, P.; Yao, S. Q.; Xu, Q.-H.; Xu, G. Q. Gold Nanorods as Dual Photo-Sensitizing and Imaging Agents for Two-Photon Photodynamic Therapy. Nanoscale 2012, 4 (24), 7712-7719.

(96) Douglas, P.; Waechter, G.; Mills, A. Ionic Strength Effects on the Ground State Complexation and Triplet State Electron Transfer Reaction between Rose Bengal and Methyl Viologen. Photochem. Photobiol. 1990, 52 (3), 473-479.

(97) Wachter, E.; Dees, C.; Harkins, J.; Scott, T.; Petersen, M.; Rush, R. E.; Cada, A. Topical Rose Bengal: Pre-Clinical Evaluation of Pharmacokinetics and Safety. Lasers Surg. Med. 2003, 32, 101-110.

(98) Sztandera, K.; Gorzkiewicz, M.; Klajnert-Maculewicz, B. Nanocarriers in Photodynamic Therapy-in Vitro and in Vivo Studies. Wiley Interdiscip. Rev. Nanomedicine Nanobiotechnology 2019, e1509.

(99) Lenard, J.; Rabsont, A.; Vanderoef, R. Photodynamic Inactivation of Infectivity of Human Immunode ficiency Virus and Other Enveloped Viruses Using Hypericin and Rose Bengal: Inhibition of Fusion and Syncytia Formation. Proc. Natl. Acad. Sci. U. S. A. 1993, 90, 158-162.

(100) Chodosh, J.; Banks, M. C.; Stroop, W. G. Rose Bengal Inhibits Herpes Simplex Virus Replication in Vero and Human Corneal Epithelial Cells in Vitro. Invest. Ophthalmol. Vis. Sci. 1992, 33 (8), 2520-2527.

(101) Dahl, T.; Midden, W. R.; Neckers, D. C. COMPARISON OF PHOTODYNAMIC ACTION BY ROSE BENGAL IN GRAM-POSITIVE AND GRAM-NEGATIVE BACTERIA. Photochem. Photobiol. 1988, 48 (5), 607-612.

(102) Morton, C. O.; Chau, M.; Stack, C. In Vitro Combination Therapy Using Low Dose Clotrimazole and Photodynamic Therapy Leads to Enhanced Killing of the Dermatophyte Trichophyton Rubrum. BMC Microbiol. 2014, 14, 261.

(103) Cruz, F. S.; Lopes, L. A.; De Souza, W.; Moreno, S. N.; Mason, R. P.; Docampo, R. The Photodynamic Action of Rose Bengal on Trypanosoma Cruzi. Acta Trop. 1984, 41 (2), 99-108.

(104) Tonogai, Y.; Ito, Y.; Iwaida, M.; Tati, M.; Ose, Y.; Sato, T. Studies on the Toxicity of Coal-Tar Dyes. I. Photodecomposed Products of Four Xanthene Dyes and Their Acute Toxicity to Fish. J. Toxicol. Sci. 1979, 4 (2), 115-125.

(105) Provectus Biopharmaceuticals web page https://www.provectusbio.com/ (accessed Jan 15, 2019).

(106) ClinicalTrials.gov database https://clinicaltrials.gov/ (accessed Jan 15, 2019).

(107) Kochevar lab web page https://wellman.massgeneral.org/faculty-kochevar-projects.htm (accessed Feb 12, 2020).

(108) Yao, M.; Yaroslavsky, A.; Henry, F. P.; Redmond, R. W.; Kochevar, I. E. Phototoxicity Is Not Associated with Photochemical Tissue Bonding of Skin. Lasers Surg. Med. 2010, 42 (2), 123-131.

(109) Tsao, S.; Yao, M.; Tsao, H.; Henry, F. P.; Zhao, Y.; Kochevar, J. J.; Redmond, R. W.; Kochevar, I. E. Light-Activated Tissue Bonding for Excisional Wound Closure: A Split-Lesion Clinical Trial. Br. J. Dermatol. 2012, 166 (3), 555-563.

(110) Lee, J. S.; Lee, B. II; Park, C. B. Photo-Induced Inhibition of Alzheimer's $\beta$-Amyloid Aggregation in Vitro by Rose Bengal. Biomaterials 2015, 38, 43-49.

(111) Watson, B. D.; Dietrich, W. D.; Prado, R.; Ginsberg, M. D. Argon Laser-Induced Arterial Photothrombosis. Characterization and Possible Application to Therapy of Arteriovenous Malformations. J. Neurosurg. 1987, 66 (5), 748-754.

(112) Nonaka, M.; Yamamoto, M.; Yoshino, S.; Umemura, S. I.; Sasaki, K.; Fukushima, T. Sonodynamic Therapy Consisting of Focused Ultrasound and a Photosensitizer Causes a Selective Antitumor Effect in a Rat Intracranial Glioma Model. Anticancer Res. 2009, 29 (3), 943-950.

(113) Costley, D.; Mc Ewan, C.; Fowley, C.; McHale, A. P.; Atchison, J.; Nomikou, N.; Callan, J. F. Treating Cancer with Sonodynamic Therapy: A Review. Int. J. Hyperth. 2015, 31 (2), 107-117.

(114) Ross, M. I. Intralesional Therapy with PV-10 (Rose Bengal) for in-Transit Melanoma. J. Surg. Oncol. 2014, 109 (4), $314-319$.

(115) Mousavi, S. H.; Tavakkol-Afshari, J.; Brook, A.; Jafari-Anarkooli, I. Direct Toxicity of Rose Bengal in MCF-7 Cell Line: Role of Apoptosis. Food Chem. Toxicol. 2009, 47 (4), 855-859.

(116) Koevary, S. B. Selective Toxicity of Rose Bengal to Ovarian Cancer Cells in Vitro. Int. J. Physiol. Pathophysiol. Pharmacol. 2012 
4 (2), 99-107.

(117) Swift, L.; Zhang, C.; Trippett, T.; Narendran, A. Potent in Vitro and Xenograft Antitumor Activity of a Novel Agent, PV-10, against Relapsed and Refractory Neuroblastoma. Onco. Targets. Ther. 2019, 12, 1293-1307.

(118) Fischer, E.; Varga, F. Hepatic Storage and Biliary Excretion of Rose Bengal in the Rat. Acta Physiol. Acad. Sci. Hung. 1979, 54 (1), 89-94.

(119) Chang, C. C.; Yang, Y. T.; Yang, J. C.; Wu, H. Da; Tsai, T. Absorption and Emission Spectral Shifts of Rose Bengal Associated with DMPC Liposomes. Dye. Pigment. 2008, 79 (2), 170-175.

(120) Pereira, P. C. de S.; Costa, P. F. do A.; Pellosi, D. S.; Calori, I. R.; Vilsinski, B. H.; Estevão, B. M.; Hioka, N.; Caetano, W Photophysical Properties and Interaction Studies of Rose Bengal Derivatives with Biomimetic Systems Based in Micellar Aqueous Solutions. J. Mol. Liq. 2017, 230, 674-685.

(121) Croce, A. C.; Wyroba, E.; Bottiroli, G. Distribution and Retention of Rose Bengal and Disulphonated Aluminium Phthalocyanine: A Comparative Study in Unicellular Eukaryote. J. Photochem. Photobiol. B Biol. 1992, 16, 318-330.

(122) Killig, F.; Stark, G.; Apell, H.-J. Photodynamic Inactivation of the Na,K-ATPase Occurs via Different Pathways. J. Membr. Biol. 2004, 200 (3), 133-144.

(123) Alarcon, E. I.; Poblete, H.; Roh, H.; Couture, J.-F.; Comer, J.; Kochevar, I. E. Rose Bengal Binding to Collagen and Tissue Photobonding. ACS Omega 2017, 2 (10), 6646-6657.

(124) Bottiroli, G.; Croce, A. C.; Balzarini, P.; Locatelli, D.; Baglioni, P.; Lo Nostro, P.; Monici, M.; Pratesi, R. Enzyme-Assisted Cell Photosensitization: A Proposal for an Efficient Approach to Tumor Therapy and Diagnosis. The Rose Bengal Fluorogenic Substrate. Photochem. Photobiol. 1997, 66 (3), 374-383.

(125) Luttrull, D. K.; Valdes-Aguilera, O.; Linden, S. M.; Paczkowski, J.; Neckers, D. C. Rose Bengal Aggregation in Rationally Synthesized Dimeric Systems. Photochem. Photobiol. 1988, 47 (4), 551-557.

(126) Lin, W.; Garnett, M. C.; Davis, S. S.; Schacht, E.; Ferruti, P.; Illum, L. Preparation and Characterisation of Rose Bengal-Loaded Surface-Modified Albumin Nanoparticles. J. Control. Release 2001, 71, 117-126.

(127) Fadel, M.; Kassab, K. Evaluation of the Photostability and Photodynamic Efficacy of Rose Bengal Loaded in Multivesicular Liposomes. Trop. J. Pharm. Res. 2011, 10 (3), 289-297.

(128) Wang, X.-L.; Zeng, Y.; Zheng, Y.-Z.; Chen, J.-F.; Tao, X.; Wang, L.-X.; Teng, Y. Rose Bengal-Grafted Biodegradable Microcapsules: Singlet-Oxygen Generation and Cancer-Cell Incapacitation. Chem. - A Eur. J. 2011, 17 (40), 11223-11229.

(129) Karthikeyan, K.; Babu, A.; Kim, S. J.; Murugesan, R.; Jeyasubramanian, K. Enhanced Photodynamic Efficacy and Efficient Delivery of Rose Bengal Using Nanostructured Poly(Amidoamine) Dendrimers: Potential Application in Photodynamic Therapy of Cancer. Cancer Nanotechnol. 2011, 2, 95-103.

(130) Uppal, A.; Jain, B.; Gupta, P. K.; Das, K. Photodynamic Action of Rose Bengal Silica Nanoparticle Complex on Breast and Oral Cancer Cell Lines. Photochem. Photobiol. 2011, 87 (5), 1146-1151.

(131) Wang, B.; Wang, J. H.; Liu, Q.; Huang, H.; Chen, M.; Li, K.; Li, C.; Yu, X. F.; Chu, P. K. Rose-Bengal-Conjugated Gold Nanorods for in Vivo Photodynamic and Photothermal Oral Cancer Therapies. Biomaterials 2014, 35, 1954-1966.

(132) Martins Estevão, B.; Cucinotta, F.; Hioka, N.; Cossi, M.; Argeri, M.; Paul, G.; Marchese, L.; Gianotti, E. Rose Bengal Incorporated in Mesostructured Silica Nanoparticles: Structural Characterization, Theoretical Modeling and Singlet Oxygen Delivery. Phys. Chem. Chem. Phys. 2015, 17 (40), 26804-26812.

(133) Gianotti, E.; Martins Estevão, B.; Cucinotta, F.; Hioka, N.; Rizzi, M.; Renò, F.; Marchese, L. An Efficient Rose Bengal Based Nanoplatform for Photodynamic Therapy. Chem. Eur. J. 2014, 20 (35), 10921-10925.

(134) Chaudhuri, S.; Sardar, S.; Bagchi, D.; Dutta, S.; Debnath, S.; Saha, P.; Lemmens, P.; Pal, S. K. Photoinduced Dynamics and Toxicity of a Cancer Drug in Proximity of Inorganic Nanoparticles under Visible Light. ChemPhysChem 2016, 17 (2), $270-277$.

(135) Rosa-Pardo, I.; Roig-Pons, M.; Heredia, A. A.; Usagre, J. V.; Ribera, A.; Galian, R. E.; Pérez-Prieto, J. Fe304@Au@mSiO2 as an Enhancing Nanoplatform for Rose Bengal Photodynamic Activity. Nanoscale 2017, 9 (29), 10388-10396.

(136) Dabrzalska, M.; Janaszewska, A.; Zablocka, M.; Mignani, S.; Majoral, J. P.; Klajnert-Maculewicz, B. Cationic Phosphorus Dendrimer Enhances Photodynamic Activity of Rose Bengal against Basal Cell Carcinoma Cell Lines. Mol. Pharm. 2017, 14 (5), 1821-1830.

(137) Kautzka, Z.; Clement, S.; Goldys, E. M.; Deng, W. Light-Triggered Liposomal Cargo Delivery Platform Incorporating Photosensitizers and Gold Nanoparticles for Enhanced Singlet Oxygen Generation and Increased Cytotoxicity. Int. J. Nanomedicine 2017, 12, 969-977.

(138) Cao, H.; Wang, L.; Yang, Y.; Li, J.; Qi, Y.; Li, Y.; Li, Y.; Wang, H.; Li, J. An Assembled Nanocomplex for Improving Both Therapeutic Efficiency and Treatment Depth in Photodynamic Therapy. Angew. Chemie - Int. Ed. 2018, 57 (26), 7759-7763.

(139) Sabri, T.; Pawelek, P. D.; Capobianco, J. A. Dual Activity of Rose Bengal Functionalized to Albumin-Coated Lanthanide-Doped Upconverting Nanoparticles: Targeting and Photodynamic Therapy. ACS Appl. Mater. Interfaces 2018, 10 (32), 26947-26953.

(140) Sun, X.; Ji, Z.; He, S. SHG-Enhanced NIR-Excited in Vitro Photodynamic Therapy Using Composite Nanoparticles of Barium Titanate and Rose Bengal. RSC Adv. 2019, 9 (14), 8056-8064.

(141) Zhang, X.; Li, L.; Liu, Q.; Wang, Y.; Yang, J.; Qiu, T.; Zhou, G. Co-Delivery of Rose Bengal and Doxorubicin Nanoparticles for Combination Photodynamic and Chemo-Therapy. J. Biomed. Nanotechnol. 2019, 15 (1), 184-195.

(142) Srivastav, A. K.; Mujtaba, S. F.; Dwivedi, A.; Amar, S. K.; Goyal, S.; Verma, A.; Kushwaha, H. N.; Chaturvedi, R. K.; Ray, R. S. Photosensitized Rose Bengal-Induced Phototoxicity on Human Melanoma Cell Line under Natural Sunlight Exposure. J. Photochem. Photobiol. B Biol. 2016, 156, 87-99. 
(143) Song, L.; Li, C.; Zou, Y.; Dai, F.; Luo, X.; Wang, B.; Ni, J.; Liu, Q. O2 and Ca2+ Fluxes as Indicators of Apoptosis Induced by Rose Bengal-Mediated Photodynamic Therapy in Human Oral Squamous Carcinoma Cells. Photomed. Laser Surg. 2015, 33 (5), 258265.

(144) Cho, K. S.; Lee, E. H.; Choi, J. S.; Joo, C. K. Reactive Oxygen Species-Induced Apoptosis and Necrosis in Bovine Corneal Endothelial Cells. Investig. Ophthalmol. Vis. Sci. 1999, 40 (5), 911-919.

(145) Chan, W.-H.; Wu, H.-J. Anti-Apoptotic Effects of Curcumin on Photosensitized Human Epidermal Carcinoma A431 Cells. J. Cell. Biochem. 2004, 92 (1), 200-212.

(146) Panzarini, E.; Inguscio, V.; Dini, L. Overview of Cell Death Mechanisms Induced by Rose Bengal Acetate-Photodynamic Therapy. Int. J. Photoenergy 2011, 713726

(147) Panzarini, E.; Inguscio, V.; Dini, L. Timing the Multiple Cell Death Pathways Initiated by Rose Bengal Acetate Photodynamic Therapy. Cell Death Dis. 2011, 2, e169.

(148) Kochevar, I. E.; Bouvier, J.; Lynch, M.; Lin, C.-W. Influence of Dye and Protein Location on Photosensitization of the Plasma Membrane. Biochim. Biophys. Acta 1994, 1196 (2), 172-180.

(149) Valenzeno, D. P.; Arriaga, E.; Trank, J.; Tarr, M. Membrane Potential Can Influence the Rate of Membrane Photomodification Photochem. Photobiol. 1993, 57 (6), 996-999.

(150) Arnau-del-Valle, C.; Felip-León, C.; Angulo-Pachón, C. A.; Galindo, F.; Miravet, J. F. Adsorption of Rose Bengal on a SelfAssembled Fibrillar Network Affords a Thermally Switchable Oxygenation Photocatalyst and a Thermochromic Soft Material. J. Photochem. Photobiol. A Chem. 2020, 387, 112142

(151) Arbeloa, E. M.; Previtali, C. M.; Bertolotti, S. G. A Comparative Study on the Photophysics and Photochemistry of Xanthene Dyes in the Presence of Polyamidoamine (PAMAM) Dendrimers. ChemPhysChem 2018, 19 (8), 934-942.

(152) Bhowmik, B. B.; Ganguly, P. Photophysics of Xanthene Dyes in Surfactant Solution. Spectrochim. Acta - Part A Mol. Biomol. Spectrosc. 2005, 61 (9), 1997-2003.

(153) Rauf, M. A.; Graham, J. P.; Bukallah, S. B.; Al-Saedi, M. A. S. Solvatochromic Behavior on the Absorption and Fluorescence Spectra of Rose Bengal Dye in Various Solvents. Spectrochim. Acta - Part A 2009, 72, 133-137.

(154) Rodríguez, H. B.; Lagorio, M. G.; San Román, E. Rose Bengal Adsorbed on Microgranular Cellulose: Evidence on Fluorescent Dimers. Photochem. Photobiol. Sci. 2004, 3 (7), 674-680.

(155) Daraio, M. E.; San Román, E. Aggregation and Photophysics of Rose Bengal in Alumina-Coated Colloidal Suspensions. Helv. Chim. Acta 2001, 84 (9), 2601-2614.

(156) Mirenda, M.; Dicelio, L. E.; San Román, E. Effect of Molecular Interactions on the Photophysics of Rose Bengal in Polyelectrolyte Solutions and Self-Assembled Thin Films. J. Phys. Chem. B 2008, 112 (39), 12201-12207.

(157) Hussain, S. A.; Dey, D.; Bhattacharjee, D. Layer-by-Layer Self-Assembled Films of Rose Bengal. Int. J. Mod. Phys. B 2011, 25 (29), 4039-4046.

(158) Valdes-Aguilera, O.; Neckers, D. C. Aggregation of Rose Bengal Ethyl Ester Induced by Alkali Metal Cations in Aqueous Solution J. Photochem. Photobiol. A Chem. 1989, 47 (2), 213-222.

(159) Rohatgi, K. K.; Mukhopadhyay, A. K. Isolation of Unique Dimer Spectra of Dyes From the Composite Spectra of Aggregated Solutions. Photochem. Photobiol. 1971, 14 (4), 551-559.

(160) Valdes-Aguilera, O.; Neckers, D. C. Rose Bengal Ethyl Ester Aggregation in Aqueous Solution. J. Phys. Chem. 1988, 92 (15), 4286-4289.

(161) Xu, D.; Neckers, D. C. Aggregation of Rose Bengal Molecules in Solution. J. Photochem. Photobiol. A Chem. 1987, 40, 361-370.

(162) Chen, Y.; Urano, T.; Karatsu, T.; Takahara, S.; Yamaoka, T.; Tokumaru, K. Effect of Aggregates on the Photochemical Behavior of Rose Bengal Peroxybenzoate Initiator in PMMA Film. J. Chem. Soc. Perkin Trans. 2 1998, No. 10, 2233-2237.

(163) Martínez-Izquierdo, M. E.; Durand-Alegría, J. S.; Cabrera-Martín, A.; Gallego-Andreu, R. Spectrophotometric and Spectrofluorimetric Study of Rose Bengal B and Its Reaction with Platinum(IV). Analyst 1984, 109 (3), 377-379.

(164) Islam, S. D. M.; Ito, O. Solvent Effects on Rates of Photochemical Reactions of Rose Bengal Triplet State Studied by Nanosecond Laser Photolysis. J. Photochem. Photobiol. A Chem. 1999, 123, 53-59.

(165) Buchner, M.; García Calavia, P.; Muhr, V.; Kröninger, A.; Baeumner, A. J.; Hirsch, T.; Russell, D. A.; Marín, M. J. Photosensitiser Functionalised Luminescent Upconverting Nanoparticles for Efficient Photodynamic Therapy of Breast Cancer Cells. Photochem. Photobiol. Sci. 2019, 18, 98-109.

(166) Wang, M.; Huang, L.; Sharma, S. K.; Jeon, S.; Thota, S.; Sperandio, F. F.; Nayka, S.; Chang, J.; Hamblin, M. R.; Chiang, L. Y. Synthesis and Photodynamic Effect of New Highly Photostable Decacationically Armed [60]- and [70]Fullerene Decaiodide Monoadducts to Target Pathogenic Bacteria and Cancer Cells. J. Med. Chem. 2012, 55 (9), 4274-4285.

(167) Felip-León, C.; Puche, M.; Miravet, J. F.; Galindo, F.; Feliz, M. A Spectroscopic Study to Assess the Photogeneration of Singlet Oxygen by Graphene Oxide. Mater. Lett. 2019, 251, 45-51.

(168) Beltrán, A.; Mikhailov, M.; Sokolov, M. N.; Pérez-Laguna, V.; Rezusta, A.; Revillo, M. J.; Galindo, F. A Photobleaching Resistant Polymer Supported Hexanuclear Molybdenum lodide Cluster for Photocatalytic Oxygenations and Photodynamic Inactivation of Staphylococcus Aureus. J. Mater. Chem. B 2016, 4 (36), 5975-5979.

(169) Hackbarth, S.; Röder, B. Singlet Oxygen Luminescence Kinetics in a Heterogeneous Environment - Identification of the Photosensitizer Localization in Small Unilamellar Vesicles. Photochem. Photobiol. Sci. 2015, 14, 329-334.

(170) Abrahamse, H.; Hamblin, M. New Photosensitizers for Photodynamic Therapy. Biochem J 2016, 473 (4), $347-364$ 
(171) Theodossiou, T. A.; Hothersall, J. S.; De Witte, P. A.; Pantos, A.; Agostinis, P. The Multifaceted Photocytotoxic Profile of Hypericin. Mol. Pharm. 2009, 6 (6), 1775-1789.

(172) Lavie, G.; Mazur, Y.; Lavie, D.; Meruelo, D. The Chemical and Biological Properties of Hypericin - a Compound with a Broad Spectrum of Biological Activities. Med. Res. Rev. 1995, 15 (2), 111-119.

(173) Durán, N.; Song, P. S. Hypericin and Its Photodynamic Action. Photochem. Photobiol. 1986, 43 (6), 677-680.

(174) Schempp, C. M.; Winghofer, B.; Langheinrich, M.; Schöpf, E.; Simon, J. C. Hypericin Levels in Human Serum and Interstitial Skin Blister Fluid after Oral Single-Dose and Steady-State Administration of Hypericum Perforatum Extract (St. John's Wort). Skin Pharmacol. Appl. Skin Physiol. 1999, 12, 299-304.

(175) Schempp, C. M.; Müller, K.; Winghofer, B.; Schulte-Mönting, J.; Simon, J. C. Single-Dose and Steady-State Administration of Hypericum Perforatum Extract (St John's Wort) Does Not Influence Skin Sensitivity to UV Radiation, Visible Light, and SolarSimulated Radiation. Arch. Dermatol. 2001, 137 (4), 512-513.

(176) Yechiam, E.; Ben-Eliezer, D.; Ashby, N. J. S.; Bar-Shaked, M. The Acute Effect of Hypericum Perforatum on Short-Term Memory in Healthy Adults. Psychopharmacology (Berl). 2019, 236 (2), 613-623.

(177) Ehrenberg, B.; Anderson, J. L.; Foote, C. S. Kinetics and Yield of Singlet Oxygen Photosensitized by Hypericin in Organic and Biological Media. Photochem. Photobiol. 1998, 68 (2), 135-140.

(178) Diwu, Z.; Lown, J. W. Photosensitization with Anticancer Agents 17. EPR Studies of Photodynamic Action of Hypericin: Formation of Semiquinone Radical and Activated Oxygen Species on Illumination. Free Radic. Biol. Med. 1993, 14 (2), 209 215.

(179) Thomas, C.; Pardini, R. S. Oxygen Dependence of Hypericin-Induced Phototoxicity To EMT6 Mouse Mammary Carcinoma Cells. Photochem. Photobiol. 1992, 55 (6), 831-837.

(180) Vandenbogaerde, A. L.; Cuveele, J. F.; Proot, P.; Himpens, B. E.; Merlevede, W. J.; De Witte, P. A. Differential Cytotoxic Effects Induced after Photosensitization by Hypericin. J. Photochem. Photobiol. B Biol. 1997, 38, 136-142.

(181) Miadokova, E.; Chalupa, I.; Vlckova, V.; Sevcovicova, A.; Nadova, S.; Kopaskova, M.; Hercegova, A.; Gasperova, P.; Alfoldiova L.; Komjatiova, M.; Csanyiova, Z.; Galova, E.; Cellarova, E.; Vlcek, D. Genotoxicity and Antigenotoxicity Evaluation of NonPhotoactivated Hypericin. Phyther. Res. 2010, 24 (1), 90-95.

(182) Jendželovská, Z.; Jendželovský, R.; Hilovská, L.; Koval, J.; Mikeš, J.; Fedoročko, P. Single Pre-Treatment with Hypericin, a St. John's Wort Secondary Metabolite, Attenuates Cisplatin- and Mitoxantrone-Induced Cell Death in A2780, A2780cis and HL60 Cells. Toxicol. Vitr. 2014, 28 (7), 1259-1273.

(183) Feruszova, J.; Imreová, P.; Bodnárová, K.; Ševčovičová, A.; Kyzek, S.; Chalupa, I.; Gálová, E.; Miadoková, E. Photoactivated Hypericin Is Not Genotoxic. Gen. Physiol. Biophys. 2016, 35, 223-230.

(184) Kamuhabwa, A. A. R.; Cosserat-Gerardin, I.; Didelon, J.; Notter, D.; Guillemin, F.; Roskams, T.; D’Hallewin, M.-A.; Baert, L.; de Witte, P. A. M. Biodistribution of Hypericin in Orthotopic Transitional Cell Carcinoma Bladder Tumors: Implication for Whole Bladder Wall Photodynamic Therapy. Int. J. Cancer 2002, 97 (2), 253-260.

(185) Noell, S.; Mayer, D.; Strauss, W. S. L.; Tatagiba, M. S.; Ritz, R. Selective Enrichment of Hypericin in Malignant Glioma: Pioneering in Vivo Results. Int. J. Oncol. 2011, 38 (5), 1343-1348.

(186) Rezusta, A.; López-Chicón, P.; Paz-Cristobal, M. P.; Alemany-Ribes, M.; Royo-Díez, D.; Agut, M.; Semino, C.; Nonell, S.; Revillo, M. J.; Aspiroz, C.; Gilaberte, Y. In Vitro Fungicidal Photodynamic Effect of Hypericin on Candida Species. Photochem. Photobiol. 2012, 88 (3), 613-619.

(187) Paz-Cristobal, M. P.; Gilaberte, Y.; Alejandre, C.; Pardo, J.; Revillo, M. J.; Rezusta, A. In Vitro Fungicidal Photodynamic Effect of Hypericin on Trichophyton Spp. Mycopathologia 2014, 178, 221-225.

(188) Kashef, N.; Borghei, Y. S.; Djavid, G. E. Photodynamic Effect of Hypericin on the Microorganisms and Primary Human Fibroblasts. Photodiagnosis Photodyn. Ther. 2013, 10 (2), 150-155.

(189) García, I.; Ballesta, S.; Gilaberte, Y.; Rezusta, A.; Pascual, Á. Antimicrobial Photodynamic Activity of Hypericin against Methicillin-Susceptible and Resistant Staphylococcus Aureus Biofilms. Future Microbiol. 2015, 10 (3), 347-356.

(190) Hudson, J. B.; Harris, L.; Towers, G. H. N. The Importance of Light in the Anti-HIV Effect of Hypericin. Antiviral Res. 1993, 20 (2), 173-178.

(191) Prince, A. M.; Pascual, D.; Meruelo, D.; Liebes, L.; Mazur, Y.; Dubovi, E.; Mandel, M.; Lavie, G. Strategies for Evaluation of Enveloped Virus Inactivation in Red Cell Concentrates Using Hypericin. Photochem. Photobiol. 2000, 71 (2), $188-195$.

(192) Karioti, A.; Bilia, A. R. Hypericins as Potential Leads for New Therapeutics. Int. J. Mol. Sci. 2010, 11 (2), $562-594$.

(193) Saw, C. L. L.; Olivo, M.; Soo, K. C.; Heng, P. W. S. Delivery of Hypericin for Photodynamic Applications. Cancer Lett. 2006, 241, 23-30.

(194) Jendželovská, Z.; Jendželovský, R.; Kuchárová, B.; Fedoročko, P. Hypericin in the Light and in the Dark: Two Sides of the Same Coin. Front. Plant Sci. 2016, 7, 560.

(195) D’Hallewin, M. A.; Kamuhabwa, A. R.; Roskams, T.; De Witte, P. A. M.; Baert, L. Hypericin-Based Fluorescence Diagnosis of Bladder Carcinoma. BJU Int. 2002, 89, 760-763.

(196) Kubin, A.; Meissner, P.; Wierrani, F.; Burner, U.; Bodenteich, A.; Pytel, A.; Schmeller, N. Fluorescence Diagnosis of Bladder Cancer with New Water Soluble Hypericin Bound to Polyvinylpyrrolidone: PVP-Hypericin. Photochem. Photobiol. 2008, 84 (6), 1560-1563.

(197) Thong, P. S. P.; Olivo, M.; Chin, W. W. L.; Bhuvaneswari, R.; Mancer, K.; Soo, K. C. Clinical Application of Fluorescence Endoscopic Imaging Using Hypericin for the Diagnosis of Human Oral Cavity Lesions. Br. J. Cancer 2009, 101 (9), 1580-1584. 
(198) Abbaci, M.; Casiraghi, O.; Temam, S.; Ferchiou, M.; Bosq, J.; Dartigues, P.; De Leeuw, F.; Breuskin, I.; Laplace-Builhé, C. Red and Far-Red Fluorescent Dyes for the Characterization of Head and Neck Cancer at the Cellular Level. J. Oral Pathol. Med. 2015, 44 (10), 831-841.

(199) Ritz, R.; Daniels, R.; Noell, S.; Feigl, G. C.; Schmidt, V.; Bornemann, A.; Ramina, K.; Mayer, D.; Dietz, K.; Strauss, W. S. L.; Tatagiba, M. Hypericin for Visualization of High Grade Gliomas: First Clinical Experience. Eur. J. Surg. Oncol. 2012, 38 (4), 352360.

(200) Sattler, S.; Schaefer, U.; Schneider, W.; Hoelzl, J.; Lehr, C. M. Binding, Uptake, and Transport of Hypericin by Caco-2 Cell Monolayers. J. Pharm. Sci. 1997, 86 (10), 1120-1126.

(201) Siboni, G.; Weitman, H.; Freeman, D.; Mazur, Y.; Malik, Z.; Ehrenberg, B. The Correlation between Hydrophilicity of Hypericins and Helianthrone: Internalization Mechanisms, Subcellular Distribution and Photodynamic Action in Colon Carcinoma Cells. Photochem. Photobiol. Sci. 2002, 1 (7), 483-491.

(202) Weber, N. D.; Murray, B. K.; North, J. A.; Wood, S. G. The Antiviral Agent Hypericin Has in Vitro Activity against HSV-1 through Non-Specific Association with Viral and Cellular Membranes. Antivir. Chem. Chemother. 1994, 5, 83-90.

(203) English, D. S.; Doyle, R. T.; Petrich, J. W.; Haydon, P. G. Subcellular Distributions and Excited-State Processes of Hypericin in Neurons. Photochem. Photobiol. 1999, 69, 301-305.

(204) Ali, S. M.; Olivo, M. Bio-Distribution and Subcellular Localization of Hypericin and Its Role in PDT Induced Apoptosis in Cancer Cells. Int. J. Oncol. 2002, 21 (3), 531-540.

(205) Galanou, M. C.; Theodossiou, T. A.; Tsiourvas, D.; Sideratou, Z.; Paleos, C. M. Interactive Transport, Subcellular Relocation and Enhanced Phototoxicity of Hypericin Encapsulated in Guanidinylated Liposomes via Molecular Recognition. Photochem. Photobiol. 2008, 84 (5), 1073-1083.

(206) Mikeš, J.; Hýzalová, M.; Kocí, L.; Jendzelovský, R.; Koval, J.; Vaculová, A.; Hofmanová, J.; Kozubík, A.; Fedorocko, P. Lower Sensitivity of FHC Fetal Colon Epithelial Cells to Photodynamic Therapy Compared to HT-29 Colon Adenocarcinoma Cells despite Higher Intracellular Accumulation of Hypericin. Photochem. Photobiol. Sci. 2011, 10 (4), 626-632.

(207) Vantieghem, A.; Xu, Y.; Declercq, W.; Vandenabeele, P.; Denecker, G.; Vandenheede, J. R.; Merlevede, W.; De Witte, P. A.; Agostinis, P. Different Pathways Mediate Cytochrome c Release After Photodynamic Therapy with Hypericin. Photochem. Photobiol. 2001, 74 (2), 133-142.

(208) Hadjur, C.; Richard, M. J.; Parat, M. O.; Jardon, P.; Favier, A. Photodynamic Effects of Hypericin on Lipid Peroxidation and Antioxidant Status in Melanoma Cells. Photochem. Photobiol. 1996, 64 (2), 375-381.

(209) Mikešová, L.; Mikeš, J.; Koval', J.; Gyurászová, K.; Čulka, L.; Vargová, J.; Valeková, B.; Fedoročko, P. Conjunction of Glutathione Level, NAD(P)H/FAD Redox Status and Hypericin Content as a Potential Factor Affecting Colon Cancer Cell Resistance to Photodynamic Therapy with Hypericin. Photodiagnosis Photodyn. Ther. 2013, 10 (4), 470-483.

(210) Mikeš, J.; Jendželovský, R.; Fedoročko, P. Cellular Aspects of Photodynamic Therapy with Hypericin. In Photodynamic Therapy: New Research; Elsaie, M. L. T., Ed.; Nova Science Publishers: New York, NY, 2013; pp 111-147.

(211) Garg, A. D.; Agostinis, P. ER Stress, Autophagy and Immunogenic Cell Death in Photodynamic Therapy-Induced Anti-Cancer Immune Responses. Photochem. Photobiol. Sci. 2014, 13 (3), 474-487.

(212) Du, H.-Y.; Olivo, M.; Tan, B. K.-H.; Bay, B.-H. Hypericin-Mediated Photodynamic Therapy Induces Lipid Peroxidation and Necrosis in Nasopharyngeal Cancer. Int. J. Oncol. 2003, 23 (5), 1401-1405.

(213) Mikeš, J.; Kleban, J.; Sačková, V.; Horváth, V.; Jamborová, E.; Vaculová, A.; Kozubík, A.; Hofmanová, J.; Fedoročko, P. Necrosis Predominates in the Cell Death of Human Colon Adenocarcinoma HT-29 Cells Treated under Variable Conditions of Photodynamic Therapy with Hypericin. Photochem. Photobiol. Sci. 2007, 6, 758-766.

(214) Mikeš, J.; Koval', J.; Jendelovský, R.; Saková, V.; Uhrinová, I.; Kello, M.; Kuliková, L.; Fedoroĉko, P. The Role of P53 in the Efficiency of Photodynamic Therapy with Hypericin and Subsequent Long-Term Survival of Colon Cancer Cells. Photochem. Photobiol. Sci. 2009, 8, 1558-1567.

(215) Buytaert, E.; Callewaert, G.; Hendrickx, N.; Scorrano, L.; Hartmann, D.; Missiaen, L.; Vandenheede, J. R.; Heirman, I.; Grooten, J.; Agostinis, P. Role of Endoplasmic Reticulum Depletion and Multidomain Proapoptotic BAX and BAK Proteins in Shaping Cell Death after Hypericin-Mediated Photodynamic Therapy. FASEB J. 2006, 20 (6), 756-758.

(216) Rubio, N.; Coupienne, I.; Di Valentin, E.; Heirman, I.; Grooten, J.; Piette, J.; Agostinis, P. Spatiotemporal Autophagic Degradation of Oxidatively Damaged Organelles after Photodynamic Stress Is Amplified by Mitochondrial Reactive Oxygen Species. Autophagy 2012, 8 (9), 1312-1324.

(217) Garg, A. D.; Krysko, D. V.; Verfaillie, T.; Kaczmarek, A.; Ferreira, G. B.; Marysael, T.; Rubio, N.; Firczuk, M.; Mathieu, C.; Roebroek, A. J. M.; Annaert, W.; Golab, J.; De Witte, P.; Vandenabeele, P.; Agostinis, P. A Novel Pathway Combining Calreticulin Exposure and ATP Secretion in Immunogenic Cancer Cell Death. EMBO J. 2012, 31 (5), 1062-1079.

(218) Xie, X.; Hudson, J. B.; Guns, E. S. Tumor-Specific and Photodependent Cytotoxicity of Hypericin in the Human LNCaP Prostate Tumor Model. Photochem. Photobiol. 2001, 74 (2), 221-225.

(219) Head, C. S.; Luu, Q.; Sercarz, J.; Saxton, R. Photodynamic Therapy and Tumor Imaging of Hypericin-Treated Squamous Cell Carcinoma. World J. Surg. Oncol. 2006, 4, 87

(220) Sačková, V.; Fedoročko, P.; Szilárdiová, B.; Mikeš, J.; Kleban, J. Hypericin-Induced Photocytotoxicity Is Connected with G2/M Arrest in HT-29 and S-Phase Arrest in U937 Cells. Photochem. Photobiol. 2006, 82, 1285-1291.

(221) Koval', J.; Mikeš, J.; Jendželovský, R.; Kello, M.; Solár, P.; Fedoročko, P. Degradation of HER2 Receptor through HypericinMediated Photodynamic Therapy. Photochem. Photobiol. 2010, 86, 200-205. 
(222) Kleemann, B.; Loos, B.; Scriba, T. J.; Lang, D.; Davids, L. M. St John's Wort (Hypericum Perforatum L.) Photomedicine: Hypericin-Photodynamic Therapy Induces Metastatic Melanoma Cell Death. PLoS One 2014, 9 (7), e103762.

(223) Vandenbogaerde, A. L.; Geboes, K. R.; Cuveele, J. F.; Agostinis, P. M.; Merlevede, W. J.; De Witte, P. A. Antitumour Activity of Photosensitized Hypericin on A431 Cell Xenografts. Anticancer Res. 1996, 16, 1619-1625.

(224) Thong, P. S. P.; Watt, F.; Ren, M. Q.; Tan, P. H.; Soo, K. C.; Olivo, M. Hypericin-Photodynamic Therapy (PDT) Using an Alternative Treatment Regime Suitable for Multi-Fraction PDT. J. Photochem. Photobiol. B Biol. 2006, 82, 1-8.

(225) Bhuvaneswari, R.; Gan, Y. K.; Lucky, S. S.; Chin, W. W. L.; Ali, S. M.; Soo, K. C.; Olivo, M. Molecular Profiling of Angiogenesis in Hypericin Mediated Photodynamic Therapy. Mol. Cancer 2008, 7, 56.

(226) Liu, C. D.; Kwan, D.; Saxton, R. E.; McFadden, D. W. Hypericin and Photodynamic Therapy Decreases Human Pancreatic Cancer in Vitro and in Vivo. J. Surg. Res. 2000, 93, 137-143.

(227) Alecu, M.; Ursaciuc, C.; Hãlãlãu, F.; Coman, G.; Merlevede, W.; Waelkens, E.; de Witte, P. Photodynamic Treatment of Basal Cell Carcinoma and Squamous Cell Carcinoma with Hypericin. Anticancer Res. 1998, 18, 4651-4654.

(228) Kacerovská, D.; Pizinger, K.; Majer, F.; Šmíd, F. Photodynamic Therapy of Nonmelanoma Skin Cancer with Topical Hypericum Perforatum Extract - A Pilot Study. Photochem. Photobiol. 2008, 84, 779-785.

(229) Rook, A. H.; Wood, G. S.; Duvic, M.; Vonderheid, E. C.; Tobia, A.; Cabana, B. A Phase II Placebo-Controlled Study of Photodynamic Therapy with Topical Hypericin and Visible Light Irradiation in the Treatment of Cutaneous T-Cell Lymphoma and Psoriasis. J. Am. Acad. Dermatol. 2010, 63 (6), 984-990.

(230) Wynn, J. L.; Cotton, T. M. Spectroscopic Properties of Hypericin in Solution and at Surfaces. J. Phys. Chem. 1995, 99 (12), 43174323.

(231) Bánó, G.; Staničová, J.; Jancura, D.; Marek, J.; Bánó, M.; Uličný, J.; Strejčková, A.; Miškovský, P. On the Diffusion of Hype ricin in Dimethylsulfoxide/Water Mixtures-the Effect of Aggregation. J. Phys. Chem. B 2011, 115 (10), 2417-2423.

(232) Dumas, S.; Leprêtre, J. C.; Lepellec, A.; Darmanyan, A.; Jardon, P. Reactivity of the Photo Excited Forms of Hypericin, Hypocrellin A, Hypocrellin B and Methylated Hypericin towards Molecular Oxygen - The Role of Charge Transfer Interaction. J. Photochem. Photobiol. A Chem. 2004, 163 (3), 297-306.

(233) Arabei, S. M.; Pavich, T. A.; Galaup, J. P.; Jardon, P. Influence of the Nature of Sol-Gel Matrices on Absorption and FineStructure Fluorescence Spectra of Hypericin. Chem. Phys. Lett. 1999, 306, 303-313.

(234) Falk, H. From the Photosensitizer Hypericin to the Photoreceptor Stentorin - The Chemistry of Phenanthroperylene Quinones. Angew. Chem. Int. Ed. 1999, 38, 3116-3136.

(235) Lajos, G.; Jancura, D.; Miskovsky, P.; García-Ramos, J. V.; Sanchez-Cortes, S. Surface-Enhanced Fluorescence and Raman Scattering Study of Antitumoral Drug Hypericin: An Effect of Aggregation and Self-Spacing Depending on PH. J. Phys. Chem. C 2008, 112, 12974-12980.

(236) Kapinus, E. I.; Falk, H.; Tran, H. T. N. Spectroscopic Investigation of the Molecular Structure of Hypericin and Its Salts. Monatshefte für Chemie 1999, 130 (5), 623-635.

(237) Liu, X.; Jiang, C.; Li, Y.; Liu, W.; Yao, N.; Gao, M.; Ji, Y.; Huang, D.; Yin, Z.; Sun, Z.; Ni, Y.; Zhang, J. Evaluation of Hypericin: Effect of Aggregation on Targeting Biodistribution. J. Pharm. Sci. 2015, 104, 215-222.

(238) Falk, H.; Meyer, J. On the Homo- and Heteroassociation of Hypericin. Monatshefte für Chemie 1994, 125 (6), $753-762$.

(239) Sevenants, M. Pigments of Blepharisma Undulans Compared with Hypericin. J. Protozool. 1965, 12 (2), $240-245$.

(240) Yamazaki, T.; Ohta, N.; Yamazaki, I.; Song, P. S. Excited-State Properties of Hypericin: Electronic Spectra and Fluorescence Decay Kinetics. J. Phys. Chem. 1993, 97 (30), 7870-7875.

(241) Keša, P.; Antalík, M. Determination of PKa Constants of Hypericin in Aqueous Solution of the Anti-Allergic Hydrotropic Drug Cromolyn Disodium Salt. Chem. Phys. Lett. 2017, 676, 112-117.

(242) Gai, F.; Fehr, M. J.; Petrich, J. W. Observation of Excited-State Tautomerization in the Antiviral Agent Hypericin and Identification of Its Fluorescent Species. J. Phys. Chem. 1994, 98 (22), 5784-5795.

(243) Van De Putte, M.; Roskams, T.; Bormans, G.; Verbruggen, A.; De Witte, P. A. M. The Impact of Aggregation on the Biodistribution of Hypericin. Int. J. Oncol. 2006, 28 (3), 655-660.

(244) Kascakova, S.; Refregiers, M.; Jancura, D.; Sureau, F.; Maurizot, J.-C.; Miskovsky, P. Fluorescence Spectroscopic Study of Hypericin-Photosensitized Oxidation of Low-Density Lipoproteins. Photochem. Photobiol. 2005, 81, 1395-1403.

(245) Theodossiou, T.; Spiro, M. D.; Jacobson, J.; Hothersall, J. S.; MacRobert, A. J. Evidence for Intracellular Aggregation of Hypericin and the Impact on Its Photocytotoxicity in PAM 212 Murine Keratinocytes. Photochem. Photobiol. 2004, 80, 438-443.

(246) Nakajima, N.; Kawashima, N. A Basic Study on Hypericin-PDT in Vitro. Photodiagnosis Photodyn. Ther. 2012, 9, 196-203.

(247) Nafee, N.; Youssef, A.; El-Gowelli, H.; Asem, H.; Kandil, S. Antibiotic-Free Nanotherapeutics: Hypericin Nanoparticles Thereof for Improved in Vitro and in Vivo Antimicrobial Photodynamic Therapy and Wound Healing. Int. J. Pharm. 2013, 454 (1), 249258.

(248) Zeisser-Labouèbe, M.; Delie, F.; Gurny, R.; Lange, N. Benefits of Nanoencapsulation for the Hypercin-Mediated Photodetection of Ovarian Micrometastases. Eur. J. Pharm. Biopharm. 2009, 71, 207-213.

(249) Huygens, A.; Kamuhabwa, A. R.; De Witte, P. A. M. Stability of Different Formulations and Ion Pairs of Hypericin. Eur. J. Pharm. Biopharm. 2005, 59, 461-468.

(250) Kamuhabwa, A. R.; Geboes, K. R.; De Witte, P. A. Investigation of the Absorption of Hypericin into the Skin of Hairless Mice. J. Pharm. Pharmacol. 2000, 52, 487-494. 
(251) Derycke, A. S. L.; De Witte, P. A. M. Transferrin-Mediated Targeting of Hypericin Embedded in Sterically Stabilized PEGLiposomes. Int. J. Oncol. 2002, 20, 181-187.

(252) Zeisser-Labouèbe, M.; Mattiuzzo, M.; Lange, N.; Gurny, R.; Delie, F. Quenching-Induced Deactivation of Photosensitizer by Nanoencapsulation to Improve Phototherapy of Cancer. J. Drug Target. 2009, 17 (8), 619-626.

(253) Fadel, M.; Kassab, K.; Youssef, T. Photodynamic Efficacy of Hypericin Targeted by Two Delivery Techniques to Hepatocellular Carcinoma Cells. Lasers Med. Sci. 2010, 25, 675-683.

(254) Mondon, K.; Zeisser-Labouèbe, M.; Gurny, R.; Möller, M. MPEG-HexPLA Micelles as Novel Carriers for Hypericin, a Fluorescent Marker for Use in Cancer Diagnostics. Photochem. Photobiol. 2011, 87, 399-407.

(255) Huntosova, V.; Buzova, D.; Petrovajova, D.; Kasak, P.; Nadova, Z.; Jancura, D.; Sureau, F.; Miskovsky, P. Development of a New LDL-Based Transport System for Hydrophobic/Amphiphilic Drug Delivery to Cancer Cells. Int. J. Pharm. 2012, 436, $463-471$.

(256) Youssef, T.; Fadel, M.; Fahmy, R. H.; Kassab, K. Evaluation of Hypericin-Loaded Solid Lipid Nanoparticles: Physicochemical Properties, Photostability and Phototoxicity. Pharm. Dev. Technol. 2012, 17 (2), 177-186.

(257) Yang, X.; Xiao, Q.; Niu, C.; Jin, N.; Ouyang, J.; Xiao, X.; He, D. Multifunctional Core-Shell Upconversion Nanoparticles for Targeted Tumor Cells Induced by near-Infrared Light. J. Mater. Chem. B 2013, 1, 2757-2763.

(258) Barras, A.; Boussekey, L.; Courtade, E.; Boukherroub, R. Hypericin-Loaded Lipid Nanocapsules for Photodynamic Cancer Therapy in Vitro. Nanoscale 2013, 5, 10562-10572.

(259) Lima, A. M.; Pizzol, C. D.; Monteiro, F. B. F.; Creczynski-Pasa, T. B.; Andrade, G. P.; Ribeiro, A. O.; Perussi, J. R. Hypericin Encapsulated in Solid Lipid Nanoparticles: Phototoxicity and Photodynamic Efficiency. J. Photochem. Photobiol. B Biol. 2013, $125,146-154$

(260) Unterweger, H.; Subatzus, D.; Tietze, R.; Janko, C.; Poettler, M.; Stiegelschmitt, A.; Schuster, M.; Maake, C.; Boccaccini, A. R.; Alexiou, C. Hypericin-Bearing Magnetic Iron Oxide Nanoparticles for Selective Drug Delivery in Photodynamic Therapy. Int. J. Nanomedicine 2015, 10, 6985-6996.

(261) Penjweini, R.; Deville, S.; D’Olieslaeger, L.; Berden, M.; Ameloot, M.; Ethirajan, A. Intracellular Localization and Dynamics of Hypericin Loaded PLLA Nanocarriers by Image Correlation Spectroscopy. J. Control. Release 2015, 218, 82-93.

(262) Li, Y.; Zhang, J.; Wang, B.; Shen, Y.; Ouahab, A. Co-Delivery of SiRNA and Hypericin into Cancer Cells by Hyaluronic Acid Modified PLGA-PEI Nanoparticles. Drug Dev. Ind. Pharm. 2016, 42 (5), 737-746.

(263) Cheng, W.; Fan, F.; Zhang, Y.; Pei, Z.; Wang, W.; Pei, Y. A Facile Approach for Fabrication of Core-Shell Magnetic Molecularly Imprinted Nanospheres towards Hypericin. Polymers (Basel). 2017, 9 (4).

(264) Lopera, A. A.; Montoya, A.; Vélez, I. D.; Robledo, S. M.; Garcia, C. P. Synthesis of Calcium Phosphate Nanostructures by Combustion in Solution as a Potential Encapsulant System of Drugs with Photodynamic Properties for the Treatment of Cutaneous Leishmaniasis. Photodiagnosis Photodyn. Ther. 2018, 21, 138-146.

(265) Barras, A.; Skandrani, N.; Gonzalez Pisfil, M.; Paryzhak, S.; Dumych, T.; Haustrate, A.; Héliot, L.; Gharbi, T.; Boulahdour, H.; Lehen'kyi, V.; Bilyy, R.; Szunerits, S.; Bidaux, G.; Boukherroub, R. Improved Photodynamic Effect through Encapsulation of Two Photosensitizers in Lipid Nanocapsules. J. Mater. Chem. B 2018, 6 (37), 5949-5963.

(266) Plenagl, N.; Seitz, B. S.; Pinnapireddy, S. R.; Jedelská, J.; Brüßler, J.; Bakowsky, U. Hypericin Loaded Liposomes for AntiMicrobial Photodynamic Therapy of Gram-Positive Bacteria. Phys. Status Solidi Appl. Mater. Sci. 2018, 1700837.

(267) Plenagl, N.; Seitz, B. S.; Duse, L.; Pinnapireddy, S. R.; Jedelska, J.; Brüßler, J.; Bakowsky, U. Hypericin Inclusion Complexes Encapsulated in Liposomes for Antimicrobial Photodynamic Therapy. Int. J. Pharm. 2019, 570, 118666

(268) de Morais, F. A. P.; Gonçalves, R. S.; Vilsinski, B. H.; de Oliveira, É. L.; Rocha, N. L.; Hioka, N.; Caetano, W. Hypericin Photodynamic Activity in DPPC Liposome. PART I: Biomimetism of Loading, Location, Interactions and Thermodynamic Properties. J. Photochem. Photobiol. B Biol. 2019, 190, 118-127.

(269) Plenagl, N.; Duse, L.; Seitz, B. S.; Goergen, N.; Pinnapireddy, S. R.; Jedelska, J.; Brüßler, J.; Bakowsky, U. Photodynamic Therapy-Hypericin Tetraether Liposome Conjugates and Their Antitumor and Antiangiogenic Activity. Drug Deliv. 2019, 26 (1), 23-33.

(270) Joniova, J.; Rebič, M.; Strejčková, A.; Huntosova, V.; Staničová, J.; Jancura, D.; Miskovsky, P.; Bánó, G. Formation of Large Hypericin Aggregates in Giant Unilamellar Vesicles-Experiments and Modeling. Biophys. J. 2017, 112 (5), 966-975.

(271) Brown, R. B.; Audet, J. Current Techniques for Single-Cell Lysis. J. R. Soc. Interface 2008, 5, S131-S138.

(272) Shapiro, H. M. Practical Flow Cytometry; Alan R. Liss, Inc.: New York, 1985.

(273) Flow Cytometry Fundamentals, Molecular Probes School of Fluorescence, Invitrogen by Thermo Fisher Scientifics. https://www.thermofisher.com/es/es/home/life-science/cell-analysis/cell-analysis-learning-center/molecular-probesschool-of-fluorescence/flow-cytometry-basics/flow-cytometry-fundamentals/how-flow-cytometer-works.html (accessed Mar 23, 2020)

(274) Flow Cytometry Fundamental Principle, How FACS Works, Boster. https://www.bosterbio.com/protocol-andtroubleshooting/flow-cytometry-principle (accessed Mar 23, 2020)

(275) Technical Bulletin : Multiple Methods for Detecting Apoptosis on the BD Accuri ${ }^{\text {TM }}$ C6 Flow Cytometer; 2012.

(276) Van Engeland, M.; Nieland, L. J. W.; Ramaekers, F. C. S.; Schutte, B.; Reutelingsperger, C. P. M. Annexin V-Affinity Assay: A Review on an Apoptosis Detection System Based on Phosphatidylserine Exposure. Cytometry 1998, 31, 1-9.

(277) Hama, Y.; Urano, Y.; Koyama, Y.; Bernardo, M.; Choyke, P.; Kobayashi, H. A Comparison of the Emission Efficiency of Four Common Green Fluorescence Dyes after Internalization into Cancer Cells. Bioconjug. Chem. 2006, 17 (6), 1426-1431. 
(278) Martin, S. J.; Reutelingsperger, C. P. M.; McGahon, A. J.; Rader, J. A.; Van Schie, R. C. A. A.; LaFace, D. M.; Green, D. R. Early Redistribution of Plasma Membrane Phosphatidylserine Is a General Feature of Apoptosis Regardless of the Initiating Stimulus: Inhibition by Overexpression of BCL-2 and Abl. J. Exp. Med. 1995, 182, 1545-1556.

(279) Wlodkowic, D.; Skommer, J.; Darzynkiewicz, Z. Flow Cytometry-Based Apoptosis Detection. Methods Mol. Biol. 2009, 559, 1932.

(280) Glisic-Milosavljevic, S.; Waukau, J.; Jana, S.; Jailwala, P.; Rovensky, J.; Ghosh, S. Comparison of Apoptosis and Mortality Measurements in Peripheral Blood Mononuclear Cells (PBMCs) Using Multiple Methods. Cell Prolif. 2005, 38, 301-311.

(281) Healy, E.; Dempsey, M.; Lally, C.; Ryan, M. P. Apoptosis and Necrosis: Mechanisms of Cell Death Induced by Cyclosporine A in a Renal Proximal Tubular Cell Line. Kidney Int. 1998, 54, 1955-1966.

(282) Wlodkowic, D.; Telford, W.; Skommer, J.; Darzynkiewicz, Z. Apoptosis and Beyond: Cytometry in Studies of Programmed Cell Death. Methods Cell Biol. 2011, 103, 55-98.

(283) Breitenbach, T.; Kuimova, M. K.; Gbur, P.; Hatz, S.; Schack, N. B.; Pedersen, B. W.; Lambert, J. D. C.; Poulsen, L.; Ogilby, P. R. Photosensitized Production of Singlet Oxygen: Spatially-Resolved Optical Studies in Single Cells. Photochem. Photobiol. Sci. 2009, 8 (4), 442-452.

(284) MolecularProbes; Invitrogen. Protocol of Membrane Permeability / Dead Cell Apoptosis Kit with YO-PRO ${ }^{-1}$ and PI for Flow Cytometry (Catalog No. V13243); 2010.

(285) Daly, J. M.; Jannot, C. B.; Beerli, R. R.; Graus-Porta, D.; Maurer, F. G.; Hynes, N. E. Neu Differentiation Factor Induces ErbB2 Down-Regulation and Apoptosis of ErbB2-Overexpressing Breast Tumor Cells. Cancer Res. 1997, 57, 3804-3811.

(286) Fish, K. N.; Schmid, S. L.; Damke, H. Evidence That Dynamin-2 Functions as a Signal-Transducing GTPase. J. Cell Biol. 2000, 150, 145-154.

(287) Idziorek, T.; Estaquier, J.; De Bels, F.; Ameisen, J. C. YOPRO-1 Permits Cytofluorometric Analysis of Programmed Cell Death (Apoptosis) without Interfering with Cell Viability. J. Immunol. Methods 1995, 185, 249-258.

(288) Estaquier, J.; Tanaka, M.; Suda, T.; Nagata, S.; Golstein, P.; Ameisen, J. C. Fas-Mediated Apoptosis of CD4+ and CD8+ T Cells from Human Immunodeficiency Virus-Infected Persons: Differential in Vitro Preventive Effect of Cytokines and Protease Antagonists. Blood 1996, 87 (12), 4959-4966.

(289) Estaquier, J.; Idziorek, T.; Zou, W.; Emilie, D.; Farber, C.; Bourez, J.; Ameisen, J. C. T Helper Type 1/T Helper Type 2 Cytokines and T Cell Death: Preventive Effect of Interleukin 12 on Activation-Induced and CD95 (FAS/APO-1)-Mediated Apoptosis of CD4+ T Cells from Human Immunodeficiency Virus-Infected Persons. J. Exp. Med. 1995, 182, 1759-1767.

(290) Fujisawa, S.; Romin, Y.; Barlas, A.; Petrovic, L. M.; Turkekul, M.; Fan, N.; Xu, K.; Garcia, A. R.; Monette, S.; Klimstra, D. S.; Erinjeri, J. P.; Solomon, S. B.; Manova-Todorova, K.; Sofocleous, C. T. Evaluation of YO-PRO-1 as an Early Marker of Apoptosis Following Radiofrequency Ablation of Colon Cancer Liver Metastases. Cytotechnology 2014, 66, 259-273.

(291) Matsuzaki, T.; Suzuki, T.; Fujikura, K.; Takata, K. Nuclear Staining for Laser Confocal Microscopy. Acta Histochem. Cytochem. 1997, 30 (3), 309-314

(292) Irminger-Finger, I.; Soriano, J. V.; Vaudan, G.; Montesano, R.; Sappino, A. P. In Vitro Repression of Brca1-Associated RING Domain Gene, Bard1, Induces Phenotypic Changes in Mammary Epithelial Cells. J. Cell Biol. 1998, 143 (5), 1329-1339.

(293) Suzuki, T.; Matsuzaki, T.; Takata, K. Fluorescence Counter-Staining of Cell Nuclear DNA for Multi-Color Laser Confocal Microscopy. Acta Histochem. Cytochem. 1998, 31 (4), 297-301.

(294) Coriat, R.; Marut, W.; Leconte, M.; Ba, L. B.; Vienne, A.; Chéreau, C.; Alexandre, J.; Weill, B.; Doering, M.; Jacob, C.; Nicco, C.; Batteux, F. The Organotelluride Catalyst LAB027 Prevents Colon Cancer Growth in the Mice. Cell Death Dis. 2011, 2, e191.

(295) Lv, Z.; Bian, Z.; Shi, L.; Niu, S.; Ha, B.; Tremblay, A.; Li, L.; Zhang, X.; Paluszynski, J.; Liu, M.; Zen, K.; Liu, Y. Loss of Cell Surface CD47 Clustering Formation and Binding Avidity to SIRP $\alpha$ Facilitate Apoptotic Cell Clearance by Macrophages. J. Immunol. 2015, 195, 661-671.

(296) Plantin-Carrenard, E.; Bringuier, A.; Derappe, C.; Pichon, J.; Guillot, R.; Bernard, M.; Foglietti, M. J.; Feldmann, G.; Aubery, M.; Braut-Boucher, F. A Fluorescence Microplate Assay Using Yopro-1 to Measure Apoptosis: Application to HL60 Cells Subjected to Oxidative Stress. Cell Biol. Toxicol. 2003, 19, 121-133.

(297) Kamuhabwa, A. R.; Agostinis, P. M.; D’Hallewin, M. A.; Baert, L.; de Witte, P. A. Cellular Photodestruction Induced by Hypericin in AY-27 Rat Bladder Carcinoma Cells. Photochem. Photobiol. 2001, 74 (2), 126-132.

(298) Piette, J.; Volanti, C.; Vantieghem, A.; Matroule, J. Y.; Habraken, Y.; Agostinis, P. Cell Death and Growth Arrest in Response to Photodynamic Therapy with Membrane-Bound Photosensitizers. Biochem. Pharmacol. 2003, 66, 1651-1659.

(299) Agostinis, P.; Assefa, Z.; Vantieghem, A.; Vandenheede, J. R.; Merlevede, W.; De Witte, P. Apoptotic and Anti-Apoptotic Signaling Pathways Induced by Photodynamic Therapy with Hypericin. Adv. Enzyme Regul. 2000, 40, 157-182.

(300) Ali, S. M.; Olivo, M. Mechanisms of Action of Phenanthroperylenequinones in Photodynamic Therapy (Review). Int. J. Oncol. 2003, 22 (6), 1181-1191.

(301) Marchal, S.; Fadloun, A.; Maugain, E.; D’Hallewin, M. A.; Guillemin, F.; Bezdetnaya, L. Necrotic and Apoptotic Features of Cell Death in Response to Foscan ${ }^{\circledR}$ Photosensitization of HT29 Monolayer and Multicell Spheroids. Biochem. Pharmacol. 2005, 69, 1167-1176.

(302) Vuong, T. T. K.; Vever-Bizet, C.; Bonneau, S.; Bourg-Heckly, G. Hypericin Incorporation and Localization in Fixed HeLa Cells for Various Conditions of Fixation and Incubation. Photochem. Photobiol. Sci. 2011, 10, 561-568.

(303) Timm, M.; Saaby, L.; Moesby, L.; Hansen, E. W. Considerations Regarding Use of Solvents in in Vitro Cell Based Assays. Cytotechnology 2013, 65 (5), 887-894. 
REFERENCES 
NANOGELS ENCAPSULATING NITRIC OXIDE PROBES 



\section{CHAPTER 5. NANOGELS ENCAPSULATING NITRIC OXIDE PROBES}

Some of the results presented in this chapter were outlined and performed under the supervision of Dr María J. Marín during a research stay (September - December 2018) in the School of Chemistry at the University of East Anglia (Norwich, UK).

\subsection{INTRODUCTION TO NITRIC OXIDE PROBES}

Nitric oxide $(\cdot \mathrm{N}=\mathrm{O}, \mathrm{NO})$ is a widespread intracellular and intercellular signalling molecule that has been attracting interest in the past 20 years due to its vast role in human health and biology.

\section{NITRIC OXIDE BIOSYNTHESIS}

In mammals, nitric oxide is biosynthesized endogenously by the oxidation of the amino acid Larginine (Figure 5.1). The nitrogen atom in nitric oxide derives from the guanidino nitrogen atom of the amino acid and the oxygen atom originates from molecular oxygen. ${ }^{1,2} \mathrm{~N}$-hydroxy-Larginine is an intermediate, and the final organic product is L-citrulline, which regenerates as Larginine as part of the urea cycle. These oxidation processes require calcium ions, the reduced form of nicotinamide adenine dinucleotide phosphate (NADPH) and tetrahydrobiopterin as cofactors for the enzyme nitric oxide synthase (NOS). ${ }^{3,4}$

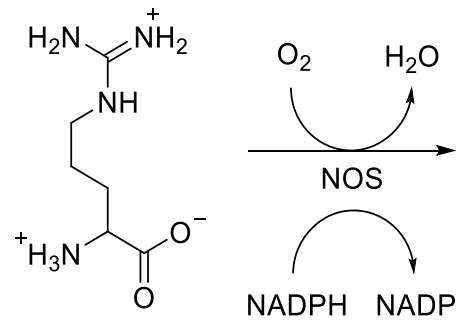

L-arginine<smiles>N/C(=N/O)NCCCC([NH3+])C(=O)[O-]</smiles>

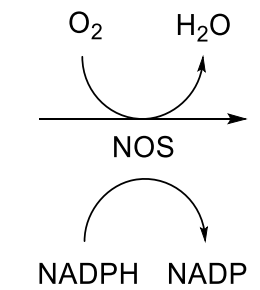<smiles>NC(=O)NCCCC([NH3+])C(=O)[O-]</smiles>

L-citrulline

Figure 5.1. Production of nitric oxide as the intermediate of the oxidation of L-arginine to produce L-citrulline. ${ }^{3}$

Three different isoforms of NOS have been identified as involved in the synthesis of NO in mammalian cells: neuronal (nNOS), endothelial (eNOS) and inducible NOS (iNOS). ${ }^{3}$ Although the enzymes were named according to the tissue in which they were first found, further research 
indicated the presence of the different isoforms in other tissues. ${ }^{5-7}$ The three isoforms of NOS present differences between them, such as their constitutive or inducible character and their $\mathrm{Ca}^{2+}$ dependence. For example, eNOS and nNOS are $\mathrm{Ca}^{2+}$ dependent and the enzymes are activated when the $\mathrm{Ca}^{2+}$ concentration inside the cell is increased above the norm. In contrast, iNOS is independent of the concentration of $\mathrm{Ca}^{2+}$ present and can be activated in a wide range of cells by bacterial lipopolysaccharides, cytokines or other agents. ${ }^{6}$ Furthermore, the amount of NO produced also changes, as eNOS and nNOS give a picomolar concentration of NO, whereas iNOS produces higher amounts of NO (nanomolar concentration) when activated by a foreign body. ${ }^{8,9}$

\section{NITRIC OXIDE IN THE HUMAN BODY}

NO is involved in a wide range of physiological functions. It has a high diffusion capacity and can permeate various cellular membranes due to its hydrophobic nature. ${ }^{10}$ Nearly 35 years ago, NO was identified as an endothelial relaxing factor responsible for vasodilation and blood pressure regulation, a discovery which garnered Robert F. Furchgott, Ferid Murad and Louis J. Ignarro a Nobel prize for medicine or physiology in $1998 .{ }^{11}$ However, it plays a significant role in a multitude of other normal physiological processes. ${ }^{12}$ In the central nervous system, NO acts as a neurotransmitter (it has been shown to regulate feelings of pain, appetite, the sleep-wake cycle, thermoregulation, synaptic plasticity and neural secretion ${ }^{13-17}$ ) and may also act as a neuroprotective or degradative agent, depending upon its concentration within the surrounding tissue. ${ }^{13}$ Aside from its role in vasodilation, NO also plays a significant role in modulating platelet aggregation, smooth muscle cell proliferation and LDL (low density lipoprotein) cholesterol oxidation in the cardiovascular system. ${ }^{11,18,19}$ As for the gastrointestinal tract, NO protects the stomach epithelium by stimulating mucosal and bicarbonate secretions ${ }^{20,21}$ and may also confer protection to the mucosal layer by inhibiting gastric acid secretion and leukocyte adhesion to the epithelium. ${ }^{20,22}$ In the renal system, NO is principally responsible for regulating blood pressure and flow to the kidneys ${ }^{23}$ and has also been implicated in natriuresis and diuresis due to its ability to inhibit sodium transport within the nephron. ${ }^{24}$

Apart from NO signalling functions, it is also remarkable its action in the immune system. NO is generated in intracellular organelles at high concentrations, where it is used to destroy foreign agents. For instance, immune cells as macrophages synthesize this molecule to destroy or neutralise foreign bodies responsible for their activation, ${ }^{8}$ causing tumour cells senescence or 
death $^{25}$ or showing antimicrobial properties. Epithelial cells have also shown to decrease replication and kill infectious agents. ${ }^{25,26}$

The dual activity of NO, as a signalling molecule or as a cytotoxic response, has been explained based on its reactivity. This comes from the free radical nature of $\mathrm{NO}$, with the unpaired electron in a $\pi$ antibonding $\left(\pi^{*}\right)$ orbital. ${ }^{27} \mathrm{NO}$ is a relatively stable free radical (as the unpaired electron is delocalised between the $\mathrm{N}$ and the $\mathrm{O}$ atoms), with a lifetime between $2 \mathrm{~ms}$ and $2 \mathrm{~s}$ depending on the availability of intracellular reactants. ${ }^{28}$ If NO is in oxygenated media, it has a short lifetime as it reacts with dissolved oxygen, and only fast reactions between biological molecules and NO take place. These reactions are known as direct reactions or direct effects ${ }^{29}$ and occur when NO is present at low concentrations $(<200 \mathrm{nM}){ }^{30}$ The reactions between $\mathrm{NO}$ and oxygen or superoxide generate reactive nitrogen species (RNS). The reactions in which RNS are involved are known as indirect reactions or indirect effects ${ }^{29}$ and occur when NO is present at high concentrations (> $400 \mathrm{nM}$ ). ${ }^{30}$ As previously described, cell types containing nNOS and eNOS produce less NO than cells containing iNOS. Therefore, direct effects are the predominant reactions in the nNOS and eNOS cell types and indirect effects are the predominant reactions in the iNOS cell type.

An example of direct effect is the reaction of NO with ions of transition metals, such as Fe (II), forming stable complexes. ${ }^{31}$ For instance, in the case of smooth muscle cells and neurons, the role of NO as a signalling agent consists of forming a Fe-nitrosyl complex with the enzyme soluble guanylate cyclase (sGC) to activate the protein. ${ }^{29}$ This protein catalyses the conversion of guanosine-5-triphosphate (GTP) into cyclic guanosine-3,5-monophosphate (cGMP), ${ }^{32}$ which is a messenger ${ }^{33}$ involved in many regulatory functions such as vasodilation. ${ }^{34}$

Indirect effects are mediated by RNS and have been the subject of intense research interest. ${ }^{35,36}$ The major pathway for the metabolism of $\mathrm{NO}$ is its oxidation (Figure 5.2). NO reacts with $\mathrm{O}_{2}$ to form nitrogen dioxide $\left(\mathrm{NO}_{2}\right)$, one of the most common RNS. The $\mathrm{NO}_{2}$ formed can react with another $\mathrm{NO}$ molecule to yield dinitrogen trioxide $\left(\mathrm{N}_{2} \mathrm{O}_{3}\right)$, which produces $\mathrm{NO}_{2}{ }^{-}$and $\mathrm{H}^{+}$in water. ${ }^{3,8}$ Both $\mathrm{NO}_{2}$ and $\mathrm{N}_{2} \mathrm{O}_{3}$ can damage biological tissues. ${ }^{29}$ The dimerisation product (dinitrogen tetraoxide, $\mathrm{N}_{2} \mathrm{O}_{4}$ ) could be formed when $\mathrm{NO}_{2}$ accumulates in large quantities, and this product would react with water to form $\mathrm{NO}_{2}{ }^{-}$and $\mathrm{NO}_{3}{ }^{-} \cdot{ }^{37}$ Nitrite and nitrate are eventually eliminated by urinary excretion. ${ }^{10}$ 


$$
\begin{aligned}
& \mathrm{NO}+1 / 2 \mathrm{O}_{2} \longrightarrow \mathrm{NO}_{2} \\
& \mathrm{NO}_{2}+\mathrm{NO} \rightleftarrows \mathrm{N}_{2} \mathrm{O}_{3} \\
& \mathrm{~N}_{2} \mathrm{O}_{3}+\mathrm{H}_{2} \mathrm{O} \longrightarrow 2 \mathrm{HNO}_{2} \longrightarrow 2 \mathrm{H}^{+}+2 \mathrm{NO}_{2}^{-} \\
& 2 \mathrm{NO}_{2} \longrightarrow \mathrm{N}_{2} \mathrm{O}_{4} \\
& \mathrm{~N}_{2} \mathrm{O}_{4}+\mathrm{H}_{2} \mathrm{O} \longrightarrow \mathrm{NO}_{2}^{-}+\mathrm{NO}_{3}^{-}+2 \mathrm{H}^{+}
\end{aligned}
$$

Figure 5.2. Reactive nitrogen species generated from the reaction between NO and oxygen.

Another RNS involved in the toxicity of the NO derivatives is peroxynitrite $\left(\mathrm{ONOO}^{-}\right)$, formed by the reaction between $\mathrm{NO}$ and superoxide (Figure 5.3). ${ }^{38} \mathrm{ONOO}^{-}$is a powerful oxidant and reacts with various biological targets (including nitration of tyrosine, the rupture of DNA strands and the oxidation of haem proteins), generating cell damage. ${ }^{39}$

$$
\mathrm{NO}+\mathrm{O}_{2}^{-} \longrightarrow \mathrm{ONOO}^{-}
$$

Figure 5.3. Reactive nitrogen species generated from the reaction between NO and superoxide.

\section{NITRIC OXIDE IN THE ILLNESS}

Due to the importance of this molecule in physiological processes, NO homeostasis is crucial to its proper functions and altered NO production is implicated in a large number of pathologies. ${ }^{40}$ NO has been associated with neurodegenerative disorders such as Alzheimer's disease, Parkinson's disease, Huntington's disease, multiple sclerosis and amyotrophic lateral sclerosis, among others. ${ }^{41,42}$ It also plays an important role in many psychiatric disorders such as schizophrenia, ${ }^{43}$ bipolar affective disorders, ${ }^{44}$ substance abuse, ${ }^{45}$ autism, ${ }^{46,47}$ disruptive behavioural disorder ${ }^{48}$ and depression. ${ }^{49}$ Due to its role in the vascular system, NO is also involved in strokes and cerebral ischemia. ${ }^{42} \mathrm{Also}, \mathrm{NO}$ is involved in inflammatory diseases, ${ }^{50}$ as psoriasis. $^{51}$

If the alteration in NO production that leads to the illness is known, the pharmacological modulation of NO levels in tissues can help to create satisfactory therapeutic procedures. ${ }^{10}$ For example, inhibition of nNOS in patients of Parkinson's disease can reduce the neurotoxicity responsible for the disease. ${ }^{52}$ In the case of stroke and angina pectoris, the risk is reduced by increasing the bioavailability of NO using NO-donors as nitroglycerine. ${ }^{3,53,54}$ Also, inhalation of gaseous NO can reverse hypoxic pulmonary vasoconstriction. ${ }^{55}$ 
NO has also been associated with cancer, ${ }^{56,57}$ exerting dual effects (pro- and anti-tumour activity) on tumour development depending on the concentration. ${ }^{30,58}$ Low levels of NO produced by tumour cells (which induce the expression of iNOS ${ }^{59}$ ) promote cell growth, antiapoptotic responses and neovascularization. However, exogenously high levels of NO lead to cytotoxic functions and are likely to induce cell cycle arrest and apoptosis. ${ }^{60}$ Cancer therapies that are being developed nowadays include NO-donating agents to deliver a high level of NO to tumour sites and iNOS inhibitors to reduce tumour growth. ${ }^{61}$

\section{DETECTION OF NITRIC OXIDE IN BIOLOGICAL SYSTEMS}

Due to the significant role that NO plays in biological functions, many strategies have been developed for real-time monitoring the NO levels to understand the action of NO in living organisms and develop diagnostic tools for its detection. Among these approaches, fluorescence spectroscopy, chemiluminescence, electrochemistry, amperometry, colorimetry and electron paramagnetic resonance spectroscopy can be found. ${ }^{62-66}$ The fluorescence-based bioimaging method has attracted attention owing to its excellent sensitivity and selectivity, high spatiotemporal resolution, non-invasiveness and experimental convenience, providing in vivo and in situ visualization of NO in cells and tissues. ${ }^{62,67-73}$ The ideal fluorescent probes for this purpose should have the following characteristics: be water-soluble and membrane-permeable so that they can readily enter tissues, cells and organelles; be non-cytotoxic and should not interfere with other biological processes; show dynamic changes of fluorescence properties upon reaction with NO or NO products; be sensitive to the change of cellular NO concentration from the low nanomolar range; be excitable at low-energy wavelengths so that the induced auto-fluorescence can be effectively prevented.

Organic-based small-molecule fluorescent NO probes are the more commonly used for NO bioimaging. These probes adopt a turn-on mechanism to enhance imaging resolution: no or weak emission before treatment with NO and strong emission after NO trigger. To this end, these molecules often comprise of two elements, a NO-reactive moiety and a fluorophore. The NO-reactive moiety serves as a modulator in the photoinduced electron transfer (PET) mechanism, which quenches the fluorescence of the fluorophore until it reacts with the NO or NO oxidized products. ${ }^{72,74}$ Some approaches used for NO recognition are listed in Figure $\mathbf{5 . 4}$ and include the conversion of ortho-phenylenediamine to give triazole derivatives, ${ }^{75-78}$ the conversion of dihydropyridine (Hantzsch ester) into corresponding pyridines, ${ }^{79,80}$ direct 
nitrosation of secondary aromatic amines, ${ }^{81-83}$ deamination of primary aromatic amines, $N$ nitrosation reaction of aromatic amines to give diazo ring compounds and the transformation of thiosemicarbazide into oxadiazole..$^{70,71}$
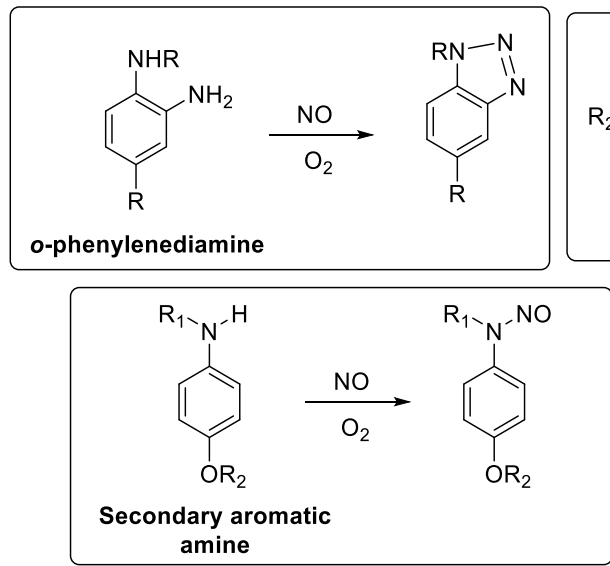

amine

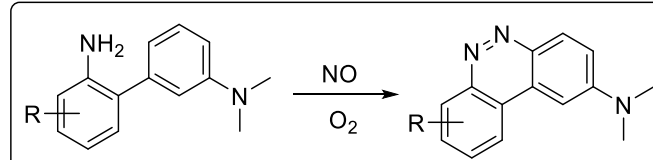

Aromatic amine
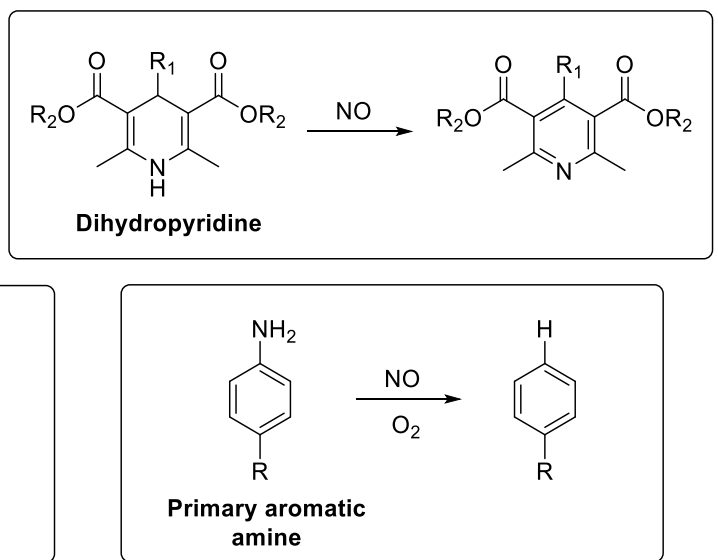

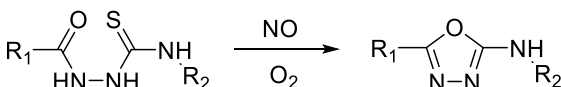

Thiosemicarbazide

Figure 5.4. Some strategies for NO recognition used in fluorescent probes.

Among these possibilities, the most frequently used strategy is the utilization of probes containing the ortho-phenylenediamine structure. ${ }^{62,84}$ As can be seen in Figure 5.5, the relatively non-fluorescent ortho-diamino compounds are nitrosylated to yield the corresponding fluorescent 1,2,3-triazole derivative. ${ }^{85,86}$ As in oxygenated aqueous solutions NO is rapidly converted into nitrous anhydride $\left(\mathrm{N}_{2} \mathrm{O}_{3}\right)$ (Figure 5.2), ${ }^{87}$ this reactive nitrosating species has been proposed as the responsible for this process. ${ }^{62,88}$ The fact that 0 -diamino probes react with oxidized NO products and not with NO itself has the advantage of detect NO without the inhibition of its function. ${ }^{75,89}$ The precise mechanisms leading to triazole formation when 0 diamino compounds react with NO in the presence of oxygen inside the cells are still unknown. The proposed mechanism of nitrosation and dehydration is given in Figure 5.6. ${ }^{85,90}$ Alternatively, a radical-radical reaction between $\mathrm{NO}$ and a radical intermediate of the probe, which is oxidized by ROS within the cells, has also been proposed..$^{91}$ 

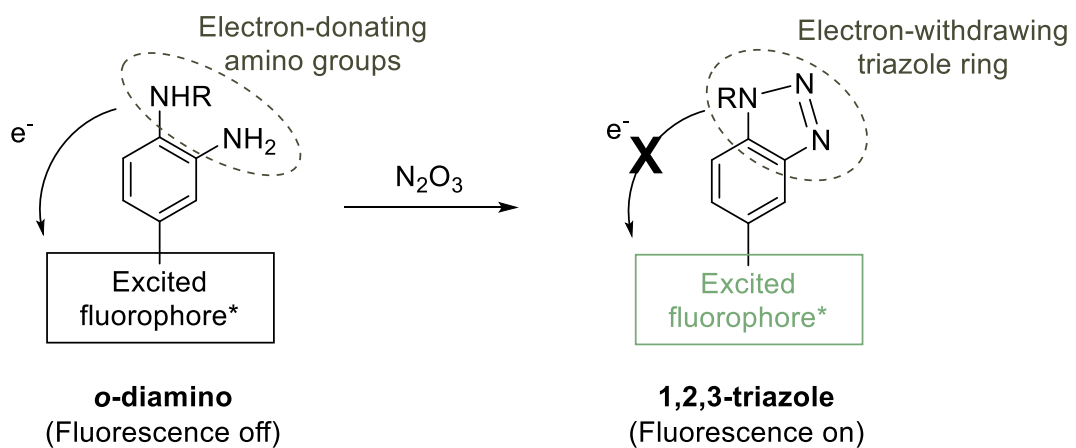

1,2,3-triazole

(Fluorescence on)

Figure 5.5. Sensing mechanism of NO fluorescent probes based on cyclization of $o$-phenylenediamine. ${ }^{68}$

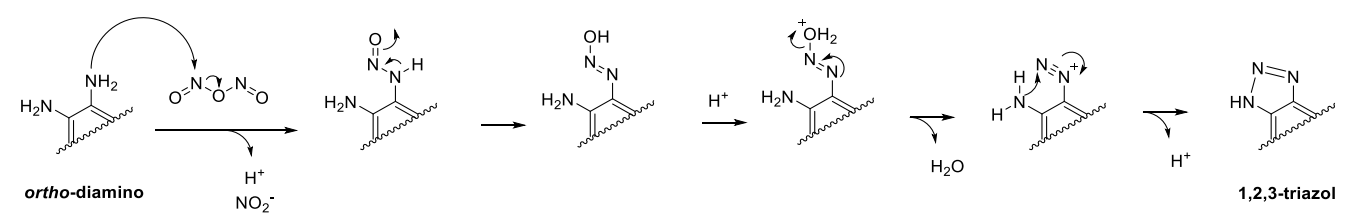

Figure 5.6. Proposed mechanism of the formation of a 1,2,3-triazole derivative when the corresponding o-diamino compound reacts with $\mathrm{N}_{2} \mathrm{O}_{3} \cdot{ }^{85,86}$

As explained above, in the absence of NO a photoinduced electron transfer process from electron-donating $o$-phenylenediamine group to the fluorophore quenches the fluorescence (Figure 5.5). However, when the corresponding triazole derivative is formed, the electronwithdrawing benzotriazole ring blocks the PET process and the fluorescence of the fluorophore is turned on. The PET mechanism is generally divided into two categories, acceptor-excited PET (a-PET) and donor-excited PET (d-PET). ${ }^{84}$ The a-PET process (Figure 5.7 left) employs NO trapping group as electron donor and fluorophore as electron acceptor. The highest occupied molecular orbital (HOMO) energy level of the NO trapping group is higher than that of the fluorophore, which results in intramolecular electron transfer from HOMO of NO trapping group to HOMO of the excited fluorophore. This process blocks the lowest unoccupied molecular orbital (LUMO) $\rightarrow$ HOMO electron transition that should occur in excited fluorophore, thereby quenching its fluorescence. After reacting with NO, the HOMO energy level of the NO trapping group becomes lower than that of the fluorophore (Figure $\mathbf{5 . 7}$ centre), which activates the LUMO $\rightarrow$ HOMO electron transition in excited fluorophore with significant fluorescent emission. Oppositely, the d-PET process employs the fluorophore as an electron donor and NO trapping group as an electron acceptor (Figure 5.7 right), and the LUMO energy level of the NO trapping group is lower than that of the fluorophore. Consequently, the intramolecular electron transfer 
occurs from LUMO of the excited fluorophore to LUMO of NO trapping group. Reaction with NO results in higher LUMO energy level of NO trapping group than that of the fluorophore, which is accompanied with the LUMO $\rightarrow$ HOMO electron transition in excited fluorophore and significant fluorescent emission (Figure 5.7 centre)..$^{70}$

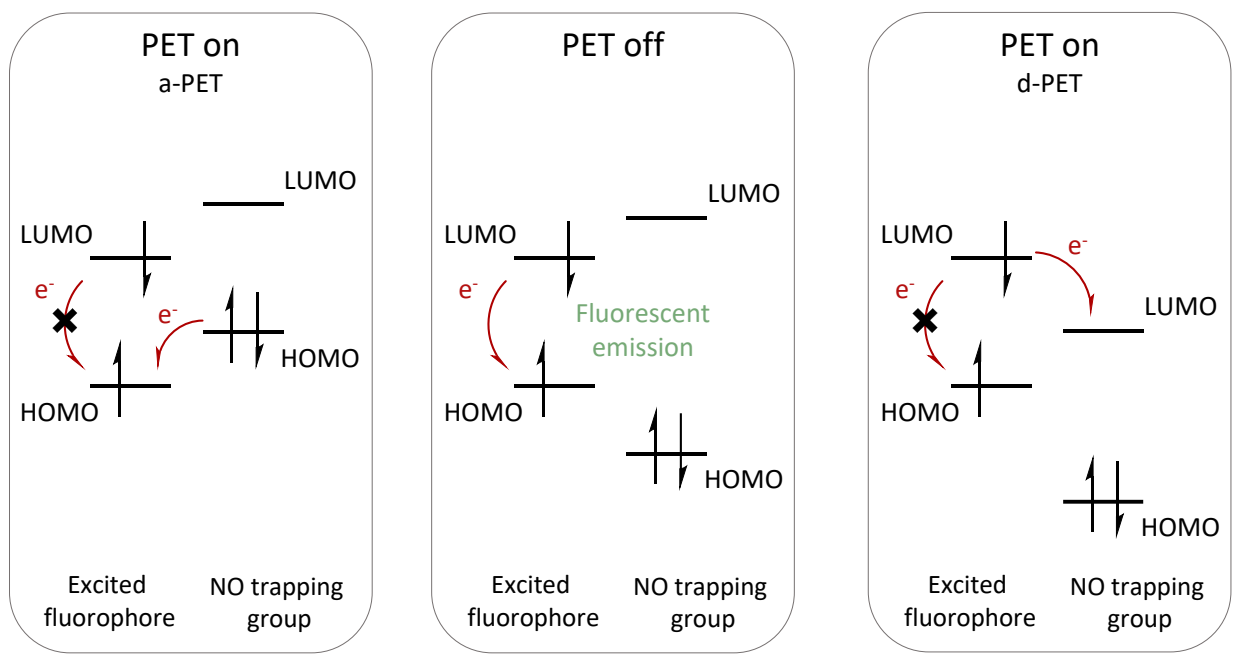

Figure 5.7. Proposed PET mechanism between the fluorophore and the NO trapping group: a-PET process (left), blocking of the PET process (centre) and d-PET process (right). ${ }^{70}$

Nagano and co-workers developed the first fluorescent indicators for NO in living cells with these characteristics. The detection of NO with 2,3-diaminophthalene (DAN) had been reported, but poor cellular uptake was observed..${ }^{92,93}$ For this reason, an ester derivative (DAN-1 EE, Figure 5.8) was developed, which improved the performance. ${ }^{89,94}$ However, DAN-1 EE presented disadvantages such as cytotoxicity, strong autofluorescence due to UV light excitation, a small extinction coefficient and low solubility in neutral $\mathrm{pH} .{ }^{95}$

Then, Nagano and co-workers reported a new family of probes based on the fluorescein chromophore, diaminofluoresceins (DAFs, Figure 5.8). ${ }^{75,96}$ As expected from the convenient characteristics of fluorescein, the $N$-nitrosation of DAFs yields highly green-fluorescent triazole forms, with fluorescence quantum efficiencies increased more than 100 times after the transformation and an increase of fluorescence intensity dependent on the concentration of NO. ${ }^{75}$ Also, DAFs possess favourable properties for cellular imaging applications, as noncytotoxicity ${ }^{75}$ and a visible excitation wavelength, which is less damaging to cells and is not subject to interference from the autofluorescence of biological samples. The specificity for NO 
is high: DAFs do not react in neutral solution with oxidized forms of $\mathrm{NO}, \mathrm{NO}_{2}^{-}$and $\mathrm{NO}_{3}{ }^{-}$, or other reactive oxygen species, such as $\mathrm{O}_{2}{ }^{-}, \mathrm{H}_{2} \mathrm{O}_{2}$ and $\mathrm{ONOO}^{-}$, to yield any fluorescent product, and, under physiological conditions, triazolofluorescein is not formed in the absence of NO. Therefore, DAFs provide a rapid, easy and sensitive assay for detecting NO, both intra- as well as extracellular, ${ }^{75,85,96}$ in living cells, such as macrophages ${ }^{75}$ or endothelial cells, even when the concentration of NO released from these later is low. ${ }^{85}$

However, DAF-Ts ("T" meaning triazol) fluorescence intensity shows pH dependence: the fluorescence emission is almost completely quenched when the phenolic OH group is protonated, which makes challenging to monitor intracellular NO changes after a stimulus that shifts the $\mathrm{pH}$ level. Dichloro derivatives of DAF (DAF-4, DAF-5, DAF-6) were synthesized for this reason, as chlorine atoms lower the $\mathrm{pK}_{\mathrm{a}}$ value of the adjacent phenolic $\mathrm{OH}$ group due to their electronegativity. However, they showed varying fluorescence intensity around neutral $\mathrm{pH} .{ }^{90}$ DAF-FM, with fluorine atoms at the $2^{\prime}$ and $6^{\prime}$ positions and an $N$-methyl group, was also developed based on that fluorination lowers the $\mathrm{pK}_{\mathrm{a}}$. Consequently, the spectrum of DAF-FM-T is essentially independent of $\mathrm{pH}$ above 5.5 ( $\mathrm{pK}_{\mathrm{a}}$ of the phenolic hydroxyl group 4.38 \pm 0.05 ). Together with DAF-2, which is also stable at neutral $\mathrm{pH}$ ( $\mathrm{pK}_{\mathrm{a}}$ of the phenolic proton $6.27 \pm 0.02$ ), this probe is a commercially available fluorescent NO-indicator for living cell imaging. ${ }^{90,97}$

Nagano's group reported other generations of 0 -diamino derivative NO probes with improved characteristics and no $\mathrm{pH}$ dependence. For instance, diaminorhodamines (DARs, Figure 5.8), rhodamine B fluorophore-based probes, which show longer excitation wavelength and higher photostability, ${ }^{98}$ or DAMBOs (Figure 5.8), BODIPY-based NO probes, which have a higher sensitivity. ${ }^{99}$ Diaminocyanines (DACs, Figure 5.8) contain a fluorophore that is excited with near infrared (NIR) light (700-900 nm), which penetrates more deeply into the tissues as is absorbed less by biological tissues and, besides, auto-fluorescence originated from indigenous biomolecules does not interfere with NIR emission. ${ }^{76}$ 


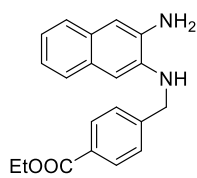

DAN-1 EE

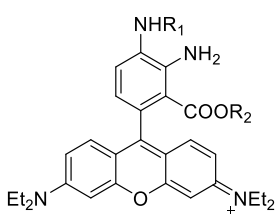

DAR

DAR-1: $\mathrm{R}_{1}=\mathrm{R}_{2}=\mathrm{H}$

DAR-4M AM: $\mathrm{R}_{1}=\mathrm{CH}_{3}, \mathrm{R}_{2}=$ Acetoxymethyl

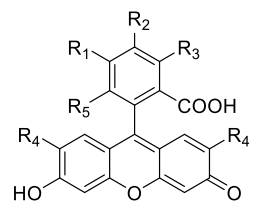

DAF

DAF-1: $\mathrm{R}_{2}=\mathrm{R}_{3}=\mathrm{NH}_{2}, \mathrm{R}_{1}=\mathrm{R}_{4}=\mathrm{R}_{5}=\mathrm{H}$

DAF-2: $\mathrm{R}_{1}=\mathrm{R}_{2}=\mathrm{NH}_{2}, \mathrm{R}_{3}=\mathrm{R}_{4}=\mathrm{R}_{5}=\mathrm{H}$

DAF-3: $\mathrm{R}_{1}=\mathrm{R}_{5}=\mathrm{NH}_{2}, \mathrm{R}_{2}=\mathrm{R}_{3}=\mathrm{R}_{4}=\mathrm{H}$

DAF-4: $\mathrm{R}_{2}=\mathrm{R}_{3}=\mathrm{NH}_{2}, \mathrm{R}_{1}=\mathrm{R}_{5}=\mathrm{H}, \mathrm{R}_{4}=\mathrm{Cl}$

DAF-5: $\mathrm{R}_{1}=\mathrm{R}_{2}=\mathrm{NH}_{2}, \mathrm{R}_{3}=\mathrm{R}_{5}=\mathrm{H}, \mathrm{R}_{4}=\mathrm{Cl}$

DAF-6: $R_{1}=R_{5}=N_{2}, R_{2}=R_{3}=H, R_{4}=C l$

DAF-FM: $\mathrm{R}_{2}=\mathrm{NHCH}_{3}, \mathrm{R}_{3}=\mathrm{NH}_{2}, \mathrm{R}_{1}=\mathrm{R}_{5}=\mathrm{H}, \mathrm{R}_{4}=\mathrm{F}$

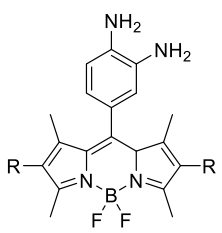

DAMBO

DAMBO: $\mathrm{R}=\mathrm{H}$

DAMBO-PH: $\mathrm{R}=\mathrm{CH}_{2} \mathrm{CH}_{2} \mathrm{COOH}$

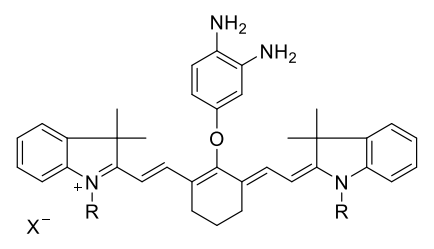

DAC

DAC-P: $\mathrm{R}=\mathrm{CH}_{2} \mathrm{CH}_{2} \mathrm{CH}_{3}, \mathrm{X}=\mathrm{I}$

DAC-S: $\mathrm{R}=\mathrm{CH}_{2}\left(\mathrm{CH}_{2}\right)_{3} \mathrm{SO}_{3} \mathrm{Na}, \mathrm{CH}_{2}\left(\mathrm{CH}_{2}\right)_{3} \mathrm{SO}_{3}^{-}$

Figure 5.8. Some representative NO probes based on the ortho-phenylenediamine moiety developed by Nagano's group.

In addition to the contributions of Nagano's group, a huge variety of NO fluorescent probes containing the $o$-phenylenediamine group has been reported in the past decades. ${ }^{10}$ Besides high selectivity and sensitivity and low cytotoxicity, these molecules were designed with attractive in vivo NO imaging features. For example, subcellular localisation, as not only concentration but also location are key determinants for $\mathrm{NO}$ to activate cellular compartment-specific signalling pathways. ${ }^{72}$ Organelle targeting ability can be achieved by adding some functional groups to the molecule (Figure 5.9), ${ }^{100}$ as in LysoNO-Naph, in which morpholine group helps to selectively image NO in lysosomes, ${ }^{101}$ or in DSDMHDAB, designed for cell membrane targeting, with a hydrophilic BODIPY part and a hydrophobic lipid tail, for imaging extracellular NO released from cells. ${ }^{102}$

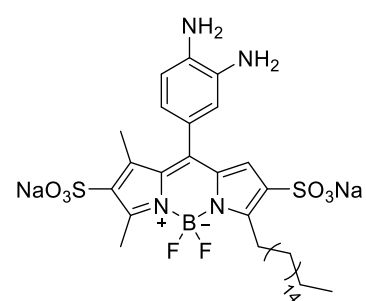

DSDMHDAB

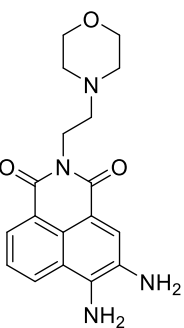

LysoNO-Naph

Figure 5.9. Structure of NO fluorescent probes DSDMHDAB and LysoNO-Naph. 
Probe-drug conjugates have also been developed. For example, $\mathrm{CCH}$ can simultaneously image hypoxia and NO in live tumour cells (hypoxia can induce overproduction of NO in tumour cells, which further leads to the proliferation and metastasis of tumour cells ${ }^{103,104}$ ), and delivery the antitumor drug chlorambucil under photoirradiation to induce tumour cell death (Figure 5.10).

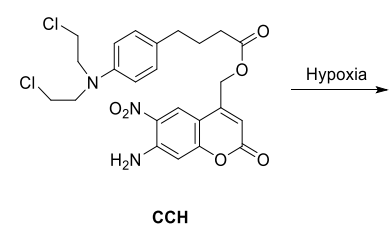

$\mathrm{CCH}$

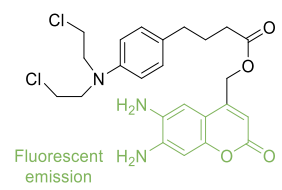

CCH-1

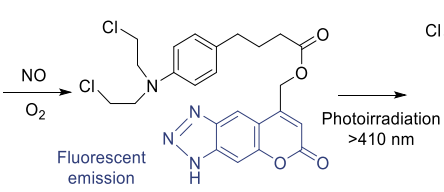

CCH-2

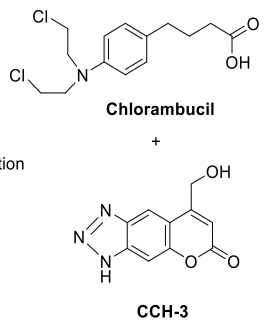

$\mathrm{CCH}-3$

Figure 5.10. Fluorescent probe-drug conjugate $\mathrm{CCH} .{ }^{70}$ 


\subsection{NANOGELS ENCAPSULATING 4,5- DIAMINOFLUORESCEIN (DAF-2)}

\subsubsection{INTRODUCTION}

Probably the most popular probe for NO sensing in living cells is 4,5-diaminofluorescein (or 5,6diaminofluorescein, DAF-2) because of its temporal and spatial resolution and an easy working protocol. ${ }^{72}$ The nitrosylation of DAF-2 (with low fluorescence) by $\mathrm{N}_{2} \mathrm{O}_{3}$ results in the highly green-fluorescent triazolofluorescein DAF-2T (Figure 5.11), whose fluorescence quantum efficiency is increased more than 180 times (0.005 for DAF-2 and 0.92 for DAF-2T). ${ }^{96} \mathrm{~A}$ linear correlation exists between the fluorescence response of DAF-2T and the concentration of NO up to around $1000 \mathrm{nM}$, and the detection limit is less than $5 \mathrm{nM} .^{75,85}$ Thus, it is possible to measure NO in cells which produce small amounts of NO, such as endothelial cells, as well as in cells which generate a large amount of NO, such as macrophages. In addition to its sensitivity, DAF-2 shows no cytotoxicity and the specificity characteristic of DAFs. ${ }^{96,105}$

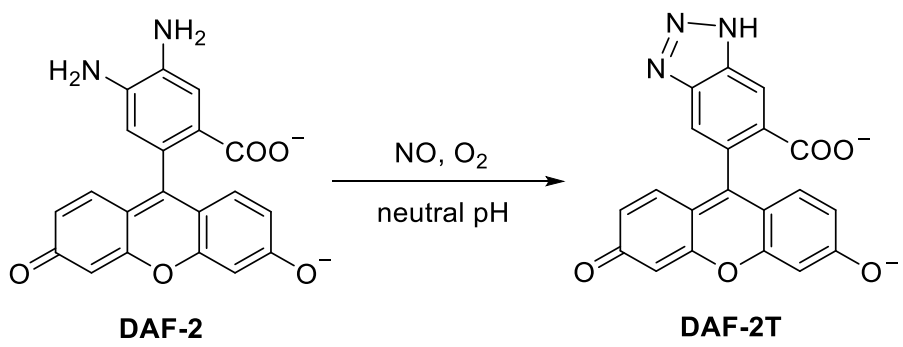

Figure 5.11. Chemical structures of DAF-2 and DAF-2T. 85

As DAF-2 is membrane-impermeable, its diacetate derivative (DAF-2 DA) was developed to improve accumulation in cells (Figure 5.12). DAF-2 DA, which is a fluorogenic substrate with no fluorescence, ${ }^{106}$ passively diffuses across cellular membranes. Once inside cells, ester bonds are readily hydrolysed by intracellular esterases in the cytosol, generating the water-soluble DAF-2 trapped within the cell. ${ }^{75,90,106}$ Diacetate form enhances cell membrane permeability and is necessary to load the cells efficiently with the probe, but also improves specificity. DAF-2 DA has been widely used for NO measurement in cultured cells (endothelial cells, ${ }^{107-111}$ aortic smooth muscle cells, ${ }^{75}$ cardiomyocytes, ${ }^{111}$ immune cells, ${ }^{112}$ hepatocarcinoma cells ${ }^{113}$ ), isolated vessel segments ${ }^{114}$ and intact vessels. ${ }^{115-121}$ 


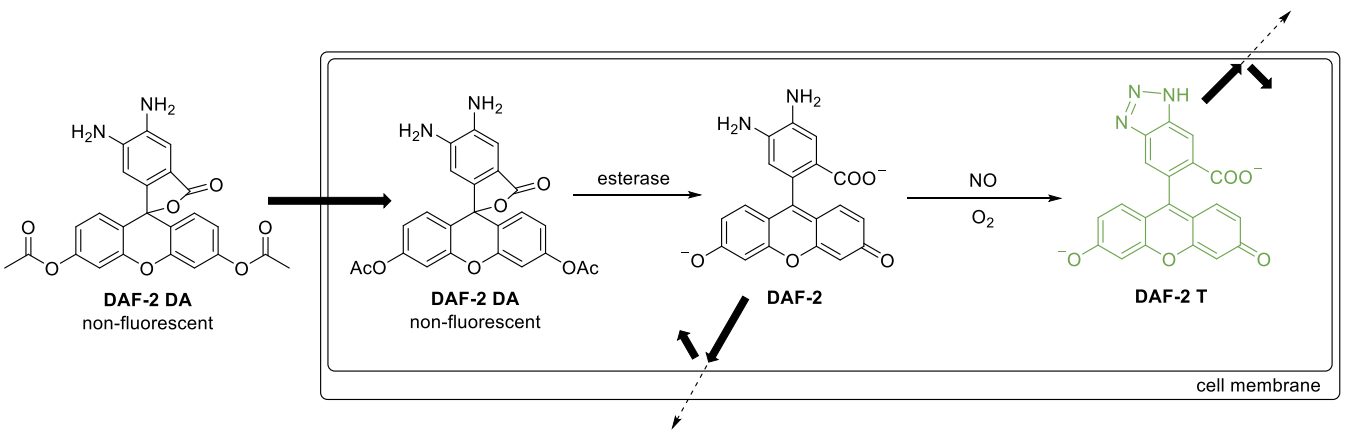

Figure 5.12. Schematic representation of DAF-2 DA behaviour in the cell. DAF-2 DA permeates through the cell membrane and is hydrolysed to yield DAF-2, which is retained in the cell owing to its relatively low permeating ability. DAF-2 reacts with NO to form fluorescent DAF-2T. ${ }^{75}$

However, in addition to the characteristic $\mathrm{pH}$ sensitivity of DAFs (although DAF-2T can be used at $\mathrm{pH}$ values above 7 , as has a $\mathrm{pK}_{\mathrm{a}}$ of $6.27 \pm 0.02$ ), DAF-2 has some shortcomings. First, DAF-2 reacts with dehydroascorbic acid (DHA) and ascorbic acid (AA), which often colocalize with NOS in the central nervous, cardiovascular and immune systems with concentrations in the low millimolar range, yielding a fluorescent adduct indistinguishable from the NO-derived product (1 mM DHA yielded fluorescence signals that are comparable to those for $300 \mathrm{nM} \mathrm{NO}$ ). ${ }^{122}$ They also attenuate the formation of DAF-2T because they compete with NO for DAF-2 or affect the amount of available $\mathrm{N}_{2} \mathrm{O}_{3}$ generated from $\mathrm{NO}$ due to their reducing activity. ${ }^{123}$ Additionally, DAF2 also reacts with methylglyoxal (MGO). ${ }^{124}$ Second, DAF-2T suffers from photobleaching. For example, the fluorescence intensity of DAF-2T was reduced to $58 \%$ of the initial intensity after exposure to sunlight for $1 h^{90}$

Encapsulation of DAF-2 into nanocarriers could solve some of the mentioned drawbacks. Up to our knowledge, only an example of DAF-2 encapsulation has been reported. ${ }^{125}$ DAF-2 was embedded by electrostatic interactions with the amino group of chitosan of a chitosan/dextran sulfate film $\left(40 \mathrm{~nm}\right.$ ) over mesoporous silica particles of $1.6 \mu \mathrm{m}$ diameter $\left(3.9 \times 10^{-19} \mathrm{~g}\right.$ of DAF-2 per particle). These sensor particles were found to show a high nitric oxide sensitivity (5-500 nM $\mathrm{NO}$ ), with the same detection limit that original DAF-2 and with a higher cytocompatibility in fibroblast cells, so they were aimed to be used for the analysis of extracellular NO molecules from living cells. 


\subsubsection{AIM OF THE RESEARCH REPORTED IN THIS SECTION}

In this section, the encapsulation of two molecules of different nature, 4,5-diaminofluorescein (DAF-2) and its diacetate derivative (DAF-2 DA), into our nanoparticles is studied. Due to its ionic nature in water, DAF-2 is membrane impermeable and suffers from poor intracellular uptake ability. Its diacetate derivative DAF-2 DA, which includes an additional step of hydrolysis before triazole formation, has been the probe used for in vitro NO imaging. Encapsulation would help DAF-2 to cross the cell membrane and would improve accumulation in cells. Other drawbacks of the probe could be solved: nanogels network could act as a barrier for bulky interfering species (as DHA or MGO), blocking secondary reactions, and could protect the probe from photobleaching. On the other hand, the hydrophobic character of DAF-2 DA limits its solubility in water, being only able to use reduced concentrations of this fluorogenic substrate in NO imaging assays. Encapsulation of DAF-2 DA in our nanogels would help to increase its concentration in physiological solutions and, in addition, could also change the cellular uptake mechanism and increase its internalisation or target some subcellular localisation of interest.

DAF-2 and DAF-2 DA were loaded into nanogels of 1 prepared by sonication method by introducing these species in a toluene suspension to prepare the gels, as this strategy has proved successful encapsulation of hydrophobic and hydrophilic molecules (Chapter 4). The spectroscopic characteristics of both probes were studied in different environments to be able to determine if they were encapsulated into the nanoparticles. Calibration curves using standards were also performed to determine the quantity of probe encapsulated. Finally, the response of these new DAF2@1 or DAF2DA@1 systems to NO was evaluated both in solution and in vitro.

\subsubsection{RESULTS AND DISCUSSION}

\subsubsection{SPECTROSCOPIC CHARACTERIZATION OF DAF-2 AND DAF-2T}

A solution of DAF-2 in PBS showed an absorption band at $486 \mathrm{~nm}$ and a fluorescence emission $\left(\lambda_{\text {ex }} 450 \mathrm{~nm}\right.$ ) band at $512 \mathrm{~nm}$ (Figure 5.13). On the other hand, the fluorogenic substrate DAF-2 DA showed an absorption maxima at $340 \mathrm{~nm}$ in DMSO and no fluorescence (Figure 5.14). ${ }^{75}$ 


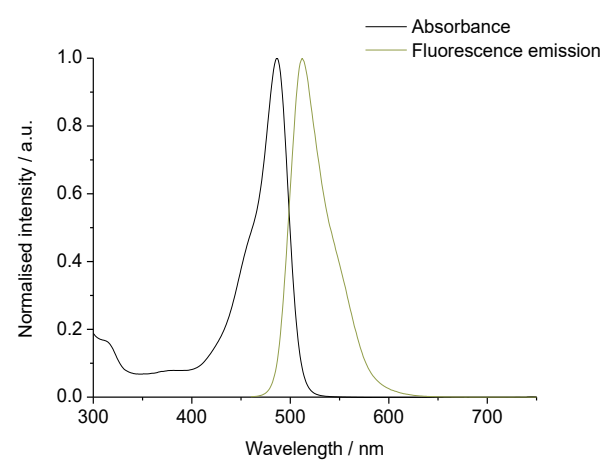

Figure 5.13. Normalised absorption (black) and fluorescence emission spectra (green, $\lambda_{\mathrm{ex}} 450 \mathrm{~nm}$ ) of a $10 \mu \mathrm{M}$ DAF-2 solution in PBS-0.2\%DMSO.

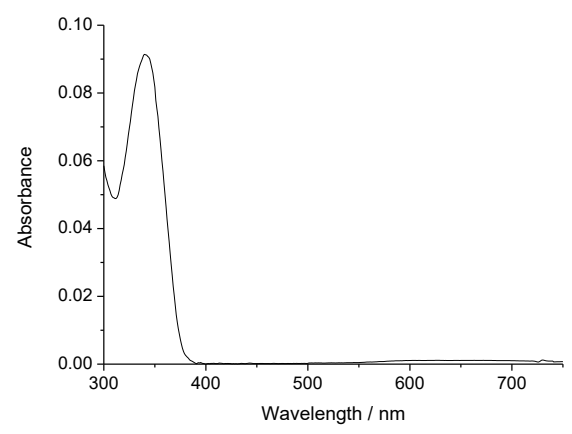

Figure 5.14. Absorption spectra of a $10 \mu \mathrm{M}$ DAF2 DA solution in DMSO.

The absorption of a series of $10 \mu \mathrm{M}$ DAF-2 solutions in PBS with different DMSO proportions $(100,75,50,25,10,5,1,0.4 \%$ DMSO) was studied to determine the dependence of optical properties on the environment of the probe (Figure 5.16). From a colourless solution in DMSO, a coloured species was obtained when DMSO proportion was below $75 \%$, with solutions going from orange to yellow (Figure 5.15). This was translated to the apparition of a band at $500 \mathrm{~nm}$ in absorption spectra, which linearly shifted to $486 \mathrm{~nm}$ when water proportion was increased (from $75 \%$ to $0.4 \%$ DMSO). $100 \%$ and $75 \%$ DMSO solutions showed a peak at $340 \mathrm{~nm}$. Additionally, the $490 \mathrm{~nm}$ band shows a shoulder, whose ratio of intensity with the maximum, $\mathrm{I}\left(\lambda_{\max }\right) / \mathrm{I}\left(\lambda_{\text {shoulder }}\right)$, decreases from 2.7 to 2 as PBS proportion is increased (the shoulder increases).

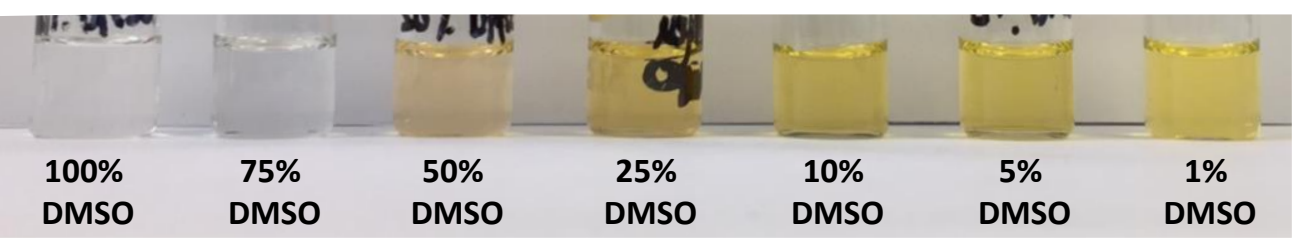

Figure 5.15. $10 \mu \mathrm{M}$ DAF-2 in PBS with different percentages of DMSO. 


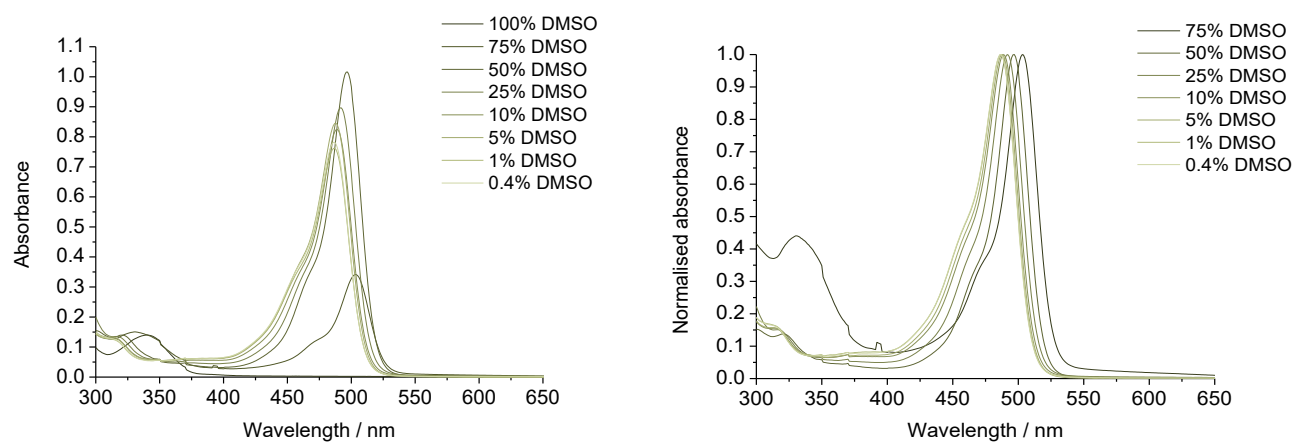

Figure 5.16. Absorption (left) and normalised absorption (right) spectra of $10 \mu \mathrm{M}$ DAF-2 solutions in different PBS-DMSO mixtures.

Therefore, the existence of a peak at $490 \mathrm{~nm}$, its exact $\lambda_{\max }$ and the ratio with its shoulder depend on the environment of DAF-2. This probe could be existing as a colourless neutral lactonic molecule in DMSO, and the coloured anionic form would be appearing with increasing water proportion (Figure 5.17). This would be parallel to what happens with Rose Bengal, a molecule quite similar to DAF-2 in structure, solubility, absorption and fluorescence emission spectra. For Rose Bengal, the anionic form in water reaches a concentration in which it begins to aggregate driven by hydrophobic effects, and a shoulder appears in absorption spectra. If we consider the quotient of absorption of the peak to the shoulder, a smaller ratio value indicates more significant aggregation. ${ }^{126,127}$ Although it has not been reported in the literature, DAF-2 could also be aggregated in aqueous solution. Xanthene dyes, as Rose Bengal and DAF-2, exhibit substantial aggregation effects at high concentrations, being polar protic solvents the ones promoting aggregation and polar aprotic solvents those hindering the aggregation process. ${ }^{128}$ Therefore, a shift towards lower wavelengths of the anionic DAF-2 absorption peak and an increase of the shoulder (decreased I $\left(\lambda_{\max }\right) / I\left(\lambda_{\text {shoulder}}\right)$ ratio) would mean aggregation and a more hydrophobic environment. As a hypsochromic shift is observed, these aggregates would be, thus, of the H-type. ${ }^{127}$
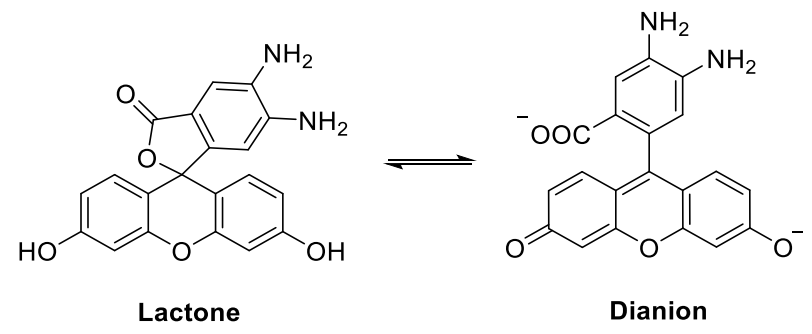

Figure 5.17. Protolytic equilibrium of tautomeric structures of DAF-2. 
In the same way that for DAF-2, the dependence of the optical properties of DAF-2 DA with the environment was studied, measuring the absorption of $10 \mu \mathrm{M}$ DAF-2 DA solutions in PBS with different DMSO proportions (100, 75, 50, 25, 10, 5\% DMSO). A peak around 330-340 nm was obtained for all solutions, whose maximum changed from $341 \mathrm{~nm}$ to $325 \mathrm{~nm}$ when increasing PBS proportion (see Figure 5.18). Therefore, there is again an environment dependency of the position of the maximum for DAF-2 DA.

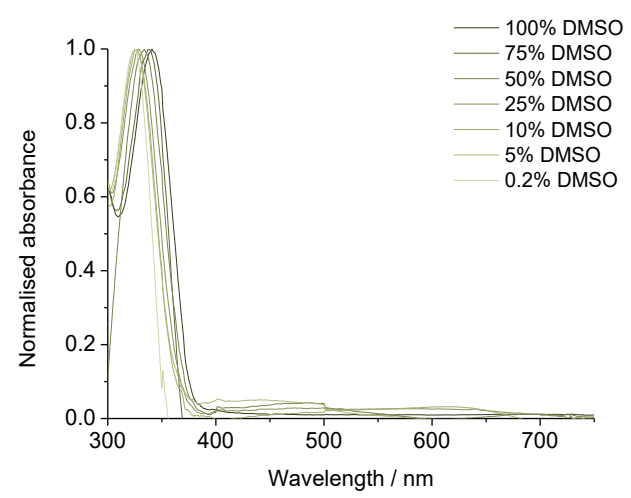

Figure 5.18. Normalised absorption spectra of $10 \mu \mathrm{M}$ DAF-2 DA solution in different PBS-DMSO mixtures.

\subsubsection{DAF-2 AND DAF-2 DA QUANTIFICATION}

The calibration curve to quantify DAF-2 was obtained after measuring the absorbance at 487 $\mathrm{nm}$ of a series of standards with concentrations ranging from 0.25 to $20 \mu \mathrm{M}$ of DAF-2 in PBS0.4\%DMSO (Figure 5.19).

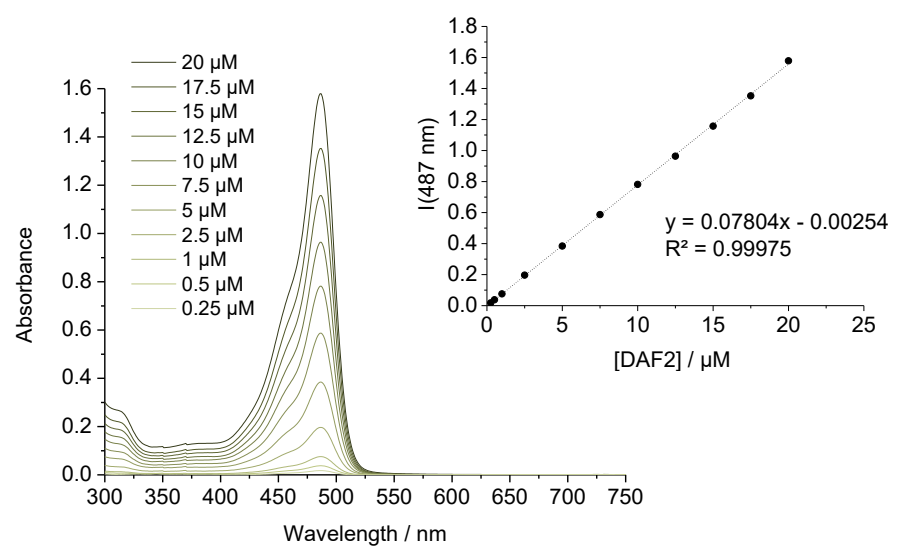

Figure 5.19. Absorption spectra of DAF-2 standards in PBS-0.4\%DMSO and calibration curve at $487 \mathrm{~nm}$. 
Similarly, the quantification of DAF-2 DA could be quickly done with a calibration curve using the intensity of absorption at $340 \mathrm{~nm}$. However, our nanoparticles show an enhanced baseline due to the particle dispersion effect, which is more notable in the lower wavelengths (Figure 5.20) and overlaps with the DAF-2 DA absorption band. Therefore, a protocol in which DAF-2 DA was hydrolysed to DAF-2 in a basic medium was developed. The addition of $20 \mu \mathrm{L} 1 \mathrm{M} \mathrm{NaOH}$ solution in water to $1 \mathrm{~mL}$ of a DAF-2 DA sample, which readily affords the hydrolysis reaction, was followed by the determination of the amount of hydrolysed DAF-2 using the DAF-2 calibration curve and, then, the estimation of the amount of DAF-2 DA that had been in the system. The hydrolysis of known DAF-2 DA solutions $(5,10,15$ and $20 \mu \mathrm{M}$ DAF-2 DA solutions in PBS-0.2\%DMSO) by this method revealed that the percentage of recovery of DAF-2 DA as DAF2 was an average of $68.4 \%$ (Figure 5.21 and Table 5.1). Therefore, this percentage was applied once we wanted to determine the quantity of DAF-2 DA encapsulated in our systems using the basification protocol.

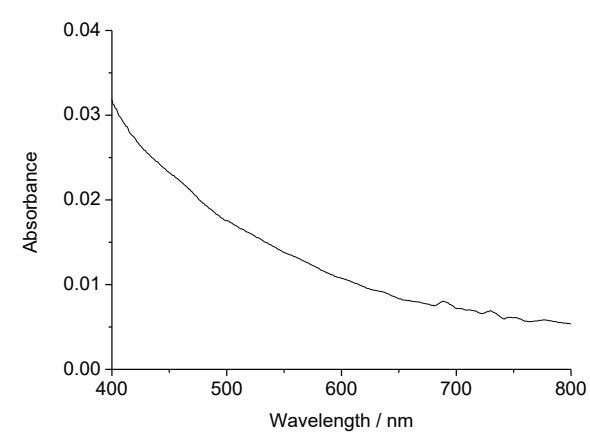

Figure 5.20. Nanoparticles dispersion shown in absorption spectra.

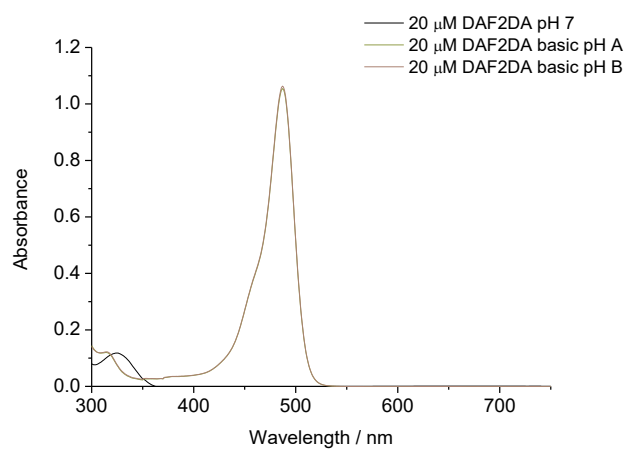

Figure 5.21. Absorption spectra of the hydrolysis of a $20 \mu \mathrm{M}$ DAF-2 DA solution in PBS-0.2\%DMSO.

Table 5.1. Quantified [DAF-2] after the basification of DAF-2 DA standards.

\begin{tabular}{ccc}
\hline Initial [DAF-2 DA] / $\boldsymbol{\mu M}$ & \multicolumn{2}{c}{ Quantified [DAF-2] / $\boldsymbol{\mu M}$} \\
repetition A & repetition B \\
\hline 20 & 13.54 & 13.65 \\
15 & 9.90 & 9.91 \\
10 & 6.85 & 6.93 \\
5 & 3.53 & 3.52 \\
\hline
\end{tabular}




\subsubsection{DAF-2 ENCAPSULATION INTO NANOGELS}

DAF2-loaded nanogels (DAF2@1) were prepared following the procedure described in Section 3.1 using a DAF-2 solution in toluene-0.5\%DMSO to prepare the gel. Firstly, an optimization of the loading was performed by preparing DAF2@1 samples at nominal concentrations ranging from 5 to $25 \mu \mathrm{M}$ (Figure 5.22). For comparison purposes, control experiments (DAF2-prep controls) of the DAF2@1 samples preparation procedure without gelator were also performed.

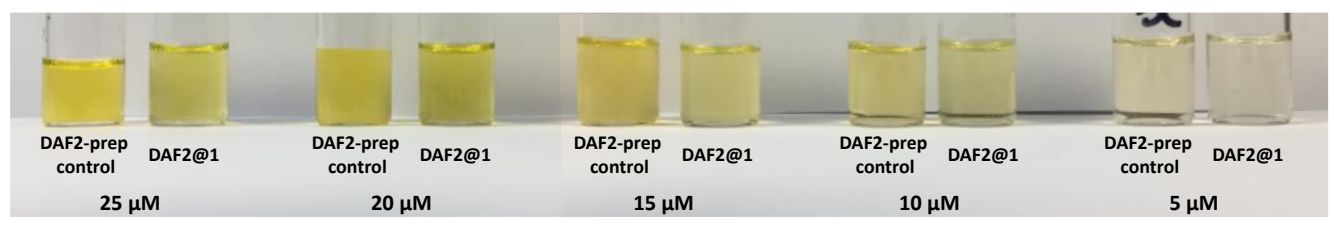

Figure 5.22. DAF2@1 and the corresponding DAF2-prep controls at the same nominal concentration.

Absorption and fluorescence emission $\left(\lambda_{\mathrm{ex}} 450 \mathrm{~nm}\right)$ of the obtained yellowish samples were recorded, with representative results in Figure 5.23. Absorption spectra revealed different environments for DAF-2 in the DAF2@1 sample and in DAF2-prep control, proving that the encapsulation of the probe in the nanoparticles had taken place. A shift in the absorption maximum occurred, from $487 \mathrm{~nm}$ for controls to $484 \mathrm{~nm}$ for nanoparticle samples. Additionally, an increase of the shoulder was observed, with an average peak-shoulder ratio $\left(I\left(\lambda_{\max }\right) / I\left(\lambda_{\text {shoulder }}\right)\right)$ value of $1.33 \pm 0.11$ for nanoparticles and $1.99 \pm 0.06$ for controls. An increase of the absorption band shoulder and a shift towards lower wavelengths were suggested in Section 5.2.3.1 to be a consequence of the formation of aggregates and a more hydrophobic environment for the probe. Therefore, two options are possible: DAF-2 is rather forming aggregates into nanoparticles than being in monomeric form, or the probe is in a more hydrophobic environment when it is encapsulated. This latter explanation would match what was observed for Rose Bengal, which was stabilised into the hydrophobic pockets of nanoparticles. The quantification of DAF-2 in the samples can be seen in Figure 5.24. 

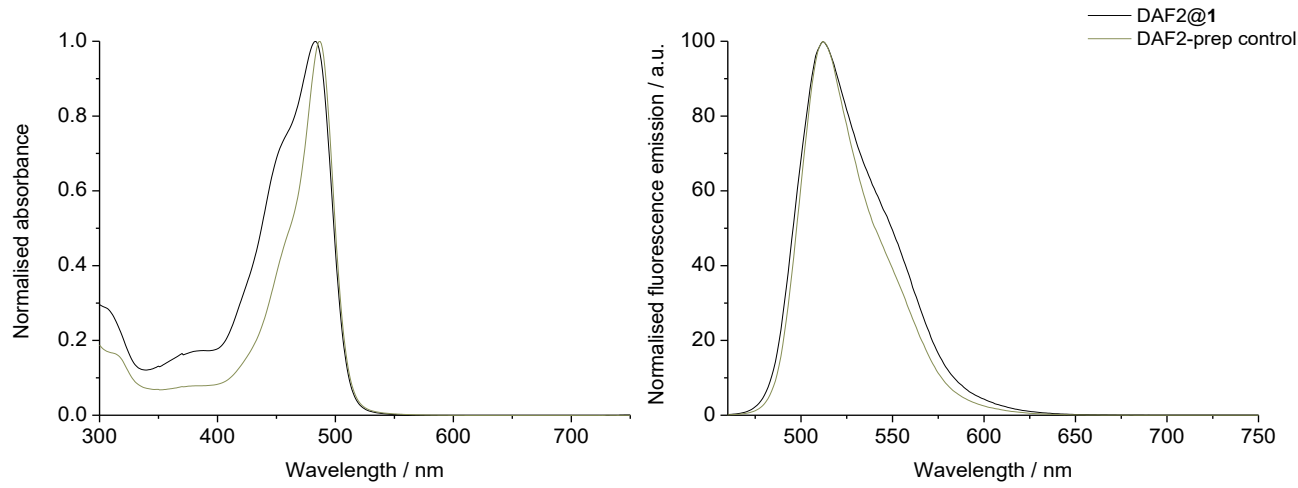

Figure 5.23. Normalised absorption and fluorescence emission $\left(\lambda_{\mathrm{ex}} 450 \mathrm{~nm}\right)$ spectra of a representative DAF2@1 sample (black) and its corresponding DAF2-prep control (green) ( $25 \mu \mathrm{M}$ nominal concentration).

Regarding fluorescence emission, there are no differences in the position of the emission maxima for nanoparticles and control samples $(511 \mathrm{~nm})$. However, nanoparticle samples show a sharper shoulder, indicating that the probe is in a different environment and that the encapsulation has been achieved. Regarding intensity, a quenching is observed for nominal concentrations higher than $10 \mu \mathrm{M}$ (Figure 5.25). Therefore, to achieve higher fluorescence results, $10 \mu \mathrm{M}$ was chosen as the optimum nominal concentration for DAF2@1 samples preparation. An average concentration of 5.2 $\pm 1.1 \mu \mathrm{M}$ DAF-2 was determined for these DAF2@1 samples, representing a $0.2-0.3 \% \mathrm{w} / \mathrm{w}$ of probe loading in the nanoparticles.

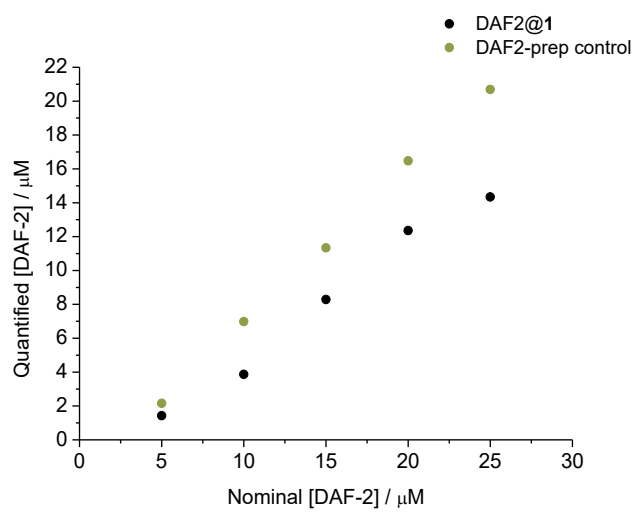

Figure 5.24. [DAF-2] in DAF2@1 (black) and DAF2prep controls (green) for a representative set of experiments.

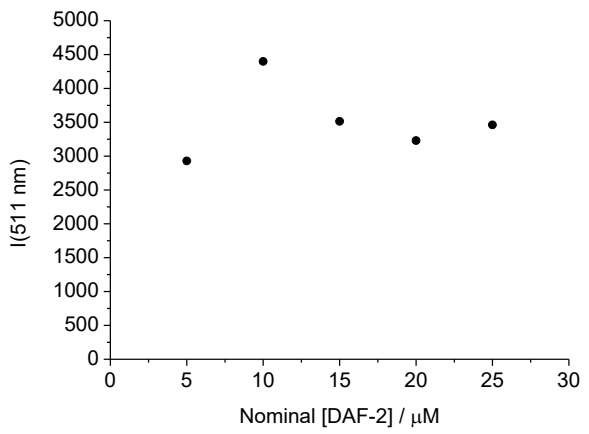

Figure 5.25. Fluorescence emission $\left(\lambda_{\mathrm{ex}} 450 \mathrm{~nm}\right)$ intensity at 511 nm of DAF2@1 samples at different nominal concentrations. 


\subsubsection{DAF-2 DA ENCAPSULATION INTO NANOGELS}

The encapsulation of DAF-2 diacetate in our nanoparticles (DAF2DA@1) was performed as for DAF-2, using a DAF-2 DA solution in toluene-0.5\%DMSO to prepare the gel. Again, an optimization of the loading was performed by the preparation of DAF2DA@1 samples and the corresponding DAF2DA-prep controls at nominal concentrations ranging from 5 to $25 \mu \mathrm{M}$.

The absorption spectra of some representative samples can be seen in Figure 5.26. The expected DAF-2 DA absorption band at 320-340 nm could only be observed for DAF2DA-prep controls as the increased baseline of particle dispersion overlaps in the case of DAF2DA@1 samples. Besides, a slightly yellow colour (double peak at 450-480 nm) was observed both for nanoparticles and controls, due to some hydrolysed DAF-2 from the preparation protocol. A shift towards lower wavelengths for nanoparticles (479 nm vs. $482 \mathrm{~nm}$ for controls) and differences in the peak-shoulder ratio of the DAF-2 band are revealed, which would mean a different environment for the probe consequence of the encapsulation of hydrolysed DAF-2 in the nanoparticles. Therefore, the original DAF-2 DA would also be encapsulated. It should be noted that the spontaneous hydrolysis found when preparing the samples is low (around 10\%), being the main amount of the compound still as the diacetate form.
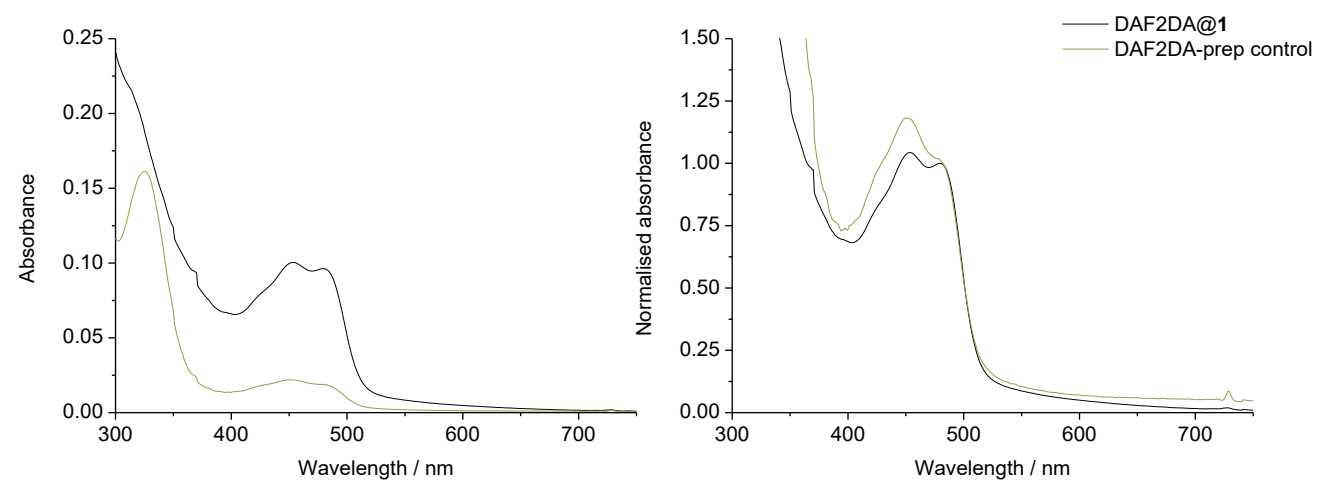

Figure 5.26. Absorption spectra and normalized absorption spectra of a representative DAF2DA@1 sample (black) and its corresponding DAF2DA-prep control (green) ( $25 \mu \mathrm{M}$ nominal concentration).

The quantification of DAF-2 DA in the samples was performed by basification to transform DAF2 DA in DAF-2, quantification of hydrolysed DAF-2 and application of the recovery percentage to obtain [DAF-2 DA] in the original sample. The absorption spectra for both DAF2DA@1 and DAF2DA-prep control at basic pH (Figure $\mathbf{5 . 2 7}$ left) show a maximum around $488 \mathrm{~nm}$ and a 
peak/shoulder ratio of 2.7, indicating that the nanoparticles have been disassembled, and DAF2 is well solubilised in both cases (additionally, these were the values obtained for DAF- 2 in a PBS solution with a high percentage of DMSO). The original concentration of DAF-2 DA (Figure 5.27 right) was obtained for each sample, being quite similar for nanoparticles and control samples at the same initial concentration.
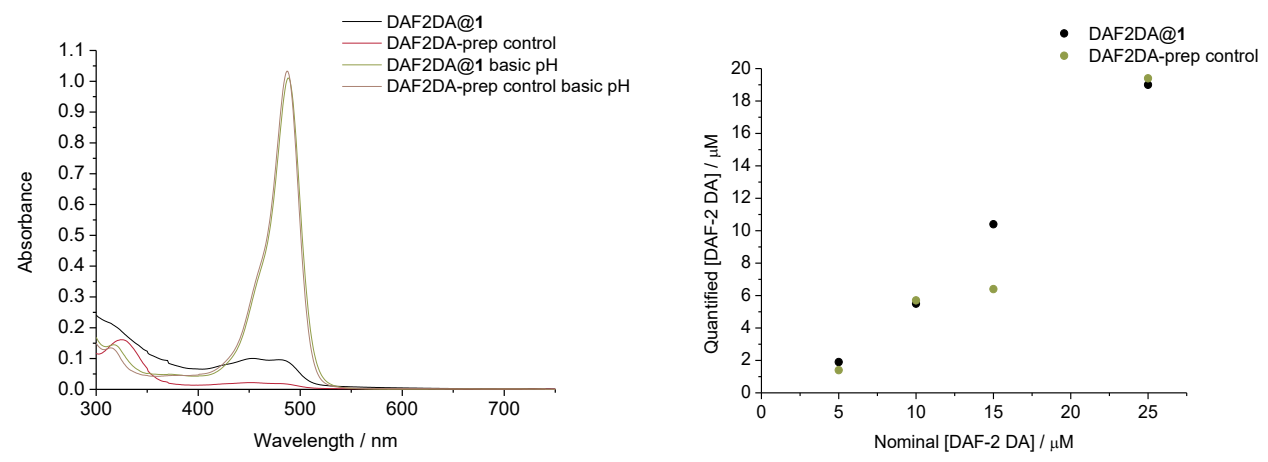

Figure 5.27. Left: Absorption spectra of a representative DAF2DA@1 sample (black) and its corresponding DAF2DA-prep control (red) after basification (green and light brown, respectively) (25 $\mu \mathrm{M}$ nominal concentration). Right: Quantification of DAF-2 DA in DAF2DA@1 (black) and DAF2DA-prep controls (green).

Moreover, the yellow colour of the samples was enhanced after days, meaning a spontaneous hydrolysis process for DAF-2 DA samples. Absorption for both DAF2DA@1 and DAF2DA-prep control was studied after 2 and 7 days (Figure 5.28), and the relative amount of DAF-2 DA/hydrolysed DAF-2 was calculated. Although there is more hydrolysed DAF-2 in freshly prepared nanoparticle samples, as days go by, the quantity of hydrolysed DAF-2 increases from $10 \%$ to $26 \%$ for nanoparticles and from $4 \%$ to $40 \%$ for controls. Therefore, the gelator could be interacting with DAF-2 DA, and nanoparticles would somehow avoid the hydrolysis of the probe found in solution. 

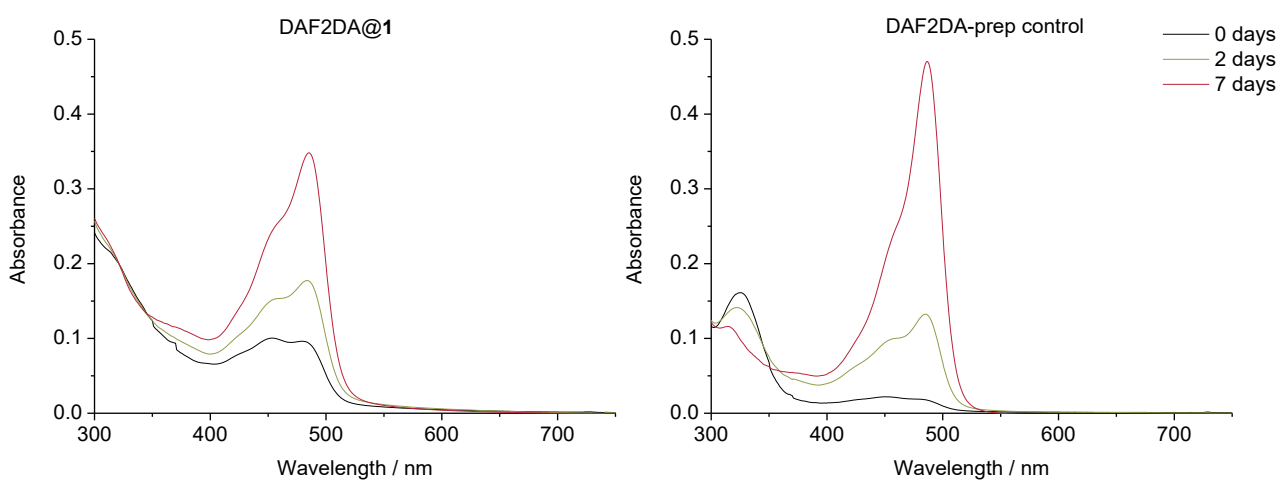

Figure 5.28. Absorption spectra for a representative DAF2DA@1 sample (left) and its corresponding DAF2DA-prep control (right) over time (0, 2 and 7 days) (25 $\mu \mathrm{M}$ nominal concentration).

To work as for DAF-2 samples, $10 \mu \mathrm{M}$ was chosen as the optimum nominal concentration. An average concentration of 5.8 $\pm 1.0 \mu \mathrm{M}$ DAF-2 DA was determined for these DAF2DA@1 samples, representing a $0.3-0.4 \% \mathrm{w} / \mathrm{w}$ of probe loading in the nanoparticles.

\subsubsection{DAF2-LOADED NANOPARTICLES RESPONSE TO NITRIC OXIDE}

Next, the fluorescence response of DAF2@1 systems towards NO was studied. To this end, the commercially available NO donor DEA NONOate (1,1-diethyl-2-hydroxy-2-nitroso-hydrazine sodium salt) was used to check the fluorescent triazole formation. ${ }^{3,129,130}$ DEA NONOate is a stable complex of diethylamine with nitric oxide in basic solution and, when $\mathrm{pH}$ is lowered to 7.4, decomposes to give 1.5 molecules of NO. ${ }^{131}$ Only DAF2@1 samples were tested to know if the nanoparticles as carriers influence DAF-2 response to NO, as DAF-2 DA needs intracellular esterases to give DAF-2 and be able to form the triazole.

In our experiment, incremental volumes of a $15.6 \mathrm{mM}$ stock solution of NONOate in $0.01 \mathrm{M}$ $\mathrm{NaOH}$ were added to $2 \mathrm{~mL}$ aliquots of a DAF2@1 sample (6.4 $\mu \mathrm{M}$ DAF-2), to reach solutions with NO concentrations ranging from 12 to $572 \mu \mathrm{M}$. For control purposes, the titration was repeated with a DAF2-prep control (8.9 $\mu \mathrm{M}$ DAF-2) to test what happens to DAF-2 in an aqueous solution. Samples were air-equilibrated so the reaction of triazole formation could proceed, and the $\mathrm{pH}$ was checked to be 7 for solutions after NONOate addition. The response of DAF-2 to NO was checked by absorption and fluorescence emission $\left(\lambda_{\text {ex }} 450 \mathrm{~nm}\right)$ spectroscopy. Results after 10 min of NONOate titration are shown in Figure 5.29, Figure 5.30 and Figure 5.31. 


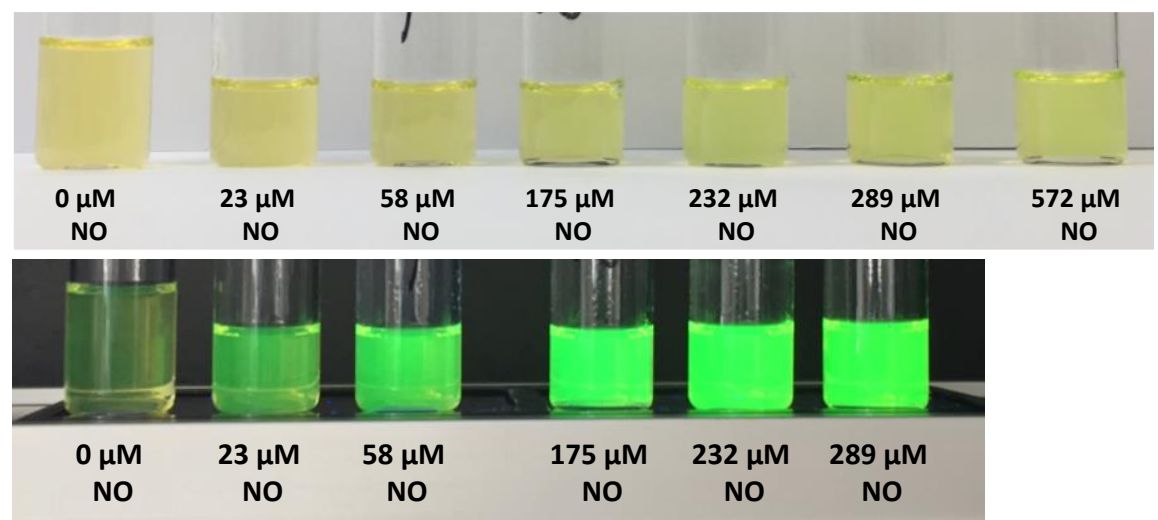

Figure 5.29. NO titration of a DAF2@1 sample observed under ambient light (top) or UV light (bottom).
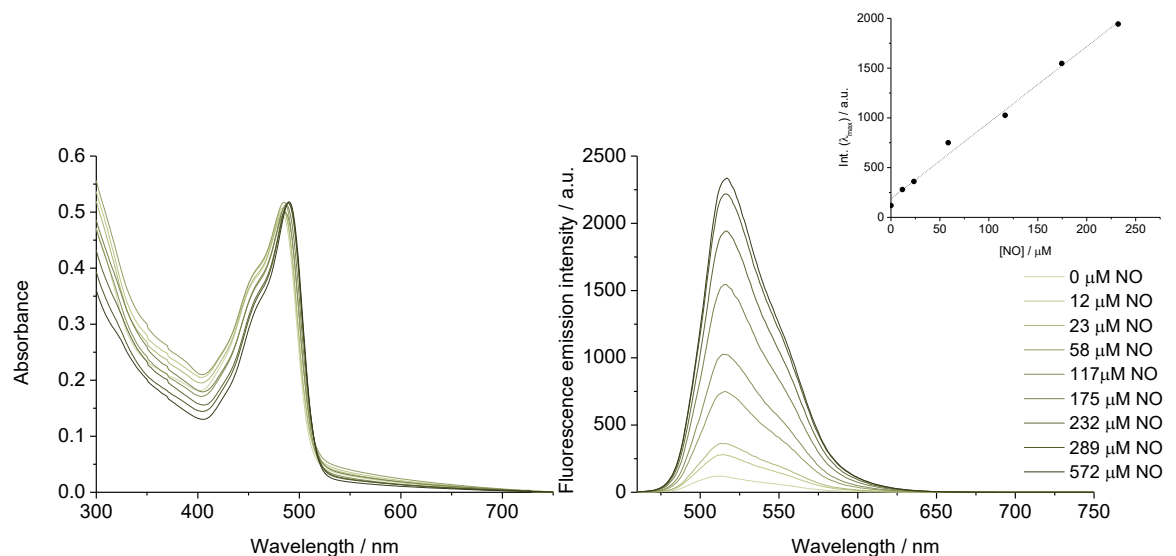

Figure 5.30. Absorption and fluorescence emission spectra $\left(\lambda_{\mathrm{ex}} 450 \mathrm{~nm}\right)$ for a DAF2@1 sample in the presence of increasing amounts of NO (from 0 to $572 \mu \mathrm{M}$ ).

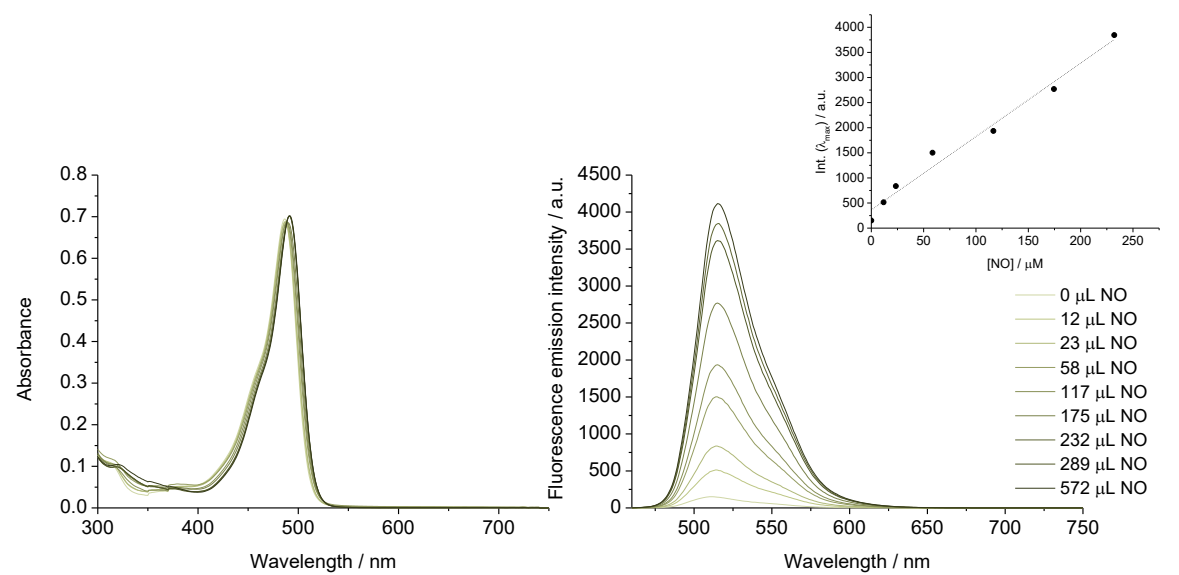

Figure 5.31. Absorption and fluorescence emission spectra $\left(\lambda_{\mathrm{ex}} 450 \mathrm{~nm}\right)$ for a DAF2-prep control in the presence of increasing amounts of NO (from 0 to $572 \mu \mathrm{M}$ ). 
After NO addition, the remarkable turn-on fluorescence response of DAF-2 after nitrosylation to give the triazole DAF-2T was displayed for the solubilised probe and the encapsulated molecule. A linear correlation was obtained in both cases between the fluorescence response and the concentration of NO until $232 \mu \mathrm{M}$. Photophysical properties are summarised in Table 5.2. A shift in absorption maximum for control and loaded nanoparticles was observed, as reported in the literature (absorption maximum from $486 \mathrm{~nm}$ for diamine to $491 \mathrm{~nm}$ for triazole; fluorescence emission at $513 \mathrm{~nm}$ for both ${ }^{75}$ ). These data confirm the formation of the triazole DAF-2T for DAF-2 into nanoparticles.

Table 5.2. Photophysical properties of DAF-2 and its triazole DAF-2T in nanoparticles and preparation control before and after $572 \mu \mathrm{M}$ NO addition.

\begin{tabular}{lcccc}
\hline \multirow{2}{*}{ Sample } & \multicolumn{2}{c}{ Absorption $\boldsymbol{\lambda}_{\max } / \mathbf{n m}$} & \multicolumn{2}{c}{ Emission $\boldsymbol{\lambda}_{\max } / \mathbf{n m}$} \\
& Before NO & After NO & Before NO & After NO \\
\hline DAF2@1 & 483 & 490 & 512 & 517 \\
DAF2-prep control & 486 & 491 & 511 & 515 \\
\hline
\end{tabular}

Fluorescence intensity at $\lambda_{\max }$ after NO addition to DAF2@1 sample and DAF2-prep control was normalized by concentration for comparison purposes (Figure 5.32). A 20-fold increase in the intensity of the fluorescence signal relative to initial fluorescence was found for DAF2@1 samples after reaction with $572 \mu \mathrm{M}$ of NO, whereas a 27 -fold increase was obtained for DAF2prep control. Therefore, DAF-2 encapsulated into our nanogels show a similar response that free DAF-2, revealing that encapsulation does not hinder NO signalling.

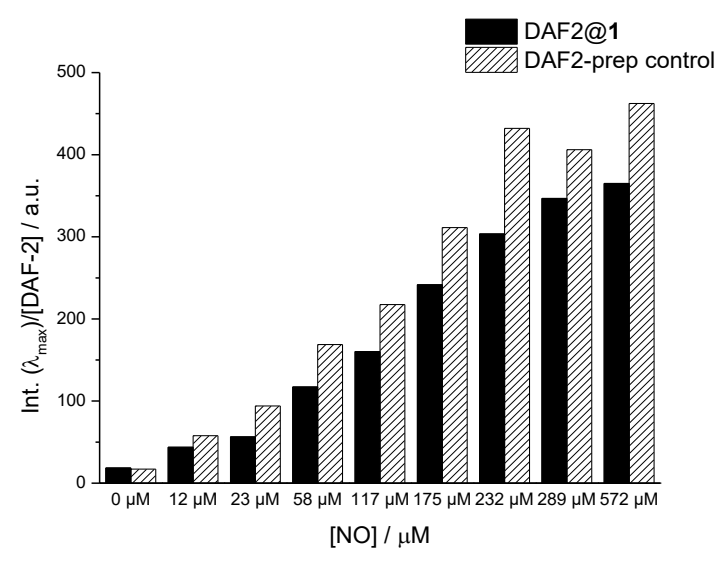

Figure 5.32. Fluorescence emission intensity $\left(\lambda_{\mathrm{ex}} 450 \mathrm{~nm}\right)$ at $\lambda_{\max }$ normalised by DAF-2 concentration for DAF2@1 (black) and DAF2-prep control (pattern) after NO addition at different concentrations. 


\subsubsection{INTRACELLULAR DAF-2 RESPONSE TO NITRIC OXIDE WHEN INTERNALISED AS DAF2/DAF2DA@1}

The applicability of our DAF2/DAF2DA@1 systems to image NO in vitro was studied by flow cytometry and confocal laser fluorescence microscopy in mouse macrophage RAW 264.7 cells. An improvement of the cellular uptake and the response to NO when DAF-2 was internalised as DAF2@1 or DAF2DA@1 were studied. The vehicle of the samples, PBS, was used as negative control, and a solution of the probe at the same concentration in PBS was used as a control of the free probe behaviour. Macrophages were chosen as they are integral to the immune response and, when activated by a foreign body, increase their production of NO to destroy or neutralise the foreign matter. ${ }^{132,133}$ The external stimulation of RAW 264.7 cells to produce NO requires interferon- $\gamma$ (INF- $\gamma$ ) and lipopolysaccharide (LPS), which activate the inducible nitric oxide synthase (iNOS). ${ }^{134,135}$ The cytotoxicity of the nanoparticles was tested before these studies to work in harmless conditions although is described later in the text (Section 5.3.3.5).

\section{CELLULAR UPTAKE ASSAYS AND RESPONSE TO NITRIC OXIDE}

The internalisation of loaded nanoparticles into RAW 264.7 cells was studied by flow cytometry, considering the intracellular fluorescence emission. For each experiment, RAW 264.7 cells were incubated for $24 \mathrm{~h}$ with a DAF2/DAF2DA@1 sample and the corresponding controls in a 1:6 dilution with FBS-, phenol red-free DMEM. After incubation, samples were removed, and cells were harvested in fresh FBS-, phenol red-free DMEM. Intracellular fluorescence emission of the cell suspension was analysed by flow cytometry ( $\lambda_{\text {ex }} 488 \mathrm{~nm}, \lambda_{\text {em }} 518-548 \mathrm{~nm}$ ).

RAW 264.7 cells were incubated with three batches of DAF2@1 (4.2, 5.1 and 5.7 $\mu$ M DAF-2) and DAF2DA@1 (5.3, 5.6 and 7.6 $\mu \mathrm{M}$ DAF-2 DA) samples and their corresponding controls. Results of intracellular fluorescence emission intensity per cell, expressed as the average of two replicas and normalised as the percentage over the negative control, are shown in Figure 5.33. In all cases, fluorescence intensity per cell over negative control is higher when the probe has been incubated encapsulated into nanoparticles. Specifically, an average of 11-times higher fluorescence intensity was achieved for DAF-2 and an average of 12-times higher for DAF-2 DA. As it has been reported that the DAF-2T triazole is formed for DAF-2 internalised into cells, this species is expected to be the fluorescence source. So, if we assume a correlation between intracellular concentration and emission, the internalisation of the DAF-2T probe is higher when

using nanoparticles. Therefore, nanoparticles encapsulation would be solving the cellular 
uptake issues of both probes: the low internalisation of DAF-2 due to its anionic nature and the low solubility of DAF-2 DA in water due to its hydrophobic character. These results could be a consequence of a different internalisation mechanism, as the probes would enter by a diffusion process and nanoparticles by endocytosis and carrying a high local loading.
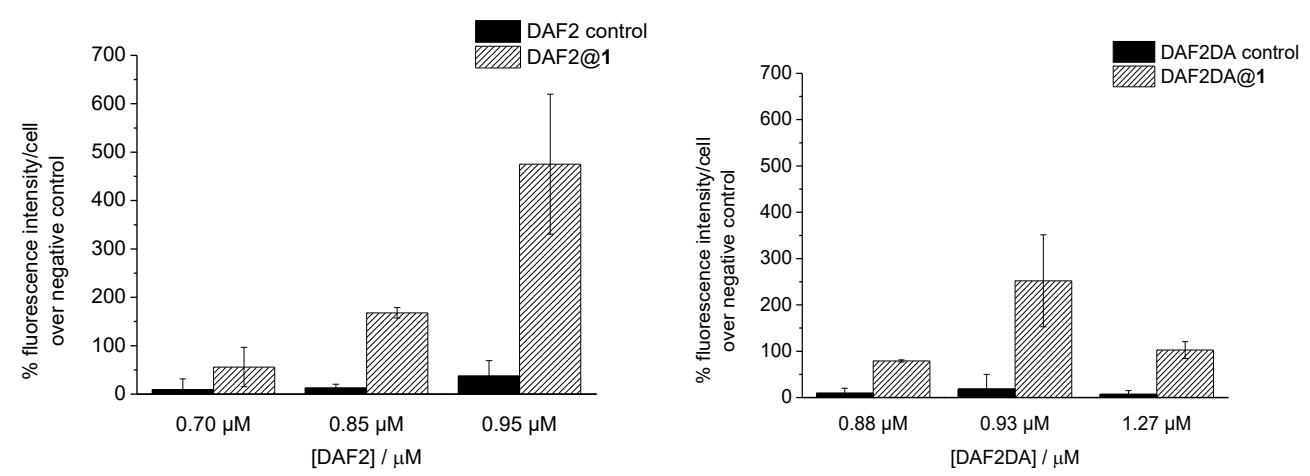

Figure 5.33. Fluorescence intensity/cell over negative control for different batches of DAF2/DAF2DA@1 (pattern) and their corresponding DAF2/DAF2DA solution controls (black) at the same concentration.

\section{NITRIC OXIDE BIOIMAGING}

The internalisation of loaded nanoparticles into RAW 264.7 cells was also studied by confocal laser fluorescence microscopy. RAW 264.7 cells were incubated overnight with a DAF2/DAF2DA@1 sample, its corresponding DAF2/DAF2DA control and the negative control in a 1:6 dilution with FBS-, phenol red-free DMEM. A DAF2@1 sample (5.3 $\mu \mathrm{M}$ DAF-2) and a DAF2DA@1 sample (5.1 MM DAF-2 DA) were tested. After incubation, cells were washed with PBS and visualised in FBS-, phenol red-free DMEM under the confocal laser fluorescence microscope ( $\lambda_{\text {ex }} 488 \mathrm{~nm}, \lambda_{\text {em }} 500-560 \mathrm{~nm}$ ), using the same parameter settings for each cell population.

Some representative images of the different populations of cells can be found in Figure 5.34. An increased intracellular fluorescence emission intensity can be observed when the probe is internalised encapsulated into nanoparticles in agreement with flow cytometry results. Moreover, cells incubated with control solutions of the probe at the same concentration showed negligible fluorescence. 10 MM DAF-2 DA are conventionally used for NO bioimaging. ${ }^{85}$ In contrast, our systems have proved to show acceptable fluorescence intensity levels using less than $1 \mu \mathrm{M}$ of the probe, which is a positive result as using the lowest dye concentration is desirable in imaging. ${ }^{10}$ The quantification of the fluorescence intensity of the cells can be seen 
in Figure 5.35. The mean fluorescence intensity/cell was 10-times higher when DAF2DA@1 were internalised than for the negative control, and 17-times higher in the case of DAF2@1.

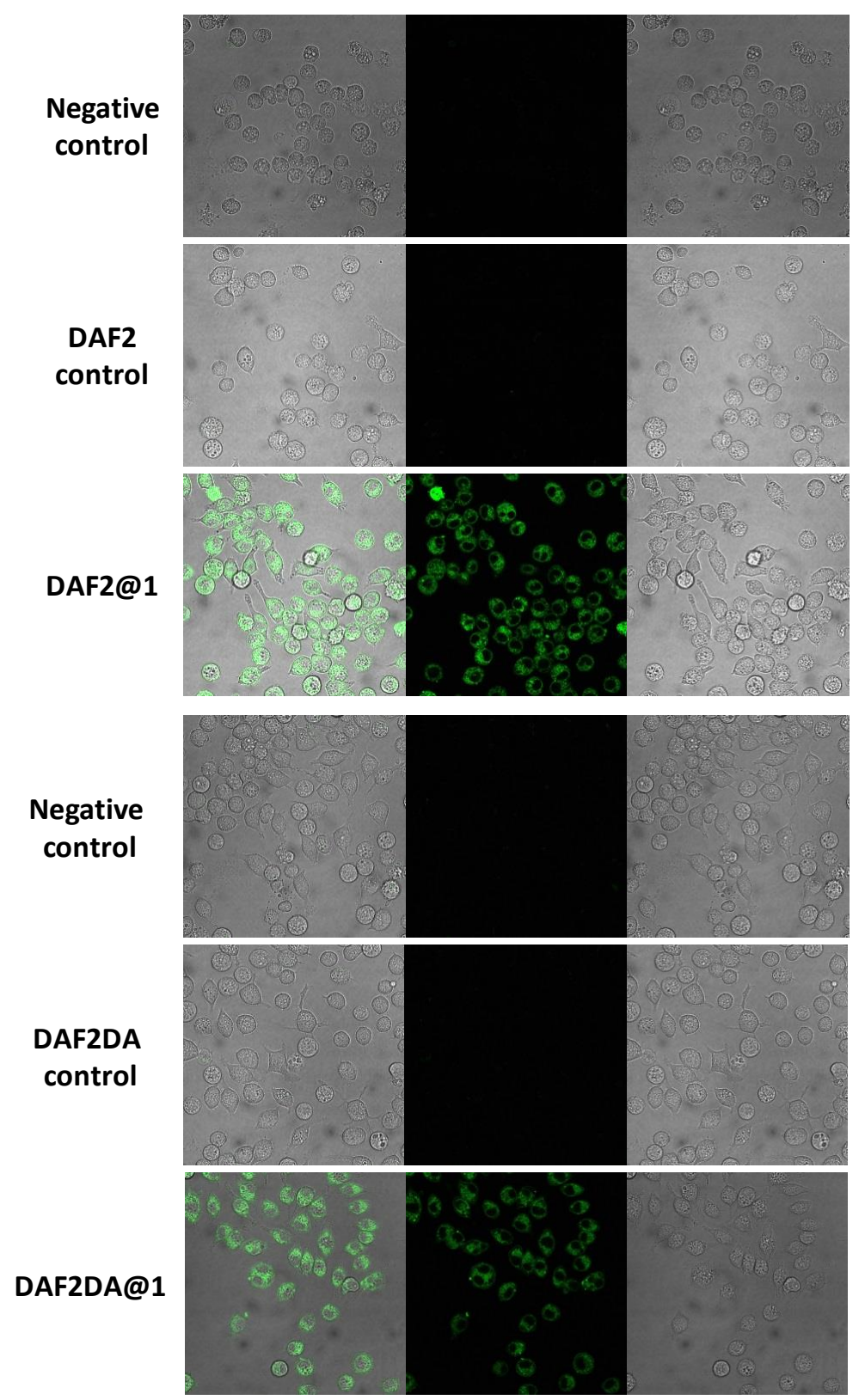

Figure 5.34. Confocal microscopy images of cells incubated overnight with a DAF2@1 sample $(0.88 \mu \mathrm{M}$ DAF-2) and a DAF2DA@1 sample (0.85 $\mathrm{MM}$ DAF-2 DA) with their corresponding controls. Bright field (right), green channel (center), merged (left). 

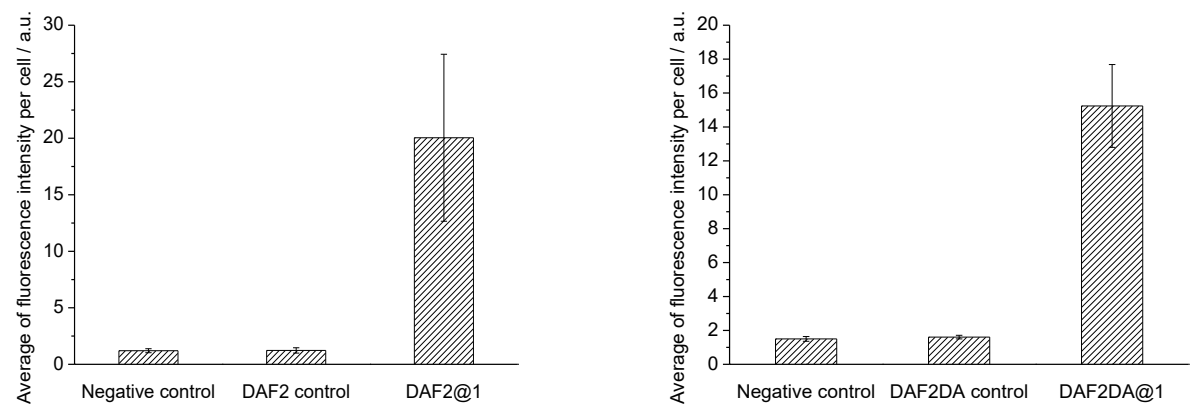

Figure 5.35. Quantification of the fluorescence intensity of the confocal microscopy images of cells (at least 100 cells per sample) incubated overnight with a DAF2@1 sample (0.88 $\mu \mathrm{M} \mathrm{DAF-2,} \mathrm{left)} \mathrm{and} \mathrm{a}$ DAF2DA@1 sample (0.85 $\mu \mathrm{M}$ DAF-2 DA, right) and their corresponding controls.

It is also noteworthy that a difference in the localisation of the triazole in cells incubated with DAF2@1 and DAF2DA@1 can be derived from the confocal microscopy images (Figure 5.36). When the DAF-2T triazole is internalised as DAF2DA@1, cell and nuclear membrane and cytoplasm are stained, whereas when DAF2@1 were used, only a cytosolic distribution was obtained. Most likely, DAF-2 DA molecules accumulate in the membrane considering their lipophilic nature. On the other hand, DAF-2 has an affinity for aqueous environments and would remain in the cytoplasm once the nanoparticles release their cargo. Finally, it should be noted that some organelles seem to be more stained, as indicated by bright points in the cytoplasm for both probes. It would be interesting to perform some co-localization experiments with probes that stain a known organelle to shed light on the triazole localisation.

DAF2@1

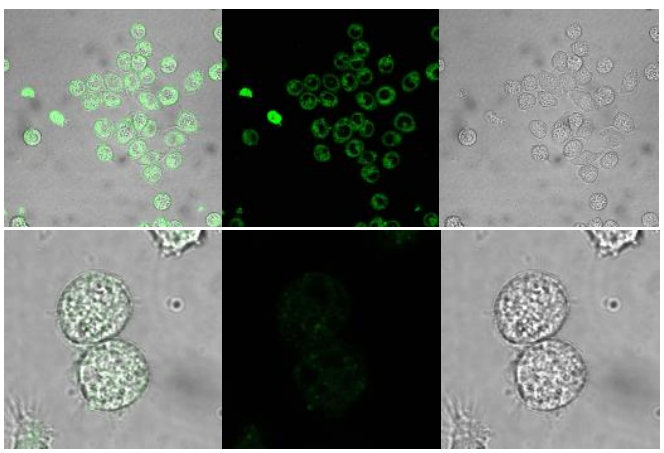

DAF2DA@1

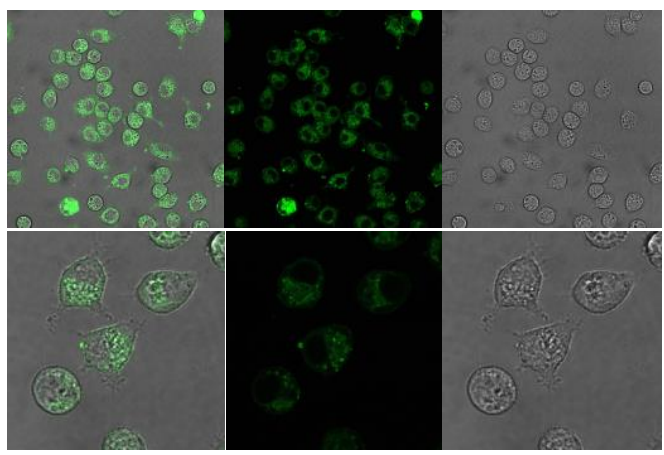

Figure 5.36. Confocal microscopy images of cells incubated overnight with a

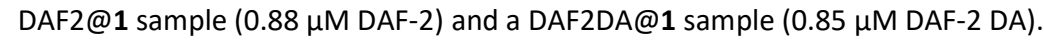
Bright field (right), green channel (centre), merged (left). 


\subsection{NANOGELS ENCAPSULATING 1,2- DIAMINOANTHRAQUINONE (DAQ)}

\subsubsection{INTRODUCTION}

Another commercially available ortho-diamino compound is 1,2-diaminoanthraquinone (DAQ or DAA). The relatively non-fluorescent DAQ (fluorescence quantum yield of $0.0004^{136}$ ) yields the triazole derivative, DAQ-TZ, after the nitrosation reaction with nitrous anhydride (Figure 5.37). ${ }^{85,86,132,136-138}$ DAQ is also sensitive to nitrite in an acid medium $\left(\mathrm{NO}_{2}{ }^{-}\right.$gives $\mathrm{N}_{2} \mathrm{O}_{3}$ as in Figure 5.38), an anion of enormous importance for its involvement in biological processes. ${ }^{87,132,136,139-}$ 143 The distinctive optical characteristics of DAQ and its triazole have been used for the NO colorimetric detection. ${ }^{132,136,139-141,143}$ DAQ in DMSO is characterized by a charge-transfer (CT) absorption band at about $540 \mathrm{~nm}$ (responsible for the intense pink-red colour of this probe in solution), which arises from the transition from the HOMO centred at one of the amino groups to the LUMO centred at the carbonyl moiety. ${ }^{144,145}$ On the contrary, DAQ-TZ exhibits no CT band and is colourless due to the absence of a donor group (free amine). ${ }^{132}$ Naked-eye colorimetric sensors consisting of DAQ into a polymeric matrix have been reported, for example in a series of poly(2-hydroxyethyl methacrylate) (PHEMA) films, as indicators of nitrite ${ }^{146}$ and nitric oxide, or both. ${ }^{137,138}$ Other example includes electrospun nanofibers from blends of poly(NIPAAm-coNMA) with DAQ. ${ }^{147}$ A real-time solid-state fluorescence type sensor for $\mathrm{NO}^{148}$ and a real-time NO sensing tip were also developed using DAQ. ${ }^{149}$

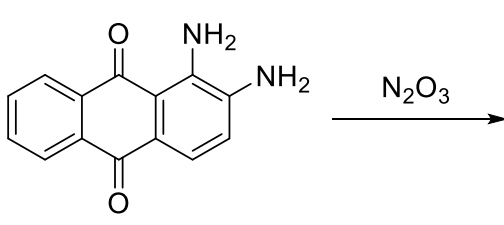

DAQ<smiles>O=C1c2ccccc2C(=O)c2c1ccc1nn[nH]c21</smiles>

DAQ-TZ

Figure 5.37. Structure of DAQ and its triazole derivative DAQ-TZ after reaction with $\mathrm{N}_{2} \mathrm{O}_{3} \cdot{ }^{132,137,138}$

$$
\begin{aligned}
\mathrm{NO}_{2}{ }^{-}+\mathrm{H}^{+} & \rightleftarrows \mathrm{HNO}_{2} \\
2 \mathrm{HNO}_{2} & \rightleftarrows \mathrm{N}_{2} \mathrm{O}_{3}+\mathrm{H}_{2} \mathrm{O}
\end{aligned}
$$

Figure 5.38. Nitrite gives $\mathrm{N}_{2} \mathrm{O}_{3}$ in acid medium. 
DAQ has also been used for the imaging of NO production in cultured hippocampal neurons ${ }^{143}$ and in rat brain slices to investigate the involvement of NO in long-term potentiation. ${ }^{139,150}$ Some advantages of the use of DAQ for NO bioimaging are its compatibility with the physiological conditions and low cost, and that is non-toxic, with high sensitivity and specificity. ${ }^{140}$

Nevertheless, DAQ has been scarcely described as a nitric oxide probe (for example, 19 papers were found in the search at Scopus "1,2-diaminoanthraquinone" "nitric oxide", whereas "4,5diaminofluorescein" and "nitric oxide" reports 376 papers). Galindo and co-workers studied the use of this compound as NO sensor to clarify, in their words, "apparent inconsistencies about the use of DAQ for the detection of NO". ${ }^{132,136}$ They found that the formation of the triazole derivative DAQ-TZ did not result in increased fluorescence in diluted solutions, but rather that DAQ-TZ shows aggregation-induced emission (AIE) at concentrations above $500 \mu \mathrm{M}$ in solution. The formation of fluorescent aggregates is a common photophysical phenomenon with many examples in the literature. ${ }^{151,152}$ Aggregates are useful for NO detection as once formed, they remain in the same place as they cannot passively diffuse, which allows the examination of the fluorescence pattern even in fixed tissues. ${ }^{35}$ However, the requirement of such a high concentration of triazole to form them represents a severe limitation to the practical use of DAQ as NO sensor. Additionally, this molecule has a notable hydrophobic character and displays a low aqueous solubility and a high tendency to aggregate in water, limiting its widespread practical application. ${ }^{139,140,143}$

\subsubsection{AIM OF THE RESEARCH REPORTED IN THIS SECTION}

In this section, the encapsulation of 1,2-diaminoanthraquinone (DAQ) into our nanoparticles is studied. Although its useful features for detecting NO under physiological conditions, DAQ widespread practical application is limited due to its hydrophobic character, which displays low aqueous solubility and a high tendency to aggregate in water, and the requirement of a high concentration of triazole for its fluorescent detection. The encapsulation aims for an improved performance of this compound as a NO sensor.

Nanogels of 1 prepared by sonication method were used and two approaches were studied to obtain DAQ@1 samples: adding DAQ as a solution to a nanoparticles sample and introducing $\mathrm{DAQ}$ in the solvent to prepare the gel. Different concentrations were tested to find the 
optimised DAQ@1 sample. The spectroscopic characteristics of the probe and its triazole derivative were studied in different environments to determine if DAQ is encapsulated into the nanoparticles and how the system changes in the presence of NO. A calibration curve using standards was also performed to determine the quantity of probe encapsulated. Finally, the response of this new DAQ@1 system to NO was evaluated both in solution and in vitro.

\subsubsection{RESULTS AND DISCUSSION}

\subsubsection{SPECTROSCOPIC CHARACTERIZATION OF DAQ AND DAQ-TZ}

Although the absorption and fluorescence emission spectra of DAQ in some solvents had been previously reported, ${ }^{132,136,137,144,145}$ the behaviour of this probe in a variety of different solvents was studied (Figure 5.39). DAQ showed to be poorly soluble in hexane and water, but is soluble in the other chosen solvents (toluene, dichloromethane, ethyl acetate, acetonitrile, acetone, tetrahydrofuran, methanol, ethanol and dimethyl sulfoxide) at the tested concentrations. DAQ absorption, fluorescence emission and excitation spectra were recorded (Figure 5.40), and maximum wavelengths of the bands for each solvent are listed in Table 5.3.

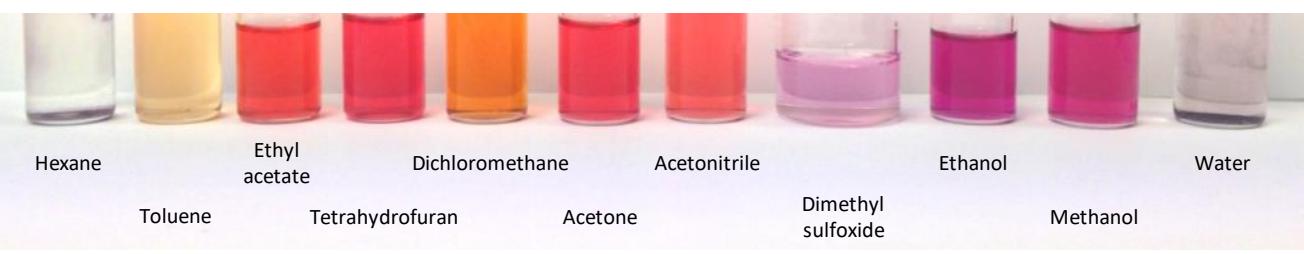

Figure 5.39. DAQ in different solvents.
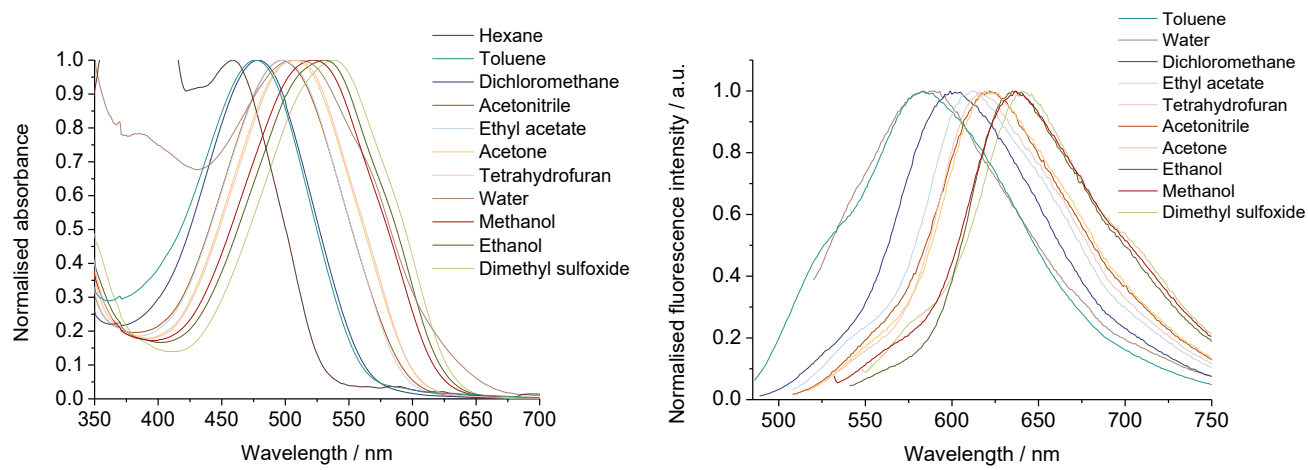

Figure 5.40. Normalised absorption (left) and fluorescence emission (right, $\lambda_{\text {ex }}$ depending on absorption $\lambda_{\max }$ for each solvent) spectra of DAQ in different solvents. 
Table 5.3. Wavelength of maximum intensity for the absorption, excitation and emission bands of DAQ in different solvents.

\begin{tabular}{lc}
\hline \multicolumn{1}{c}{ Solvent } & $\begin{array}{c}\text { Absorption } \\
\boldsymbol{\lambda}_{\max } / \mathbf{n m}\end{array}$ \\
\hline Hexane & 459 \\
Toluene & 476 \\
Dichloromethane & 479 \\
Ethyl acetate & 498 \\
Acetonitrile & 498 \\
Acetone & 506 \\
Tetrahydrofuran & 508 \\
Water & 508 \\
Methanol & 522 \\
Ethanol & 531 \\
DMSO & 538 \\
\hline
\end{tabular}

\begin{tabular}{lc}
\hline \multicolumn{1}{c}{ Solvent } & $\begin{array}{c}\text { Excitation } \\
\boldsymbol{\lambda}_{\max } / \mathbf{n m}\end{array}$ \\
\hline Hexane & 463 \\
Dichloromethane & 472 \\
Water & 491 \\
Acetonitrile & 493 \\
Ethyl acetate & 494 \\
Acetone & 501 \\
Tetrahydrofuran & 512 \\
Methanol & 533 \\
Toluene & 535 \\
Ethanol & 536 \\
DMSO & 545 \\
\hline
\end{tabular}

\begin{tabular}{lc}
\hline \multicolumn{1}{c}{ Solvent } & $\begin{array}{c}\text { Emission } \\
\lambda_{\max } / \mathbf{n m}\end{array}$ \\
\hline Hexane & 548 \\
Toluene & 583 \\
Water & 589 \\
Dichloromethane & 599 \\
Ethyl acetate & 611 \\
Tetrahydrofuran & 619 \\
Acetonitrile & 622 \\
Acetone & 623 \\
Ethanol & 635 \\
Methanol & 637 \\
DMSO & 640 \\
\hline
\end{tabular}

The $\lambda_{\max }$ in the absorption and emission spectra of DAQ depends on the solvent. In the case of absorption spectra of anthraquinones, the CT transition is greatly affected by dipolar interactions because of the large permanent dipole moment of the CT state. Changing from a non-polar to a polar medium produces a considerable reduction in the energy of the CT state and, consequently, the absorption spectrum is markedly displaced towards the red. ${ }^{153}$ Besides, DAQ shows a single absorption band in some solvents in which it is soluble (as toluene, dichloromethane, ethyl acetate, acetonitrile, acetone, tetrahydrofuran) and a band with a shoulder in alcohols (methanol and ethanol), DMSO and water (Figure 5.41). It is known that DAQ is aggregated in water, therefore the mentioned shoulder could be a measure of DAQ aggregation. Thus, although it seems to the naked eye that DAQ is soluble in alcohols and DMSO, maybe some aggregation occurs. These are the most polar solvents, so it would make sense that DAQ was not completely soluble at the concentrations tested as the aggregation of anthraquinones decreases with a decrease in the polarity of the medium. ${ }^{153}$

To confirm this hypothesis, DAQ was encapsulated in micelles of sodium dodecyl sulfate (SDS), an anionic surfactant that self-assembles in water at concentrations above $8.2 \mathrm{mM}$ (critical micelle concentration, CMC). ${ }^{154}$ From a shouldered absorption band for a suspension of aggregated DAQ in water $(100 \mu \mathrm{M})$, the addition of $20 \mathrm{mM}$ SDS provides the encapsulation of DAQ into the self-assembled micelles, which gives a pink solution and a DAQ absorption spectra of a single band (Figure 5.42). Therefore, whilst monomeric DAQ shows a single absorption band, aggregated DAQ can be detected by the apparition of a shoulder. Additionally, a shift in the $\lambda_{\max }$ towards the blue was observed for encapsulated DAQ (form $527 \mathrm{~nm}$ to $521 \mathrm{~nm}$ ). 


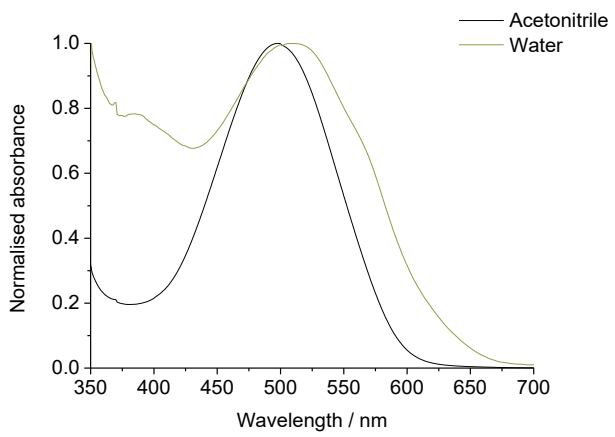

Figure 5.41. Normalised absorption spectra of $\mathrm{DAQ}$ in acetonitrile (black) and water (green).

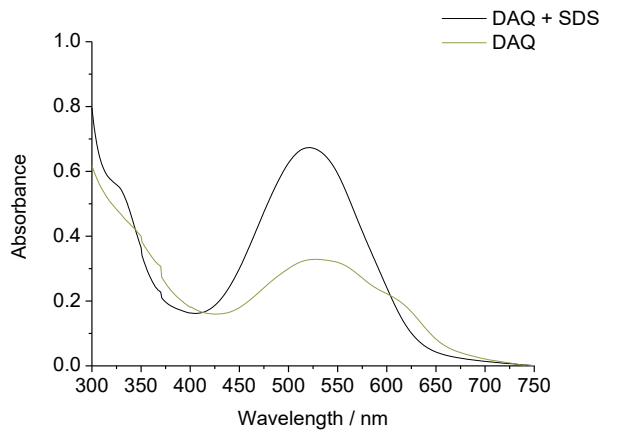

Figure 5.42. Absorption spectra of aggregated $\mathrm{DAQ}$ in water (green) and DAQ encapsulated in SDS (black).

In addition to DAQ, the spectroscopic characterization of DAQ-TZ was aimed to find differences in the properties of both molecules and determine if encapsulated DAQ has reacted to NO. As previously reported, ${ }^{132}$ DAQ-TZ was obtained from a solution of DAQ in ethanol bubbled with gaseous NO. ${ }^{129,155}$ As DAQ and DAQ-TZ are soluble in DMSO, they have been mainly studied in this solvent. ${ }^{132,136}$ The CT band of DAQ electronic absorption spectrum ( $c a .540 \mathrm{~nm}$ ) is not observed in DMSO for DAQ-TZ ${ }^{132}$ and, only at high concentrations $(>500 \mu \mathrm{M})$, the absorption spectrum of a DAA-TZ solution presents a band at $c a .350 \mathrm{~nm}$ and a shoulder at about $475-500$ $\mathrm{nm}$ (Figure 5.43 left). ${ }^{136,156}$ At these high concentrations, a tremendous increment in fluorescence emission intensity ( $\lambda_{\mathrm{ex}} 480 \mathrm{~nm}$ ) is observed (Figure $\mathbf{5 . 4 3}$ right) due to DAQ-TZ aggregation. If the concentration of DAQ-TZ is lower, fluorescence emission intensity is similar to that of DAQ at the same concentration. However, DAQ shows an emission band at $625 \mathrm{~nm}$ and a shoulder at $581 \mathrm{~nm}$, whereas DAQ-TZ emission is centred at $565 \mathrm{~nm}$ (Figure 5.44). Differences in DAQ fluorescence emission spectrum shape from that reported for the different solvent studies result from the excitation wavelength used. ${ }^{136}$ 

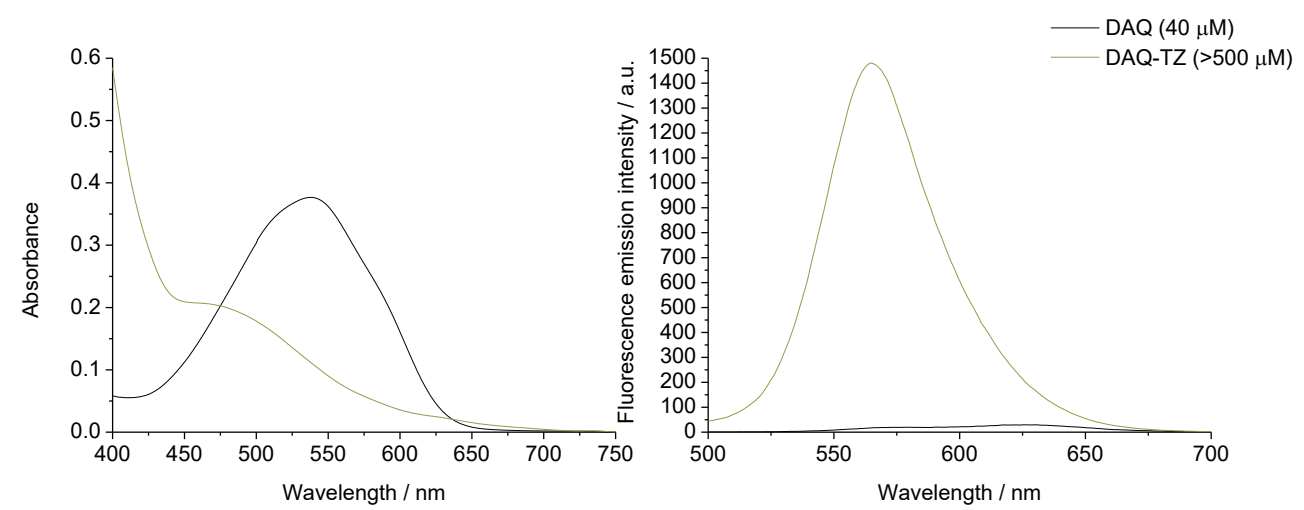

Figure 5.43. Absorption and fluorescence emission $\left(\lambda_{\mathrm{ex}} 480 \mathrm{~nm}\right)$ spectra of DAQ ( $40 \mu \mathrm{M}$, black) and DAQ$\mathrm{TZ}(>500 \mu \mathrm{M}$, green) in DMSO.

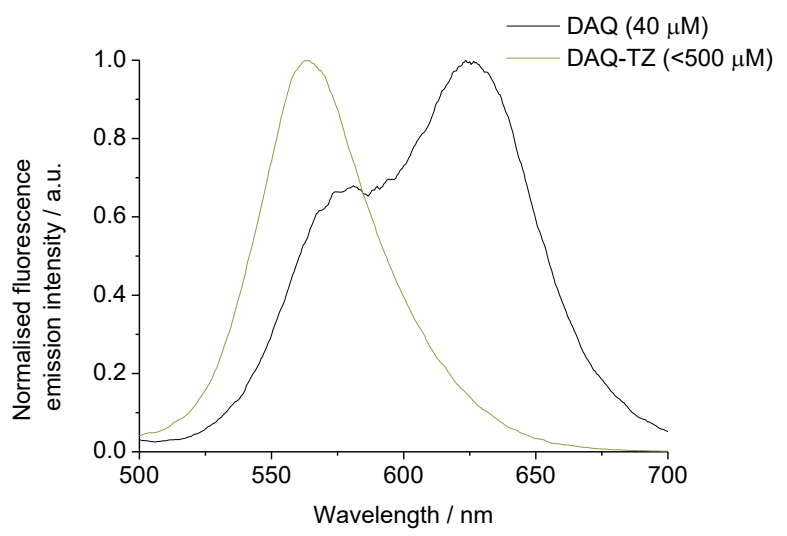

Figure 5.44. Normalised fluorescence emission $\left(\lambda_{\text {ex }} 480 \mathrm{~nm}\right)$ spectra of DAQ ( $40 \mu \mathrm{M}$, black) and DAQ-TZ $(<500 \mu \mathrm{M}$, green) in DMSO.

Triazole spectra a in a PBS-DMSO mixture were also recorded, as, up to our knowledge, it was not previously reported by its own (Figure 5.45). Although we could not obtain enough DAQ-TZ to prepare a $>500 \mu \mathrm{M}$ solution, we observed some differences between DAQ and nonaggregated DAQ-TZ. Again, the CT band of DAQ electronic absorption spectrum (ca. $520 \mathrm{~nm})^{137}$ was not observed for DAQ-TZ. As for fluorescence emission $\left(\lambda_{\mathrm{ex}} 480 \mathrm{~nm}\right)$, the intensity is similar and low for both DAQ and DAQ-TZ at these concentrations. DAQ shows a band at $569 \mathrm{~nm}$ with a shoulder at $620 \mathrm{~nm}$ and DAQ-TZ a band at $566 \mathrm{~nm}$. However, when DAQ concentration is decreased, the fluorescence emission spectrum loses that shoulder and becomes more similar to DAQ-TZ one. The way of distinguishing them would be the fluorescence intensity, much higher for DAT-TZ (when aggregated). 


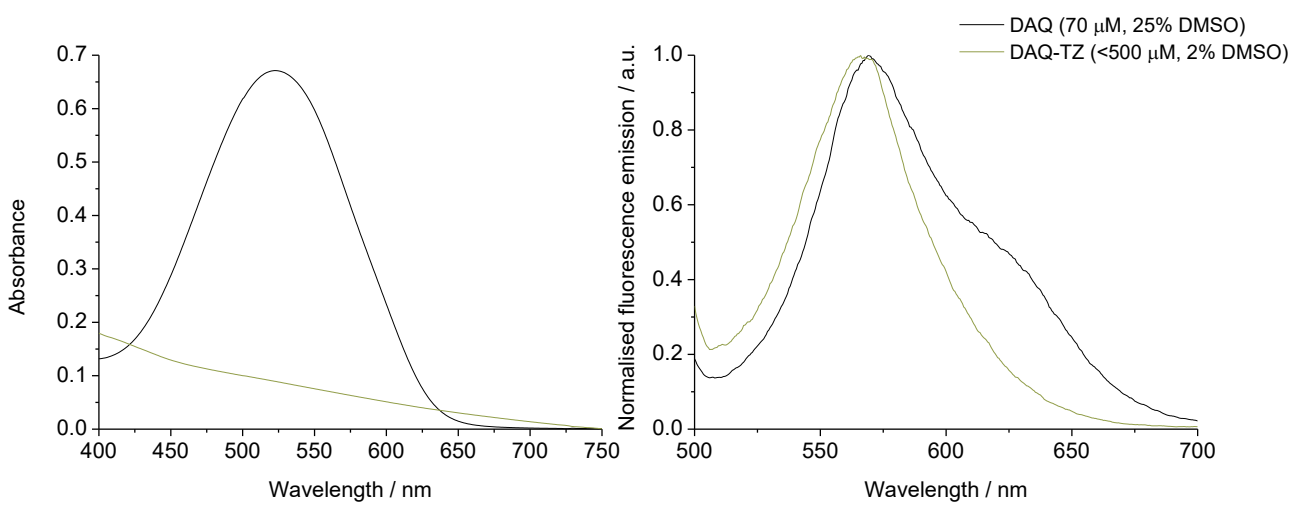

Figure 5.45. Absorption and normalised fluorescence emission $\left(\lambda_{\mathrm{ex}} 480 \mathrm{~nm}\right)$ spectra of DAQ (70 $\mu \mathrm{M}, 25 \%$ DMSO, black) and DAQ-TZ ( $<500 \mu \mathrm{M}, 2 \%$ DMSO, green) in DMSO-PBS mixtures.

\subsubsection{DAQ QUANTIFICATION}

A calibration curve to quantify DAQ encapsulated into nanoparticles was obtained after measuring the absorbance at $520 \mathrm{~nm}$ of a series of standards with concentrations ranging from 0.75 to $8 \mu \mathrm{M}$ DAQ in PBS-1\%DMSO to ensure the presence of monomeric DAQ (Figure 5.46).

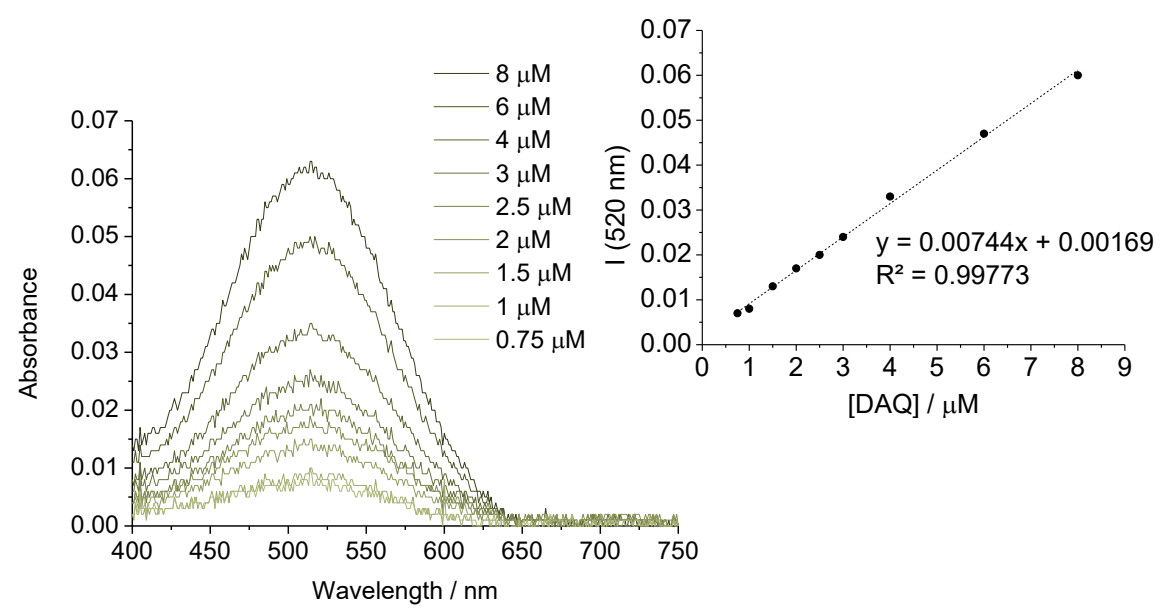

Figure 5.46. Absorption spectra of DAQ solutions in PBS-1\%DMSO at different concentrations (left) and the calibration curve to quantify its concentration (right). 


\subsubsection{DAQ ENCAPSULATION INTO NANOGELS}

The encapsulation of DAQ in nanogels (DAQ@1 samples) was approached by different strategies, together with an optimisation of the loading. The probe was successfully loaded into the nanoparticles in all of them. Changes in absorption spectra were followed to determine the encapsulation. Quantifications of the samples were performed with the calibration curve described above using absorption intensity at $520 \mathrm{~nm}$.

\section{ADDITION OF DAQ TO NANOGELS}

The first approach tested was to introduce the probe in a suspension of nanoparticles. As DAQ is hydrophobic, it was expected that it would find a place in the hydrophobic pockets of the nanoparticles rather than forming aggregates in water. DAQ was added in different volumes as a DMSO solution to $2 \mathrm{~mL}$ aliquots of nanoparticle samples to achieve different nominal concentrations. For control purposes, the process was repeated with $2 \mathrm{~mL}$ aliquots of PBS (DAQ control). Concentrated solutions of DAQ in DMSO were used to do not change the final volume of the sample significantly, and a maximum of $1.25 \%$ DMSO was used to do not interfere with nanoparticles stability. Several nanoparticle samples were prepared and mixed to achieve a composed, homogeneous sample, to avoid the effect of possible differences between batches.

Samples with DAQ nominal concentrations in the range 20-250 $\mu \mathrm{M}$ were prepared (Figure 5.47). Except for the lower concentrations of DAQ@1 (20 and $50 \mu \mathrm{M})$, all absorption spectra were shouldered, and DAQ@1 and DAQ control had a similar intensity. The aggregates in suspension precipitated after $24 \mathrm{~h}$, being the solubilized quantity of DAQ always higher for nanoparticles: DAQ in PBS is only soluble until $4 \mu \mathrm{M}$, whereas when nanoparticles are present values around $20 \mu \mathrm{M}$ could be achieved (5 times higher) (Figure 5.48). Single absorption bands for DAQ controls and shouldered bands for DAQ@1 (except for 20 and $50 \mu \mathrm{M}$ ) were obtained after precipitation of aggregates. Therefore, it seems that DAQ is being encapsulated as aggregates in nanoparticles, except for lower concentrations, in which monomeric DAQ is encapsulated. 

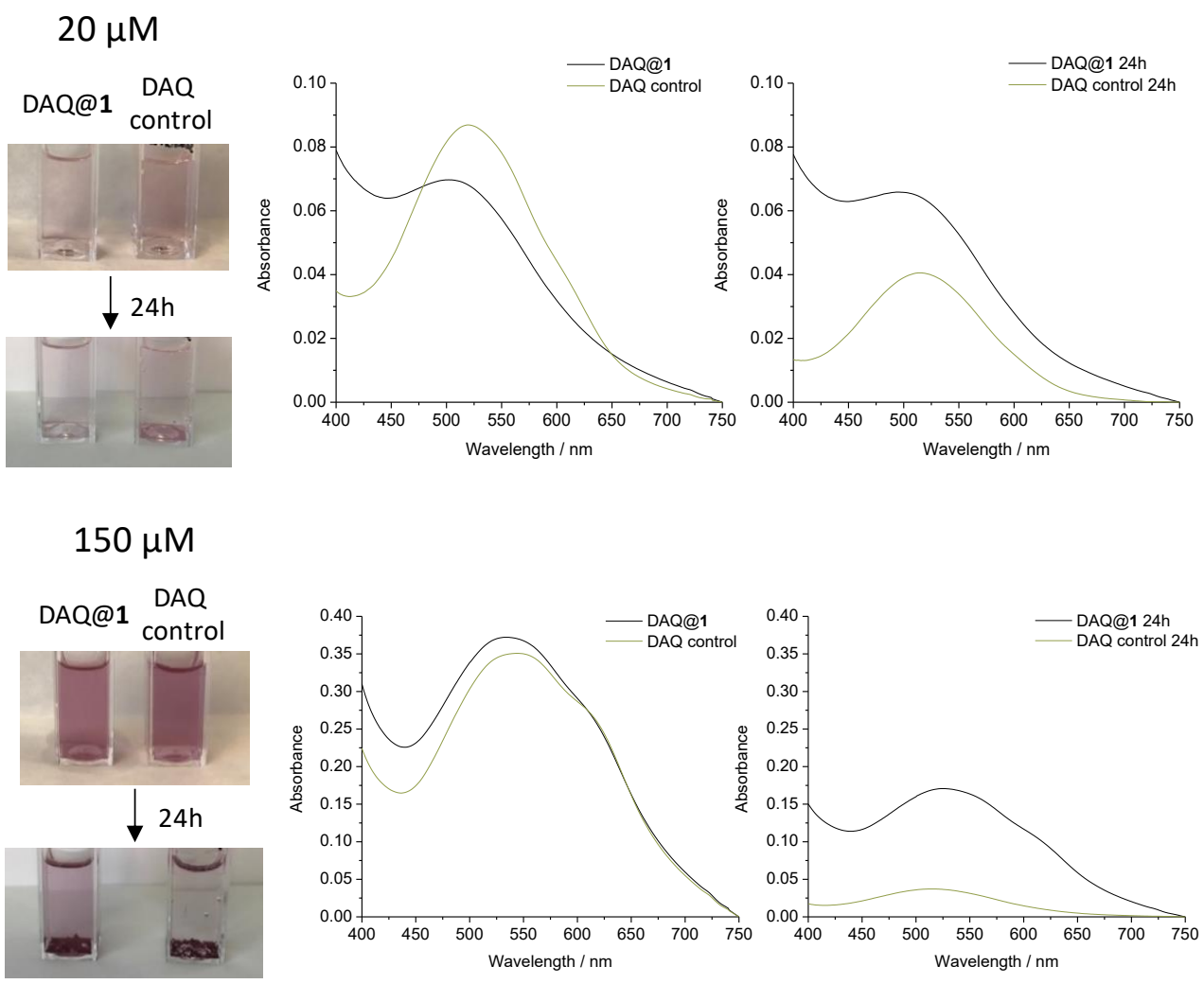

Figure 5.47. Images of the samples and absorption spectra of DAQ@1 (black) and DAQ control (green) after DAQ addition (left) and after $24 \mathrm{~h}$ (right) at two representative nominal concentrations (top: 20 $\mu \mathrm{M}$, bottom: $150 \mu \mathrm{M})$.

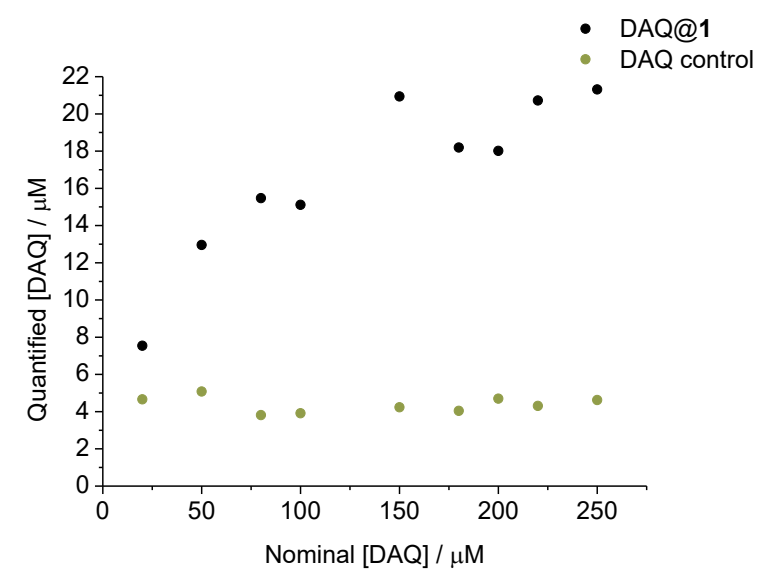

Figure 5.48. Quantification of DAQ in DAQ@1 (black) and DAQ control (green) samples at different nominal concentrations after $24 \mathrm{~h}$. 
Even though DAQ is encapsulated in the monomeric form at the lower concentrations, there is no simple way of knowing if some DAQ remains in solution in DAQ@1 samples. Vivaspin ${ }^{\mathrm{TM}}$ and centrifugation were unsuccessfully tested to remove the potential unencapsulated DAQ. For this reason, other strategies to encapsulate DAQ were preferred.

\section{ADDITION OF A DAQ SOLUTION IN THE GEL SOLVENT}

In this approach, DAQ was introduced into the system when preparing the solution of the gelator in an organic solvent in the initial step of nanoparticles preparation. DAQ@1 and DAQprep controls (following the same protocol as for DAQ@1 without gelator) were prepared at different nominal concentrations, and different solvents were tested to prepare the gel (toluene, toluene/ethyl acetate mixture and ethyl acetate), aiming to obtain a higher encapsulation depending on the initial solubility of the probe. In toluene, DAQ is mixed with gelator as a suspension, but a solution is obtained when heating. The addition of ethyl acetate increases the presence of dissolved DAQ when mixed with the gelator. Nonetheless, pink solutions were always obtained as final samples and DAQ was in monomeric form in all cases, as confirmed by a band without shoulder as absorption spectra. For all DAQ@1 samples, a shift to longer wavelengths than the corresponding to DAQ-prep controls was observed, confirming a change in the probe environment and its encapsulation. Also, DAQ was always solubilised in a higher quantity when using nanoparticles. Therefore, DAQ was encapsulated in monomeric form into the nanoparticles and increasing the solubility of DAQ in water, so this method was chosen to prepare optimised samples. Results for the different solvents are presented in the following lines. Selected spectra are representative of different samples.

Toluene gel: 2 mL DAQ@1 samples and DAQ-prep controls with nominal concentrations raging 7 to $300 \mu \mathrm{M}$ were prepared using a suspension of DAQ in toluene to prepare the gels (Figure 5.49). An average of $2.2 \mu \mathrm{M}$ DAQ was solubilised in DAQ-prep controls and even 12.7 $\mu \mathrm{M}$ when DAQ was encapsulated (Figure 5.51). For the example in Figure 5.50, maximum absorption shifted from $491 \mathrm{~nm}$ for DAQ-prep control to 503 nm for DAQ@1.

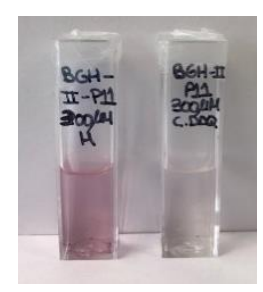

Figure 5.49. DAQ@1 (left) and DAQ-prep control (right) prepared from a toluene gel $(300 \mu \mathrm{M}$ nominal concentration). 


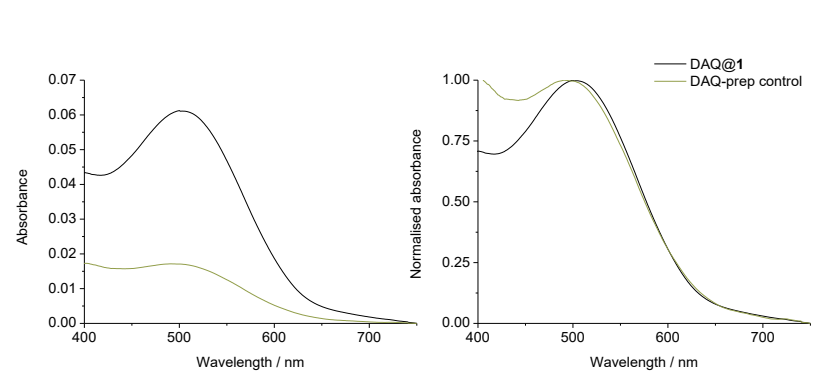

Figure 5.50. Absorption spectra of DAQ@1 (black) and DAQprep control (green) prepared from a toluene gel $(100 \mu \mathrm{M}$ nominal concentration).

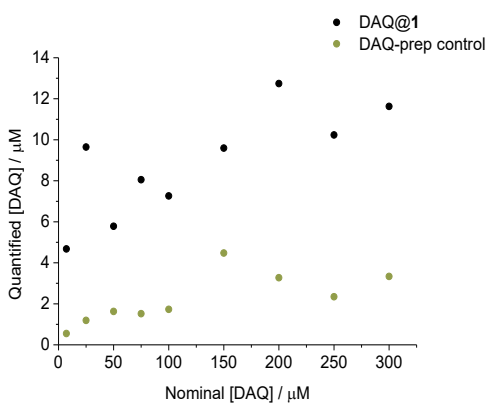

Figure 5.51. Quantification of DAQ in DAQ@1 (black) and DAQ-prep controls (green) prepared from a toluene gel.

Toluene/ethyl acetate gel: $2 \mathrm{~mL}$ DAQ@1 samples and DAQ-prep controls with nominal concentrations ranging from 1 to $250 \mu \mathrm{M} \mathrm{DAQ}$ were prepared from gels of toluene-7\%AcOEt (Figure 5.52). The average quantity of solubilised DAQ was $0.7 \mu \mathrm{M}$ for DAQ-prep controls, whereas nanoparticles help to solubilise until $14 \mu \mathrm{M}$ (20 times higher) (Figure 5.54). For the example in Figure 5.53, a $6 \mathrm{~nm}$ shift to longer wavelengths is observed when DAQ is encapsulated, from $502 \mathrm{~nm}$ for DAQ-prep control to 508 nm for DAQ@1.

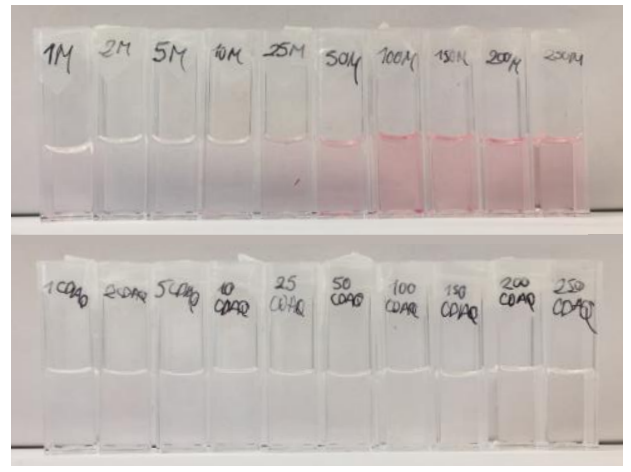

Figure 5.52. DAQ@1 samples (top) and DAQprep controls (bottom) prepared from gels of toluene-7\%AcOEt at increasing nominal concentrations.

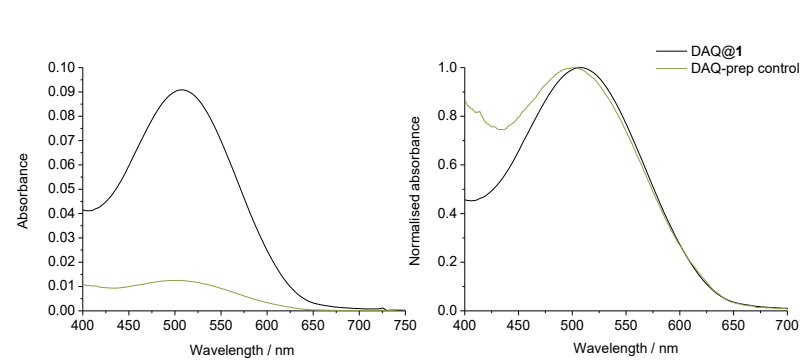

Figure 5.53. Absorption spectra of DAQ@1 (black) and DAQprep control (green) prepared from a toluene/AcOEt gel (250 $\mu \mathrm{M}$ nominal concentration).

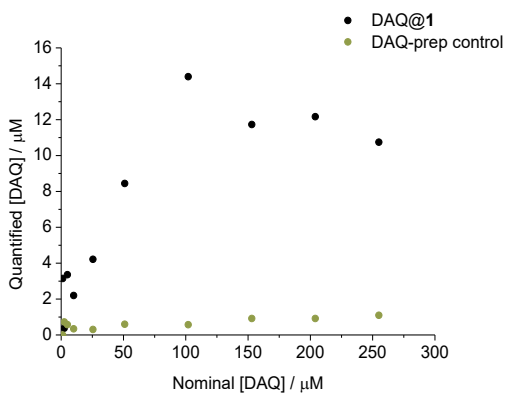

Figure 5.54. Quantification of DAQ in DAQ@1 (black) and DAQ-prep controls (green) prepared from a toluene/AcOEt gel. 
Ethyl acetate gel: 2mL DAQ@1 samples and DAQ-prep controls with nominal concentrations ranging from 1 to $250 \mu \mathrm{M}$ DAQ were prepared using solutions of DAQ in AcOEt to prepare the gels. Average solubilisation of $0.5 \mu \mathrm{M}$ DAQ was obtained for DAQ-prep controls, whereas even 16.4 $\mu \mathrm{M}$ could be solubilized when using nanoparticles (33 times higher) (Figure 5.56). Absorption spectra showed an average shift of $c a .5 \mathrm{~nm}$ to longer wavelengths when DAQ was encapsulated (DAQ@1 has a maximum at 504 nm whereas DAQ-prep control has it at 500 nm in the example of Figure $\mathbf{5 . 5 5}$ right).

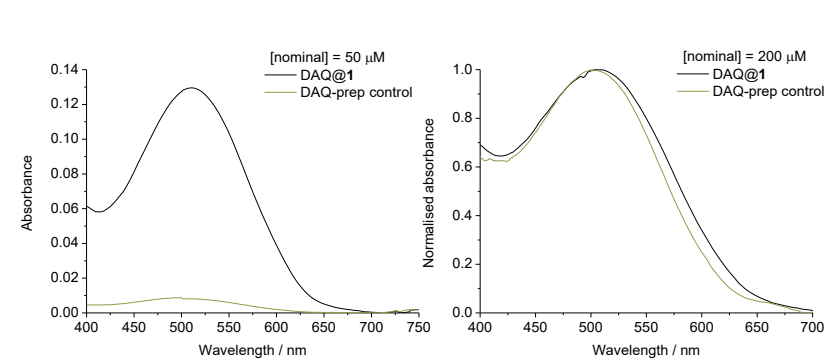

Figure 5.55. Absorption spectra of DAQ@1 (black) and DAQprep control (green) prepared from an AcOEt gel.

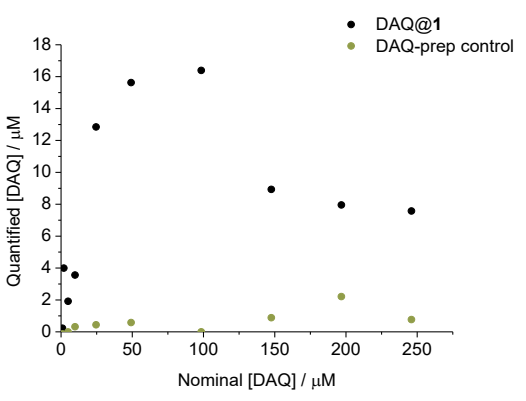

Figure 5.56. Quantification of DAQ in DAQ@1 (black) and DAQ-prep controls (green) prepared from an AcOEt gel.

A higher loading was obtained when the probe was more solubilised in the solvent to prepare the gel, maybe due to a higher interaction time of the gelator and individual DAQ molecules. However, although the highest encapsulation can be found for ethyl acetate, a toluene7\%AcOEt solution was chosen to prepare optimised samples because aggregates (and not a gel) are obtained in the AcOEt method. As for the nominal concentration of these optimised samples, $100 \mu \mathrm{M}$ DAQ was chosen as is the lowest nominal concentration with which the highest concentration of encapsulated DAQ can be obtained. Some pictures of the optimised sample preparation process and a representative example of absorption and fluorescence emission of these samples are shown in Figure 5.57 and Figure 5.58. DAQ loaded into nanoparticles shows a low fluorescence emission band at $c a .630 \mathrm{~nm}$ with a shoulder at $c a .580 \mathrm{~nm}$. This would be another confirmation that DAQ is in monomeric form, as it is the same spectrum as DAQ dissolved in DMSO (Figure 5.44). On the other hand, DAQ-prep controls only show a band at $c a$. $580 \mathrm{~nm}$, as expected for DAQ in water at low concentrations (Figure 5.45). The quantified DAQ concentration for different DAQ@1 samples and DAQ-prep controls of $100 \mu \mathrm{M}$ nominal concentration (Figure 5.59) revealed an average of 9.3 $\pm 2.2 \mu \mathrm{M}$ DAQ for DAQ@1 samples (0.2- 
$0.4 \% \mathrm{w} / \mathrm{w}$ of probe loading in the nanoparticles), whereas $2.1 \pm 0.9 \mu \mathrm{M} \mathrm{DAQ}$ was found in $\mathrm{DAQ}$ prep controls.

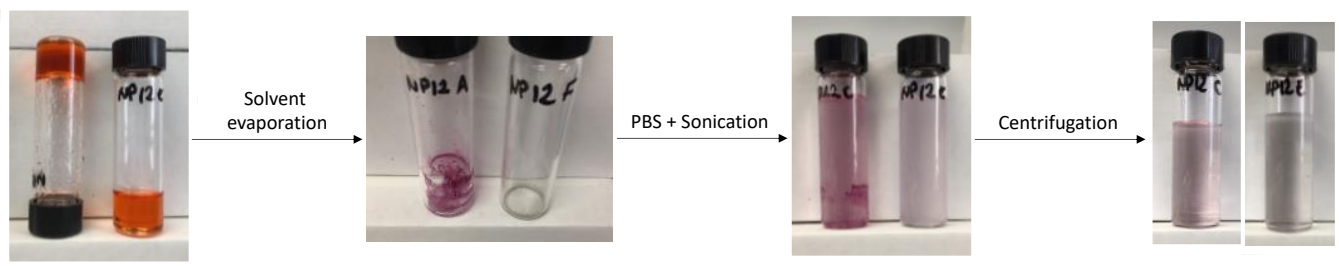

Figure 5.57. Preparation process of a DAQ@1 sample (left) and its DAQ-prep control (right).

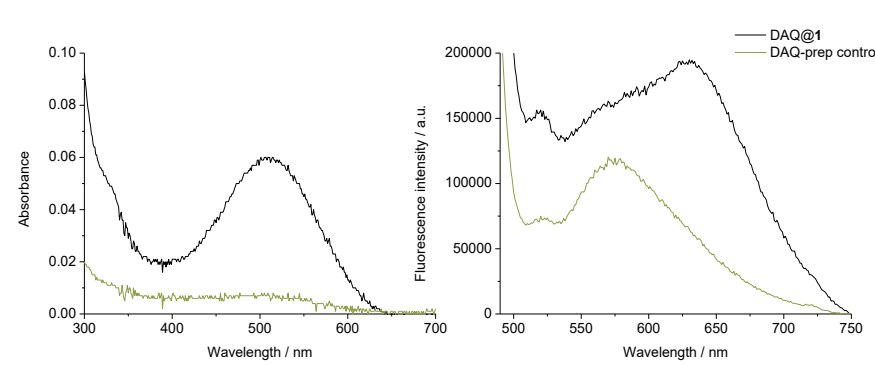

Figure 5.58. Absorption (left) and fluorescence emission (right, $\lambda_{\text {ex }} 480 \mathrm{~nm}$ ) spectra of a DAQ@1 sample (black, $7.7 \mu \mathrm{M}$ DAQ) and a DAQ-prep control (green, $0.7 \mu \mathrm{M} \mathrm{DAQ}$ ).

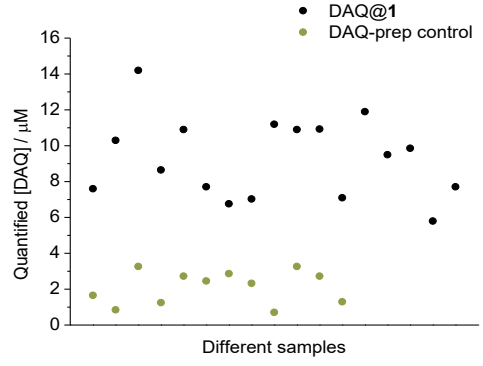

Figure 5.59. Quantified DAQ concentration for different DAQ@1 (black) and DAQ-prep controls (green) of $100 \mu \mathrm{M}$ nominal concentration.

The size of DAQ@1 samples was measured by DLS (Figure 5.60), revealing no changes due to DAQ encapsulation. A representative example of the size distribution is shown in Figure 5.61. For 10 different DAQ-loaded nanogel samples, an average of $170 \pm 40 \mathrm{~nm}$ was obtained as intensity averaged diameter $\left(D_{\mathrm{i}}\right)$ and of $43 \pm 17 \mathrm{~nm}$ as number averaged diameter $\left(D_{\mathrm{n}}\right)$, with a PDI in the range $0.2-0.4$. 

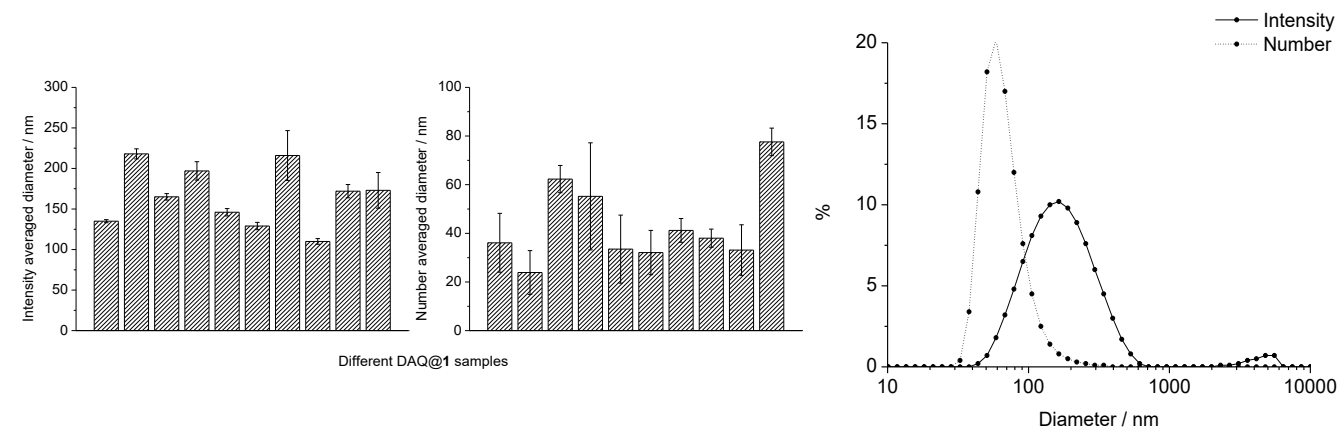

Figure 5.60. Intensity and number averaged diameters of 10 different DAQ@1 samples obtained by DLS.

Figure 5.61. Size distribution by intensity (solid line) and by number (dotted line) of a representative DAQ@1 sample obtained by DLS analysis.

\subsubsection{DAQ-LOADED NANOPARTICLES RESPONSE TO NITRIC OXIDE}

Next, the fluorescence response of DAQ-loaded nanogels towards NO was studied. As in Section 5.2.3.5, the commercial NO donor DEA NONOate was used to check the triazole formation.

In our experiment, incremental volumes of a $15 \mathrm{mM}$ stock solution of DEA NONOate in $0.01 \mathrm{M}$ $\mathrm{NaOH}$ were added to $2 \mathrm{~mL}$ aliquots of a DAQ@1 sample (6.8 $\mu \mathrm{M} \mathrm{DAQ})$, to reach solutions with NO concentrations ranging from 20 to $1000 \mu \mathrm{M}$. For control purposes, the titration was repeated with a DAQ-prep control (2.2 $\mu \mathrm{M} \mathrm{DAQ})$ to test what happens to DAQ in an aqueous solution. Samples were air-equilibrated so the triazole formation reaction could proceed, and the $\mathrm{pH}$ was checked to be 6.7-7.1 for final solutions after NONOate addition. The response of DAQ to NO was checked by absorption and fluorescence emission ( $\lambda_{\text {ex }} 480 \mathrm{~nm}$ ) spectroscopy. Overnight results of NONOate titrations are shown in Figure $\mathbf{5 . 6 2 .}$

According to Section 5.3.3.1, a decrease of the DAQ absorption band and an increase of fluorescence emission due to the formation of DAQ-TZ were expected. In our case, a decrease of DAQ maxima (ca. $505 \mathrm{~nm}$ ) in absorption spectra and the apparition of a peak at ca. $340 \mathrm{~nm}$ were observed for DAQ@1 sample and DAQ-prep control for increasing NO concentrations. Fluorescence emission shows a decrease in both samples when NO is added, in ca. $630 \mathrm{~nm}$ peak for DAQ@1 and in ca. 580 nm peak for DAQ-prep control, with a similar decrease (24\%) in both cases. 

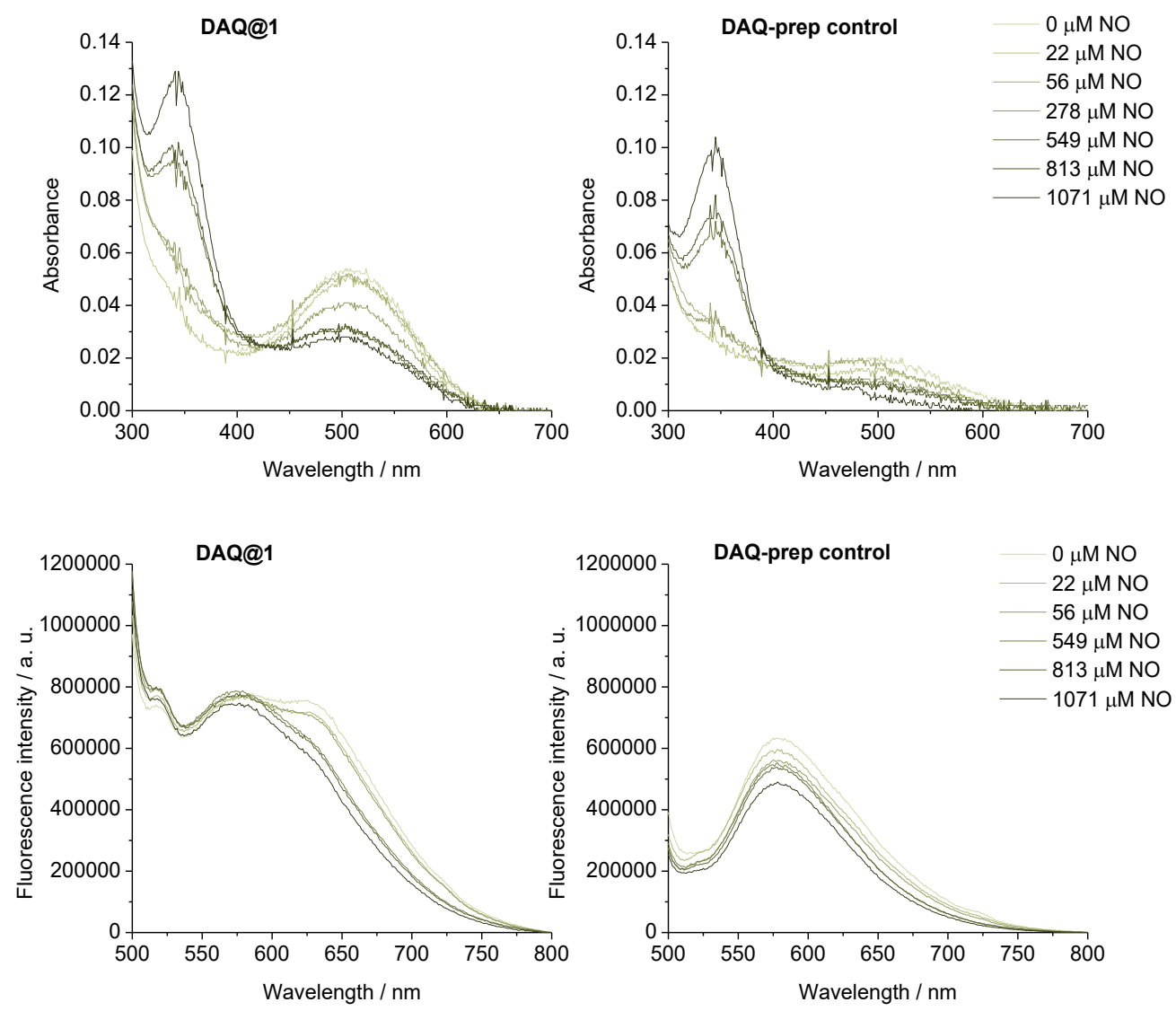

Figure 5.62. Changes in absorption (top) and fluorescence emission (bottom, $\lambda_{\mathrm{ex}} 480 \mathrm{~nm}$ ) spectra of a DAQ@1 sample (left, 6.8 $\mu \mathrm{M}$ DAQ) and a DAQ-prep control (right, $2.2 \mu \mathrm{M} \mathrm{DAQ}$ ) after $24 \mathrm{~h}$ of the addition of 22-1071 $\mu \mathrm{M}$ NO.

The band formed in absorption spectra corresponds to some derivative of NONOate or NO in water, as the apparition of a new band at $c a .350 \mathrm{~nm}$ was also seen for a PBS control solution to which a final $1071 \mu \mathrm{M}$ NO was added (Figure 5.63). This band apparition has also been observed in literature. ${ }^{136,137,146}$ 


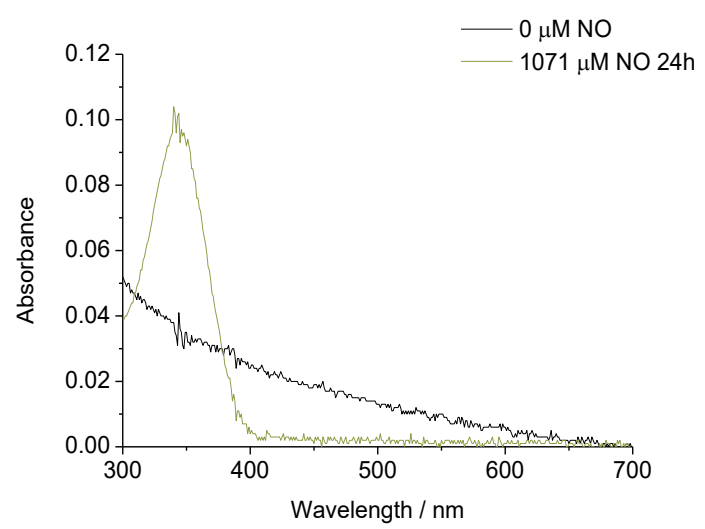

Figure 5.63. Absorption spectra of a PBS control before (black) and after $24 \mathrm{~h}$ (green) addition of NO.

The change in the volume and $\mathrm{pH}$ of the sample aliquot once NONOate solution is added was also checked not to decrease the DAQ band. With this objective, $100 \mu \mathrm{L}$ of NONOate (which gives $1071 \mu \mathrm{M}$ NO) or $\mathrm{NaOH}$ 0.01M were added to $2 \mathrm{~mL}$ aliquots of a DAQ@1 sample (7.7 $\mu \mathrm{M}$ DAQ), and the effect over time was checked by absorption spectroscopy (Figure 5.64). A decrease in absorption peak was only observed for NO, meaning no effect of $\mathrm{NaOH}$.
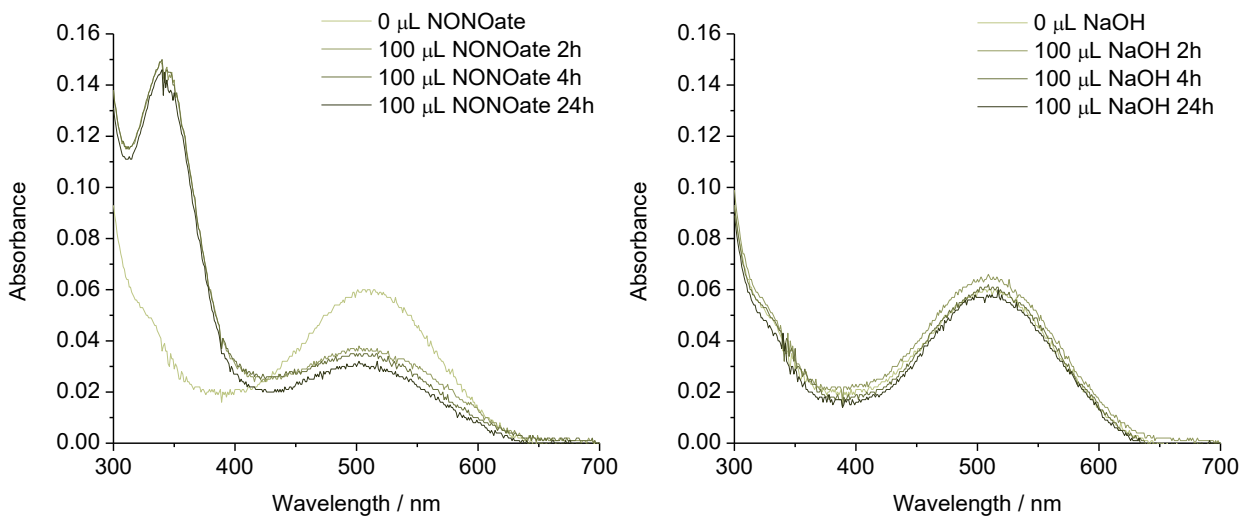

Figure 5.64. Changes in absorption spectra of a DAQ@1 sample (7.7 $\mu \mathrm{M} \mathrm{DAQ})$ after addition of $100 \mu \mathrm{L}$ NONOate (1071 $\mu \mathrm{M}$ NO) (left) or $100 \mu \mathrm{L} \mathrm{NaOH} 0.01 \mathrm{M}$ (right) at different times.

Finally, the response of DAQ to NO was also studied for a control of DAQ in DMSO at a concentration as in DAQ@1 (10 $\mu \mathrm{M}$, DAQ-DMSO control), to emulate the environment of DAQ inside the nanoparticles. Absorption and fluorescence emission ( $\lambda_{\text {ex }} 500 \mathrm{~nm}$ ) measures were performed over time after the addition of $1071 \mu \mathrm{M}$ NO (Figure 5.65). Although this study was performed over time instead of over different concentrations, the last time corresponds to DAQ@1 sample reaction time. Results are quite similar to those of DAQ@1 sample: a decrease 
in DAQ absorption band ( $535 \mathrm{~nm}$ ) and in ca. $640 \mathrm{~nm}$ fluorescence emission band (60\% of the original), whereas $c a .570 \mathrm{~nm}$ remains without changes.
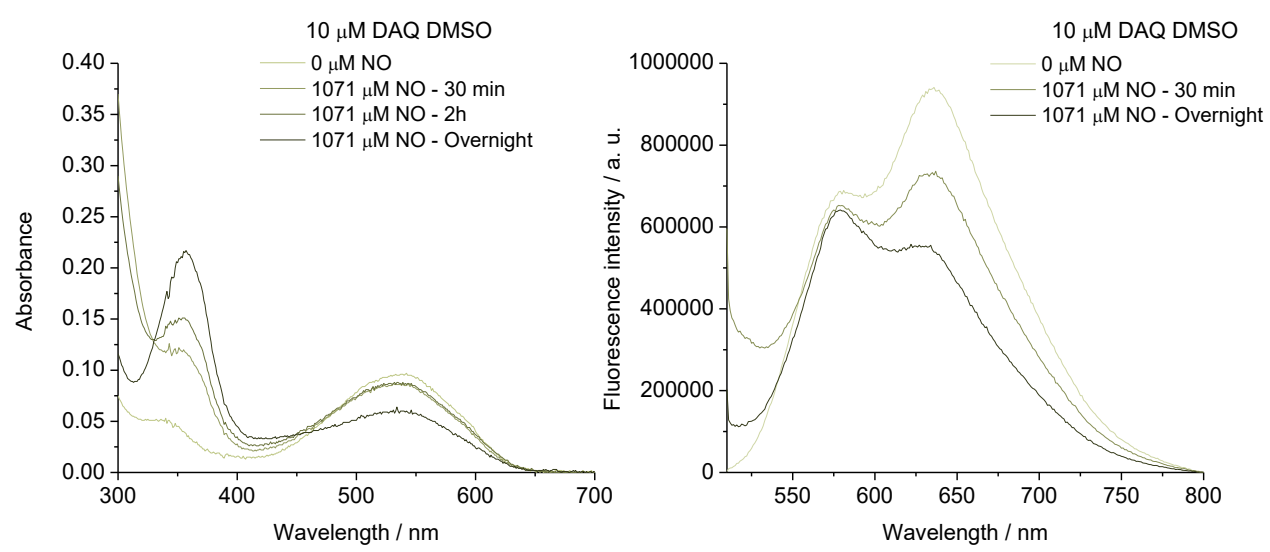

Figure 5.65. Changes in absorption (left) and fluorescence emission (right, $\lambda_{\text {ex }} 500 \mathrm{~nm}$ ) spectra of a 10 $\mu \mathrm{M}$ DAQ solution in DMSO after addition of $1071 \mu \mathrm{M}$ NO at different times.

Therefore, it seems that DAQ in DAQ@1 responds to NO as when it is in a DMSO solution. The decrease of the absorption band means that DAQ is reacting to form the colourless triazole, which is also observed by the decrease of its fluorescence (and the maintenance of the $580 \mathrm{~nm}$ peak). The increase of the fluorescence of the triazole was most probably not seen in these experiments because of the concentrations used (a concentration of $500 \mu \mathrm{M}$ has been reported to be needed to observe DAQ-TZ fluorescence emission in solution ${ }^{132}$ ). Nevertheless, DAQ is reacting, and higher concentrations are expected to be reached inside cells in biological experiments.

\subsubsection{INTRACELLULAR DAQ RESPONSE TO NITRIC OXIDE WHEN INTERNALISED AS DAQ@1}

The applicability of our DAQ@1 systems to image NO in vitro was studied by confocal laser fluorescence microscopy in RAW 264.7 macrophage cells as in Section 5.2.3.6. An improvement of the cellular uptake and the response to NO when DAQ was internalised as DAQ@1 were studied. 


\section{NANOGELS TOXICITY}

The toxicity of our nanoparticles on this cell line was assessed using CellTiter-Blue ${ }^{\circledR}$ cell viability assay, ${ }^{157-159}$ a fluorometric method for estimating the number of viable cells (Figure 5.66). Resazurin is used to measure the metabolic capacity of cells as an indicator of cell viability: viable cells retain the ability to reduce the non-fluorescent resazurin into resorufin, which is highly fluorescent, whereas non-viable cells rapidly lose metabolic capacity, do not reduce the indicator dye, and thus do not generate a fluorescent signal. Therefore, the fluorescence produced is proportional to the number of viable cells.

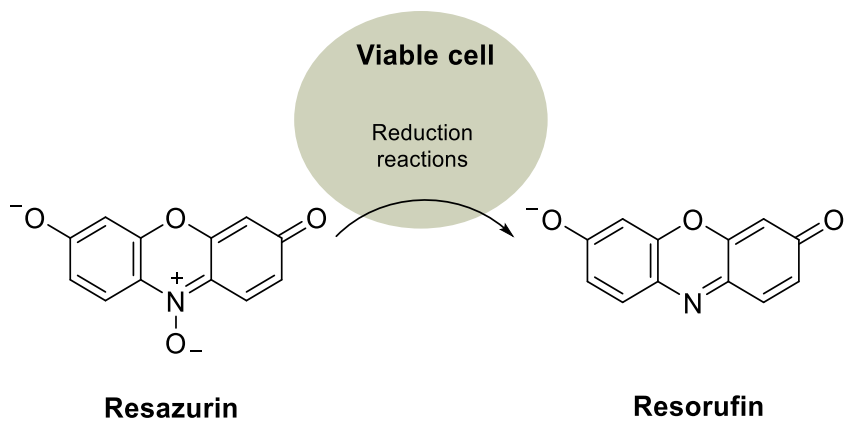

Figure 5.66. Reduction reaction of resazurin to resorufin by metabolically active cells. ${ }^{158}$

RAW 264.7 cells were incubated for $48 \mathrm{~h}$ with serial dilutions (from 1:2.5 to 1:80) of a DAQ@1 sample in medium. After the addition of the CellTiter-Blue ${ }^{\circledR}$ reagent and $4 \mathrm{~h}$ incubation, fluorescence emission was measured (at $590 \mathrm{~nm}$ following excitation at $560 \mathrm{~nm}$ ). Cell viability was calculated as the percentage of non-treated cells (negative control) average. All samples were tested in triplicate and Grubbs test was used to remove outliers. ${ }^{160}$ Several controls were also tested: a positive control for cytotoxicity ( $20 \mu \mathrm{M}$ staurosporine in DMEM-2\%DMSO, which is completely toxic to the cells by induction of apoptosis ${ }^{159,161}$ ) and the vehicle of the samples at the same proportions (PBS). Results can be seen in Figure 5.67. 

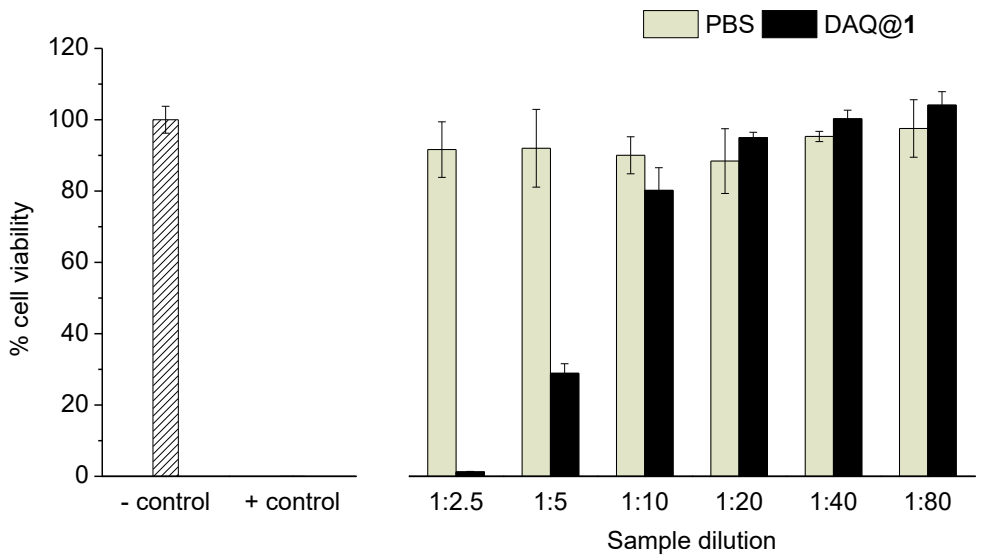

Figure 5.67. CellTiter-Blue ${ }^{\circledR}$ cell viability assay for RAW 264.7 cells incubated with different dilutions of a DAQ@1 sample (black) and the vehicle of the samples (PBS, green) at the same proportion. Error bars represent the standard deviation $(n=3)$. Staurosporine was used as a positive control for cytotoxicity.

Taking into consideration that negative control (cells treated with DMEM) showed a $100 \%$ cell viability and that positive control (staurosporine), a 0\% cell viability, results show that DAQ@1 have some kind of toxicity after $48 \mathrm{~h}$ incubation at the two smallest dilutions (1:2.5 and 1:5). Almost no decrease in viability is observed for higher dilutions than 1:10.

To be more precise about the dilution chosen, viability of the cells for 1:6 and 1:8 dilutions was determined using the Trypan blue exclusion method as in Section 4.4.2 for HT-29 cells. RAW 264.7 cells were incubated for $24 \mathrm{~h}$ with the different dilutions of two nanoparticle samples and PBS at the same proportions as negative control. The percentage of viable and non-viable cells is shown in Figure 5.68, expressed as the average of two replicas. The percentage of viability is quite high for cells incubated at both dilutions. Therefore, a 1:6 dilution, representing about 400 $\mu \mathrm{M}$ of gelator, was chosen for the following experiments using nanogels in RAW 264.7 cells. 


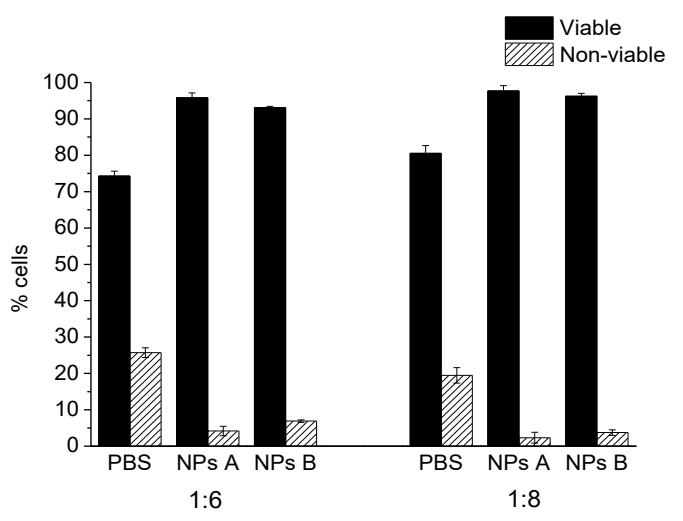

Figure 5.68. Percentage of viable (black) and non-viable (pattern) cells after $24 \mathrm{~h}$ incubation with negative control and two nanoparticle samples. Error bars represent the standard deviation $(n=2)$.

\section{NITRIC OXIDE BIOIMAGING}

The internalisation of DAQ-loaded nanoparticles into RAW 264.7 cells was studied by confocal laser fluorescence microscopy. For imaging experiments, RAW 264.7 cells were incubated overnight with a DAQ@1 sample (9.5 $\mu \mathrm{M}$ DAQ) in a 1:6 dilution in FBS-, phenol red-free DMEM. The vehicle of the sample, PBS, was used as negative control, and a solution of DAQ at the same concentration in PBS-0.5\%DMSO (DAQ-DMSO control) was used as a control of free probe behaviour. The external stimulation of the macrophage cells to produce nitric oxide was achieved by the addition of interferon- $\gamma($ INF- $\gamma, 0.01 \mu \mathrm{g} / \mathrm{mL}$ ) and lipopolysaccharide (LPS, 0.5 $\mu \mathrm{g} / \mathrm{mL}){ }^{132,135}$ For comparison purposes, incubations were duplicated, one replica stimulated and the other not. After incubation, cells were imaged under confocal microscopy ( $\lambda_{\text {ex }} 488 \mathrm{~nm}$, $\left.\lambda_{\mathrm{em}} 505-750\right)$ at $37^{\circ} \mathrm{C}$ in imaging medium. Imaging medium was used instead of DMEM as this latter is known to interfere with cell imaging (contains fluorescent riboflavins and phenol red, which quenches fluorescence and strongly absorbs light in the red region of the electromagnetic spectrum $^{134,162}$ ) and incubations were performed in a medium free of FBS to promote starving conditions or avoid fluorescence interferences. The probes internalised in cells were excited at $488 \mathrm{~nm}$ in agreement with previous NO-imaging reports of DAQ. ${ }^{136}$ Some representative images of stimulated and non-stimulated cells incubated with the indicated samples can be seen in

Figure 5.69. As all cell populations were visualised using the same parameter settings, the fluorescence intensity analysis is shown in Figure $\mathbf{5 . 7 0 .}$ 


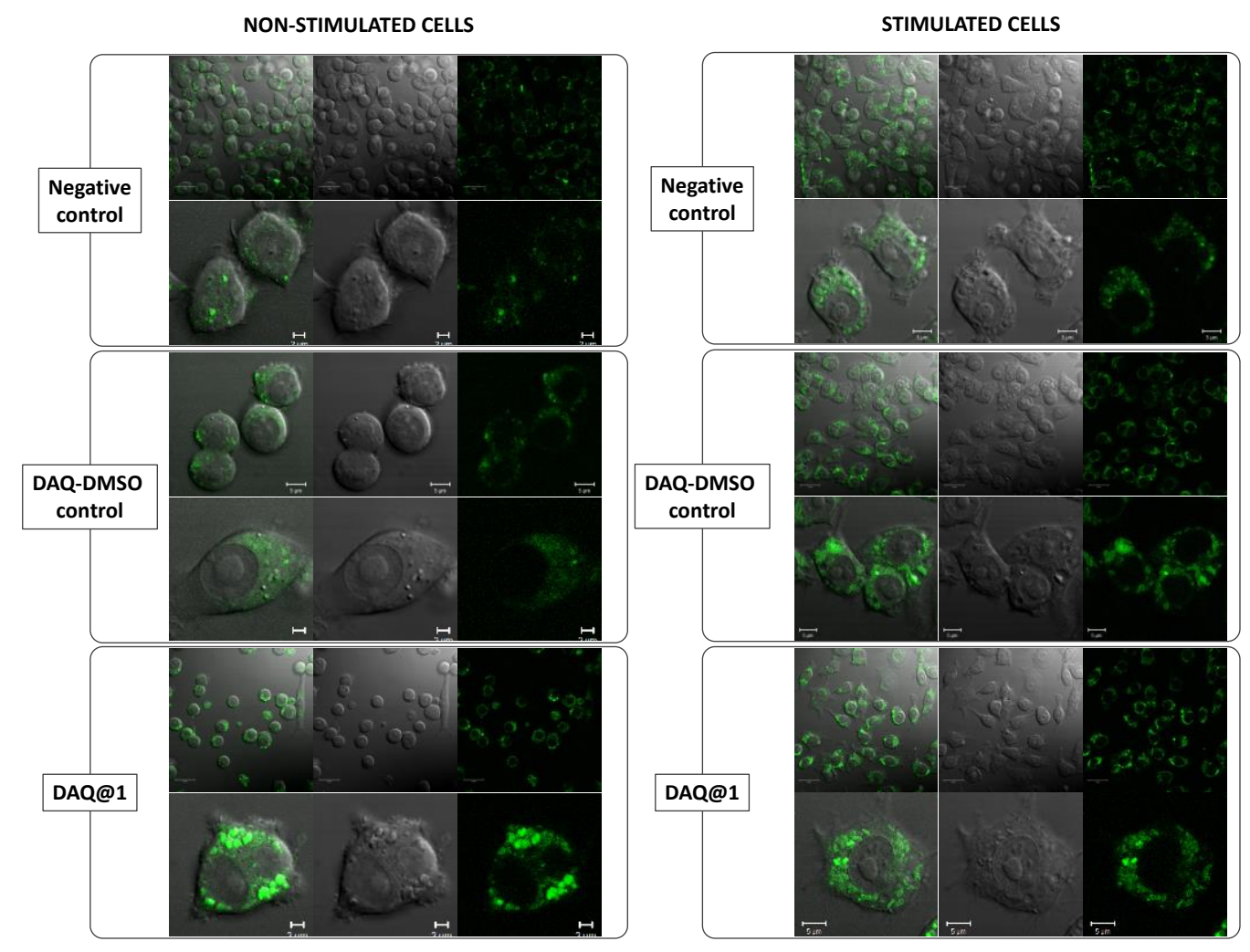

Figure 5.69. Confocal microscopy images $\left(\lambda_{\mathrm{ex}} 488 \mathrm{~nm}\right.$ ) of non-stimulated (left) and stimulated (right) RAW 264.7 cells incubated overnight with a DAQ@1 sample (1.6 $\mu \mathrm{M})$, DAQ-DMSO control (1.6 $\mu \mathrm{M})$ and a negative control. Green channel (right), bright field/DIC channel (center), merged (left).

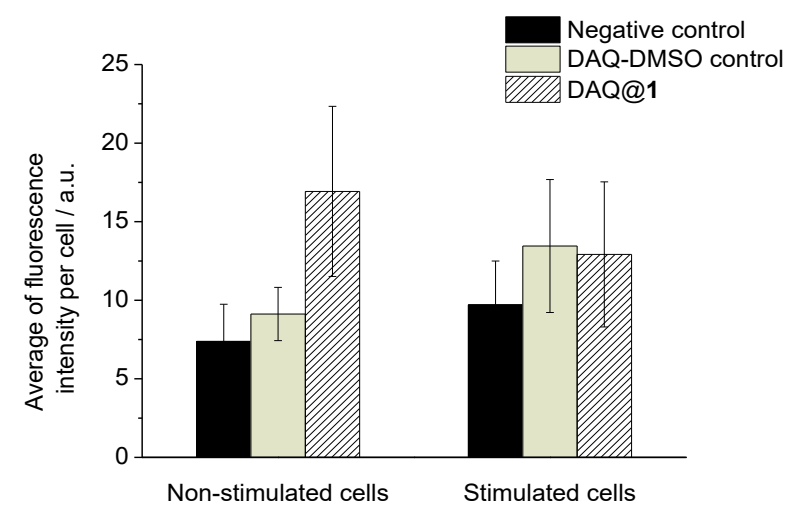

Figure 5.70. Fluorescence emission intensity per cell $\left(\lambda_{\mathrm{ex}} 488 \mathrm{~nm}\right)$ from confocal microscopy images of stimulated and non-stimulated RAW 264.7 cells incubated overnight with a DAQ@1 sample (pattern, 1.6 $\mu \mathrm{M}), \mathrm{DAQ}-\mathrm{DMSO}$ control (green, $1.6 \mu \mathrm{M}$ ) and a negative control (black). Error bars indicate the standard deviation ( $n=40-140$ cells/sample). 
As can be seen both in confocal images and fluorescence emission intensity analysis, the intracellular fluorescent emission is quite similar for cells incubated with DAQ@1, DAQ-DMSO control and the negative control, either for stimulated or non-stimulated cells. Negative control emission corresponds to the weak residual autofluorescence of cells, ${ }^{132}$ so our samples results are meagre. The low recorded fluorescence prevented from obtaining intracellular DAQ/DAQTZ spectra. A DAQ-prep control was also assayed, but results are not shown as they are also similar to the negative control. Nevertheless, fluorescence intensity is always higher for DAQ@1 and DAQ-DMSO control, so DAQ has been internalised, and a probe (DAQ or DAQ-TZ) is emitting fluorescence.

When DAQ is internalised into cells, the formation of the triazole DAQ-TZ is expected, accompanied by an increase of fluorescence emission due to its aggregation. ${ }^{132}$ So, the low fluorescence of the probe into cells can be consequence of the following issues: a low internalisation of DAQ, that the reaction with NO has not been produced, or that the concentration inside cells is not enough to form DAQ-TZ aggregates. The two first possibilities were rejected as experiments were performed following a reported protocol with successful results for DAQ NO-imaging in RAW 264.7 cells, proving DAQ-TZ formation. ${ }^{132}$ Additionally, we had also proved the formation of the triazole in our DAQ@1 systems after exposure to NO. However, the concentration of DAQ and DAQ-TZ used in the reported protocol was $27.4 \mu \mathrm{M}$, which is 17 times higher than in our experiments. Therefore, the low fluorescence observed from DAQ-TZ should be ascribed to the fact that its concentration inside cells is not enough to produce the highly fluorescent aggregates.

Under the light of these results, we cannot thus conclude that the nanoparticles help to internalise the probe in cells or improve NO response, as cell uptake for DAQ@1 and DAQ-DMSO control seems quite similar (with fluorescence values that sometimes overlap). Although the solubility of DAQ, and probably the internalisation, has been enhanced using nanoparticles, we cannot check this due to the necessity of DAQ-TZ to aggregate to show fluorescence. Therefore, DAQ would not be an adequate probe for nanoparticle-increased NO sensing, as DAQ quantity cannot be increased into DAQ@1 to the amount required for DAQ-TZ aggregation to occur. 


\subsection{REFERENCES}

Hibbs, J. B. J.; Taintor, R. R.; Vavrin, Z. Macrophage Cytotoxicity: Role for L-Arginine Deiminase and Imino Nitrogen Oxidation to Nitrite. Science. 1987, 235 (4787), 473-476.

lyengar, R.; Stuehr, D. J.; Marletta, M. A. Macrophage Synthesis of Nitrite, Nitrate, and N-Nitrosamines: Precursors and Role of the Respiratory Burst. Proc. Natl. Acad. Sci. U. S. A. 1987, 84 (18), 6369-6373.

Williams, D. L. H. A Chemist's View of the Nitric Oxide Story. Org. Biomol. Chem. 2003, 1, 441-449.

Stuehr, D. J.; Kwon, N. S.; Nathan, C. F.; Griffith, O. W.; Feldman, P. L.; Wiseman, J. N( $\omega)$-Hydroxy-L-Arginine Is an Intermediate in the Biosynthesis of Nitric Oxide from L-Arginine. J. Biol. Chem. 1991, 266 (10), 6259-6263.

Förstermann, U.; Closs, E. I.; Pollock, J. S.; Nakane, M.; Schwarz, P.; Gath, I.; Kleinert, H. Nitric Oxide Synthase Isozymes. Characterization, Purification, Molecular Cloning, and Functions. Hypertension 1994, 23, 1121-1131.

Förstermann, U.; Sessa, W. C. Nitric Oxide Synthases: Regulation and Function. Eur. Heart J. 2012, 33 (7), 829-837.

Villanueva, C.; Giulivi, C. Subcellular and Cellular Locations of Nitric Oxide Synthase Isoforms as Determinants of Health and Disease. Free Radic. Biol. Med. 2010, 49 (3), 307-316.

Butler, A. R.; Williams, D. L. H. The Physiological Role of Nitric Oxide. Chem. Soc. Rev. 1993, 22 (4), 233-241.

Geller, D. A.; Billiar, T. R. Molecular Biology of Nitric Oxide Synthases. Cancer Metastasis Rev. 1998, 17, 7-23.

Csonka, C.; Páli, T.; Bencsik, P.; Görbe, a; Ferdinandy, P.; Csont, T. Measurement of NO in Biological Samples. Br. J. Pharmacol. 2015, 172 (6), 1620-1632.

Yetik-Anacak, G.; Catravas, J. D. Nitric Oxide and the Endothelium: History and Impact on Cardiovascular Disease. Vascul. Pharmacol. 2006, 45 (5), 268-276.

Iverson, N. M.; Hofferber, E. M.; Stapleton, J. A. Nitric Oxide Sensors for Biological Applications. Chemosensors 2018, 6 (8), 113.

Calabrese, V.; Mancuso, C.; Calvani, M.; Rizzarelli, E.; Butterfield, D. A.; Stella, A. M. G. Nitric Oxide in the Central Nervous System: Neuroprotection versus Neurotoxicity. Nat. Rev. Neurosci. 2007, 8 (10), 766-775.

Yamamoto, T.; Shimoyama, N.; Mizuguchi, T. Nitric Oxide Synthase Inhibitor Blocks Spinal Sensitization Induced by Formalin Injection into the Rat Paw. Anesth. Analg. 1993, 77 (5), 886-890.

, J. E.; Farr, S. A.; Sell, R. L.; Hileman, S. M.; Banks, W. A. Nitric Oxide Is a Central Component in Neuropeptide Regulation of Appetite. Peptides 2011, 32 (4), 776-780.

Monti, J. M.; Jantos, H. Effects of L-Arginine and SIN-1 on Sleep and Waking in the Rat during Both Phases of the Light-Dark Cycle. Life Sci. 2004, 75 (17), 2027-2034. Neuroendocrine Integration? Prog. Biophys. Mol. Biol. 2004, 84 (2-3), 197-215.

Wang, G. R.; Zhu, Y.; Halushka, P. V; Lincoln, T. M.; Mendelsohn, M. E. Mechanism of Platelet Inhibition by Nitric Oxide: In Vivo Phosphorylation of Thromboxane Receptor by Cyclic GMP-Dependent Protein Kinase. Proc. Natl. Acad. Sci. U. S. A. 1998, 95 (9), 4888-4893.

Chen, L. Y.; Mehta, P.; Mehta, J. L. Oxidized LDL Decreases L-Arginine Uptake and Nitric Oxide Synthase Protein Expression in Human Platelets. Circulation 1996, 93 (9), 1740-1746.

Lanas, A. Role of Nitric Oxide in the Gastrointestinal Tract. Arthritis Res. Ther. 2008, 10, S4.

Brown J. F. Keates, A. C.; Hanson, P. J.; Whittle, B. J. Nitric Oxide Generators and CGMP Stimulate Mucus Secretion by Rat Gastric Mucosal Cells. Am. J. Physiol. 1993, 265, G418-G422.

Kubes, P.; Suzuki, M.; Granger, D. N. Nitric Oxide: An Endogenous Modulator of Leukocyte Adhesion. Proc. Natl. Acad. Sci. U. S. A. 1991, 88 (11), 4651-4655.

Majid, D. S.; Navar, L. G. Nitric Oxide in the Control of Renal Hemodynamics and Excretory Function. Am. J. Hypertens. 2001, $14,74 \mathrm{~S}-82 \mathrm{~S}$

Mount, P. F.; Power, D. A. Nitric Oxide in the Kidney: Functions and Regulation of Synthesis. Acta Physiol. 2006, 187 (4), $433-$ 446.

Bogdan, C. Nitric Oxide and the Immune Response. Nat. Immunol. 2001, 2 (10), 907-916.

Jones, M. L.; Ganopolsky, J. G.; Labbé, A.; Wahl, C.: Prakash, S. Antimicrobial Properties of Nitric Oxide and Its Application in Antimicrobial Formulations and Medical Devices. Appl. Microbiol. Biotechnol. 2010, 88 (2), 401-407.

McCleverty, J. A. Chemistry of Nitric Oxide Relevant to Biology. Chem. Rev. 2004, 104 (2), 403-418. Dynamics of $\mathrm{NO}$ and O2. Proc. Natl. Acad. Sci. U. S. A. 2001, 98 (1), 355-360. Mechanisms of Nitric Oxide. Free Radic. Biol. Med. 1998, 25 (4-5), 434-456.

Thomas, D. D.; Ridnour, L. A.; Isenberg, J. S.; Flores-Santana, W.; Switzer, C. H.; Donzelli, S.; Hussain, P.; Vecoli, C.; Paolocci, N.; Ambs, S.; Colton, C. A.; Harris, C. C.; Roberts, D. D.; Wink, D. A. The Chemical Biology of Nitric Oxide: Implications in Cellular Signaling. Free Radic. Biol. Med. 2008, 45 (1), 18-31. 
(31) Wink, D. A.; Grisham, M. B.; Mitchell, J. B.; Ford, P. C. B. T.-M. in E. Direct and Indirect Effects of Nitric Oxide in Chemical Reactions Relevant to Biology. In Nitric Oxide Part A: Sources and Detection of NO; NO Synthase; Academic Press, $1996 ;$ Vol. 268, pp 12-31.

(32) Hardman, J. G.; Beavo, J. A.; Gray, J. P.; Chrisman, T. D.; Patterson, W. D.; Sutherland, E. W. The Formation and Metabolism of Cyclic GMP. Ann. N. Y. Acad. Sci. 1971, 185, 27-35.

(33) Hobbs, A. J.; Ignarro, L. J. Nitric Oxide-Cyclic GMP Signal Transduction System. In Nitric Oxide Part B: Physiological and Pathological Processes; Academic Press, 1996; Vol. 269, pp 134-148.

(34) Furchgott, R. F.; Zawadzki, J. V. The Obligatory Role of Endothelial Cells in the Relaxation of Arterial Smooth Muscle by Acetylcholine. Nature 1980, 288, 373-376.

(35) Gomes, A.; Fernandes, E.; Lima, J. L. F. C. Use of Fluorescence Probes for Detection of Reactive Nitrogen Species: A Review. J. Fluoresc. 2006, 16 (1), 119-139.

(36) Yao, D.; Vlessidis, A. G.; Evmiridis, N. P. Determination of Nitric Oxide in Biological Samples. Microchim. Acta 2004, 147,1 -20.

(37) Pfeiffer, S.; Mayer, B.; Hemmens, B. Nitric Oxide: Chemical Puzzles Posed by a Biological Messenger. Angew. Chem. Int. Ed. Engl. 1999, 38 (12), 1714-1731.

(38) Huie, R. E.; Padmaja, S. The Reaction of NO with Superoxide. Free Radic. Res. Commun. 1993, 18 (4), $195-199$.

(39) Pryor, W. A.; Squadrito, G. L. The Chemistry of Peroxynitrite: A Product from the Reaction of Nitric Oxide with Superoxide. Am. J. Physiol. Cell. Mol. Physiol. 1995, 268 (5), L699-L722.

(40) Yuan, S.; Patel, R. P.; Kevil, C. G. Working with Nitric Oxide and Hydrogen Sulfide in Biological Systems. Am. J. Physiol. Lung Cell. Mol. Physiol. 2015, 308 (5), L403-L415.

(41) Dawson, V. L.; Dawson, T. M. Nitric Oxide Neurotoxicity. J. Chem. Neuroanat. 1996, 10 (3), 179-190.

(42) Pacher, P.; Beckman, J. S.; Liaudet, L. Nitric Oxide and Peroxynitrite in Health and Disease. Physiol. Rev. 2007, 87, 315-424.

(43) Bernstein, H.-G.; Bogerts, B.; Keilhoff, G. The Many Faces of Nitric Oxide in Schizophrenia. A Review. Schizophr. Res. 2005, 78 (1), 69-86.

(44) Savaş, H. A.; Herken, H.; Yürekli, M.; Uz, E.; Tutkun, H.; Zoroğlu, S. S.; Ozen, M. E.; Cengiz, B.; Akyol, O. Possible Role of Nitric Oxide and Adrenomedullin in Bipolar Affective Disorder. Neuropsychobiology 2002, 45 (2), 57-61.

(45) Tayfun Uzbay, I.; Oglesby, M. W. Nitric Oxide and Substance Dependence. Neurosci. Biobehav. Rev. 2001, 25, 43-52.

(46) Söğüt, S.; Zoroğlu, S. S.; Özyurt, H.; Yilmaz, H. R.; Özuǧurlu, F.; Sivasli, E.; Yetkin, Ö.; Yanik, M.; Tutkun, H.; Savaş, H. A.; Tarakçioğlu, M.; Akyol, Ö. Changes in Nitric Oxide Levels and Antioxidant Enzyme Activities May Have a Role in the Pathophysiological Mechanisms Involved in Autism. Clin. Chim. Acta 2003, 331, 111-117.

(47) Sweeten, T. L.; Posey, D. J.; Shankar, S.; McDougle, C. J. High Nitric Oxide Production in Autistic Disorder: A Possible Role for Interferon-Gamma. Biol. Psychiatry 2004, 55 (4), 434-437.

(48) Tas, F. V.; Guvenir, T.; Tas, G.; Cakaloz, B.; Ormen, M. Nitric Oxide Levels in Disruptive Behavioral Disorder. Neuropsychobiology 2006, 53 (4), 176-180.

(49) Dhir, A.; Kulkarni, S. K. Nitric Oxide and Major Depression. Nitric oxide 2011, 24 (3), 125-131.

(50) Szabo, C. Gasotransmitters in Cancer: From Pathophysiology to Experimental Therapy. Nat. Rev. Drug Discov. 2016, 15 (3), 185-203.

(51) Coto-Segura, P.; Coto, E.; Mas-Vidal, A.; Morales, B.; Alvarez, V.; Díaz, M.; Alonso, B.; Santos-Juanes, J. Influence of Endothelial Nitric Oxide Synthase Polymorphisms in Psoriasis Risk. Arch. Dermatol. Res. 2011, 303 (6), 445-449.

(52) Olanow, C. W.; Jenner, P.; Brooks, D. Dopamine Agonists and Neuroprotection in Parkinson's Disease. Ann. Neurol. 1998, 44 (S1), S167-S174.

(53) R. J. Thatcher, G. NO Problem for Nitroglycerin: Organic Nitrate Chemistry and Therapy. Chem. Soc. Rev. 1998, 27 (5), 331337.

(54) Endres, M.; Laufs, U.; Liao, J. K.; Moskowitz, M. A. Targeting ENOS for Stroke Protection. Trends Neurosci. 2004, 27 (5), 283289.

(55) Frostell, C.; Fratacci, M. D.; Wain, J. C.; Jones, R.; Zapol, W. M. Inhaled Nitric Oxide. A Selective Pulmonary Vasodilator Reversing Hypoxic Pulmonary Vasoconstriction. Circulation 1991, 83 (6), 2038-2047.

(56) López-Sánchez, L. M.; Aranda, E.; Rodríguez-Ariza, A. Nitric Oxide and Tumor Metabolic Reprogramming. Biochem. Pharmacol. 2020, 176, 113769.

(57) Özenver, N.; Efferth, T. Small Molecule Inhibitors and Stimulators of Inducible Nitric Oxide Synthase in Cancer Cells from Natural Origin (Phytochemicals, Marine Compounds, Antibiotics). Biochem. Pharmacol. 2020, 176, 113792.

(58) Vahora, H.; Khan, M. A.; Alalami, U.; Hussain, A. The Potential Role of Nitric Oxide in Halting Cancer Progression Through Chemoprevention. J. cancer Prev. 2016, 21, 1-12.

(59) Fukumura, D.; Kashiwagi, S.; Jain, R. K. The Role of Nitric Oxide in Tumour Progression. Nat. Rev. Cancer 2006, 6 (7), 521-534.

(60) Singh, S.; Gupta, A. K. Nitric Oxide: Role in Tumour Biology and INOS/NO-Based Anticancer Therapies. Cancer Chemother Pharmacol. 2011, 67 (6), 1211-1224.

(61) Wang, H.; Wang, L.; Xie, Z.; Zhou, S.; Li, Y.; Zhou, Y.; Sun, M. Nitric Oxide (NO) and NO Synthases (NOS)-Based Targeted Therapy for Colon Cancer. Cancers (Basel). 2020, 12, 1881.

(62) Nagano, T.; Yoshimura, T. Bioimaging of Nitric Oxide. Chem. Rev. 2002, 102, 1235-1269. 
(63) Privett, B. J.; Shin, J. H.; Schoenfisch, M. H. Electrochemical Nitric Oxide Sensors for Physiological Measurements. Chem. Soc. Rev. 2010, 39 (6), 1925-1935.

(64) Brown, F. O.; Finnerty, N. J.; Bolger, F. B.; Millar, J.; Lowry, J. P. Calibration of NO Sensors for In-Vivo Voltammetry: Laboratory Synthesis of NO and the Use of UV-Visible Spectroscopy for Determining Stock Concentrations. Anal. Bioanal. Chem. 2005, 381 (4), 964-971.

(65) Hogg, N. Detection of Nitric Oxide by Electron Paramagnetic Resonance Spectroscopy. Free Radic. Biol. Med. 2010, 49 (2) 122-129.

(66) Katayama, Y.; Soh, N.; Maeda, M. A New Strategy for the Design of Molecular Probes for Investigating Endogenous Nitric Oxide Using an EPR or Fluorescent Technique. ChemPhysChem 2001, 2 (11), 655-661.

(67) Ueno, T.; Nagano, T. Fluorescent Probes for Sensing and Imaging. Nat. Methods 2011, 8 (8), 642-645.

(68) Chen, X.; Tian, X.; Shin, I.; Yoon, J. Fluorescent and Luminescent Probes for Detection of Reactive Oxygen and Nitrogen Species. Chem. Soc. Rev. 2011, 40 (9), 4783-4804.

(69) McQuade, L. E.; Lippard, S. J. Fluorescent Probes to Investigate Nitric Oxide and Other Reactive Nitrogen Species in Biology (Truncated Form: Fluorescent Probes of Reactive Nitrogen Species). Curr. Opin. Chem. Biol. 2010, 14 (1), $43-49$.

(70) Wang, L.; Zhang, J.; An, X.; Duan, H. Recent Progress on the Organic and Metal Complex-Based Fluorescent Probes for Monitoring Nitric Oxide in Living Biological Systems. Org. Biomol. Chem. 2020, 18 (8), 1522-1549.

(71) Chen, Y. Recent Developments of Fluorescent Probes for Detection and Bioimaging of Nitric Oxide. Nitric Oxide - Biol. Chem 2020, 98, 1-19.

(72) Li, H.; Wan, A. Fluorescent Probes for Real-Time Measurement of Nitric Oxide in Living Cells. Analyst 2015, 140, 7129-7141.

(73) Hetrick, E. M.; Schoenfisch, M. H. Analytical Chemistry of Nitric Oxide. Annu. Rev. Anal. Chem. 2009, 2, 409-433.

(74) Hall, C. N.; Garthwaite, J. What Is the Real Physiological NO Concentration in Vivo? Nitric oxide Biol. Chem. 2009, 21 (2), $92-$ 103.

(75) Kojima, H.; Nakatsubo, N.; Kikuchi, K.; Kawahara, S.; Kirino, Y.; Nagoshi, H.; Hirata, Y.; Nagano, T. Detection and Imaging of Nitric Oxide with Novel Fluorescent Indicators: Diaminofluoresceins. Anal. Chem. 1998, 70 (13), 2446-2453.

(76) Sasaki, E.; Kojima, H.; Nishimatsu, H.; Urano, Y.; Kikuchi, K.; Hirata, Y.; Nagano, T. Highly Sensitive Near-Infrared Fluorescent Probes for Nitric Oxide and Their Application to Isolated Organs. J. Am. Chem. Soc. 2005, 127 (11), 3684-3685.

(77) Yu, H.; Xiao, Y.; Jin, L. A Lysosome-Targetable and Two-Photon Fluorescent Probe for Monitoring Endogenous and Exogenous Nitric Oxide in Living Cells. J. Am. Chem. Soc. 2012, 134 (42), 17486-17489.

(78) Zhang, H.-X.; Chen, J.-B.; Guo, X.-F.; Wang, H.; Zhang, H.-S. Highly Sensitive Low-Background Fluorescent Probes for Imaging of Nitric Oxide in Cells and Tissues. Anal. Chem. 2014, 86 (6), 3115-3123.

(79) Ma, S.; Fang, D.-C.; Ning, B.; Li, M.; He, L.; Gong, B. The Rational Design of a Highly Sensitive and Selective Fluorogenic Probe for Detecting Nitric Oxide. Chem. Commun. 2014, 50 (49), 6475-6478.

(80) Ma, S.-F.; Wang, Q.-H.; Liu, F.-T.; Wang, H.-L.; Fang, D.-C.; Gong, B.; He, L.; Lu, Z.-L. Dihydropyridine-Based Fluorescence Probe for Nitric Oxide. RSC Adv. 2016, 6 (89), 85698-85703.

(81) Miao, J.; Huo, Y.; Lv, X.; Li, Z.; Cao, H.; Shi, H.; Shi, Y.; Guo, W. Fast-Response and Highly Selective Fluorescent Probes for Biological Signaling Molecule NO Based on N-Nitrosation of Electron-Rich Aromatic Secondary Amines. Biomaterials 2016, 78 11-19.

(82) Yu, Z.; Zhou, J.; Dong, X.; Zhao, W.; Chen, Z. Visualizing Nitric Oxide in Mitochondria and Lysosomes of Living Cells with NNitrosation of BODIPY-Based Fluorescent Probes. Anal. Chim. Acta 2019, 1067, 88-97.

(83) Mao, Z.; Jiang, H.; Song, X.; Hu, W.; Liu, Z. Development of a Silicon-Rhodamine Based Near-Infrared Emissive Two-Photon Fluorescent Probe for Nitric Oxide. Anal. Chem. 2017, 89 (18), 9620-9624.

(84) Nagano, T. Development of Fluorescent Probes for Bioimaging Applications. Proc. Japan Acad. Ser. B Phys. Biol. Sci. 2010, 86 (8), 837-847.

(85) Nakatsubo, N.; Kojima, H.; Kikuchi, K.; Nagoshi, H.; Hirata, Y.; Maeda, D.; Imai, Y.; Irimura, T.; Nagano, T. Direct Evidence of Nitric Oxide Production from Bovine Aortic Endothelial Cells Using New Fluorescence Indicators: Diaminofluoresceins. FEBS Lett. 1998, 427, 263-266.

(86) Balcerczyk, A.; Soszynski, M.; Bartosz, G. On the Specificity of 4-Amino-5-Methylamino-2',7'- Difluorofluorescein as a Probe for Nitric Oxide. Free Radic. Biol. Med. 2005, 39 (3), 327-335.

(87) Ignarro, L. J.; Fukuto, J. M.; Griscavage, J. M.; Rogers, N. E.; Byrns, R. E. Oxidation of Nitric Oxide in Aqueous Solution to Nitrite but Not Nitrate: Comparison with Enzymatically Formed Nitric Oxide from L-Arginine. Proc. Natl. Acad. Sci. U. S. A. 1993, 90 (17), 8103-8107.

(88) von Bohlen und Halbach, O. Nitric Oxide Imaging in Living Neuronal Tissues Using Fluorescent Probes. Nitric Oxide 2003, 9, 217-228.

(89) Kojima, H.; Sakurai, K.; Kikuchi, K.; Kawahara, S.; Kirino, Y.; Nagoshi, H.; Hirata, Y.; Akaike, T.; Maeda, H.; Nagano, T. Development of a Fluorescent Indicator for the Bioimaging of Nitric Oxide. Biol. Pharm. Bull. 1997, 20 (12), 1229-1232.

(90) Kojima, H.; Urano, Y.; Kikuchi, K.; Higuchi, T.; Hirata, Y.; Nagano, T. Fluorescent Indicators for Imaging Nitric Oxide Production Angew. Chemie - Int. Ed. 1999, 38 (21), 3209-3212.

(91) Wardman, P. Fluorescent and Luminescent Probes for Measurement of Oxidative and Nitrosative Species in Cells and Tissues: Progress, Pitfalls, and Prospects. Free Radic. Biol. Med. 2007, 43, 995-1022. 
(92) Ji, X.-B.; Hollocher, T. C. Mechanism for Nitrosation of 2,3-Diaminonaphthalene by Escherichia Coli: Enzymatic Production of NO Followed by O2-Dependent Chemical Nitrosation. Appl. Environ. Microbiol. 1988, 54 (7), 1791-1794.

(93) Miles, A. M.; Chen, Y.; Owens, M. W.; Grisham, M. B. Fluorometric Determination of Nitric Oxide. Methods A Companion to Methods Enzymol. 1995, 7, 40-47.

(94) Tsien, R. Y. A Non-Disruptive Technique for Loading Calcium Buffers and Indicators into Cells. Nature 1981, 290 (5806), 527528.

(95) Nagano, T. Practical Methods for Detection of Nitric Oxide. Luminescence 1999, 14 (6), 283-290.

(96) Kojima, H.; Sakurai, K.; Kikuchi, K.; Kawahara, S.; Kirino, Y.; Nagoshi, H.; Hirata, Y.; Nagano, T. Development of a Fluorescent Indicator for Nitric Oxide Based on the Fluorescein Chromophore. Chem. Pharm. Bull. 1998, 46 (2), 373-375.

(97) Itoh, Y.; Ma, F. H.; Hoshi, H.; Oka, M.; Noda, K.; Ukai, Y.; Kojima, H.; Nagano, T.; Toda, N. Determination and Bioimaging Method for Nitric Oxide in Biological Specimens by Diaminofluorescein Fluorometry. Anal. Biochem. 2000, 287, $203-209$.

(98) Kojima, H.; Hirotani, M.; Nakatsubo, N.; Kikuchi, K.; Urano, Y.; Higuchi, T.; Hirata, Y.; Nagano, T. Bioimaging of Nitric Oxide with Fluorescent Indicators Based on the Rhodamine Chromophore. Anal. Chem. 2001, 73 (9), 1967-1973.

(99) Gabe, Y.; Urano, Y.; Kikuchi, K.; Kojima, H.; Nagano, T. Highly Sensitive Fluorescence Probes for Nitric Oxide Based on Boron Dipyrromethene Chromophore-Rational Design of Potentially Useful Bioimaging Fluorescence Probe. J. Am. Chem. Soc. 2004, 126 (10), 3357-3367.

(100) Xu, W.; Zeng, Z.; Jiang, J.-H.; Chang, Y.-T.; Yuan, L. Discerning the Chemistry in Individual Organelles with Small-Molecule Fluorescent Probes. Angew. Chem. Int. Ed. Engl. 2016, 55 (44), 13658-13699.

(101) Feng, W.; Qiao, Q.-L.; Leng, S.; Miao, L.; Yin, W.-T.; Wang, L.-Q.; Xu, Z.-C. A 1,8-Naphthalimide-Derived Turn-on Fluorescent Probe for Imaging Lysosomal Nitric Oxide in Living Cells. Chinese Chem. Lett. 2016, 27 (9), 1554-1558.

(102) Yao, H.-W.; Zhu, X.-Y.; Guo, X.-F.; Wang, H. An Amphiphilic Fluorescent Probe Designed for Extracellular Visualization of Nitric Oxide Released from Living Cells. Anal. Chem. 2016, 88 (18), 9014-9021.

(103) Burrows, N.; Cane, G.; Robson, M.; Gaude, E.; Howat, W. J.; Szlosarek, P. W.; Pedley, R. B.; Frezza, C.; Ashcroft, M.; Maxwell, P. H. Hypoxia-Induced Nitric Oxide Production and Tumour Perfusion Is Inhibited by Pegylated Arginine Deiminase (ADIPEG20). Sci. Rep. 2016, 6, 22950.

(104) Mishra, O. P.; Zanelli, S.; Ohnishi, S. T.; Delivoria-Papadopoulos, M. Hypoxia-Induced Generation of Nitric Oxide Free Radicals in Cerebral Cortex of Newborn Guinea Pigs. Neurochem. Res. 2000, 25 (12), 1559-1565.

(105) Ye, X.; Kim, W. S.; Rubakhin, S. S.; Sweedler, J. V. Measurement of Nitric Oxide by 4,5-Diaminofluorescein without Interferences. Analyst 2004, 129 (12), 1200-1205.

(106) Rotman, B.; Papermaster, B. W. Membrane Properties of Living Mammalian Cells as Studied by Enzymatic Hydrolysis of Fluorogenic Esters. Proc. Natl. Acad. Sci. 1966, 55, 134-141.

(107) Berkels, R.; Dachs, C.; Roesen, R.; Klaus, W. Simultaneous Measurement of Intracellular Ca2+ and Nitric Oxide: A New Method. Cell Calcium 2000, 27 (5), 281-286.

(108) Chen, H.; Montagnani, M.; Funahashi, T.; Shimomura, I.; Quon, M. J. Adiponectin Stimulates Production of Nitric Oxide in Vascular Endothelial Cells. J. Biol. Chem. 2003, 278 (45), 45021-45026.

(109) Montagnani, M.; Chen, H.; Barr, V. A.; Quon, M. J. Insulin-Stimulated Activation of ENOS Is Independent of Ca2+ but Requires Phosphorylation by Akt at Ser1179. J. Biol. Chem. 2001, 276 (32), 30392-30398.

(110) Qiu, W.; Kass, D. A.; Hu, Q.; Ziegelstein, R. C. Determinants of Shear Stress-Stimulated Endothelial Nitric Oxide Production Assessed in Real-Time by 4,5-Diaminofluorescein Fluorescence. Biochem. Biophys. Res. Commun. 2001, 286 (2), $328-335$.

(111) Strijdom, H.; Jacobs, S.; Hattingh, S.; Page, C.; Lochner, A. Nitric Oxide Production Is Higher in Rat Cardiac Microvessel Endothelial Cells than Ventricular Cardiomyocytes in Baseline and Hypoxic Conditions: A Comparative Study. FASEB J. 2006, 20 (2), 314-316.

(112) Tiscornia, A.; Cairoli, E.; Marquez, M.; Denicola, A.; Pritsch, O.; Cayota, A. Use of Diaminofluoresceins to Detect and Measure Nitric Oxide in Low Level Generating Human Immune Cells. J. Immunol. Methods 2009, 342, 49-57.

(113) Liu, T.; Hu, J.; Ma, X.; Kong, B.; Wang, J.; Zhang, Z.; Guo, D. S.; Yang, X. Hollow Double-Layered Polymer Nanoparticles with SNitrosothiols for Tumor Targeted Therapy. J. Mater. Chem. B 2017, 5 (36), 7519-7528.

(114) Yi, F.; Zhang, A. Y.; Campbell, W. B.; Zou, A.; Van Breemen, C.; Li, P. Simultaneous in Situ Monitoring of Intracellular Ca2+ and NO in Endothelium of Coronary Arteries. Am J Physiol Hear. Circ Physiol 2002, 283, H2725-H2732.

(115) Eksakulkla, S.; Suksom, D.; Siriviriyakul, P.; Patumraj, S. Increased NO Bioavailability in Aging Male Rats by Genistein and Exercise Training: Using 4,5-Diaminofluorescein Diacetate. Reprod. Biol. Endocrinol. 2009, 7, 93.

(116) Kashiwagi, S.; Kajimura, M.; Yoshimura, Y.; Suematsu, M. Nonendothelial Source of Nitric Oxide in Arterioles but Not in Venules: Alternative Source Revealed in Vivo by Diaminofluorescein Microfluorography. Circ. Res. 2002, 91, e55-e64.

(117) Zhou, X.; He, P. Endothelial [Ca2+]i and Caveolin-1 Antagonistically Regulate ENOS Activity and Microvessel Permeability in Rat Venules. Cardiovasc. Res. 2010, 87 (2), 340-347.

(118) Zhu, L.; He, P. Platelet-Activating Factor Increases Endothelial [Ca2+]i and NO Production in Individually Perfused Intact Microvessels. Am. J. Physiol. - Hear. Circ. Physiol. 2005, 288, H2869-H2877.

(119) Zhou, X.; He, P. Improved Measurements of Intracellular Nitric Oxide in Intact Microvessels Using 4,5-Diaminofluorescein Diacetate. Am. J. Physiol. - Hear. Circ. Physiol. 2011, 301, H108-H114.

(120) Zhang, L.; Zeng, M.; Fu, B. M. Inhibition of Endothelial Nitric Oxide Synthase Decreases Breast Cancer Cell MDA-MB-231 
Adhesion to Intact Microvessels under Physiological Flows. Am. J. Physiol. - Hear. Circ. Physiol. 2016, 310 (11), H1735-H1747. Yen, W.; Cai, B.; Yang, J.; Zhang, L.; Zeng, M.; Tarbell, J. M.; Fu, B. M. Endothelial Surface Glycocalyx Can Regulate Flow-Induced Nitric Oxide Production in Microvessels in Vivo. PLoS One 2015, 10 (1), e0117133.

Ye, X.; Rubakhin, S. S.; Sweedler, J. V. Simultaneous Nitric Oxide and Dehydroascorbic Acid Imaging by Combining Diaminofluoresceins and Diaminorhodamines. J. Neurosci. Methods 2008, 168 (2), 373-382.

Zhang, X.; Kim, W.-S.; Hatcher, N.; Potgieter, K.; Moroz, L. L.; Gillette, R.; Sweedler, J. V. Interfering with Nitric Oxide Measurements: 4,5-Diaminofluorescein Reacts with Dehydroascorbic Acid and Ascorbic Acid. J. Biol. Chem. 2002, 277 (50), 48472-48478.

Shaheen, F.; Shmygol, A.; Rabbani, N.; Thornalley, P. J. A Fluorogenic Assay for Methylglyoxal. Biochem. Soc. Trans. 2014, 42 (2), 548-555.

Amemori, S.; Matsusaki, M.; Akashi, M. Biocompatible and Highly Sensitive Nitric Oxide Sensor Particles Prepared by Layerby-Layer Assembly. Chem. Lett. 2010, 39, 42-43.

Valdes-Aguilera, O.; Neckers, D. C. Rose Bengal Ethyl Ester Aggregation in Aqueous Solution. J. Phys. Chem. 1988, 92 (15), 4286-4289.

Xu, D.; Neckers, D. C. Aggregation of Rose Bengal Molecules in Solution. J. Photochem. Photobiol. A Chem. 1987, 40, 361-370. Valdes-Aguilera, O.; Neckers, D. C. Aggregation Phenomena in Xanthene Dyes. Acc. Chem. Res. 1989, 22, $171-177$.

Beltrán, A.; Burguete, M. I.; Abánades, D. R.; Pérez-Sala, D.; Luis, S. V.; Galindo, F. Turn-on Fluorescent Probes for Nitric Oxide Sensing Based on the Ortho-Hydroxyamino Structure Showing No Interference with Dehydroascorbic Acid. Chem. Commun. 2014, 50 (27), 3579-3581.

Felip-León, C.; Angulo-Pachón, C. A.; Miravet, J. F.; Galindo, F. Self-Assembly Controls Reactivity with Nitric Oxide: Implications for Fluorescence Sensing. ACS Omega 2018, 3 (11), 15538-15545.

Maragos, C. M.; Morley, D.; Wink, D. A.; Dunams, T. M.; Saavedra, J. E.; Hoffman, A.; Bove, A. A.; Isaac, L.; Hrabie, J. A.; Keefer L. K. Complexes of NO with Nucleophiles as Agents for the Controlled Biological Release of Nitric Oxide. Vasorelaxant Effects. J. Med. Chem. 1991, 34 (41), 3242-3247.

Marín, M. J.; Thomas, P.; Fabregat, V.; Luis, S. V.; Russell, D. a.; Galindo, F. Fluorescence of 1,2-Diaminoanthraquinone and Its Nitric Oxide Reaction Product within Macrophage Cells. ChemBioChem 2011, 12 (16), 2471-2477.

Norby, S. W.; Weyhenmeyer, J. A.; Clarkson, R. B. Stimulation and Inhibition of Nitric Oxide Production in Macrophages and Neural Cells as Observed by Spin Trapping. Free Radic. Biol. Med. 1997, 22, 1-9.

Leggett, R.; Thomas, P.; Marín, M. J.; Gavrilovic, J.; Russell, D. A. Imaging of Compartmentalised Intracellular Nitric Oxide, Induced during Bacterial Phagocytosis, Using a Metalloprotein-Gold Nanoparticle Conjugate. Analyst 2017, 142 (21), 40994105.

Murphy, W. J.; Muroi, M.; Zhang, C. X.; Suzuki, T.; Russell, S. W. Both Basal and Enhancer KB Elements Are Required for Full Induction of the Mouse Inducible Nitric Oxide Synthase Gene. J. Endotoxin Res. 1996, 3 (5), 381-393.

Galindo, F.; Kabir, N.; Gavrilovic, J.; Russell, D. a. Spectroscopic Studies of 1,2-Diaminoanthraquinone (DAQ) as a Fluorescent Probe for the Imaging of Nitric Oxide in Living Cells. Photochem. Photobiol. Sci. 2008, 7 (1), 126-130.

Bru, M.; Burguete, M. I.; Galindo, F.; Luis, S. V.; Marín, M. J.; Vigara, L. Cross-Linked Poly(2-Hydroxyethylmethacrylate) Films Doped with 1,2-Diaminoanthraquinone (DAQ) as Efficient Materials for the Colorimetric Sensing of Nitric Oxide and Nitrite Anion. Tetrahedron Lett. 2006, 47 (11), 1787-1791.

Fabregat, V.; Burguete, M. I.; Galindo, F.; Luis, S. V. Influence of Polymer Composition on the Sensitivity towards Nitrite and Nitric Oxide of Colorimetric Disposable Test Strips. Environ. Sci. Pollut. Res. 2017, 24 (4), 3448-3455.

von Bohlen und Halbach, O.; Albrecht, D.; Heinemann, U.; Schuchmann, S. Spatial Nitric Oxide Imaging Using 1,2Diaminoanthraquinone to Investigate the Involvement of Nitric Oxide in Long-Term Potentiation in Rat Brain Slices. Neuroimage 2002, 15 (3), 633-639.

Heiduschka, P.; Thanos, S. NO Production during Neuronal Cell Death Can Be Directly Assessed by a Chemical Reaction in Vivo. Neuroreport 1998, 9 (18), 4051-4057.

Centelles, J. J.; Fernández-Cancio, M.; Imperial, S. Spectrophotometric Determination of Nitrites in Biological Samples Using 1,2-Diaminoanthraquinone. Potential Application to the Determination of Nitric Oxide Synthase Activity. Anal. Lett. 2003, 36 (10), 2139-2149.

Maia, L. B.; Moura, J. J. G. How Biology Handles Nitrite. Chem. Rev. 2014, 114 (10), 5273-5357.

Chen, X.; Sheng, C.; Zheng, X. Direct Nitric Oxide Imaging in Cultured Hippocampal Neurons with Diaminoanthraquinone and Confocal Microscopy. Cell Biol. Int. 2001, 25 (7), 593-598.

Miyaji, H.; Sessler, J. L. Off-the-Shelf Colorimetric Anion Sensors. Angew. Chemie Int. Ed. 2001, 40, 154-157.

Khan, M. S.; Khan, Z. H. Electronic Absorption Spectra of Amino Substituted Anthraquinones and Their Interpretation Using the ZINDO/S and AM1 Methods. Spectrochim. Acta. A. Mol. Biomol. Spectrosc. 2003, 59 (7), 1409-1426.

Burguete, M. I.; Fabregat, V.; Galindo, F.; Izquierdo, M. A.; Luis, S. V. Improved PolyHEMA-DAQ Films for the Optical Analysis of Nitrite. Eur. Polym. J. 2009, 45 (5), 1516-1523.

Chen, L.; Chiu, Y.; Hung, J.; Kuo, C.-C.; Chen, W.-C. Multifunctional Electrospun Nanofibers Prepared from Poly((Nisopropylacrylamide)-co-(N-hydroxymethylacrylamide)) and Their Blends with 1, 2-Diaminoanthraquinone for NO Gas Detection. Macromol. Chem. Phys. 2014, 215, 286-294. 
(148) Hong, W. Y.; Yang, T. W.; Wang, C. M.; Syu, J. H.; Lin, Y. C.; Meng, H. F.; Tsai, M. J.; Cheng, H.; Zan, H. W.; Horng, S. F. Conversion of Absorption to Fluorescence Probe in Solid-State Sensor for Nitric Oxide and Nitrite. Org. Electron. 2013, 14, 1136-1141.

(149) Chung, C. W.; Tsai, M. J.; Lin, P. W.; Huang, D. W.; Wang, K. H.; Chen, Y. A.; Meng, H. F.; Zan, H. W.; Cheng, H.; Tong, L.; Zhang, L.; Horng, S. F.; Hung, C. H. Accurate Real-Time Sensing Tip for Aqueous NO with Optical Fibers Embedded in Active Hydrogel Waveguide. AIP Adv. 2018, 8, 025207.

(150) Schuchmann, S.; Albrecht, D.; Heinemann, U.; von Bohlen und Halbach, O. Nitric Oxide Modulates Low-Mg2+-Induced Epileptiform Activity in Rat Hippocampal-Entorhinal Cortex Slices. Neurobiol. Dis. 2002, 11 (1), 96-105.

(151) Tong, H.; Hong, Y.; Dong, Y.; Häußler, M.; Lam, J. W. Y.; Li, Z.; Guo, Z.; Guo, Z.; Tang, B. Z. Fluorescent "Light-up" Bioprobes Based on Tetraphenylethylene Derivatives with Aggregation-Induced Emission Characteristics. Chem. Commun. 2006, No. 35, 3705-3707.

(152) Hong, Y.; Lam, J. W. Y.; Tang, B. Z. Aggregation-Induced Emission: Phenomenon, Mechanism and Applications. Chem. Commun. 2009, No. 29, 4332-4353.

(153) Navas Diaz, A. Absorption and Emission Spectroscopy and Photochemistry of 1,10-Anthraquinone Derivatives: A Review. J. Photochem. Photobiol. A Chem. 1990, 53 (2), 141-167.

(154) Mukerjee, P.; Mysels, K. J. Critical Micelle Concentrations of Aqueous Surfactant Systems; US Government Printing Office: Washington DC., 1971; Vol. 36

(155) Mowery, K. A.; Meyerhoff, M. E. The Transport of Nitric Oxide through Various Polymeric Matrices. Polymer (Guildf). 1999 40 (22), 6203-6207.

(156) Marín, M. J. Intracellular Analysis of PH and Nitric Oxide Using Fluorescence Based Nanosensors and Molecular Probes, University of East Anglia, Norwich, UK, 2012.

(157) O'Brien, J.; Wilson, I.; Orton, T.; Pognan, F. Investigation of the Alamar Blue (Resazurin) Fluorescent Dye for the Assessment of Mammalian Cell Cytotoxicity. Eur. J. Biochem. 2000, 267 (17), 5421-5426.

(158) Promega Cooperation. CellTiter-Blue Cell Viability Assay Technical Bulletin (TB317). 2016, 16.

(159) Buchner, M.; García Calavia, P.; Muhr, V.; Kröninger, A.; Baeumner, A. J.; Hirsch, T.; Russell, D. A.; Marín, M. J. Photosensitiser Functionalised Luminescent Upconverting Nanoparticles for Efficient Photodynamic Therapy of Breast Cancer Cells. Photochem. Photobiol. Sci. 2019, 18, 98-109.

(160) García Calavia, P.; Marín, M. J.; Chambrier, I.; Cook, M. J.; Russell, D. A. Towards Optimisation of Surface Enhanced Photodynamic Therapy of Breast Cancer Cells Using Gold Nanoparticle-Photosensitiser Conjugates. Photochem. Photobiol. Sci. 2018, 17 (3), 281-289.

(161) Belmokhtar, C. A.; Hillion, J.; Ségal-Bendirdjian, E. Staurosporine Induces Apoptosis through Both Caspase-Dependent and Caspase-Independent Mechanisms. Oncogene 2001, 20 (26), 3354-3362.

(162) Hollingworth, S.; Baylor, S. M. Changes in Phenol Red Absorbance in Response to Electrical Stimulation of Frog Skeletal Muscle Fibers. J. Gen. Physiol. 1990, 96, 473-491. 
REFERENCES 
CHAPTER 6

CONCLUSIONS 



\section{CHAPTER 6. CONCLUSIONS}

\section{1) Molecular gel nanoparticles were prepared from a low molecular weight hydrogelator by sonication of a xerogel}

Merging the interest in polymeric nanogels as nanocarriers and in molecular gels as new soft materials, the preparation of novel molecular nanogels by self-assembly of a low molecular weight gelator is reported in this thesis. Using the $\mathrm{pH}$-triggered molecular hydrogelator $\mathbf{1}$, molecular nanogels were obtained by two different strategies: ultrasound promoted formation and preparation using liposomes as a template.

In the first approach, nanosized particles of $c a .50 \mathrm{~nm}$ and in concentrations as high as $c a .2$ $\mathrm{mM}$ were reproducibly prepared by sonication of a xerogel of $\mathbf{1}$ in aqueous media. These spherical nanogels, composed mainly of water as the analogous macroscopic material, would correspond to the initial stages of the aggregation of molecular gels, and seem to constitute an intermediate state between free molecules and self-assembled fibrillar networks. Nanogels show good temporal stability, in agreement with their Z-potential, and kinetically stable nanogels can be found after $24 \mathrm{~h}$ at 10-times below the critical aggregation concentration of 1. Also, the particles present accessible hydrophobic domains in which Nile red was entrapped.

As for the $\mathrm{pH}$-responsiveness of the nanoparticles, loaded non-polar substances were released at $\mathrm{pH}$ values above 11. However, no discernible size changes of the nanoparticles could be detected, suggesting a structural change of nanogels to objects with similar size at basic $\mathrm{pH}$, but with a lower hydrophobic encapsulation power that would release Nile red. Additionally, the nanogels showed partial solubilisation above $50^{\circ} \mathrm{C}$ and were stable in solutions of sodium chloride with a concentration similar to that of physiological media. Besides, high biocompatibility of the gelator and the nanoparticles was found in studies with human lung carcinoma cells (A549).

2) Molecular gel nanoparticles were prepared using liposomes as a template, affording intermediate lipogels

In the second strategy, nanogel particles of $\mathbf{1}$ were also obtained using liposomes as a template. Molecular gel formation into liposomes loaded with the ionized gelator at pH 9 was 
triggered by acidification with D-glucono-1,5-lactone to $\mathrm{pH}$ 5-6, and provided hybrid gel@liposome particles. Removal of the phospholipid bilayer with SDS afforded nanogel particles, with a total concentration of the gelator in the sample of $0.7 \mathrm{mM}$, a size of $c a .50 \mathrm{~nm}$ and keeping the acidic $\mathrm{pH}$ in the interior of the particle. Both lipogels and nanogels showed no size changes for at least a month.

Moreover, the hydrogel@liposome particles were loaded with doxorubicin, showing a similar release than that observed for liposomes. The hybrid particles described here constitute the first case of preparation of a molecular, nonpolymeric, gel inside liposomes. This type of nanocarriers merges the benefits of increased stability of liposomal vehicles with the inherent stimuli-specific release of hydrogels formed by low-molecular weight molecules. This foretells a potential use in the transport of bioactive substances and environmentally sensitive drug release of these hybrid systems.

\section{3) Nanogels loaded with the photosensitizers Rose Bengal and hypericin were prepared}

Encapsulation of Rose Bengal and hypericin was achieved in monomeric form and maintaining photoactivity characteristics. Loading into nanogel particles of 1 solves the aggregation-caused quenching of the excited states of these photosensitizers, which is detrimental for their use in photodynamic therapy. Additionally, in the case of hypericin, nanoparticles increase ca. 10 times the maximum concentration of this molecule that it is possible to solubilise in water.

\section{4) The photosensitizers loaded in the nanogels show enhanced cellular uptake}

The low cellular uptake ability, due to RB anionic nature and HYP low solubility in water, was improved. HYP@1 and RB@1 show a higher cell uptake in HT-29 cells than the controls, obtaining even 70 times higher intracellular fluorescence intensity for RB@1.

5) The photosensitizers loaded in the nanogels show improved performance in photodynamic therapy

Preliminary in vitro results in HT-29 cells show enhanced phototoxicity for RB@1 compared to RB in solution, probably ascribed to the higher internalization of RB in cells when incubated with RB@1. Moreover, the phototoxicity of hypericin using HYP@1 in HT-29 cells is maintained compared to the use of DMSO as cosolvent, so our nanoparticles would be a safer alternative 
formulation. Also, both RB@1 and HYP@1 show no dark toxicity, making them suitable for clinical applications and promising PDT agents.

6) The nitric oxide probes DAF-2 and DAF-2 DA were loaded in the nanogels, resulting in enhanced cellular uptake

Nanoparticle encapsulation improved the cellular uptake issues of the fluorescent NO sensors DAF-2 (due to its anionic nature) and DAF-2 DA (because its low solubility in water). DAF2/DAF2DA@1 samples increased 12- to 17-times the intracellular fluorescence emission intensity for RAW 264.7 macrophage cells. These results were obtained using a 10-times lower probe concentration than conventionally used for NO imaging by DAF-2 DA. It was checked that the encapsulation does not hinder NO signalling, as the formation of the triazole was demonstrated for DAF2@1 in solution after NO addition. Additionally, the encapsulation of DAF-2 DA reduced the spontaneous hydrolysis of the probe to DAF-2 with time in solution.

\section{7) The solubility in water of the nitric oxide probe DAQ is improved by encapsulation into nanogels}

Encapsulation of the NO sensor DAQ into nanogels increased 4.5-times the solubility in water of the probe, which has a hydrophobic character. However, nanoparticles could not help to increase the cellular uptake of DAQ in RAW 264.7 cells to fulfil the requirement of a high concentration of triazole for its fluorescent detection.

\section{FINAL REMARKS}

The use of molecular nanogels formed by compound 1 to carry photoactive molecules has been demonstrated, enhancing their solubilisation and cellular uptake and maintaining their photoactivity. As a result, the reported nanogel systems overcome some of the inherent unfavourable pharmacological properties of these molecules for a clinical application. The improvement of the intracellular performance observed for most molecules could be a consequence of a different internalisation mechanism, as the free fluorophores would enter in the cells by a diffusion process and nanoparticles by endocytosis. As future work, it would be interesting to study if a disassemble is being produced into cells or photoactive molecules are still encapsulated during its action. 
The promising results of these first molecular nanogels show their great potential to be used as new nanocontainers for biomedical applications. In the so-called race for drug delivery, the use of polymeric nanogels presents challenges associated with stimuli-triggered release, biodegradation, polydispersity and batch-to-batch reproducibility in polymers preparation. The particles described here show intrinsic advantages compared to polymeric nanogels, such as stimuli-triggered disassembly and improved biodegradability due to their molecular nature. Additionally, the extensive library of molecular gelators available paves the way for the preparation of molecular nanogels with tailored properties, such as stimuli responsiveness or the presence of the desired functional groups in their structure. 



\section{CHAPTER 7. EXPERIMENTAL SECTION}

\subsection{PREPARATION OF MOLECULAR NANOGELS}

\subsubsection{GENERAL REMARKS}

Commercially available reagents and HPLC grade solvents were used as received from SigmaAldrich, Acros Organics and Fisher Scientific. Phosphate buffered saline (PBS) solution (0.01 M phosphate buffer, $0.0027 \mathrm{M} \mathrm{KCl}, 0.137 \mathrm{M} \mathrm{NaCl}$ ) was prepared from tablets, adjusting the $\mathrm{pH}$ to 7.0 and filtering the solution using a $0.22 \mu \mathrm{m}$ pore nylon filter. Toluene, ethyl acetate and other buffers were also filtered with a $0.45 \mu \mathrm{m}$ pore nylon filter. MiliQ water was always used. All samples and solutions containing fluorophores were protected from light to avoid photobleaching. For sonication, an Elmasonic S $60 \mathrm{H}$ (Elma) or a Fisherbrand FB15053 (Fisher Scientific) ultrasonic units were used. Centrifugation was performed in the vial using a Hettich ${ }^{\circledR}$ EBA 20 centrifuge at room temperature or in Eppendorf tubes $(1.5 \mathrm{~mL})$ using a Beckman Coulter Allegra ${ }^{\mathrm{TM}} \mathrm{X}-22 \mathrm{R}$ centrifuge at $15^{\circ} \mathrm{C}$.

\subsubsection{SYNTHESIS AND CHARACTERIZATION OF 1}

General remarks: Reactions which required an inert atmosphere were carried out under $\mathrm{N}_{2}$. ${ }^{1} \mathrm{H}-\mathrm{NMR}$ and ${ }^{13} \mathrm{C}-\mathrm{NMR}$ spectra were recorded on an Agilent VNMR System spectrometer (500 $\mathrm{MHz}$ for ${ }^{1} \mathrm{H}-\mathrm{NMR}$ and $126 \mathrm{MHz}$ for ${ }^{13} \mathrm{C}-\mathrm{NMR}$ ) or a Bruker Avance III HD spectrometer (400 MHz and $300 \mathrm{MHz}$ for ${ }^{1} \mathrm{H}-\mathrm{NMR}$, and $101 \mathrm{MHz}$ and $75 \mathrm{MHz}$ for ${ }^{13} \mathrm{C}-\mathrm{NMR}$ ) in the indicated solvent at $30^{\circ} \mathrm{C}$. The signals of the deuterated solvent (DMSO- $d_{6}$ ) were taken as the reference, the singlet at $\delta 2.50 \mathrm{ppm}$ and the quadruplet centred at $39.52 \mathrm{ppm}$ for ${ }^{1} \mathrm{H}$ and ${ }^{13} \mathrm{C}$, respectively. The ${ }^{1} \mathrm{H}$ NMR chemical shifts $\left(\delta_{H}\right)$ and ${ }^{13} \mathrm{C}-\mathrm{NMR}$ chemical shifts $\left(\delta_{\mathrm{C}}\right)$ are quoted in parts per million (ppm) downfield from trimethylsilane (TMS) and coupling constants $(J)$ are quoted in Hertz $(\mathrm{Hz})$. The abbreviations for NMR data are $s$ (singlet), $d$ (doublet), $t$ (triplet), q (quartet), quin (quintet) and $\mathrm{m}$ (multiplet). The ${ }^{1} \mathrm{H}$ and ${ }^{13} \mathrm{C}$ signals were assigned with the aid of $2 \mathrm{D}$ methods (COSY, HSQC and HMBC). The data were processed with the software Mestrenova. The mass spectra were run by the electrospray mode (ESMS) and were recorded using a Mass Spectrometry triple Quadrupole Q-TOF Premier (Waters) with simultaneous Electrospray and an APCI Probe. 
(S)-2,5-Dioxopyrrolidin-1-yl-2-(((benzyloxy)carbonyl)amino)-3-methylbutanoate (4). A solution of $N$-carbobenzyloxy-L-valine 5 (10 g, $40 \mathrm{mmol})$ and $N$-hydroxysuccinimide $(4.61 \mathrm{~g}, 40$ mmol, 1.0 eq.) in THF (200 mL) was added dropwise under $\mathrm{N}_{2}$ at $0^{\circ} \mathrm{C}$ to a solution of $N, N^{\prime}-$ dicyclohexylcarbodiimide (8.33 g, $40.4 \mathrm{mmol}, 1.01 \mathrm{eq}$ ) in THF (100 mL). The mixture was further stirred for $2 \mathrm{~h}$ at $0^{\circ} \mathrm{C}$. The solution was then kept in the refrigerator at $4^{\circ} \mathrm{C}$ for $16 \mathrm{~h}$, which caused precipitation of $N, N^{\prime}$-dicyclohexylurea. Then the mixture was filtered under vacuum, the solvent was removed under reduced pressure and the crude residue was purified by crystallization in isopropanol to yield $4(13.1 \mathrm{~g}, 37.6 \mathrm{mmol}, 94 \%)$ as a white solid. The NMR spectra were consistent with those reported in the literature. ${ }^{1}$

Benzyl (S)-(3-methyl-1-(nonylamino)-1-oxobutan-2-yl)carbamate (3). A solution of compound $4(7.58 \mathrm{~g}, 20 \mathrm{mmol})$ in THF $(90 \mathrm{~mL})$ was added dropwise under $\mathrm{N}_{2}$ at $25^{\circ} \mathrm{C}$ to a solution of $n$ nonylamine ( $4.1 \mathrm{~mL}, 22.8 \mathrm{mmol}, 1.1$ eq.) in THF (55 mL). The mixture was further stirred for 5 $\mathrm{h}$ at $50^{\circ} \mathrm{C}$. The white solid obtained was filtered under vacuum and washed with $\mathrm{HCl} 0.1 \mathrm{M}$ $(100 \mathrm{~mL})$ and water $(200 \mathrm{~mL})$. The compound was dried under reduced pressure at $50^{\circ} \mathrm{C}$ to yield 3 (5.8 g, $15.4 \mathrm{mmol}, 77 \%$ ) as a white solid. ${ }^{1} \mathbf{H}$ NMR (300 MHz, DMSO-d 6 ): $\delta 7.82$ (dd, $J=$ 5.2, $5.4 \mathrm{~Hz}, 1 \mathrm{H}), 7.42-7.24(\mathrm{~m}, 5 \mathrm{H}), 7.15(\mathrm{~d}, J=8.9 \mathrm{~Hz}, 1 \mathrm{H}), 5.02(\mathrm{~s}, 2 \mathrm{H}), 3.78(\mathrm{dd}, J=7.5,6.6$ $\mathrm{Hz}, 1 \mathrm{H}), 3.19-2.88(\mathrm{~m}, 2 \mathrm{H}), 1.92(\mathrm{~m}, 1 \mathrm{H}), 1.37(\mathrm{~m}, 2 \mathrm{H}), 1.23(\mathrm{~m}, 12 \mathrm{H}), 0.85(\mathrm{~m}, 9 \mathrm{H}) .{ }^{13} \mathrm{C}$ NMR (75 MHz, DMSO-d $\left.\boldsymbol{d}_{6}\right): \delta 170.8(\mathrm{C}=\mathrm{O}), 156.0(\mathrm{C}=\mathrm{O}), 137.1(\mathrm{C}), 128.2(2 \times \mathrm{CH}), 127.7(\mathrm{CH}), 127.5(2 \times$ $\mathrm{CH}), 65.3\left(\mathrm{CH}_{2}\right), 60.3(\mathrm{CH}), 38.3\left(\mathrm{CH}_{2}\right), 31.2\left(\mathrm{CH}_{2}\right), 30.2(\mathrm{CH}), 28.9\left(\mathrm{CH}_{2}\right), 28.9\left(\mathrm{CH}_{2}\right), 28.7\left(\mathrm{CH}_{2}\right)$, 28.6 $\left(\mathrm{CH}_{2}\right), 26.3\left(\mathrm{CH}_{2}\right), 22.0\left(\mathrm{CH}_{2}\right), 19.1\left(\mathrm{CH}_{3}\right), 18.2\left(\mathrm{CH}_{3}\right), 13.9\left(\mathrm{CH}_{3}\right)$. HRMS (ESI-TOF): $\mathrm{m} / z \mathrm{calcd}$ for $\left[\mathrm{C}_{22} \mathrm{H}_{37} \mathrm{~N}_{2} \mathrm{O}_{3}\right]^{+}:$377.2804; found: $377.2802[\mathrm{M}+\mathrm{H}]^{+}(\Delta=0.3 \mathrm{ppm})$.

(S)-2-Amino-3-methyl-N-nonylbutanamide (2). Palladium catalyst (10\% w/w Pd/C, $580 \mathrm{mg}$ ) and compound 3 (5.8 g, $15.4 \mathrm{mmol})$ were suspended in $\mathrm{CH}_{3} \mathrm{OH}(350 \mathrm{~mL})$ and stirred under $\mathrm{N}_{2}$ at room temperature for $10 \mathrm{~min}$. Subsequently, the system was kept under low vacuum and then filled with hydrogen from a latex balloon. Then the mixture was stirred at room temperature for $4 \mathrm{~h}$. After this time $\mathrm{Pd} / \mathrm{C}$ was removed by filtration through Celite, and the solvent was removed under reduced pressure to yield 2 (3.54 g, $14.6 \mathrm{mmol}, 94 \%)$ as a white solid. The compound was used without further purification for the next reaction. ${ }^{1} \mathbf{H}$ NMR (500 MHz, DMSO-d $)): \delta 7.75(\mathrm{~m}, 1 \mathrm{H}), 3.12-2.97(\mathrm{~m}, 2 \mathrm{H}), 2.88(\mathrm{~d}, J=5.1 \mathrm{~Hz}, 1 \mathrm{H}), 1.83(\mathrm{~m}, 1 \mathrm{H}), 1.38$ $(\mathrm{m}, 2 \mathrm{H}), 1.24(\mathrm{~m}, 12 \mathrm{H}), 0.87(\mathrm{~m}, 6 \mathrm{H}), 0.77(\mathrm{~d}, J=6.8 \mathrm{~Hz}, 3 \mathrm{H})$. The amine signals $\left(\mathrm{NH}_{2}\right)$ are very broad and cannot be distinguished in the spectrum. ${ }^{13} \mathrm{C}$ NMR (126 MHz, DMSO-d6): $\delta 174.2$ 
$(\mathrm{C}=\mathrm{O}), 60.0(\mathrm{CH}), 38.2\left(\mathrm{CH}_{2}\right), 31.6\left(\mathrm{CH}_{2}\right), 31.2(\mathrm{CH}), 29.2\left(\mathrm{CH}_{2}\right), 28.9\left(\mathrm{CH}_{2}\right), 28.7\left(\mathrm{CH}_{2}\right), 28.6\left(\mathrm{CH}_{2}\right)$, $26.4\left(\mathrm{CH}_{2}\right), 22.1\left(\mathrm{CH}_{2}\right), 19.5\left(\mathrm{CH}_{3}\right), 17.1\left(\mathrm{CH}_{3}\right), 13.9\left(\mathrm{CH}_{3}\right)$.

(S)-4-((3-Methyl-1-(nonylamino)-1-oxobutan-2-yl)amino)-4-oxobutanoic acid (1). Powdered $\mathrm{K}_{2} \mathrm{CO}_{3}(7.4 \mathrm{~g}, 53.5 \mathrm{mmol}, 3.8$ eq.) was added to a solution of amine 2 (3.54 g, $14.6 \mathrm{mmol})$ in THF $(290 \mathrm{~mL})$ at $0^{\circ} \mathrm{C}$ under $\mathrm{N}_{2}$ and stirred for 15 minutes. Then a solution of succinic anhydride ( $2.92 \mathrm{~g}, 29.2 \mathrm{mmol}, 2.0$ eq.) in THF (100 mL) was added dropwise and the mixture was stirred vigorously for $16 \mathrm{~h}$ at room temperature. After this, the solution was concentrated under reduced pressure and the crude residue was dissolved in water $(200 \mathrm{~mL})$. Finally, concentrated hydrochloric acid was added dropwise at $0^{\circ} \mathrm{C}$ until the formation of a white precipitate at $\mathrm{pH}=$ 3. The white solid obtained was filtered under vacuum, and the residue was washed with water $(300 \mathrm{~mL})$. The compound was dried under reduced pressure at $50^{\circ} \mathrm{C}$ to yield $1(4.86 \mathrm{~g}$, $14.2 \mathrm{mmol}, 97 \%)$ as a white solid. ${ }^{1} \mathbf{H}$ NMR (500 MHz, DMSO-d $)$ : $\delta 11.99$ (br s, $\left.1 \mathrm{H}\right), 7.80$ (m, $2 \mathrm{H}), 4.07(\mathrm{t}, J=7.9 \mathrm{~Hz}, 1 \mathrm{H}), 3.15-2.90(\mathrm{~m}, 2 \mathrm{H}), 2.47-2.32(\mathrm{~m}, 4 \mathrm{H}), 1.92(\mathrm{~m}, 1 \mathrm{H}), 1.36(\mathrm{~m}, 2 \mathrm{H})$, $1.23(\mathrm{~m}, 12 \mathrm{H}), 0.85(\mathrm{t}, J=6.7 \mathrm{~Hz}, 3 \mathrm{H}), 0.81(\mathrm{~d}$, overlapped, $J=5.3 \mathrm{~Hz}, 6 \mathrm{H}) .{ }^{13} \mathrm{C} \mathrm{NMR}(126 \mathrm{MHz}$, DMSO- $\left.d_{6}\right): \delta 174.3(\mathrm{C}=\mathrm{O}), 171.5(\mathrm{C}=\mathrm{O}), 171.2(\mathrm{C}=\mathrm{O}), 58.3(\mathrm{CH}), 38.8\left(\mathrm{CH}_{2}\right), 31.7\left(\mathrm{CH}_{2}\right), 30.9$ $\left(\mathrm{CH}_{2}\right), 30.4\left(\mathrm{CH}_{2}\right), 29.8(\mathrm{CH}), 29.4\left(\mathrm{CH}_{2}\right), 29.4\left(\mathrm{CH}_{2}\right), 29.1\left(\mathrm{CH}_{2}\right), 29.1\left(\mathrm{CH}_{2}\right), 26.8\left(\mathrm{CH}_{2}\right), 22.5$ $\left(\mathrm{CH}_{2}\right), 19.6\left(\mathrm{CH}_{3}\right), 18.6\left(\mathrm{CH}_{3}\right), 14.4\left(\mathrm{CH}_{3}\right)$. HRMS (ESI-TOF): $\mathrm{m} / z$ calcd for $\left[\mathrm{C}_{18} \mathrm{H}_{35} \mathrm{~N}_{2} \mathrm{O}_{4}\right]^{+}$: 343.2597; found: $343.2591[\mathrm{M}+\mathrm{H}]^{+}(\Delta=1.7 \mathrm{ppm})$. 

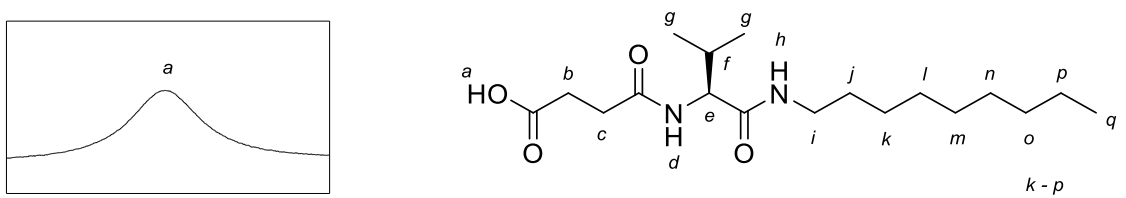

$\begin{array}{lllll}12.4 & 12.2 & 12.0 & 11.8 & 11.6\end{array}$
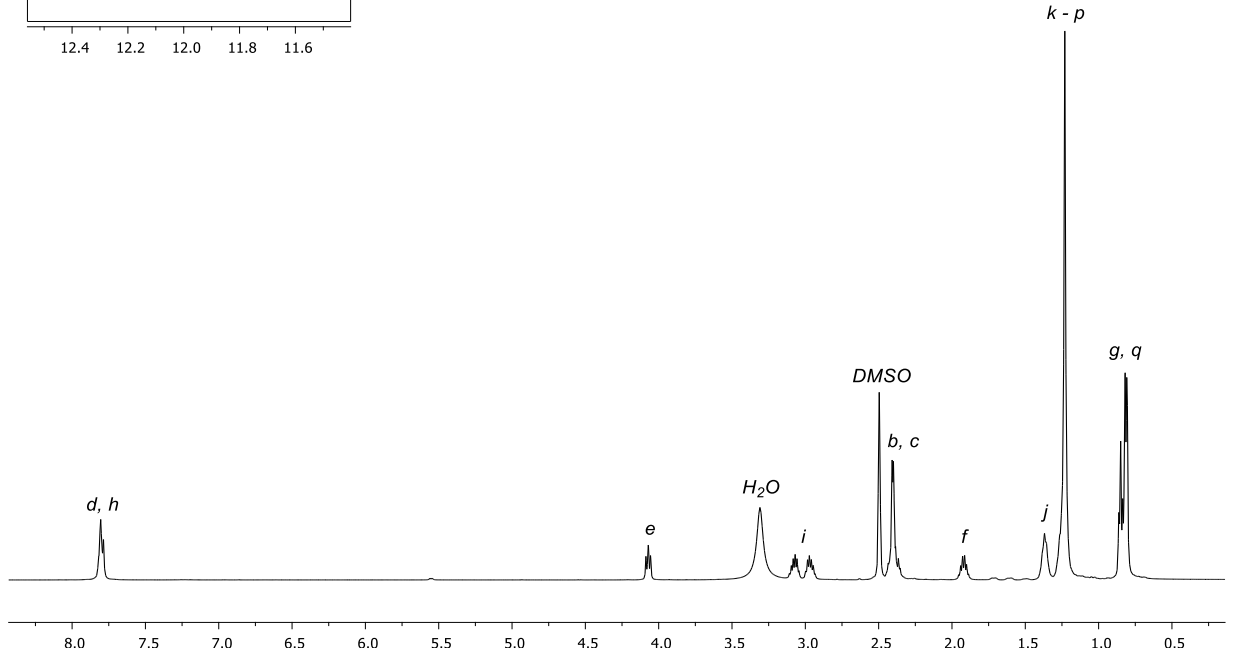

Figure 7.1. ${ }^{1} \mathrm{H}$ NMR spectrum of 1 in DMSO- $\mathrm{d}_{6}$.
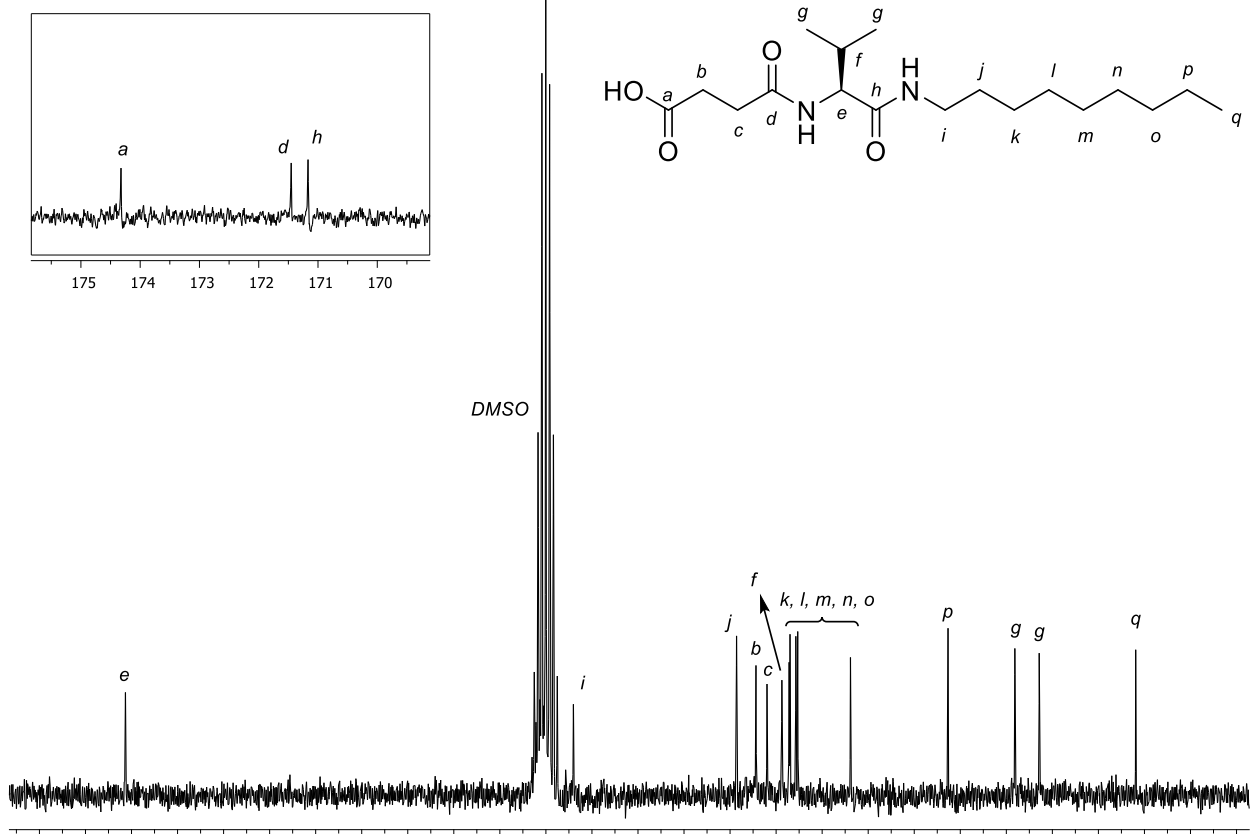

$\begin{array}{lllllllllllllllllllllllllllllllll}62 & 60 & 58 & 56 & 54 & 52 & 50 & 48 & 46 & 44 & 42 & 40 & 38 & 36 & 34 & 32 & 30 & 28 & 26 & 24 & 22 & 20 & 18 & 16 & 14 & 12 & 10\end{array}$

Figure 7.2. ${ }^{13} \mathrm{C}$ NMR spectrum of 1 in DMSO- $d_{6}$. 


\subsubsection{GEL PREPARATION AND CHARACTERISATION GELS OBTAINED BY HEATING-COOLING PROCESS}

In a typical experiment, $5 \mathrm{mg}$ of compound $\mathbf{1}$ and $1 \mathrm{~mL}$ of solvent were introduced into a cylindrical screw-capped glass vial $(8 \mathrm{~mL}$, diameter $=1.5 \mathrm{~cm})$. The system was sonicated to obtain a homogeneous suspension and the vial was heated (heat gun, $250^{\circ} \mathrm{C}$ ) until a clear solution was obtained. Then, the vial was introduced into a thermostatic bath at $25^{\circ} \mathrm{C}$. After 10-20 minutes, the formation of the gel was assessed by turning the vial upside down (all the solvent remains entrapped).

\section{GELS OBTAINED BY PH CHANGE}

A $20 \mathrm{mg} / \mathrm{mL}$ stock solution of compound 1 in $\mathrm{NaOH} 0.1 \mathrm{M}$ was used to prepare $500 \mu \mathrm{L}$ solutions of lower concentration in cylindrical screw-capped glass vials $(4 \mathrm{~mL}$, diameter $=1.3$ $\mathrm{cm}) .500 \mu \mathrm{L}$ of $\mathrm{HCl} 0.1 \mathrm{M}$ were added to each one and, after $24 \mathrm{~h}$, gelation was checked by vial inversion. For D-glucono-1,5-lactone gelation, compound $\mathbf{1}$ was dissolved in 10 equivalents of $\mathrm{K}_{2} \mathrm{CO}_{3}$ and gelation was triggered by addition of 60 equivalents of D-glucono-1,5-lactone to yield a $1 \mathrm{~mL}$ macroscopic gel.

\section{TRANSMISSION ELECTRON MICROSCOPY (TEM)}

Transmission electron micrographs were obtained using a JEOL 2100 microscope with a thermionic gun LaB6 200 kV equipped with a Gatan Orius high resolution CCD camera. TEM samples were prepared over a formvar/carbon film on 200 mesh copper grids. Fresh gels were applied directly onto the grid and the expelled solvent was carefully removed by capillary action with paper. The grids were immediately stained with one drop of $1 \%$ aqueous phosphotungstic acid for 2 min and the liquid was subsequently removed by capillary action.

\section{POTENTIOMETRIC TITRATION}

Potentiometric titrations to determine $\mathrm{p} K_{\mathrm{a}}$ of 1 were carried out at $25^{\circ} \mathrm{C}$. An aqueous solution $(0.5 \mathrm{mg} / \mathrm{mL}, 5 \mathrm{~mL})$ of compound 1 in sodium hydroxide $0.1 \mathrm{M}$ was titrated with vigorous stirring with a $0.1 \mathrm{M}$ normalized solution of hydrochloric acid. The addition rate was $6 \mathrm{~mL} / \mathrm{h}$ and $\mathrm{pH}$ was monitored every $10 \mathrm{~s}$ (in a S220 Seven Compact pH meter, Mettler Toledo). pKa was calculated by fitting the experimental data to calculated titration curves with the program HYPERQUAD. ${ }^{2}$ 


\section{DETERMINATION OF THE CRITICAL AGGREGATION CONCENTRATION AND THE CRITICAL MICELLE CONCENTRATION}

The critical aggregation concentration (cac) of compound 1 at $\mathrm{pH} 1$ was determined using pyrene fluorescence changes (peak I/peak III ratio). A series of solutions of pyrene $(1 \mu \mathrm{g} / \mathrm{mL}$ ) and gelator 1 at different concentrations $(0-500 \mu \mathrm{g} / \mathrm{mL})$ were prepared in $0.1 \mathrm{M}$ aqueous $\mathrm{NH}_{4} \mathrm{OH}$. Then, the samples were acidified to $\mathrm{pH} 1$ with $2 \mathrm{M} \mathrm{H}_{2} \mathrm{SO}_{4}$ (promoting aggregation) and fluorescence was recorded ( $\lambda_{\mathrm{ex}} 334 \mathrm{~nm}$ ). The critical micelle concentration (CMC) was determined based on the same principle, by recording the fluorescence $\left(\lambda_{\text {ex }} 334 \mathrm{~nm}\right)$ of solutions of the gelator 1 at different concentrations $(0.01-5 \mathrm{mg} / \mathrm{mL}$ gelator) in a solution of pyrene in $0.05 \mathrm{M} \mathrm{K}_{2} \mathrm{CO}_{3}$ or in $0.1 \mathrm{M} \mathrm{NaOH}$.

\section{DIFFERENTIAL SCANNING CALORIMETRY}

Xerogels were placed in a sealed aluminium pan. DSC thermograms were recorded on a Mettler Toledo DSC2 apparatus at a heating rate of $1^{\circ} \mathrm{C} / \mathrm{min}$.

\subsubsection{NANOGELS PREPARED BY ULTRASONICATION}

\section{PREPARATION OF MOLECULAR NANOGELS BY ULTRASONICATION}

To prepare a $2 \mathrm{~mL}$ nanogel sample, a suspension of $7.3 \mu \mathrm{mol}$ of compound $\mathbf{1} \mathrm{in} 1 \mathrm{~mL}$ toluene in a screw-capped vial $\left(4 \mathrm{~mL}\right.$, diameter $=1.3 \mathrm{~cm}$ ) was heated (heat gun, $250^{\circ} \mathrm{C}$ ) until complete solution. After cooling (thermostatic bath, $25^{\circ} \mathrm{C}$ ), a gel was obtained. Then, the solvent was removed under vacuum and the xerogel was hydrated in $2 \mathrm{~mL}$ of PBS for $10 \mathrm{~min}$. At this point, the system was ultrasonicated for $c a .10$ minutes until a homogenous suspension was obtained, with a final $\mathrm{pH}$ of 6.4. Then, large particles were removed by centrifugation at 6000 $\mathrm{rpm}$ for $60 \mathrm{~min}$ to yield a clear solution of the nanogel particles. $7 \mathrm{~mL}$ nanogel samples were also prepared using $25.6 \mu \mathrm{mol}$ of $\mathbf{1}$ to prepare a $2 \mathrm{~mL}$ gel in a $8 \mathrm{~mL}$ screw-capped glass vial and hydrating with $7 \mathrm{~mL}$ of PBS.

\section{GENERAL METHOD FOR PREPARATION OF FLUOROPHORE@1 SAMPLES}

Following the protocol in the section above, a fluorophore@1 sample at a X/2 $\mu \mathrm{M}$ nominal concentration was obtained using $7.3 \mu \mathrm{mol}$ of compound 1 and $1 \mathrm{~mL}$ of a $\mathrm{X} \mu \mathrm{M}$ solution of fluorophore to prepare the gel. 


\section{NILE RED LOADING}

Preparation of nanogels in the presence of the cargo: $10 \mu \mathrm{M}$ Nile red solution in toluene was used to prepare the gel.

Addition of the cargo once nanoparticles were formed: $1.5 \mathrm{~mL}$ of a $10 \mu \mathrm{M}$ Nile red solution in PBS-0.4\%EtOH were mixed with $1.5 \mathrm{~mL}$ of nanogels sample. Nile red solution in PBS- $0.4 \% \mathrm{EtOH}$ was prepared from a $2.4 \mathrm{mM}$ Nile red in ethanol and used after $1 \mathrm{~h}$ stabilization. ${ }^{3}$

\section{LOADED NANOGEL SAMPLES FOR CELLULAR ASSAYS}

Rose Bengal: A $40 \mu \mathrm{M}$ RB suspension in toluene was used to obtain RB@1 samples of $20 \mu \mathrm{M}$ nominal concentration. The pH of RB@1 was adjusted to 7 by $\mathrm{NaOH} 0.1 \mathrm{M}$ addition and the sample was quantified. For comparison purposes, for each RB@1 sample, a RB solution in PBS at a higher concentration was prepared (RB control) from a $550 \mu \mathrm{M} R B$ stock in PBS.

Hypericin: $25 \mu \mathrm{M}$ nominal concentration HYP@1 samples were prepared using $1 \mathrm{~mL}$ of $50 \mu \mathrm{M}$ ( $2 \mathrm{~mL}$ samples) or $3 \mathrm{~mL}$ of $55 \mu \mathrm{M}$ ( $7 \mathrm{~mL}$ samples) HYP suspension in toluene. For comparison purposes, for each HYP@1 sample, a HYP solution in PBS-1\%DMSO at the same concentration was prepared (HYP-DMSO control) from a $1 \mathrm{mM}$ HYP stock in DMSO. No pH change was performed, HYP@1 were assayed at pH 6.4 and controls at pH 7.

DAF-2 DA: $10 \mu \mathrm{M}$ nominal concentration DAF2DA@1 samples were prepared using $1 \mathrm{~mL}$ of 20 $\mu M$ DAF-2 DA solution in toluene-0.5\%DMSO. For comparison purposes, for each DAF2DA@1 sample, a DAF-2 DA solution in PBS-X\%DMSO at the same concentration (DAF2DA control) was prepared from a $1 \mathrm{mM}$ DAF-2 DA stock in DMSO. The percentage of DMSO was always kept to the minimum quantity possible. No pH change was performed, DAF2DA@1 samples were assayed at $\mathrm{pH} 6.4$ and controls at $\mathrm{pH} 7$.

DAF-2: $10 \mu \mathrm{M}$ nominal concentration DAF2@1 samples were prepared using $1 \mathrm{~mL}$ of $20 \mu \mathrm{M}$ DAF-2 solution in toluene-0.4\%DMSO ( $2 \mathrm{~mL}$ samples) or $2 \mathrm{~mL}$ of $35 \mu \mathrm{M}$ DAF-2 solution in toluene-0.7\%DMSO (7 mL samples). For comparison purposes, for each DAF2@1 sample, a DAF-2 solution in PBS-0.1\%DMSO at the same concentration (DAF2 control) was prepared from a $20 \mu \mathrm{M}$ DAF-2 stock in PBS-0.4\%DMSO. No pH change was performed, DAF2@1 samples were assayed at $\mathrm{pH} 6.4$ and controls at $\mathrm{pH} 7$. 
DAQ: $100 \mu \mathrm{M}$ nominal concentration DAQ@1 samples were prepared using $1 \mathrm{~mL}$ of $200 \mu \mathrm{M}$ (2 $\mathrm{mL}$ samples) or $1.75 \mathrm{~mL}$ of a $400 \mu \mathrm{M}$ (7mL samples) DAQ solution in toluene-7\%AcOEt. For comparison purposes, for each DAQ@1 sample, a DAQ solution at the same concentration in PBS-0.5\%DMSO (DAQ-DMSO control) was prepared from a $5 \mathrm{mM}$ DAQ stock in DMSO. No pH change was performed, DAQ@1 was assayed at pH 6.4 and controls at pH 7.

\subsubsection{NANOGELS PREPARED USING LIPOSOMES AS A TEMPLATE LIPOSOME PREPARATION}

L- $\alpha-P C(10 \mathrm{mg})$ and cholesterol $(1.25 \mathrm{mg})$ were mixed in a $25 \mathrm{~mL}$ round-bottom flask in a total volume of $2 \mathrm{~mL}$ chloroform (8:2 molar ratio). L- $\alpha-P C$ from egg yolk was equally used as a 100 $\mathrm{mg} / \mathrm{mL}$ solution in chloroform ( $99 \%$ purity) or as a solid ( $60 \%$ purity), and cholesterol was used as a $1 \mathrm{mg} / \mathrm{mL}$ solution in chloroform. Then, chloroform was removed first using a rotary evaporator and then under vacuum until dryness $(5 \mathrm{~h})$. The dry lipid film was rehydrated with $2 \mathrm{~mL} \mathrm{0.1} \mathrm{M} \mathrm{phosphate} \mathrm{buffer} \mathrm{pH}$ 9. Then, the mixture was vigorously stirred for $30 \mathrm{~min}$ at $55^{\circ} \mathrm{C}$ and later, transferred to an $8 \mathrm{~mL}$ screw-capped glass vial and sonicated for $1 \mathrm{~h}$. The nonencapsulated materials were removed using SEC (Sepharose ${ }^{\star} 4 \mathrm{~B}, 0.1 \mathrm{M}$ phosphate buffer pH 9 as mobile phase) and the final sample was filtered (nylon $0.45 \mu \mathrm{m}$ filter). Samples were kept at $4^{\circ} \mathrm{C}$.

\section{NANOGEL@LIPOSOME PREPARATION}

L- $\alpha-P C(10 \mathrm{mg})$ and cholesterol $(1.25 \mathrm{mg})$ were mixed in a $25 \mathrm{~mL}$ round-bottom flask in a total volume of $2 \mathrm{~mL}$ chloroform (8:2 molar ratio). Then, chloroform was removed first using a rotary evaporator and then under vacuum until dryness (5 h). The dry lipid film was rehydrated with $2 \mathrm{~mL}$ of a solution of $1(10 \mathrm{mg} / \mathrm{mL})$ in $0.1 \mathrm{M}$ phosphate buffer $\mathrm{pH}$ 9. Then, the mixture was vigorously stirred for $30 \mathrm{~min}$ at $55^{\circ} \mathrm{C}$ and later, transferred to an $8 \mathrm{~mL}$ screwcapped glass vial and sonicated for $1 \mathrm{~h}$. The nonencapsulated materials were removed using SEC (Sepharose 4 B, $0.1 \mathrm{M}$ phosphate buffer $\mathrm{pH} 9$ as mobile phase) and the final sample was filtered (nylon $0.45 \mu \mathrm{m}$ filter). To obtain gel@liposomes, gelation inside vesicles was promoted adding $18 \mathrm{mg} / \mathrm{mL}$ of D-glucono-1,5-lactone. After waiting overnight, dialysis against $1 \mathrm{~L}$ of water was performed to remove excess of salts. To obtain naked nanogels, vesicles were disassembled and phospholipids removed. For this purpose, SDS (10 mg/mL) was added and, 
after $10 \mathrm{~min}$, dialysis against $1 \mathrm{~L}$ of water was repeated. Samples were kept at $4^{\circ} \mathrm{C}$. Liposomes for control purposes were prepared following the same protocol without gelator 1.

\section{PYRANINE}

Loading: Pyranine was introduced in the system in the hydration medium $(2 \mathrm{~mL} 0.1 \mathrm{M}$ phosphate buffer $\mathrm{pH}$ 9), using a $20 \mu \mathrm{M}$ pyranine solution for liposomes and a $100 \mu \mathrm{M}$ for gel@liposomes. The protocol was followed as indicated above.

pH calibration curve: A calibration curve was obtained from $1 \mu \mathrm{M}$ solutions of pyranine in 0.1 $\mathrm{M}$ phosphate buffer at $\mathrm{pH}$ values ranging from 5 to 11 . The fluorescence intensity at $511 \mathrm{~nm}$ was recorded after excitation at two different wavelengths (415 and $460 \mathrm{~nm}$ ), and the ratio between both measures plotted against $\mathrm{pH}$ was fitted to a sigmoidal curve.

\section{DOXORUBICIN}

Loading: To introduce doxorubicin in the liposomal systems, $1 \mathrm{mM}$ doxorubicin solution (2\% DMSO) was used as a hydration medium. The protocol was followed as indicated above.

Release studies: Doxorubicin release from liposomes and nanogel@liposome systems was studied by dialysis. Samples $(1.5 \mathrm{~mL})$ were introduced in a dialysis membrane (Spectra/Por 3 dialysis membrane, MWCO 3.5 kD, Spectrum Labs) and were dialyzed against $40 \mathrm{~mL}$ of $0.1 \mathrm{M}$ phosphate buffer $\mathrm{pH}$ 7. Then, $3 \mathrm{~mL}$ aliquots from the outside solution were taken at selected time intervals for spectroscopic analysis and then returned to the dialysis system. Doxorubicin concentration was calculated based on fluorescence (at $556 \mathrm{~nm}$, excitation at $480 \mathrm{~nm}$ ) or absorption intensity (at $478 \mathrm{~nm}$ ).

\subsubsection{METODOLOGY OF NANOGELS CHARACTERISATION DYNAMIC LIGHT SCATTERING}

Size measurements of the particles were performed by dynamic light scattering (DLS) using a Zetasizer Nano ZS (Malvern). Analyses were carried out using a He-Ne laser (633 nm) at a fixed scattering angle of $173^{\circ}$. Automatic optimization of beam focusing and attenuation was applied for each sample. When measurements were performed at $25^{\circ} \mathrm{C}$, nanogel suspensions were introduced into $3 \mathrm{~mL}$ disposable PMMA cuvettes (10 mm optical path length). Otherwise, the samples were placed in $3 \mathrm{~mL}$ optical glass cuvettes (10 $\mathrm{mm}$ optical path) and $5 \mathrm{~min}$ of 
sample stabilization were used each time the temperature was changed. The particle size was reported as the average of three measurements.

\section{Z-POTENTIAL}

Z-Potential measurements were performed at $25^{\circ} \mathrm{C}$ by Laser Doppler Micro-electrophoresis using a Zetasizer Nano ZS (Malvern). $1 \mathrm{~mL}$ of the nanogel suspension was taken in disposable folded capillary zeta cells (Malvern, DTS1070). The Z-Potential was reported as the average of six measures per sample of five different samples.

\section{TRANSMISSION ELECTRON MICROSCOPY}

TEM without staining: Transmission electron micrographs were obtained using a JEOL 2100 microscope with a thermionic gun LaB6 200 kV equipped with a Gatan Orius high-resolution CCD camera. TEM samples were prepared over a formvar/carbon film on 200 mesh copper grids. A drop of the sample was added on the grid and incubated for $2 \mathrm{~min}$. Then, the solvent was removed with filter paper by capillary action.

$\underline{T E M ~ O s O_{4}}$ staining: Images were obtained using a JEOL JEM-1010 transmission electron microscope of $100 \mathrm{kV}$ equipped with an AMT RX80 digital camera (8 Mpx). TEM samples were prepared over the formvar/carbon film on 200 mesh copper grids. A drop of the sample was added on the grid and incubated for $2 \mathrm{~min}$. Then, the solvent was removed with filter paper by capillary action and a drop of $\mathrm{OsO}_{4} 0.1 \%$ was added. After 5 min incubation, the staining solution was removed by capillary action, and the grid was washed with a drop of Milli-Q water.

Cryo-TEM: For the cryo-TEM technique, a JEM-2200FS/CR transmission electron microscope (JEOL, Japan) equipped with an UltraScan 4000 SP (4008 $\times 4008$ pixels) cooled slow-Scan CCD camera (GATAN, UK) was used. A drop of the sample was placed on the TEM grid, and an automated vitrification robot Vitrobot was used to freeze the sample in liquid ethane.

Size distribution of the particles obtained from TEM and cryo-TEM images was determined with ImageJ software and at least 100 particles were measured. 


\section{SINGLE ANGLE STATIC LIGHT SCATTERING}

Single angle static light scattering (SALS) measurements were performed using a Zetasizer Nano ZS (Malvern). The samples were introduced into $3 \mathrm{~mL}$ optical glass cuvettes (10 mm optical path) and toluene was used as a standard. The refractive index increment $(\mathrm{d} n / \mathrm{d} C$ ) for the nanogel particles was set to $0.063 \mathrm{~mL} / \mathrm{g}$, according to the results obtained using an AF2000 system (Postnova Analytics) and a refractive index detector in batch mode.

\section{GELATOR QUANTIFICATION USING NMR}

Nanogels by ultrasonication: The concentration of $\mathbf{1}$ in a nanogel suspension was quantified using ${ }^{1} \mathrm{H}$ NMR (Bruker Avance III HD spectrometer $400 \mathrm{MHz}$ ) after lyophilization and solubilization of 1 in a 6:1 mixture of $\mathrm{CDCl}_{3} /$ hexafluoroisopropanol. A calibrated electronic signal (ERETIC) at $\delta 11.0 \mathrm{ppm}$ was used for this purpose.

Nanogels prepared using liposomes as a template: The concentration of $\mathbf{1}$ in a sample was quantified using ${ }^{1} \mathrm{H}$ NMR (Bruker AVANCE III HD spectrometer $400 \mathrm{MHz}$ ) after lyophilization and solubilization of 1 in a $6: 1$ mixture of $\mathrm{CDCl}_{3} /$ hexafluoroisopropanol. A calibrated signal at $\delta$ $9.76 \mathrm{ppm}$ (quartet) of acetaldehyde contained in a concentric tube was used for this purpose. Acetaldehyde signal was calibrated using a $5 \mathrm{mg} / \mathrm{mL}$ standard of compound 1 in a 6:1 mixture of $\mathrm{CDCl}_{3} /$ hexafluoroisopropanol. Signals of the deuterated solvent $\left(\mathrm{CDCl}_{3}\right)$ were taken as the reference (singlet at $\delta 7.26)$.

\section{UV-VIS SPECTROSCOPY}

UV-Vis absorption spectra were recorded using a JASCO V-630 spectrophotometer equipped with a Peltier accessory ETCS- 761 at $25^{\circ} \mathrm{C}$ (samples in $1.5 \mathrm{~mL}$ quartz SUPRASIL ${ }^{\mathrm{TM}}$ cuvettes, 10 $\mathrm{mm}$ optical path length) or a Hitachi U-3000 spectrophotometer at room temperature (samples in $1.5 \mathrm{~mL}$ optical glass cuvettes, $10 \mathrm{~mm}$ optical path length).

\section{FLUORESCENCE SPECTROSCOPY}

The excitation and emission spectra were obtained using a JASCO FP-8300 spectrofluorometer equipped with a Peltier accessory ETC- 815 at $25^{\circ} \mathrm{C}$ (samples in $3 \mathrm{~mL}$ optical glass cuvettes, 10 $\mathrm{mm}$ optical path length), an Agilent Cary Eclipse fluorescence spectrophotometer at r.t. (samples in $1.5 \mathrm{~mL}$ or $3 \mathrm{~mL}$ quartz SUPRASIL ${ }^{\mathrm{TM}}$ cuvettes, $10 \mathrm{~mm}$ optical path length) or a Horiba 
Jobin Yvon FL3-11 FluoroLog fluorescence spectrometer at r.t. (spectra recorded in right angle mode using $1.5 \mathrm{~mL}$ optical glass cuvettes, $10 \mathrm{~mm}$ optical path length).

\subsubsection{QUANTIFICATION OF FLUOROPHORES}

Rose Bengal: A calibration curve was determined from the absorption spectra of a series of RB standard solutions in PBS (0.1-15 $\mu \mathrm{M})$ at $549 \mathrm{~nm}$. The quantification in samples was performed using the absorption intensity at the corresponding $\lambda_{\max }$ ( $549 \mathrm{~nm}$ for solutions and $569 \mathrm{~nm}$ for RB into nanogels), assuming the same molar extinction coefficient $(\varepsilon)$ for RB and RB@1. In the case the absorbance exceeded the calibration curve range 0.1-15 $\mu \mathrm{M}$, samples were diluted with PBS prior their absorbance measurement.

Hypericin: A calibration curve was determined from the absorption spectra of hypericin standards $(0.1-10 \mu \mathrm{M})$ in DMSO at $599 \mathrm{~nm}$. Using the calibration curve and the absorption intensity at the $\lambda_{\max }$ for each sample (596 nm for HYP@1 samples and 605 nm for HYP-PBS controls), the concentration of hypericin in samples was calculated. The same molar extinction coefficient $(\varepsilon)$ for HYP in both media was assumed.

DAF-2: A calibration curve was determined from the absorption spectra of DAF-2 standards $(0.25-20 \mu \mathrm{M})$ in PBS-0.4\%DMSO at $487 \mathrm{~nm}$. Using the calibration curve and the absorption intensity at the $\lambda_{\max }$ for each sample, the concentration of DAF-2 in samples was calculated. In the case the absorbance exceeded the calibration curve range $0.25-20 \mu \mathrm{M}$, samples were diluted with PBS prior their absorbance measurement.

DAF-2 DA: For DAF-2 DA quantification, first DAF-2 DA was hydrolysed to DAF-2 by addition of $20 \mu \mathrm{L}$ of $1 \mathrm{M} \mathrm{NaOH}$ solution to $1 \mathrm{~mL}$ of sample. Then, the concentration of hydrolysed DAF- 2 in samples was calculated and the original DAF-2 DA concentration was obtained considering a percentage of recovery of diacetate as DAF- 2 of $68.4 \%$.

DAQ: A calibration curve was determined from the absorption spectra of DAQ standards in PBS-1\%DMSO $(0.75-8 \mu \mathrm{M})$ at $520 \mathrm{~nm}$. Using the calibration curve and the absorption intensity at $520 \mathrm{~nm}$ of a sample, the concentration of DAQ in that sample was calculated. In the case the absorbance exceeded the calibration curve range 0.75-8 $\mu \mathrm{M}$, samples were diluted with PBS prior their absorbance measurement. 


\subsubsection{SYNTHESIS OF THE TRIAZOLE DAQ-TZ}

DAQ-TZ was synthesised as previously reported. ${ }^{4}$ An excess of gaseous $\mathrm{NO}(5 \mathrm{~mL} / \mathrm{mmol} \mathrm{DAQ})$ was injected to a DAQ (50 mg, $0.21 \mathrm{mmol})$ solution in aerated ethanol $(20 \mathrm{~mL} / \mathrm{mmol} \mathrm{DAQ})$, changing the colour of the solution from deep purple to dark brown. After 30 min stirring, centrifugation of the mixture afforded a small amount of unreacted DAQ (precipitated) and soluble DAQ-TZ. After rotary evaporation and vacuum drying, a brown solid corresponding to DAQ-TZ was obtained.

Gaseous nitric oxide was synthesized by a redox reaction in which sodium nitrite is reduced to nitric oxide and sodium iodide is oxidized to iodine in the presence of concentrated sulfuric acid: $:^{5,6}$

$$
2 \mathrm{NaNO}_{2(a q)}+2 \mathrm{NaI}_{(a q)}+2 \mathrm{H}_{2} \mathrm{SO}_{4(a q)} \rightarrow \mathrm{I}_{2(s)}+2 \mathrm{NO}_{(g)}+2 \mathrm{H}_{2} \mathrm{O}_{(l)}
$$

$250 \mathrm{~mL}$ of a $0.1 \mathrm{M} \mathrm{NaNO}_{2}$ solution and $250 \mathrm{~mL}$ of a $0.1 \mathrm{M} \mathrm{Nal}$ solution were introduced in a $500 \mathrm{~mL}$ separation funnel. After the funnel was isolated from the exterior with a septum and introducing the tip in a water bath (stopcock opened), air between the septum and the liquid was purged with nitrogen. Then, concentrated sulfuric acid was slowly added and the gas formation was seen at the same time that iodine was being formed (purple). ${ }^{7}$

\subsubsection{MEASUREMENT OF SINGLET OXYGEN PHOTOGENERATION}

Production of singlet oxygen was measured using the singlet oxygen probe 9,10anthracenediyl-bis (methylene) dimalonic acid (ABMA). $2 \mathrm{~mL}$ samples were placed in a $10 \times 10$ mm fluorescence quartz cuvette together with $1 \mu \mathrm{M}$ of ABMA (3.2 $\mu \mathrm{L}$ of a $0.625 \mathrm{mM}$ solution in methanol) and a magnetic stirrer bar. Oxygen was bubbled through the solution and the cuvette was closed with the stopper and Parafilm. The fluorescence emission spectrum of the initial solution was recorded using an excitation wavelength of $380 \mathrm{~nm}$. The sample was then irradiated, under continuous stirring, with visible light using a LED light for the RB study (8.6 W, ca. 400-700 nm emission output, the lamp placed as close to the cuvette as possible) and two LED lamps for the HYP study (11 W each one, ca. 400-700 nm emission output, the lamps placed perpendicular to each other at $1 \mathrm{~cm}$ from the cuvette). The evolution of the photoreaction was monitored over time by fluorescence spectroscopy ( $\lambda_{\text {ex }} 380 \mathrm{~nm}$ ), following the decrease of ABMA emission intensity at $407 \mathrm{~nm}$. The cuvette was protected from external light during all the process. The initial points of the kinetic traces were fitted to a pseudo-first 
order model ( $\ln C / C_{0}=-k_{\text {obs }} t$, where $C$ is the concentration of ABMA at a specific time $t$ and $C_{0}$ is the initial concentration of ABMA).

\subsubsection{STUDY OF THE RESPONSE TO NITRIC OXIDE IN SOLUTION}

To study the response to NO of the nanogel-loaded probe, a titration with NO was performed by addition of different volumes of a $15 \mathrm{mM}$ stock solution of DEA NONOate in $0.01 \mathrm{M} \mathrm{NaOH}$ to $2 \mathrm{~mL}$ aliquots of the sample. DEA NONOate decomposes at $\mathrm{pH} 7.4$ to give 1.5 molecules of NO. ${ }^{5,8-10}$ NO concentrations ranging from 12 to $1071 \mu \mathrm{M}$ were tested by addition of 1-100 $\mu \mathrm{L}$ NONOate. To test all NO concentrations in the same nanogel sample, several samples were prepared and mixed to achieve a composed, homogeneous probe@1 sample. After NONOate addition, samples were air-equilibrated so the reaction of triazole formation could proceed and the $\mathrm{pH}$ was checked to be 7 . The response to NO was studied over time by absorption and fluorescence emission spectroscopy. 


\subsection{CELLULAR STUDIES}

\subsubsection{A549 CELLS}

\section{GENERAL REMARKS AND CELL CULTURE}

A549 cell line was maintained in Dulbecco's modified Eagle's medium (DMEM) containing glucose $(1 \mathrm{~g} / \mathrm{L})$, glutamine $(2 \mathrm{mM})$, penicillin $(50 \mu \mathrm{g} / \mathrm{mL})$, streptomycin $(50 \mu \mathrm{g} / \mathrm{mL})$ and amphotericin $B(1.25 \mu \mathrm{g} / \mathrm{mL})$, supplemented with $10 \%$ fetal bovine serum (FBS). Cells were grown and incubated at $37^{\circ} \mathrm{C}$ in a $5 \% \mathrm{CO}_{2}$ humid atmosphere, otherwise indicated. Cell culture media were purchased form Gibco (Thermo Fisher Scientific), FBS was obtained from HarlanSeralab and supplements and other chemicals not listed in this section were obtained from Sigma-Aldrich. Plastics for cell culture were supplied by Thermo Fisher Scientific. All tested compounds were dissolved in DMSO at a concentration of $10 \mathrm{mM}$ and stored at $-20^{\circ} \mathrm{C}$ until use.

\section{INSTRUMENTAL TECHNIQUES}

UV-Vis spectroscopy: Absorption of the samples in plates was measured using a Multiskan ${ }^{\mathrm{TM}} \mathrm{FC}$ Microplate Photometer (Thermo Fisher Scientific) at room temperature.

\section{CELL PROLIFERATION ASSAY}

A549 cells in a 96-well plate $\left(3 \times 10^{3}\right.$ cells/well) were incubated with serial dilutions of the tested compounds in a total volume of $100 \mu \mathrm{L}$ of the grown medium. After 2 days of incubation, $10 \mu \mathrm{L}$ of MTT ( $5 \mathrm{mg} / \mathrm{mL}$ in PBS) were added to each well and the plate was further incubated for $3 \mathrm{~h}$. The supernatant was discarded and replaced by $100 \mu \mathrm{L}$ of DMSO to dissolve formazan crystals. The absorbance was then read at $540 \mathrm{~nm}$ by spectrophotometry. At least, three independent experiments were performed, and the $\mathrm{IC}_{50}$ values (i.e., concentration half inhibiting cell proliferation) were graphically determined using GraphPad Prism 4 software.

\subsubsection{HT-29 CELLS}

\section{GENERAL REMARKS AND CELL CULTURE}

HT-29 cell line was obtained from the American Type Culture Collection (ATCC) and cultured as explained for A549 cells (Section 7.2.1). The medium was changed every 2-3 days and subcultured again when cell population density reached $70-80 \%$ confluence. To resuspend 
attached HT-29 cells, a protocol of using a commercial accutase ${ }^{\mathrm{TM}}$ cell detachment solution was followed. Phenol red- or FBS-free medium with the same supplements were used when indicated to avoid fluorescence interferences or to promote starving conditions, respectively. Sterilised PBS was used to wash or irradiate cells. Chambered coverslips from Ibidi ( $\mu$-slide 8well plates, 80826) were used for confocal microscopy.

\section{INSTRUMENTAL TECHNIQUES}

Flow cytometer: Flow cytometry analysis was performed with a BD Accuri ${ }^{\mathrm{TM}}$ C6 flow cytometer, equipped with three bandpass filters: $533 / 30 \mathrm{~nm}, 610 / 20 \mathrm{~nm}$ and $670 \mathrm{LP}$. The data were recorded for at least 30.000 events per sample. A gate was applied in the forward scatter (FSC) vs. side scatter (SSC) dot plot to restrict the analysis to cells. When fluorescent probes were used, positive and negative populations were set up using an untreated or negative control cell population.

Confocal laser scanning microscopy (CLSM): Experiments were performed on an inverted confocal microscope Leica TCS SP8. Images were obtained with an HC PL APO CS2 63x/1.40 oil immersion objective. Excitation of samples was performed with a diode laser excitation and fluorescence was acquired with a $\mathrm{HyD}$ detector, also recording the transmission. Microspectroscopy was performed with $\Delta \lambda=5 \mathrm{~nm}$.

\section{ASSAYS}

Study of nanogels toxicity: HT-29 cells in a 24 -well plate (60-70\% confluence) were incubated for $24 \mathrm{~h}$ with several dilutions of a sample of nanoparticles (grown medium as diluent). A negative control using the vehicle of the sample (PBS) was also incubated in the same dilutions. Each set of conditions was tested at least three times. Then, cells were resuspended in fresh medium and a Trypan blue solution was prepared (10 $\mu \mathrm{L}$ cell solution $+10 \mu \mathrm{L}$ Trypan blue $0.4 \%$ PBS). The number of viable/non-viable cells $/ \mathrm{mL}$ was determined using a Neubauer chamber and a microscope. Thus, knowing the total number of cells we could determine the percentage of viable and non-viable cells for each incubation.

Flow cytometry evaluation of the cellular uptake of loaded nanogels: HT-29 cells in a 6-/12well plate (60-70\% confluence) were incubated for $24 \mathrm{~h}$ with the solutions to be tested in a 1:3 dilution with FBS-, phenol red-free medium. Two replicas were performed of each incubation. After incubation, cells were harvested from the culture plates, washed three times with PBS 
and analysed by flow cytometry suspended in fresh FBS-, phenol red-free medium. The internalised probe was excited at $488 \mathrm{~nm}$ and the fluorescence emission per cell was measured using 670LP filter for both RB and HYP. To compare the data obtained between different experiments, results were normalised as the percentage of fluorescence intensity/cell over the negative control. Errors were obtained from the standard deviation of the duplicates and their transformation using partial derivatives based on propagation of uncertainty.

Confocal microscopy evaluation of the cellular uptake of loaded nanogels: HT-29 cells in a $\mu$ slide 8-well Ibidi plate (60-70\% confluence) were incubated overnight with the solutions to be tested in a 1:3 dilution with FBS-, phenol red-free medium. After washing four times with PBS, cells were kept in FBS-, phenol red-free medium and visualised under the confocal microscope (RB: $\lambda_{\text {ex }} 514 \mathrm{~nm}, \lambda_{\text {em }}$ 551-680 nm, HYP: $\lambda_{\text {ex }} 561 \mathrm{~nm}, \lambda_{\text {em }} 585-700 \mathrm{~nm}$ ), using the same parameter settings for each cell population. The quantification of the fluorescence intensity of the cells was performed using ImageJ software. Results are expressed as the average of fluorescence intensity/cell. The intracellular spectra of the imaged cells were acquired and reported using the Leica software as the average of the spectra of several identical circles drawn were cells show fluorescence.

PDT assays: HT-29 cells in a 6-/12-well plate (60-70\% confluence) were incubated for $24 \mathrm{~h}$ with the solutions to be tested in a 1:3 dilution with phenol red-free medium. Two replicas were performed of each incubation. After incubation, cells were washed three times with PBS and, while kept in PBS, were irradiated for 2 min with two LED lamps (11W each one, ca. 400-700 $\mathrm{nm}$ emission output) placed as close to the lid of the cells plate as possible. In non-irradiated controls, the plate was kept covered in aluminium foil under the same conditions. After another $24 \mathrm{~h}$ incubation in fresh phenol red-free medium, cells were washed with PBS and harvested. Then, cells were stained according to the instructions of the corresponding apoptosis detection kit and the fluorescence of the obtained cell solutions was analysed by flow cytometry. Cells populations were classified into viable (FITC Annexin V/YO-PRO ${ }^{\circledR}-1$ and PI negative), apoptotic (FITC Annexin V/YO-PRO ${ }^{\circledR}-1$ positive and $\mathrm{PI}$ negative) and non-viable cells (PI positive).

FITC-Annexin V/Propidium lodide staining: For the FITC Annexin V apoptosis detection kit I (BD Pharmingen $\left.{ }^{\mathrm{TM}}, 556547\right)$, cells were resuspended in binding buffer (0.01 M HEPES/NaOH 
( $\mathrm{pH}$ 7.4), $0.14 \mathrm{M} \mathrm{NaCl}, 2.5 \mathrm{mM} \mathrm{CaCl}$ ) at a concentration of $10^{6}$ cells/mL. Then, $100 \mu \mathrm{L}$ of the cell solution were transferred to a $5 \mathrm{~mL}$ culture tube and $5 \mu \mathrm{L}$ of FITC Annexin V (aqueous buffered solution containing BSA and $<0.09 \%$ sodium azide) and $5 \mu \mathrm{L}$ PI (50 $\mu \mathrm{g} / \mathrm{mL}$ in PBS, $\mathrm{pH} 7.4$ ) were added. The cells were gently vortexed and incubate for $15 \mathrm{~min}$ at r.t. in the dark. Finally, $400 \mu \mathrm{L}$ of binding buffer were added to each tube and cells were analysed by flow cytometry. Excitation of both species was performed at $488 \mathrm{~nm}$, collecting FITC and PI fluorescence emission using 533/30 nm and 610/20 nm bandpass filters, respectively.

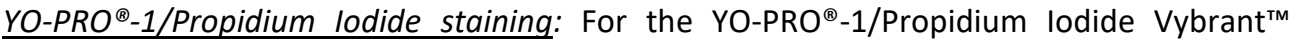
apoptosis assay kit \#4 (V13243 Invitrogen ThermoFisher), cells were resuspended in PBS (10 cells $/ \mathrm{mL}$ ). To $1 \mathrm{~mL}$ of cell suspension, $1 \mu \mathrm{L}$ YO-PRO ${ }^{\circledR}-1$ stock solution (100 $\mu \mathrm{M}$ in DMSO) and $1 \mu \mathrm{L} \mathrm{PI} \mathrm{stock} \mathrm{solution} \mathrm{(1.5} \mathrm{mM} \mathrm{in} \mathrm{deionised} \mathrm{water)} \mathrm{were} \mathrm{added.} \mathrm{Cells} \mathrm{were}$ incubated on ice for 20-30 minutes and analysed by flow cytometry. Excitation of both species was performed at $488 \mathrm{~nm}$, collecting YO-PRO ${ }^{\circledR}-1$ and propidium iodide fluorescence emission using 533/30 nm and 670LP bandpass filters, respectively.

\subsubsection{RAW 264.7 CELLS}

\section{GENERAL REMARKS}

RAW 264.7 mouse macrophage cells were purchased from American Type Culture Collection (ATCC). DMEM containing $4.5 \mathrm{~g} / \mathrm{L}$ D-glucose, FBS and Trypan blue stain were from Gibco (Thermo Fisher Scientific). Penicillin-streptomycin, L-Glutamine, lipopolysaccharide (LPS) from Escherichia coli 026:B6 and interferon- $\gamma$ (INF- $\nu$ ) were obtained from Sigma-Aldrich. CellTiterBlue $^{\circledR}$ cell viability assay (G8080) was purchased from Promega. Staurosporine free base (>99\%) was purchased from LC Laboratories. Plastics for cell culture were also from Thermo Fisher Scientific. Millex GP syringe driven filter units $(0.22 \mu \mathrm{m})$ were purchased from Millipore Corporation. $18 \mathrm{~mm}$ diameter, round glass coverslips (thickness no.1.5) were form VWR. Chambered coverslips were from Ibidi ( $\mu$-slide 4-well plates, 80426). Costar ${ }^{\text {TM }}$ sterile disposable reagent reservoirs, sterile centrifuge tubes, sterile disposable serological pipettes and cell scrapers were purchased from Corning.

For DAQ experiments, sterile PBS was prepared by dissolving 10 PBS tablets in water $(1 \mathrm{~L})$. The solution was sterilised by autoclaving at $110^{\circ} \mathrm{C}$ for $10 \mathrm{~min}$. The final PBS solution (pH 7.3) 
contained $\mathrm{Na}_{2} \mathrm{HPO}_{4}(8 \mathrm{mM}), \mathrm{KH}_{2} \mathrm{PO}_{4}(1 \mathrm{mM}), \mathrm{NaCl}(160 \mathrm{mM}), \mathrm{KCl}(3 \mathrm{mM})$. Imaging medium based on Hank's balanced salt solution (HBSS) was prepared in water containing $\mathrm{NaCl}(120$ $\mathrm{mM}), \mathrm{KCl}(5 \mathrm{mM}), \mathrm{CaCl}_{2} \cdot 2 \mathrm{H}_{2} \mathrm{O}(2 \mathrm{mM}), \mathrm{MgCl}_{2} \cdot 6 \mathrm{H}_{2} \mathrm{O}(1 \mathrm{mM}), \mathrm{NaH}_{2} \mathrm{PO}_{4}(1 \mathrm{mM}), \mathrm{NaHCO}_{3}(1 \mathrm{mM})$, HEPES (25 mM), D-glucose (11 mM) and bovine serum albumin (BSA; $1 \mathrm{mg} / \mathrm{mL})$. The $\mathrm{pH}$ of the imaging medium was adjusted to 7.4 using an aqueous solution of $1 \mathrm{M} \mathrm{NaOH}$. Prior to use, the solution was sterilised by filtration through a Millex GP syringe filter $(0.22 \mu \mathrm{m})$. For DAF-2 biological experiments, sterile PBS was bought from Sigma-Aldrich.

\section{CELl CULTURE}

The RAW 264.7 mouse macrophage cell line was cultured in DMEM containing $4.5 \mathrm{mg} / \mathrm{L} \mathrm{D}-$ glucose and phenol red indicator and supplemented with 1\% L-Glutamine (200 mM), 1\% penicillin-streptomycin (100 U/mL and $100 \mu \mathrm{g} / \mathrm{mL}$, respectively) and $10 \% \mathrm{FBS}$. This media would be referred simply as DMEM in the following sections. Phenol red- or FBS-free DMEM were used when indicated to avoid fluorescence interferences or to promote starving conditions, respectively. Cells were grown and incubated at $37^{\circ} \mathrm{C}$ in a $5 \% \mathrm{CO}_{2}$ atmosphere in $75 / 25 \mathrm{~cm}^{2}$ Nunc Easy flasks with porous caps. Subcultures (1:3) were made every 3 days by dislodging the cells from the flask surface using a cell scraper (18 $\mathrm{mm}$ blade).

To ensure the availability of a stock of the RAW 264.7 cell line, cells were stored in liquid nitrogen. In order to freeze, cells were harvested, transferred to a $15 \mathrm{~mL}$ sterile centrifuge tube and centrifuged at 1.000 rcf for $5 \mathrm{~min}$. The pellet containing the RAW 264.7 cells was resuspended in $9.5 \mathrm{~mL}$ of freezing medium (90\% DMEM and 10\% culture grade DMSO). Cells in the freezing medium were placed in $1.8 \mathrm{~mL}$ Nunc cryo tubes ( $1 \mathrm{~mL}$ in each tube) and frozen to $-80^{\circ} \mathrm{C}$ overnight in a cryogenic freezing container filled with propan-2-ol. The following day, the tubes were placed in a sample box and stored in liquid nitrogen for long term storage. For cell defrosting, a cryo tube was defrosted in a water bath at $37^{\circ} \mathrm{C}$, cells were transferred into a $15 \mathrm{~mL}$ centrifuge tube containing fresh DMEM $(9 \mathrm{~mL})$ and centrifuged at $1.000 \mathrm{rcf}$ for $5 \mathrm{~min}$. The cell pellet was resuspended in fresh DMEM $(12 \mathrm{~mL})$ and cultured as previously explained.

\section{INSTRUMENTAL TECHNIQUES}

Fluorescence spectroscopy: Fluorescence emission measurements of the plates were performed using a CLARIOstar ${ }^{\circledR}$ (BMG Labtech) microplate reader at room temperature.

Flow cytometer: see HT-29 cells (Section 7.2.2). 
Confocal laser scanning microscopy: Cells were imaged using a Carl Zeiss LSM 510 META confocal microscope (University of East Anglia) or an inverted confocal microscope Leica TCS SP8 (Universitat Jaume I). In the Carl Zeiss microscope, images were acquired with a planapochromat $63 x / 1.4$ oil-immersion objective and processed using the Zeiss LSM Image Browser Version 4.2.0.121 software and ImageJ. Differential interference contrast (DIC) images were collected simultaneously with fluorescence images, with transmitted light by excitation using an argon-ion laser. Intracellular fluorescence emission spectra were obtained using the lambda scan mode. For experiments performed on the Leica microscope, see HT-29 cells (Section 7.2.2). In both cases, fluorescence intensity analysis of the collected images was performed with ImageJ software by considering the whole cell. Results are expressed as the average of fluorescence intensity/cell.

\section{ASSAYS}

Study of nanogels toxicity: For viability studies using CellTiter-Blue ${ }^{\circledR}$ assay, RAW 264.7 cells seeded onto white-bottom Nunc Nunclon ${ }^{\mathrm{TM}} \Delta$ Surface 96 -well microplates (9.000 cells/well) were incubated for $48 \mathrm{~h}$ with serial dilutions of the samples to be tested ( $60 \mu \mathrm{L} /$ well DMEM and $40 \mu \mathrm{L} /$ well of solutions of the samples in DMEM). Additionally, each plate also contained a negative control (100 $\mu \mathrm{L} /$ well DMEM), a positive control (100 $\mu \mathrm{L} /$ well $20 \mu \mathrm{M}$ staurosporine in DMEM-2\%DMSO) and a background control (100 $\mu \mathrm{L} /$ well DMEM, no cells). All samples were tested in triplicate. CellTiter-Blue ${ }^{\circledR}$ reagent $(20 \mu \mathrm{L} /$ well) was added and cells were further incubated for $4 \mathrm{~h}$. Fluorescence emission was then measured at $590 \mathrm{~nm}$ following excitation at $560 \mathrm{~nm}$. Background fluorescence was corrected by subtracting fluorescence emission from the control of DMEM alone (to which CellTiter-Blue ${ }^{\circledR}$ had been added as for cells). Cell viability was calculated as the percentage of non-treated cells (negative control) average. Grubbs test was used to remove outliers.

For viability studies using the Trypan blue exclusion method, RAW 264.7 cells seeded onto 12 well plates (65\% confluence) were incubated for $24 \mathrm{~h}$ with dilutions of the samples to be tested in DMEM. Two replicas of each incubation were performed. Then, the same protocol than for HT-29 cells was followed to stain with Trypan blue and determine the percentage of viable and non-viable cells (Section 7.2.2).

Nitric oxide bioimaging by DAQ: RAW 264.7 cells were seeded onto round glass coverslips (18 $\mathrm{mm}$ diameter): a sterile coverslip was placed in each well of a 6 -well plate and $3 \mathrm{~mL} / \mathrm{well}$ of a 
solution of RAW 264.7 cells in phenol red-free DMEM (ca. 60.000 cells/mL) were added. After $24 \mathrm{~h}$ incubation, culture medium was removed and cells were incubated overnight with the solutions to be tested in a 1:6 dilution with FBS-, phenol red-free DMEM. Incubations were performed in duplicate, one replica stimulated $(0.01 \mu \mathrm{g} / \mathrm{mL}$ IFN $-\gamma$ and $0.5 \mu \mathrm{g} / \mathrm{mL}$ LPS) and the other not. After incubation, cell medium was removed and $3 \mathrm{~mL} /$ well of phenol red-free DMEM were added. For imaging cells, coverslips were placed in a Ludin chamber (Life Imaging Services) and washed twice with ca. $2 \mathrm{~mL}$ of imaging medium. After adding fresh imaging medium, the chamber was fitted on a heating stage at $37^{\circ} \mathrm{C}$ and cells were visualised in the Carl Zeiss LSM 510 META confocal laser scanning microscope $\left(\lambda_{\text {ex }} 488 \mathrm{~nm}, \lambda_{\text {em }} 505-750\right)$ using the same parameter settings for each cell population.

Flow cytometry evaluation of the cellular uptake of DAF2/DAF2DA@1: RAW 264.7 cells seeded onto 6-well plates (65\% confluence) were incubated for $24 \mathrm{~h}$ with the solutions to be tested in a 1:6 dilution with FBS-, phenol red-free DMEM. Two replicas of each incubation were performed. After incubation, samples were removed and cells were harvested in fresh FBS-, phenol red-free DMEM. Intracellular fluorescence emission of the cell suspension was analysed by flow cytometry ( $\lambda_{\text {ex }} 488 \mathrm{~nm}, \lambda_{\mathrm{em}} 518-548 \mathrm{~nm}$ ). A value of fluorescence intensity/cell was obtained for each tested cell population and was normalised as the percentage of fluorescence intensity/cell over negative control. Errors were obtained from the standard deviation of the duplicates and their transformation using partial derivatives based on propagation of uncertainty.

Nitric oxide bioimaging by DAF-2: RAW 264.7 cells seeded onto a $\mu$-slide 4 -well Ibidi plate (65\% confluence) were incubated overnight with the solutions to be tested in a 1:6 dilution with FBS-, phenol red-free DMEM. After washing three times with PBS, cells were visualised in FBS-, phenol red-free DMEM under the Leica TCS SP8 confocal laser fluorescence microscope ( $\lambda_{\text {ex }}$ $\left.488 \mathrm{~nm}, \lambda_{\mathrm{em}} 500-560 \mathrm{~nm}\right)$, using the same parameter settings for each cell population. 


\subsection{REFERENCES}

(1)

Becerril, J.; Bolte, M.; Burguete, M. I.; Galindo, F.; García-España, E.; Luis, S. V.; Miravet, J. F. Efficient Macrocyclization of UTurn Preorganized Peptidomimetics: The Role of Intramolecular H-Bond and Solvophobic Effects. J. Am. Chem. Soc. 2003, 125 (22), 6677-6686.

Alderighi, L.; Gans, P.; lenco, A.; Peters, D.; Sabatini, A.; Vacca, A. Hyperquad Simulation and Speciation (HySS): A Utility Program for the Investigation of Equilibria Involving Soluble and Partially Soluble Species. Coord. Chem. Rev. 1999, 184, 311-318.

Mishra, R.; Sjölander, D.; Hammarström, P. Spectroscopic Characterization of Diverse Amyloid Fibrils in Vitro by the Fluorescent Dye Nile Red. Mol. Biosyst. 2011, 7 (4), 1232-1240.

Marín, M. J.; Thomas, P.; Fabregat, V.; Luis, S. V.; Russell, D. a.; Galindo, F. Fluorescence of 1,2-Diaminoanthraquinone and Its Nitric Oxide Reaction Product within Macrophage Cells. ChemBioChem 2011, 12 (16), 2471-2477.

Beltrán, A.; Burguete, M. I.; Abánades, D. R.; Pérez-Sala, D.; Luis, S. V.; Galindo, F. Turn-on Fluorescent Probes for Nitric Oxide Sensing Based on the Ortho-Hydroxyamino Structure Showing No Interference with Dehydroascorbic Acid. Chem. Commun. 2014, 50 (27), 3579-3581.

Mowery, K. A.; Meyerhoff, M. E. The Transport of Nitric Oxide through Various Polymeric Matrices. Polymer (Guildf). 1999, 40 (22), 6203-6207.

Fabregat Tena, V. Nuevas Matrices Poliméricas Hidrofílicas Con Aplicaciones En Bioanálisis y Fotocatálisis, Universitat Jaume I, 2013.

Williams, D. L. H. A Chemist's View of the Nitric Oxide Story. Org. Biomol. Chem. 2003, 1, 441-449.

Felip-León, C.; Angulo-Pachón, C. A.; Miravet, J. F.; Galindo, F. Self-Assembly Controls Reactivity with Nitric Oxide: Implications for Fluorescence Sensing. ACS Omega 2018, 3 (11), 15538-15545.

Maragos, C. M.; Morley, D.; Wink, D. A.; Dunams, T. M.; Saavedra, J. E.; Hoffman, A.; Bove, A. A.; Isaac, L.; Hrabie, J. A.; Keefer, L. K. Complexes of NO with Nucleophiles as Agents for the Controlled Biological Release of Nitric Oxide. Vasorelaxant Effects. J. Med. Chem. 1991, 34 (41), 3242-3247. 
ANNEXE I RESUMEN EN CASTELLANO 



\section{ANNEXE. RESUMEN EN CASTELLANO}

\section{INTRODUCCIÓN GENERAL}

La nanotecnología es la ciencia que estudia y desarrolla sistemas en el rango nanométrico. Las nanopartículas se definen generalmente como cualquier material particulado con al menos una dimensión en el rango de 1 a $100 \mathrm{~nm}$. En concreto, la nanomedicina ha surgido en los últimos años como un área interdisciplinar que busca abordar los retos y carencias que afronta la medicina convencional. Además de presentar interesantes propiedades por sí mismas, las nanopartículas sirven como excelentes portadores de agentes activos moleculares o macromoleculares, que pueden incorporarse en su interior y/o en sus poros o pueden unirse a su superficie. La razón de utilizar nanoportadores surge del creciente número de agentes terapéuticos y de diagnóstico cuya eficacia se ve afectada por una biodistribución inespecífica en células y tejidos, una toxicidad sistémica, escasa solubilidad y biodisponibilidad o una rápida metabolización y excreción del cuerpo.

Las nanopartículas tienen varias ventajas clave sobre los agentes moleculares convencionales en medicina:

- permiten dispersiones acuosas estables de agentes poco solubles en agua, mejorando la solubilidad y estabilidad de estos activos.

- pueden proteger a los agentes encapsulados de la degradación producida por los mecanismos de defensa endógenos del cuerpo.

- se puede conseguir que las nanopartículas se dirijan no solo a órganos o tejidos específicos del cuerpo, sino también que presenten una especificidad celular y subcelular.

- las nanopartículas proponen una forma diferente de entrada a la célula, lo que puede modificar el destino final del agente que portan.

- la matriz de la nanopartícula puede diseñarse para la liberación controlada de fármacos en áreas diana, incluyendo liberación sostenida, liberación sensible a estímulos y liberación activada externamente.

- las nanopartículas permiten la multimodalidad, que implica realizar varias funciones diagnósticas y/o terapéuticas a la vez. 
- los nanoportadores no deben presentar toxicidad y deben ser excretados de forma segura del cuerpo.

Entre los tipos de nanopartículas, se pueden distinguir orgánicas e inorgánicas según su naturaleza. Entre las orgánicas encontramos, por citar algunas, nanopartículas poliméricas, micelas, liposomas, dendrímeros o nanogeles poliméricos. Los nanogeles se describen como nanopartículas formadas por redes poliméricas tridimensionales capaces de retener grandes cantidades de agua. La preparación de estos sistemas se ha descrito por medio de diferentes procedimientos (Figura A1). La mayoría de los nanogeles descritos están constituidos por redes reticuladas covalentemente, formadas, por ejemplo, por polimerización en emulsión. Alternativamente, se pueden preparar nanogeles a partir de polímeros como precursores, que se entrecruzan con grupos lábiles para mejorar la biodegradabilidad y la respuesta a los estímulos. También se han reportado nanogeles basados en interacciones físicas, por ejemplo, el autoensamblaje de copolímeros de bloques anfifílicos, polisacáridos o ADN.

Los nanogeles han recibido mucha atención recientemente como portadores debido a sus ventajas sobre otras nanopartículas, como una mayor flexibilidad y biocompatibilidad, su sencilla preparación, alto contenido de agua y alta estabilidad. Las características de los nanogeles pueden modularse alterando su tamaño, densidad de reticulación y propiedades superficiales. Además, han demostrado una alta capacidad de carga tanto para moléculas hidrofílicas como hidrofóbicas debido a su estructura altamente porosa, que proporciona un gran volumen para cargar fármacos. Las especies encapsuladas pueden ser retenidas por diferentes fuerzas intermoleculares. Las especies poco solubles en agua se adsorben en los dominios hidrofóbicos de la red polimérica. Los agentes terapéuticos también pueden unirse covalentemente para conseguir mayor estabilidad. Además, los nanogeles pueden diseñarse para liberar su carga como respuesta a factores ambientales, por medio de un aumento de volumen del sistema o su degradación. Estos cambios pueden producirse en respuesta a estímulos externos como la temperatura, la luz y los ultrasonidos, o a desencadenantes biológicos tras la internalización celular, como diferencias en el pH o potencial de reducción. 
A. Polymerization in Emulsion

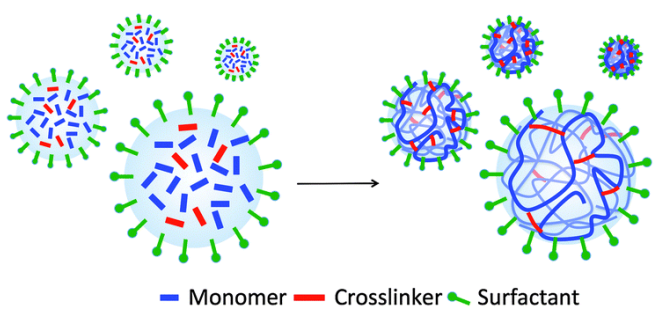

C. Self-Assembly through Physical interaction

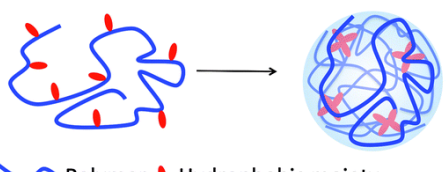

Polymer Hydrophobic moiety
B. Chemical/ Physical Crosslinking of Polymer in Emulsion

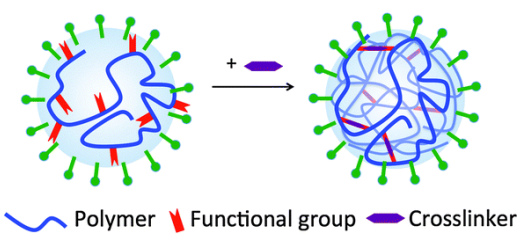

D. Self-Assembly through Electrostatic Interaction

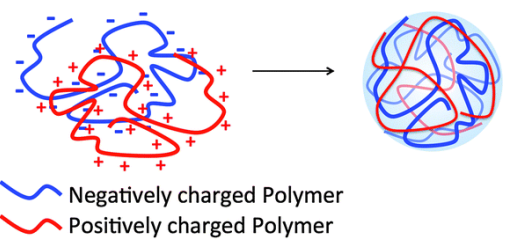

Figura A1. Estrategias de preparación de nanogeles poliméricos. ${ }^{81}$

En la intersección entre liposomas y nanogeles, en los últimos años ha surgido un gran interés en el desarrollo de liposomas rellenos de hidrogel o lipogeles, con el objetivo de mejorar algunos aspectos de las formulaciones liposómicas convencionales. La presencia de un núcleo de hidrogel proporcionaría una estabilidad mecánica mejorada al liposoma, una liberación de fármaco sensible al medio y una mayor capacidad de carga debido a la red polimérica. Por otro lado, la bicapa lipídica alrededor del nanogel mejoraría su biodisponibilidad y biocompatibilidad.

\section{GELES MOLECULARES}

A diferencia de los geles poliméricos formados por redes macromoleculares, los geles moleculares (también denominados geles supramoleculares) están formados por moléculas de bajo peso molecular (100-1000 Da) (Figura A2). Estos gelantes de bajo peso molecular (LMWG) se autoensamblan en agregados unidimensionales anisotrópicos similares a fibras, que evolucionan a redes tridimensionales a través de interacciones no covalentes, como enlaces de hidrógeno, coordinación metal-ligando, interacciones de van der Waals, interacciones de apilamiento $\pi-\pi$, fuerzas solvofóbicas... La transición de solución a gel ocurre cuando la concentración de gelante está por encima de un valor crítico, la concentración mínima de gelación (mgc). Normalmente, se requiere una cantidad mínima de gelante, menos del $1 \%$ en peso, para inmovilizar el disolvente y se ha visto que moléculas de únicamente 200 Dalton pueden formar geles fuertes. Por lo tanto, a diferencia de los geles poliméricos, se pueden obtener materiales muy interesantes siguiendo una estrategia de abajo hacia arriba, usando 
moléculas baratas y sintéticamente simples que contienen la información de autoensamblaje programada a nivel molecular.

Además, los geles moleculares son sistemas supramoleculares dinámicos en los que las entidades moleculares libres y los agregados están en un equilibrio gobernado por la solubilidad de la fase gel. Este equilibrio es rápido en comparación con los análogos poliméricos y permite una rápida respuesta al medio. Esta naturaleza dinámica de las interacciones no covalentes y el hecho de que son termodinámicamente reversibles, les da a estos geles moleculares la capacidad inherente de responder a estímulos externos, recuperándose las moléculas libres originales.

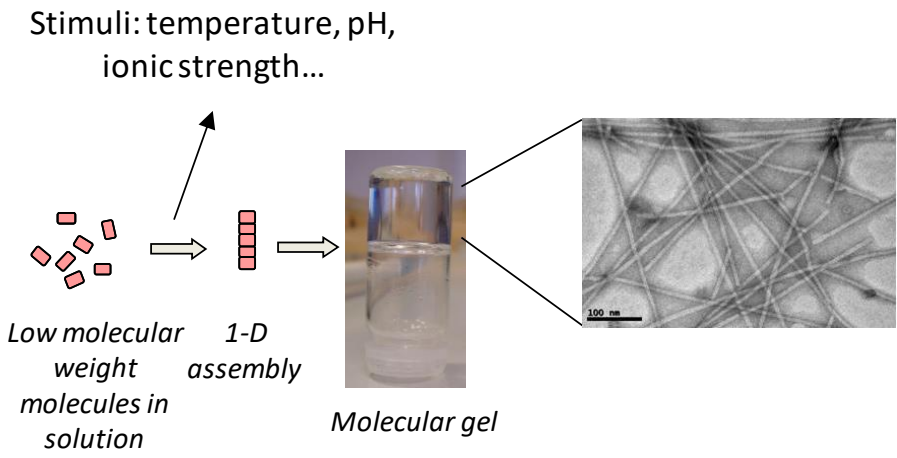

Figura A2. Representación esquemática del proceso de autoensamblado de gelantes de bajo peso molecular.

\section{TERAPIA FOTODINÁMICA}

La fotosensibilización para producir especies reactivas de oxígeno (ROS) se usa en una gran variedad de campos, como fotocatálisis, inactivación fotodinámica de microorganismos o bacterias o terapia fotodinámica (TFD) en medicina. En concreto, la TFD se lleva utilizando en clínica para el tratamiento de tumores sólidos durante los últimos 25 años. La terapia fotodinámica requiere de tres componentes: un fotosensibilizador (PS), luz y oxígeno en el tejido. El proceso general comienza con la administración del fotosensibilizador por vía sistémica o tópica, seguida su irradiación en un lugar específico con la longitud de onda adecuada, lo que genera especies reactivas de oxígeno que hacen que las células cancerosas perezcan. Realmente, el efecto antitumoral de la TFD está mediado por una combinación de tres acciones principales: a) efecto citotóxico directo sobre las células cancerosas, b) la destrucción de los vasos sanguíneos del tumor y c) la estimulación de la inmunidad antitumoral. 
Dado que los fotosensibilizadores solo producen ROS tras la irradiación con un tipo en concreto de luz y que la difusión de ROS es limitada, el daño citotóxico solo se produce en la región específica irradiada y el área circundante inmediata. Por esa razón, se ha denominado a la TFD como una "bala mágica" que afecta selectivamente a tumores malignos, sin afectar los órganos sanos. Así pues, la TFD se ha convertido en una solución para el tratamiento del cáncer ya que evita la frecuente toxicidad sistémica grave y los efectos adversos de los dos tratamientos clínicos principales, la quimioterapia y la radioterapia. Sin embargo, todavía existen algunos desafíos en su práctica clínica como la naturaleza química de los compuestos utilizados como fotosensibilizadores. Así pues, la generación más novedosa de fotosensibilizadores está basada en nanopartículas con el objetivo de solventar estos problemas.

\section{SONDAS DE ÓXIDO NÍTRICO}

El óxido nítrico (NO) es una molécula de señalización intra e intercelular muy estudiada durante los últimos 20 años debido al gran papel que tiene en el cuerpo humano. Hace casi 35 años, el NO fue identificado como un factor relajante endotelial responsable de la vasodilatación y la regulación de la presión arterial, un descubrimiento por el que Robert F. Furchgott, Ferid Murad y Louis J. Ignarro recibieron el premio Nobel de medicina en 1998. Sin embargo, el NO juega un papel significativo en una multitud de otros procesos fisiológicos normales. Por ejemplo, actúa como neurotransmisor en el sistema nervioso central o está muy involucrado en el sistema cardiovascular, el tracto gastrointestinal o el sistema renal. También es destacable su acción en el sistema inmunológico: las células inmunes como macrófagos sintetizan esta molécula para destruir o neutralizar los cuerpos extraños responsables de su activación.

Debido a la importancia de esta molécula en los procesos fisiológicos, la homeostasis del NO es crucial para sus funciones adecuadas y la producción alterada de NO está implicada en un gran número de patologías. El NO se ha asociado con trastornos neurodegenerativos, como la enfermedad de Alzheimer o la enfermedad de Parkinson, y juega un papel importante en muchos trastornos psiquiátricos. Por su papel en el sistema vascular, el NO también está implicado en los accidentes cerebrovasculares y la isquemia cerebral. Asimismo, el NO está implicado en enfermedades inflamatorias, como la psoriasis. También se ha asociado esta molécula con el cáncer, ejerciendo efectos duales (actividad pro y antitumoral) sobre el desarrollo tumoral dependiendo de la concentración. Si se conoce la alteración en la producción 
de NO que conduce a la enfermedad, la modulación farmacológica de los niveles de NO en los tejidos puede llevar a solucionarla.

Debido a este papel tan importante del NO en las funciones biológicas, se han desarrollado muchas estrategias para monitorear en tiempo real los niveles de NO, tanto para comprender su acción en los organismos vivos como para desarrollar herramientas de diagnóstico para su detección. El método de bioimagen basado en fluorescencia es de los más utilizados. Las sondas de NO fluorescentes usan un mecanismo de encendido para mejorar la resolución de la imagen: pasan de una emisión débil o nula a una emisión intensa después de la activación por NO. Estas moléculas a menudo comprenden dos elementos, un elemento que reacciona con $\mathrm{NO}$ y un fluoróforo. El elemento que reacciona con NO sirve como modulador y apaga la fluorescencia del fluoróforo hasta que reacciona con el NO. La estrategia utilizada con más frecuencia es la utilización de sondas que contienen la estructura de orto-fenilendiamina. Los compuestos con este elemento son no fluorescentes hasta que sufren una nitrosilación y dan lugar a los correspondientes derivados triazol fluorescentes.

\section{PLANTEAMIENTO, METOdOLOGÍA Y OBJETIVOS}

El objetivo principal de esta tesis ha sido el desarrollo de un nuevo tipo de nanopartículas: nanogeles moleculares. Los nanogeles moleculares fusionan el interés en los nanogeles poliméricos como nanoportadores y en los geles moleculares como materiales blandos. Estas partículas, obtenidas por autoensamblaje de un compuesto de bajo peso molecular, se intentaron preparar con una estabilidad termodinámica o cinética apropiada y de forma reproducible. Además, se idearon para su aplicación en la solución de problemas biomédicos relacionados con la administración de fármacos y detección.

Aunque hay muchos ejemplos de nanogeles poliméricos en la literatura, no hay referencias al estudio de nanogeles moleculares. El presente trabajo tiene como objetivo ser el primero sobre su preparación y caracterización detallada. El uso de nanogeles poliméricos en la administración de fármacos presenta desafíos asociados con la liberación debida a estímulos, la biodegradación, la polidispersidad y la reproducibilidad de lote a lote en la preparación de polímeros. Los nanogeles moleculares pueden representar una alternativa interesante a estos análogos poliméricos teniendo en cuenta sus características intrínsecas: (i) un ensamblaje 
totalmente reversible para dar las moléculas originales libres que puede ser regulado por estímulos, (ii) fácil biodegradación porque están compuestos por moléculas de bajo peso molecular, y (iii) excelente biocompatibilidad ya que están constituidos principalmente por productos naturales. Además, la extensa biblioteca de gelantes moleculares disponible allana el camino para la preparación de nanogeles moleculares con propiedades personalizadas, como la capacidad de respuesta a estímulos o la presencia de los grupos funcionales deseados en su estructura.

En este trabajo se siguieron dos estrategias diferentes para preparar nanogeles moleculares: la formación de nanogeles promovida por ultrasonidos y el uso de liposomas como plantilla (Capítulo 3). Este último método incluye la preparación de nuevas partículas de hidrogel@liposoma como intermedio, obtenidas por formación de un gel molecular dentro de liposomas. Todos los lipogeles reportados están constituidos por polímeros, siendo la interacción de geles moleculares y liposomas solo previamente descrita para la formación de liposomas dentro de un gel supramolecular macroscópico. Nuevamente, las características inherentes de los geles moleculares mejorarían la versión polimérica de los lipogeles como sistemas de administración de fármacos, con sensibilidad a estímulos, ideal para la preparación de portadores sensibles al medio, y biocompatibilidad mejorada.

Finalmente, otro objetivo crucial de este trabajo ha sido el estudio de la capacidad de los nanogeles moleculares para incorporar y liberar activos y actuar como portadores intracelulares. Se seleccionaron especies fluorescentes para este propósito: sondas de óxido nítrico (Capítulo 5) y fotosensibilizadores para terapia fotodinámica anticancerosa (Capítulo 4). En concreto, se eligieron fluoróforos comerciales con diferente naturaleza en cuanto a su solubilidad en agua. El estudio de las propiedades de los nuevos sistemas cargados en disolución y en células (sensing y fotoactividad) se planteó como objetivo adicional en este trabajo, buscando mejorar la eficiencia de las moléculas fotoactivas en su uso biomédico.

Respecto a las moléculas para terapia fotodinámica, tanto el Rosa de Bengala como la hipericina son fotosensibilizadores muy potentes y conocidos. A pesar de ello, presentan propiedades farmacológicas inherentes desfavorables que han obstaculizado su desarrollo clínico como agentes contra el cáncer. En particular, el Rosa de Bengala tiene una naturaleza aniónica a pH fisiológico, por lo que no puede atravesar las membranas celulares y tiene una baja internalización celular. Además, tienen tendencia a agregar en dímeros y multímeros, lo que 
disminuye el rendimiento de formación de especies reactivas de oxígeno y causa problemas para la administración intravenosa. Este último problema lo comparte con la hipericina, que en ambiente acuoso forma agregados, por lo que su solubilidad es baja.

Por lo que respecta a las sondas fluorescentes para detección de NO, la 4,5-diaminofluoresceína (DAF-2) es una de las más populares debido a sus características. Sin embargo, debido a su naturaleza iónica en el agua, DAF-2 no puede atravesar la membrana celular y tiene una capacidad de internalización en células deficiente. Su derivado diacetato (DAF-2 DA), desarrollado para mejorar este problema, presenta por otro lado un carácter hidrofóbico que limita su solubilidad en agua. Otros inconvenientes que podrían solucionarse al encapsular en nanogeles sería que la red evitara la entrada de especies más voluminosas y bloqueara reacciones secundarias, o que se protegiera a la sonda del fotoblanqueo. Por otra parte, la 1,2diaminoantraquinona (DAQ) es otro compuesto orto-diamino disponible comercialmente que se ha utilizado en NO imaging, con ventajas como su compatibilidad con las condiciones fisiológicas, su bajo costo, baja toxicidad, alta sensibilidad y especificidad. Sin embargo, su aplicación práctica es limitada debido a su carácter hidrofóbico, con baja solubilidad y una alta tendencia a agregar en agua, y el requerimiento de una alta concentración de triazol para su detección fluorescente.

\section{APORTACIONES ORIGINALES, CONCLUSIONES Y FUTURAS LÍNEAS DE INVESTIGACIÓN}

\section{1) Se prepararon nanogeles moleculares a partir de un hidrogelante de bajo peso molecular mediante sonicación de un xerogel}

Fusionando el interés en los nanogeles poliméricos como nanoportadores y en los geles moleculares como nuevos materiales blandos, en esta tesis se prepararon nuevos nanogeles moleculares mediante el autoensamblaje de un gelante de bajo peso molecular. Utilizando el hidrogelante molecular $\mathbf{1}$, con propiedades gelantes por cambios de $\mathrm{pH}$, se obtuvieron nanogeles moleculares mediante dos estrategias diferentes: preparación promovida por ultrasonidos y utilizando liposomas como plantilla. En la primera metodología, se obtuvieron partículas nanométricas de $50 \mathrm{~nm}$ de forma reproducible y en concentraciones de $2 \mathrm{mM}$, mediante sonicación de un xerogel de 1 en medio acuoso. Estos nanogeles esféricos, 
compuestos principalmente de agua como el material macroscópico análogo, corresponderían a las etapas iniciales de la agregación de geles moleculares, constituyendo un estado intermedio entre las moléculas libres y las redes fibrilares autoensambladas. Los nanogeles muestran una buena estabilidad temporal, de acuerdo con su potencial Z, y son estables cinéticamente tras 24 horas a concentraciones 10 veces por debajo de la concentración crítica de agregación de 1. Además, las partículas presentan dominios hidrofóbicos accesibles con los que interacciona el rojo Nilo. En cuanto a la capacidad de respuesta al pH de las nanopartículas, las sustancias no polares cargadas se liberaron a valores de $\mathrm{pH}$ superiores a 11 . Sin embargo, no se pudieron detectar cambios discernibles en el tamaño de las nanopartículas, lo que sugiere un cambio estructural de los nanogeles a objetos de tamaño similar a pH básico, pero con un menor poder de encapsulación hidrofóbico que liberaría el rojo Nilo. Además, los nanogeles mostraron solubilización parcial por encima de $50^{\circ} \mathrm{C}$ y fueron estables en soluciones de cloruro de sodio con una concentración similar a la del medio fisiológico. Además, se probó la alta biocompatibilidad del gelante y las nanopartículas en células de carcinoma de pulmón humano (A549).

\section{2) Se prepararon nanogeles moleculares usando liposomas como plantilla, proporcionando "lipogeles" intermedios}

En la segunda estrategia, se obtuvieron partículas de nanogel de $\mathbf{1}$ utilizando liposomas como plantilla. La formación de geles moleculares dentro de liposomas cargados con el gelante ionizado a pH 9 se desencadenó tras acidificación con D-glucono-1,5-lactona hasta pH 5-6, y proporcionó partículas híbridas de gel@liposoma. La eliminación de la bicapa de fosfolípidos con SDS proporcionó partículas de nanogel, con una concentración total del gelante en la muestra de $0.7 \mathrm{mM}$, un tamaño de $50 \mathrm{~nm}$ y manteniendo el $\mathrm{pH}$ ácido en el interior de la partícula. Tanto los lipogeles como los nanogeles no mostraron cambios de tamaño al menos durante un mes.

Además, las partículas de hidrogel@liposoma se cargaron con doxorrubicina, mostrando una liberación similar a la observada para los liposomas. Las partículas híbridas aquí descritas constituyen el primer caso de preparación de un gel molecular, no polimérico, dentro de los liposomas. Este tipo de nanoportadores fusiona los beneficios de una mayor estabilidad de los vehículos liposomales con la liberación específica frente a estímulos inherente de los hidrogeles formados por moléculas de bajo peso molecular. En consecuencia se prevé que estos sistemas 
híbridos tengan gran utilidad en el transporte de sustancias bioactivas y en la liberación de fármacos en respuesta a estímulos.

\section{3) Se prepararon nanogeles cargados con los fotosensibilizadores Rosa de Bengala e hipericina}

La encapsulación de Rosa de Bengala e hipericina se consiguió en forma monomérica y manteniendo su fotoactividad. De esta forma, la carga en nanogeles moleculares resuelve los problemas de agregación de estos fotosensibilizadores, perjudiciales para su uso en terapia fotodinámica. Además, en el caso de la hipericina, las nanopartículas aumentan unas 10 veces la concentración máxima de esta molécula en agua.

4) Los fotosensibilizadores cargados en los nanogeles muestran una internalización celular mejorada

Se mejoró la baja capacidad de internalización celular, debida a la naturaleza aniónica del RB y la baja solubilidad de HYP en agua. HYP@1 y RB@1 muestran una mayor internalización en células HT-29 que los controles, obteniendo una intensidad de fluorescencia intracelular incluso 70 veces mayor para RB@1.

\section{5) Los fotosensibilizadores cargados en los nanogeles muestran un rendimiento mejorado en} terapia fotodinámica

Los resultados preliminares in vitro en células HT-29 muestran una mayor fototoxicidad para RB@1 en comparación con RB en solución, probablemente atribuida a la mayor internalización de RB en las células cuando se incuban con RB@1. Además, la fototoxicidad de la hipericina usando HYP@1 en células HT-29 se mantiene en comparación con el uso de DMSO como cosolvente, por lo que nuestras nanopartículas constituirían una formulación alternativa más segura. Además, tanto RB@1 como HYP@1 no muestran toxicidad en oscuridad, lo que los hace adecuados para aplicaciones clínicas y prometedores como agentes de TFD.

6) Las sondas de óxido nítrico DAF-2 y DAF-2 DA se cargaron en los nanogeles, lo que resultó en una mayor internalización celular

La encapsulación en nanopartículas mejoró los problemas de internalización celular de los sensores fluorescentes de óxido nítrico DAF-2 (debido a su naturaleza aniónica) y DAF-2 DA (debido a su baja solubilidad en agua). Las muestras de DAF2/DAF2DA@1 aumentaron de 12 a 
17 veces la intensidad de fluorescencia intracelular en macrófagos RAW 264.7. Estos resultados se obtuvieron utilizando una concentración de sonda 10 veces menor a la convencionalmente utilizada para el bioimaging de NO usando DAF-2 DA. Se comprobó que la encapsulación no obstaculiza la señalización del NO, ya que se demostró la formación del triazol para DAF2@1 en solución tras la adición de NO. Además, la encapsulación de DAF-2 DA redujo la hidrólisis espontánea de la sonda a DAF-2 en disolución.

\section{7) La solubilidad en agua de la sonda de óxido nítrico DAQ se mejora mediante la} encapsulación en nanogeles

La encapsulación del sensor de óxido nítrico DAQ en nanogeles aumentó 4.5 veces la solubilidad en agua de la sonda, que tiene un carácter hidrofóbico. Sin embargo, las nanopartículas no consiguieron aumentar la internalización celular de DAQ en células RAW 264.7 y cumplir con el requisito de la alta concentración de triazol necesaria para su detección por fluorescencia.

\section{OBSERVACIONES FINALES}

Se ha demostrado el uso de nanogeles moleculares formados por el compuesto 1 para transportar moléculas fotoactivas, incrementando su solubilización en agua e internalización celular y manteniendo su fotoactividad. Como resultado, los sistemas reportados mejoran algunas de las propiedades farmacológicas desfavorables inherentes de estas moléculas para su aplicación clínica. La mejora del comportamiento intracelular observada para la mayoría de moléculas podría ser consecuencia de un mecanismo de internalización diferente, ya que los fluoróforos libres entrarían en las células por un proceso de difusión y las nanopartículas por endocitosis. Como trabajo futuro, sería interesante estudiar si se está produciendo un desensamblaje de las nanopartículas en las células o si las moléculas fotoactivas ejercen su acción encapsuladas. Los prometedores resultados de estos primeros nanogeles moleculares muestran su gran potencial para ser utilizados como nuevos nanoportadores para aplicaciones biomédicas. En el campo de la liberación controlada de fármacos, el uso de nanogeles poliméricos presenta desafíos asociados con la liberación desencadenada por estímulos, la biodegradación, la polidispersidad y la reproducibilidad de lote a lote en la preparación de polímeros. Las partículas aquí descritas muestran ventajas intrínsecas en comparación con los nanogeles poliméricos, como el desensamblaje provocado por estímulos y una biodegradabilidad mejorada debido a su naturaleza molecular. Además, la extensa biblioteca de gelantes moleculares disponible allana el camino para la preparación de nanogeles 
moleculares con propiedades personalizadas, como la capacidad de respuesta a estímulos o la presencia de los grupos funcionales deseados en su estructura. 
ANNEXE II REPRINT OF PUBLISHED PAPERS 

Check for updates

Cite this: Soft Matter, 2019, 15, 3565

\section{In between molecules and self-assembled fibrillar networks: highly stable nanogel particles from a low molecular weight hydrogelator $\dagger$}

\author{
Ana Torres-Martínez, (D) César A. Angulo-Pachón, (D) Francisco Galindo (D) * and \\ Juan F. Miravet (D) *
}

\begin{abstract}
The preparation of molecular, non-polymeric nanogels from a low molecular weight hydrogelator is reported. The molecular nanogels are expected to overcome issues associated with the use of polymeric nanogels in biomedicine such as biodegradability, stimuli responsiveness, polydispersity, and batch-to-batch reproducibility. Nanogels formed by compound 1 were reproducibly prepared by sonication of a xerogel in PBS, with a total concentration of ca. $2 \mathrm{mM}$. The intensity averaged diameter of ca. $200 \mathrm{~nm}$ was determined by DLS. Electron microscopy (TEM and cryo-TEM) showed spherical particles. Light scattering (SALS) indicates that water is the main component of the nanoparticles, and the concentration of $\mathbf{1}$ in the nanogels is ca. $3 \mathrm{mg} \mathrm{mL}^{-1}$. These particles can be considered to constitute an intermediate state between free molecules and self-assembled fibrillar networks. The nanogels present excellent temporal and thermal stability and accessible hydrophobic domains, as demonstrated by the incorporation of the fluorescent dye Nile Red.
\end{abstract}

Received 4th February 2019 Accepted 25th March 2019

DOI: $10.1039 / \mathrm{c} 9 \mathrm{sm} 00252 \mathrm{a}$

rsc.li/soft-matter-journal by covalently crosslinked networks, formed, for example, by emulsion polymerization, ${ }^{24}$ following the seminal work of Vinogradov with poly(ethylene glycol)-polyethyleneimine particles. ${ }^{25,26}$ Alternatively, nanogels can be prepared from polymer precursors, which are crosslinked with labile groups to improve biodegradability and stimuli responsiveness. ${ }^{27}$ Physically crosslinked nanogels have been reported based, for example, on the self-assembly of amphiphilic block copolymers, ${ }^{28}$ hydrophobized polysaccharides ${ }^{29}$ or DNA. ${ }^{30}$

In opposition to polymeric gels, molecular gels (also named supramolecular gels) are formed by low molecular weight molecules and their study has blossomed in the last two decades. ${ }^{31-37}$ These gels are formed by 1-D anisotropic molecular aggregation, resulting in self-assembled fibrillar networks. Distinct from polymeric gels, the fibres are fully reversible, yielding original free molecules upon changes of temperature or other physicochemical stimuli. ${ }^{38}$ These materials were attractive for application in areas such as controlled release, catalysis, tissue engineering and optoelectronics, among others. In particular, special interest has grown regarding molecular hydrogels in a biomedical context. ${ }^{36}$

The preparation of molecular nanogels is an exciting challenge taking into account their envisaged unique properties in comparison with polymeric analogues. Some critical issues in the use of polymeric nanogels such as biodegradability, stimuli responsiveness, polymer polydispersity, and batch-to-batch reproducibility are intrinsically solved by molecular gels. Here, we report the formation of stable nanogel particles of compound 1 (Scheme 1), a molecular hydrogelator. This work follows our initial efforts in this 


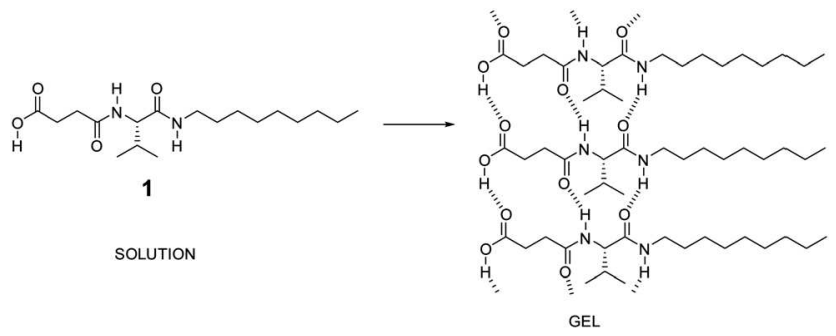

Scheme 1 Structure of compound 1 and a proposed aggregation model.

direction, which afforded nanoparticles from a molecular gelator derived from naphthalimide. ${ }^{39}$ In this case, the nature of the nanoparticles, solid or gel-like, could not be clarified, and the particles showed poor stability towards aggregation at concentrations above $c a .20 \mu \mathrm{M}$. To our knowledge, the present work is the first report on the preparation and detailed characterization of molecular nanogels.

\section{Results and discussion}

Compound 1 (Scheme 1) consists of an L-valine unit modified with a nonylamide at the carboxylic acid unit and acylated with succinic acid at the amine function. In previous work from our group, related amphiphilic structures with variations of the aliphatic chain length or the amino acid unit have been shown to form pH-responsive hydrogels. The aggregation in water of this type of molecule should be based on hydrophobic forces complemented with multiple H-bonds as reported for related systems. ${ }^{40}$ A tentative aggregation model is shown in Scheme 1, based on those proposed for closely related molecules previously described. ${ }^{41,42}$

Gels of 1 could be formed in water by cooling down hot solutions of the gelator, with a minimum gelation concentration value $(\mathrm{mgc})$ of $5 \mathrm{mg} \mathrm{mL} \mathrm{m}^{-1}(16 \mathrm{mM})$. Additionally, gels can also be prepared in different organic solvents with $\mathrm{mgc}$ in the range 3-38 $\mathrm{mM}$ (see the $\mathrm{ESI} \dagger$ ). The transmission electron microscopy images of the xerogel in water and toluene (Fig. 1) showed a fibrillar structure, as commonly described for molecular gels. Noticeably the fibers in the xerogel in water are straight and monodisperse, with a diameter of $c a .20 \mathrm{~nm}$, while those obtained in toluene show more flexibility, and are curved and entangled. These morphological differences probably reflect the different arrangements of the gelator molecules in the fibers, and hydrophobic forces are dominant for the aggregation in water and polar interactions, namely hydrogen
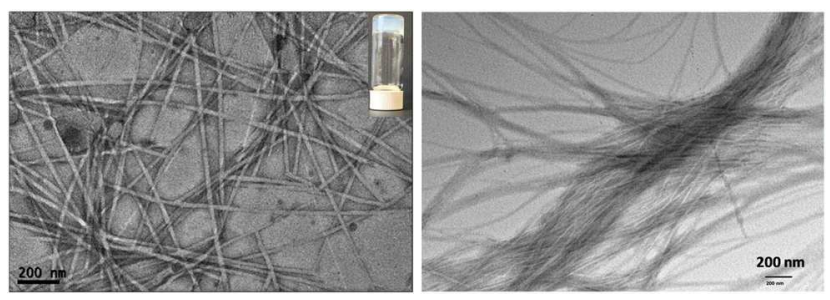

Fig. 1 TEM image of the xerogels obtained from 1 in water (left) and toluene (right). Inset: The macroscopic image of the hydrogel. bonding, predominant in organic solvents. Indeed, differential scanning microscopy reveals that the xerogels obtained in water and toluene are polymorphic, presenting different melting points and endothermic polymorphic transitions (see the ESI $\dagger$ ).

The presence of an ionizable carboxylic unit in 1 results in $\mathrm{pH}$-dependent gelation properties. Potentiometric titrations were carried out to evaluate the $\mathrm{pH}$ range of existence of neutral species of 1, revealing an apparent $\mathrm{p} K_{\mathrm{a}}$ of 7.6 (see the ESI $\dagger$ ). As has been previously reported, this $\mathrm{p} K_{\mathrm{a}}$ value is considerably shifted when compared to soluble, non-aggregating carboxylic acids, which present $\mathrm{p} K_{\mathrm{a}}$ values around $3-5 .{ }^{43-45}$ The reduced acidity of 1 could be ascribed to the thermodynamic stabilisation of neutral species gained from the aggregation process.

Following a report on the formation of hybrid peptidequantum dot colloidal spheres, ${ }^{46}$ nanogel particles were prepared by sonication of a xerogel of $\mathbf{1}$ suspended in aqueous medium. For this purpose, a gel was prepared in toluene, and the corresponding xerogel was obtained by solvent evaporation under vacuum. Upon sonication in phosphate buffer saline (PBS, $10 \mathrm{mM}, \mathrm{pH}=7$ ), a colloidal suspension of the nanoparticles with a final $\mathrm{pH}$ of 6.4 was obtained. Centrifugation to remove large fragments of the xerogel particles afforded an optically clear suspension of the nanogels, which was analysed by dynamic light scattering (DLS).

As can be seen in Fig. 2, a bimodal distribution of intensity averaged diameters $\left(D_{\mathrm{i}}\right)$ with maxima at $c a .200$ and $50 \mathrm{~nm}$ was obtained (see the correlogram in the ESI $\dagger$ ). The conversion of these data to number averaged diameters $\left(D_{n}\right)$ shows that particles with a diameter around $50 \mathrm{~nm}$ are predominant. The procedure for the preparation of the nanoparticles was found to be quite reproducible, and a set of 29 preparations afforded similar results, as shown in Fig. 2.

The efficiency of the transformation of the xerogel into nanoparticles was evaluated determining the concentration of 1 in the colloids by ${ }^{1} \mathrm{H}$ NMR. For this purpose, the signals were integrated using a calibrated electronic signal (ERETIC). ${ }^{47}$ The concentration of the nanogel particles was found to be reproducible. For a set of 10 experiments, using an initial concentration of xerogel of $1.2 \mathrm{mg} \mathrm{mL}^{-1}$, an average concentration of 1 in the sample of $0.71 \pm 0.14 \mathrm{mg} \mathrm{mL}^{-1}$, namely $2.1 \pm 0.4 \mathrm{mM}$, was obtained.

The same procedure was applied for the xerogel obtained in an aqueous medium, and again particles with a similar size
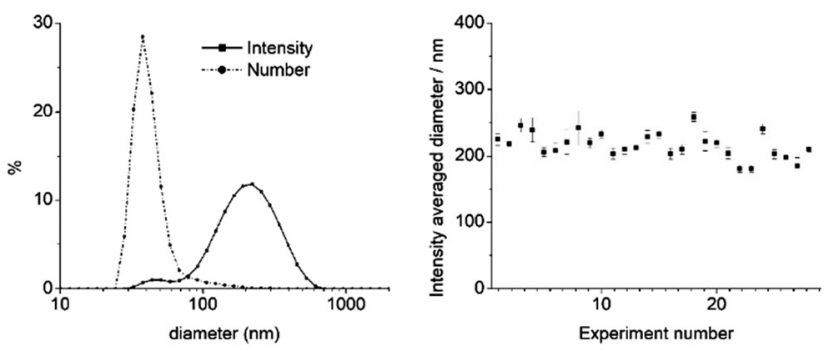

Fig. 2 Left: Size distribution by intensity (solid line) and by number (dasheddotted line) of a representative sample of nanogel particles obtained by DLS analysis. Right: Intensity averaged diameters of 29 different nanogel samples. 


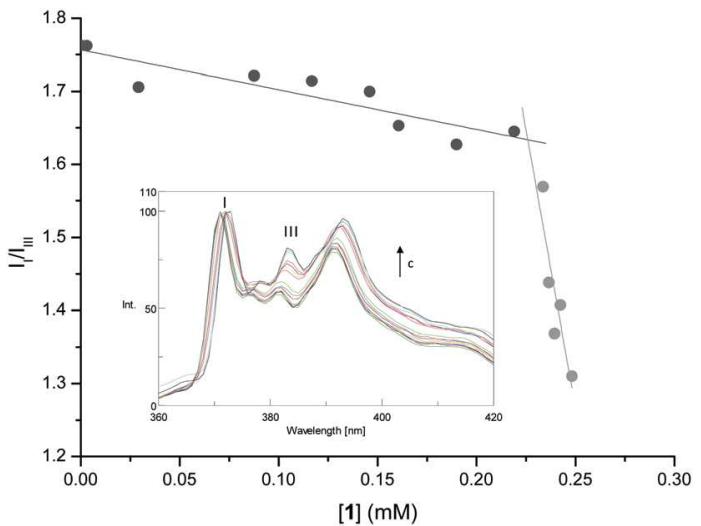

Fig. 3 Variation of the relative intensity of emission bands I and III of pyrene in the presence of increasing concentrations of compound $\mathbf{1}$ in water. $\lambda_{\mathrm{exc}}=334 \mathrm{~nm}$.

distribution were obtained (see the ESI $\dagger$ ). These results highlight that the solvent in which the xerogel is obtained is not, a priori, a key parameter in the preparation of the nanoparticles. However, sonication of a finely powered solid gelator did not permit us to obtain the nanoparticles, revealing that a high surface ratio of the fibrillar xerogels is essential for their transformation into nanogels. We decided to focus on the study of the particles obtained from the xerogel in toluene because, for future envisaged studies, the use of an organic solvent should be favourable for the entrapment of actives which are poorly soluble in water.

Unlike mgc, which determines the amount of compound required to form a sample spanning network, the critical aggregation concentration, cac, indicates the onset of self-assembly and represents the dilution limit for thermodynamically stable aggregates. Determination of cac in acidic medium for $\mathbf{1}$ was carried out using pyrene as a fluorescent probe. The incorporation of pyrene into hydrophobic environments leads to an increase of intensity of its emission band III (see Fig. 3). ${ }^{48}$ Fluorescence spectra were recorded for samples with increasing concentrations of $\mathbf{1}$.

As can be seen in Fig. 3, the measured fluorescence ratio shows a moderate decrease with concentration up to a point where a dramatic tendency change is observed, with a much steeper slope, resulting in a cac value of $0.22 \mathrm{mM}$.

Transmission electron microscopy (TEM) analysis revealed the presence of irregular spherical objects with diameters around $50 \mathrm{~nm}$ (Fig. 4, top). The particles were also observed by cryo-TEM (Fig. 4, bottom), showing diameters of $c a .200 \mathrm{~nm}$. The cryo-TEM images show a sponge-like structure with dark dots that might correspond to non-vitreous water trapped inside the particles. ${ }^{49}$ The smaller particle diameter observed by TEM when compared to that by cryo-TEM could be ascribed to drying effects associated with the former technique.

To evaluate the gel-like nature of the nanoparticles, an average concentration of 1 within the particle $\left([\mathbf{1}]_{\mathrm{NP}}\right)$ can be calculated using eqn (1), where $M_{\mathrm{w}}$ is the apparent molecular weight of the particle, $r$ is the radius of the particle and $N_{\mathrm{A}}$ is Avogadro's number. ${ }^{29,50-52}$

$$
[1]_{\mathrm{NP}}=M_{\mathrm{w}} / N_{\mathrm{A}}\left(4 / 3 \pi r^{3}\right)^{-1}
$$

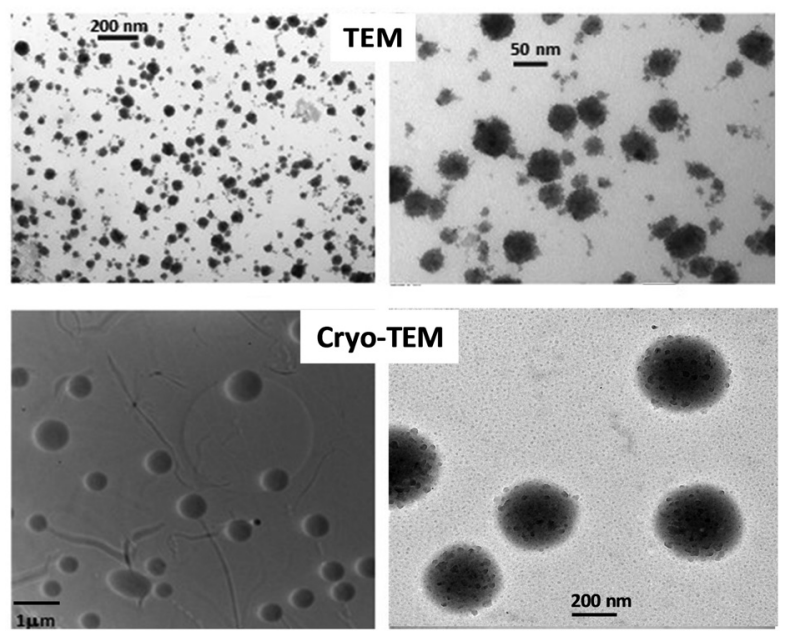

Fig. 4 Electron microscopy images of nanogel particles formed by 1 .

$M_{\mathrm{w}}$ was obtained by single angle static light scattering (SALS) measurements performed using the DLS equipment available in our laboratories. ${ }^{53}$ The light scattering intensity was measured at different concentrations and analysed using the Debye-Zimm relation (see eqn (2)). ${ }^{54}$ In eqn (2), $K$ is an experimental constant (see eqn (3)) that depends on the wavelength of the incident light $\left(\lambda_{0}\right)$, the refractive index of the solvent $\left(n_{0}\right)$ and the variation of the refractive index with particle concentration $(\mathrm{d} n / \mathrm{d} c)$. Additionally, $c$ is the sample concentration, $R_{\theta}$ is the ratio of scattered light to incident light, $A_{2}$ is the thermodynamic 2nd virial nonideality coefficient, and $P(\theta)$ is the angular dependence of scattering intensity.

$$
\begin{aligned}
& K c / R_{\theta}=\left(1 / M_{\mathrm{w}}+2 A_{2} c\right) P(\theta) \\
& K=\left(4 \pi^{2} / \lambda_{0} 4 N_{\mathrm{A}}\right)\left(n_{0} \mathrm{~d} n / \mathrm{d} c\right)^{2}
\end{aligned}
$$

For the study of the nanogels of $1, \mathrm{~d} n / \mathrm{d} c$ was found to be $0.063 \mathrm{~mL} \mathrm{~g}^{-1}$ and the scattering intensity of four samples at different concentrations was measured (Table 1). It has to be noted that given the supramolecular nature of the nanoparticles and their pH-sensitivity, the concentration of $\mathbf{1}$ in the form of nanoparticles should be less than the total amount of $\mathbf{1}$ in the sample. The presence of the ionised compound, $\mathrm{p} K_{\mathrm{a}}=7.6$, and free $\mathbf{1}$ corresponding to the cac value is considered (see the ESI $\dagger$ ). The graphical representation of the value $K c / R_{\theta} v s . c$ is the so-called Debye plot, the intercept of the linear fitting at $c=0$ being the value of $1 / M_{\mathrm{w}}$ (Fig. 5). A good correlation was obtained, and the apparent $M_{\mathrm{w}}$ was calculated to be $13.8 \times$ $10^{6} \pm 8.3 \times 10^{6} \mathrm{Da}$ (see details of error estimation in the

Table 1 Light scattering intensity obtained for aqueous dispersions of nanoparticles of 1 at different concentrations

\begin{tabular}{lll}
\hline$[1] / \mathrm{mg} \mathrm{mL}^{-1}$ & Count rate $/ \mathrm{kcps}$ & $10^{4} \mathrm{KcR}_{\theta}^{-1} / \mathrm{kDa}^{-1}$ \\
\hline 0.54 & 1058 & 1.36 \\
0.45 & 922 & 1.30 \\
0.36 & 781 & 1.24 \\
0.27 & 654 & 1.11 \\
0.17 & 527 & 0.88
\end{tabular}




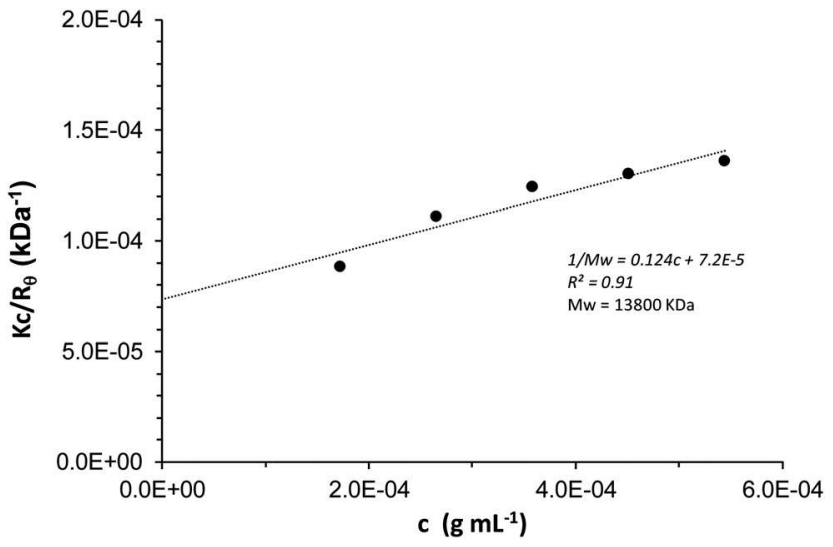

Fig. 5 Debye plot obtained for the SALS study of the nanoparticles.

Methods section). Using this value and the diameter obtained by DLS for the studied samples $(210 \mathrm{~nm})$, an average concentration of 1 in the particles of $4.7 \pm 2.8 \mathrm{mg} \mathrm{mL}^{-1}$ is calculated using eqn (1). This result reveals the gel-like nature of the nanoparticles, with water being the main component. The concentration of $\mathbf{1}$ in the particles is similar to that described for polyethylene glycol with $M_{\mathrm{w}}=2 \times 10^{6} \mathrm{Da}, 2.9 \mathrm{mg} \mathrm{mL}^{-1},{ }^{55}$ and comparable to that found, for example, in microgels ${ }^{50,56}$ or pullulan nanogels, ${ }^{52,57}$ which show a polymer concentration in the range $10-30 \mathrm{mg} \mathrm{mL}^{-1}$.

The average concentration of the gelator in the particles is closely related to the so-called overlap concentration, which is used in polymer chemistry and is defined as the point where the concentration within a given polymer particle is equal to the solution concentration. ${ }^{58}$ By analogy, $[\mathbf{1}]_{\mathrm{NP}}$ would represent the total sample concentration required for the onset of interparticle interactions, leading to gelation. Notably, there is a reasonable agreement between $[1]_{\mathrm{NP}}, 4.7 \pm 2.8 \mathrm{mg} \mathrm{mL} \mathrm{m}^{-1}$, and $\mathrm{mgc}, 5 \mathrm{mg} \mathrm{mL} \mathrm{m}^{-1}$.

The TEM images shown above do not permit distinguishing a fibrillar structure within the particles, such as that observed in the parent macroscopic gels. Indeed, the formation of such small nanoparticles by fibers of width as that found in the xerogels does not seem feasible. Consequently, the sonication, rather than fracturing the xerogel fibers, should provoke solubilisation of the monomers, affording local concentrations high enough to form seminal fibrils, which are described as precursors of fibers in the formation of macroscopic gels. ${ }^{59}$ The gel-like nature of the particles should be ascribed to the entanglement of these seminal fibrils into spherical particles. Therefore, the nanogel particles would correspond to the initial stages of the aggregation of molecular gels, and constitute an intermediate state between free molecules and fibrillar objects. In this regard, kinetic studies usually demonstrate that molecular gels are formed by a nucleation-growth mechanism, ${ }^{45,60,61}$ and it has been recently reported that fibres could be formed by scrolling of supramolecular lamellae that present asymmetric surfaces. ${ }^{62}$

The nanogels exhibited good temporal stability. Similar DLS results were obtained when stored at room temperature for 4 days. The samples stored at $4{ }^{\circ} \mathrm{C}$ were stable after ten days.
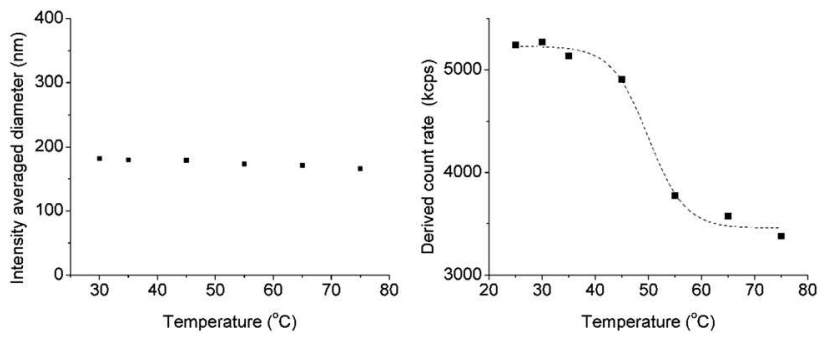

Fig. 6 Influence of temperature on the size of the nanogels (left) and on the intensity of scattered light (right).

The $Z$-potential of the particles was measured to be $-65 \mathrm{mV}$, a value in the range of those reported for highly stable colloids. ${ }^{63}$ The particles are stable upon dilution with PBS in the concentration range $0.25-2 \mathrm{mM}$, with no diameter variation (see the ESI $\dagger$ ). The stability towards temperature between 30 and $75{ }^{\circ} \mathrm{C}$ was also assayed by DLS. As shown in Fig. 6, the intensity averaged diameter was not affected by temperature changes but, on the other hand, the intensity of scattered light dropped significantly at $50{ }^{\circ} \mathrm{C}$, suggesting partial solubilisation of $\mathbf{1}$.

The results mentioned above on the determination of cac demonstrate that the initial aggregates formed upon increasing concentration of $\mathbf{1}$ can entrap hydrophobic species such as pyrene, but no nanogel preparation was carried out in that study. To test the accessibility of the hydrophobic domains of the nanogel particles, experiments using the fluorescent dye Nile Red were carried out. Nile Red is almost nonfluorescent in water and other polar solvents but shows intense fluorescence emission in nonpolar environments.

Additionally, the emission maximum of this probe is strongly affected by the polarity of the medium, changing dramatically from $666 \mathrm{~nm}$ in water to $606 \mathrm{~nm}$ in dichloromethane and $570 \mathrm{~nm}$ in cyclohexane. ${ }^{64}$ As can be seen in Fig. 7, when nanogels were prepared in the presence of the dye a notable increase in fluorescence was observed compared with a control in the absence of nanogels. The emission maximum of Nile Red@1 is shifted to $587 \mathrm{~nm}$, indicating a quite hydrophobic environment.

Therefore, these results highlight the presence of accessible hydrophobic domains in the nanogel particles, demonstrating

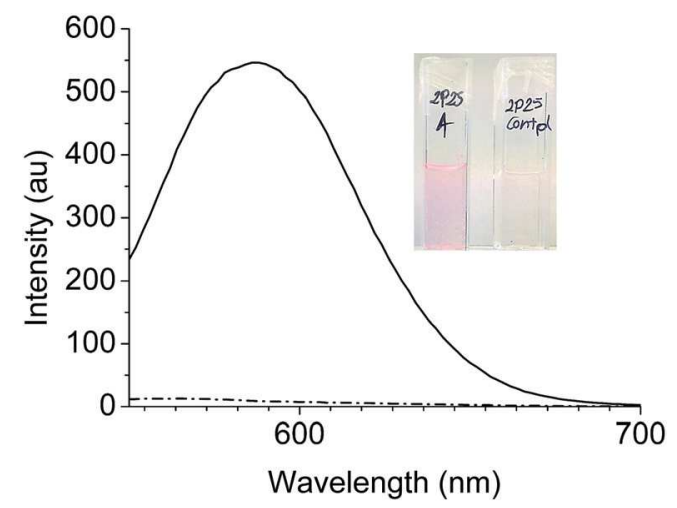

Fig. 7 Representative fluorescence emission spectra of Nile Red(a1 (solid line) and the free dye (dash-dot line). [Nile Red] $=10 \mu \mathrm{M}$. Inset: Cuvettes containing nanogel particles loaded with Nile Red (left) and free Nile Red (right). 
their potential use for the entrapment of poorly soluble organic actives.

\section{Methods}

\section{Synthesis and characterization of 1}

The synthesis of $\mathbf{1}$ is outlined in Scheme 2. Reactions which required an inert atmosphere were carried out under $\mathrm{N}_{2}$. Commercially available reagents and HPLC grade solvents were used as received.

${ }^{1} \mathrm{H}-\mathrm{NMR}$ and ${ }^{13} \mathrm{C}-\mathrm{NMR}$ spectra were recorded on an Agilent VNMR System spectrometer (500 MHz for ${ }^{1} \mathrm{H}-\mathrm{NMR}$ and $125 \mathrm{MHz}$ for ${ }^{13} \mathrm{C}-\mathrm{NMR}$ ) or a Bruker Avance III HD spectrometer (400 MHz and $300 \mathrm{MHz}$ for ${ }^{1} \mathrm{H}-\mathrm{NMR}$, and $101 \mathrm{MHz}$ and $75 \mathrm{MHz}$ for $\left.{ }^{13} \mathrm{C}-\mathrm{NMR}\right)$ in the indicated solvent at $30{ }^{\circ} \mathrm{C}$. The signals of the deuterated solvent (DMSO- $d_{6}$ ) were taken as the reference, the singlet at $\delta 2.50$ and the quadruplet centred at $39.52 \mathrm{ppm}$ for ${ }^{1} \mathrm{H}$ and ${ }^{13} \mathrm{C}$, respectively. The ${ }^{1} \mathrm{H}$-NMR chemical shifts $\left(\delta_{\mathrm{H}}\right)$ and ${ }^{13} \mathrm{C}-\mathrm{NMR}$ chemical shifts $\left(\delta_{\mathrm{C}}\right)$ are quoted in parts per million (ppm) downfield from trimethylsilane (TMS) and coupling constants $(J)$ are quoted in Hertz (Hz). The abbreviations for NMR data are s (singlet), d (doublet), $\mathrm{t}$ (triplet), q (quartet), quin (quintet) and $\mathrm{m}$ (multiplet). The ${ }^{1} \mathrm{H}$ and ${ }^{13} \mathrm{C}$ signals were assigned with the aid of $2 \mathrm{D}$ methods (COSY, HSQC and HMBC). The data were processed with the software Mestrenova.

The mass spectra were run by the electrospray mode (ESMS) and were recorded using a Mass Spectrometry triple Quadrupole Q-TOF Premier (Waters) with simultaneous Electrospray and an APCI Probe.

(S)-2,5-Dioxopyrrolidin-1-yl-2-(((benzyloxy)carbonyl)amino)-3methylbutanoate (4). A solution of $N$-carbobenzyloxy-L-valine 5 $(10 \mathrm{~g}, 40 \mathrm{mmol})$ and $N$-hydroxysuccinimide $(4.61 \mathrm{~g}, 40 \mathrm{mmol}$, 1.0 eq.) in THF (200 mL) was added dropwise under $\mathrm{N}_{2}$ at $0{ }^{\circ} \mathrm{C}$ to a solution of $N, N^{\prime}$-dicyclohexylcarbodiimide $(8.33 \mathrm{~g}, 40.4 \mathrm{mmol}$, 1.01 eq.) in THF $(100 \mathrm{~mL})$. The mixture was further stirred for $2 \mathrm{~h}$ at $0{ }^{\circ} \mathrm{C}$. The solution was then kept in the refrigerator at $4{ }^{\circ} \mathrm{C}$ for $16 \mathrm{~h}$, which caused precipitation of $N, N^{\prime}$-dicyclohexylurea. Then the mixture was filtered under vacuum, the solvent was removed under reduced pressure and the crude residue was purified by crystallization in isopropanol to yield 4 (13.1 g, $37.6 \mathrm{mmol}, 94 \%)$ as a white solid. The NMR spectra were consistent with those reported in the literature. ${ }^{65}$

Benzyl (S)-(3-methyl-1-(nonylamino)-1-oxobutan-2-yl)carbamate (3). A solution of compound $4(7.58 \mathrm{~g}, 20 \mathrm{mmol})$ in THF $(90 \mathrm{~mL})$ was added dropwise under $\mathrm{N}_{2}$ at $25{ }^{\circ} \mathrm{C}$ to a solution of

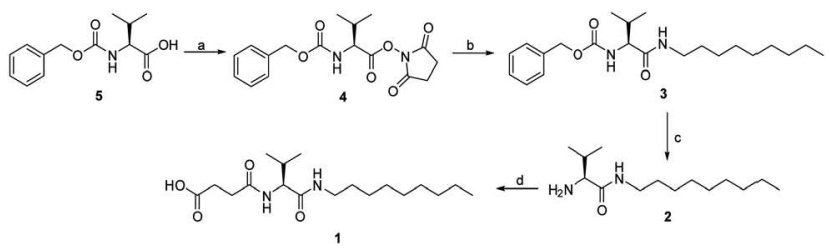

Scheme 2 Preparation of 1: (a) DCC, N-hydroxysuccinimide, THF, 2 h, 94\%; (b) n-nonylamine, THF, 5 h, 77\%; (c) $\mathrm{Pd} / \mathrm{C}, \mathrm{H}_{2}, \mathrm{CH}_{3} \mathrm{OH}, 4 \mathrm{~h}, 94 \%$; (d) succinic anhydride, $\mathrm{K}_{2} \mathrm{CO}_{3}, \mathrm{THF}, 16 \mathrm{~h}, 97 \%$. n-nonylamine (4.1 mL, $22.8 \mathrm{mmol}, 1.1$ eq.) in THF $(55 \mathrm{~mL})$. The mixture was further stirred for $5 \mathrm{~h}$ at $50{ }^{\circ} \mathrm{C}$. The white solid obtained was filtered under vacuum and washed with $\mathrm{HCl}$ $0.1 \mathrm{M}(100 \mathrm{~mL})$ and water $(200 \mathrm{~mL})$. The compound was dried under reduced pressure at $50{ }^{\circ} \mathrm{C}$ to yield $3(5.8 \mathrm{~g}, 15.4 \mathrm{mmol}$, 77\%) as a white solid. ${ }^{1} \mathrm{H}$ NMR (300 MHz, DMSO- $d_{6}$ ): $\delta 7.82$ (dd, $J=5.2,5.4 \mathrm{~Hz}, 1 \mathrm{H}), 7.42-7.24(\mathrm{~m}, 5 \mathrm{H}), 7.15(\mathrm{~d}, J=8.9 \mathrm{~Hz}, 1 \mathrm{H})$, $5.02(\mathrm{~s}, 2 \mathrm{H}), 3.78(\mathrm{dd}, J=7.5,6.6 \mathrm{~Hz}, 1 \mathrm{H}), 3.19-2.88(\mathrm{~m}, 2 \mathrm{H})$, $1.92(\mathrm{~m}, 1 \mathrm{H}), 1.37(\mathrm{~m}, 2 \mathrm{H}), 1.23(\mathrm{~m}, 12 \mathrm{H}), 0.85(\mathrm{~m}, 9 \mathrm{H})$. ${ }^{13} \mathrm{C}$ NMR (75 MHz, DMSO- $\left.d_{6}\right): \delta 170.8(\mathrm{C}=\mathrm{O}), 156.0(\mathrm{C}=\mathrm{O})$, $137.1(\mathrm{C}), 128.2(2 \times \mathrm{CH}), 127.7(\mathrm{CH}), 127.5(2 \times \mathrm{CH}), 65.3\left(\mathrm{CH}_{2}\right)$, $60.3(\mathrm{CH}), 38.3\left(\mathrm{CH}_{2}\right), 31.2\left(\mathrm{CH}_{2}\right), 30.2(\mathrm{CH}), 28.9\left(\mathrm{CH}_{2}\right), 28.9\left(\mathrm{CH}_{2}\right)$, 28.7 $\left(\mathrm{CH}_{2}\right), 28.6\left(\mathrm{CH}_{2}\right), 26.3\left(\mathrm{CH}_{2}\right), 22.0\left(\mathrm{CH}_{2}\right), 19.1\left(\mathrm{CH}_{3}\right), 18.2\left(\mathrm{CH}_{3}\right)$, $13.9\left(\mathrm{CH}_{3}\right)$. HRMS (ESI-TOF): $\mathrm{m} / \mathrm{z}$ calcd for $\left[\mathrm{C}_{22} \mathrm{H}_{37} \mathrm{~N}_{2} \mathrm{O}_{3}\right]^{+}$: 377.2804; found: $377.2802[\mathrm{M}+\mathrm{H}]^{+}(\Delta=0.3 \mathrm{ppm})$.

(S)-2-Amino-3-methyl- $\boldsymbol{N}$-nonylbutanamide (2). Palladium catalyst (10\% w/w Pd/C, $580 \mathrm{mg}$ ) and compound 3 (5.8 g, $15.4 \mathrm{mmol}$ ) were suspended in $\mathrm{CH}_{3} \mathrm{OH}(350 \mathrm{~mL})$ and stirred under $\mathrm{N}_{2}$ at room temperature for $10 \mathrm{~min}$. Subsequently, the system was kept under low vacuum and then filled with hydrogen from a latex balloon. Then the mixture was stirred at room temperature for $4 \mathrm{~h}$. After this time $\mathrm{Pd} / \mathrm{C}$ was removed by filtration through Celite, and the solvent was removed under reduced pressure to yield 2 (3.54 g, $14.6 \mathrm{mmol}$, $94 \%$ ) as a white solid. The compound was used without further purification for the next reaction. ${ }^{1} \mathrm{H}$ NMR (500 MHz, DMSO- $\left.d_{6}\right): \delta$ $7.75(\mathrm{~m}, 1 \mathrm{H}), 3.12-2.97(\mathrm{~m}, 2 \mathrm{H}), 2.88(\mathrm{~d}, J=5.1 \mathrm{~Hz}, 1 \mathrm{H}), 1.83(\mathrm{~m}, 1 \mathrm{H})$, 1.38 (m, 2H), $1.24(\mathrm{~m}, 12 \mathrm{H}), 0.87(\mathrm{~m}, 6 \mathrm{H}), 0.77$ (d, $J=6.8 \mathrm{~Hz}, 3 \mathrm{H})$. The amine signals $\left(\mathrm{NH}_{2}\right)$ are very broad and cannot be distinguished in the spectrum. ${ }^{13} \mathrm{C}$ NMR (126 MHz, DMSO- $\left.d_{6}\right): \delta 174.2$ $(\mathrm{C}=\mathrm{O}), 60.0(\mathrm{CH}), 38.2\left(\mathrm{CH}_{2}\right), 31.6\left(\mathrm{CH}_{2}\right), 31.2(\mathrm{CH}), 29.2\left(\mathrm{CH}_{2}\right), 28.9$ $\left(\mathrm{CH}_{2}\right), 28.7\left(\mathrm{CH}_{2}\right), 28.6\left(\mathrm{CH}_{2}\right), 26.4\left(\mathrm{CH}_{2}\right), 22.1\left(\mathrm{CH}_{2}\right), 19.5\left(\mathrm{CH}_{3}\right), 17.1$ $\left(\mathrm{CH}_{3}\right), 13.9\left(\mathrm{CH}_{3}\right)$.

(S)-4-((3-Methyl-1-(nonylamino)-1-oxobutan-2-yl)amino)-4-oxobutanoic acid (1). Powdered $\mathrm{K}_{2} \mathrm{CO}_{3}(7.4 \mathrm{~g}, 53.5 \mathrm{mmol}, 3.8 \mathrm{eq}$.) was added to a solution of amine $2(3.54 \mathrm{~g}, 14.6 \mathrm{mmol})$ in THF $(290 \mathrm{~mL})$ at $0{ }^{\circ} \mathrm{C}$ under $\mathrm{N}_{2}$ and stirred for 15 minutes. Then a solution of succinic anhydride (2.92 g, $29.2 \mathrm{mmol}, 2.0$ eq.) in THF $(100 \mathrm{~mL})$ was added dropwise and the mixture was stirred vigorously for $16 \mathrm{~h}$ at room temperature. After this, the solution was concentrated under reduced pressure and the crude residue was dissolved in water $(200 \mathrm{~mL})$. Finally, concentrated hydrochloric acid was added dropwise at $0{ }^{\circ} \mathrm{C}$ until the formation of a white precipitate at $\mathrm{pH}=3$. The white solid obtained was filtered under vacuum, and the residue was washed with water $(300 \mathrm{~mL})$. The compound was dried under reduced pressure at $50{ }^{\circ} \mathrm{C}$ to yield 1 (4.86 g, $14.2 \mathrm{mmol}, 97 \%)$ as a white solid. ${ }^{1} \mathrm{H}$ NMR (500 MHz, DMSO- $d_{6}$ ): $\delta 11.99$ (br s, $\left.1 \mathrm{H}\right), 7.80(\mathrm{~m}, 2 \mathrm{H}), 4.07$ $(\mathrm{t}, J=7.9 \mathrm{~Hz}, 1 \mathrm{H}), 3.15-2.90(\mathrm{~m}, 2 \mathrm{H}), 2.47-2.32(\mathrm{~m}, 4 \mathrm{H}), 1.92$ $(\mathrm{m}, 1 \mathrm{H}), 1.36(\mathrm{~m}, 2 \mathrm{H}), 1.23(\mathrm{~m}, 12 \mathrm{H}), 0.85(\mathrm{t}, J=6.7 \mathrm{~Hz}, 3 \mathrm{H}), 0.81$ (d, overlapped, $J=5.3 \mathrm{~Hz}, 6 \mathrm{H}) .{ }^{13} \mathrm{C}$ NMR (126 MHz, DMSO- $d_{6}$ ): $\delta 174.3(\mathrm{C}=\mathrm{O}), 171.5(\mathrm{C}=\mathrm{O}), 171.2(\mathrm{C}=\mathrm{O}), 58.3(\mathrm{CH}), 38.8\left(\mathrm{CH}_{2}\right)$, $31.7\left(\mathrm{CH}_{2}\right), 30.9\left(\mathrm{CH}_{2}\right), 30.4\left(\mathrm{CH}_{2}\right), 29.8(\mathrm{CH}), 29.4\left(\mathrm{CH}_{2}\right)$, $29.4\left(\mathrm{CH}_{2}\right), 29.1\left(\mathrm{CH}_{2}\right), 29.1\left(\mathrm{CH}_{2}\right), 26.8\left(\mathrm{CH}_{2}\right), 22.5\left(\mathrm{CH}_{2}\right)$, $19.6\left(\mathrm{CH}_{3}\right), 18.6\left(\mathrm{CH}_{3}\right), 14.4\left(\mathrm{CH}_{3}\right)$. HRMS (ESI-TOF): $\mathrm{m} / \mathrm{z}$ calcd for $\left[\mathrm{C}_{18} \mathrm{H}_{35} \mathrm{~N}_{2} \mathrm{O}_{4}\right]^{+}$: 343.2597; found: $343.2591[\mathrm{M}+\mathrm{H}]^{+}$ $(\Delta=1.7 \mathrm{ppm})$. 


\section{Macroscopic gel preparation}

In a typical experiment, $5 \mathrm{mg}$ of compound 1 and $1 \mathrm{~mL}$ of solvent were introduced into a cylindrical screw-capped glass vial $(8 \mathrm{~mL}$, diameter $=1.5 \mathrm{~cm}$ ). The system was sonicated to obtain a homogeneous suspension and the vial was heated (hot air at $250{ }^{\circ} \mathrm{C}$ from a heat gun) until a clear solution was obtained. Then, the vial was introduced into a thermostatic bath at $25{ }^{\circ} \mathrm{C}$. After 10-20 minutes, the formation of the gel was assessed by turning the vial upside down (all the solvent remains entrapped).

\section{Nanogel particle preparation}

As a representative example, a suspension of $7.3 \mu \mathrm{mol}$ of compound 1 in $1 \mathrm{~mL}$ toluene in a screw-capped vial $(4 \mathrm{~mL}$, diameter $=1.3 \mathrm{~cm}$ ) was heated (heat gun, $250{ }^{\circ} \mathrm{C}$ ) until complete solution. After cooling (thermostatic bath, $25{ }^{\circ} \mathrm{C}$ ), a gel was obtained. Then, the solvent was removed under vacuum and the xerogel was hydrated in $2 \mathrm{~mL}$ of phosphate buffer saline (PBS, $10 \mathrm{mM}$, pH 7, filtered through a nylon $0.45 \mu \mathrm{m}$ mesh filter) for $10 \mathrm{~min}$. At this point, the system was ultrasonicated for $c a$. 10 minutes until a homogenous suspension was obtained, with a final $\mathrm{pH}$ of 6.4. Then, large particles were removed by centrifugation at $6000 \mathrm{rpm}$ for $60 \mathrm{~min}$ to yield a clear solution of the nanogel particles.

\section{Transmission electron microscopy (TEM, Cryo-TEM)}

Transmission electron micrographs were obtained using a JEOL 2100 microscope with a thermionic gun LaB6 $200 \mathrm{kV}$ equipped with a Gatan Orius high resolution CCD camera. TEM samples were prepared over a Formvar/Carbon film on 200 mesh copper grids.

Gels. Fresh gels were applied directly onto the grid and the expelled solvent was carefully removed by capillary action with paper. The grids were immediately stained with one drop of $1 \%$ aqueous phosphotungstic acid for $2 \mathrm{~min}$ and the liquid was subsequently removed by capillary action.

Nanogels. A drop of nanogel suspension was added on the grid and incubated for $2 \mathrm{~min}$. Then, the solvent was removed with filter paper by capillary action and a drop of $\mathrm{OsO}_{4} 0.1 \%$ was added. After $5 \mathrm{~min}$, the staining solution was removed by capillary action and the grid was washed with a drop of MilliQ water.

For the Cryo-TEM technique, a JEM-2200FS/CR transmission electron microscope (JEOL, Japan) equipped with an UltraScan 4000 SP $(4008 \times 4008$ pixels $)$ cooled slow-scan CCD camera (GATAN, UK) was used. A drop of the nanogel suspension was placed on the TEM grid and an automated vitrification robot Vitrobot $^{\mathrm{TM}}$ was used to freeze the sample in liquid ethane.

\section{Potentiometric titration}

Potentiometric titrations to determine $\mathrm{p} K_{\mathrm{a}}$ of 1 were carried out at $25{ }^{\circ} \mathrm{C}$. An aqueous solution $\left(0.5 \mathrm{mg} \mathrm{mL}^{-1}, 5 \mathrm{~mL}\right)$ of compound 1 in sodium hydroxide $0.1 \mathrm{M}$ was titrated with vigorous stirring with a $0.1 \mathrm{M}$ normalized solution of hydrochloric acid. The addition rate was $6 \mathrm{~mL} \mathrm{~h}^{-1}$ and $\mathrm{pH}$ was monitored every $10 \mathrm{~s}$ (in a S220 Seven Compact $\mathrm{pH}$ meter, Mettler Toledo). $\mathrm{p} K_{\mathrm{a}}$ was calculated by fitting the experimental data to calculated titration curves with the program HYPERQUAD. ${ }^{66}$

\section{Determination of the critical aggregation concentration (cac)}

The critical aggregation concentration of compound 1 at $\mathrm{pH} 1$ was determined using pyrene fluorescence changes (peak I/ peak III ratio). A series of solutions of gelator $\mathbf{1}$ at different concentrations $\left(0-500 \mu \mathrm{g} \mathrm{mL}^{-1}\right)$ and pyrene $\left(1 \mu \mathrm{g} \mathrm{mL}{ }^{-1}\right)$ were prepared in $0.1 \mathrm{M}$ aqueous $\mathrm{NH}_{4} \mathrm{OH}$. Then, the samples were acidified to $\mathrm{pH} 1$ with $2 \mathrm{M} \mathrm{H}_{2} \mathrm{SO}_{4}$ (promoting aggregation) and fluorescence was recorded $\left(\lambda_{\mathrm{ex}}=334 \mathrm{~nm}\right)$.

\section{Nile Red loading}

A macroscopic gel of compound 1 was prepared using $1 \mathrm{~mL}$ $10 \mu \mathrm{M}$ Nile Red solution in toluene. Then, the procedure reported above was carried out to obtain nanoparticles.

\section{Dynamic light scattering}

Size measurements of nanogel particles were performed by dynamic light scattering (DLS) using a Zetasizer Nano ZS (Malvern). Analyses were carried out using a He-Ne laser $(633 \mathrm{~nm})$ at a fixed scattering angle of $173^{\circ}$. Automatic optimization of beam focusing and attenuation was applied for each sample. When measurements were performed at $25{ }^{\circ} \mathrm{C}$, nanogel suspensions were introduced into $3 \mathrm{~mL}$ disposable PMMA cuvettes (10 mm optical path length). Otherwise, the samples were placed in $3 \mathrm{~mL}$ optical glass cuvettes (10 mm optical path, Hellma Analytics). In the latter case, 5 min of sample stabilization was used each time the temperature was changed. The particle size was reported as the average of three measurements.

\section{Z-Potential}

$Z$-Potential measurements were performed at $25{ }^{\circ} \mathrm{C}$ by Laser Doppler Micro-electrophoresis using a Zetasizer Nano ZS (Malvern). $1 \mathrm{~mL}$ of the nanogel suspension was taken in disposable folded capillary zeta cells (Malvern, DTS1070). The Z-Potential is reported as the average of six measures per sample of five different samples.

\section{Single angle static light scattering}

Single angle static light scattering (SALS) measurements were performed using a Zetasizer Nano ZS (Malvern). The samples were introduced into $3 \mathrm{~mL}$ optical glass cuvettes $(10 \mathrm{~mm}$ optical path, Hellma Analytics) and toluene was used as a standard. The refractive index increment $(\mathrm{d} n / \mathrm{d} C)$ for the nanogel particles was set to $0.063 \mathrm{~mL} \mathrm{~g}^{-1}$, according to the results obtained using an AF2000 system (Postnova Analytics) and a refractive index detector in batch mode. The use of SALS requires the simplification of considering isotropic scattering, using a value of 1 for $P(\theta)$ in eqn (2). This assumption is used to generate an error of $60 \%$ in the $M_{\mathrm{w}}$ value for particles with a diameter of $200 \mathrm{~nm}$ (Zetasizer Nano application note "Molecular weight measurements with the Zetasizer Nano system").

To assess the reliability of SALS in the determination of nanoparticles $M_{\mathrm{w}}$, the measurements were carried out for a standard of monodisperse polystyrene latex nanoparticles $($ diameter $=100 \mathrm{~nm})$. Using a value of $\mathrm{d} n / \mathrm{d} c$ of $0.159 \mathrm{~mL} \mathrm{~g}^{-167}$ the $M_{\mathrm{w}}$ of the PS nanoparticles was calculated to be $6.1 \times$ $10^{5} \pm 2 \times 10^{5} \mathrm{kDa}$. This value is in reasonable agreement with 
that obtained considering solid-like particles with a density of $1.04 \mathrm{~g} \mathrm{~mL}^{-1}$ and a diameter of $100 \mathrm{~nm}$, which results in $M_{\mathrm{w}}=3.3 \times 10^{5} \mathrm{kDa}$ (see the ESI $\dagger$ ).

\section{Gelator quantification using NMR ERETIC}

The concentration of $\mathbf{1}$ in a nanogel suspension was quantified using ${ }^{1} \mathrm{H}$ NMR (Bruker Avance III HD spectrometer $400 \mathrm{MHz}$ ) after lyophilization and solubilization of 1 in a $6: 1$ mixture of $\mathrm{CDCl}_{3}$ /hexafluoroisopropanol. A calibrated electronic signal (ERETIC) at $\delta 11.0 \mathrm{ppm}$ was used for this purpose.

\section{Fluorescence spectroscopy}

The fluorescence measurements were carried out using a JASCO FP-8300 spectrofluorometer equipped with a Peltier accessory ETC-815 at $25{ }^{\circ} \mathrm{C}$. The samples were placed in $3 \mathrm{~mL}$ disposable PMMA cuvettes (10 $\mathrm{mm}$ optical path length).

\section{Conclusions}

Merging the interest in polymeric nanogels as nanocarriers and in molecular gels as new soft materials, the preparation of molecular nanogel particles by self-assembly of a low molecular weight compound is reported. Compound $\mathbf{1}$ forms macroscopic gels in water and a variety of organic solvents. Nanosized particles in concentrations as high as ca. $2 \mathrm{mM}$ were prepared reproducibly by sonication of a xerogel of $\mathbf{1}$ in aqueous media. Electron microscopy reveals spherical particles that would correspond to the initial stages of the aggregation into fibers. SALS shows that the nanogels are composed mainly of water, as the analogous macroscopic material. The described nanogels seem to constitute an intermediate state between free molecules and self-assembled fibrillar networks. The particles show good temporal and thermal stability and present accessible hydrophobic domains that entrap Nile Red. In the so-called race for drug delivery, the use of polymeric nanogels presents challenges associated with stimuli-triggered release, biodegradation, and batch-to-batch reproducibility in the preparation of polymers. ${ }^{68}$ The particles described here show intrinsic advantages compared to polymeric nanogels, such as stimuli-triggered disassembly and improved biodegradability due to their molecular nature. Further work will be carried out in the future to assess the feasibility of loading different bioactive substrates in the particles and their use as nanocarriers in cells. It has to be considered that the extensive library of molecular gelators available paves the way for the preparation of molecular nanogels with tailored properties, such as stimuli responsiveness or the presence of the desired functional groups in their structure.

\section{Conflicts of interest}

There are no conflicts to declare.

\section{Acknowledgements}

Ministerio de Economía y Competitividad of Spain (grant CTQ2015-71004-R) and Universitat Jaume I (grant P1.1B2015-76) are thanked for financial support. A. T.-M. thanks Ministerio de Educación, Cultura y Deporte of Spain for a FPU fellowship (FPU14/05974). Technical support from SCIC of University Jaume I and Dr David Gil (CIC bioGUNE) for his assistance in cryo-TEM measurements are acknowledged.

\section{Notes and references}

1 K. Öztürk-Atar, H. Eroğlu and S. Çalış, J. Drug Targeting, 2018, 26, 633-642.

2 V. P. Torchilin, Nat. Rev. Drug Discovery, 2005, 4, 145-160.

3 B. Aryasomayajula, G. Salzano and V. P. Torchilin, Methods Mol. Biol., 2017, 1530, 41-61.

4 M. Geszke-Moritz and M. Moritz, Mater. Sci. Eng., C, 2016, 68, 982-994.

5 B. L. Banik, P. Fattahi and J. L. Brown, Wiley Interdiscip. Rev.: Nanomed. Nanobiotechnol., 2016, 8, 271-299.

6 A. P. Sherje, M. Jadhav, B. R. Dravyakar and D. Kadam, Int. J. Pharm., 2018, 548, 707-720.

7 M. Elsabahy, G. S. Heo, S. M. Lim, G. Sun and K. L. Wooley, Chem. Rev., 2015, 115, 10967-11011.

8 S. Wang, P. Huang and X. Chen, ACS Nano, 2016, 10, 2991-2994.

9 D. Bobo, K. J. Robinson, J. Islam, K. J. Thurecht and S. R. Corrie, Pharm. Res., 2016, 33, 2373-2387.

10 M. Hamidi, A. Azadi and P. Rafiei, Adv. Drug Delivery Rev., 2008, 60, 1638-1649.

11 J. K. Oh, R. Drumright, D. J. Siegwart and K. Matyjaszewski, Prog. Polym. Sci., 2008, 33, 448-477.

12 A. V. Kabanov and S. V. Vinogradov, Angew. Chem., Int. Ed., 2009, 48, 5418-5429.

13 R. T. Chacko, J. Ventura, J. Zhuang and S. Thayumanavan, Adv. Drug Delivery Rev., 2012, 64, 836-851.

14 K. S. Soni, S. S. Desale and T. K. Bronich, J. Controlled Release, 2016, 240, 109-126.

15 I. Neamtu, A. G. Rusu, A. Diaconu, L. E. Nita and A. P. Chiriac, Drug Delivery, 2017, 24, 539-557.

16 J. Estelrich, M. Quesada-Pérez, J. Forcada and J. CallejasFernández, Soft Nanoparticles for Biomedical Applications, Royal Society of Chemistry, 2014.

17 A. Vashist, A. K. Kaushik, S. Ahmad and M. Nair, Nanogels for Biomedical Applications, Royal Society of Chemistry, 2017.

18 K. Raemdonck, J. Demeester and S. De Smedt, Soft Matter, 2009, 5, 707-715.

19 L. Zha, B. Banik and F. Alexis, Soft Matter, 2011, 7, 5908-5916.

20 J. Ramos, A. Imaz, J. Callejas-Fernández, L. Barbosa-Barros, J. Estelrich, M. Quesada-Pérez and J. Forcada, Soft Matter, 2011, 7, 5067-5082.

21 C. C. Lin and A. T. Metters, Adv. Drug Delivery Rev., 2006, 58, 1379-1408.

22 Q. Li, Z. Ning, J. Ren and W. Liao, Curr. Med. Chem., 2018, 25, 963-981.

23 S. Khoee and H. Asadi, Nanogels: Chemical Approaches to Preparation, Encyclopedia of Biomedical Polymers and Polymeric Biomaterials, Taylor and Francis, 2016. 
24 K. Landfester, A. Musyanovych and V. Mailänder, J. Polym. Sci., Part A: Polym. Chem., 2010, 48, 493-515.

25 S. Vinogradov, E. Batrakova and A. Kabanov, Colloids Surf., B, 1999, 16, 291-304.

26 R. Lupitskyy and S. Minko, Soft Matter, 2010, 6, 4396-4402.

27 X. Zhang, S. Malhotra, M. Molina and R. Haag, Chem. Soc. Rev., 2015, 44, 1948-1973.

28 A. Rösler, G. W. M. Vandermeulen and H.-A. Klok, Adv. Drug Delivery Rev., 2001, 53, 95-108.

29 K. Akiyoshi, S. Deguchi, H. Tajima, T. Nishikawa and J. Sunamoto, Macromolecules, 1997, 30, 857-861.

30 H. V. P. Thelu, S. K. Albert, M. Golla, N. Krishnan, D. Ram, S. M. Srinivasula and R. Varghese, Nanoscale, 2018, 10, 222-230.

31 P. Terech and R. G. Weiss, Chem. Rev., 1997, 97, 3133-3159.

32 L. A. Estroff and A. D. Hamilton, Chem. Rev., 2004, 104, 1201-1217.

33 A. R. Hirst, B. Escuder, J. F. Miravet and D. K. Smith, Angew. Chem., Int. Ed., 2008, 47, 8002-8018.

34 S. Banerjee, R. K. Das and U. Maitra, J. Mater. Chem., 2009, 19, 6649-6687.

35 J. W. Steed, Chem. Commun., 2011, 47, 1379-1383.

36 X. Du, J. Zhou, J. Shi and B. Xu, Chem. Rev., 2015, 115, 13165-13307.

37 E. R. Draper and D. J. Adams, Chem, 2017, 3, 390-410.

38 M. D. Segarra-Maset, V. J. Nebot, J. F. Miravet and B. Escuder, Chem. Soc. Rev., 2013, 42, 7086-7098.

39 C. Felip-León, R. Cejudo-Marín, M. Peris, F. Galindo and J. F. Miravet, Langmuir, 2017, 33, 10322-10328.

40 V. J. Nebot, J. Armengol, J. Smets, S. F. Prieto, B. Escuder and J. F. Miravet, Chem. - Eur. J., 2012, 18, 4063-4072.

41 M. Fontanillo, C. A. Angulo-Pachón, B. Escuder and J. F. Miravet, J. Colloid Interface Sci., 2013, 412, 65-71.

42 C. A. Angulo-Pachón and J. F. Miravet, Chem. Commun., 2016, 52, 5398-5401.

43 C. Tang, A. M. Smith, R. F. Collins, R. V. Ulijn and A. Saiani, Langmuir, 2009, 25, 9447-9453.

44 L. Chen, S. Revel, K. Morris, L. C. Serpell and D. J. Adams, Langmuir, 2010, 26, 13466-13471.

45 M. Tena-Solsona, B. Escuder, J. F. Miravet, V. Casttelleto, I. W. Hamley and A. Dehsorkhi, Chem. Mater., 2015, 27, 3358-3365.

46 X. Yan, Y. Cui, W. Qi, Y. Su, Y. Yang, Q. He and J. Li, Small, 2008, 4, 1687-1693.
47 S. Akoka, L. Barantin and M. Trierweiler, Anal. Chem., 1999, 71, 2554-2557.

48 M. I. Burguete, F. Galindo, E. García-Verdugo, N. Karbass and S. V. Luis, Chem. Commun., 2007, 3086-3088.

49 E. D. Sone, S. Weiner and L. Addadi, J. Struct. Biol., 2007, 158, 428-444.

50 C. Wu and S. Zhou, J. Macromol. Sci., Phys., 1997, 36, 345-355.

51 R. Pelton, Macromol. Symp., 2004, 207, 57-65.

52 N. Morimoto, S. Hirano, H. Takahashi, S. Loethen, D. H. Thompson and K. Akiyoshi, Biomacromolecules, 2013, 14, 56-63.

53 S. Islam, D. L. Inglefield and O. D. Velev, Soft Matter, 2018, 14, 2118-2130.

54 B. H. Zimm, J. Chem. Phys., 1948, 16, 1093-1099.

55 N. Zȩibacz, S. A. Wieczorek, T. Kalwarczyk, M. Fiałkowski and R. Hołyst, Soft Matter, 2011, 7, 7181-7186.

56 S. Peng and C. Wu, Macromolecules, 2001, 34, 568-571.

57 Y. Sekine, H. Endo, H. Iwase, S. Takeda, S. A. Mukai, H. Fukazawa, K. C. Littrell, Y. Sasaki and K. Akiyoshi, J. Phys. Chem. B, 2016, 120, 11996-12002.

58 Q. Ying and B. Chu, Macromolecules, 1987, 20, 362-366.

59 A. Aggeli, I. A. Nyrkova, M. Bell, R. Harding, L. Carrick, T. C. B. McLeish, A. N. Semenov and N. Boden, Proc. Natl. Acad. Sci. U. S. A., 2001, 98, 11857-11862.

60 X. Huang, P. Terech, S. R. Raghavan and R. G. Weiss, J. Am. Chem. Soc., 2005, 127, 4336-4344.

61 M. A. Rogers, X. Liu, V. A. Mallia and R. G. Weiss, CrystEngComm, 2015, 17, 8085-8092.

62 C. D. Jones, S. R. Kennedy, M. Walker, D. S. Yufit and J. W. Steed, Chem, 2017, 3, 603-628.

63 J. Stachurski and M. MichaŁek, J. Colloid Interface Sci., 1996, 184, 433-436.

64 K. A. Fletcher, I. A. Storey, A. E. Hendricks, S. Pandey and S. Pandey, Green Chem., 2001, 3, 210-215.

65 J. Becerril, M. Bolte, M. I. Burguete, F. Galindo, E. GarcíaEspaña, S. V. Luis and J. F. Miravet, J. Am. Chem. Soc., 2003, 125, 6677-6686.

66 L. Alderighi, P. Gans, A. Ienco, D. Peters, A. Sabatini and A. Vacca, Coord. Chem. Rev., 1999, 184, 311-318.

67 J. Jacobs, A. Byrne, N. Gathergood, T. E. Keyes, J. P. A. Heuts and A. Heise, Macromolecules, 2014, 47, 7303-7310.

68 S. V. Vinogradov, Nanomedicine, 2010, 5, 165-168. 


\section{SUPPLEMENTARY MATERIAL}

In between molecules and self-assembled fibrillar networks: Highly stable nanogel particles from a low molecular weight hydrogelator

Ana Torres-Martínez, César A. Angulo-Pachón, Francisco Galindo* and Juan F. Miravet $^{*}$

\section{NMR spectra}
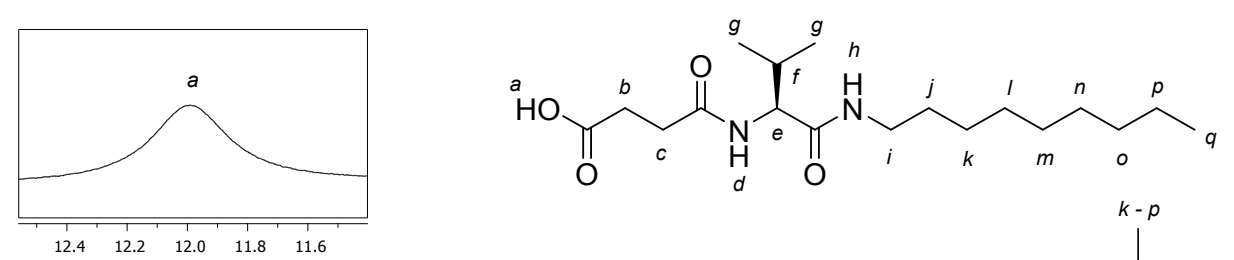

(1)

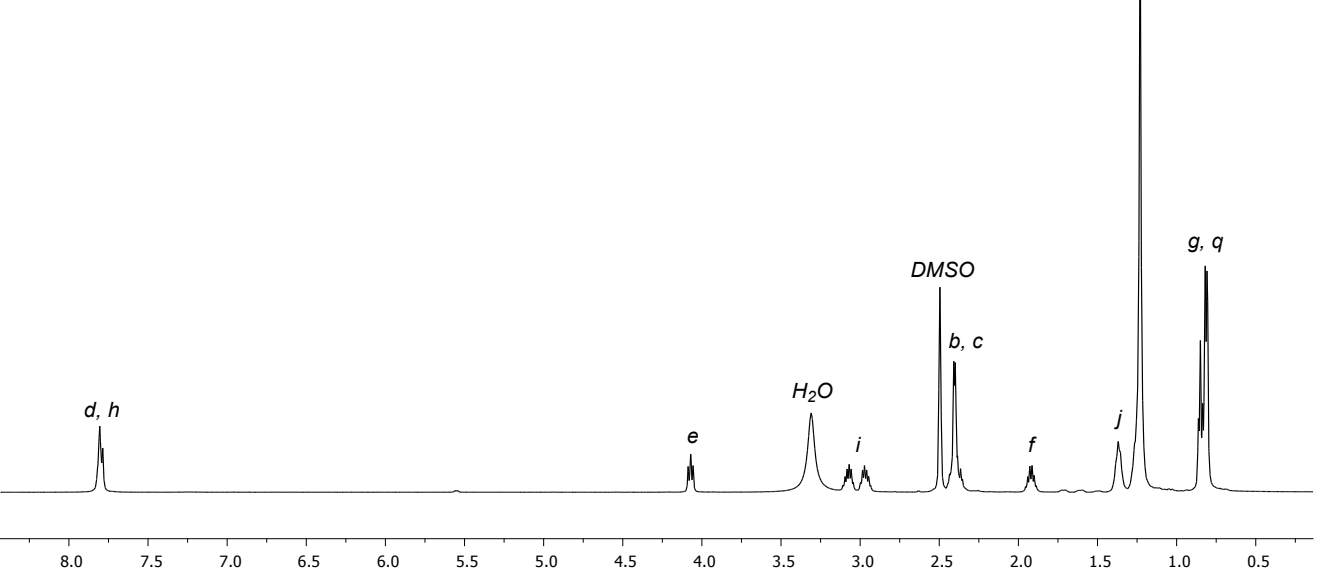

re S1. ${ }^{1} \mathrm{H}$ NMR spectrum of 1 in $\mathrm{D}_{6}$-DMSO. 

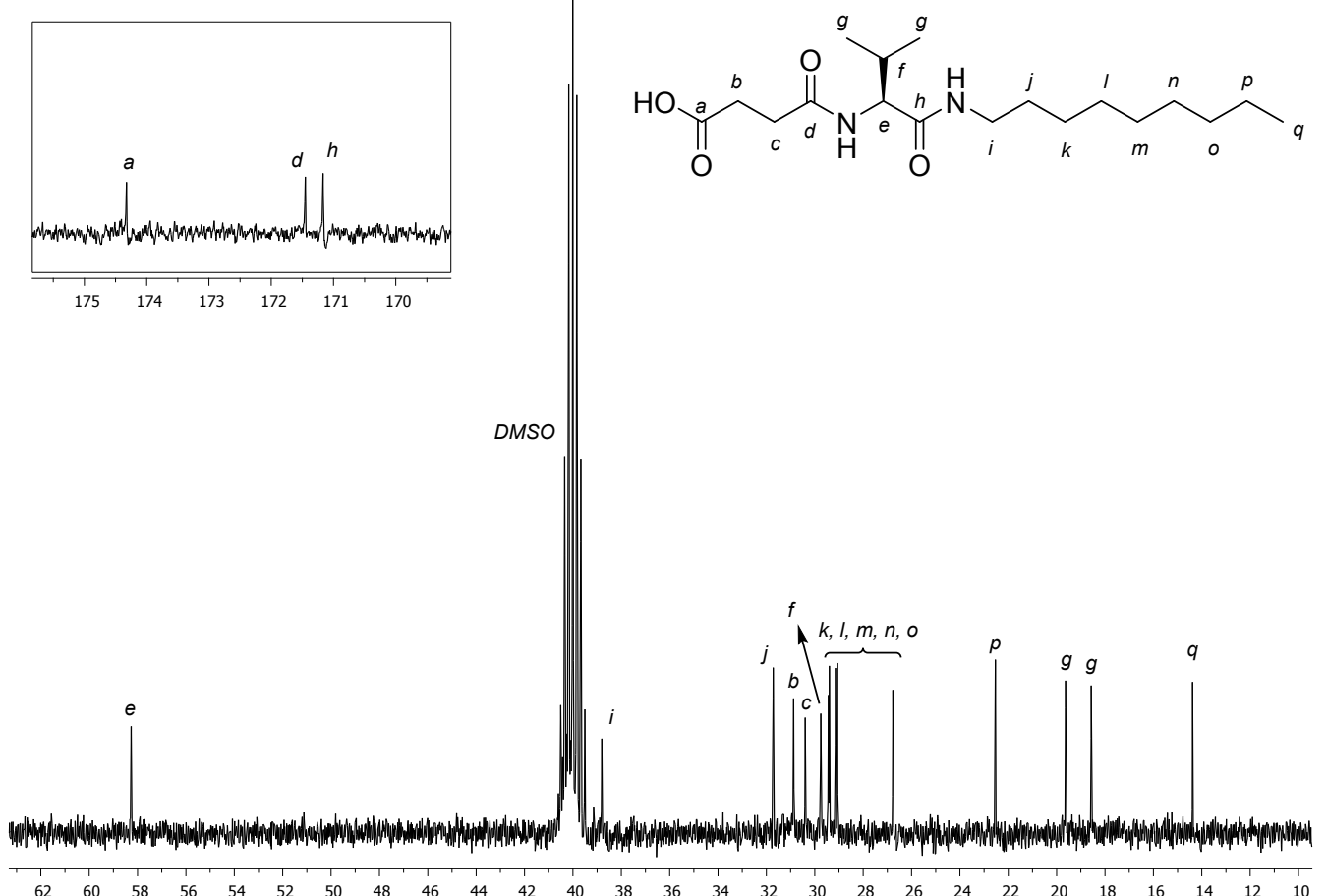

ure S2. ${ }^{13} \mathrm{C}$ NMR spectrum of 1 in $\mathrm{D}_{6}$-DMSO.

\section{Determination of minimum gelation concentration (mgc)}

Table S1. Minimum gelation concentration of compound $\mathbf{1}$ in different solvents.

\begin{tabular}{|c|c|c|}
\hline \multirow{2}{*}{ Solvent } & \multicolumn{2}{|c|}{ mgc } \\
\hline & $(\mathrm{mg} / \mathrm{mL})$ & (mM) \\
\hline Acetonitrile & 6 & 18 \\
\hline Dicloromethane & 6 & 18 \\
\hline Toluene & 1 & 3 \\
\hline Water & 5 & 16 \\
\hline Ethyl acetate & 13 & 38 \\
\hline Chloroform & \multicolumn{2}{|c|}{ Soluble } \\
\hline Tetrahydrofurane & \multicolumn{2}{|c|}{ Soluble } \\
\hline Ethanol & \multicolumn{2}{|c|}{ Soluble } \\
\hline Methanol & \multicolumn{2}{|c|}{ Soluble } \\
\hline
\end{tabular}




\section{3. pKa determination}

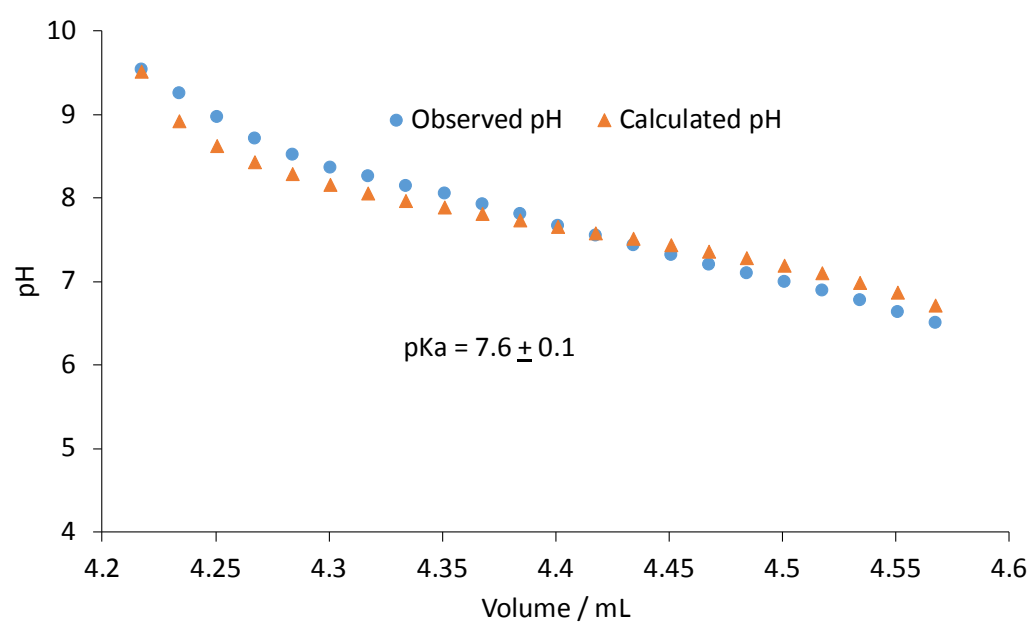

Figure S3. Calculated (Hyperquad) vs observed $\mathrm{pH}$ for the potentiometric titration of 1 with $\mathrm{HCl}$ $0.1 \mathrm{M}$.

\section{Dynamic light scattering}

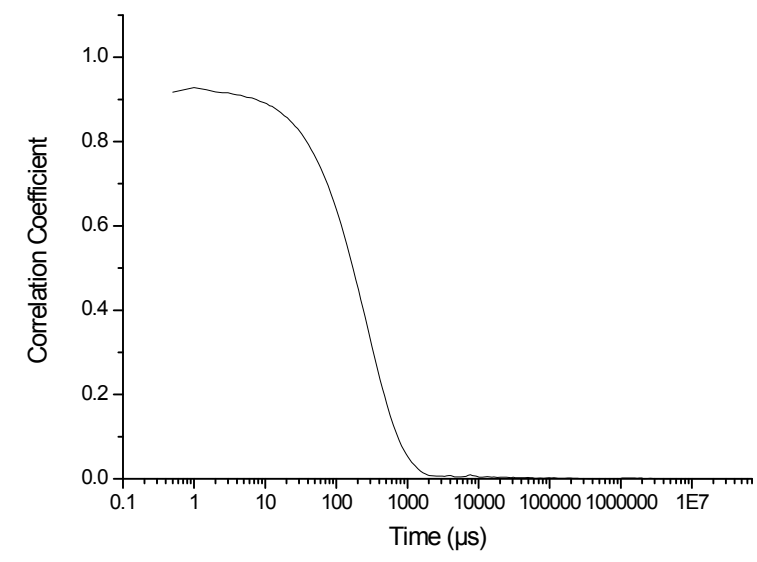

Figure S4. Correlation function of a representative DLS measurement of nanogel particles of 1 obtained from the xerogel from toluene.

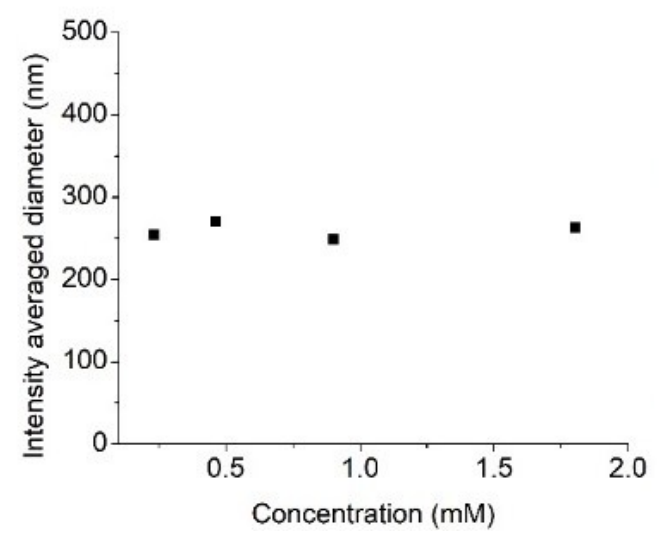

Figure S5. Influence of the concentration in the diameter of the nanoparticles formed by 1 in PBS. 


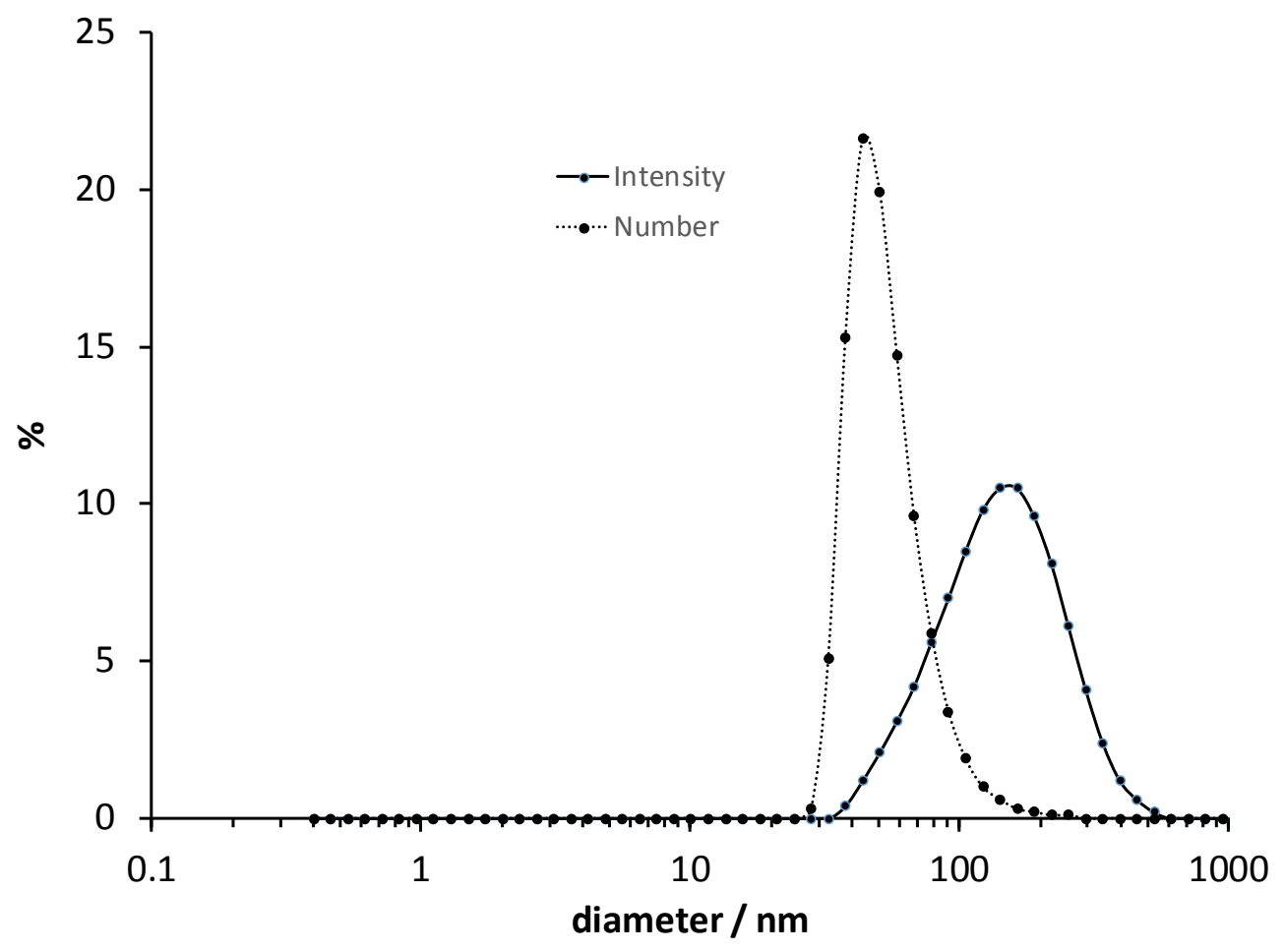

Figure S6. DLS analysis of size distribution by intensity (solid line) and by number (dash-dot line) of a representative sample of nanogel particles obtained from a xerogel from water.

\section{Static light scattering}

Table S2. Calculated concentration $\left(\mathrm{mg} \mathrm{mL}^{-1}\right)$ of aggregated 1 in the samples used in SALS. ${ }^{a}$

\begin{tabular}{|c|c|c|c|}
\hline$[\mathbf{1}]_{\text {total }}$ & {$[\mathbf{1}]_{\text {ionized }}$} & {$[\mathbf{1}]_{\text {free, neutral }}$} & {$[\mathbf{1}]_{\text {nanoparticles }}$} \\
\hline 0.274 & 0.026 & 0.075 & 0.173 \\
\hline 0.377 & 0.036 & 0.075 & 0.266 \\
\hline 0.480 & 0.046 & 0.075 & 0.359 \\
\hline 0.582 & 0.055 & 0.075 & 0.452 \\
\hline 0.685 & 0.065 & 0.075 & 0.545 \\
\hline
\end{tabular}

${ }^{a}[1]_{\text {ionized }}$ is calculated for a system with $\mathrm{pH}=6.4$ and $\mathrm{pKa}=7.6$; [1] free, neutral corresponds to the cac value, $0.2 \mathrm{mM}, 0.075 \mathrm{mg}$ $\mathrm{mL}^{-1}$ 


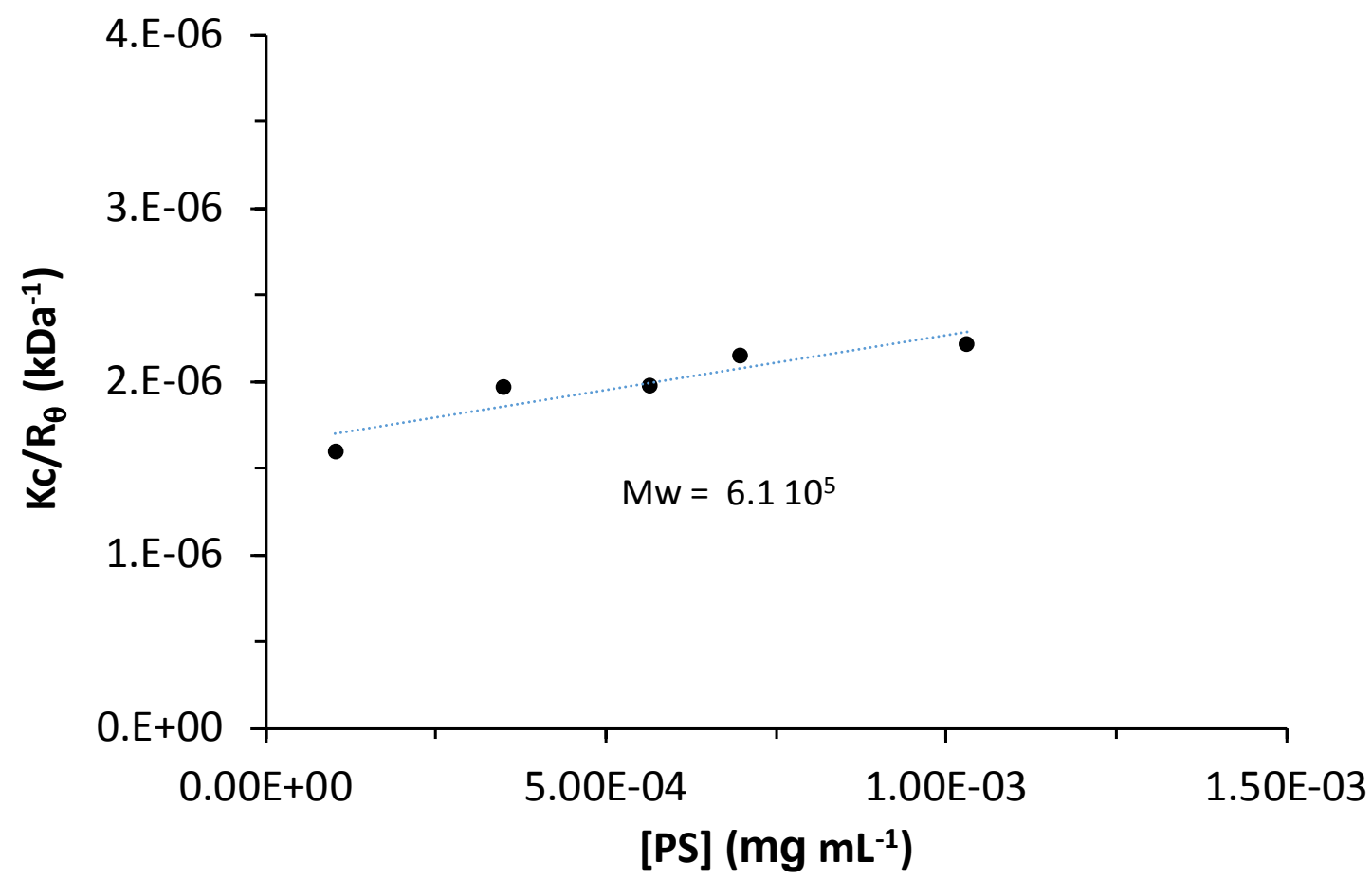

Figure S7. Debye plot for determination of $M_{w}$ of standard polystyrene latex particles $(d=100$ $\mathrm{nm})$.

\section{Differential scanning calorimetry}
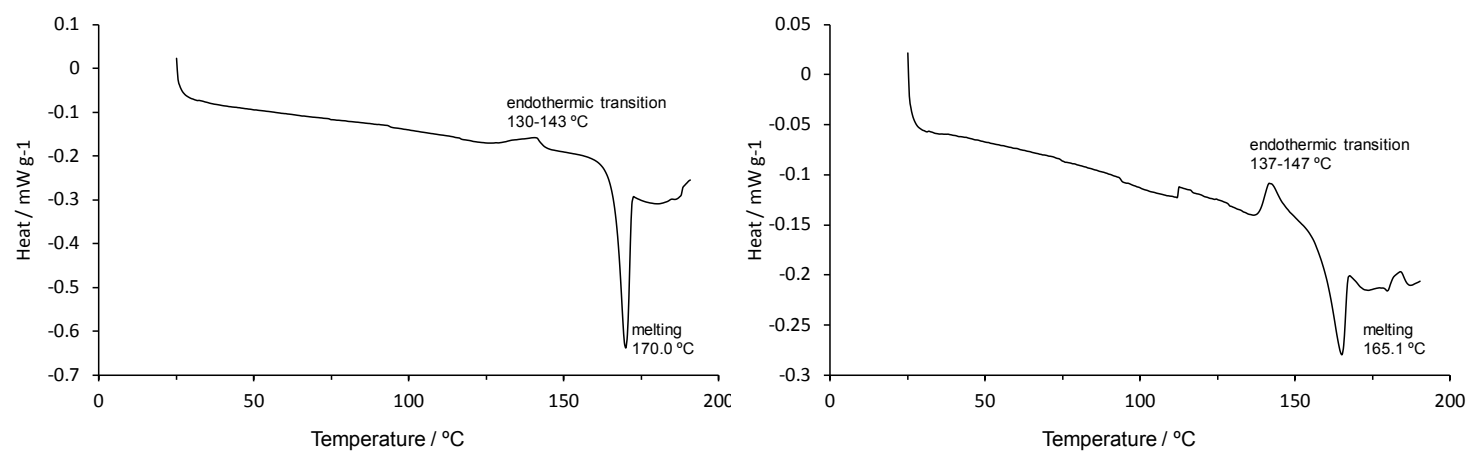

Figure S8. DSC traces of the xerogels from toluene (left) and water (right). 


\title{
Liposome-Enveloped Molecular Nanogels
}

\author{
Ana Torres-Martínez, César A. Angulo-Pachón, Francisco Galindo, ${ }^{\circledR}$ and Juan F. Miravet*잉 \\ Departament de Química Inorgànica i Orgànica, Universitat Jaume I, Avda. Sos Baynat s/n, 12071 Castelló de la Plana, Spain
}

Supporting Information

ABSTRACT: Novel hydrogel@liposome particles were prepared by $\mathrm{pH}$ triggered molecular gel formation inside of liposomes loaded with a lowmolecular weight gelator derived from L-valine (1). Liposome formation was carried out using L- $\alpha$-phosphatidylcholine (PC) and cholesterol as components of the lipid bilayer. Molecular hydrogelator 1 and pyranine, a ratiometric fluorescent $\mathrm{pH}$ probe, were entrapped in the liposomes at $\mathrm{pH} 9$ and posterior acidification with D-glucono-1,5-lactone to $\mathrm{pH} \mathrm{5-6}$ provoked intraliposomal gel formation. Removal of the lipid bilayer with sodium

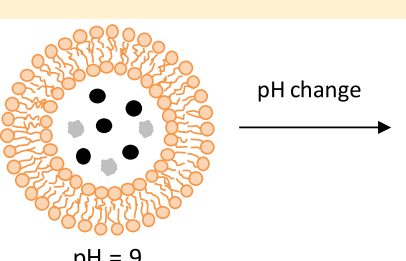

$\mathrm{pH}=9$

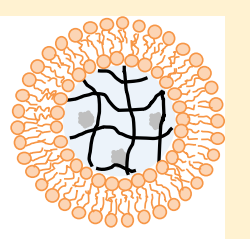

$\mathrm{pH}=5$ dodecyl sulfate yielded naked nanogel particles. The systems were characterized by transmission electron microscopy and dynamic light scattering. The hydrogel@liposomes were loaded with doxorubicin, showing a similar release than that observed for liposomes. The hybrid particles described here are the first case of nonpolymeric hydrogel@liposome systems reported. This type of nanocarriers merges the benefits of liposomal vehicles with the inherent stimuli responsiveness and enhanced biocompatibility of hydrogels formed by low-molecular weight molecules, foretelling a potential use in environmentally sensitive drug release.

\section{INTRODUCTION}

Different organic nanoparticles such as liposomes, solid lipid nanoparticles, ${ }^{1}$ polymeric nanoparticles, ${ }^{2}$ and dendrimers ${ }^{3}$ have been increasingly studied as vehicles for the delivery of actives in a biomedical context. ${ }^{4}$ In this regard, liposomes undoubtedly represent the most successful type of nanocarrier, with several liposomal drug formulations currently approved for clinical practice. ${ }^{5-7}$ In addition to the abovementioned nanocarriers, nanogels are a particular group of organic nanostructures that has lately received much attention. ${ }^{8-13}$ Nanogels are formed by three-dimensional polymeric networks capable of retaining large quantities of water, most of them being constituted by covalently cross-linked networks.

At the intersection of liposomes and nanogels, a growing interest has emerged in recent years regarding the development of hydrogel-filled liposomes, aiming to improve some aspects of conventional liposomal formulations. As several reviews state, the presence of a hydrogel core is intended to provide improved mechanical stability to the liposome and environmentally sensitive drug release. ${ }^{14-18}$ On the other hand, the lipid bilayer around the nanogel is envisaged to enhance its bioavailability and biocompatibility. Different denominations have been used for hydrogel@liposome hybrid systems such as lipobeads, ${ }^{19}$ gel core liposomes, ${ }^{20}$ core-shell lipid-polymer nanoparticles, ${ }^{15}$ lipogels, ${ }^{21}$ or liposome-camouflaged nanogels. $^{22}$ The first report on liposome-gel hybrids dates back to 1987 when Torchilin's group described the encapsulation of a cross-linked polyacrylamide gel in the inner compartment of vesicles. $^{23}$ They prepared liposomes in the presence of a monomer and, after liposome separation by size exclusion chromatography (SEC), UV-promoted polymerization was carried out. UV polymerization is the most common approach for the preparation of lipogels that followed that initial work.
The polymeric hydrogel@liposome particles prepared in this way include, for example, poly( $\mathrm{N}$-isopropylacrylamide $),^{24-27}$ cross-linked polymers derived from dextran, ${ }^{28,29}$ polyethylene glycol,$~^{30-32}$ polyacrylic acid, ${ }^{33}$ polyglycidol, ${ }^{34}$ and ethyl methacrylate derivatives. ${ }^{21}$ Thermally initiated polymerization, in the absence of UV irradiation, has been used for the formation of a redox-responsive nanogel ${ }^{22}$ and a polymethacrylate derivative inside liposomes. ${ }^{35}$ Alternatively, hydrogels of alginate and polyacrylic acid were prepared into liposomes by the addition of $\mathrm{Ca}^{2+}$ and $\mathrm{pH}$ change, respectively. ${ }^{20,36}$ Moreover, temperature change provoked gel formation in liposomes containing poloxamer ${ }^{37}$ and, in a different approach, gel@liposomes were prepared by microfluidics. ${ }^{38}$

Significant results in the use of lipogels embrace, among others, the preparation of a synthetic mimic of the secretory granule for the delivery of doxorubicin, ${ }^{39}$ entrapment of hemoglobin, ${ }^{27}$ release of proteins, ${ }^{29}$ intramuscular delivery of a malaria antigen, ${ }^{20}$ enhancement of tumor immunotherapy ${ }^{31}$ and responsive intracellular release of doxorubicin. ${ }^{35}$

The common point of all the reported gel@liposome systems is that they are constituted by polymers. Nonpolymeric gels, namely, molecular gels (also known as supramolecular gels) are formed by the supramolecular assembly of low-molecular weight species ${ }^{40-46}$ and are intrinsically responsive to temperature or other physicochemical stimuli. ${ }^{47}$ The interaction of molecular gels and liposomes was first described by van Esch et al. Contrary to the systems reported here, which describe gel formation inside liposomes, that work indicated liposome formation within a macroscopic

Received: July 22, 2019

Revised: September 12, 2019

Published: September 24, 2019 
supramolecular gel. ${ }^{48}$ Following our previous experience in molecular gels, we recently approached the preparation of nanoparticles from low-molecular weight gelators. ${ }^{49-51}$ Here, as a step forward, the incorporation of molecular, nonpolymeric, gels into liposomes is reported. The substitution of polymeric hydrogels by molecular ones in gel@liposome particles is designed to overcome some issues associated with polymers in their biomedical use, such as biodegradability or polymer structure variations resulting from polydispersity or cross-linking degree. More importantly, the inherent stimuliresponsiveness of molecular gels is ideal for the preparation of environment-sensitive carriers.

\section{RESULTS AND DISCUSSION}

Compound $\mathbf{1}$ (Scheme 1) is a molecular hydrogelator formed by L-valine, modified as a nonylamide at the carboxylic acid

Scheme 1. Structure of Some the Organic Compounds Mentioned in the Main Text

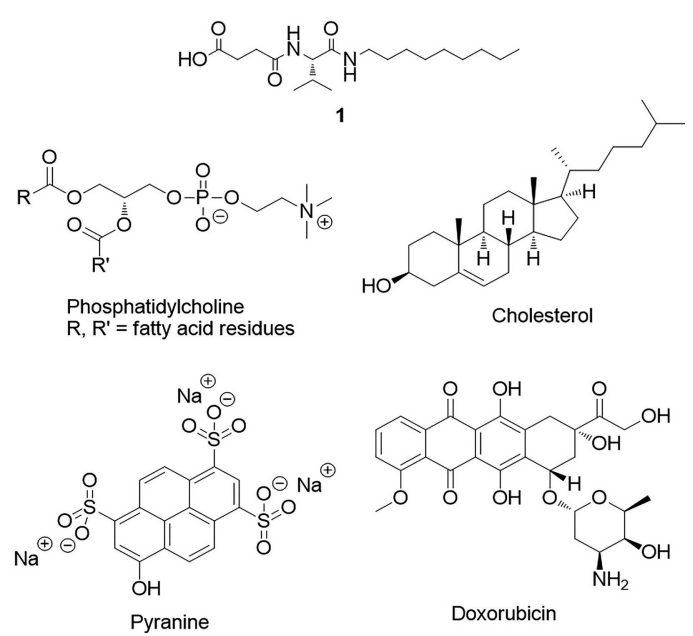

unit and acylated with succinic acid at the amine function. As we recently reported, this compound can form nanogel particles in water with a critical aggregation concentration of $0.07 \mathrm{mg} \mathrm{mL}^{-1}$. Also, macroscopic gels can be prepared in water at neutral or acidic $\mathrm{pH}$ with a critical gelation concentration of $5 \mathrm{mg} \mathrm{mL}{ }^{-1}$, being soluble in its deprotonated, anionic form $\left(\mathrm{p} K_{\mathrm{a}}=7.6\right){ }^{51}$ The strategy followed to prepare nanogel@ liposome particles using compound $\mathbf{1}$ is depicted in Scheme 2.

Before nanogel@liposome development, liposome formation and changes in their internal $\mathrm{pH}$ were assayed. Liposomes were made using L- $\alpha$-phosphatidylcholine (PC) and cholesterol (8:2 molar ratio). The addition of cholesterol provides a more robust bilayer, reducing leakage from the liposomes. ${ }^{52}$ The preparation process involved the hydration of the dry lipid film with a $\mathrm{pH} 9$ phosphate buffer and sonication. ${ }^{53,54}$ Small unilamellar vesicles were obtained after SEC, as could be visualized by transmission electron microscopy (TEM, Figure $1)$.

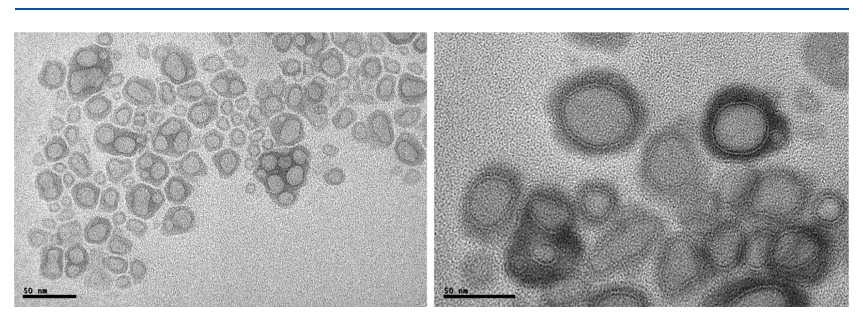

Figure 1. TEM images (no staining) of the liposomes prepared at $\mathrm{pH}$ $=9$ (without hydrogelator).

The changes in the intraliposomal $\mathrm{pH}$ were studied using pyranine, a water-soluble, membrane-impermeable fluorescent molecule. Pyranine-loaded liposomes were prepared at $\mathrm{pH} 9$ and isolated by SEC, in which pyranine was used as a marker to follow the separation process of the vesicles. Then, the system was acidified by the addition of D-glucono-1,5-lactone, which affords progressive, smooth acidification, useful in molecular gel formation. ${ }^{55}$ The changes of $\mathrm{pH}$ inside the liposomes could be evaluated from the pyranine excitation spectrum which consists of two independent contributions: the neutral molecule $(400 \mathrm{~nm})$ and its deprotonated conjugate base $(450 \mathrm{~nm})$ (Figure 2, left). The ratio of fluorescence excitation at 460 and $415 \mathrm{~nm}$, monitoring emission at $511 \mathrm{~nm}$, thereby gives a ratiometric $\mathrm{pH}$ measure. ${ }^{56-58}$ In this case, after $2 \mathrm{~h}$, the $\mathrm{pH}$ inside the vesicles changed from 9 to 6.3 (see Figure 2, right, and Figure S2), a value compatible with gel formation in the case of compound $1\left(\mathrm{p} K_{\mathrm{a}}=7.6\right)$. After $\mathrm{pH}$ change, liposomes were stable regarding their size.

Next, following the preparation of nanogel@liposomes outlined in Scheme 2, liposomes were assembled at $\mathrm{pH} 9$ in the presence of the ionized, soluble form of gelator $\mathbf{1}$ and pyranine. After separation of the loaded liposomes from the nonencapsulated molecules by SEC, dynamic light scattering (DLS) revealed the formation of particles with a number averaged diameter of $41 \pm 24 \mathrm{~nm}$ (Figure 3, top). Then, the system was acidified to protonate gelator 1 , promoting selfassembly and affording gel@liposome hybrid particles. The particles were analyzed by TEM (Figure 4) and DLS (Figure 3, middle), revealing a very similar size distribution to that found

\section{Scheme 2. Outline of the Preparation of Molecular Gel@Liposomes Entrapping a Cargo}

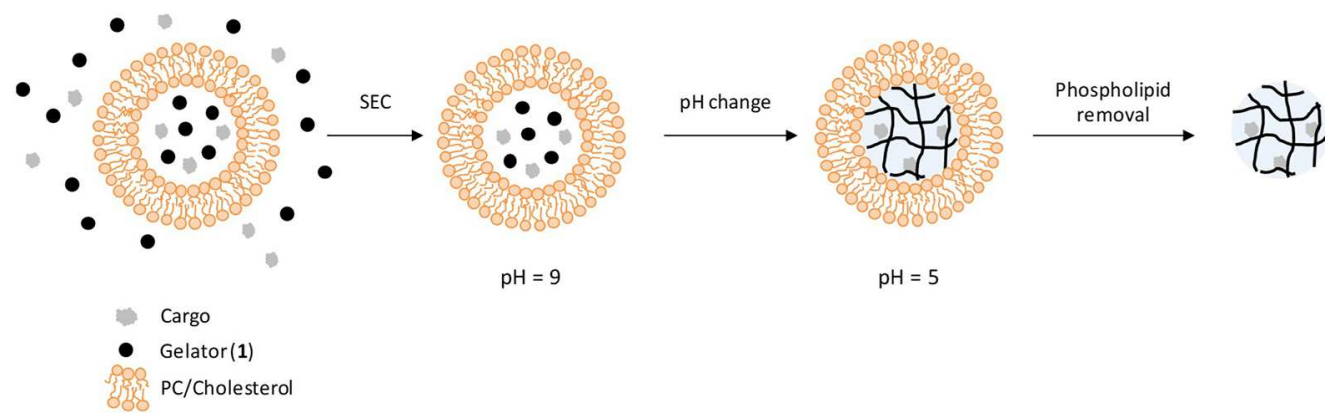



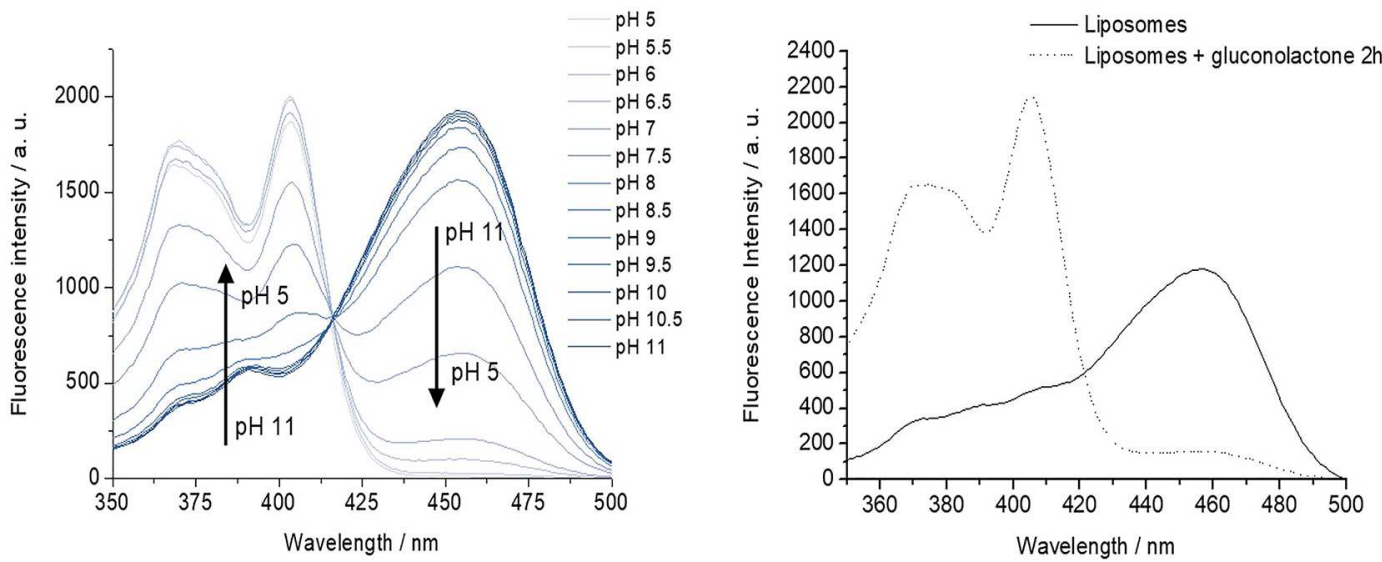

Figure 2. Fluorescence excitation spectra $\left(\lambda_{\mathrm{em}}=511 \mathrm{~nm}\right)$ of pyranine standards $(1 \mu \mathrm{M})$ at different $\mathrm{pH}$ (from 5 to 11) (left) and of pyranine@ liposomes before (solid line, $\mathrm{pH}$ 9) and after $2 \mathrm{~h}$ of external $\mathrm{pH}$ change with gluconolactone (red dotted line, $\mathrm{pH}$ 6.3) (right).
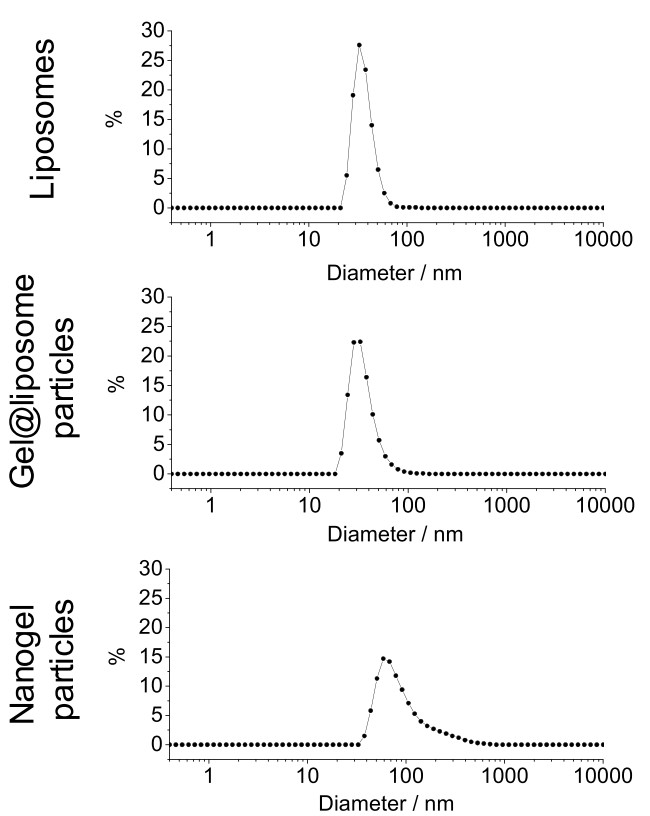

Figure 3. Number size distribution of a representative sample of gelator@liposomes ( $\mathrm{pH}$ 9), gel@liposome particles, and nanogel particles obtained by DLS analysis.
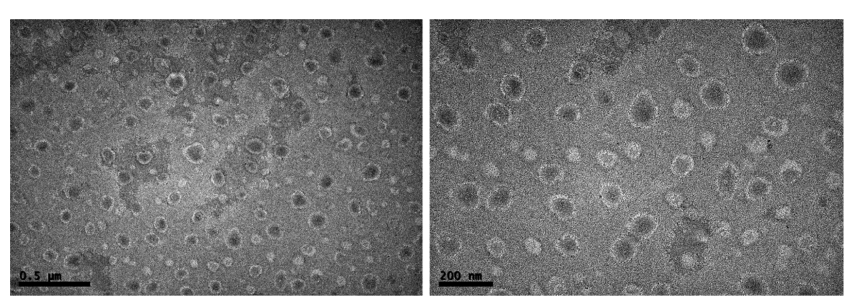

Figure 4. TEM image (no staining) of gel@liposome particles.

for the initial liposomes, with number averaged diameters obtained by DLS and TEM (Supporting Information, Figure S6), respectively, of $37 \pm 10 \mathrm{~nm}$ and $95 \pm 19 \mathrm{~nm}$. Additionally, cryo-TEM images could be obtained (Supporting Information, Figure S1), revealing larger diameters with an average value of $293 \pm 90 \mathrm{~nm}$. Such differences between diameters obtained by DLS, TEM, and cryo-TEM might be caused by aggregation phenomena associated with sample preparation in the electron microscopy techniques.
Liposome disassembly permitted to obtain naked nanogel particles. For this purpose, sodium dodecyl sulfate (SDS) was added, and dialysis against water was performed to remove the mixed micelles of SDS-PC and any free gelator that could have leaked from the particles. TEM images corroborated the formation of nanogel particles (Figure 5), and DLS revealed a

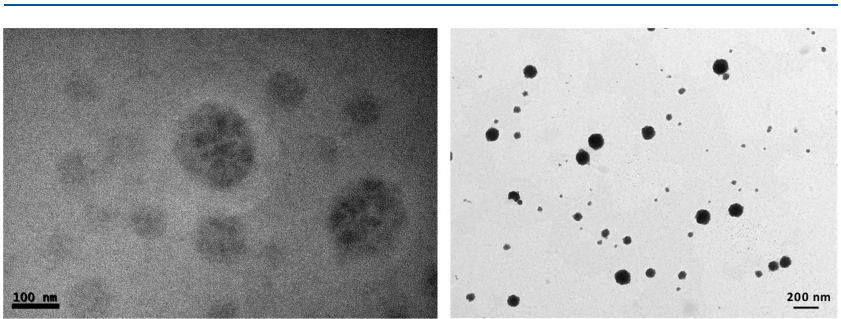

Figure 5. TEM images of nanogel particles. Left: no staining; right: $\mathrm{OsO}_{4}$ staining.

number averaged diameter of $51 \pm 25 \mathrm{~nm}$ (Figure 3, bottom; an average of ten samples), presenting the size distribution a broad tail in the region of big particles. These larger particles may arise from aggregates formed between SDS and the phospholipid $^{59}$ which were not removed by dialysis, or from the interaction of SDS with the nanogel particles. ${ }^{60}$

The quantification of the concentration of the gelator in the aqueous samples containing naked hydrogel particles was found to be $0.7 \mathrm{mM}$. This determination was carried out dissolving a lyophilized sample in $\mathrm{CDCl}_{3}$ and measuring the integration of the signals in the ${ }^{1} \mathrm{H}$ NMR spectrum against an external standard. Furthermore, the study of different samples using pyranine revealed $\mathrm{pH}$ values of 5-6 for both lipogels and nanogel particles (Figure S3). Regarding stability, gel@ liposome particles and nanogels were stable at least for a month at $4{ }^{\circ} \mathrm{C}$ according to DLS measurements, which revealed a similar size distribution although slightly shifted towards larger particles, presumably because of aggregation (Supproting Information, Figure S5).

Finally, the ability of the gel@liposome particles to entrap and release doxorubicin was tested. Doxorubicin is the most effective chemotherapeutic drug developed against a broad range of cancers. ${ }^{61}$ Because of its cardiotoxicity, doxorubicin is loaded into liposomes (MyocetV) and pegylated liposomes (DOXIL) in clinically approved formulations. Aiming to improve its therapeutic efficiency, hundreds of papers have 
explored the use of different nanocarriers for doxorubicin, including, among others, dextran, polylactic acid, solid-lipid nanoparticles, or polymeric nanogels. ${ }^{62}$ The preparation of the lipogel particles was carried out using a $1 \mathrm{mM}$ doxorubicin solution as hydration medium. UV-vis and fluorescence spectroscopy revealed its incorporation into the lipogel particles. As can be seen in Figure 6, fluorescence spectra for

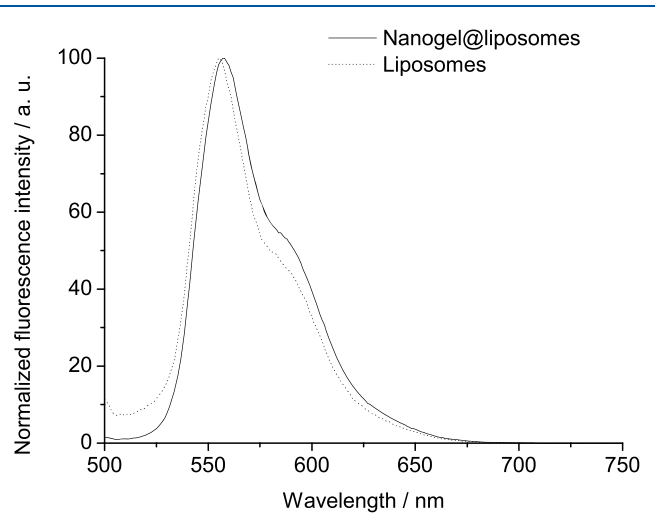

Figure 6. Comparison of doxorubicin emission spectra $\left(\lambda_{\mathrm{ex}}=480\right.$ $\mathrm{nm}$ ) in liposomes (dotted line) and lipogels (solid line).

free and lipogel-loaded doxorubicin are quite similar, but the emission of doxorubicin in the lipogel is slightly red-shifted compared to that of plain liposomes at the same $\mathrm{pH}$. It has to be considered that the hydrophilic nature of the drug probably precludes its complete adsorption on the nanogel network, being solvated in the aqueous pools. However, this shift implies a small difference in solvation, which would indicate that there is an interaction with the gel fibers, although the environment remains essentially aqueous. Additionally, doxorubicin release from the lipogels and liposomes was studied using a dialysis membrane against a $\mathrm{pH} 7$ solution. ${ }^{63-65}$ The amount of doxorubicin outside the dialysis tubing was quantified by UV-vis and fluorescence spectroscopy, revealing a rather similar release from both systems (ca. $20 \%$ after $24 \mathrm{~h}$, see Figure S4). Therefore, the lipogels presented here maintain the capability of entrapping doxorubicin found in pure liposomes. It seems reasonable that the gel does not alter the doxorubicin release considering the hydrophilic nature of this drug and its low molecular weight, which does not limit the diffusion through the gel network. Also, it has to be considered that the dialysis process at $\mathrm{pH} 7$ may, at least partially, disassemble the nanogel core considering that the $\mathrm{p} K_{\mathrm{a}}$ of the gelator is 7.6 .

\section{CONCLUSIONS}

Molecular gel formation inside liposomes was triggered by the acidification of liposomes loaded with gelator 1. Small unilamellar liposomes were prepared by hydration and sonication of a dry lipid film in the presence of the ionized gelator at $\mathrm{pH} 9$ and pyranine, which permitted to isolate loaded liposomes after SEC. Addition of D-glucono-1,5-lactone

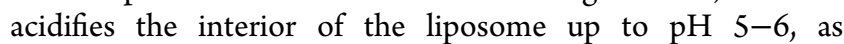
confirmed by pyranine as a ratiometric fluorescent $\mathrm{pH}$ probe, triggering molecular gel formation. TEM and DLS analysis revealed that the hybrid gel@liposome particles have a similar size distribution as the parent liposomes. Removal of the phospholipid bilayer affords nanogel particles, with a total concentration of the gelator in the sample of $0.7 \mathrm{mM}$ and keeping the acidic $\mathrm{pH}$ in the interior of the particle. Both lipogels and nanogels showed no size changes at least for a month. Therefore, liposomes can be used as templates for the preparation of molecular nanoparticles. The studied molecular gel@liposome system can be loaded with doxorubicin.

In the context of the interest in gel@liposomes particles, the results presented here constitute the first case of preparation of a molecular, nonpolymeric, gel inside liposomes. The noncovalent nature of the hydrogel offers a priori some attractive advantages related to biocompatibility and stimuli responsiveness. Therefore, these hybrid systems could be used for the transport of bioactive substances and would benefit from increased liposome stability together with stimuli specific release when using molecular gelators with the appropriate functionality such redox or photoresponsive groups among others. $^{47}$ In the case of the gelator presented here, $\mathrm{pH}$ triggered disassembly could be studied. The application of these new vehicles as carriers in biological media will be evaluated in future work.

\section{EXPERIMENTAL SECTION}

General Methods. Commercially available reagents and highperformance liquid chromatography grade solvents were used as received. Buffers were prepared and filtered $(0.45 \mu \mathrm{m}$ nylon filter) before their use. Milli- $Q$ water was used in all processes. $\mathrm{L}-\alpha$-PC from egg yolk was equally used as a $100 \mathrm{mg} / \mathrm{mL}$ solution in chloroform ( $99 \%$ purity) or as a solid (60\% purity). Cholesterol was used as a 1 $\mathrm{mg} \mathrm{mL} \mathrm{m}^{-1}$ solution in chloroform. Compound $\mathbf{1}$ was synthesized as previously reported. ${ }^{51}$

Liposome Preparation. L- $\alpha$-PC (10 mg) and cholesterol (1.25 $\mathrm{mg}$ ) were mixed in a $25 \mathrm{~mL}$ round-bottom flask in a total volume of 2 $\mathrm{mL}$ chloroform (8:2 molar ratio). Then, chloroform was removed first using a rotary evaporator and then under vacuum until dryness $(5 \mathrm{~h})$. The dry lipid film was rehydrated with $2 \mathrm{~mL}$ phosphate buffer $0.1 \mathrm{M}$ $\mathrm{pH}$ 9. Then, the mixture was vigorously stirred for $30 \mathrm{~min}$ at $55^{\circ} \mathrm{C}$ and later, transferred to an $8 \mathrm{~mL}$ screw-capped glass vial and sonicated for $1 \mathrm{~h}$. The nonencapsulated materials were removed using SEC (Sepharose 4B, mobile phase phosphate buffer $\mathrm{pH} 90.1 \mathrm{M}$ ) and the final sample was filtered (nylon $0.45 \mu \mathrm{m}$ filter). Samples were kept at $4{ }^{\circ} \mathrm{C}$. When using liposomes for control purposes, these were subjected to the same protocol as the nanogel@liposomes.

Nanogel@Liposome Preparation. L- $\alpha$-PC $(10 \mathrm{mg})$ and cholesterol $(1.25 \mathrm{mg})$ were mixed in a $25 \mathrm{~mL}$ round-bottom flask in a total volume of $2 \mathrm{~mL}$ chloroform (8:2 molar ratio). Then, chloroform was removed first using a rotary evaporator and then under vacuum until dryness $(5 \mathrm{~h})$. The dry lipid film was rehydrated with $2 \mathrm{~mL}$ of a solution of $\mathbf{1}\left(10 \mathrm{mg} \mathrm{mL}^{-1}\right)$ in phosphate buffer $0.1 \mathrm{M}$ $\mathrm{pH}$ 9. Then, the mixture was vigorously stirred for $30 \mathrm{~min}$ at $55^{\circ} \mathrm{C}$ and later, transferred to an $8 \mathrm{~mL}$ screw-capped glass vial and sonicated for $1 \mathrm{~h}$. The nonencapsulated materials were removed using SEC (Sepharose 4B, mobile phase phosphate buffer $\mathrm{pH} 90.1 \mathrm{M}$ ) and the final sample was filtered (nylon $0.45 \mu \mathrm{m}$ filter). To obtain gel@ liposomes, gelation inside vesicles was promoted adding $18 \mathrm{mg} \mathrm{mL}^{-1}$ of D-glucono-1,5-lactone. After waiting overnight, dialysis against $1 \mathrm{~L}$ of water was performed to remove excess of salts. To obtain naked nanogels, vesicles were disassembled and phospholipids removed. For this purpose, SDS $\left(10 \mathrm{mg} \mathrm{mL}^{-1}\right)$ was added and, after $10 \mathrm{~min}$, dialysis against $1 \mathrm{~L}$ of water was repeated. Samples were kept at $4{ }^{\circ} \mathrm{C}$.

Pyranine Loading. Pyranine was introduced in the systems in the hydration medium ( $2 \mathrm{~mL}$ phosphate buffer $0.1 \mathrm{M} \mathrm{pH} 9$ ), using a 20 $\mu \mathrm{M}$ pyranine solution for liposomes and a $100 \mu \mathrm{M}$ for gel@liposomes. The rest of the protocol was followed as indicated above.

Pyranine Calibration Curve. A calibration curve was obtained using $1 \mu \mathrm{M}$ solutions of pyranine in $0.1 \mathrm{M}$ phosphate buffer at different values of $\mathrm{pH}$ (from 5 to 11). The fluorescence intensity at $511 \mathrm{~nm}$ was recorded after excitation at two different wavelengths (415 and $460 \mathrm{~nm}$ ), and the ratio between both measures was plotted 
against $\mathrm{pH}$ and fitted to a sigmoidal curve to obtain the calibration curve (Figure S2).

Doxorubicin Loading and Release Studies. To introduce doxorubicin in the liposomal systems, $1 \mathrm{mM}$ doxorubicin solution ( $2 \%$ dimethyl sulfoxide) was used as a hydration medium. The rest of the protocol was followed as indicated above.

Doxorubicin release from liposomes and nanogel@liposome systems was studied by dialysis. Samples $(1.5 \mathrm{~mL})$ were introduced in a dialysis membrane (Spectra/Por 3 dialysis membrane, MWCO $3.5 \mathrm{kD}$, Spectrum Labs) and were dialyzed against $40 \mathrm{~mL}$ of phosphate buffer $0.1 \mathrm{M} \mathrm{pH}$ 7. Then, $3 \mathrm{~mL}$ aliquots from the outside solution were taken at selected time intervals for spectroscopic analysis and then returned to the dialysis system. Doxorubicin concentration was calculated based on fluorescence (at $556 \mathrm{~nm}$, excitation at $480 \mathrm{~nm}$ ) or absorbance intensity (at $478 \mathrm{~nm}$ ).

Transmission Electron Microscopy. Without Staining. Transmission electron micrographs were obtained using a JEOL 2100 microscope with a thermionic gun LaB6 $200 \mathrm{kV}$ equipped with a Gatan Orius high-resolution CCD camera. TEM samples were prepared over a formvar/carbon film on 200 mesh copper grids. A drop of the sample was added on the grid and incubated for $2 \mathrm{~min}$. Then, the solvent was removed with filter paper by capillary action.

$\mathrm{OsO}_{4}$ Staining. Images were obtained using a JEOL JEM-1010 transmission electron microscope of $100 \mathrm{kV}$ equipped with an AMT RX80 digital camera ( $8 \mathrm{Mpx}$ ). TEM samples were prepared over the formvar/carbon film on 200 mesh copper grids. A drop of the sample was added on the grid and incubated for $2 \mathrm{~min}$. Then, the solvent was removed with filter paper by capillary action and a drop of $\mathrm{OsO}_{4} 0.1 \%$ was added. After $5 \mathrm{~min}$ incubation, the staining solution was removed by capillary action, and the grid was washed with a drop of Milli-Q water.

Cryo-TEM. For the cryo-TEM technique, a JEM-2200FS/CR transmission electron microscope (JEOL, Japan) equipped with an UltraScan 4000 SP $(4008 \times 4008$ pixels $)$ cooled slow-scan CCD camera (GATAN, UK) was used. A drop of the sample was placed on the TEM grid, and an automated vitrification robot Vitrobot was used to freeze the sample in liquid ethane.

Dynamic Light Scattering. Size measurements of the particles were performed by DLS using a Zetasizer Nano ZS (Malvern). Analyses were carried out using a $\mathrm{He}-\mathrm{Ne}$ laser $(633 \mathrm{~nm})$ at a fixed scattering angle of $173^{\circ}$. Automatic optimization of beam focusing and attenuation was applied for each sample. Measurements were performed at $25{ }^{\circ} \mathrm{C}$, and samples were introduced into $3 \mathrm{~mL}$ disposable PMMA cuvettes (10 mm optical path length). The particle size was reported as the average of three measurements.

Fluorescence Spectroscopy. Fluorescence measurements were carried out using a JASCO FP-8300 spectrofluorometer equipped with a Peltier accessory ETC- 815 at $25{ }^{\circ} \mathrm{C}$. The samples were placed in a $3 \mathrm{~mL}$ optical glass cuvette $(10 \mathrm{~mm}$ optical path length, Hellma Analytics).

UV-Vis Spectroscopy. UV-vis measurements were carried out in a JASCO V-630 spectrophotometer equipped with a Peltier accessory ETCS-761 at $25{ }^{\circ} \mathrm{C}$. Samples were placed in a quartz SUPRASIL cuvette (10 mm optical path length, Hellma Analytics). Data interval acquisition was set to $\Delta \lambda=1 \mathrm{~nm}$, and scan speed set at $1000 \mathrm{~nm} \mathrm{~min}{ }^{-1}$.

Gelator Quantification Using NMR. The concentration of $\mathbf{1}$ in a sample was quantified using ${ }^{1} \mathrm{H}$ NMR (Bruker AVANCE III HD spectrometer $400 \mathrm{MHz}$ ) after lyophilization and solubilization of $\mathbf{1}$ in a 6:1 mixture of $\mathrm{CDCl}_{3} /$ hexafluoroisopropanol. A calibrated signal at $\delta 9.76 \mathrm{ppm}$ (quartet) of acetaldehyde contained in a concentric tube was used for this purpose. Acetaldehyde signal was calibrated using a $5 \mathrm{mg} \mathrm{mL}{ }^{-1}$ standard of compound 1 in a $6: 1$ mixture of $\mathrm{CDCl}_{3} /$ hexafluoroisopropanol. Signals of the deuterated solvent $\left(\mathrm{CDCl}_{3}\right)$ were taken as the reference (singlet at $\delta$ 7.26).

\section{ASSOCIATED CONTENT}

\section{(5) Supporting Information}

The Supporting Information is available free of charge on the ACS Publications website at DOI: 10.1021/acs.langmuir.9b02282.

Cryo-TEM images, fluorescence data used for $\mathrm{pH}$ determination, and results in the study of doxorubicin release (PDF)

\section{AUTHOR INFORMATION}

\section{Corresponding Author}

*E-mail: miravet@uji.es (J.F.M.).

ORCID

Francisco Galindo: 0000-0003-0826-6084

Juan F. Miravet: 0000-0003-0946-3784

\section{Author Contributions}

The manuscript was written through the contributions of all authors. All authors have given approval to the final version of the manuscript.

Notes

The authors declare no competing financial interest.

\section{ACKNOWLEDGMENTS}

Ministerio de Economía y Competitividad of Spain (grant CTQ2015-71004-R) and Universitat Jaume I (grant UJIB2018-54) are thanked for financial support. A.T.-M. thanks Ministerio de Educación, Cultura y Deporte of Spain for a FPU fellowship (FPU14/05974). Technical support from SCIC of Universitat Jaume I and Dr. David Gil (CIC bioGUNE) for his assistance in cryo-TEM measurements are acknowledged.

\section{REFERENCES}

(1) Geszke-Moritz, M.; Moritz, M. Solid lipid nanoparticles as attractive drug vehicles: Composition, properties and therapeutic strategies. Mater. Sci. Eng., C 2016, 68, 982-994.

(2) Banik, B. L.; Fattahi, P.; Brown, J. L. Polymeric nanoparticles: The future of nanomedicine. Wiley Interdiscip. Rev.: Nanomed. Nanobiotechnol. 2016, 8, 271-299.

(3) Sherje, A. P.; Jadhav, M.; Dravyakar, B. R.; Kadam, D. Dendrimers: A versatile nanocarrier for drug delivery and targeting. Int. J. Pharm. 2018, 548, 707-720.

(4) Öztürk-Atar, K.; Eroğlu, H.; Çalış, S. Novel advances in targeted drug delivery. J. Drug Targeting 2018, 26, 633-642.

(5) Torchilin, V. P. Recent advances with liposomes as pharmaceutical carriers. Nat. Rev. Drug Discovery 2005, 4, 145-160.

(6) Aryasomayajula, B.; Salzano, G.; Torchilin, V. P. Multifunctional liposomes. Methods Mol. Biol. 2017, 1530, 41-61.

(7) Pattni, B. S.; Chupin, V. V.; Torchilin, V. P. New Developments in Liposomal Drug Delivery. Chem. Rev. 2015, 115, 10938-10966.

(8) Hamidi, M.; Azadi, A.; Rafiei, P. Hydrogel nanoparticles in drug delivery. Adv. Drug Delivery Rev. 2008, 60, 1638-1649.

(9) Oh, J. K.; Drumright, R.; Siegwart, D. J.; Matyjaszewski, K. The development of microgels/nanogels for drug delivery applications. Prog. Polym. Sci. 2008, 33, 448-477.

(10) Kabanov, A. V.; Vinogradov, S. V. Nanogels as pharmaceutical carriers: Finite networks of infinite capabilities. Angew. Chem., Int. Ed. 2009, 48, 5418-5429.

(11) Chacko, R. T.; Ventura, J.; Zhuang, J.; Thayumanavan, S. Polymer nanogels: A versatile nanoscopic drug delivery platform. Adv. Drug Delivery Rev. 2012, 64, 836-851.

(12) Soni, K. S.; Desale, S. S.; Bronich, T. K. Nanogels: An overview of properties, biomedical applications and obstacles to clinical translation. J. Control. Release 2016, 240, 109-126. 
(13) Neamtu, I.; Rusu, A. G.; Diaconu, A.; Nita, L. E.; Chiriac, A. P. Basic concepts and recent advances in nanogels as carriers for medical applications. Drug Delivery 2017, 24, 539-557.

(14) Hadinoto, K.; Sundaresan, A.; Cheow, W. S. Lipid-polymer hybrid nanoparticles as a new generation therapeutic delivery platform: A review. Eur. J. Pharm. Biopharm. 2013, 85, 427-443.

(15) Mandal, B.; Bhattacharjee, H.; Mittal, N.; Sah, H.; Balabathula, P.; Thoma, L. A.; Wood, G. C. Core-shell-type lipid-polymer hybrid nanoparticles as a drug delivery platform. Nanomed. Nanotechnol. Biol. Med. 2013, 9, 474-491.

(16) Tan, S.; Li, X.; Guo, Y.; Zhang, Z. Lipid-enveloped hybrid nanoparticles for drug delivery. Nanoscale 2013, 5, 860-872.

(17) Raemdonck, K.; Braeckmans, K.; Demeester, J.; De Smedt, S. C. Merging the best of both worlds: hybrid lipid-enveloped matrix nanocomposites in drug delivery. Chem. Soc. Rev. 2014, 43, 444-472.

(18) Kazakov, S. Liposome-Nanogel Structures for Future Pharmaceutical Applications: An Updated Review. Curr. Pharm. Des. 2016, 22, 1391-1413.

(19) Jin, T.; Pennefather, P.; Lee, P. I. Lipobeads: a hydrogen anchored lipid vesicle system. FEBS Lett. 1996, 397, 70-74.

(20) Tiwari, S.; Goyal, A. K.; Mishra, N.; Khatri, K.; Vaidya, B.; Mehta, A.; Wu, Y.; Vyas, S. P. Development and characterization of novel carrier gel core liposomes based transmission blocking malaria vaccine. J. Control. Release 2009, 140, 157-165.

(21) Homyak, C. C.; Fernandez, A.; Touve, M. A.; Zhao, B.; Anson, F.; Hardy, J. A.; Vachet, R. W.; Gianneschi, N. C.; Ross, J. L.; Thayumanavan, S. Lipogels for Encapsulation of Hydrophilic Proteins and Hydrophobic Small Molecules. Biomacromolecules 2018, 19, 132140.

(22) Ma, J.; Deng, H.; Zhao, F.; Deng, L.; Wang, W.; Dong, A.; Zhang, J. Liposomes-Camouflaged Redox-Responsive Nanogels to Resolve the Dilemma between Extracellular Stability and Intracellular Drug Release. Macromol. Biosci. 2018, 18, 1800049.

(23) Torchilin, V. P.; Klibanov, A. L.; Ivanov, N. N.; Ringsdorf, H.; Schlarb, B. Polymerization of liposome-encapsulated hydrophilic monomers. Macromol. Rapid Commun. 1987, 8, 457-460.

(24) Kazakov, S.; Kaholek, M.; Teraoka, I.; Levon, K. UV-induced gelation on nanometer scale using liposome reactor. Macromolecules 2002, 35, 1911-1920.

(25) Kazakov, S.; Kaholek, M.; Kudasheva, D.; Teraoka, I.; Cowman, M. K.; Levon, K. Poly(N-isopropylacrylamide-co-1-vinylimidazole) Hydrogel Nanoparticles Prepared and Hydrophobically Modified in Liposome Reactors: Atomic Force Microscopy and Dynamic Light Scattering Study. Langmuir 2003, 19, 8086-8093.

(26) Patton, J. N.; Palmer, A. F. Photopolymerization of Bovine Hemoglobin Entrapped Nanoscale Hydrogel Particles within Liposomal Reactors for Use as an Artificial Blood Substitute. Biomacromolecules 2005, 6, 414-424.

(27) Patton, J. N.; Palmer, A. F. Engineering Temperature-Sensitive Hydrogel Nanoparticles Entrapping Hemoglobin as a Novel Type of Oxygen Carrier. Biomacromolecules 2005, 6, 2204-2212.

(28) Van Thienen, T. G.; Lucas, B.; Flesch, F. M.; van Nostrum, C. F.; Demeester, J.; De Smedt, S. C. On the Synthesis and Characterization of Biodegradable Dextran Nanogels with Tunable Degradation Properties. Macromolecules 2005, 38, 8503-8511.

(29) Van Thienen, T. G.; Raemdonck, K.; Demeester, J.; De Smedt, S. C. Protein Release from Biodegradable Dextran Nanogels. Langmuir 2007, 23, 9794-9801.

(30) An, S. Y.; Bui, M.-P. N.; Nam, Y. J.; Han, K. N.; Li, C. A.; Choo, J.; Lee, E. K.; Katoh, S.; Kumada, Y.; Seong, G. H. Preparation of monodisperse and size-controlled poly(ethylene glycol) hydrogel nanoparticles using liposome templates. J. Colloid Interface Sci. 2009, 331, 98-103.

(31) Park, J.; Wrzesinski, S. H.; Stern, E.; Look, M.; Criscione, J.; Ragheb, R.; Jay, S. M.; Demento, S. L.; Agawu, A.; Licona Limon, P.; Ferrandino, A. F.; Gonzalez, D.; Habermann, A.; Flavell, R. A.; Fahmy, T. M. Combination delivery of TGF- $\beta$ inhibitor and IL-2 by nanoscale liposomal polymeric gels enhances tumour immunotherapy. Nat. Mater. 2012, 11, 895-905.
(32) Petralito, S.; Spera, R.; Pacelli, S.; Relucenti, M.; Familiari, G.; Vitalone, A.; Paolicelli, P.; Casadei, M. A. Design and development of PEG-DMA gel-in-liposomes as a new tool for drug delivery. React. Funct. Polym. 2014, 77, 30-38.

(33) Wang, Y.; Tu, S.; Pinchuk, A. N.; Xiong, M. P. Active drug encapsulation and release kinetics from hydrogel-in-liposome nanoparticles. J. Colloid Interface Sci. 2013, 406, 247-255.

(34) Lockhart, J. N.; Beezer, D. B.; Stevens, D. M.; Spears, B. R.; Harth, E. One-pot polyglycidol nanogels via liposome master templates for dual drug delivery. J. Control. Release 2016, 244, 366374.

(35) Zhao, X.; Deng, L.; Deng, H.; Dong, A.; Wang, W.; Zhang, J. In Situ Template Polymerization to Prepare Liposome-Coated PDMAEMA Nanogels with Controlled Size, High Stability, Low Cytotoxicity, and Responsive Drug Release for Intracellular DOX Release. Macromol. Chem. Phys. 2018, 219, 1800071.

(36) Monshipouri, M.; Rudolph, A. S. Liposome-encapsulated alginate: controlled hydrogel particle formation and release. $J$. Microencapsulation 1995, 12, 117-127.

(37) Rudolph, B.; Chen, J.; Lu, Y.; Qi, J.; Wu, W. Liposomes interiorly thickened with thermosensitive nanogels as novel drug delivery systems. Int. J. Pharm. 2013, 455, 276-284.

(38) Hong, J. S.; Stavis, S. M.; DePaoli Lacerda, S. H.; Locascio, L. E.; Raghavan, S. R.; Gaitan, M. Microfluidic Directed Self-Assembly of Liposome-Hydrogel Hybrid Nanoparticles. Langmuir 2010, 26, 11581-11588.

(39) Kiser, P. F.; Wilson, G.; Needham, D. A synthetic mimic of the secretory granule for drug delivery. Nature 1998, 394, 459-462.

(40) Terech, P.; Weiss, R. G. Low molecular mass gelators of organic liquids and the properties of their gels. Chem. Rev. 1997, 97, 31333160.

(41) Estroff, L. A.; Hamilton, A. D. Water gelation by small organic molecules. Chem. Rev. 2004, 104, 1201-1218.

(42) Hirst, A. R.; Escuder, B.; Miravet, J. F.; Smith, D. K. High-tech applications of self-assembling supramolecular nanostructured gelphase materials: From regenerative medicine to electronic devices. Angew. Chem., Int. Ed. 2008, 47, 8002-8018.

(43) Banerjee, S.; Das, R. K.; Maitra, U. Supramolecular gels 'in action'. J. Mater. Chem. 2009, 19, 6649-6687.

(44) Steed, J. W. Supramolecular gel chemistry: Developments over the last decade. Chem. Commun. 2011, 47, 1379-1383.

(45) Du, X.; Zhou, J.; Shi, J.; Xu, B. Supramolecular Hydrogelators and Hydrogels: From Soft Matter to Molecular Biomaterials. Chem. Rev. 2015, 115, 13165-13307.

(46) Draper, E. R.; Adams, D. J. Low-Molecular-Weight Gels: The State of the Art. Chem 2017, 3, 390-410.

(47) Segarra-Maset, M. D.; Nebot, V. J.; Miravet, J. F.; Escuder, B. Control of molecular gelation by chemical stimuli. Chem. Soc. Rev. 2013, 42, 7086-7098.

(48) Boekhoven, J.; Brizard, A. M.; Stuart, M. C. A.; Florusse, L.; Raffy, G.; Del Guerzo, A.; van Esch, J. H. Bio-inspired supramolecular materials by orthogonal self-assembly of hydrogelators and phospholipids. Chem. Sci. 2016, 7, 6021-6031.

(49) Felip-León, C.; Galindo, F.; Miravet, J. F. Insights into the aggregation-induced emission of 1,8-naphthalimide-based supramolecular hydrogels. Nanoscale 2018, 10, 17060.

(50) Felip-León, C.; Cejudo-Marín, R.; Peris, M.; Galindo, F.; Miravet, J. F. Sizing Down a Supramolecular Gel into Micro- and Nanoparticles. Langmuir 2017, 33, 10322-10328.

(51) Torres-Martínez, A.; Angulo-Pachón, C. A.; Galindo, F.; Miravet, J. F. In between molecules and self-assembled fibrillar networks: highly stable nanogel particles from a low molecular weight hydrogelator. Soft Matter 2019, 15, 3565-3572.

(52) McIntosh, T. J. The effect of cholesterol on the structure of phosphatidylcholine bilayers. Biochim. Biophys. Acta 1978, 513, 4358.

(53) Patil, Y. P.; Jadhav, S. Novel methods for liposome preparation. Chem. Phys. Lipids 2014, 177, 8-18. 
(54) Akbarzadeh, A.; Rezaei-Sadabady, R.; Davaran, S.; Joo, S. W.; Zarghami, N.; Hanifehpour, Y.; Samiei, M.; Kouhi, M.; Nejati-Koshki, K. Liposome: Classification, preparation, and applications. Nanoscale Res. Lett. 2013, 8, 102.

(55) Adams, D. J.; Butler, M. F.; Frith, W. J.; Kirkland, M.; Mullen, L.; Sanderson, P. A new method for maintaining homogeneity during liquid-hydrogel transitions using low molecular weight hydrogelators. Soft Matter 2009, 5, 1856-1862.

(56) Avnir, Y.; Barenholz, Y. pH determination by pyranine: Medium-related artifacts and their correction. Anal. Biochem. 2005, 347, 34-41.

(57) Clement, N. R.; Gould, J. M. Pyranine (8-hydroxy-1,3,6pyrenetrisulfonate) as a probe of internal aqueous hydrogen ion concentration in phospholipid vesicles. Biochemistry 1981, 20, 15341538.

(58) Kano, K.; Fendler, J. H. Pyranine as a sensitive $\mathrm{pH}$ probe for liposome interiors and surfaces. $\mathrm{pH}$ gradients across phospholipid vesicles. Biochim. Biophys. Acta, Biomembr. 1978, 509, 289-299.

(59) Chen, Y.; Qiao, F.; Fan, Y.; Han, Y.; Wang, Y. Interactions of Phospholipid Vesicles with Cationic and Anionic Oligomeric Surfactants. J. Phys. Chem. B 2017, 121, 7122-7132.

(60) Nebot, V. J.; Escuder, B.; Miravet, J. F.; Smets, J.; FernándezPrieto, S. Interplay of molecular hydrogelators and SDS affords responsive soft matter systems with tunable properties. Langmuir 2013, 29, 9544-9550.

(61) Sun, J.; Wei, Q.; Zhou, Y.; Wang, J.; Liu, Q.; Xu, H. A systematic analysis of FDA-approved anticancer drugs. BMC Syst. Biol. 2017, 11, 87.

(62) Kanwal, U.; Irfan Bukhari, N.; Ovais, M.; Abass, N.; Hussain, K.; Raza, A. Advances in nano-delivery systems for doxorubicin: an updated insight. J. Drug Targeting 2018, 26, 296-310.

(63) Sun, H.; Guo, B.; Cheng, R.; Meng, F.; Liu, H.; Zhong, Z. Biodegradable micelles with sheddable poly(ethylene glycol) shells for triggered intracellular release of doxorubicin. Biomaterials 2009, 30, 6358-6366.

(64) Shuai, X.; Ai, H.; Nasongkla, N.; Kim, S.; Gao, J. Micellar carriers based on block copolymers of poly ( $\varepsilon$-caprolactone $)$ and poly(ethylene glycol) for doxorubicin delivery. J. Control. Release 2004, 98, 415-426.

(65) Fugit, K. D.; Xiang, T.-X.; Choi, D. H.; Kangarlou, S.; Csuhai, E.; Bummer, P. M.; Anderson, B. D. Mechanistic model and analysis of doxorubicin release from liposomal formulations. J. Control. Release 2015, 217, 82-91. 


\section{SUPPORTING INFORMATION}

\section{Liposome-enveloped molecular nanogels}

Ana Torres-Martínez, César A. Angulo-Pachón Francisco Galindo, and Juan F. Miravet*

CONTENTS (3 pages including this one):

Figure S1. Cryo-TEM images, page S2

Figure S2. Pyranine $\mathrm{pH}$ calibration, page S2

Figure S3. Normalized fluorescence excitation spectra, page S2

Figure S4. Doxorubicin release, page S3

Figure S5. DLS study of temporal stability, page S3

Figure S6. Size distributions from electron microscopy images, page S3 


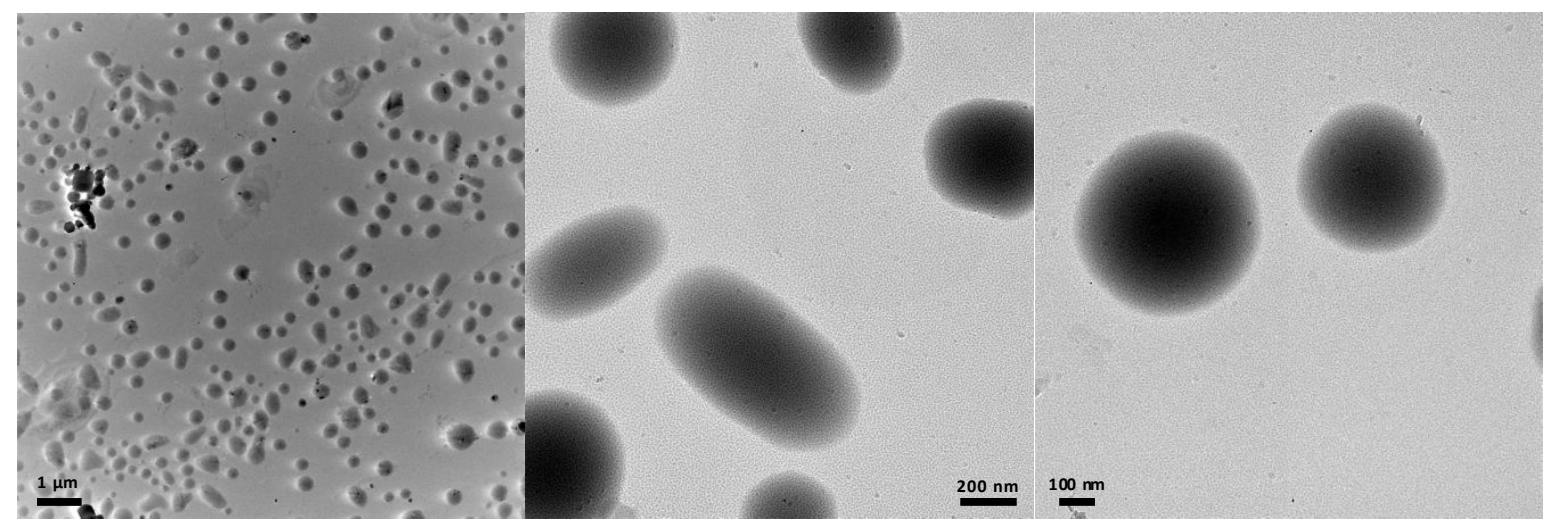

Figure S1. Cryo-TEM images of gel@liposome particles.

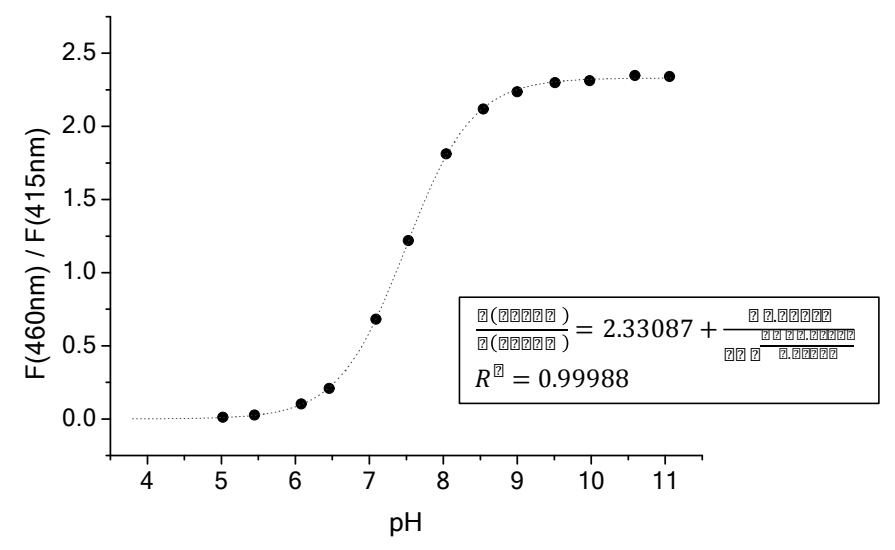

Figure S2. Pyranine pH calibration curve using $1 \mu \mathrm{M}$ solutions in phosphate buffer $0.1 \mathrm{M}$ at pH 5-11.

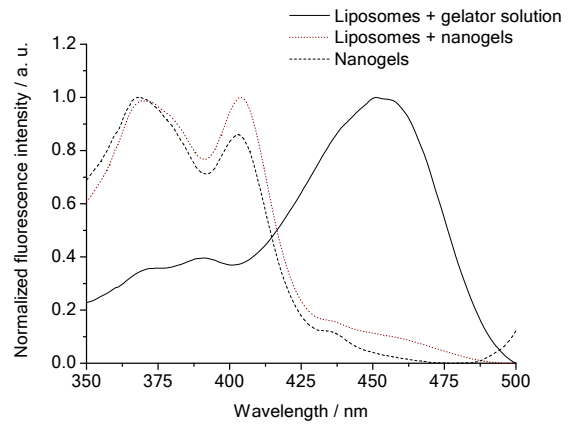

Figure S3. Normalized fluorescence excitation spectra $\left(\lambda_{\mathrm{em}}=511 \mathrm{~nm}\right)$ of a gelator@liposomes sample (solid line, pH 8.2), gel@liposome particles (red dotted line, pH 6.5) and nanogel particles (black dotted line, $\mathrm{pH}$ 6.4). 


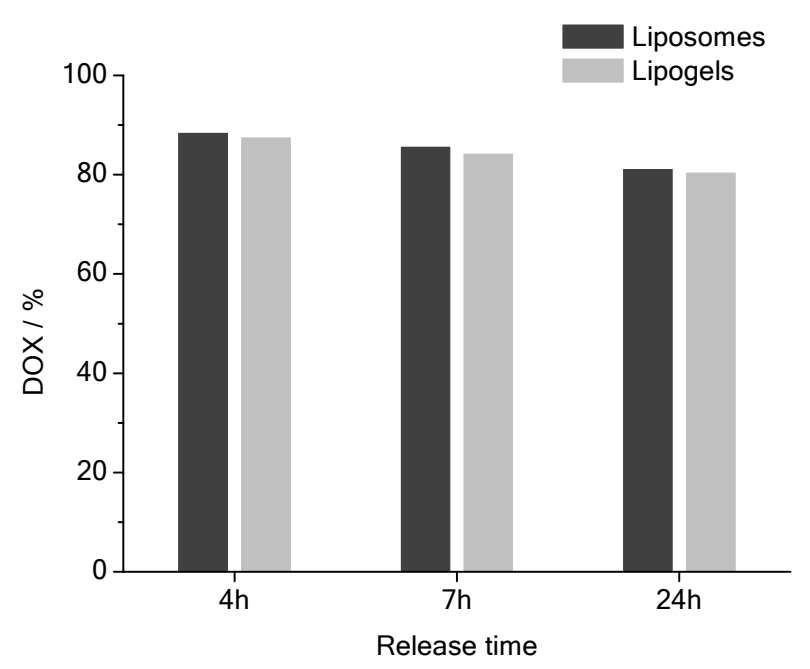

Figure S4. Percentage of doxorubicin kept in the dialysis membrane after the indicated times of dialysis for doxorubicin@liposomes and doxorubicin@lipogels.

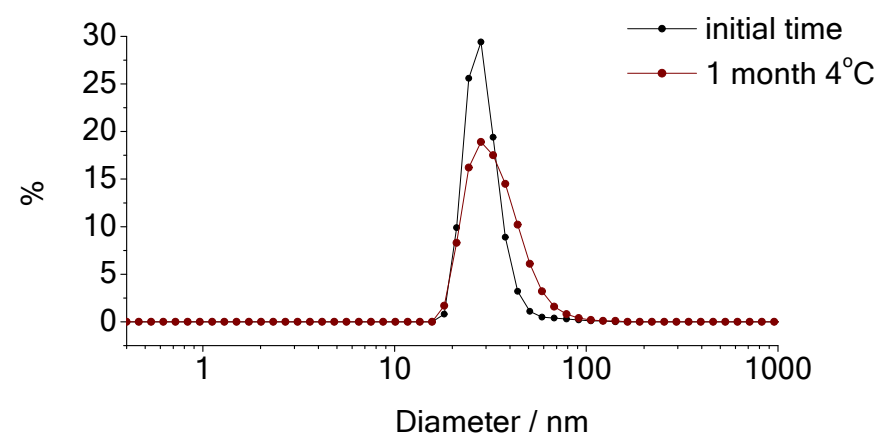

Figure S5. Number averaged size distribution of a representative sample of gel@liposome particles obtained by DLS analysis: immediately after preparation (black) and one month later kept at $4{ }^{\circ} \mathrm{C}$ (red line).
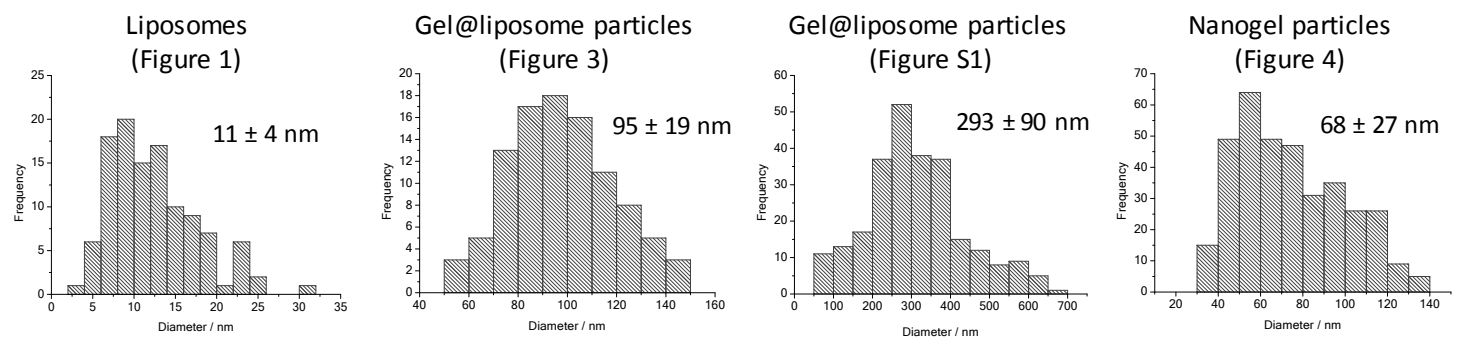

Figure S6. Size distribution of the particles obtained from TEM and cryo-TEM images determined with ImageJ software. The selected micrographs can be seen in Figures 1, 3, 4 and S1. Mean particle size indicated for each histogram. 